\title{
Suberin-Associated Fatty Acyl-CoA Reductases from Arabidopsis thaliana
}

By

\section{Sollapura J. Vishwanath}

A thesis submitted to the Faculty of Graduate and Postdoctoral Affairs in partial fulfillment of the requirements for the degree of

\section{Doctor of Philosophy \\ in}

Biology

\author{
Department of Biology \\ Carleton University \\ Ottawa, Ontario, Canada
}

C)2013, Sollapura J. Vishwanath 
Library and Archives

Canada

Published Heritage

Branch

395 Wellington Street

Ottawa ON K1A ON4

Canada
Bibliothèque et

Archives Canada

Direction du

Patrimoine de l'édition

395 , rue Wellington

Ottawa ON K1A ON4

Canada
Your file Votre référence

ISBN: 978-0-494-94538-4

Our file Notre référence

ISBN: $978-0-494-94538-4$
NOTICE:

The author has granted a nonexclusive license allowing Library and Archives Canada to reproduce, publish, archive, preserve, conserve, communicate to the public by telecommunication or on the Internet, loan, distrbute and sell theses worldwide, for commercial or noncommercial purposes, in microform, paper, electronic and/or any other formats.

The author retains copyright ownership and moral rights in this thesis. Neither the thesis nor substantial extracts from it may be printed or otherwise reproduced without the author's permission.
AVIS:

L'auteur a accordé une licence non exclusive permettant à la Bibliothèque et Archives Canada de reproduire, publier, archiver, sauvegarder, conserver, transmettre au public par télécommunication ou par l'Internet, prêter, distribuer et vendre des thèses partout dans le monde, à des fins commerciales ou autres, sur support microforme, papier, électronique et/ou autres formats.

L'auteur conserve la propriété du droit d'auteur et des droits moraux qui protege cette thèse. $\mathrm{Ni}$ la thèse ni des extraits substantiels de celle-ci ne doivent être imprimés ou autrement reproduits sans son autorisation.
In compliance with the Canadian Privacy Act some supporting forms may have been removed from this thesis.

While these forms may be included in the document page count, their removal does not represent any loss of content from the thesis.
Conformément à la loi canadienne sur la protection de la vie privée, quelques formulaires secondaires ont été enlevés de cette thèse.

Bien que ces formulaires aient inclus dans la pagination, il n'y aura aucun contenu manquant. 


\section{ABSTRACT}

Suberin is a biopolymer deposited in the cell walls of specific internal and external tissues layers of plants, such as root endodermis, root and tuber peridermis, and seed coats. Suberin serves as a hydrophobic barrier controlling water and ion transport in these tissue layers. It is also a wound-induced antimicrobial barrier. The suberin polymer consists of phenolics, glycerol, and a variety of fatty acid derivatives, including C18:0-C22:0 primary fatty alcohols. In this thesis, I report on the identification and characterization of fatty acyl reductase (FAR) enzymes that synthesize primary alcohols associated with suberin. Three genes encoding alcohol-forming fatty acyl reductases (FARs) from the model plant Arabidopsis thaliana, FAR1, FAR4 and FAR5, were found to be expressed at sites of suberin deposition. Suberin composition in loss-of-function far mutants was uniquely modified; specifically, $\mathrm{C} 18: 0-\mathrm{OH}$ was reduced in far5, C20:0-OH was reduced in far4, and $\mathrm{C} 22: 0-\mathrm{OH}$ was reduced in far1, indicating that FAR1, FAR4, and FAR5 each generates a distinct chain-length of fatty alcohol found in root, seed coat, and wound-induced suberin. Heterologous expression of FAR1, FAR4, and FAR5 in yeast and Nicotiana benthamiana leaves confirmed their preferred substrate specificity to C22:0, C20:0, and C18:0 acyl chains, respectively. Double and triple far mutant lines were developed using conventional genetic crossing and artificial microRNA (amiRNA)-mediated gene silencing. The double mutants far1 far4, far1 far5, and far4 far $5^{*}$ (*denotes amiRNA line) had reduced levels of both respective chain lengths of fatty alcohols in root and seed coat suberin, and the triple mutant far1 far 4 far $5^{*}$ had reduced levels of all three C18:0 to C22:0 primary fatty alcohols. 
The C18:0-C22:0 alkyl hydroxycinnamates of suberin-associated root waxes were also significantly reduced in these lines. The triple mutant seeds were found to have increased seed coat permeability and increased sensitivity to abscisic acid (ABA) hormone during germination. Domain swaps of the FAR1, FAR4, and FAR5 proteins and their heterologous expression in yeast showed that the substrate specificities of these enzymes are largely governed by the region between amino acid positions 225-385. Transient expression of a fusion of Arabidopsis FAR1 with green fluorescent protein in Nicotiana benthamiana leaves revealed localization to the endoplasmic reticulum. FAR1, FAR4, FAR5 and other suberin biosynthetic genes showed differential inductions at the transcript level when subjected to various abiotic stresses (ABA, cold, salt, osmotic, and wounding). All the genes were highly induced by abscisic acid, while they showed the least amount of induction by cold stress. Altogether, the results reported in this thesis have advanced our understanding of the suberin biosynthetic pathway and its regulated deposition. This knowledge may lead to novel approaches for the development of drought, salt, and pathogen resistant crop plants. Furthermore, the engineering of FAR enzymes with desired substrate specificities would enable the production of high-value fatty alcohols or wax esters in an industrial oilseed crop or engineered microbe. 


\section{PREFACE}

This thesis follows the integrated thesis format and, as such, the main chapters represent work that has been published in a peer-reviewed journal at the time of submission of this thesis (Chapter 2), or will soon be submitted for publication. I formally acknowledge the contributions of the co-authors of the manuscript that comprise the research chapters of my thesis. My supervisor, Dr. 0 . Rowland contributed his expertise by guiding me in the formulation of the research questions and experimental design, and by assisting with the analysis and interpretation of data, and the preparation of manuscripts for each of the research chapters of my thesis.

Chapter 2 is part of a published research paper:

Domergue, F.*, Vishwanath, S.J.*, Joubès, J., Ono, J., Lee, J., Bourdon, M., Alhattab, R., Lowe, C., Pascal, S., Lessire, R., and Rowland, O. (2010). Three Arabidopsis Fatty AcylCoA Reductases, FAR1, FAR4, and FAR5, Generate Primary Fatty Alcohols Associated with Suberin Deposition. Plant Physiology, 153: 1539-1554. (*These authors contributed equally to this work).

All data including figures and tables are extracted from the above paper and reprinted with permission of the American Society of Plant Biologists.

\section{Statement of contribution:}

The experiments reported in the Results section of this chapter were conceived and designed by me, F. Domergue, and 0 . Rowland. The manuscript was 
written by me, F. Domergue, and O. Rowland, and then updated and modified for thesis format by me.

I generated the following data reported in this chapter:

- FAR promoter: GUS expression analysis of various tissues under normal and wound stress conditions, and root sectioning presented in Figure 2.3.

- Semi-quantitative RT-PCR analysis of FAR1, FAR4 and FAR5 gene induction under wound and salt stress conditions presented in Figure 2.4.

- Confirmation of T-DNA single mutant lines by semi-quantitative RT-PCR and identification of T-DNA insertion sites by DNA sequencing analysis presented in Figure 2.5.

- Analysis of fatty acyl chain profiles of yeast heterolgously expressing FAR1, FAR4 and FAR5 by GC-FID, presented in Figure 2.10.

The other authors' contributions were:

- J. Ono and J. Lee were Carleton University undergraduate students who first identified the T-DNA far mutant lines, which were subsequently characterized by me.

- J. Ono, J. Lee, and C. Lowe were Carleton University undergraduate students who generated the FAR promoter:GUS constructs and transformed Arabidopsis plants, and then the reported GUS assay and microscopic analyses were performed by me.

- R. Alhattab, a Carleton University undergraduate student, cloned the FAR4 cDNA and generated the FAR4 yeast expression construct, along with preliminary experimental data on fatty alcohol production in yeast. 
- F. Domergue, J. Joubès, M. Bourdon, and S. Pascal carried out polyester depolymerizations, method development, and GC-MS analysis at Laboratoire de Biogenèse Membranaire, CNRS, University of Bordeaux, France with plant materials provided by me.

- R. Lessire is the head of the Laboratoire de Biogenèse Membranaire, CNRS, University of Bordeaux and raised the funds, in part, to carry out the experiments and provided intellectual input.

- Dr. 0. Rowland raised the funds, in part, to carry out the experiments and provided intellectual input.

\section{Chapter 3}

\section{Statement of contribution:}

- I generated the far 1 far 4 and far 1 far 5 double mutants by conventional genetic crossing, and far 4 far $5 *$ ami double and far 1 far 4 far $5 *$ ami triple mutant lines by artificial microRNA gene silencing technique.

- I performed the delipidations, lipid profiling and data analysis of root and seed coat suberin polyesters of the far double and triple mutants.

- I performed the data collection and analysis of the phenotypic experiments and the tetrazolium (Formazan) assay with far double and triple mutants.

- Dr. Frederic Domergue (CNRS, University of Bordeaux) performed the lipid profiling and data analysis of soluble and polyester bound fractions of ColWT roots. 
- Dr. Dylan Kosma (Michigan State University) performed the lipid profiling and data analysis of the root waxes (AHCs) by GC-MS from the far double and triple mutants that I provided.

- The manuscript (thesis chapter) was written by me under the guidance of Dr. 0. Rowland.

- Dr. 0. Rowland raised the funds, in part, to carry out the experiments and provided intellectual input.

\section{Chapter 4}

\section{Statement of contribution:}

- All experiments and data reported in the results section of this chapter were designed, performed and analyzed by me.

- F. Tran, a Carleton University undergraduate student, cloned FAR5 into pYES2-His6x/T7 yeast expression vector.

- A. Fournier, a Carleton University graduate student, cloned FAR4 into pYES2His6x/T7 yeast expression vector.

- The manuscript (thesis chapter) was written by me under the guidance of $\mathrm{Dr}$. 0. Rowland.

- Dr. O. Rowland raised the funds, in part, to carry out the experiments and provided intellectual input. 


\section{Chapter 5}

\section{Statement of contribution:}

- All experiments and data reported in the results section of this chapter were designed, performed and analyzed by me.

- The manuscript (thesis chapter) was written by me under the guidance of Dr. O. Rowland.

- Dr. O. Rowland raised the funds, in part, to carry out the experiments and provided intellectual input.

I have obtained copyright permission from each co-author to reproduce published manuscripts and to use collaborative works in this thesis. Work that has been published in Plant Physiology® (Chapter 2) has been cited with "Copyright American Society of Plant Biologists" notice www.plantphysiol.org (http://my.aspb.org/?P Permission) according to the publisher grant. 


\section{ACKNOWLEDGEMENTS}

There are a number of people without whom this dissertation might not have been written, and to whom I am greatly indebted. First and foremost, I would like to express my sincere gratitude to my advisor and mentor, Dr. Owen Rowland, for giving me an opportunity to pursue my $\mathrm{PhD}$ studies under his expert guidance. I am deeply grateful to him for his unconditional support, encouragement and patience for the last six years. Discussions with him during my PhD studies have taught me how to think critically like a scientist and have led to my professional development as a researcher. I would also like to thank my committee members, Dr. Susan Aitken and Dr. Linda Bonen, for their valuable comments and advice on my research project throughout the course of this work.

The accomplishment of this project is a result of collaborative efforts with many people. I would like to thank Dr. Frédéric Domergue (Centre National de la Recherche Scientifique, Laboratoire de Biogenèse Membranaire, Université Victor Ségalen Bordeaux, Bordeaux, France), for sharing his scientific expertise with me, providing training and resources, helping in the manuscript preparation, and for coauthoring my first paper. I appreciate his help in the GC-MS analysis of the many samples that I have sent him. I would like to thank Dr. Dylan Kosma (Michigan State University, USA) for his help in the analysis of root waxes. I am grateful to Dr. Shelley Hepworth for training and helping me with the stereomicroscope for the imaging work. I would like to thank Dr. Ann F. Yang (Agriculture and Agri-Food Canada, Ottawa) for training me and letting me use the microtome facility, and Dr. 
Denise Chabot (Agriculture and Agri-Food Canada, Ottawa) for her help with the confocal microscopy.

I would like to thank my colleague and fellow graduate student, Ian Pulsifer, for sharing his technical expertise with me and for all his help with the GC machine. His sense of humor made my lab life a lot more fun. I would also like to thank all my other labmates and friends from both the Rowland and Hepworth labs, both present and past members, especially: Christine Lowe, Micaela Chacon, Ashley Fournier, Fakhria M. Razeq, Dr. Jhadeswar Murmu, Dr. Mingli Xu, Madiha Khan, and Emily Giroux for providing their input and valuable assistance in my work. I would also like to thank the administrative staff of the Biology Department, Laura Thomas, Lisa Chiarelli, Caitlyn McKenzie, Jane McArthur, and Marija Gojmerac, who were always helpful with administrative tasks.

I wish to thank all my friends, near and far, for the great times together and for their help in relieving work stress. Thanks in particular to Dr. Pratik Lodha and Vaishali Jain for all the help and support they gave me from the day I landed in Canada. Their company made my life in Ottawa more enjoyable and memorable. I am most grateful to my parents for their unconditional love, hard work, sacrifices, and prayers for my success. I express my sincere gratitude to my dear wife, Preethi Subramanyam, for all her love, understanding, patience and support throughout the duration of my PhD studies. Thanks for being such an awesome person in my life.

I would like to thank the Carleton Department of Biology and Faculty of Graduate and Postgraduate Affairs for providing the financial support through graduate scholarships and endowment awards. The project was funded by the 
Natural Sciences and Engineering Research Council of Canada, the Embassy of France in Canada (France-Canada Research Fund), and by an European Commission (EC) Framework 7 project entitled 'Industrial crops producing added value oils for novel chemicals' (ICON). Finally, I would like to acknowledge Plant Physiology journal for their permission for reproducing previously copyrighted material in Chapter 2. 


\section{TABLE OF CONTENTS}

ABSTRACT

1 CHAPTER 1: GENERAL INTRODUCTION AND LITERATURE REVIEW ...................................

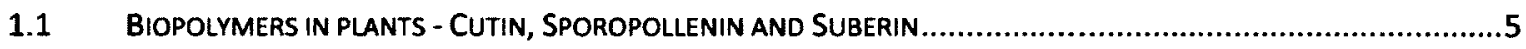

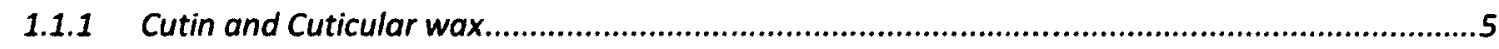

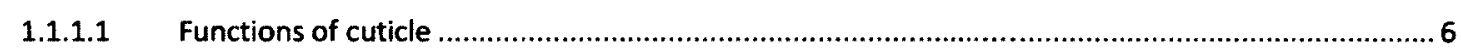

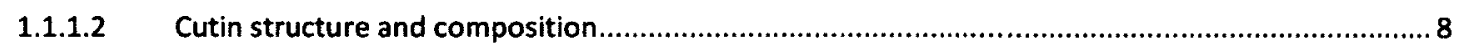

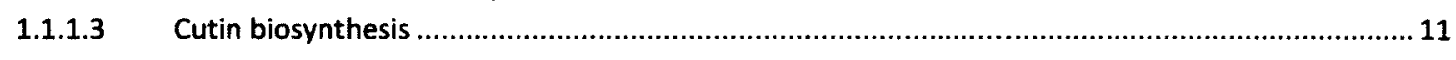

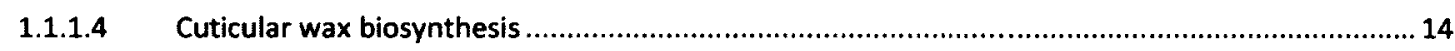

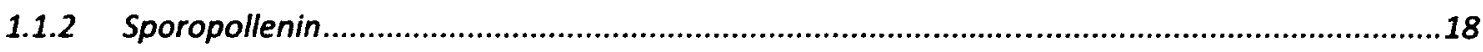

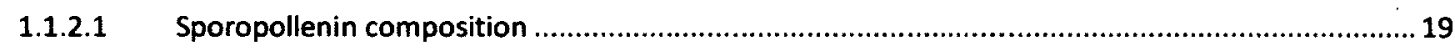

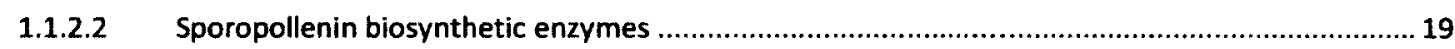

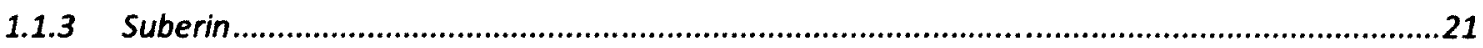

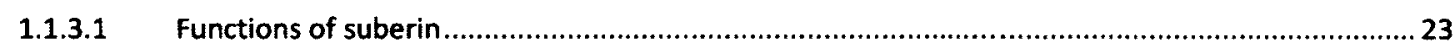

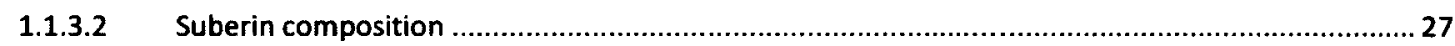

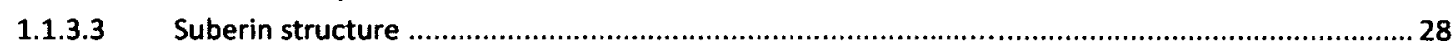

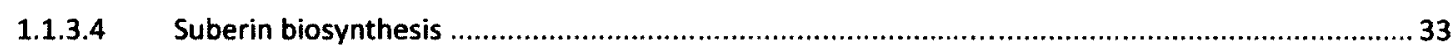

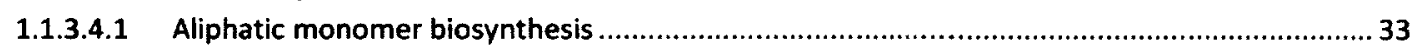

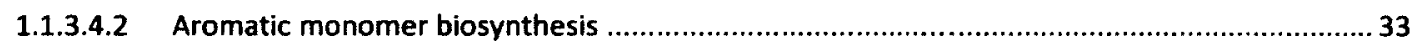

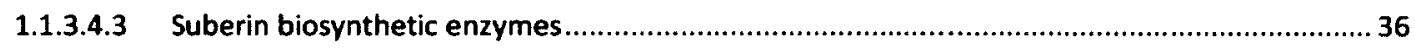

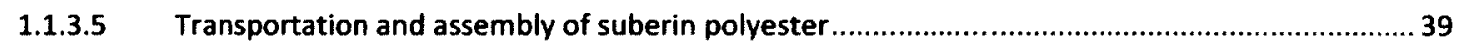

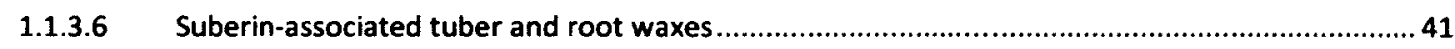

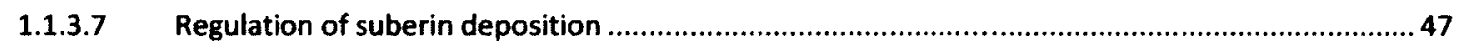

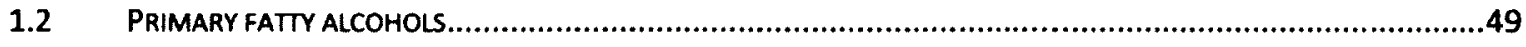

1.2.1 Fatty alcohols- free and combined forms ..............................................................49

1.2.2 Fatty alcohols in plants: biosynthesis and functions...................................................51

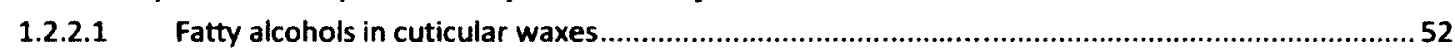

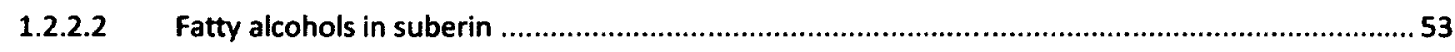

1.2.2.3 Fatty alcohols in suberin-associated alkyl hydroxycinnamates ..............................................5.5

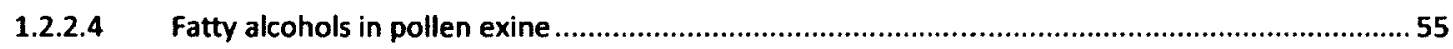

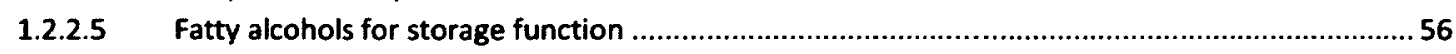

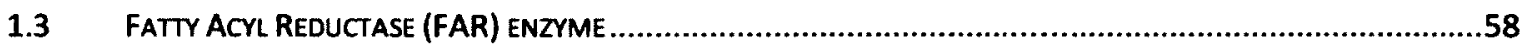

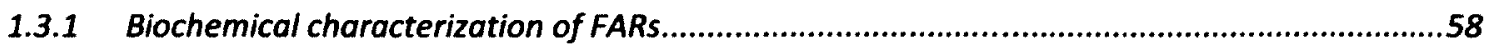




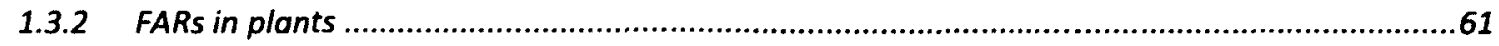

1.3.3 Substrate specificities of plant FAR enzymes..........................................................66

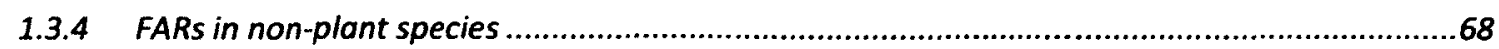

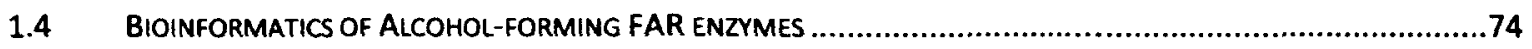

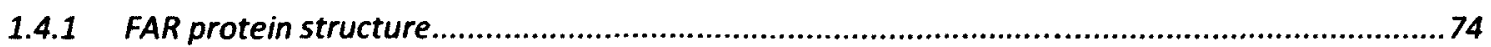

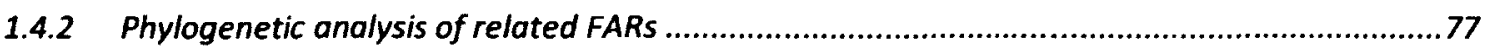

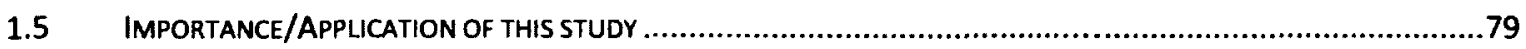

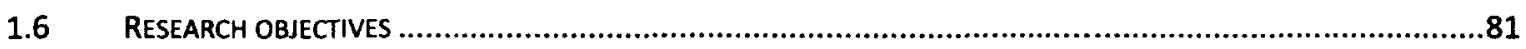

2 CHAPTER 2: THREE ARABIDOPSIS FATTY ACYL-COENZYME A REDUCTASES, FAR1, FAR4, AND FAR5, GENERATE PRIMARY FATTY ALCOHOLS ASSOCIATED WITH SUBERIN DEPOSITION. 83

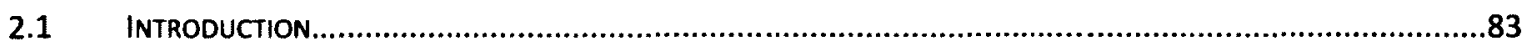

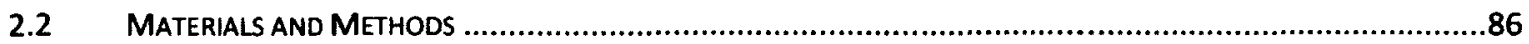

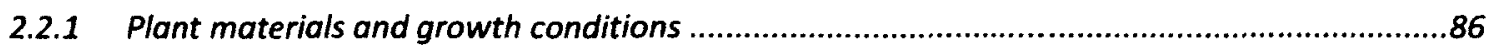

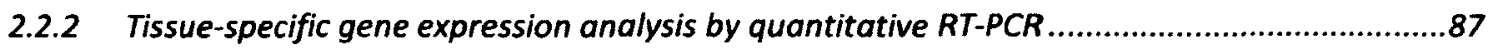

2.2.3 Preparation of constructs with promoter-glucuronidase (GUS) fusions ...............................88

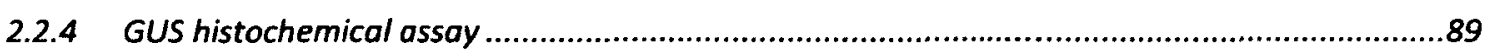

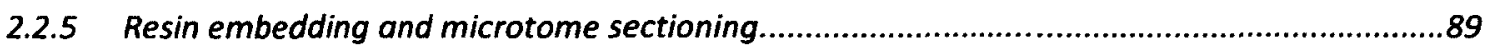

2.2.6 RNA extraction and cDNA synthesis ....................................................................90

2.2.7 Gene expression analysis by RT-PCR after wounding and salt treatment ..........................91

2.2.8 Characterization of FAR activity in Saccharomyces cerevisiae ............................................92

2.2.9 Characterization of T-DNA insertion lines.................................................................94

2.2.10 Analysis of lipid polyester composition (Suberin and Cutin) .......................................95

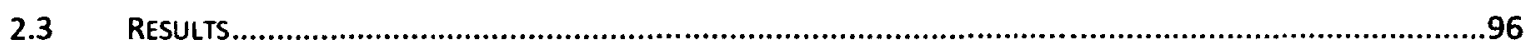

2.3.1 FAR1, FAR4, and FAR5 gene expression is associated with suberin deposition.....................96

2.3.2 Isolation of single mutants disrupted in the FAR1, FAR4, and FAR5 genes .......................100

2.3.3 Alterations of fatty alcohol composition associated with suberin isolated from roots and seed coats of far single mutants ................................................................................................101

2.3.4 Alterations of fatty alcohol composition in leaves of far single mutants after wounding ....104

2.3.5 Heterologous Expression of FAR1, FAR4, and FAR5 in Yeast ..........................................106

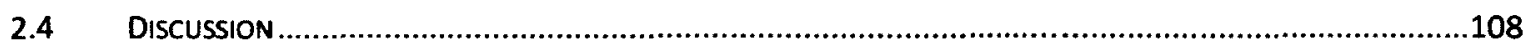

2.4.1 FAR1, FAR4, and FAR5 are expressed at sites of suberin deposition. .............................108

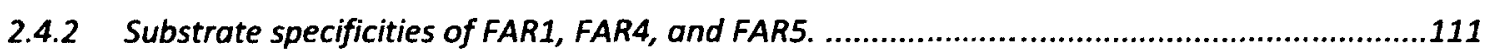

2.4.3 Production of primary alcohols associated with suberin is affected in far 1, far4, and far5

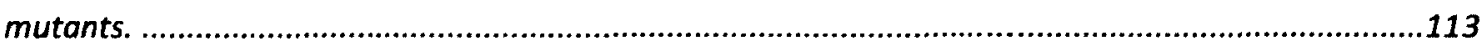

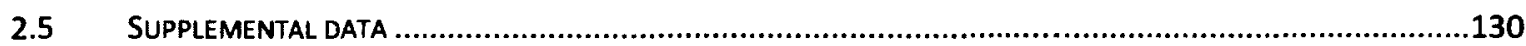

3 CHAPTER 3: BIOSYNTHESIS AND FUNCTIONS OF FREE AND COMBINED FATTY ALCOHOLS

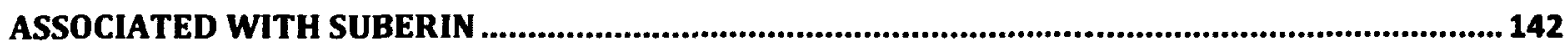

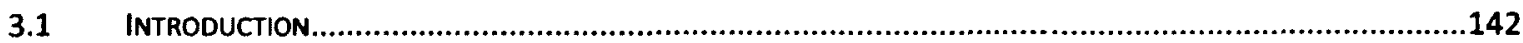

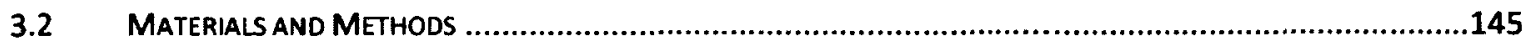

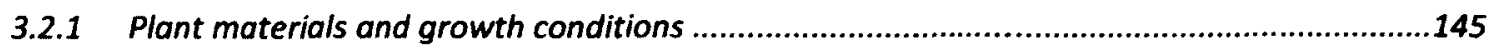

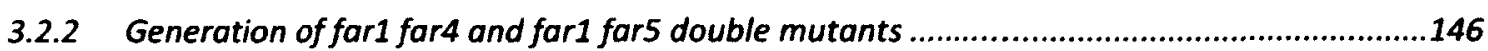

3.2.3 Generation of artificial microRNA (amiRNA) lines. .........................................................146

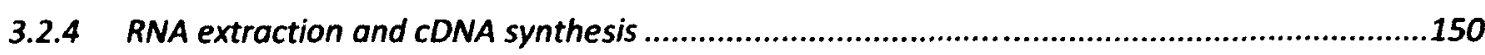




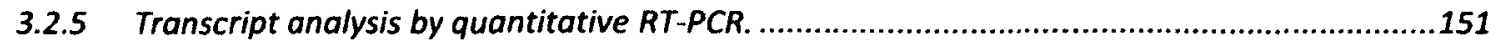

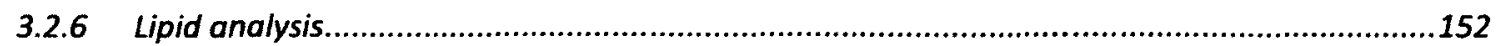

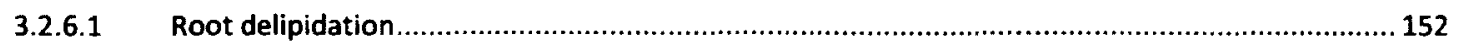

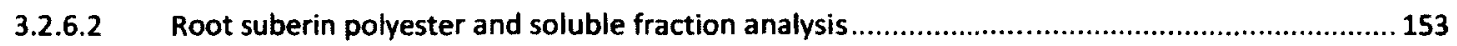

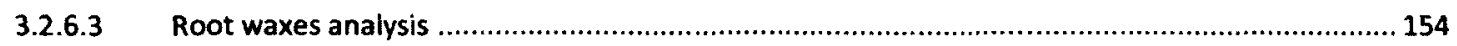

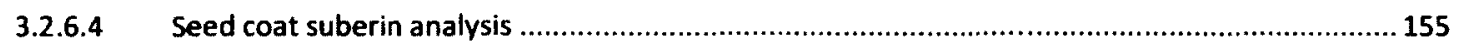

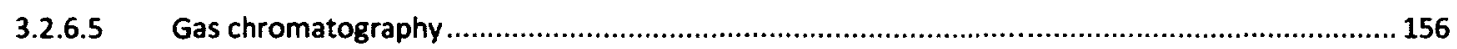

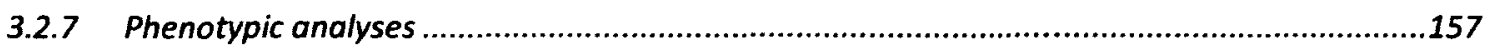

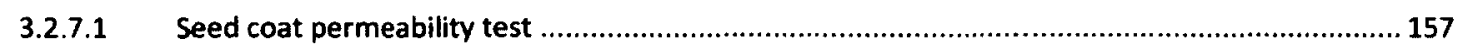

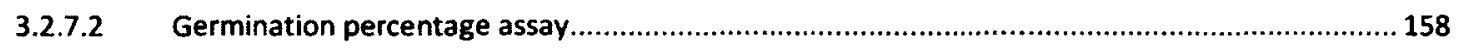

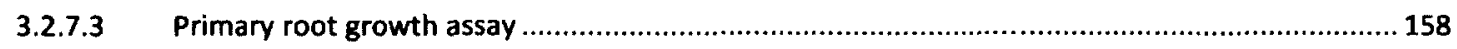

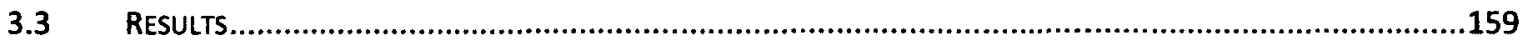

3.3.1 Distribution of lipids and phenolics in suberin polymer and soluble lipid fractions of

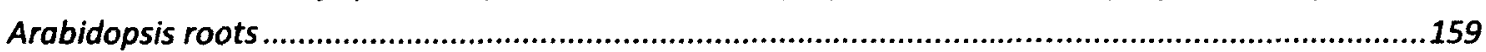

3.3.2 Isolation of double and triple mutants disrupted in the FAR1, FAR4, and FAR5 genes .........160

3.3.3 Alterations of fatty alcohol composition associated with suberin polyester and soluble

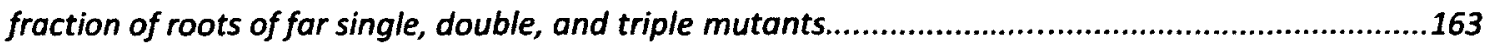

3.3.4 Alterations of fatty alcohol composition and alkyl hydroxycinnamates in root waxes of far

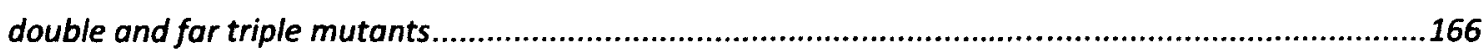

3.3.5 Alterations of fatty alcohol composition associated with seed coat suberin of far single,

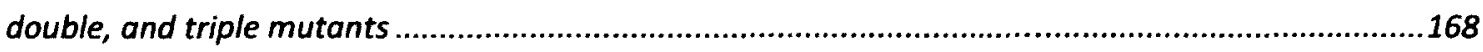
3.3.6 Alteration in seed coat permeability and germination percentage in far double and far triple

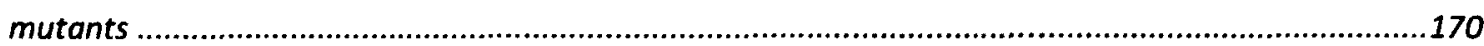

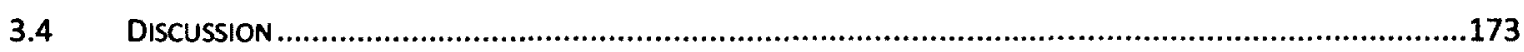

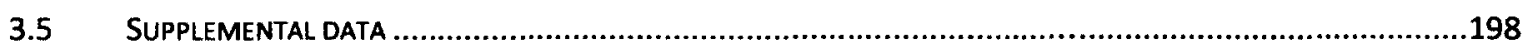

\section{CHAPTER 4: SUBSTRATE SPECIFICITIES AND SUBCELLULAR LOCALISATION STUDIES OF}

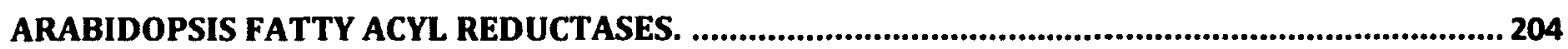

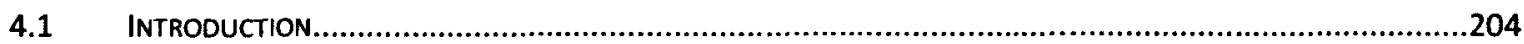

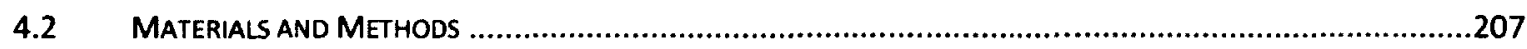

4.2.1 Cloning of FAR1, FAR4, FARS and FAR chimeric genes into yeast expression vector.............207

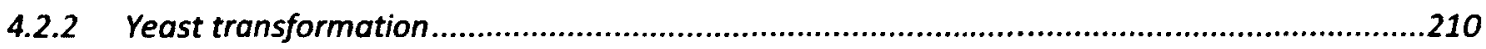

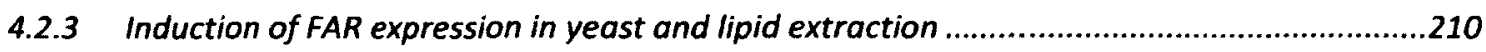

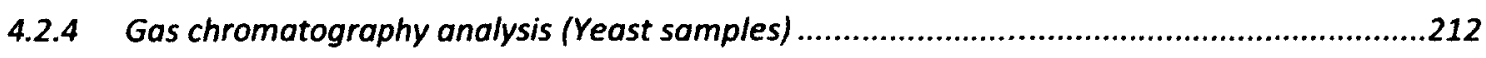

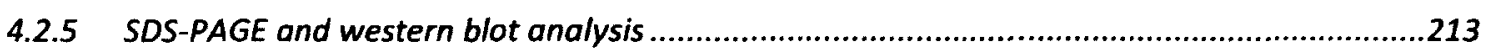

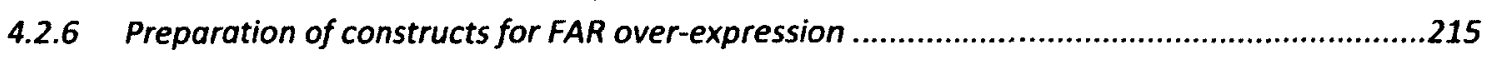

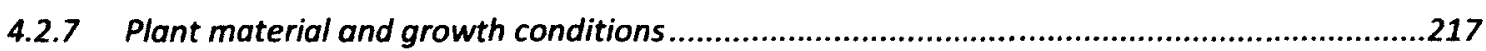

4.2.8 Transient expression of Arabidopsis FAR1, FAR4 and FAR5 in Nicotiana benthamiana leaves 218

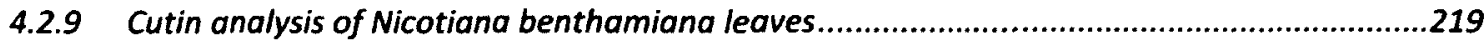

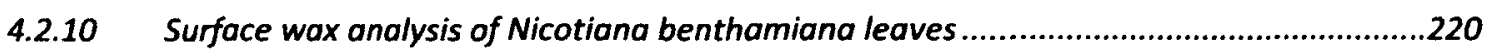

4.2.11 Total internal lipid analysis of Nicotiana benthamiana leaves ......................................221

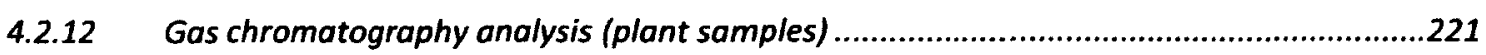

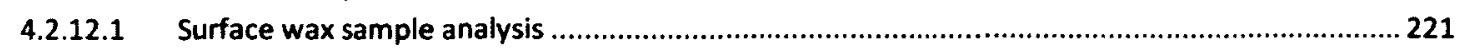

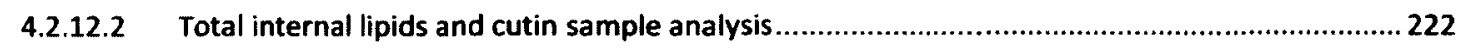

4.2.13 Preparation of AtFAR1-GFP fusion expression construct ............................................222 
4.2.14 Transient expression of AtFAR1-GFP in N. benthamiana leaves and analysis by confocal

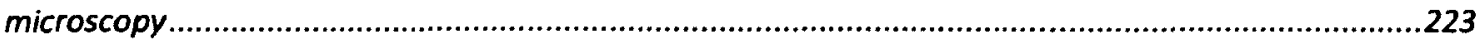

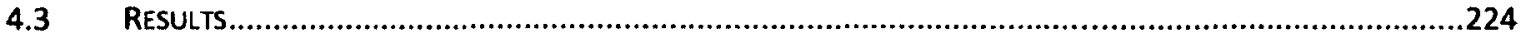

4.3.1 Heterologous expression of Arabidopsis FAR1, FAR4, and FAR5 and FAR1/4/5 domain swap

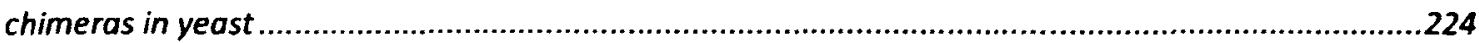

4.3.2 Transient overexpression of Arabidopsis FAR1, FAR4 and FAR5 in Nicotiana benthamiana leaves 227

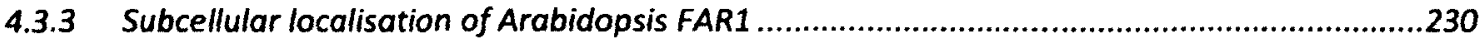

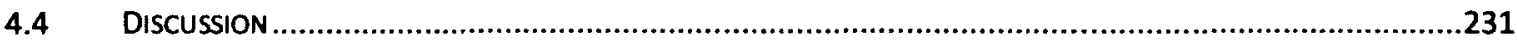

4.4.1 Substrate specificities of plant FARs are governed by both the Rossmann fold domain and the FAR_C domain.

4.4.2 FAR1, FAR4 and FAR5 specificities in planta and influence of acyl-CoA pool on fatty alcohol production

4.4.3 Arabidopsis FAR1 is localised to the Endoplasmic Reticulum (ER)...................................237

5 CHAPTER 5: TRANSCRIPTIONAL REGULATION OF ARABIDOPSIS FAR1, FAR4, AND FAR5 AND OTHER SUBERIN BIOSYNTHETIC GENES UNDER ABIOTIC STRESS CONDITIONS............. 256

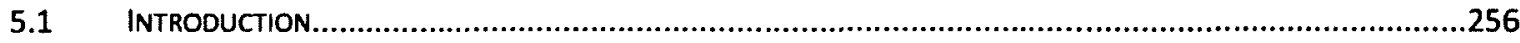

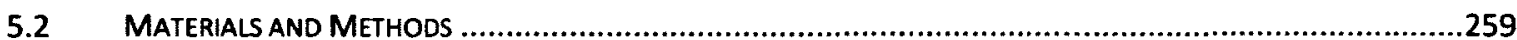

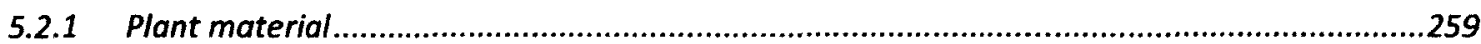

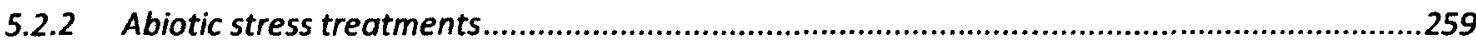

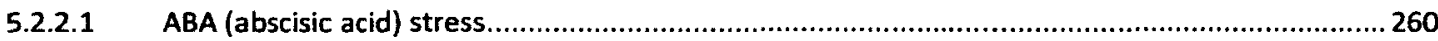

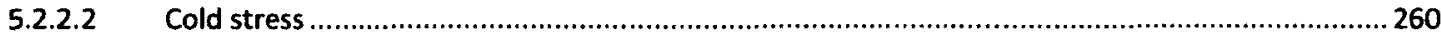

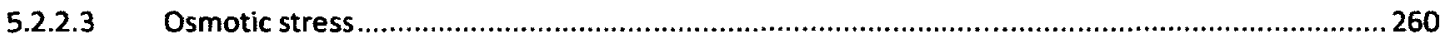

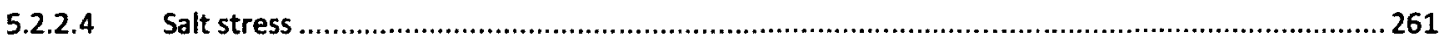

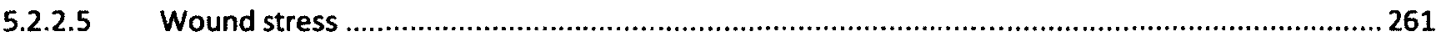

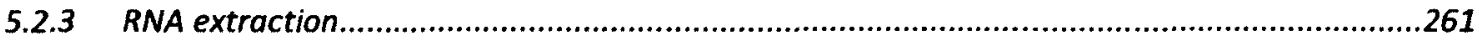

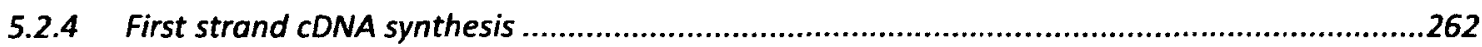

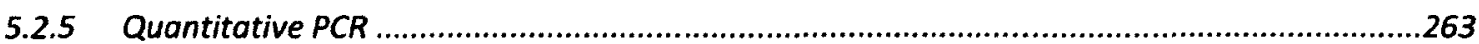

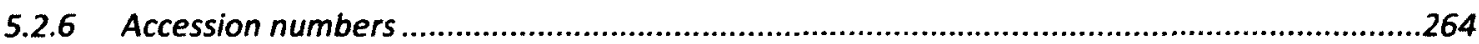

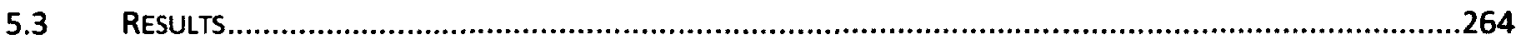

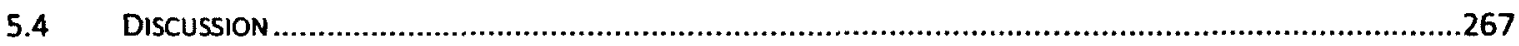

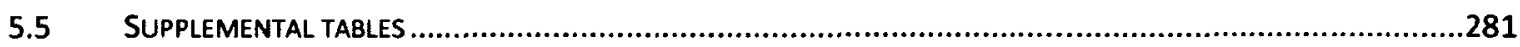

6 CHAPTER 6: CONCLUSIONS AND FUTURE DIRECTIONS................................................ 284

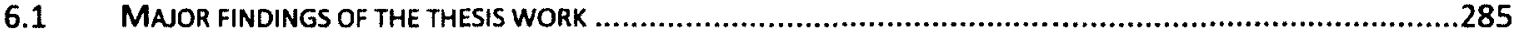

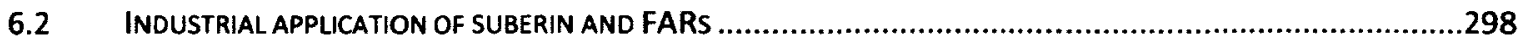

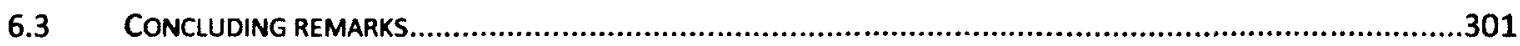

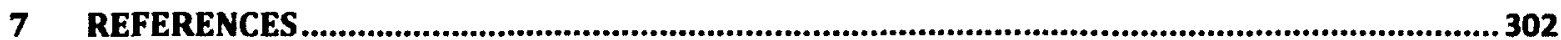




\section{LIST OF TABLES}

Table 1.1. Summary of common cutin, suberin and sporopollenin monomers, identified by abundance and functional group for each biopolymer. 10

Table 1.2. Summary of identified genes involved in the suberin polyester biosynthetic pathway in Arabidopsis roots, periderms and seed coat.

Table 1.3. Characterized Fatty Acyl Reductases (FARs) from plants .65

Table 2.1. Aliphatic suberin composition in roots of tissue culture-grown wildtype, far 1-1, far4-1 and far 5-1 plants

Table 2.2. Internal fatty acyl-chain composition of the different FAR expressing yeast strains

Table 3.1. The oligonucleotide primer combinations used for amplification of each amiRNA fragment, the template used, and their expected PCR product sizes to form the full-length amiRNA sequences.......................................................................................................185

Table 3.2. Fatty alcohol composition in insoluble suberin polymer fraction versus soluble fraction of roots of 4-week-old tissue culture grown wild-type plants

Table 3.3. Total primary fatty alcohol loads (suberin polymer bound and delipidated soluble fraction combined) and total quantity of particular fatty alcohol chain length in the roots of 4-weeks-old tissue culture grown wild-type plants.

Table 3.4. Percent of fatty alcohol bound to suberin polymer in the roots of 4week-old, tissue culture grown wild-type and far mutant plants.......193

Table 4.1. Summary table of amino acid sequence identity between the Arabidopsis FAR family and jojoba FAR. 239 


\section{LIST OF SUPPLEMENTAL TABLES}

Table S2.1. Fatty Acyl-CoA Reductase (FAR) genes in Arabidopsis thaliana .......135

Table S2.2. Composition of residual bound lipids in whole roots of soil-grown wild-type, far1, far4, and far5 plants 136

Table S2.3. Composition of residual bound lipids in seeds of wild-type, far1-1, far 4-1, and far5-1 plants

Table S2.4. Composition of residual bound lipids in unwounded and wounded leaves of wild-type, far 1-1, far 4-1, and far5-1.

Table S2.5. Sequences of DNA primers used for PCR in Chapter-2. 140

Table S3.1. Composition of residual bound lipids in roots of wild-type, far1, far4, far5 and far double and far triple mutant plants.

Table S3.2. Composition of suberin-associated waxes in roots of wild-type, and far double and far triple mutant plants

Table S3.3. Composition of residual bound lipids in seed coat of wild-type, far1, far4, far 5 and far double and far triple mutant plants. Each value is the mean shown in $\mu \mathrm{g} / \mathrm{mg}$ delipidated dry weight of residue $\pm \mathrm{SD}$ of three biological replicates. 202

Table S3.4. List of primers used in the qPCR analysis of far mutants 203

Table S4.1. Composition of fatty alcohols in transgenic yeast cells expressing Arabidopsis FAR1, FAR4 and FAR5 251

Table S4.2. Composition of total lipids in $N$. benthamiana leaves expressing Arabidopsis FAR1, FAR4 and FAR5 252

Table S4.3. Composition of surface wax in $N$. benthamiana leaves expressing Arabidopsis FAR1, FAR4 and FAR5. .253

Table S4.4. List of primers used for generation of FAR chimera constructs. 254

Table S5.1. Modulation of expression (fold change) of FAR1, FAR4, and FAR5 and five other suberin genes in Arabidopsis root tissue subjected to various abiotic stresses. 281

Table S5.2. List of primers used for the quantitative-PCR study of FAR1, FAR4, FAR5 and five other suberin genes under abiotic stresses. 282 


\section{LIST OF FIGURES}

Figure 1.1. Plant surface barriers: cuticle, pollen exine, and suberin. Electron microscopic views of (A) Arabidopsis epidermal cell wall featuring cuticle, (B) exine of pollen grains, and (C) suberized root cell wall

Figure 1.2. Schematic representation of cutin and epicuticular wax deposition in a leaf.

Figure 1.3. Schematic of a matured root cross section.

Figure 1.4. Locations of suberized barriers in a typical root and their functions. 25

Figure 1.5. Transesterification of suberin polyester using methanolic$\mathrm{HCl}$......

Figure 1.6. Bernards (2002) model of suberin lamellae .32

Figure 1.7. Phenolic precursors of suberized tissues 35

Figure 1.8. Proposed pathways for the synthesis of suberin polyester monomers

Figure 1.9. Generalized structures of primary fatty alcohols, wax esters, alkyl hydroxycinnamates, and ether lipids....

Figure 1.10. Fatty alcohols and their derivatives. .57

Figure 1.11. Enzymatic reaction catalyzed by Fatty Acyl Reductase (FAR) .58

Figure 1.12. FAR structural domains . .76

Figure 1.13. Phylogenetic tree of related FAR protein sequences .78

Figure 2.1. The Arabidopsis FAR gene family on chromosomes I-V 116

Figure 2.2. Tissue-specific gene expression patterns of Arabidopsis FAR genes

Figure 2.3. Expression patterns of FAR1 (A), FAR4 (B), and FAR5 (C) detected in transgenic promoter:GUS lines. 
Figure 2.4. Induction of FAR1, FAR4, and FAR5 gene expression by wounding and salt stress.

Figure 2.5. T-DNA insertion lines of FAR1, FAR4 and FAR5, and transcript levels in the far mutants 120

Figure 2.6. Aliphatic suberin composition in roots of soil-grown wild-type, far1, far4, and far5 single mutant plants 122

Figure 2.7. Lipid polyester composition in seeds of wild-type, far1, far4, and far5 single mutant plants 124

Figure 2.8. Composition of residual bound lipids in unwounded and wounded leaves of wild-type, far1-1, far 4-1, and far 5-1 125

Figure 2.9. Fatty alcohol content of residual bound lipids in unwounded and wounded leaves of wild-type, far 1-1, far 4-1, and far 5-1 126

Figure 2.10. Fatty acyl-chain profiles of transgenic yeast expressing Arabidopsis FARs.

Figure 3.1. Schematic showing PCR amplification of artificial miRNA precursors 184

Figure 3.2. Distribution of aliphatics (fatty acids and derivatives), phenolics and sterols in suberin polymer and soluble fraction of roots from 4-weekold wild-type (A), GC chromatogram with peak relevant to fatty alcohol identified shown in both suberin polymer and soluble lipids fractions of roots of wild-type plants (B) 186

Figure 3.3. Schematic showing the FAR5-amiRNA sequence-1 and the cleavage site on FAR5 cDNA sequence (A) and the FAR5-amiRNA-sequence1 (reverse compliment) aligned with the coding sequence of rootexpressing FARs: FAR1, FAR3, FAR4, and FAR5 gene (B). Semiquantitative RT-PCR transcript analysis of two independent transgenic lines of far5 single, far 4 far 5 double, and far 1 far 4 far 5 triple mutants with FAR5-amiRNA-1 (C) 188

Figure 3.4. Transcript levels of FAR genes in wild type, far1, far4, far5, and far double, and far triple mutant lines determined by quantitative RTPCR 189

Figure 3.5. Aliphatic suberin residual-bound monomer composition in roots of wild-type, far 1, far 4, far5, far $5^{*}$ and far double, and far triple mutant plants 190 
Figure 3.6. Total fatty alcohol content of suberin in roots of wild-type, far1, far4, far 5, far $5^{*}$ as well as far double and far triple mutant plants (A). Fatty alcohols sorted into individual chain lengths, where mean values are shown in $\mu \mathrm{g} / \mathrm{mg}$ delipidated dry residue (DR) of three replicates (B). 191

Figure 3.7. Fatty alcohol content of suberin in root delipidation (soluble) fraction of wild-type, far1, far4, far5, far $5^{*}$ as well as far double and far triple mutant plants. 192

Figure 3.8. Levels of total amounts of waxes (A), and primary fatty alcohols and alkyl hydroxycinnamates (AHCs) (B) in root waxes of wild-type, far double, and far triple mutant plants. .194

Figure 3.9. Suberin polyester composition in seed coat of wild-type, far1, far4, far5, as well as far double and far triple mutant plants (A), Amounts of seed coat suberin-associated fatty alcohols, sorted into individual chain lengths in Col-0 and far mutants (B) 195

Figure 3.10. Tetrazolium penetration assays. (A) Time course of formazans produced by reduction of tetrazolium salts by the embryos of Arabidopsis wild-type (WT) and far double and triple mutants, (B) Staining pattern in seeds of Col-0 and far mutants incubated in 1\% tetrazolium red. 196

Figure 3.11. Seed germination rate of wild type, far1, far4, far5, and far double, and far triple mutants subjected to varying abscisic acid (ABA) concentration 197

Figure 4.1. Alignment of Arabidopsis FAR1, FAR4 and FAR5 protein sequences. 240

Figure 4.2a. Domain swap site shown in the alignment of FAR1, FAR4 and FAR5 protein sequence. 241

Figure 4.2b. Schematic showing domains of native FAR1, FAR4 and FAR5 protein and the resultant FAR chimeras after domain swaps. .242

Figure 4.3. SDS-PAGE and western blot analysis of transgenic yeast expressing tagged Arabidopsis FAR1, FAR4, FAR5 and FAR chimeras. .243

Figure 4.4. GC-FID chromatograms (zoomed in) of lipids extracted from transgenic yeast expressing tagged Arabidopsis FAR1, FAR4, FAR5 and FAR chimeras 245

Figure 4.5. Internal fatty alcohol composition of yeast cells expressing $\mathbf{x x}$ 
Arabidopsis FAR1, FAR4, FAR5 and FAR1/4/5 chimeras, measured by gas chromatography (GC-FID). 246

Figure 4.6. Composition of total internal lipids in Nicotiana benthamiana leaves over-expressing Arabidopsis FAR1, FAR4 and FAR5

Figure 4.7. Composition of surface wax of Nicotiana benthamiana leaves transiently over-expressing Arabidopsis FAR1, FAR4 and FAR5 248

Figure 4.8. Composition of cutin polyester of Nicotiana benthamiana leaves transiently over-expressing Arabidopsis FAR1, FAR4 and FAR5 249

Figure 4.9. Localisation of AtFAR1-GFP fusion protein to Endoplasmic Reticulum (ER) in Nicotiana benthamiana leaves examined 3-days post infiltration 250

Figure 5.1. Modulation of expression (fold change) of FAR1, FAR4, and FAR5 and five other suberin genes in the Arabidopsis root tissue subjected to ABA stress $(10 \mu \mathrm{M})$ for 24 hours 276

Figure 5.2. Modulation of expression (fold change) of FAR1, FAR4, and FAR5 and five other suberin genes in the Arabidopsis root tissue subjected to cold stress $\left(4^{\circ} \mathrm{C}\right)$ for 24 hours 277

Figure 5.3. Modulation of expression (fold change) of FAR1, FAR4, and FAR5 and five other suberin genes in the Arabidopsis root tissue subjected to osmotic stress (150mM Mannitol) for 24 hours .278

Figure 5.4. Modulation of expression (fold change) of FAR1, FAR4, and FAR5 and five other suberin genes in the Arabidopsis root tissue subjected to salt stress $(200 \mathrm{mM} \mathrm{NaCl})$ for 24 hours. 279

Figure 5.5. Modulation of expression (fold change) of FAR1, FAR4, and FAR5 and five other suberin genes in the Arabidopsis stem tissue subjected to wounding stress for 24 hours. 280 


\section{LIST OF SUPPLEMENTAL FIGURES}

Figure S2.1. The developmental expression pattern of FAR1, FAR4 and FAR5 genes in various plant tissues analysed using DNA microarray (AtGenExpress) data

Figure S2.2. Expression patterns of FAR1 (A), FAR4 (B), and FAR5 (C) detected in root and aerial tissues of transgenic promoter:GUS lines

Figure S2.3. Aliphatic suberin composition in roots of tissue culture-grown wildtype, far1-1, far4-1, and far5-1 plants

Figure S2.4. Identification by gas chromatography-mass spectrometry of primary fatty alcohols produced in transgenic yeast expressing Arabidopsis FAR1 133

Figure S3.1. Seed germination rate of wild type, far1, far4, far5, far double, far triple and other suberin mutants subjected to varying salt concentrations

Figure S3.2. Primary root growth of wild type, far1, far4, far5, far double, far triple and other suberin mutants subjected salt stress 


\section{LIST OF ABBREVIATIONS}

\begin{tabular}{|c|c|}
\hline $\mathbf{2 O H}$ & : 2-hydroxy fatty acid \\
\hline $\mathbf{2}^{\circ}-\mathbf{O H}$ & : Secondary alcohol \\
\hline $\mathbf{A B C}$ & : ATP binding cassette transporter \\
\hline ACP & : Acyl Carrier Protein \\
\hline AHCs & : Alkyl Hydroxycinnamates \\
\hline amiRNA & : artificial microRNA \\
\hline APS & : ammonium persulfate \\
\hline ASFT & : Aliphatic Suberin Feruloyl Transferase \\
\hline ATT1 & : Aberrant induction of Type Three genes \\
\hline AVG & : Average \\
\hline BSTFA+TMCS & $\begin{array}{l}\text { : } \mathrm{N}, \mathrm{O} \text {-bis (trimethylsilyl) trifluoroacetamide+ } \\
\text { trimethylchlorosilane }\end{array}$ \\
\hline C & :Carbon \\
\hline cDNA & : Complementary deoxyribonucleic acid \\
\hline CER & : Eceriferum \\
\hline CoA & : Coenzyme A \\
\hline Col-0 & : Columbia-0 wild-type Arabidopsis thaliana \\
\hline Ct & : Threshold Cycle \\
\hline CYP & : Cytochrome p450 \\
\hline DCA & : Dicarboxylic acid \\
\hline DEPC & : Diethylpyrocarbonate \\
\hline DNA & : Deoxyribonucleic acid \\
\hline dNTP & : Deoxyribonucleotide triphosphate \\
\hline DPW & : Defective Pollen Wall \\
\hline DTT & : Dithiothreitol \\
\hline DR & : Dry Residue \\
\hline EDTA & : Ethylenediamine tetraacetic Acid \\
\hline ER & : Endoplasmic Reticulum \\
\hline EV & : Empty Vector \\
\hline FA & : Fatty Acid \\
\hline FACT & : Fatty Alcohol:Caffeoyl-CoA Caffeoyl Transferase \\
\hline FAE & : Fatty Acid Elongase \\
\hline FAR & : Fatty Acyl-CoA Reductase \\
\hline FAS & : Fatty Acid Synthase \\
\hline FAT & : Fatty Acyl-ACP Thioesterase \\
\hline FFA & : Free Fatty Acids \\
\hline FHT & $\begin{array}{l}\text { : Omega-hydroxyacid - Fatty Alcohol Hydroxycinnamoyl } \\
\text { transferase }\end{array}$ \\
\hline $\begin{array}{l}\text { GAPC } \\
\text { GC }\end{array}$ & $\begin{array}{l}\text { : Glyceraldehyde-3-Phosphate Dehydrogenase C Subunit } \\
\text { : Gas Chromatography }\end{array}$ \\
\hline GC-FID & : Gas Chromatography-Flame Ionization Detector \\
\hline GC-MS & : Gas Chromatography-Mass Spectrophotometry \\
\hline GFP & : Green Fluorescent Protein \\
\hline
\end{tabular}




\begin{tabular}{|c|c|}
\hline $\begin{array}{l}\text { GPAT } \\
\text { GUS }\end{array}$ & $\begin{array}{l}: \text { Glycerol-3-Phosphate Acyltransferase } \\
: \beta \text {-glucuronidase }\end{array}$ \\
\hline his & : Histidine \\
\hline IPTG & : Isopropyl $\beta$-D-1-thiogalactopyranoside \\
\hline $\mathrm{K}_{3} \mathrm{Fe}(\mathrm{CN})_{6}$ & : Potassium ferricyanide \\
\hline $\mathrm{K}_{4} \mathrm{Fe}(\mathrm{CN})_{6}$ & : Potassium ferrocyanide \\
\hline $\mathbf{K C l}$ & : Potassium Chloride \\
\hline KCS & : $\beta$-ketoacyl-CoA synthase \\
\hline kDa & : Kilodalton \\
\hline $\mathbf{k V}$ & : Kilovolt \\
\hline LACS & : Long-Chain Acyl-CoA Synthetase \\
\hline LB & : Luria-Bertani \\
\hline LCR & : Laçerata \\
\hline leu & : Leucine \\
\hline LiAc & : Lithium Acetate \\
\hline $\mathrm{MeOH} / \mathrm{HCl}$ & : Methanolic $\mathrm{HCl}$ \\
\hline $\mathrm{MgCl}_{2}$ & : magnesium chloride \\
\hline MS2 & : MALE STERILITY2 \\
\hline NABD & : Nicotinamide Adenine Dinucleotide Binding \\
\hline $\mathrm{NaCl}$ & : Sodium Chloride \\
\hline NADH & : Nicotinamide Adenine Dinucleotide \\
\hline NADPH & : Nicotinamide Adenine Dinucleotide Phosphate \\
\hline NaPO4 & : Sodium Phosphate \\
\hline$\left(\mathrm{NH}_{4}\right)_{2} \mathrm{SO}_{4}$ & : Ammonium Sulphate \\
\hline OD & : Optical Density \\
\hline $\mathbf{O H}$ & : Alcohol \\
\hline ORF & : Open Reading Frame \\
\hline PBS & : Phosphate Buffered Saline \\
\hline PCR & : Polymerase Chain Reaction \\
\hline PEG & : Poly(Ethylene Glycol) \\
\hline PMSF & : Phenylmethylsulfonyl fluoride \\
\hline qPCR & : Quantitative PCR \\
\hline RNA & : Ribonucleic Acid \\
\hline RT-PCR & : Reverse transcription PCR \\
\hline ScFAR & : Simmondsia chinensis FAR \\
\hline SD & : Synthetic Media Containing D-glucose \\
\hline SDS-PAGE & : Sodium Dodecyl Sulfate-Polyacrylamide Gel Electrophoresis \\
\hline SG & : Synthetic Media Containing Galactose \\
\hline SSC & : Saline sodium citrate \\
\hline ST DEV & : Standard Deviation \\
\hline Taq & : Thermus aquaticus DNA Polymerase \\
\hline TBST & : TRIS Base buffered Sodium Chloride solution with Tween 20 \\
\hline TCA & : Trichloroacetic acid \\
\hline TE & : Tris EDTA \\
\hline TEM & : Transmission electron microscopy \\
\hline
\end{tabular}


TEMED : Tetramethylethylenediamine

Tris-HCl : Tris(Hydroxymethyl) Aminomethane- Hydrochloride

trp : Tryptophan

ura : Uracil

VLCFA : Very-Long-Chain Fatty Acids

$\omega-\mathbf{O H} \quad: \omega$-hydroxy fatty acid

WS : Wax Synthase

WSD : Was Synthase/Diacylglycerol Acyltransferase

X-Gluc $\quad$ : 5-bromo-4-chloro-3-indolyl $\beta$-D-glucuronide

YPAD : Yeast Extract, Peptone, Adenine Hemisulphate, D-glucose 


\section{CHAPTER 1: General Introduction and Literature Review}

Vascular plants have three major lipidic extracellular barriers: cuticle coating their aerial surfaces, sporopollenin in the outer layer of pollen grains, and suberin within the cell walls of various endodermal and peridermal cell layers (Figure 1.1). These three barriers are biopolymers consisting of lipids and phenolics and were key adaptations for plant life in a terrestrial environment.

Cutin is mostly a lipid polyester and constitutes the structural component of cuticle, which represents one of the largest biological-environmental interfaces (Bargel et al., 2006). Suberin is a heteropolymer of lipids and phenolics constitutively produced by various internal and external cells, such as shoot and root periderms. Suberin is also induced to form a barrier in response to environmental stresses such as wounding and pathogen attack (Kolattukudy, 2001a; Bernards, 2002). In addition, both cutin and suberin are found to have noncovalently linked waxes associated with them (Schreiber, 2010). These protective barriers play an important role at the plant-environment interface by preventing the loss of water and solutes to the environment and helping plants to cope with various abiotic stresses, such as drought, extreme temperature, and salinity, as well as biotic stresses such as microbial pathogen attack and insect predation (Priestley and North, 1922; Franke and Schreiber, 2007; Samuels et al., 2008, Schreiber, 2010). 

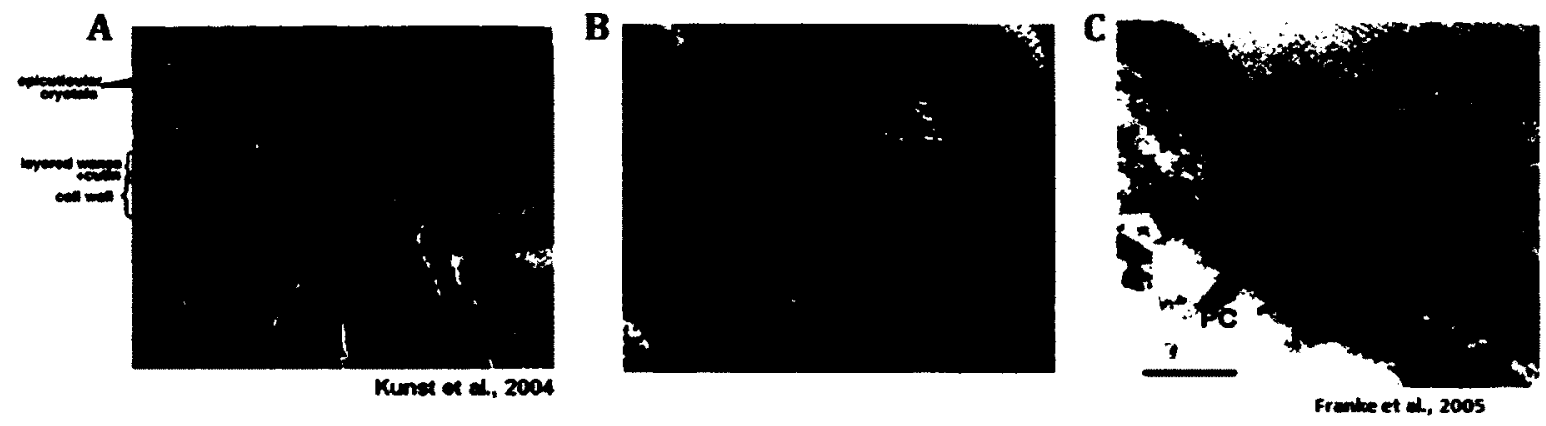

Figure 1.1. Plant surface barriers: cuticle, pollen exine, and suberin. Scanning electron microscopic views of Arabidopsis epidermal cell wall featuring cuticle (A) and exine of pollen grains (B). Transmission electron microscopic view of Arabidopsis suberized root cell wall (C). Suberized peridermal and endodermal cells typically have a lamellar appearance within the cell walls (alternative light and dark layers). CW, cell wall; PC, peridermal cell. Bar: $100 \mathrm{~nm}$ (panel C). The SEM image of cuticle was adapted from Kunst et al. (2004) and the TEM image of suberin lamellae from Franke et al. (2005). Image reproduced with permission of American Society of Plant Biologists (Copyright (C) ASPB). 
Although both cutin and suberin have structural similarities and analogous functions in the protection of plant organs, they are distinguished by their sites of deposition and chemical composition. Cutin is composed largely of C16 and C18 chains (e.g. hydroxy and hydroxy-epoxy fatty acids), while the aliphatic part of suberin is composed of $\mathrm{C} 16$ and $\mathrm{C} 18$ chains plus high amounts of C20-C24 chain lengths. In addition, suberin contains a significant phenolic (aromatic) component consisting of hydroxycinnamic acids, especially ferulic acid (Kolattukudy, 2001b), which is much less abundant in cutin. Glycerol is a component of both polyesters (Moire et al., 1999; Graça and Pereira, 2000; Graça and Santos, 2007).

Lipid polyesters are agriculturally important because of their importance in crop physiology. Although the compositional knowledge of cutin and suberin is almost complete, little is known about the intact polymeric structures. It is difficult to isolate these polymers because of their structural complexity and insolubility, and hence progress in understanding the structure, enzymology and genetics of lipid polyesters has been slow. Significant progress has been made in the last decade with the availability of molecular genetic tools developed mostly for the model plant Arabidopsis thaliana. One advantage of using Arabidopsis as a model is that economically important oilseed crops such as Brassica napus and Camelina sativa are members of the same family. Suberin present in the roots of Arabidopsis is also similar to other more distantly related plant species, indicating that Arabidopsis can serve as a model for suberized tissues (Franke et al., 2005). In recent years, research regarding the influence of cutin and suberin polymers on physiological and mechanical properties as well as microbial susceptibility has increased by studying 
Arabidopsis mutants and overexpressing lines (Nawrath, 2006; Isaacson et al., 2009).

Primary fatty alcohols are aliphatic hydrocarbons that contain a hydroxyl group at the terminal carbon. Long-chain (C16-C18) and very-long-chain (>C18) primary fatty alcohols are found in nature either as free alcohols (as found in cuticular and root waxes) or, for example, combined with fatty acids to give wax esters (found in cuticular waxes and jojoba seeds) or with hydroxycinnamic acids to give alkyl hydroxycinnamates (found in tuber periderm waxes and root waxes). Saturated primary alcohols of chain lengths C18-C22 constitute about $6-10 \%$ of the total suberin polyester aliphatic load depending on the species (Pollard et al., 2008). Primary alcohols are synthesized by the acyl reduction pathway in plants, where the substrates fatty acyl-CoA (coenzymeA) or fatty acyl-ACP (acyl carrier protein) are

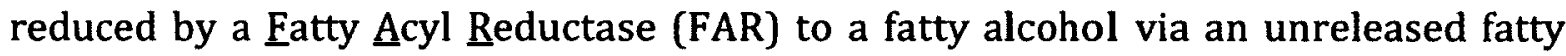
aldehyde intermediate. The Arabidopsis genome has an eight-member FAR gene family of which two of the FAR genes, FAR2 and FAR3/CER4, are involved in production of primary alcohols associated with sporopollenin (Aarts et al., 1997) and cuticular wax (Rowland et al., 2006), respectively. Several aspects of this Arabidopsis FAR enzyme family remain elusive. The $F A R$ genes involved in production of primary alcohols associated with suberin polyester were unknown up until the present study. We were interested in how suberin-associated free and combined fatty alcohols are formed and their biological functions. In this thesis, I report on the characterization of three root-expressed Arabidopsis FAR genes, FAR1, 
FAR4 and FAR5, with an emphasis on their roles in suberin biosynthesis in root tissues and seeds.

The following sections summarize our current knowledge of the structure and biosynthesis of the lipid polyesters cutin, sporopollenin and suberin, and provide some background on their functions and chemistries. In addition, I also present an overview of FAR enzymology.

\subsection{Biopolymers in plants - Cutin, Sporopollenin and Suberin}

Cutin, sporopollenin and suberin are distinct types of plant biopolymers made up of polymerized lipids and phenolics with their associated waxes. These lipid- and phenolic-based polyesters form protective barriers for plants. Many important transport processes between the plant and the environment take place at these interfaces (Nawrath, 2006; Franke and Schreiber, 2007). Recently, significant progress has been made in identifying and characterizing the enzymes involved in cutin, sporopollenin and suberin biosynthesis.

\subsubsection{Cutin and Cuticular wax}

The cuticle, consisting primarily of cutin and waxes, is a protective and waterproofing barrier deposited at the outermost surface of epidermal cells of all land plants and some bryophytes. Cutin consists mostly of polymerized fatty acids and forms the structural skeleton of the cuticle (Figure 1.2). Plant epidermal cells synthesize and secrete cutin monomers and waxes. The functions and characteristics of the cuticular layer are strongly influenced by the insoluble cuticular polymers cutin and cutan (cutan is a hydrocarbon polymer also known as 
'non-ester cutin'], as well as by cuticular polysaccharides and soluble cuticular waxes (Kolattukudy, 2001c).

\subsubsection{Functions of cuticle}

The cuticle covers the external surfaces of the epidermal cell walls of leaves, primary stems, flowers, and fruits. Internal cuticles also occur, occuring within seed coats and lining the substomatal cavity (Molina et al., 2006; Samuels et al., 2008). Cuticle protects the underlying tissues from non-stomatal water loss and gas exchange (Riederer and Schreiber, 2001). It acts as a barrier against pathogens, insects, and UV radiation (Kolattukudy, 1987; Jenks et al., 1994). In addition, the cuticle influences plant development by preventing organs from fusing (Sieber et al., 2000). It also serves as a waterproofing barrier that causes shedding of water

droplets, thereby minimizing deposition of dust particles and spores (Reiderer and Muller, 2006). 


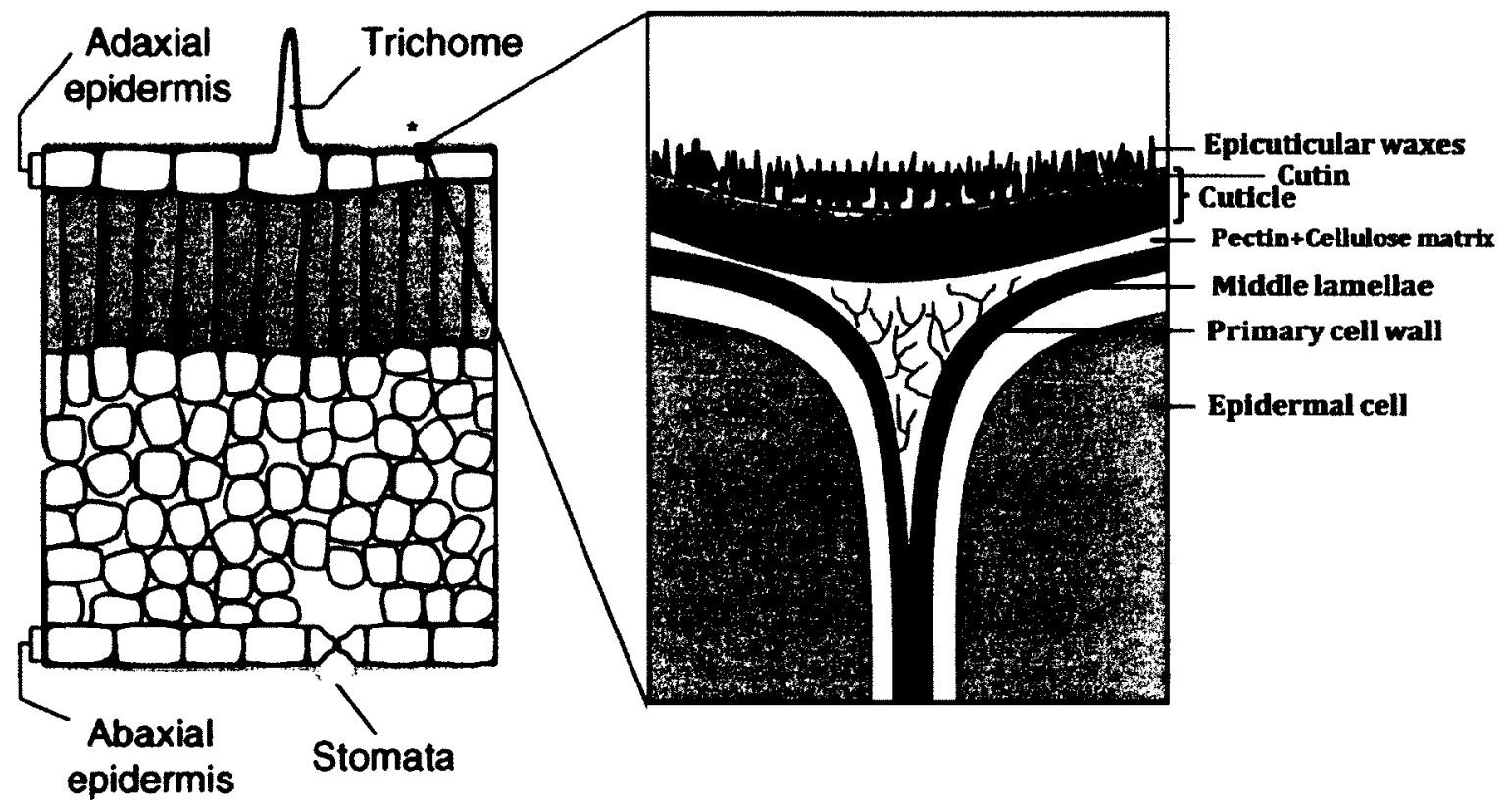

Figure 1.2. Schematic representation of cutin and epicuticular wax deposition in a leaf (not to scale). Modified from Pollard et al, (2008). Reproduced with permission. Copyright 2008 Elsevier. 


\subsubsection{Cutin structure and composition}

The mature cuticle consists of two distinct parts: (1) a largely lipid-based cutin layer embedded with various intracuticular waxes, and (2) an outer layer composed primarily of epicuticular waxes. This cuticle proper is attached to the epidermal cell via a pectin and polysaccharide layer (Figure 2) (Kolattukudy, 2001c). The thickness of cuticle layers and their composition depend on the plant species and the developmental stage. The overall cuticular thickness ranges from 0.1 to $14 \mu \mathrm{m}$ in leaves and the weight of an isolated cuticle ranges from $450-800$ $\mu \mathrm{g} / \mathrm{cm} 2$ (leaves) to $2000 \mu \mathrm{g} / \mathrm{cm} 2$ (fruits), of which usually $40-80 \%$ corresponds to cutin (Kolattukudy, 2001c). The insoluble nature of cutin has left the analysis of the three-dimensional structure unresolved. Knowledge on the composition of this material has been based on the monomers obtained after depolymerization reactions (Bonaventure et al., 2004).

Cutin is a polyester matrix containing glycerol and is mostly composed of C16 and C18 $\omega$-hydroxyacids, polyhydroxyacids, $\omega$-hydroxy-epoxyacids, $\alpha, \omega$-dicarboxylic acids (DCA) and minor amounts of hydroxycinnamic acids (Kolattukudy, 2001c; Graca et al., 2002; Pollard et al., 2008; Beisson et al., 2012) (Table 1.1). Cutin also contains other less abundant components, such as unsubstituted fatty acids and primary alcohols. The monomer acyl groups are transferred to hydroxyl groups in the growing polymer at the extracellular location. The proportions of these various ester-linked fatty acid derivatives differ greatly between species and even between organs within the same species. For example, in 
Arabidopsis thaliana the cutin of leaves and stems is dominated by dicarboxylic acids (DCAs), the most abundant being C18:2 dicarboxylic acid, while the cutin of flowers is mainly composed of polyhydroxyacids (Bonaventure et al., 2004; Franke et al., 2005). Cutan is the polymer that remains as the non-depolymerizable fraction after ester-bond hydrolysis of cutin; it is possibly composed of cutin monomers linked by ether and C-C bonds (Kolattukudy, 2001c). 
Table 1.1. Summary of common cutin, suberin and sporopollenin monomers, identified by abundance and functional group for each biopolymer.

\begin{tabular}{|c|c|c|c|}
\hline Common monomer type & Cutin & Suberin & Sporopollenin \\
\hline Unsubstituted fatty acids & $\begin{array}{c}\text { Major } \\
(\mathrm{C} 16, \mathrm{C} 18, \\
\mathrm{C} 18: 1, \mathrm{C} 18: 2) \\
(1-25 \%)\end{array}$ & $\begin{array}{c}\text { Minor } \\
(\mathrm{C} 18-\mathrm{C} 20) \\
(1-10 \%)\end{array}$ & $\begin{array}{l}\text { Major } \\
(\sim \text { C10 })\end{array}$ \\
\hline VLCFA (>C20) & Minor & Major & Major \\
\hline Fatty alcohols $/ \alpha, \omega$-diols & $\begin{array}{l}\text { Minor } \\
(\text { C16:0- } \\
\text { C18:1) } \\
(1-8 \%)\end{array}$ & $\begin{array}{c}\text { Minor } \\
\text { (C18:0-C22:0) } \\
(1-10 \%)\end{array}$ & $\begin{array}{c}\text { Minor } \\
(\mathrm{C} 14-\mathrm{C} 16) \\
(1-3 \%)\end{array}$ \\
\hline$\omega$-Hydroxy fatty acid & $\begin{array}{c}\text { Major } \\
\text { (C16:0-C18:1, } \\
\text { C18:2) } \\
(1-32 \%)\end{array}$ & $\begin{array}{c}\text { Major }^{2} \\
(\mathrm{C} 18: 1 ; \mathrm{C} 16- \\
\text { C26) } \\
(11-43 \%)\end{array}$ & Major \\
\hline$\alpha, \omega$-dicarboxylic fatty acid & $\begin{array}{c}\text { Minor }{ }^{1} \\
\text { (C16:0,C18:0, } \\
\text { C18:1) } \\
(1-5 \%)\end{array}$ & $\begin{array}{c}\text { Major } \\
\text { (C18:1, C18:2; } \\
\text { C16-C26) } \\
(24-45 \%)\end{array}$ & N/A \\
\hline Phenolic & $\begin{array}{c}\text { Minor } \\
(0-1 \%) \\
\text { (ferulate) }\end{array}$ & $\begin{array}{c}\text { Major } \\
(1-10 \%) \\
\text { (mostly } \\
\text { ferulate/ } \\
\text { Smaller } \\
\text { amounts of } \\
\text { coumarate, } \\
\text { caffeate, } \\
\text { sinapate) }\end{array}$ & $\begin{array}{l}\text { Oxygenated } \\
\text { phenyl- } \\
\text { propanoid } \\
\text { (p-coumaric, } \\
\text { caffeic acid) }\end{array}$ \\
\hline & $\begin{array}{c}\text { Major } \\
(1-14 \%)\end{array}$ & $\begin{array}{c}\text { Major } \\
(14-26 \%)\end{array}$ & N/A \\
\hline Funtional group & Cutin & Suberin & Sporopollenin \\
\hline Ketones & $\overline{\text { Yes }}$ & $\mathbf{N} / \mathbf{A}$ & Yes \\
\hline Epoxide & Yes & N/A & N/A \\
\hline Ester & Yes & Yes & Yes \\
\hline Ether & $\mathrm{N} / \mathrm{A}$ & N/A & Yes \\
\hline
\end{tabular}


[1] C16-C18 dicarboxylates are unusually major monomers in Arabidopsis and Brassica napus cutin ( $>50 \%)$

[2] Substituted $\omega$-hydroxy fatty acids such as dihydroxyacids and epoxyacids are more frequently found in cutin, while substituted dicarboxylic acids and $\alpha, \omega$-diols are more frequently found in suberin.

N/A, No information available VLCFA, Very-long-chain (longer than C18) fatty acids

Modified from Pollard et al., (2008)

\subsubsection{Cutin biosynthesis}

The synthesis of all lipid polymers begins with de novo biosynthesis of C16 and C18 fatty acyl-acyl carrier protein (ACP) chains within the plastid by the fatty acid synthase (FAS) complex (Ohlrogge and Jaworski, 1997). This process begins with a condensation reaction between acetyl-coenzyme A $(\mathrm{CoA})$ and malonyl-ACP. Following this condensation, the fatty acyl chain undergoes two separate reduction reactions and a dehydration step, resulting in a fatty acyl chain that is two carbons longer. Subsequent rounds of $\mathrm{C} 2$ additions from malonyl-CoA yield fatty acyl chains that are $\mathrm{C} 16$ and $\mathrm{C} 18$ in length. Once these molecules reach either C16 or C18 in length, the fatty acids are hydrolyzed from the ACP by acyl-ACP thioesterases (e.g. FATB and FATA) and exported from the plastid (Bonaventure et al. 2003). Free fatty acids at the outer envelope of the plastid may be activated through the addition of CoA by a long-chain acyl-CoA synthase (LACS) (Schnurr, et al., 2002). A portion of the $\mathrm{C} 16 / \mathrm{C} 18$ fatty acyl pool is used for plastidial membrane lipid synthesis within the plastid, while the majority is relocated to the ER for modifications (e.g. desaturation, oxidation, elongation). These modifications include the synthesis of cutin and cuticular waxes that make up the cuticle, as well as other membrane and storage lipids (Ohlrogge and Jaworski, 1997). 
A number of enzymes involved in cutin biosynthesis have been identified and characterized in the last decade mostly by studying Arabidopsis mutants with defective cuticle. LCR (for Lacerata) and ATT1 (for $\underline{A b e r r a n t}$ induction of $\underline{\text { Type }}$ Three genes) are two cytochrome P450 monooxygenases (hydroxylases) from the CYP86 family of cytochromes that have been reported to hydroxylate free fatty acids into $\omega$-hydroxy fatty acids or $\alpha, \omega$-dicarboxylic acids. Plants with mutations in these genes show characteristics of cutin defects, such as increased susceptibility to disease in the case of att1 and organ fusions in the case of lcr (Xiao et al., 2004; Wellesen et al., 2001). Two long chain acyl-CoA synthetases, LACS1 and LACS2 have also been shown to be important for cutin synthesis in Arabidopsis. Lacs2 mutants have a thinner cuticle layer than wild-type and exhibit typical features of cutin defects, such as increased chlorophyll leaching (Schnurr et al., 2004), while lacs1 mutants have a less dramatic cutin defect, but are deficient in C16 monomer derivatives (Lü et al., 2009). It has been reported that LACS2 has a higher in vitro activity with hydroxylated fatty acids over non-hydroxylated fatty acids (Schnurr et al., 2004). These LACs acylate hydroxy-fatty acids to provide acyl-CoA substrates for the glycerol-3-phosphate acyltransferase (GPAT) to form the mono-glycerolipids of cutin (Lü et al., 2009). Two of the GPATs, GPAT4 and GPAT8, have been reported to be involved in this latter activity (Li et al., 2007). The gpat4 gpat8 double mutant displays major physiological changes indicative of cuticular defects, such as increased non-stomatal water loss. Panikashvili et al. (2009) reported a cytosolic acyltransferase, DCR (for Defective in Cuticular Ridges), that plays a role in the incorporation of 9,10,16-hydroxy-hexadecanedioic acid into the cutin matrix, as the 
dcr mutant had a marked decrease in this compound. HOTHEAD (HTH) and BODYGUARD (BDG) encode a putative oxidoreductase and carboxyesterase/synthase, respectively, both of which were reported to be involved in cutin biosynthesis, although their roles may be indirect (Kurdyukov et al., 2006a and 2006b). The hth and $b d g$ mutants present organ fusions in flowers and/or leaves.

Cutin monomers constitute a complex network by forming ester linkages between their primary and secondary hydroxyl groups with carboxyl groups. Esters formed between the carboxyl group of one fatty acid and a hydroxyl group of glycerol or a secondary hydroxyl group of another fatty acid allow for a branched structure (Kolattukudy, 2001c; Graca and Lamosa, 2010). The order of events in cutin biosynthesis and the site of polymerization of the cutin precursors has been a major unknown in polyester biosynthesis (Beisson et al., 2012). Recently, tomato cutin synthase (CD1), the first extracellular enzyme forming polyester linkages that are central to the assembly of cutin, was identified (Yeats et al., 2012). CD1 encodes a member of the glycine-aspartic acid-serine-leucine motif lipase/hydrolase (GDSL) family of proteins. This extracellular acyltransferase showed in vitro polyester synthesis activity and was able to catalyze acyltransferase reactions using the substrate 2-mono (10, 16-dihydroxyhexadecanoyl) glycerol, resulting in the formation of oligomers with up to seven dihydroxypalmitoyl groups. The fruit of cutin deficient 1 (cd1) mutant has an extremely thin cuticle and increased sensitivity to water loss and pathogen susceptibility (Yeats et al., 2012). 


\subsubsection{Cuticular wax biosynthesis}

Waxes are an integral part of the cuticle and provide the hydrophobic nature of the polymer. They are found on the outer surface of the cuticle layer, forming epicuticular wax crystals that often macroscopically appear as whitish blooms on plant surfaces (Figures 1 and 2). Cuticular waxes are typically complex mixtures of mostly very-long-chain fatty acid derivatives, such as fatty acids, aldehydes, primary and secondary alcohols, ketones, and alkanes with chain lengths of C20-C36 (Samuels et al., 2008).

Wax components are synthesized in the endoplasmic reticulum (ER) of leucoplasts found in epidermal cells and require the coordinated activity of a large number of enzymes. Wax biosynthesis comprises three distinct stages. First, C16 and C18 fatty acyl-ACPs are synthesized de novo by Fatty Acid Synthase (FAS), a multi-protein complex of four soluble, dissociable enzymes in the stroma of the plastid (Buchanan et al., 2000). These ubiquitous fatty acids serve as central intermediates for all lipid classes, and thus this first stage of wax formation is shared with other lipid biosynthetic processes. The second stage of the biosynthetic pathway involves the elongation of $\mathrm{C} 16$ and $\mathrm{C} 18$ fatty acids in the endoplasmic reticulum (ER). For further extension of $\mathrm{C} 16-\mathrm{C} 18$ fatty acids, it is cleaved from ACP by an acyl-ACP thioesterase within the plastid and the exported acyl chain is esterified to Coenzyme A ( $\mathrm{CoA})$, forming acyl-chain CoA thioesters by a long-chain acyl-CoA synthetase (LACS). The fatty acyl-CoAs can then undergo further elongation in the ER into very-long-chain fatty acids (VLCFAs) of C20-C34 chains. This is catalyzed by a multienzyme system known as Fatty Acid Elongase (FAE), 
which is localized to the ER membrane. The FAE is composed of four enzymes in series: 1) the 3-ketoacyl-CoA synthetase (KCS) that condenses a 2-carbon unit from malonyl-CoA to a long-chain acyl-CoA, producing 3-ketoacyl-CoA, 2) the 3-ketoacylCoA reductase (KCR) that reduces 3-ketoacyl-CoA, resulting in 3-hydroxyacyl-CoA, 3) the 3-hydroxyacyl-CoA dehydratase that dehydrates 3-hydroxyacyl-CoA, giving rise to trans-2,3-enoyl-CoA, and 4] the trans-2-enoyl-CoA Reductase that reduces trans-2,3-enoyl-CoA, and generates a fully reduced and saturated very-long-chain acyl-CoA that is two carbons longer than when it entered the FAE (Kunst and Samuels, 2003; Franke and Schreiber, 2007; Samuels et al., 2008; Franke et al., 2009; Lee et al., 2009). Wax components have chain-lengths varying from C22 to C36 suggesting that VLCFA precursors might result from a series of elongase complexes (Beittenmiller, 1996). The extent of elongation of the long-chain fatty acyl-CoAs is controlled by the $\beta$-ketoacyl-CoA synthase (KCS), the first enzyme in the FAE complex (Miller and Kunst, 1997). Based on the expression and phenotype, CER6 is the only KCS gene reported as strictly wax-specific (Millar et al., 1999; Hooker et al., 2002; Bernard and Joubes, 2013). In the final stage, derivatization of VLCFAs to other waxes occurs in the ER; a proportion of the elongated acyl-CoAs are modified into primary and secondary alcohols, wax esters, aldehydes, alkanes, and ketones (Beittenmiller, 1996).

In Arabidopsis thaliana stems, a proportion of the elongated fatty acyl-CoAs enter (1) an alkane-forming (or decarbonylation) pathway that produces aldehydes, alkanes, secondary alcohols and ketones, while a smaller proportion enters (2) an acyl reduction pathway that produces primary alcohols and wax esters (Kunst and 
Samuels, 2003; Jetter et al., 2006). In some plants, the elongated fatty acid precursors can enter another less widespread pathway, the, $\beta$-ketoacyl elongation pathway, which produces $\beta$-diketones, methylketones and alkan-2-ol acyl esters (Kunst and Samuels, 2003). The alkane-forming pathway leads to the formation of predominantly odd-chain alkanes along with secondary alcohols and ketones of similar chain length distribution. Arabidopsis cuticles are dominated by nonacosane (C29 alkane), which together with its secondary alcohol and ketone derivatives, accounts for $\sim 80 \%$ of the stem cuticular wax content (Jetter et al., 2006).

It has been proposed that very-long-chain (VLC) fatty acyl-CoAs are converted into aldehydes by an aldehyde-forming fatty acyl-CoA reductase (Vioque and Kolattukudy, 1997), followed by decarbonylation by an aldehyde decarbonylase to yield odd-chain length alkanes (Cheesborough and Kolattukudy, 1984; Bernard and Joubes, 2013). The alkanes are then sequentially oxidized into secondary alcohols by an alkane hydroxylase and then to ketones (Samuels et al. 2008). Based on the biochemical phenotypes of eceriferum 1 (cer1) and cer3 mutants, the Arabidopsis CER1 and CER3 proteins have been proposed to play an essential metabolic role in wax alkane biosynthesis (Kunst and Samuel, 2003). Recently, biochemical confirmation of the role of CER1 in alkane biosynthesis was provided by reconstitution of the plant alkane biosynthetic pathway in yeast (Bernard et al. 2012). Heterologous coexpression of CER 1 and CER3 in a yeast strain producing VLC acyl-CoAs resulted in VLC alkane synthesis, which was further enhanced in the presence of cytochrome b5 proteins (CYTB5s) (Bernard et al. 2012). This also suggested that synthesis of VLC alkanes in plants is a redox-dependent process 
similar to the alkane formation in cyanobacteria (Schirmer et al., 2010). Based on site-directed mutagenesis studies and by analogy to the alkane biosynthesis pathway described in cyanobacteria, it is speculated that CER3 may function as an aldehyde-forming acyl-COA reductase, and CER1 as an aldehyde decarbonylase, although there is no direct evidence for that exists (Sakuradani et al., 2013). Midchain Alkane Hydroxylase 1 (MAH1) enzyme, a cytochrome P450 hydroxylase, was identified that performs both the hydroxylation of odd-chain alkanes to secondaryalcohols and their further oxidation to ketones (Greer et al., 2007). The beginning steps of this pathway, however, are still not well understood.

The acyl reduction pathway (alcohol-forming), on the other hand, produces derivatives with even numbers of carbons. The VLC fatty acyl-CoAs are reduced to primary alcohols by a single alcohol-forming fatty acyl-CoA reductase (FAR3/CER4) in a NADPH requiring reaction (Vioque and Kolattukudy, 1997, Metz et al., 2000, Rowland et al., 2006). Some of the primary alcohols are then esterified with long chain fatty acids through the action of a wax synthase WSD1 to form wax esters (Lardizabal et al., 2000; Li et al., 2008). All primary alcohols reported to occur in Arabidopsis cuticular wax are saturated with chain lengths of 22 to 34 carbons and predominant chain lengths of 26 to 30 carbons (Jenks et al., 1995; Rowland et al., 2006; Lai et al., 2007).

A portion of VLC fatty acyl-CoAs enter neither of these two pathways and are cleaved to form free fatty acids, which make-up approximately $3.2 \%$ of the total cuticular wax in Arabidopsis stems (Jenks et al., 1995; Pollard et al., 2008). No thioesterase enzyme responsible for the synthesis of VLC free fatty acids has been 
identified. It is notable, however, that CER8/LACS1 activates VLC free fatty acids with CoA to form very-long-chain fatty acyl-CoAs and is highly active for C30 acyl chains (Lü et al., 2009). These very-long-chain fatty acyl-CoAs can then be either further elongated or modified by one of the two primary wax biosynthetic pathways into other cuticular wax compounds (Lü et al., 2009). The balanced activities of the thioesterase and synthetase enzymes may be an important control step in dictating the proportions of VLC free fatty acids and VLC acyl-CoAs that can be further metabolized.

\subsubsection{Sporopollenin}

Microsporogenesis and pollen development are complex processes that take place during flower development, starting with the initiation of stamen primordia and leading to the formation of two microgametes per mature pollen grain $\mathrm{CMa}$, 2005). A critical event during pollen maturation is the formation of the pollen surface structure. The pollen wall consists of two layers: the intine and the exine. The intine is mostly synthesized by the haploid microspore itself. However, the tapetal cells are responsible for the production and secretion of the exine, which is a mixture of protein, lipids and aromatic molecules (Sanders et al., 1999; Ma, 2005).

A major component of the exine layer is termed sporopollenin, which makes up the protective layer that protects pollen grains from desiccation and makes them extremely resistant to degradation (Piffanelli et al., 1998). It is a very durable biopolymer and is considered "the most resistant organic material known"(Brooks and Shaw, 1978). This substance is extremely resistant to physical, biological and chemical degradation procedures, including acetolysis. Because of this resistance, its 
definitive chemical structure and the biochemical pathways involved in its synthesis remain poorly characterized (Dominguez et al., 1999; Barrena and Wilson, 2006).

\subsubsection{Sporopollenin composition}

NMR studies have demonstrated that sporopollenin is a complex biopolymer derived mainly from saturated long-chain fatty acids and derivatives as well as phenolics (Scott, 2004; Souza et al., 2009). The chemical composition shows that fatty acid elongation (FAE) systems are involved in sporopollenin synthesis, and that sporopollenin and waxes share certain biosynthetic enzymes (Ariizumi and Toriyama, 2011). Short carbon chain $\alpha$, $\omega$-dicarboxylic acids (C6-C9) were found as major components of sporopollenin from Pinus pinaster, Ambrosia elatior and Capsicum annuum, representing $28.8 \%, 63.2 \%$ and $88.5 \%$, respectively, of the total compounds obtained (Domniguez et al., 1999). Available data indicates that the sporopollenin polymer consists of fatty acid derivatives and poorly characterised

phenolic constituents that are covalently coupled by ether and ester linkages (Scott et al., 2004; Ariizumi and Toriyama, 2011).

\subsubsection{Sporopollenin biosynthetic enzymes}

One of the molecular mechanisms in sporopollenin synthesis first came to light through identification of the Arabidopsis male sterility2 (ms2) mutant, which produces nonviable pollen with no sign of the exine layer (Aarts et al., 1993, 1997). The MS2 protein sequence is similar to ECERIFERUM4 (CER4), which encodes a fatty acyl reductase (FAR) (Rowland and Domergue, 2012). MS2 is a plastidlocalized fatty acyl-ACP reductase that is specific to C16:0-ACP (Chen et al., 2012), while CER4 is a fatty acyl-CoA reductase generating C24:0-C30:0 primary alcohols 
(Rowland et al., 2006). The fact that cer4 mutants have normal pollen exine structure, suggests that very-long chain length alcohol groups are not used for synthesis of sporopollenin precursors. A loss of function mutation of the FACELESS POLLEN1/WAX2/YRE/CER3 gene results in defects in both pollen exine and epicuticular wax deposition (Ariizumi et al., 2003; Rowland et al., 2007; Bernards et al., 2012). FACELESS POLLEN1/WAX2/YRE/CER3 is a membrane-bound protein involved in aldehyde decarbonylation, producing alkanes, although the actual biochemical activities of the protein are currently undefined (Ariizumi et al., 2003; Bernard et al., 2013). Disruption of the Arabidopsis ACYL-CoA SYNTHETASE 5 (ACOS5) gene drastically hampers exine formation (Souza et al., 2009). ACOS5 functions as a medium- to long-chain (C8-C16) fatty acyl CoA-synthetase with a substrate preference for substituted fatty acids carrying an omega-hydroxy group (Souza et al., 2009). This enzymatic reaction produces the associated CoA-esters from hydroxylated fatty acids. Recently, Arabidopsis genes encoding the cytochrome P450 enzymes CYP704B1 and CYP703A2 have been shown to be involved in the biosynthesis of sporopollenin, since mutants show moderate to severe defects in exine deposition and pollen grain development (Morant et al., 2007; Dobritsa et al., 2009). In vitro assays indicate that these enzymes catalyze hydroxylation of medium- to long-chain fatty acid substrates. While CYP703A2 is an in-chain hydroxylase with substrate preference for lauric acid (C12:0), CYP704B1 catalyzes the w-hydroxylation of long-chain (C16 and C18) fatty acids (Morant et al, 2007; Dobritsa et al., 2009). 


\subsubsection{Suberin}

Suberin is another complex polyester matrix that acts as a barrier between plants and the environment. But unlike cutin, it is deposited within the primary cell wall of suberized cells (Figure 1.1, 1.3). It is often observed under transmission electron microscopy as alternating light and dark bands (lamellae). The location of suberin deposition in plants is highly variable. The tissue distribution of suberized cell walls in plants suggests that suberization occurs wherever and whenever a plant needs to form a barrier (Kolattukudy, 2001a, 2001b; Franke and Schreiber, 2007). Suberin is constitutively present in the secondary growth periderm and wound periderm of aerial and root tissues. In primary roots, suberin depositions also occur in endodermal cell walls (ECW), separating the vascular tissue of the central cylinder from the root cortex, and in peridermal cells, rhizodermal and hypodermal cell walls (RHCW), building the interface to the soil environment (Franke and Schreiber, 2007). Exodermis, which forms a continuous tangential layer of rectangular, radially flattened cells outside of the phloem but usually inside the epidermis is also characterized with suberin lamellae (Kolattukudy, 1980). A familiar example of suberized tissue is cork periderm in the outer bark cells (phellogen) of cork oak (Quercus suber) (Soler et al., 2007). It is also deposited in the seed chalazae micropyle region, the abscission zone of petals, and in the bundle sheaths of C4 plants (Kolattukudy, 1981, Beisson et al., 2007). Suberin is also deposited in response to wounding and pathogen attack (Kolattukudy, 2001a). 


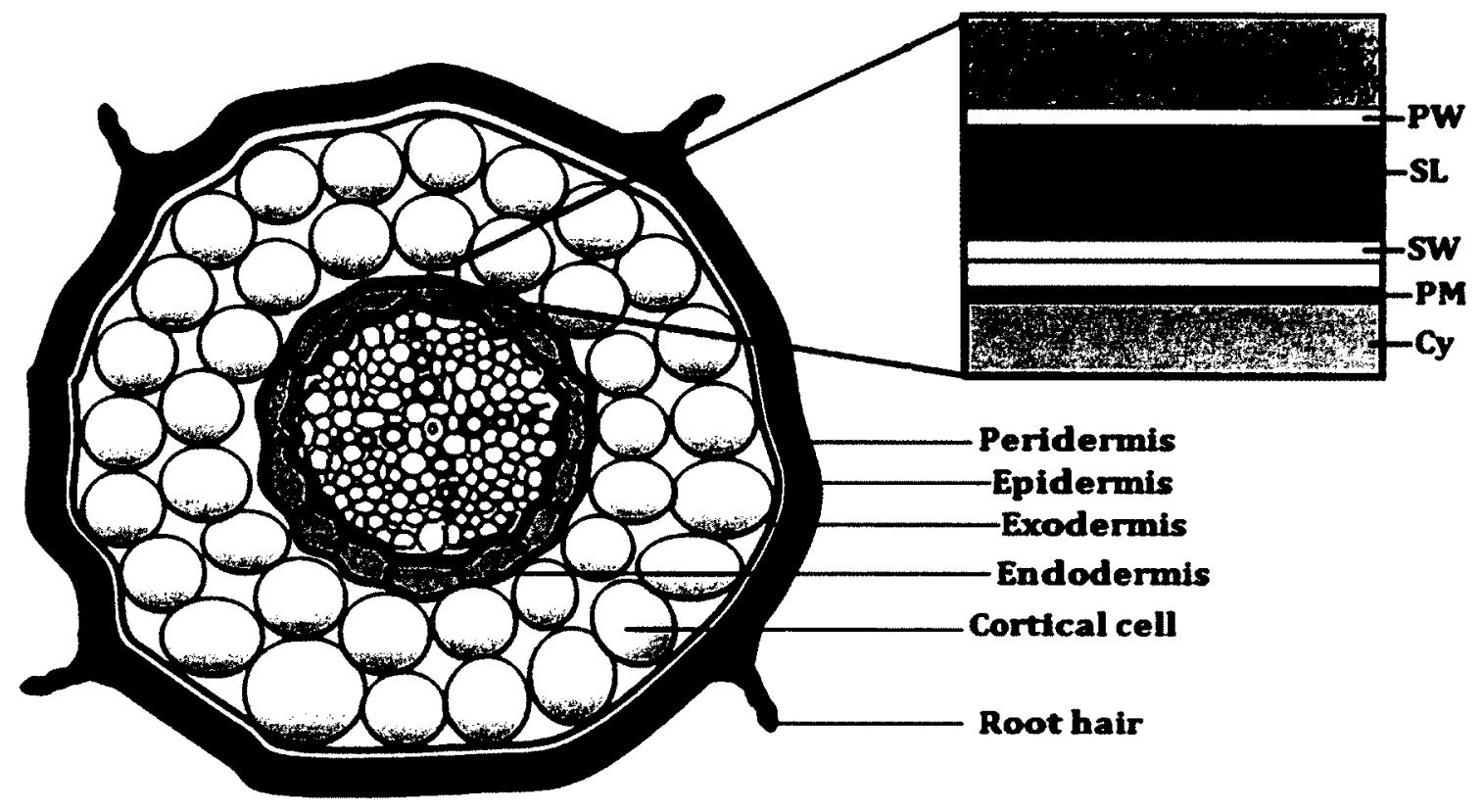

Figure 1.3. Schematic of a matured root cross section. Suberized endodermal cells present the typical lamellar deposition within the cell walls (inset). CW-Cell wall, PW-Primary wall, SL- Suberin lamellae, SW-Secondary wall, PM-Plasma membrane, Cy-Cytoplasm. Red dot represents Casparian band. Figure by Sollapura J. Vishwanath. 


\subsubsection{Functions of suberin}

Plants synthesize suberin to create a hydrophobic barrier to water and solute diffusion through cell walls during normal development, or to provide a barrier in response to environmental stresses. In roots, there are three parallel, radial transport pathways: 1) apoplastic - within cell walls and lumens of dead cells, 2) symplastic - via cytoplasms of neighboring cells connected by plasmodesmata, and 3) transcellular - via cell cytoplasms and vacuoles. In the absence of wall-modifying structures, radial transport of dissolved solutes occurs mainly through the apoplast, while the flow of water is through all three paths (Steudle and Peterson, 1998). The symplastic and transcellular pathways are often collectively referred to as the cellto-cell pathway. Exodermal and endodermal cell layers (the outermost and innermost layers of the root cortex, respectively) have a pronounced ability to limit apoplastic solute and water transport due to their characteristic cell wall-modifying structures (Figure 1.4). These structures are the Casparian bands which limit the apoplastic flow of solutes (Enstone et al., 2003) and the suberin lamellae which may limit the transcellular flow of water and solutes (Hose et al., 2001; Franke and Schreiber, 2007). Suberin deposition in the endodermis of roots has been proposed to significantly reduce uncontrolled transport of water and dissolved ions (e.g. nutrients and toxins) through the apoplast, and is thought to be also crucial for selective nutrient uptake and exclusion of pathogens (Baxter et al., 2009). Correlative permeability studies carried out between Col-0 and suberin mutants (horst1, esb1) revealed that the amount of root suberin negatively correlating with 
permeability is not always true, but rather the aliphatic monomer arrangement in the suberin biopolymer and its microstructure is key to apoplastic barrier formation (Ranathunge and Schreiber, 2011). Recently, Naseer et al., (2012) reported the presence of functional Casparian strips in Arabidopsis plants devoid of any detectable suberin, and confirming the presence of archetypal lignin in Casparian strips using lignin-specific chemical analysis. Suberin deposition was found to occur later. 


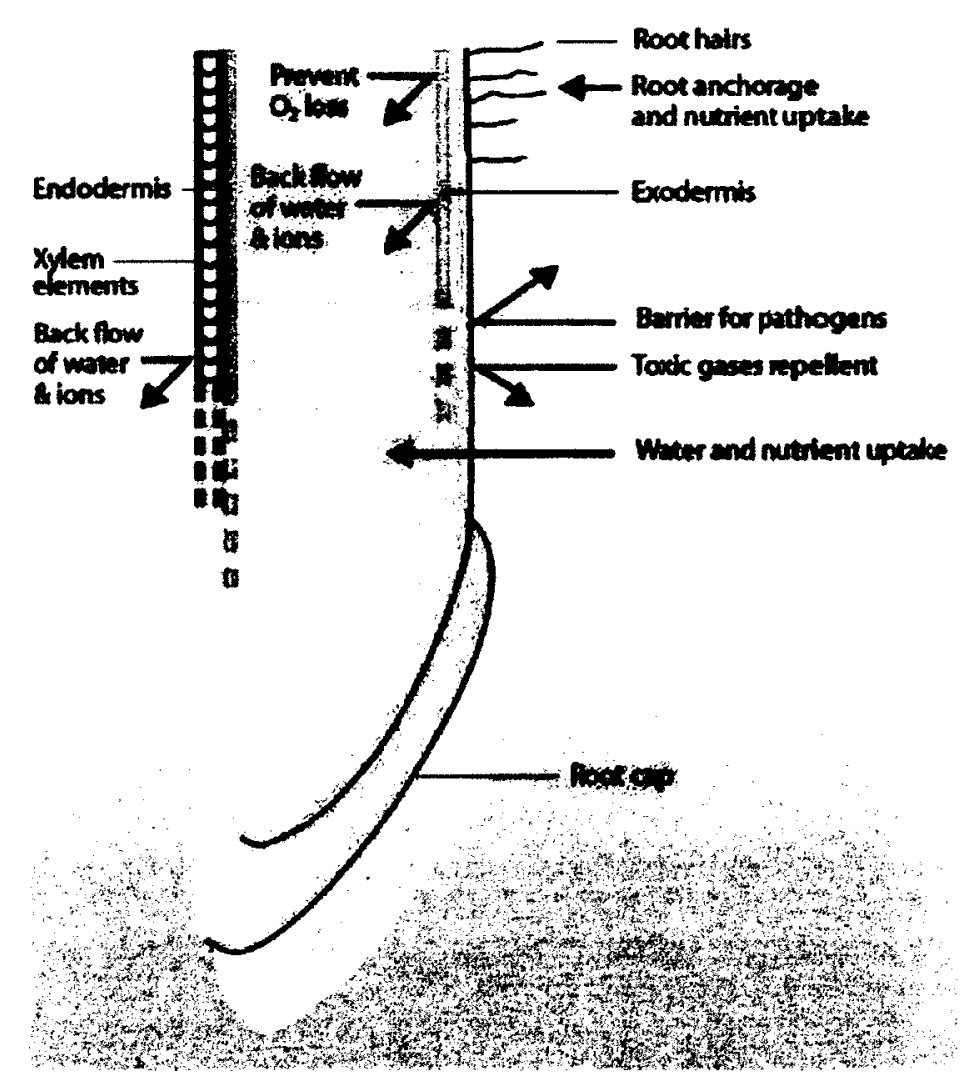

Figure 1.4. Locations of suberized barriers in a typical root and their functions. Schematic diagram of longitudinal view of a root (not to a scale) showing the locations of suberized barriers (shown as yellow band) such as endo- and exodermis with their typical functions. (Adapted from Ranathunge et al., 2011) Image reproduced with permission (C ASPB, 2011. 
Suberin plays an important role in protecting plants from various stresses (Figure 1.4). It is deposited in the peridermis of matured roots and underground storage tubers, which is the direct interface between plant and soil environment and thus it protects plants from biotic and abiotic stresses such as exposure to cold, mineral stress and microbial infection. For example, potato (Solanum tuberosum) with an immature or wounded periderm is susceptible to infection and skinning (excoriation of the skin), making the crop vulnerable during harvest and postharvest storage, potentially causing significant economical losses (Lulai and Corsini, 1998; Lulai and Freeman, 2001; Sabba and Lulai, 2002; Schreiber et al., 2005c).

Epidermal cells that normally synthesize cutin can also deposit suberin in response to wound injury to seal tissues and preventing loss of solutes and further infection. In mature seeds, suberin has been found in seed coat layers and sealing off the chalazal region of the inner seed coat after disconnection from vascular tissue (Beisson et al., 2007; Ranathunge et al., 2011). Heavily suberized phellem minimizes mechanical freezing injury to grapevines by maintaining low, constant moisture content in tree trunks through the winter (Meiering et al., 1980). It has been postulated that suberin lamella represent distinct polyaliphatic and polyaromatic domains, each of which has a separate role in the overall barrier property of the polymer. The proposed suberin poly-aliphatic domain (SPAD; after Bernards, 2002) due to its lipophilic nature and intercalated waxes, would be important for restricting radial water and solute transport (Kolattukudy and Dean, 1974; Soliday et al., 1979; Zimmermann et al., 2000; Hose et al., 2001; Schreiber et al., 2005a). The proposed suberin poly-phenolic domain (SPPD; after Bernards, 2002), similar in 
chemical composition to lignin, consists of aromatic compounds that are linked covalently to primary cell wall carbohydrates. Such features would make the SPPD important for limiting the penetration of pathogenic bacteria, fungi, and oomycetes (Kolattukudy, 1980; Lulai and Corsini, 1998). However, the SPAD/SPPD model may not be accurate and further structure/function studies need to be carried out.

\subsubsection{Suberin composition}

Suberin is glycerol-containing polymer consisting of polyaliphatic polyester associated with aromatic components and embedded waxes (Kolattukudy, 1981; Bernards, 2002; Nawrath, 2002; Franke et al., 2007; Pollard et al., 2008) (Figure 1.1c). The aromatic component is derived from the phenylpropanoid pathway and mainly consists of ferulates ( $p$-hydroxycinnamic acid derivatives) and monolignols (Bernards et al., 1995; Kolattukudy, 2001b, Franke et al., 2009). The aliphatic portion is comprised of mainly $\omega$-hydroxy fatty acids, $\alpha, \omega$-dicarboxylic acids, midchain oxygenated fatty acids, unsubstituted fatty acids, and primary fatty alcohols (Pollard et al., 2008; Kolattukudy, 2001b), along with glycerol (Moire et al., 1999). Aliphatic acids have dominant chain lengths of $16,18,20$ and 22 carbons, of which the C16 and C22 are mostly saturated, while the C18-compounds have mid-chain unsaturation, hydroxy or epoxy groups (Graca and Santos, 2006). Considerable variability is observed in overall composition and relative amounts of suberin monomers between plant species (Matzke and Riederer 1991; Zeier et al., 1999). Glycerol in the suberin matrix is found esterified to $\omega$-hydroxy and $\alpha, \omega$-dicarboxylic fatty acids (Graça and Pereira, 2000; Graça and Santos, 2006). The overall 
organization of the inter-unit linkages, including those involving unsubstituted fatty acids and primary fatty alcohols, remains to be determined.

\subsubsection{Suberin structure}

Because suberin is an insoluble heteropolymer deposited within the plant cell wall, the native polymeric structure of suberin still remains unresolved. Proposed three-dimensional arrangements based on monomer composition remain largely speculative. Complex procedures must be used to study suberin composition since this polymer is very resistant to enzymatic and chemical degradation (Ranathunge et al., 2011). Monomers are traditionally quantified and identified using gas chromatography coupled with mass spectrometry. The mature polyaliphatic polyester is subjected to depolymerisation followed by transesterification using acid (eg. methanolic $\mathrm{HCl}$ ) or base [e.g. methanolic sodium methoxide (NaOMe)] yielding methyl esters of the monomers (Figure 1.5) (Browse et al., 1986). The released monomers are then usually derivatized with BFTSA ( $N, O$ bis(trimethylsilyl)-trifluoroacetamide) or other sylylating reagents to convert hydroxyl groups to the corresponding trimethylsilyl derivatives, and these are subjected to gas chromatography/mass spectrometry (GC-MS). Upon transesterification of suberin, the common monomers released include $C_{16}-C_{28} \omega$ hydroxy fatty acids and $\mathrm{C}_{16}-\mathrm{C}_{26} \alpha, \omega$-dicarboxylic acids, which are diagnostic monomers of suberin, and also unsubstituted very-long-chain fatty acids (VLCFAs; > $\mathrm{C}_{18}$ ), C18:0-C22:0 primary fatty alcohols, glycerol and ferulate (Table 1.1, Figure 1.5). 
Models proposed to explain the macromolecular structure of suberin have been based on data generated by partial or incomplete depolymerisation of tree bark and tuber periderms, as well as solid state ${ }^{13} \mathrm{C}$ NMR spectroscopy of native and wound periderm of potato (Ranathunge et al., 2011). Identified structures in partial depolymerisation experiments included dimers of glycerol linked to $\omega$-hydroxy acids, $\alpha, \omega$-dicarboxylic acids (Graca and Pereira, 2000; Graca and Santos, 2006), ferulic acid linked to $\omega$-hydroxy acids (Graca and Pereira, 2000), and trimeric diesters built of glycerol linked to two $\alpha, \omega$-dicarboxylic acids or ferulic acid linked to $\omega$-hydroxy acids linked to glycerol (Graca and Santos, 2006). Long chain aliphatics, cross-linked phenolics, and cell wall polysaccharides were reported to be covalently linked to each other (Stark et al., 1994). The phenolics were assumed to be linked to the primary cell wall and hydroxycinnamic acids are proposed to covalently link the aliphatic suberin polyester to the cell wall, possibly with some monolignols (Kolattukudy, 2001b; Bernards, 2002). In these models, the aliphatic monomers are linearly arranged, likely forming the electron-translucent bands. Ferulic acid has been proposed to provide a covalent link between cell-wall embedded polyaromatics and the suberin polyester. A recent macromolecular structure model for suberin proposed that the monomeric blocks contain long-chain $\alpha, \omega$ dicarboxyolic acids esterified to glycerol at both ends as the core of the suberin macromolecule (Franke and Schreiber, 2007). These polyol glycerols would provide a cross-linked two- and three-dimensional network by the formation of ester linkages to additional $\alpha, \omega$-dicarboxylic acids and $\omega$-hydroxy fatty acids, leading to an insoluble polymer (Franke and Schreiber, 2007). The presence of glycerol in 
suberin with its three esterifying position, may generate a significantly cross-linked matrix (Beisson et al., 2007; Li et al., 2007b).

Bernards proposed suberin to be composed of two spatially distinct but covalently-linked domains; the poly(phenolic) domain (SPPD) embedded in the primary cell wall, and the poly(aliphatic) domain (SPAD) (Figure 1.6) (Bernards, 2002). When observed under a transmission electron microscope, SPAD in the model would represent the lamellar structure with alternating bands of translucent, electron-light (aliphatic-rich) and opaque, electron-dense (aromatic or ester linkage-rich) compounds. The nature of the opaque bands is less understood and it is thought that these may correspond to glycerol, esterified phenolics, and possibly waxes (Bernards, 2002). However, the compositional data and phenotypes of recently characterized Arabidopsis knockout mutants does not support this model. For example, the number of monofunctional aliphatics (acids and alcohols) exceeds the number of bifunctional monomers ( $\omega$-OHAs and $\alpha, \omega$-DCAs) in the seed coat polyester of the cyp86b2 mutant (Molina et al., 2009; Campagnon et al, 2009). Further, insertion mutants in ASFT/AtHHT and FHT with major reductions in esterlinked ferulic acid in the suberin polyesters, are not affected the insolubility of suberin nor the lamellar appearance observed by TEM (Molina et al., 2009; Serra et al., 2010). This contradicts the proposed role of ferulic acid in linking the polyaromatic with the aliphatic domain, as well as the presence of an extended aliphatic polyester. 

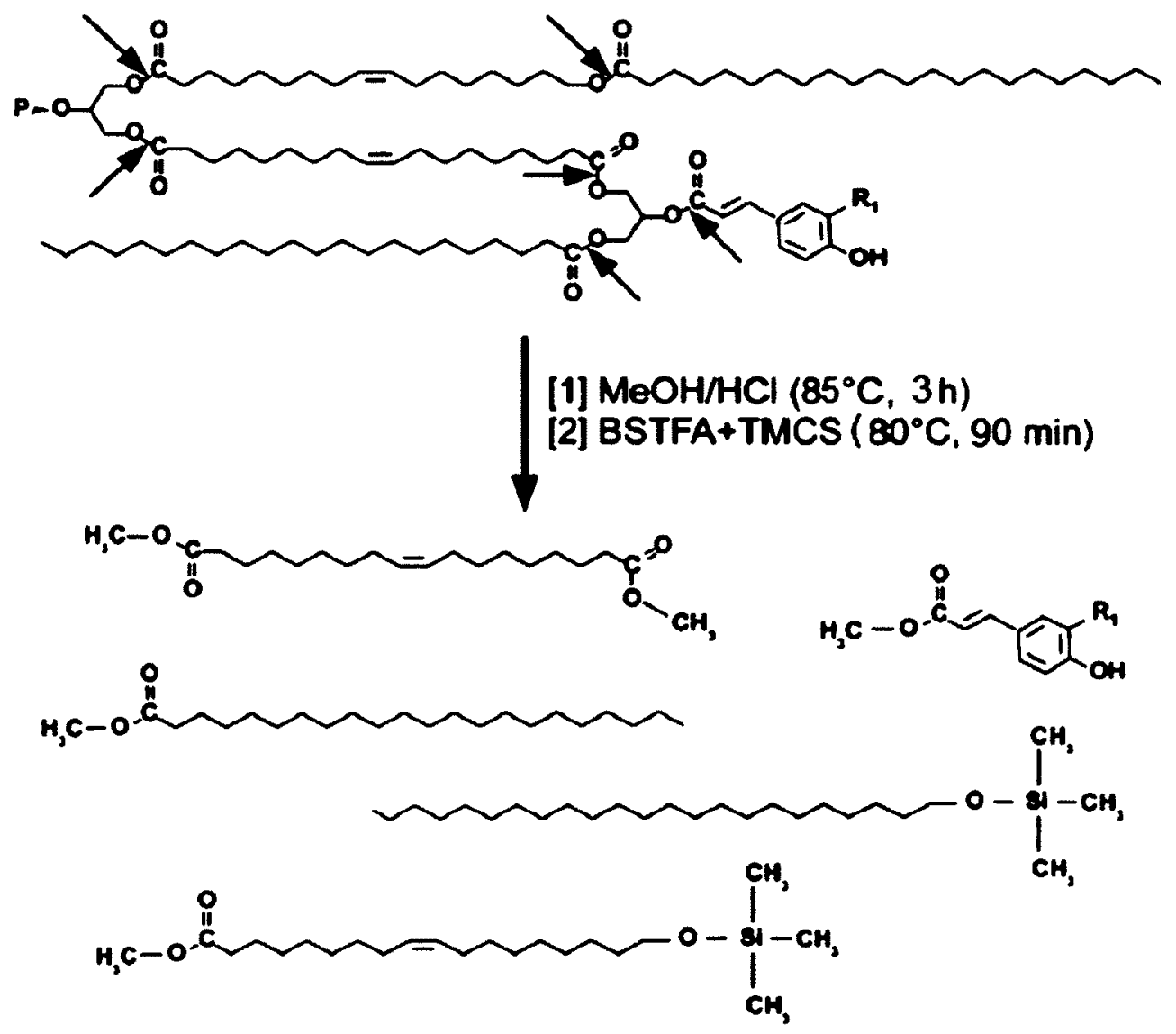

Figure 1.5. Transesterification of the suberin using $\mathrm{MeOH} / \mathrm{HCl}$. The polymeric structure is hypothetical and is based on the typical products that are released. Aliphatic monomers released are analyzed as methyl ester or trimethylsilyl ether derivatives. Modified from Browse et al., (1986). 


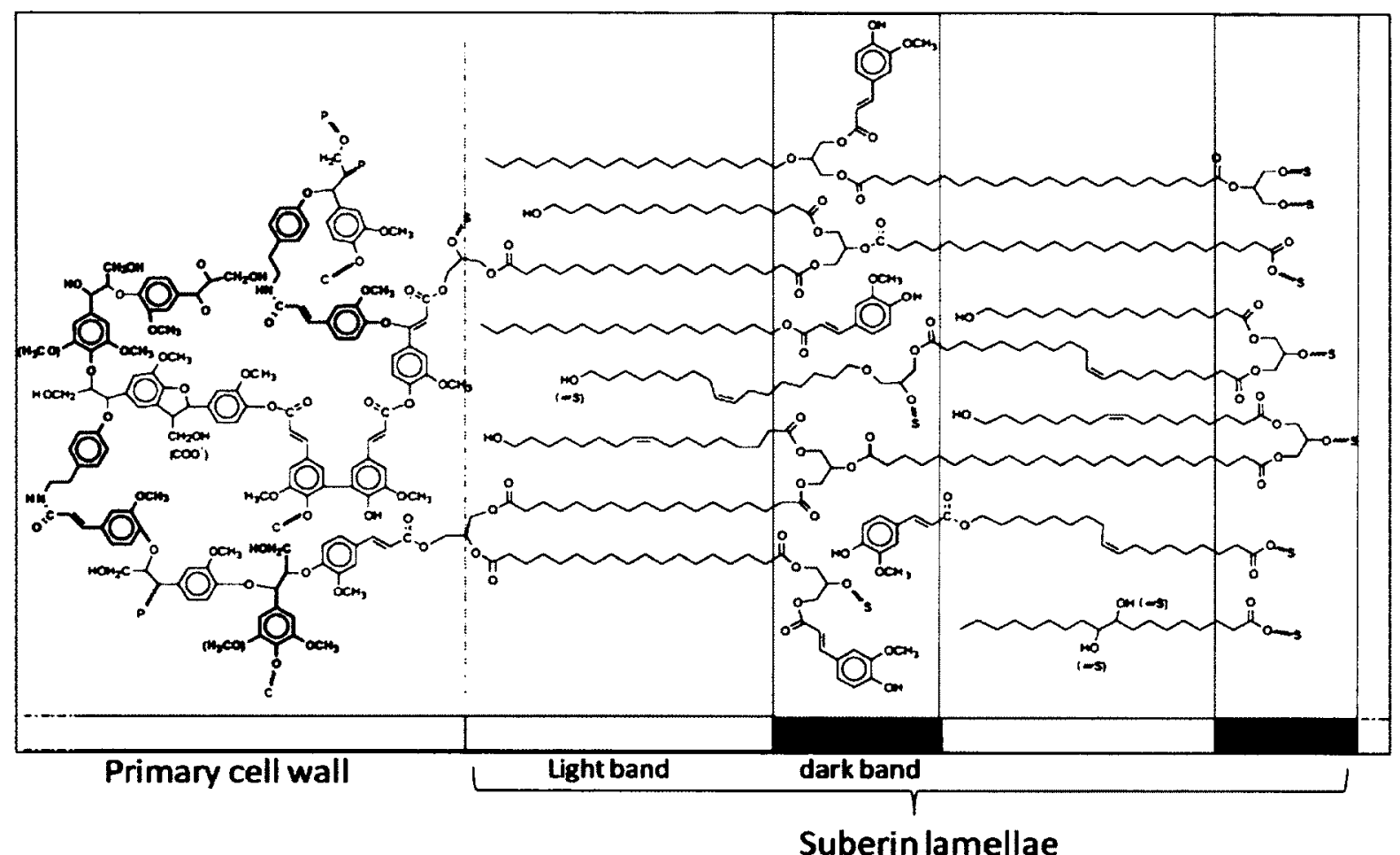

Figure 1.6. Bernards (2002) model of suberin lamellae. The poly(phenolic) domain is attached to carbohydrates in primary cell wall. The glycerol based poly(aliphatic) domain is located between the cell wall and plasma membrane. Light bandAliphatics; Grey-shading = dark regions rich in phenolics and/or ester-linkages. The light plus dark bands is about 3-4 $\mathrm{nm}$ in this model. Adapted from Bernards (2002). Image reproduced with permission $\mathcal{C} 2002$ NRC, Canada. 


\subsubsection{Suberin biosynthesis}

\subsection{Aliphatic monomer biosynthesis}

The biosynthetic routes for the major aliphatic monomers of suberin have been studied in the past using incorporation of labeled oleic acid or acetate in wound-healed potato slices (Dean and Kolattukudy, 1977). Aliphatic monomers are mostly derived from 16:0 and 18:1 fatty acyl-CoAs. Briefly, aliphatic suberin monomer production begins with the plastidic synthesis of saturated C16 and C18 fatty acids driven by fatty acid synthases. A part of these fatty acids are desaturated and $\omega$-hydroxylated into $18: 1 \omega$-hydroxy fatty acids. Fatty acid $\omega$-hydroxylation is catalyzed by NADPH-dependent cytochrome P450 monooxygenases (P450) from the CYP86 and CYP94 families (Soliday and Kolattukudy, 1979; Duan and Schuler 2005). The $\omega$-hydroxy fatty acids can then be oxidized into $18: 1 \alpha$, $\omega$-dicarboxylic acids by an w-hydroxy fatty acid dehydrogenase (Agrawal and Kolattukudy, 1978a, 1978b; Kurdyukov et al., 2006a). A sub pool of the C18 fatty acids is elongated to C20-C28 by means of a microsomal malonyl-CoA dependent pathway (Schreiber et al., 2005a). This pathway is catalyzed by a fatty acid elongation complex (FAE) located in the endoplasmic reticulum (described above). The non-elongated and elongated chains are either reduced into primary alcohols, or oxidized into $\omega$ hydroxy and 2-hydroxy fatty acids (Figure 1.8).

\subsection{Aromatic monomer biosynthesis}

The suberin phenolic domain consists of monolignols ( $p$-coumaryl, coniferyl and sinapyl alcohols), hydroxycinnamic acids ( $p$-coumaric, caffeic, and ferulic acids), 
ferulic acid esters of long-chain alcohols (ferulates), and $\mathrm{N}$-feruloyltyramine (Bernards, 2002). Hydroxycinnamates (C-C and ether-linked hydroxycinnamic acids) are thought to play an important role in suberin structure and its linkages to the other components of suberized cell walls (Graca, 2010). Partial depolymerization analysis have revealed feruloyl esters of $\omega$-hydroxy acids and glycerol (Graca and Pereira, 2000), in addition to dimeric hydroxycinnamates and dimeric structures of ferulic acid linked to hydroxycinnamic amines (Graca, 2010). As these compounds are directly bound to the aliphatic polyester of suberin, they are suggested to act as cross-linkers between the polyaliphatic domain and the polyaromatic domain (Graca and Pereira, 2000; Bernards, 2002; Graca and Santos, 2007).

The phenolics are mainly hydroxycinnamic acid derivatives with less relative amounts of monolignols and tyramine (Figure 1.7; Borg-Olivier and Monties, 1993; Bernards et al., 1995; Negrel et al., 1996). It is known that the synthesis of phenolic compounds is initiated in the shikimate pathway, which yields phenylalanine. Phenylalanine is the precursor for most phenylpropanoids including hydroxycinnamic acids. The processing of hydroxycinnamic acids involves hydroxylation at the hydroxycinnamoyl-CoA derivative level (Dixon et al., 2001) and methylation by a suberin-specific $o$-methyltransferase (Held et al., 1993), giving rise to key aromatic monomers including $p$-coumaric acid, caffeic acid, ferulic acid, and their amide derivatives (Figure 1.7; Bernards, 2002). 
<smiles>[R2]c1cc(/C=C/C(=O)[O-])cc([R2])c1O</smiles>

$\mathrm{R} 1=\mathbf{R} 2=\mathrm{H}, \mathrm{p}$-Coumaric Acid R1 $=\mathrm{OH}, \mathrm{R2}=\mathrm{H}$, Caffeic Acid $\mathrm{R} 1=\mathrm{OCH} 3, \mathrm{R} 2=\mathrm{H}$, Ferulic Acid R1=R2=0CH3, Sinapic Acid

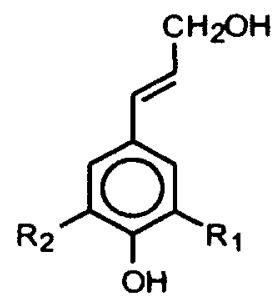

$\mathbf{R} 1=\mathbf{R} 2=\mathbf{H}, \mathbf{p}$-Coumaryl Alcohol R1 $=\mathbf{O C H} 3, \mathrm{R} 2=\mathrm{H}$, Coniferyl Alcohol R1 $=$ R2 $=0$ CH3, Sinapyl Alcohol

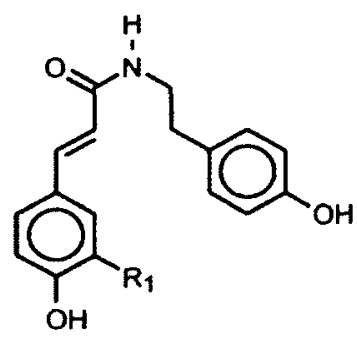

R1=H, p-Coumaroyltyramine R1 $=$ OCH3, Feruloyityramine

Figure-1.7. Phenolic precursors of suberized tissues. Adapted from Bernards (2002). Image reproduced with permission (C) 2002 NRC, Canada. 
Analogous to the oxidative cross-linking model accepted for lignification, it has been hypothesized that the phenolic component of suberized cell walls is polymerized via a peroxidase $/ \mathrm{H}_{2} \mathrm{O}_{2}$-mediated process (Kolattukudy, 1980; Nawrath, 2002). An anionic peroxidase isoform of potato involved in the polymerization of the aromatic component during wound-induced suberization was found to have a preference for feruloyl (o-methoxyphenol)-substituted substrates (order of substrate preference: feruloyl $>$ caffeoyl $>$ p-coumaryl $\sim$ syringyl) such as those that accumulate in tubers during wound healing (Espelie and Kolattukudy, 1985; Bernards et al., 1999).

\subsection{Suberin biosynthetic enzymes}

The development of analytical and quantitative analysis (GC-MS) of lipid polymers, Arabidopsis functional genomics, and the isolation of several key Arabidopsis mutants with altered suberin composition have been key for identifying several enzymes involved in suberin biosynthesis. Biosynthetic pathways have been proposed that require oxidation (e.g. $\omega$-carbon oxidation, oxidation of $\omega$-hydroxy fatty acids to $\alpha, \omega$-dicarboxylic acids), elongation of long-chain fatty acid precursors, activation of fatty acids to fatty acyl-CoA thioesters, reduction of acyl chains to primary alcohols, various acylations largely involving glycerol, aromatic compound incorporation, and polymerization of monomers (Kolattukudy, 1981; Bernards, 2002; Franke and Schreiber, 2007, Pollard et al., 2008, Ranathunge et al., 2011). 


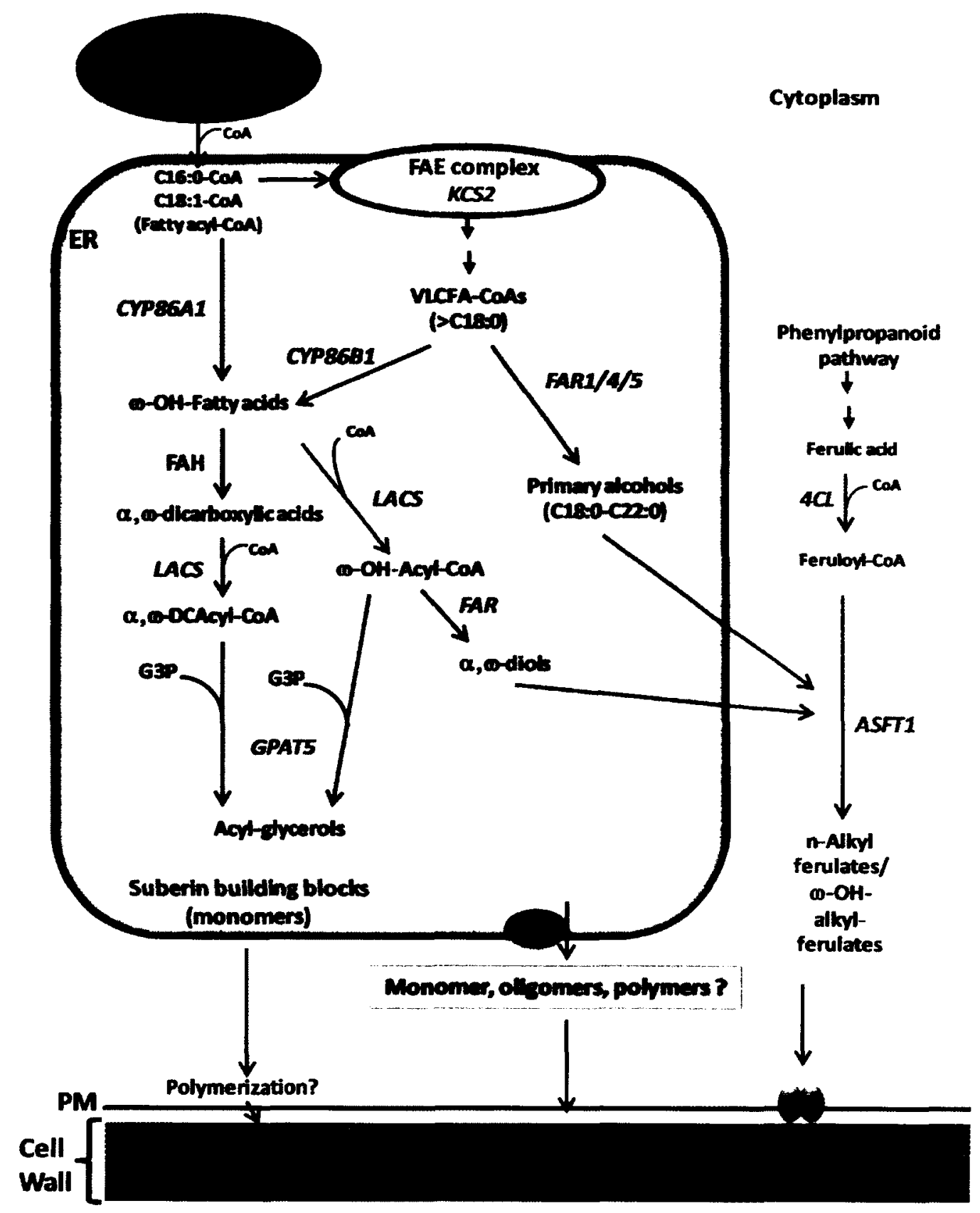

Figure 1.8. Proposed pathways for the synthesis of suberin polyester monomers. Abbreviations: 4CL, 4-coumarate ligase; $\mathrm{ABC}$, ATP binding cassette transporter; ASFT, aliphatic suberin feruloyl transferase; CYP, cytochrome $\mathrm{P} 450$ monooxygenase; DCA, dicaboxylic acid; FAE, fatty acid elongation; FAH, Fatty acyl $\omega$-hydroxylase; FAR, alcohol-forming fatty acyl-CoA reductase; G3P, glycerol-3-phosphate; GPAT, glycerol 3-phosphate acyltransferase; KCS, $\beta$-ketoacyl-CoA synthase LACS, long chain acyl-coA synthase; OH-FA, $\omega$-hydroxy fatty acid; PS, polyester synthase; PM, Plasma membrane; ER, Endoplasmic reticulum. Figure by Sollapura J. Vishwanath. 
Enzymes identified thus far for suberin monomer synthesis (Table 1.2) include: keto acyl-CoA synthases (KCS), fatty acid oxidases (FAO) of the cytochrome P450 monooxygenases (Cyp86) family, acyltransferases (AT) of the glycerol-3phosphate acyltransferase (GPAT) family, and feruloyl-CoA transferase/ aliphatic suberin feruloyl transferase (ASFT) or FHT. CYP86A1 and CYP86B1 are chain-length specific Arabidopsis cytochrome P450 dependent fatty acid oxidases (monooxygenases/ $\omega$-hydroxylases) that generate $\omega$-hydroxyacids and $\alpha, \omega$ dicarboxylic acids present in the aliphatic root suberin matrix in Arabidopsis (Li et al., 2007a, Hofer et al., 2008; Compagnon et al., 2009). Similarly, a CYP86A family member in potato (Solanum tuberosum) has been shown to be important for production of $\omega$-functionalized suberin monomers of the tuber periderm (Serra et al., 2009a). Two Arabidopsis $\beta$-ketoacyl-CoA synthases, DAISY/KCS2 and KCS20, are important for elongation of C20 acyl chain suberin precursors (Franke et al., 2009; Lee et al., 2009). Loss-of-function mutations in the corresponding $K C S$ genes result in reductions of C22 and C24 very-long-chain derivatives in root suberin, and the double mutant $k \operatorname{cs} 2 k \operatorname{cs} 20$ is affected more than either single mutant, indicating that the enzymes function in a partially redundant manner. Silencing of a potato $K C S$ gene, called StKCS6, causes reductions of suberin monomers with chain lengths of C28 and higher in the tuber peridermis (Serra et al., 2009b). Arabidopsis mutants of GPAT5 was reported to have large reductions in C20-C24 unsubstituted fatty acids, $\omega$-hydroxyacids and $\alpha, \omega$-dicarboxylic acids in both root and seed coat suberin (Beisson et al., 2007). GPAT5 encodes for an acyl-CoA:glycerol-3-phosphate acyltransferase involved in the formation of very-long-chain fatty acid-containing 
acylglycerol precursors for suberin biosynthesis (Beisson et al., 2007). A feruloylCoA transferase called AFST from Arabidopsis, as well as the ortholog from potato called fatty omega-hydroxyacid/fatty alcohol hydroxycinnamoyl transferase (FHT), are required for incorporation of ferulate into the suberin polyester (Gau et al, 2009; Molina et al., 2009; Serra et al., 2010). Periderms of FHT-RNAi silenced potato had a strong reduction in ester-linked ferulic acid, but without any structural alterations in the suberin lamellae (Serra et al., 2010). AFST and FHT enzymes catalyze the acyl transfer from feruloyl-CoA to $\omega$-OHAs and fatty alcohols (Molina et al., 2009; Serra et al., 2010). Fatty Acyl-CoA reductases, viz., FAR1, FAR4 and FAR5 from Arabidopsis have been identified to be involved in production of suberinassociated primary fatty alcohols (Domergue et al., 2010; This Study). Characterization of the first enhanced suberin mutant (esb1) revealeda two fold increase in the suberin load in roots and changes in leaf ion composition of $\mathrm{Ca}, \mathrm{Mn}$ and $\mathrm{Zn}$ along with enhanced drought tolerance (Baxter et al., 2009). The esb1 gene encodes a protein of unknown mechanistic function.

\subsubsection{Transportation and assembly of suberin polyester}

While the synthesis of suberin begins with de novo synthesis of fatty acids in the plastid, specific modifications, including oxidations, reductions and acylglycerol synthesis, likely occur in the endoplasmic reticulum (ER) (see Chapter 4 Discussion). The various suberin monomers (or possibly partially formed oligomers) then travel from the ER to the cell surface. The trafficking between the ER and the plasma membrane may occur by vesicular traffic, via soluble protein carriers, via oleophilic droplets, and/or by direct transfer between the ER and 
plasma membrane (PM) (Pollard et al., 2008). ER-PM contact sites have been identified in plants and may be involved in transfer of both cuticle and suberin lipids (Staehelin, 1997; Larsson et al., 2007). However, the intracellular trafficking of suberin monomers and intermediates has not yet been investigated. Trafficking through the plasma membrane and into the cell wall likely involves ATP-binding cassette $(A B C)$ transporters and possibly lipid transfer proteins (LTPs) (Figure 1.8). Pollard et al. (2008) proposed a plasma membrane (PM)-anchoring ER domain, which could involve a spatial coupling of monomer synthesis and transport across plasma membrane, e.g. by $\mathrm{ABC}$-binding cassette transporter proteins. Transcripts encoding $A B C$ transporters of the WBC and ATH subfamilies are highly up-regulated in phellem/cork tissue (Soler et al., 2007), and thus may be candidates for facilitating suberin transport. A silenced line (dso-4) of DSO/WBC11/ABCG11, a plasma membrane localized $\mathrm{ABC}$ transporter first identified as a wax/cutin transporter (Bird et al., 2007; Panikashvili et al., 2007), was also found to be affected in root suberin content (Panikashvili et al., 2010). A GPI-anchored LTP has been found to be involved in extracellular cuticular lipid deposition (DeBono et al., 2009), and other classes of LTP may also be involved in trafficking through the hydrophilic cell wall. LTPs may also mediate the transport of suberin precursors into the cell wall and to the site of polymerization, although there is no evidence yet for such role of LTPs. Polyester synthases (PS) are broadly defined as enzymes catalyzing the formation of ester bonds between monomers, and also between putative polyester oligomers. One member of the BAHD family of acyltransferases, encoded by the DEFECTIVE IN CUTICULAR RIDGES (DCR) gene, has been proposed to 
function in cutin polymer assembly, but biochemical evidence is lacking and it is localized to the cytoplasm (Panikashvili et al., 2009). A cell wall associated protein called BODYGUARD (BDG), which is an $\alpha, \beta$-hydrolase-fold protein, has also been proposed to be involved in polyester synthesis of cutin but again biochemical evidence is lacking (Kurdyukov et al., 2006a). Paralogues of DCR and BDG may also be involved in suberin polymerization and candidates exist but remain untested. Recently, an extracellular cutin synthase (CD1) belonging to the GDSL family of proteins, showing in vitro polyester synthesis activity was identified in tomato (Yeats et al., 2012). It is very likely that a CD1 paralog is involved in suberin polymer synthesis, but this awaits experimental evidence.

\subsubsection{Suberin-associated tuber and root waxes}

Suberin is found in association with solvent-extractable waxes (noncovalently linked). Suberin-associated waxes are considered major contributors to the barrier for water diffusion across suberized cell walls (Soliday et al., 1979). Unlike cuticular waxes, suberin-associated waxes in part resemble the structural constituents of the suberin polyester and are likely biosynthetically related. Suberin-associated waxes have been suggested to be found within the electrontranslucent region of the suberin lamellae (Soliday et al., 1979). However, this is unproven (Schmutz et al., 1994; Schreiber et al., 2005c).

Suberin-associated waxes, found in the native and wound-healing periderm of plant subterranean storage organs (tubers) include alkanes, primary alcohols, fatty acids, alkyl ferulates and monoacylglycerols ferulates (typically with C20 and C22 acyl chains) (Espelie et al., 1980b; Schreiber et al., 2005; Li et al., 2007b). The 
hydroxycinnamates in tuber periderm waxes consists mainly of alkyl ferulates. Root waxes are isolated by rapid immersion of Arabidopsis roots in organic solvents such as chloroform. A significant fraction of the aliphatics in suberin-associated root waxes are saturated fatty acids (typically C16-C22), C18:0-C22:0 fatty alcohols (16\%) and alkyl hydroxycinnamates (Pollard et al., 2008), thus showing that Arabidopsis root waxes have characteristics common to some of the monomers released upon depolymerization of root suberin (Franke et al., 2006; Beisson et al., 2007). Alkanes (typically C29 and C31) and their midchain oxidized (keto or hydroxy) derivatives have also been reported in root waxes (Kosma et al., 2012). Li et al. (2007b) reported a positive correlation between accumulation of monoacylglycerols (MAGs) and the ectopic expression of GPAT5, suggesting that the biosynthesis of aliphatic suberin and some of the suberin-associated waxes share a common pathway. Li et al. (2007b) reported that the alkanes are present at or near the surface of periderm based on the extraction kinetics, while the other root waxes are more deeply embedded in the suberized peridermal cell walls of Arabidopsis roots. The relative timing of wax and suberin deposition in peridermal tissues is unknown (Li et al., 2007b).

Root (suberin-associated) waxes contain alkyl hydroxycinnamate esters (Bernards and Lewis, 1992; Schreiber et al., 2005; Li et al., 2007b). These alkyl hydroxycinnamate esters are comprised of phenylpropanoids, typically coumaric, ferulic, or caffeic acids, esterified to fatty alcohols (Kosma et al., 2012). In Arabidopsis, alkyl ferulates comprise only a small proportion of the alkyl hydroxycinnamate esters extracted from roots (Li et al., 2007a; Molina et al., 2009). 
Instead, Arabidopsis root waxes are dominated by alkyl caffeate and alkyl coumarate esters (Kosma et al., 2012). The genes involved in alkyl ferulate synthesis identified so far are FHT, ASFT and DCF (Gou et al., 2009; Molina et al., 2009; Serra et al., 2010; Rautengarten et al., 2012; Kosma et al., 2012). All three genes encode members of the BAHD family of acyltransferases (Kosma et al., 2012). Recently, a new acyltransferase of the BAHD family named fatty alcohol:caffeoyl-CoA caffeoyl transferase (FACT) was identified in Arabidopsis, that in combination with FARs, is responsible for the synthesis of a subset of alkyl hydroxycinnamate esters, the alkyl caffeates (Kosma et al., 2012). 
Table 1.2. Summary of genes involved in the suberin polyester and root wax biosynthetic pathways in Arabidopsis roots, periderms or seed coat.

\begin{tabular}{|c|c|c|c|c|c|}
\hline $\begin{array}{l}\text { Gene } \\
\text { name }\end{array}$ & $\begin{array}{l}\text { AGI code/ } \\
\text { accession }\end{array}$ & $\begin{array}{l}\text { Punction/an } \\
\text { notation }\end{array}$ & $\begin{array}{l}\text { Chemical } \\
\text { phenotype }\end{array}$ & 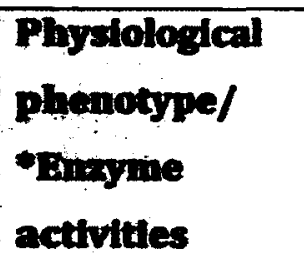 & $\begin{array}{l}\text { Refere } \\
\text { nce }\end{array}$ \\
\hline $\begin{array}{l}\text { CYP86A1 } \\
\text { /HORST }\end{array}$ & At5g58860 & $\begin{array}{l}\text { Fatty acyl } \omega \text { - } \\
\text { hydroxylase } \\
\text { (cytochrome } \\
\text { P450 } \\
\text { monooxygen } \\
\text { ase) }\end{array}$ & $\begin{array}{l}\text { Strongly reduced } \\
\text { levels of C16 and } \\
\text { C18 } \omega-\mathrm{HAs} \text { and } \alpha, \omega- \\
\text { DCAs; } 60 \% \\
\text { reduction } \\
\text { in total suberin in } \\
\text { roots }\end{array}$ & $\begin{array}{l}\text { Altered suberin } \\
\text { lamellae; } \\
\text { recombinant } \\
\text { protein exhibits } \\
\text { C16 and C18 } \\
\text { fatty acids } \\
\text { hydroxylation } \\
\text { activity }\end{array}$ & $\begin{array}{l}\text { Höfer } e t \\
\text { al., } \\
2008\end{array}$ \\
\hline $\begin{array}{l}\text { CYP86B1 } \\
/ R A L P H\end{array}$ & At5g23190 & $\begin{array}{l}\text { Fatty acyl } \omega \text { - } \\
\text { hydroxylase } \\
\text { (cytochrome } \\
\text { P450 } \\
\text { monooxygen } \\
\text { ase) }\end{array}$ & $\begin{array}{l}\text { Root and seed: } \\
\text { almost complete } \\
\text { lack of C22 } \\
\text { and C24 } \omega-\mathrm{HA} \text { and } \\
\alpha, \omega-\mathrm{DCA} ; \\
\text { accumulation of C22 } \\
\text { and C24 fatty acids }\end{array}$ & & $\begin{array}{l}\text { Compag } \\
\text { non } \text { et } \\
\text { al., } \\
2009 ; \\
\text { Molina } \\
\text { et al., } \\
2009\end{array}$ \\
\hline $\begin{array}{l}\text { KCS2/ } \\
\text { DAISY }\end{array}$ & At1g04220 & $\begin{array}{l}\beta \text {-Ketoacyl- } \\
\text { CoA synthase } \\
\text { (FAE- } \\
\text { component) }\end{array}$ & $\begin{array}{l}\text { Reduced levels of } \\
\text { suberin monomers } \\
>\mathrm{C} 20 \text {; accumulation } \\
\text { of } \leq \mathrm{C} 20 \text { monomers } \\
\text { in roots }\end{array}$ & $\begin{array}{l}\text { Reduced root } \\
\text { growth; } \\
\text { redundant } \\
\text { function } \\
\text { with KCS20; } \\
\text { KCS activity } \\
\text { when } \\
\text { heterologously } \\
\text { expressed in } \\
\text { yeast }\end{array}$ & $\begin{array}{l}\text { Franke } \\
\text { et al., } \\
2009 ; \\
\text { Lee et } \\
\text { al., } \\
2009\end{array}$ \\
\hline
\end{tabular}




\begin{tabular}{|c|c|c|c|c|c|}
\hline KCS2O & At5g43760 & $\begin{array}{l}\beta \text {-Ketoacyl- } \\
\text { CoA synthase } \\
\text { (FAE- } \\
\text { component) }\end{array}$ & $\begin{array}{l}\text { Reduced levels of } \\
\text { suberin monomers } \\
>\mathrm{C} 20 \text {; accumulation } \\
\text { of } \leq \mathrm{C} 20 \text { monomers } \\
\text { in roots }\end{array}$ & $\begin{array}{l}\text { Reduced root } \\
\text { growth; } \\
\text { redundant } \\
\text { function } \\
\text { with KCS20; } \\
\text { KCS activity } \\
\text { when } \\
\text { expressed in } \\
\text { yeast }\end{array}$ & $\begin{array}{l}\text { Lee } e t \\
\text { al., } \\
2009\end{array}$ \\
\hline GPAT5 & At3g11430 & $\begin{array}{l}\text { glycerol-3- } \\
\text { phosphate } \\
\text { acyltransfera } \\
\text { se }\end{array}$ & $\begin{array}{l}50 \% \text { reduction in } \\
\text { total suberin } \\
\text { content : mainly } \\
\text { reduction in C20- } \\
\text { C24 } \\
\omega-\text { HAs and } \alpha, \omega- \\
\text { DCAs in seeds }\end{array}$ & $\begin{array}{l}\text { Increased dye } \\
\text { permeability of } \\
\text { the seed } \\
\text { coat; increased } \\
\text { salt sensitivity }\end{array}$ & $\begin{array}{l}\text { Beisson } \\
\text { et al., } \\
\text { 2007; Li } \\
\text { et al., } \\
2007 \text {; } \\
\text { Yang et } \\
\text { al., } \\
2010\end{array}$ \\
\hline$A S F T$ & At5g41040 & $\begin{array}{l}\text { Feruloyl-CoA } \\
\text { transferase/ } \\
\text { hydroxycinna } \\
\text { moyl } \\
\text { transferase } \\
\text { (BAHD } \\
\text { acyltransfera } \\
\text { se) }\end{array}$ & $\begin{array}{l}\text { almost complete } \\
\text { lack of ferulate, } \\
\text { quantitatively } \\
\text { similar reduction in } \\
\omega-H A \text {, } \\
\text { increase } \\
\text { in } \alpha, \omega-D C A \text { in } \\
\text { seeds; major } \\
\text { reduction in ferulate } \\
\text { in roots. }\end{array}$ & $\begin{array}{l}\text { Feruloyl } \\
\text { transferase } \\
\text { activity of the } \\
\text { recombinant } \\
\text { protein to } \omega \text { - } \\
\text { HAs and } \\
\text { alcohols }\end{array}$ & $\begin{array}{l}\text { Molina } \\
\text { et al., } \\
2009 \text {; } \\
\text { Gou et } \\
\text { al., } \\
2009\end{array}$ \\
\hline FAR1 & At5g22500 & $\begin{array}{l}\text { Fatty acyl- } \\
\text { CoA } \\
\text { reductase }\end{array}$ & $\begin{array}{l}\text { Root: reduction in } \\
\text { C22:0 alcohols; } \\
\text { seed: reduction in } \\
\text { C22:0 alcohols and } \\
\text { C22:0 } \\
\text { diols }\end{array}$ & $\begin{array}{l}\text { C22:0 (and } \\
\text { C18:0) alcohol } \\
\text { formation in } \\
\text { FAR1 } \\
\text { expressing } \\
\text { yeast }\end{array}$ & $\begin{array}{l}\text { Current } \\
\text { study } \\
\text { (Domer } \\
\text { gue et } \\
\text { al., } \\
2010 \text { ) }\end{array}$ \\
\hline
\end{tabular}




\begin{tabular}{|c|c|c|c|c|c|}
\hline FAR4 & At3g44540 & $\begin{array}{l}\text { Fatty acyl- } \\
\text { CoA } \\
\text { reductase }\end{array}$ & $\begin{array}{l}\text { Root and seed: } \\
\text { specific reduction in } \\
\text { C20:0 } \\
\text { alcohols }\end{array}$ & $\begin{array}{l}\text { C20:0 (and } \\
\text { C18:0) alcohol } \\
\text { formation in } \\
\text { FAR4 } \\
\text { Expressing } \\
\text { yeast }\end{array}$ & $\begin{array}{l}\text { Current } \\
\text { study } \\
\text { (Domer } \\
\text { gue et } \\
\text { al., } \\
2010 \text { ) }\end{array}$ \\
\hline FAR5 & At3g44550 & $\begin{array}{l}\text { Fatty acyl- } \\
\text { CoA } \\
\text { reductase }\end{array}$ & $\begin{array}{l}\text { Root and seed: } \\
\text { specific reduction in } \\
\text { C18:0 } \\
\text { alcohols }\end{array}$ & $\begin{array}{l}\text { C18:0 alcohol } \\
\text { formation in } \\
\text { FAR5 } \\
\text { expressing } \\
\text { yeast }\end{array}$ & $\begin{array}{l}\text { Current } \\
\text { study } \\
\text { (Domer } \\
\text { gue et } \\
\text { al.2010) }\end{array}$ \\
\hline $\begin{array}{l}A B C G 11 \\
/ D S O / C O \\
F 1\end{array}$ & At1g17840 & $\begin{array}{l}\text { ATP-binding } \\
\text { cassette } \\
(\mathrm{ABC}) \\
\text { transporter }\end{array}$ & $\begin{array}{l}10-30 \% \text { reduction } \\
\text { in suberin } \\
\text { Monomers in roots }\end{array}$ & Cuticle defects & $\begin{array}{l}\text { Panikas } \\
\text { hvili et } \\
\text { al., } \\
2010\end{array}$ \\
\hline$E S B 1$ & At2g28670 & $\begin{array}{l}\text { Unknown } \\
\text { function } \\
\text { (Disease } \\
\text { resistance- } \\
\text { responsive } \\
\text { family } \\
\text { protein) }\end{array}$ & $\begin{array}{l}\text { Two-fold increase in } \\
\text { all suberin } \\
\text { monomers in roots }\end{array}$ & $\begin{array}{l}\text { Reduced } \mathrm{Ca}^{2+} \\
\text { content in } \\
\text { shoots, reduced } \\
\text { transpiration; } \\
\text { increased } \\
\text { drought } \\
\text { tolerance } \\
\text { and water use } \\
\text { efficiency }\end{array}$ & $\begin{array}{l}\text { Baxter } \\
\text { et al., } \\
2009\end{array}$ \\
\hline FACT1 & At5g63560 & $\begin{array}{l}\text { Fatty } \\
\text { Alcohol:Caffe } \\
\text { oyl-CoA } \\
\text { Caffeoyl } \\
\text { Transferase } \\
\text { (Acyltransfer } \\
\text { ase) }\end{array}$ & $\begin{array}{l}\text { Reduced in alkyl } \\
\text { caffeate in root } \\
\text { waxes }\end{array}$ & $\begin{array}{l}\text { Acyltransferase } \\
\text { activity of } \\
\text { recombinant } \\
\text { At5g63560p to } \\
\text { caffeoyl- } \\
\text { Coenzyme A } \\
\text { (CoA) substrate }\end{array}$ & $\begin{array}{l}\text { Kosma } \\
\text { et al., } \\
2012\end{array}$ \\
\hline
\end{tabular}

${ }^{*}$ Data from in-vitro studies/ heterologous expression studies. 


\subsubsection{Regulation of suberin deposition}

Suberin is generally deposited wherever the plant needs a persistent barrier, and such deposition varies temporally as well as spatially (Franke et al., 2009). Therefore, suberin biosynthesis and deposition must be highly and strictly regulated at the cell and tissue level. Several suberin genes that encode a variety of enzymes responsible for the formation of a diverse set of suberin monomers have been identified (Table 1.2), although the understanding of suberin regulation will need more research (Ranathunge et al., 2011).

Early suberin research was directed toward 'end point-analyses', meaning the identification and quantification of monomers that were from suberin in mature tissues having an abundant suberin deposition (see Bernards, 2002; Yang and Bernards 2006). Fewer studies on suberin composition and suberization during tissue maturation stages were carried out. Yang and Bernards (2006) reported significant differences in the abundance of aliphatic monomers in wound-induced periderm in $S$. tuberosum as it developed over the course of seven days. Histochemical studies in wound-healing potato tubers revealed that aromatic suberin deposition begins before aliphatic suberin deposition, suggesting a differential control of the aromatic and aliphatic branches of the suberin biosynthetic machinery (Lulai and Corsini, 1998). In another study, aliphatic monomers at three locations along the length of Arabidopsis thaliana roots were quantified. An increase in C18:1 $\omega$-hydroxy fatty acid abundance, a steady amount of C18:1 $\alpha, \omega$-dicarboxylic acid and a decrease in the amount of primary fatty acids, from root tip to crown was reported (Höfer et al., 2008). This approach of studying 
maturing tissue is valuable in terms of understanding the dynamic metabolic processes of suberin biosynthesis regulation.

During the development of plant tissue and the initial deposition of suberin, it is not always deposited as a uniform shield and there are often more permeable sites (Ranathunge et al., 2011). Generally, suberin is only deposited in the endodermis in seedlings and younger plants. The suberin deposited in the root exodermis and/or peridermis makes up the very outer semi-permeable barrier, while the suberin deposited in the endodermis forms a more complete and impermeable barrier. Suberin is usually deposited more completely in coordination with wax and phenylpropanoid biosynthetic pathways, in response to external environmental stimuli such as desiccation, increased salt concentration, microbial attack, wounding, and soil pollutants such as heavy metals and acidic conditions (Ranathunge et al., 2011).

To generate a superior barrier, manipulation of the entire suberin biosynthesis matrix must be achieved, as opposed to merely manipulating one biosynthetic gene at a time. Based on the correlation between their spatial distribution of gene expression and occurrence of suberin, proposed putative regulators include WRKY-, NAC- and MYB-domain-containing transcription factors (Ranathunge et al., 2011). The members of these transcription factor families have been found to be co-expressed with suberin biosynthetic genes and are preferentially expressed in tissues undergoing suberization (Kilian et al., 2007). Identifying suberin transcription factors is a major goal in suberin research since it 
will permit researchers to induce and up-regulate suberin deposition and thereby potentially making a more effective barrier.

\subsection{Primary fatty alcohols}

Primary fatty alcohols are aliphatic hydrocarbons that contain a hydroxyl group at the terminal carbon. They are ubiquitously found in nature and serve various biological functions (Liénard et al., 2010; Rowland and Domergue, 2012). Fatty alcohols and their derivatives extracted from living organisms are also important feedstock for the cosmetics, pharmaceuticals and lubricant industries. In nature, fatty alcohols are found with chain lengths from 8 to 34 carbons, with different levels of branching and/or unsaturation.

\subsubsection{Fatty alcohols- free and combined forms}

Primary fatty alcohols are found in nature either as free alcohols or in a combined state (Figure 1.9). Fatty alcohols can be combined (esterified form), for example, with fatty acids (e.g. palmitic acid) to give wax esters or with an phenolic compound (e.g. ferulic acid) to give alkyl hydroxycinnamates. These various compounds are components of plant extracellular lipid barriers like cuticle, suberin and sporopollenin (Molina et al., 2006; Pollard et al., 2008; See Above). They can also be combined with glycerophospholipids to give ether lipids, which are minor constituents of animal cells (Hajra, 1983; Wang and Kolattukudy, 1995). Primary fatty acyl alcohols possess important biological functions in living organisms. Free long-chain (C16 and C18) and very-long-chain (>C18) primary alcohols are not known to accumulate to high levels within livings cells as it is thought that they are 
detrimental to cell viability (Metz et al., 2000). Intracellular modification or combining the fatty alcohols may thus be vital for cell functioning.

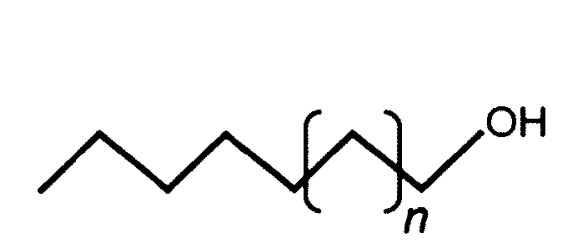

Primary Alcohol

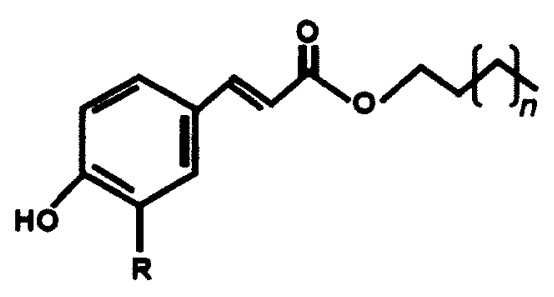

Alkyl Hydroxycinnamate

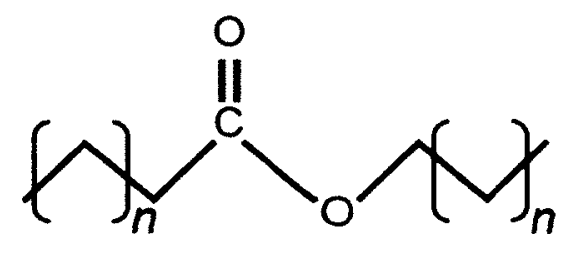

Wax Ester

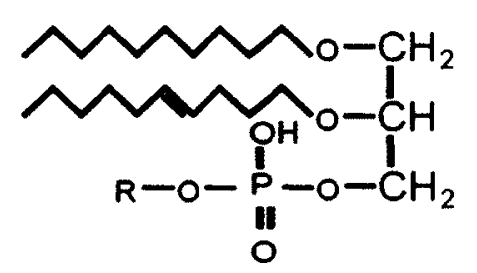

Ether Lipid

Figure 1.9. Generalized structures of primary fatty alcohols, wax esters, alkyl hydroxycinnamates, and ether lipids. $\mathrm{R}=\mathrm{H}, \mathrm{OH}$, or OCH3. 


\subsubsection{Fatty alcohols in plants: biosynthesis and functions}

In the acyl reduction pathway, the substrate fatty acyl-CoA or ACP is reduced to fatty alcohol via a fatty aldehyde intermediate by the enzyme Fatty Acyl Reductase or FAR (Figure 1.10). This reduction is a two-step process proceeding via an aldehyde intermediate, which can be carried out by a single alcohol-forming FAR (Kolattukudy, 1970; Pollard et al., 1979; Vioque and Kolattukudy, 1997; Rowland and Domergue, 2012). These primary alcohols are further esterified with long chain fatty acids through the action of a wax synthase to form wax esters (Lardizabal et al., 2000). All primary alcohols reported to occur in Arabidopsis cuticular wax are saturated with chain lengths of 22 to 34 carbons and predominant chain lengths of 26 to 30 carbons (Kunst and Samuels, 2003; Lai et al., 2007).

Primary fatty alcohols play a variety of roles in non-plant species. Some microorganisms such as Acinetobacter and Euglena gracilis accumulate fatty alcohols and/or wax esters as energy reserves (Fixter et al. 1986; Teerawanichpan and Qiu, 2010). Free fatty alcohols and their acetate ester derivatives can function as pheromones in insects and moths (Tillman et al. 1999; Moto et al., 2003; Liénard et al., 2010; Lassance et al., 2010; Teerawanichpan et al., 2010). Wax esters are important constituents of bee hive comb wax produced by honeybees (Cassier and Lensky 1995; Granier et al,, 2002). Wax esters in the diet are hydrolyzed by a bile salt-dependent pancreatic carboxyl esterase, releasing long chain alcohols, which have been reported to lower plasma cholesterol in humans (Hargrove et al., 2004). Sperm whales were hunted for spermaceti oil rich in wax esters, which possibly helped the animal in echolocation sensing and regulating buoyancy (Clarke, 1978). 
Fatty alcohols are also precursors of ether lipids (plasmalogens), which are found in various mammalian tissues and play an important role in membrane dynamics, intracellular signaling and protection from oxidative stress (Hajra, 1983; Nagan and Zoeller, 2001).

In plants, primary alcohols and derivatives are found in cuticular wax, suberin-associated waxes of tuber periderm and roots, and surface lipid polyesters (eg. cutin, sporopollenin, and suberin) (Samuels et al., 2008; Dominguez et al., 2011, Rowland and Domergue, 2012). Fatty alcohols are also found in the seeds of the jojoba plant in the form of wax esters as a seed lipid energy reserve (Metz et al., 2000). Current understanding about the distribution and possible roles of these fatty alcohols in plants is elaborated in the sections below.

\subsubsection{Fatty alcohols in cuticular waxes}

In cuticular waxes, fatty alcohols are found either in a free form or in the form of wax esters (Jetter and Kunst, 2008). The wax load on wild-type Arabidopsis stems and leaves contains $10-15 \%$ and $15-25 \%$ primary fatty alcohols, respectively, as free alcohols and wax esters (Jenks et al., 1995; Rowland et al., 2006). The primary fatty alcohols that have been reported to exist in Arabidopsis cuticular waxes are saturated and range from $\mathrm{C} 18: 0-\mathrm{C} 34: 0$, with $\mathrm{C} 26: 0-\mathrm{C} 30: 0$ being by far the dominating chain lengths (Rowland et al., 2006; Lai et al, 2007). Leaf wax of maize (Zea mays) seedlings contain up to $63 \%$ free C32:0 primary alcohol (by weight), while cuticular waxes from mature leaves contain up to $42 \%$ wax esters, with tetracosanol $(\mathrm{C} 24: 0-\mathrm{OH}, 55 \%)$ and hexacosanol $(\mathrm{C} 26: 0-\mathrm{OH}, 27 \%)$ being the major fatty alcohol moieties of these esters (Bianchi et al., 1984). Brazilian carnauba palm 
(Copernicia cerifera) accumulates massive amounts of cuticular wax on its leaf surface, of which about $85 \%$ is composed of wax esters (Vandenburg and Wildner, 1970). In several plant species, the chain length patterns of the free and esterified alcohols are similar, indicating that they are derived from a common pool of fatty alcohol precursors (Miwa, 1971; Lai et al., 2007).

Phenotypic characterization of mutants like Arabidopsis cer4 (Rowland et al., 2006) and barley cer-j.59 (Von Wettstein-Knowles, 1974), which have large reductions of primary alcohols and thereby lack of wax crystals on the surfaces, indicate that the fatty alcohols are necessary for the formation of epicuticular wax crystal structures at the plant surface (Rowland and Domergue, 2012). However, more experiments are required to elucidate the specific roles of fatty alcohols in the various interactions of plants with the biotic environment.

\subsubsection{Fatty alcohols in suberin}

Suberin is a lipid and phenylpropanoid-based protective barrier constitutively deposited in the cell walls of various external and internal tissue layers (Pollard et al., 2008) (see Section 1.1.3, above). Suberin is composed of covalently linked phenolic and aliphatic components. A large proportion of the suberin aliphatic component is composed of non-substituted fatty acids, hydroxyfatty acids ( $\omega$-hydroxy, mid-chain hydroxy, or both), $\alpha, \omega$-dicarboxylic acids and glycerol (Bernards, 2002). Fatty alcohols account for about 6-7\% of the aliphatic suberin polymer in Arabidopsis root and seed coat (Pollard et al., 2008; Rowland and Domergue, 2012) and up to $15 \%$ in potato tuber periderm (Schreiber et al., 2005b). Saturated primary fatty alcohols of chain lengths C18, C20 and C22 are the 
common components of suberin (Zeir et al., 1999; Franke et al., 2005; Schreiber et al., 2005b; Beisson et al., 2007; Domergue et al., 2010-this study).

\subsubsection{Fatty alcohols in suberin-associated alkyl hydroxycinnamates}

Free fatty alcohols are found in suberin-associated root waxes, which are similar to cuticular waxes but not covalently linked to the suberin polymer. These root waxes are extracted by 1-min immersion of 6-7 week old matured root in organic solvents like chloroform (Rowland and Domergue, 2012). Alkyl hydroxycinnamates are the most abundant class of compounds found in Arabidopsis root waxes (accounting for about 47\%), along with with free C18-C22 saturated fatty alcohols. These may play an important role in imparting barrier properties to the suberized cells. Also, alkyl hydroxycinnamates are natural antioxidants and thus may affect lipid oxidation (Kikiuzaki et al., 2002; Yunoki et al., 2004; Menezes et al., 2011). Furthermore, free fatty alcohols and alkyl-hydroxycinnamic acid conjugates (fatty alcohols esterified to a hydroxycinnamate compound such as ferulic acid) of the suberin phenolic component, have been shown to accumulate at infection sites and are important in protecting plants against pathogens (Matern and Kneusel, 1988). The solvent extracted lipids (waxes) of native and wound potato periderms was found to contain at least $50 \%$ alkyl hydroxycinnamates, mainly in the form of C16 to C32 fatty alcohols esterified with ferulic acid (Schreiber et al., 2005c). In the suberin-associated root waxes of Arabidopsis, esters of C18:0-C22:0 saturated fatty alcohols linked with $p$-coumaric, caffeic, and ferulic acids (alkyl hydroxycinnamates) are the predominating chemical components (47\% w/w) (Li et al., 2007b; Kosma et al., 2012). Cameron and Carlile (1978) reported that very long chain fatty acids, 
fatty aldehydes and fatty alcohols in root exudates act as attractants to zoospores of fungi and has a role in chemotaxis to plant roots.

\subsubsection{Fatty alcohols in pollen exine}

Sporopollenin is the major constituent of the outer cell walls in the exine of pollen grains and is made up of mixtures of fatty acid derivatives and phenolic compounds (Dominguez et al., 1999; Dobritsa et al., 2009). Sporopollenin primarily being a lipid polymer protecting the pollen grain from dessication, also plays important roles in pollen dispersal and pollen-pistil interactions (Rowland and Domergue, 2012). The exact composition of sporopollenin is unknown due to lack of material and resistance to depolymerisation for analysis by gas chromatography or other analytical methods. In Arabidopsis, MS2/FAR2 reductase enzyme was reported to be involved in production of fatty alcohols involved in sporopollenin biosynthesis (Aarts et al., 1993, 1997). The loss-of-function mutant of Defective Pollen Wall (DPW), which is the rice ortholog of MS2/FAR2, was found to have $\sim 40 \%$ reduction in $\mathrm{C} 16: 0$ and $\mathrm{C} 18: 0$ primary fatty alcohol in the soluble anther lipids (Shi et al., 2011). Both the mutants, $m s 2$ and $d p w$, were reported to have abnormal anther surface and severely altered pollen exine layer resulting in plant sterility. Recently, based on in vitro assays, C16:0 fatty alcohol was suggested to be important components of sporopollenin in Arabidopsis and rice (Doan et al., 2009; Chen et al., 2011; Shi et al., 2011) (Table 1.3), although this has not been directly measured. Primary fatty alcohols have only one functional group, and would thus terminate polymerization. It is not clear how fatty alcohols are integrated into sporopollenin structure (Rowland and Domergue, 2012). 


\subsubsection{Fatty alcohols for storage function}

Most plants usually accumulate triacylglycerol (TAG) as storage lipids in seeds for energy supply post germination. A notable exception to this is jojoba (Simmondsia chinensis), a plant native to American southwest deserts, which accumulates wax esters in its seeds as the major energy reserve. Jojoba oil accounts for up to $40-60 \%$ of seed dry weight, of which $98 \%$ is wax esters. These wax esters are the esters of unsaturated very long chain fatty acids (C40-C44) and monounsaturated long-chain alcohols, with C22:1-alcohol and C20:1 fatty acid being the most predominant constituents (Miwa, 1971; Ohlrogge et al., 1977; Metz et al., 2000; Rowland and Domergue, 2012). Fatty acyl reductases are responsible for synthesis of fatty alcohols used for wax ester biosynthesis (Figure 1.10). 


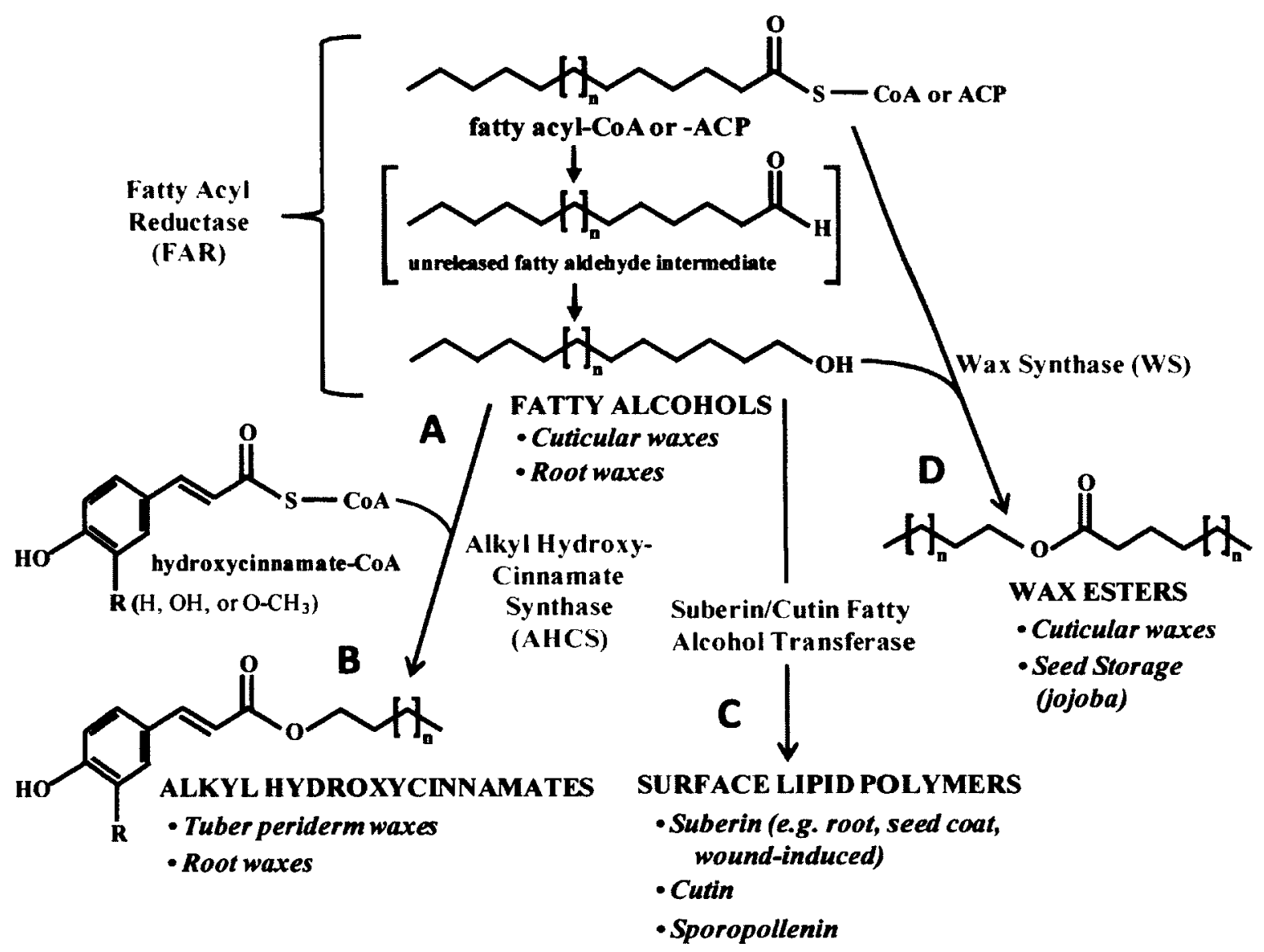

Figure 1.10. Fatty alcohols and their derivatives. (A) Fatty acyl-CoAs or fatty acylACPs are reduced by Fatty Acyl Reductases (FARs) generating fatty alcohols (C16:0C30:0 chain lengths in plants). (B) Fatty alcohols may be linked with phenolics to yield alkyl hydroxycinnamates by an alkyl hydroxycinnamate synthase (AHC). (C) Fatty alcohols can be incorporated into surface polymers (suberin, cutin, sporopollenin) by an as yet uncharacterized suberin/cutin alcohol transferase. (D) Fatty alcohols can be linked with fatty acids to yield wax esters by a wax synthase (WS). Grey = enzyme activities, uppercase $=$ major products, italics $=$ location where the compounds accumulate. Adapted from Rowland and Domergue (2012), Plant Science, reproduced with permission (C) ASPB. 


\subsection{Fatty Acyl Reductase (FAR) enzyme}

Alcohol-forming Fatty Acyl-CoA Reductase (FAR) enzymes catalyze the cleavage of the thioester bond of a fatty acyl-CoA substrate, and converts long-chain or very-long-chain acyl-CoAs into primary fatty alcohols in a NADPH dependent reaction (Vioque and Kolattukudy, 1997, Metz et al., 2000, Rowland et al., 2006) (Figure 1.11).

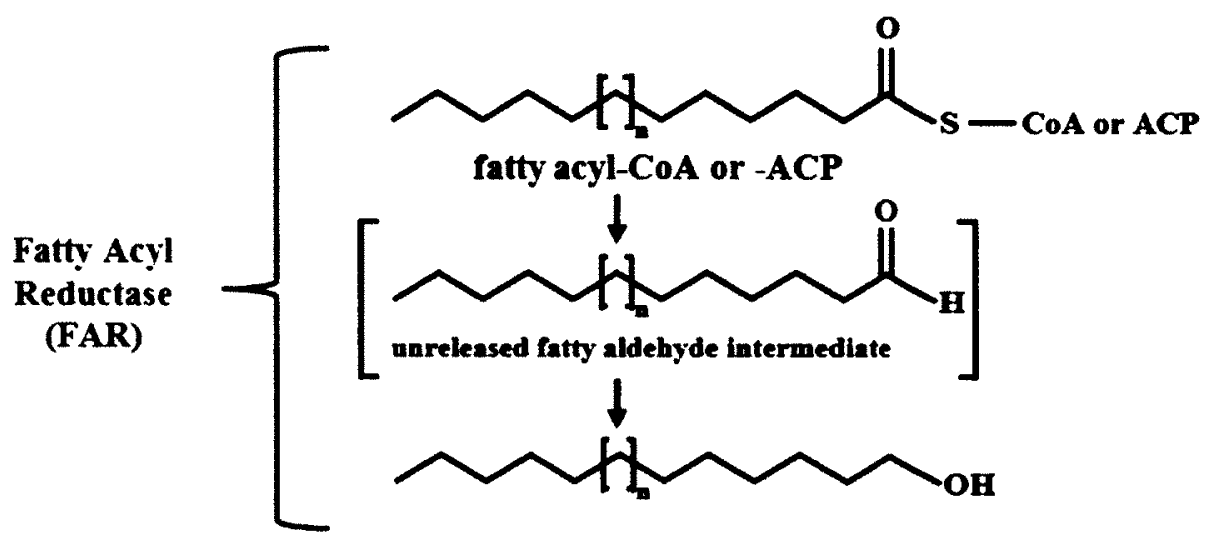

FATTY ALCOHOLS

Figure 1.11. Enzymatic reaction catalyzed by Fatty Acyl Reductase (FAR).

\subsubsection{Biochemical characterization of FARs}

Chinbal and Piper (1934) first proposed that fatty alcohols are generated by reduction of very-long chain fatty acid (VLCFA) precursors, although it must occur via intermediate aldehyde formation. Biochemical characterization of FARs, progressed with the pioneering work of Kolattukudy in 1970s.

Cell free preparation of etiolated Euglena gracilis cells was shown to reduce fatty acids to the corresponding alcohols in the presence of ATP, CoA, and NADH, where an aldehyde intermediate was detected by adding phenylhydrazine 
hydrochloride to the reaction mixture (Kolattukudy, 1970). Studies on partial purification of reducing activities from Brassica oleracea initially led to a proposal that primary alcohol production is carried out by two separate enzymes, an NADHdependent acyl-CoA reductase required for reduction of VLCFAs to aldehydes, and an NADPH-dependent aldehyde reductase required for further reduction of aldehydes to primary alcohols (Kolattukudy, 1971). Wang and Kolattukudy (1995a) reported the purification of an aldehyde-generating fatty acyl-CoA reductase with a molecular mass of $35 \mathrm{KDa}$ from the green alga Botryococcus braunii. In 1997, the first purification and characterization of alcohol-forming FAR, along with an aldehyde generating FAR from garden pea leaves (Pisum sativum L.) was reported (Vioque and Kolattukudy, 1997). This alcohol-forming FAR enzyme was found to be $58 \mathrm{KDa}$ and utilize NADPH as reductant for the production of fatty alcohol from fatty acyl-CoA.

Wax biosynthesis studies using cell-free homogenates from developing jojoba cotyledons demonstrated that the synthesis of fatty alcohols from fatty acylCoAs is NADPH dependent and occurs via an unreleased aldehyde intermediate (Pollard et al, 1979). Later in the 1990s, cloning of cDNA and characterization of ScFAR, encoding a fatty acyl-CoA reductase from jojoba (Simmondsia chinensis) that is responsible for producing fatty alcohols present in storage wax esters in developing seeds, greatly improved the understanding of fatty alcohol biosynthesis and has since become the reference for studying fatty acid reductase homologues in other species (Metz et al. 2000). Heterologous expression of jojoba FAR in rapeseed and bacteria verified that reduction of fatty acyl-CoAs to primary alcohols is indeed 
carried out by a single enzyme in plants without the release of an aldehyde intermediate (Metz et al., 2000). Identification and biochemical characterization of homologous FAR proteins from other plants such as wheat, Arabidopsis and rice, confirmed that FARs in plants exhibit the single enzyme reaction and are NADPH dependent (Wang et al., 2002; Rowland et al., 2006; Doan et al., 2009; Domergue et al., 2010; Shi et al., 2011). Expression of these FARs in various heterologous systems indicated that each has distinct substrate specificity in terms of acyl chain length and/or degree of saturation (Table 1.3). Based on the subcellular localization and acyl-linkage substrate specificity, two types of plant FARs have been described: the microsomal FAR enzymes acting on acyl-CoA substrates (e.g., Jojoba ScFAR and Arabidopsis ECERIFERUM4 (CER4)/FAR3 enzyme) (Metz et al., 2000; Rowland et al., 2006) and the plastid-localized FAR proteins that use acyl-ACPs as substrates (Chen et al., 2011, Doan et al., 2009, 2011; Shi et al., 2011).

Recently, in vitro biochemical characterization of tagged plastidial FARs was reported for Arabidopsis MS2/FAR2 (Chen et al., 2011), rice DPW (Shi et al., 2011) and Arabidopsis FAR6 (Doan et al., 2011). The results showed that Arabidopsis MS2/FAR2 and its rice homologue DPW have higher specificity for C16:0-ACP than for C16:0-CoA substrates, whereas FAR6 was found to accept both C16:0-ACP and C16:0-CoA as substrates in vitro (Doan et al., 2011). Arabidopsis MS2/FAR2 was found to accept both NADPH and NADH as reductants, but DPW and FAR6 were strictly NADPH-dependent. 


\subsubsection{FARs in plants}

Alcohol-generating reductases are ubiquitous in plants. FAR enzymes related to the jojoba FAR have subsequently been identified and characterized in wheat (Wang et al., 2002), rice (Shi et al., 2011) and Arabidopsis thaliana (Rowland et al., 2006; Doan et al., 2009, Domergue et al., 2010 (This Study), Doan et al., 2011).

\section{Jojoba}

The seed-specific FAR from jojoba, ScFAR, was the first to be cloned and characterized extensively among plant FARs (Table 1.3). The ScFAR reduces verylong-chain acyl-CoAs (mostly C20:1 and C22:1) to the corresponding fatty alcohols using NADPH as a reductant, which are further combined with long chain acyl CoAs by a fatty acyl-CoA:fatty alcohol acyltransferase (wax synthase) (Pollard et al., 1979; Lardizabal et al., 2000; Metz et al., 2000). The abundance of very-long-chain acylCoAs (mostly C20:1 and $\mathrm{C} 22: 1$ ) in the wax esters present in jojoba embryos indicates specificity of ScFAR for very-long-chain acyl-CoAs in planta (Metz et al., 2002). Expression of jojoba FAR in Brassica napus did not result in free alcohols, but resulted in accumulation of wax esters of chain lengths from C38-C44 (with mostly C22:1 fatty alcohols), in the seeds due to endogenous wax synthase activity (Lardizabal et al., 2000). The jojoba FAR along with a jojoba wax synthase (WS) was co-expressed in Arabidopsis seeds with a $\beta$-ketoacyl-CoA synthase (KCS; component of fatty acid elongase) from Lunaria annua that generates long-chain fatty acyl-CoAs (C24:1) (Lardizabal et al., 2000). The co-expression resulted in accumulation of very-long-chain acyl groups and alcohols (mostly C22:1-OH and C24:1-OH) 
accounting for $42 \%$ of seed oil content along with small amounts of wax esters (Lardizabal et al., 2000).

Wheat

Identification of three anther-specific homologous genes (TAA1a, TAA1b and TAA1c) from bread wheat (Triticum aestivum L.) was reported by Wang et al. (2002). These are the orthologs of the jojoba ScFAR (Table 1.3). TAA1a transcript was shown to be specifically expressed in the tapetum during the formation of the outer cell wall of pollen grains suggesting that these are involved in producing the lipid component in the outer pollen wall. Heterologous expression of the wheat FAR encoded by the TAA1a gene in $E$. coli resulted in accumulation of C14:0, C16:0 and C18:1 primary alcohols, while it produced long-chain and very-long-chain fatty alcohols (C18:1, C20:1, C22:1, C24:0 and C26:0) when expressed in transgenic tobacco seeds (Wang et al., 2002). Unlike Arabidopsis MS2/FAR2 and rice DPW plastid localized FARs, the predicted TAA proteins do not have a predicted chloroplast-targeting sequence and are thus likely extraplastidic, functioning in the anther most likely in tryphine formation rather than sporopollenin formation of pollen exine development (Rowland and Domergue, 2012).

\section{Rice}

Recently, using map based cloning, DEFECTIVE POLLEN WALL (DPW), which is the ortholog of Arabidopsis FAR2/MS2 was identified in rice (Oryza sativa) (Shi et al., 2011) (Table-3). DPW encodes a fatty acyl reductase that is expressed in tapetal cells and microspores. The defective pollen wall ( $d p w)$ male sterile mutant was reported to be defective in anther development and had degenerated pollen grains 
with an irregular exine (Shi et al., 2011). DPW was shown to be targeted to plastids mediated by its $\mathrm{N}$-terminal transit peptide similar to Arabidopsis FAR2/MS2. Heterologous expression of recombinant DPW enzyme was reported to have acylACP activity, with higher specificity for $\mathrm{C} 16: 0-\mathrm{ACP}$ than for $\mathrm{C} 16: 0-\mathrm{CoA}$ substrates and mainly producing $\mathrm{C} 16: 0-\mathrm{OH}$. Biochemical data indicated that DPW is involved in primary fatty alcohol synthesis for anther cuticle and pollen sporopollenin biosynthesis (Shi et al., 2011).

\section{Arabidopsis}

In the Arabidopsis genome, there are eight genes encoding FARs (FAR1-8) that are closely related to the original characterized jojoba FAR gene (Table 1.3) (Costaglioli et al., 2005; Rowland et al., 2006; Doan et al., 2009). These Arabidopsis FAR gene family members have diverse gene expression patterns and the encoded FAR enzymes have distinct substrate specificities as described below.

FAR2 (At3g11980), also known as MALE STERILITY2 (MS2/FAR2), was the first among the Arabidopsis FAR genes family to be identified and has been found to encode a tapetum-specific FAR protein involved in the synthesis of primary alcohols that are incorporated into sporopollenin of pollen exine wall (Aarts et al., 1997; Dobritsa et al., 2009). Cross-sections of mature anthers from ms2 mutants showed shrunken pollen exine layer and abnormal tapetal layer resulting in a high proportion of pollen abortion shortly after release from tetrads (Aarts et al., 1993). FAR2/MS was found to have an N-terminal transit peptide targeting it to the plastids. Biochemical analysis using purified MS2 enzyme, showed that FAR2/MS2 encodes a fatty acyl-acyl carrier protein (ACP) reductase that is able to convert 
C16:0-ACP to the corresponding C16:0 fatty alcohol and uses $N A D(P) H$ as the preferred electron donor (Chen et al., 2011). Transient expression of MS2 in tobacco (Nicotiana benthamiana) leaves resulted in the accumulation of significant levels of C16 and C18 fatty alcohols (Chen et al., 2011). Doan et al. (2009) found that FAR2/MS2 produces $\mathrm{C} 14: 0-\mathrm{OH}, \mathrm{C} 16: 0-\mathrm{OH}$ and $\mathrm{C} 18: 1-\mathrm{OH}$ using endogenous acyl-ACP pools when expressed in E. coli.

Another Arabidopsis FAR family member is FAR3 (At4g33790), also known as CER4, which encodes an alcohol-forming fatty acyl-CoA reductase and likely the only FAR that generates C24:0-C30:0 very-long-chain primary alcohols present in cuticular wax of aerial tissues (Rowland et al., 2006). cer4 mutants have glossy stems and show a major decrease in primary alcohols (C24:0, C26:0 and C28:0) and 8-fold reduced wax esters (Jenks et al., 1995; Rowland et al., 2006). FAR3/CER4 is expressed in epidermal cells of the aerial tissues and roots, although its role in roots is currently unclear (Rowland et al., 2006). FAR3/CER4 is localized to the endoplasmic reticulum (ER) when expressed in yeast cells (Rowland et al., 2006), but ER localization in planta has not yet been verified. Expression of CER4 cDNA in yeast resulted in accumulation of $\mathrm{C} 24: 0$ and C26:0 primary alcohols using endogenous acyl-CoA substrates (Rowland et al., 2006).

Arabidopsis FAR1 (At5g22500), FAR4 (At3g44540), and FAR5 (At3g44550) are responsible for generating C18:0-C22:0 primary fatty alcohols associated with suberin (Domergue et al., 2010; This Study). Characterisation of these three root- 
specific FARs is the main objective of this thesis and these have been discussed in detail in Chapters 2 - 5 of this thesis.

Table 1.3. Characterized Fatty Acyl Reductases (FARs) from plants.

Note: Characterization of FAR1, FAR4 and FAR5 genes reported is from the current study and published in Domergue et al. (2010). The Table is reproduced from Rowland and Domergue (2012) with permission.

\begin{tabular}{|c|c|c|c|c|c|c|c|}
\hline Gene name & Species & $\begin{array}{l}\text { AGI code } \\
\text { or NCBI } \\
\text { accession }\end{array}$ & $\begin{array}{l}\text { Prote } \\
\text { in } \\
\text { size } \\
\text { (a.a) }\end{array}$ & $\begin{array}{l}\text { Subcell } \\
\text { ular } \\
\text { localiza } \\
\text { tion }\end{array}$ & $\begin{array}{l}\text { Primary } \\
\text { substrate } \\
\text { specificity in } \\
\text { planta }\end{array}$ & $\begin{array}{l}\text { Functional } \\
\text { ass ociation in planta }\end{array}$ & References \\
\hline FAR1 & Arabidapsis & At5g22500 & 491 & $\mathbf{E R}^{3}$ & $\mathrm{C} 22: 0$ & $\begin{array}{l}\text { Root, seed coatand } \\
\text { wound-induced } \\
\text { suberin, cutin. }\end{array}$ & $\begin{array}{l}\text { [Domergue et } \\
\text { al, 2010] }\end{array}$ \\
\hline$F A R 2 / M S 2$ & Arabidopsis & At3g1 1980 & 616 & Plastid & $\mathrm{C} 16: 0$ & $\begin{array}{l}\text { Pollen exine (likely } \\
\text { sporopollenin) }\end{array}$ & $\begin{array}{l}\text { [Aartset al., } \\
1997 ; \text { Chen et } \\
\text { al, 2011] }\end{array}$ \\
\hline$F A R 3 / C E R 4$ & Arabidopsis & At4g33790 & 493 & $\mathbf{E R}^{4}$ & $\begin{array}{l}C 24: 0, C 26: 0 \\
C 28: 0, C 30: 0\end{array}$ & Cuticular Waxes & $\begin{array}{l}\text { [Rowland et } \\
\text { al. 2006] }\end{array}$ \\
\hline FAR4 & Arabidopsis & At3g44540 & 493 & $\mathbf{E R}^{3}$ & $\mathrm{C} 20: 0$ & $\begin{array}{l}\text { Root, seed coatand } \\
\text { wound-induced } \\
\text { suberin, cutin. }\end{array}$ & $\begin{array}{l}\text { [ Domergue et } \\
\text { aL. 2010] }\end{array}$ \\
\hline FAR5 & Arabidopsis & At3g44550 & 496 & $\mathbf{E R}^{3}$ & C18:0 & $\begin{array}{l}\text { Root, seed coatand } \\
\text { wound-induced } \\
\text { suberin, cutin }\end{array}$ & $\begin{array}{l}\text { [ Domergue et } \\
\text { al, 2010] }\end{array}$ \\
\hline FAR6 & Arabidopsis & At3g56700 & $548^{1}$ & Plastid & $\mathrm{C} 16: 0$ & Un known & $\begin{array}{l}\text { [Doan et al, } \\
\text { 2011] }\end{array}$ \\
\hline FAR7 & Arabidopsis & At5g22420 & $409^{2}$ & $\mathbf{E R}^{3}$ & None & Likely a pse udogene & $\begin{array}{l}\text { [Doan et al, } \\
\text { 2009] }\end{array}$ \\
\hline FARB & Arabidopsis & At3g44560 & 496 & $\mathbf{E R}^{3}$ & Unknown & Possible pse udogene & \\
\hline$S \subset F A R$ & Jojoba & AAD38039 & 493 & $\mathrm{ER}^{3}$ & C20: 1, C2 2: 1 & $\begin{array}{l}\text { Seed storage energy } \\
\text { (wax esters) }\end{array}$ & $\begin{array}{l}\text { [Metz et aL, } \\
\text { 2007] }\end{array}$ \\
\hline$D P W$ & Rice & BAH00399 & 608 & Plastid & C16:0 & $\begin{array}{l}\text { Anther cuticle, pollen } \\
\text { exine (likely } \\
\text { sporopollenin) }\end{array}$ & $\begin{array}{l}\text { [Shi et al. } \\
2011]\end{array}$ \\
\hline$T A A 1$ & $\begin{array}{l}\text { Bread } \\
\text { Wheat }\end{array}$ & CAD67815 & 507 & $\mathrm{ER}^{3}$ & $\begin{array}{l}\text { C18: } 1, C 20: 1 \\
C 22: 1, C 24: 0 \\
C 26: 0^{5}\end{array}$ & Pollen Wall & $\begin{array}{l}\text { [Wang et al., } \\
\text { 2002] }\end{array}$ \\
\hline $\begin{array}{l}\text { 1. Protein siz } \\
\text { inactive } 527 \\
\text { 2. Amino acid } \\
\text { codons [10] } \\
\text { 3. Predicted } \\
\text { 4. Subcellular } \\
\text { 5. Chain leng }\end{array}$ & $\begin{array}{l}\text { is for long-for } \\
\text { a. prote in [Do } \\
\text { number is from } \\
\text { bcellular local } \\
\text { ocalization pe }\end{array}$ & $\begin{array}{l}\text { splicing var } \\
\text { n et al, } 2009] \\
\text { gene predicti } \\
\text { zation, needs } \\
\text { formed in yea }\end{array}$ & $\begin{array}{l}\text { nt, a sho } \\
\text { n [ Row } \\
\text { xperime }\end{array}$ & $\begin{array}{l}\text {-form spli } \\
\text { and et al, } 2 \\
\text { tal data } \\
\text { d et al, } 20\end{array}$ & $\begin{array}{l}\text { ing variant has } \\
\text { 06], but the onl }\end{array}$ & $\begin{array}{l}\text { en identified but it enco } \\
\text { cloned cDNA has a stop }\end{array}$ & don for an 95 \\
\hline
\end{tabular}


The biochemical characterization of FAR6 (At3g56700) was reported by Doan et al., (2011). AtFAR6 is expressed in various tissues of the plant, including the stem epidermal layer (but not in the inner cortex or vascular bundles), in flower epidermis, endothecium and tapetum of anthers, (but not in the microspores), in the replum and receptacle of silique and in emerging root primordia in lateral roots (Doan et al., 2011). The N-terminal transit peptide sequence of FAR6, when transiently expressed in Nicotiana tabacum leaves showed it to be targeted to chloroplasts. FAR6 is NAD(P)H dependent and has specificity toward C16:0 and C18:0 substrates (both acyl-ACP and acyl-CoA) under in vitro conditions and in all tested expression systems (E. coli, yeast, $N$. benthamiana leaves) (Doan et al., 2009; Doan et al., 2011). Expression of full length FAR6 in Nicotiana benthamiana leaves resulted in the production a small amount of wax ester comprised of $\mathrm{C} 16: 0-0 \mathrm{H}$. Recently, Gupta et al., (2012) reported that the upstream sequence of AtFAR6 drives wound-inducible and stem-specific expression. However, the role of AtFAR6 in planta remains unclear.

The FAR7 (At5g22420) cDNA contains an early stop codon and encodes a truncated, inactive protein (Doan et al., 2009). FAR7 is thus likely a pseudogene. FAR8 has very low activity with a very low gene expression (Rowland and Domergue, 2012).

\subsubsection{Substrate specificities of plant FAR enzymes}

FARs have distinct substrate specificities with regard to chain length and acyl chain saturation (Table 1.3) (Rowland and Domergue, 2012). The substrate specificities of plant FAR enzymes was mainly studied at the in vivo level, wherein 
the conclusions regarding the substrate specificity of a certain enzyme were either based on the fatty alcohol profiles of the corresponding mutants or the expression of the FAR in a heterologous expression system, such as transiently in $N$. benthamiana leaves, in yeast ( $S$. cerevisiae), or in E. coli systems (Table 1.3).

Compositional analysis of the wax esters in jojoba seeds indicates that ScFAR mainly produces C20:1 and C22:1 primary fatty alcohols (Miwa et al., 1971), but it produced C16:0 and C18:1 111 primary alcohols, when expressed heterologously in E. coli, and C22:1 fatty alcohols were detected when expressed in the seeds of high erucic acid oilseed rape (Brassica napus) plants (Metz et al., 2000). Doan et al., (2009) expressed five of the Arabidopsis FARs (FAR1, 2, 3, 6 and 8), and the jojoba FAR in E. coli and reported that they produce $\mathrm{C} 12: 0-\mathrm{OH}, \mathrm{C} 14: 0, \mathrm{C} 16: 0$ and $\mathrm{C} 18: 1$ fatty alcohols in variable relative proportions, suggesting distinct substrate specificities of FARs, although it was not in agreement with the fatty alcohols synthesized by some of these FARs in planta. For example, heterologous expression of Arabidopsis CER4/FAR3 in yeast, results in production of C26:0-C28:0 saturated fatty alcohol, although it is responsible for the production of C24:0 to C30:0 saturated fatty alcohols in planta (Rowland et al., 2006).

The apparent substrate specificity of an enzyme can vary greatly depending upon the composition of endogenous acyl pools of the host, resulting in skewed information. The acyl pool in E. coli system consists of acyl chains linked to ACPs, while yeast mostly has acyl-CoAs along up to C26 chain length. In plants, the acyl pool in plastids (i.e. chloroplasts and leucoplasts) is mostly acyl-ACP, whereas the acyl pool outside the plastid is mostly acyl-CoA. Therefore, the yeast system is a 
more reliable indicator of in planta substrate specificity than $E$. coli for most of the plant FARs other than the plastidial FARs which most probably produce shorter chain C16:0 fatty alcohols in planta (Doan et al., 2011; Rowland and Domergue, 2012). Transient expression of FARs in leaves of Nicotiana benthamiana is another alternative currently being used for substrate specificity studies (Doan et al., 2011; Chen et al., 2011). Recently, in vitro assay to study plant FARs specificity and activity has been developed and used for study of soluble plastidial FARs (Doan et al., 2011; Shi et al., 2011). However, studying membrane localized FARs and unavailability of long chain acyl CoAs (>C18) makes in vitro assays more challenging.

\subsubsection{FARs in non-plant species}

FARs have been also identified and characterized in silkmoth (Moto et al., 2003), mouse and human (Cheng and Russell, 2004a, 2004b), honeybee (Teerawanichpan et al., 2010), birds (Hellenbrand et al., 2011), copepod (Teerawanichappan and Qiu, 2011), phytoflagellate protist (Teerawanichappan and Qiu, 2011), and marinobacter (Hofvander et al., 2011).

\section{Insects:}

In 2003, the identification of a pheromone gland-specific long-chain FAR (pgFAR) was first reported in silkmoth (Bombyx mori) (Moto et al., 2003). This FAR is the key enzyme required for the sex pheromone biosynthesis and converts the fatty-acyl pheromone precursor to bombykol (E,Z-10,12-hexadecadien-1-ol), the final active product of the pheromones. In heterologous expression studies in yeast, BmFAR was found to preferentially reduce C15 and C16 fatty acids to fatty alcohols, with a strong preference for the pheromone precursor fatty acid 10,12- 
hexadecadienoic acid (Moto et al., 2003). Subsequently, pgFARs were identified in other Lepidopteran insects, adzuki bean borer moth and small ermine moths, with each specific to substrates with certain chain lengths or degrees of saturation (Antony et al., 2009; Liénard et al., 2010). OsFARXIII, a fatty acyl-CoA reductase from bean borer moth (Ostrinia scapulalis), catalyzes the production of (Z)-11tetradecenol, which is further converted to its acetate or aldehyde derivative pheromones (Antony et al, 2009). A third insect FAR, AmFAR1, was identified and characterized in honeybees (Apis melifera) and is preferentially expressed in the head segment of the bees (Teerawanichpan et al., 2010a). Heterologous expression of AmFAR1 showed that it converts a wide range of fatty acyl-CoAs to their corresponding alcohols in the presence of NADPH, with stearic acid (18:0) as the most preferred substrate and $\mathrm{C} 18: 0-\mathrm{OH}, \mathrm{C} 20: 0-\mathrm{OH}$, and $\mathrm{C} 22: 0-\mathrm{OH}$ being the main products. These fatty alcohols could be used as precursors for synthesizing acetate esters and aldehyde derivatives by acetate esterase and alcohol oxidase, yielding the pheromone blends, suggesting that AmFAR1 is involved in pheromone biosynthesis (Teerawanichpan et al., 2010a). Lassance et al. (2010) showed that allelic variation in pheromone gland-specific fatty-acyl reductase gene accounts for the phenotypic variation in female pheromone production, leading to race-specific signals and reproductive isolation in moths. They studied the affect of divergence on the substrate specificity of two pgFARs, pgFAR-E and $p g F A R-\mathrm{Z}$, from European corn borer, Ostrinia nubilalis, known to consist of two sex pheromone races, $\mathrm{Z}$ and $\mathrm{E}$, that use different ratios of the cis and trans isomers of acetate pheromone components. Heterologous expression of these two FARs in yeast showed that pgFAR-Z almost 
exclusively converts the $Z$ isomer of the substrate (11-tetradecenoyl), with minimal amounts of the $E$ isomer being reduced, and pgFAR-E almost exclusively converts the $E$ isomer of the substrate with minimal amounts of the $Z$ isomer being reduced (Lassance et al., 2010).

Mammals:

In mammals, FARs are likely to engage the biosynthesis of both ether lipids and wax esters (Cheng and Russell, 2004a). Using a bioinformatics approach, cDNAs encoding two fatty acyl-CoA reductase isozymes, FAR1 and FAR2, were identified each in mouse (m) and human (h) (Cheng and Russell, 2004a). Both FAR1 and FAR2 were found to be highly expressed in the brain where large quantities of ether lipids are synthesized (Cheng and Russell, 2004a). FAR1 and FAR2, from both mouse and human, when expressed in human embryonic kidney (HEK) 293 cells, were reported to convert $\mathrm{C} 16: 0$ acyl-CoA to $\mathrm{C} 16: 0-\mathrm{OH}$, with FAR1 being more active than FAR2 in both human and mouse FARs (Cheng and Russell, 2004a). A similar substrate specificity, but with higher activity was observed when the mouse FAR1 and FAR2 were expressed in insect ovarian cells (Spodoptera frugiperda (Sf) 9 cells).

Subcellular localisation studies in the Chinese hamster ovary-K1 cells transfected cells revealed that FAR1 and FAR2 are localized to the peroxisome, which is consistent with these FARs being required to produce fatty alcohols for ether lipid (plasmologens) biosynthesis (Cheng and Russell, 2004a). In summary, the broadly distributed mouse FAR1 was reported to act on fatty acyl-CoAs of different chain lengths and degrees of saturation, while mouse FAR2 with narrow distribution (tissue with sebaceous glands) prefers saturated C16 and C18 fatty acyl-CoAs as 
substrates suggesting that FAR1 plays a general role in fatty alcohol synthesis and FAR2 has a more specialized function. Also, it was found that NADPH was required as a cofactor for FAR1 and there was no activity using NADH (FAR2 was not tested) (Cheng and Russell, 2004).

\section{Phytoflagellate Protist:}

Euglena gracilis is a unicellular phytoflagellate protist known to accumulate large amounts of medium and long-chain wax esters (C20-C36) under anaerobic growth conditions (62\%) (Teerawanichpan and Qiu, 2010b). Two E. gracilis genes encoding enzymes involved in wax ester biosynthesis were identified and characterized: a fatty acyl-CoA reductase (EgFAR) and a wax synthase (EgWS) (Kolattukudy, 1970; Teerawanichpan and Qiu, 2010b). Biochemical assays using a microsomal fraction of E. gracilis showed that EgFAR used C14:0, C16:0 and C18:0 as substrates and required ATP, CoA, and NADH as a cofactor (Kolattukudy, 1970). EgFAR expressed heterologously in yeast showed conversion of exogenously fed C14:0 and C16:0 fatty acids to their corresponding primary fatty alcohols, with C14:0 being the most preferred substrate. No fatty alcohols were detected when yeast were fed the saturated fatty acids $\mathrm{C} 10: 0, \mathrm{C} 12: 0$, or C18:0 or the unsaturated fatty acids C16:1n-9, C18:1n-9, or C18:2n-6. Also, co-expression of EgFAR and EgWS in yeast, in the presence of exogenously fed C14:0, resulted in myristyl myristate (14:0-14:0), myristyl palmitoleate (14:0-16:1), myristyl palmitate (14:0-16:0) and palmityl myristate (16:0-14:0) as major wax ester monomers, indicating that EgFAR and EgWS are likely involved in the biosynthesis of medium-chain wax esters in $E$. gracilis (Teerawanichpan and Qiu, 2010b). 


\section{Copepod:}

The marine copepod (Calanus finmarchicus), is a small crustacean that accumulates a high level of wax esters as carbon storage that is mainly comprised of 20:1n-9 and 22:1n-11 alcohols (Alc) linked with various kinds of fatty acids, including n-3 polyunsaturated fatty acids (Teerawanichpan and Qiu, 2012). Three FAR genes were identified in the marine copepod, CfFAR1, CfFAR2 and CfFAR3. All three CfFARs showed distinct substrate specificities upon heterologous expression in yeast: CfFAR1 is active toward saturated C18:0-C26:0 fatty acyl-CoAs, CFFAR2 is active toward C24:0 and C26:0 saturated very-long-chain fatty acyl-CoAs, and CfFAR3 is active towards both saturated (C16:0 and C18:0) and unsaturated (C18:1 and C20:1) fatty acyl-CoAs (Teerawanichpan and Qiu, 2012). CfFAR3 was suggested to be partly responsible for the observed fatty alcohol profile of $C$. finmarchicus wax esters, which consists of both saturated and monounsaturated alcohols (Teerawanichpan and Qiu, 2012). Hydropathy analysis revealed a hydrophobic region at the carboxyl terminus of CfFAR2 and CfFAR3 (but not in CfFAR1), which was also found in mammalian FARs and a honeybee FAR (Cheng and Russell 2004; Teerawanichpan et al., 2010) and was assumed to play a role in targeting the FARs to the peroxisome where the ether lipids are synthesized (Cheng and Russell 2004; Honsho et al. 2010).

\section{Birds:}

Birds clean and lubricate their feathers with waxes that are produced in the uropygial gland, a holocrine gland located on their back above the tail. The type and the composition of the secreted wax esters varies depending on the bird species 
(Hellenbrand et al., 2011). Purification and characterization of a membrane bound FAR from duck uropygial gland was reported by Wang and Kolattukudy (1995b). The enzyme purified from the microsomal preparation was found to have a molecular mass of $56 \mathrm{KDa}$ and required NADPH as electron donor for activity (Wang and Kolattukudy, 1995). Recently, Hellenbrand et al., (2011) reported cloning and heterologous expression studies of FAR enzymes from three birds: barn owl (Tyto alba), domestic chicken (Gallus gallus domesticus), and domestic goose (Anser anser domesticus). These were reported to be membrane associated enzymes catalyzing a NADPH dependent reduction of acyl-CoA thioesters to fatty alcohols. Based on feeding experiments of transgenic yeast expressing these FARs and in vitro assays, two groups of isozymes with different properties were identified, termed FAR1 and FAR2. The FAR1 group mainly synthesized 1-hexadecanol (C16:0-0H) and accepted substrates in the range between 14 and 18 carbon atoms, whereas the FAR2 group preferred stearoyl-CoA (C18:0) and accepted substrates between 16 and 20 carbon atoms (Hellenbrand et al., 2011).

\section{Marinobacter:}

In bacteria, wax esters most likely serve as energy reserves and are often found in marine environments and associated with species that can degrade crude oil or in marine sediments (Hofvander et al., 2011). In prokaryotes, reduction of acyl-CoA or acyl-ACP to fatty alcohol via a fatty aldehyde intermediate is thought to be carried out by two separate enzymes for each reduction step (Waeltermann et al., 2007), while in eukaryotes these reactions are performed by a single enzyme without the release of the intermediate fatty aldehyde (Metz, et al., 2000; Hofvander 
et al., 2011). Recently, a FAR protein named Maqu_2220, purified from Marinobacter aquaeolei VT8, was reported to catalyse both the reduction steps, thereby establishing that in certain prokaryotes these reductions can be performed in an NADPH-dependent manner by a single enzyme. In studies using in vitro assays, Marinobacter FAR showed activity with diverse substrates of different carbon chain lengths and unsaturation, with $\mathrm{C} 18: 1-\mathrm{CoA}$ being the most preferred substrate (Hofvander et al., 2011).

\subsection{Bioinformatics of Alcohol-forming FAR enzymes}

\subsubsection{FAR protein structure}

Plant FAR enzymes are about 500 amino acids in length, without the $\mathrm{N}$ terminal extension containing a plastid (e.g. chloroplast) transit peptide found in plastid localized FAR isoforms (Table 1.3). Plant FARs contain two distinct domains: a Rossmann fold NAD(P)H-binding domain and a FAR_C domain (Figure 1.12) (Rowland and Domergue, 2012), according to the protein structure prediction software- Conserved Domains Database (CDD) (Marchler-Bauer et al., 2010).

The Rossmann fold structure has an $\alpha / \beta$ folding pattern and a central $\beta$ sheet at the $\mathrm{N}$-terminus (Rossmann fold) (Rossmann, 1974; Richardson, 1981), which is commonly found in the short-chain alcohol dehydrogenase/reductase (SDR) family of proteins, also known as the single-domain reductases/epimerases/dehydrogenases (RED) family (Rowland and Domergue, 2012). These plant FARs contain the conserved motif $\operatorname{GXXGXX(G/A)}$ at their $\mathrm{N}$ terminus (Figure 1.12), which is similar to the consensus sequence for ADP binding 
domain, $[\mathrm{I} / \mathrm{V} / \mathrm{F}]-\mathrm{X}-[\mathrm{I} / \mathrm{L} / \mathrm{V}]-\mathrm{T}-\mathrm{G}-\mathrm{X}-\mathrm{T}-\mathrm{G}-\mathrm{F}-\mathrm{L}-[\mathrm{G} / \mathrm{A}]$, and is probably the NAD(P)H binding site (Wierenga et al., 1986; Eggink et al., 1990). These FARs also contain the YXXXK active site motif (predicted catalytic residue within the Rossmann fold domain), typical of the short-chain dehydrogenase/reductase superfamily and fall into the SDR117E family (Figure 1.12) (Kavanagh et al., 2008).

The FAR_C domain is located at the C-termini of FAR protein. The FAR_C (or fatty acyl-CoA reductase) domain, in databases, is also referred to as "Male Sterility 2" or "Sterile" domain based on the first cloned and characterized FAR gene encoding MALE STERILITY2 (MS2/FAR2) from Arabidopsis thaliana (Aarts et al., 1993, 1997). This domain is unique to the FAR subfamily of reductases (Aarts et al., 1997; Rowland et al., 2006; Doan et al., 2009), but the function of this domain has yet to be determined (Figure 1.12).

Some of the FARs, such as Arabiodpsis FAR2/MS2, FAR6 and the rice DPW, have an N-terminal extension of $\sim 50-120$ a.a., which contains a chloroplast targeting sequence (Figure 1.12) (Doan et al., 2009; 2011; Chen et al., 2011; Shi et al., 2011). This cleavable $\mathrm{N}$-terminal transit sequence functions as a plastid targeting domain and undergoes proteolytical processing concurrently with or shortly after translocation, by a soluble stromal processing peptidase (Soll and Tien, 1998). The C-terminal portion of this targeting sequence usually has a conserved consensus sequence I/V-X-A/C-A near the cleavage site (Gavel and von Heijne, 1990). 


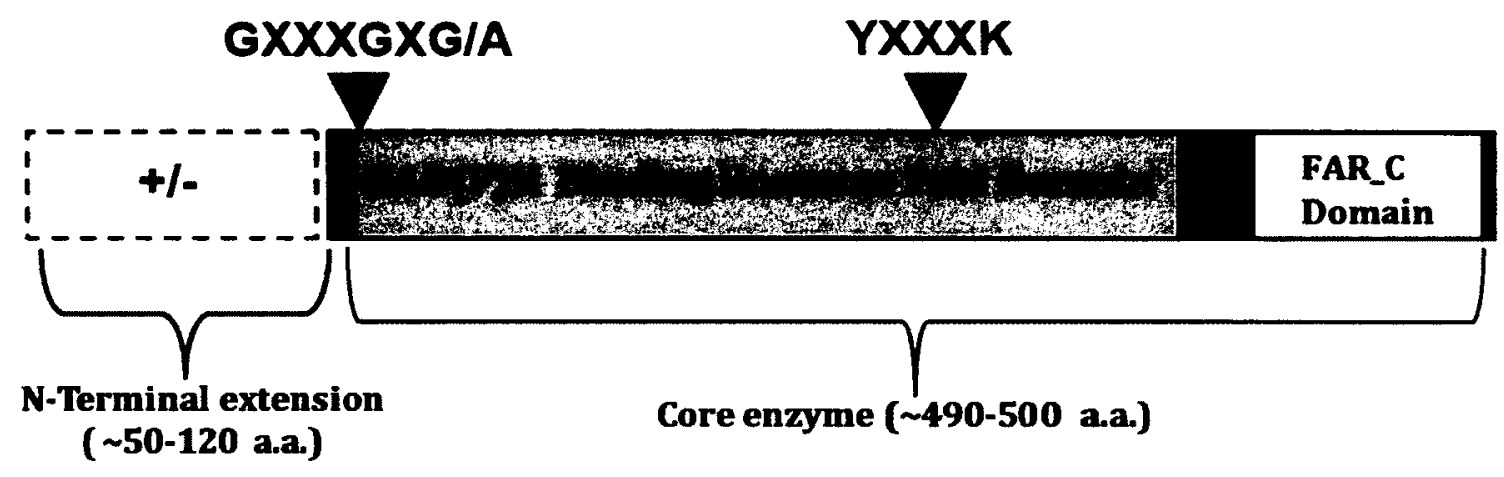

Figure 1.12. FAR structural domains. Most of the FAR enzymes have a NAD(P)Hbinding Rossmann fold domain at the $\mathrm{N}$-terminus (highlighted in grey), with the GXXXGX(G/A) sequence motif for ADP/NAD(P)H binding indicated as well as the predicted catalytic residues YXXXK (' $X$ ' represents any amino acid). The FAR_C domain (also refered as male sterile domain) at the C-terminus is common to alcohol-forming fatty acyl reductases. The regions encompassed by these two domain including the region between is about 490 amino acids. Some FARs have an $\mathrm{N}$-terminal extension ranging in size from $\sim 50$ to $\sim 120$ amino acids and contains a chloroplast targeting sequence. Adapted from Rowland and Domergue (2012). Image reproduced with permission CPlant science, ASPB. 


\subsubsection{Phylogenetic analysis of related FARs}

Evolutionarily related FARs have been identified in various species of plant and animal kingdom. The phylogenetic analysis shows clear formation of two clades separating plant FARs and the FARs from insects and animals (Figure 1.13). The plant FARs (Clade-I) separate into four groups. Most of the Arabidopsis FARs, FAR1, FAR4, FAR5, FAR7 and FAR8, which are predicted to be ER-localized, fall in the second group, while FAR3/CER4 which is more similar to jojoba FAR, falls in group-

1. These five proteins were reported to contain a cysteine at position 5 , which is recognized as a putative palmitoylation site (Rowland and Domergue, 2012). Arabidopsis FAR2/MS2 and FAR6 (Chen et al., 2011; Doan et al., 2012), which are localized in plastids and are probably soluble enzymes fall in Group 4 (Rowland and Domergue, 2012). All the species in Group 3 are monocots and the dicots are in Group 4. It is notable that Groups 1,2 and Groups 3,4 are retrieved as clades in the phylogenetic tree, based on predicted subcellular localisation (Figure 1.13). Arabidopsis FAR3/CER4, in group 1, is reported to contain an additional predicted $\mathrm{N}$-terminal transmembrane helix (amino acids 20-37) and is localized to the endoplasmic reticulum when heterologously expressed in yeast (Rowland et al., 2006). It is not known whether FAR3/CER4 has the same subcellular localisation in planta. 


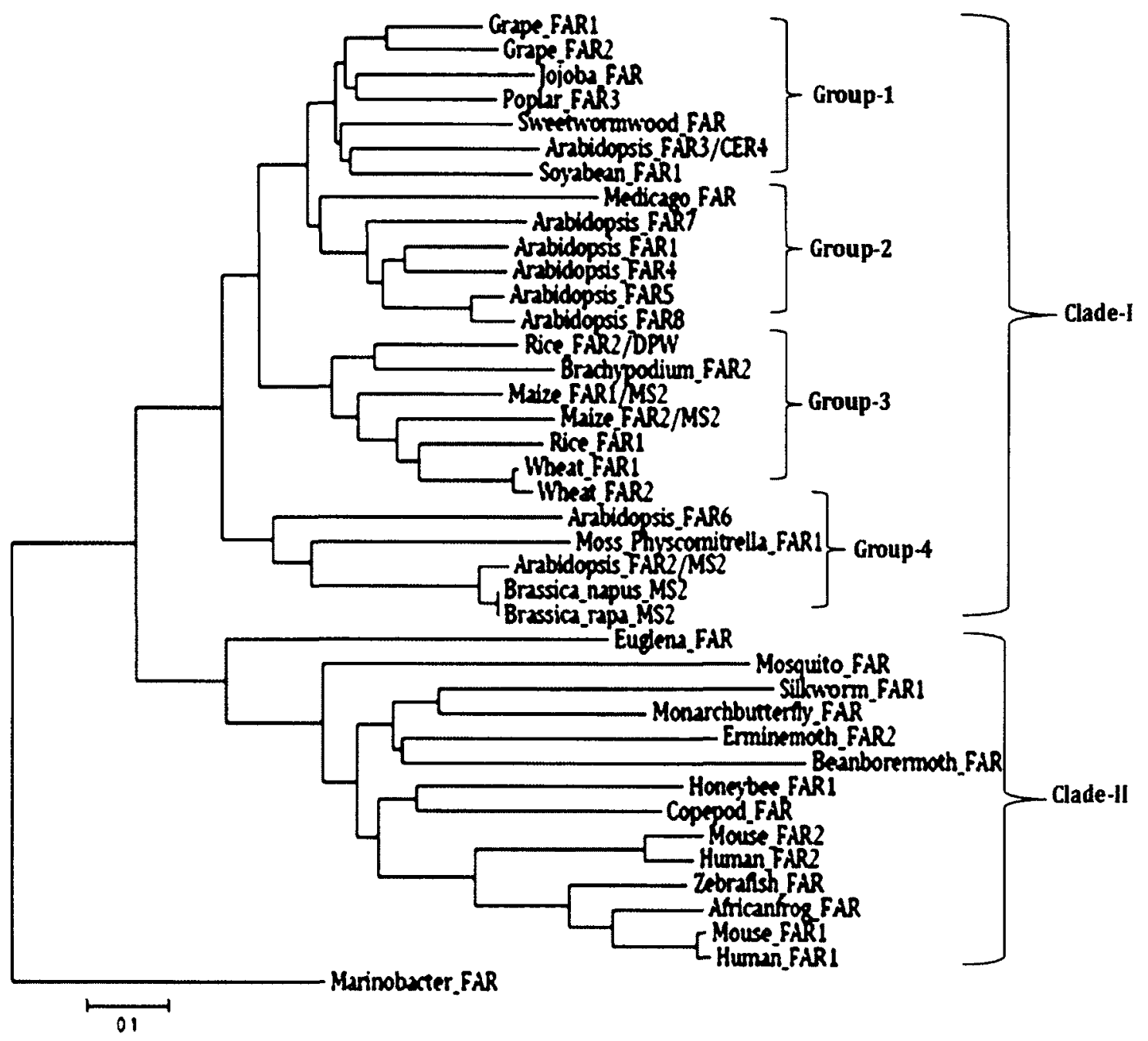

Figure 1.13. Phylogenetic tree of related FAR protein sequences. Protein sequences were aligned using ClustalW2 (Larkin et al., 2007) and phylogenetic tree generated using the Neighbor-Joining method (Saitou and Nei, 1987). Branch lengths are proportional to the amount of inferred evolutionary change. Bootstrap values were calculated from 1000 replicates. The analysis involved 40 amino acid sequences. Marinobacter-FAR is an outgroup and was used to root the tree. Evolutionary analyses were conducted in MEGA5.1 software (Tamura et al., 2011) (http://www.megasoftware.net/). The amino acid sequences of various fatty acyl reductases were obtained from NCBI protein database (U.S. National Library of Medicine, 2009). The GenBank accession identifier numbers of the sequences used for the analysis are listed in Appendix Table-1. Figure by Sollapura J. Vishwanath. 


\subsection{Importance/Application of this study}

Suberin is important to study as its inducible nature and adaptive functioning make it a crucial part of plant defence and health (Franke et al., 2009; Ranathunge et al., 2011). Knowledge of suberin structure, biosynthesis and regulated deposition would give us an invaluable repertoire to alter suberin load or properties for potentially producing agronomically superior crop plants having drought tolerance and pathogen resistance. For example, Arabidopsis esb1 (enhanced suberin1) mutant, which has double the amount of aliphatic suberin monomers, were reported to have reduced transpiration, increased drought tolerance, and increased water use efficiency (Baxter et al., 2009). Thus, identification of key suberin genes has significant implications for understanding suberization and thereby improving abiotic or biotic stress tolerance in plants using biotechnological approaches.

The polyesters present in agricultural waste may be used to produce high value polymers, and genetic engineering might be used to produce large quantities of such polymers in plants. Also, one or more of these enzymes may prove valuable for the commercial production of fatty alcohols and/or wax esters in microbes or transgenic seed oils. Wax esters in particular have important commercial applications in a variety of technical areas, including the medicine, cosmetics and food industries as well as their more traditional usage as lubricants for mechanical parts. The jojoba (Simmondsia chinensis) plant produces esters of long-chain alcohols and fatty acids (waxes) as a seed lipid energy reserve. The jojoba FAR cDNA, when expressed in embryos of Brassica napus, resulted in increased levels of 
long-chain alcohol production along with novel wax esters in the transgenic seed oils (Metz et al, 2000). High levels of wax accumulation (upto $70 \%$ of oil weight in seeds) in transgenic Arabidopsis seeds was observed on expressing jojoba FAR and WS (wax synthase) cDNA (Lassner et al., 1999; Lardizabal et al., 2000). The unique substrate specificities of the Arabidopsis FARs may be valuable for the production of novel wax esters in an oilseed crop. The substrate specificity of a FAR has been shown to have a great influence on the final product, with regards to the chain length and degree of saturation of the primary alcohols, which significantly influences the physical properties of wax esters produced by wax synthases. The FAR enzymes are being co-expressed with wax synthases in the seeds of non-food crop plants (Crambe abysinnica, Brassica carinata, and Camelina sativa) for the production of high value wax esters in a European Union Framework funded project entitled 'Industrial crops producing added value oils for novel chemicals ICON: http://icon.slu.se/ICON/.

Likewise, fatty alcohols have important commercial uses, e.g. as solvents, lubrication oil additives, plasticizers, nonionic surfactants, in pharmaceutical compositions as salves and lotions, and as ingredients in cosmetics. Certain longchain fatty alcohols have been found to have antibacterial action (Masao et al., 1987). Thus, study of FAR enzymes is of significant importance as these are involved in the production of fatty alcohols, aldehydes and wax esters. Further, the knowledge of specificity of these plant FAR enzymes would provide insights into the functioning of FARs of other organisms, such as those present in insects and mammals. 


\subsection{Research objectives}

DNA microarray studies surveying developmental gene expression patterns in various tissues (Schmid et al., 2005) indicated that FAR1, FAR4 and FAR5 genes are highly expressed in roots and floral organs. The roles for these alcohol-forming fatty acyl-CoA reductase enzymes in roots were unknown. I hypothesized that these candidate FARs are responsible for the production of long-chain and very-longchain fatty alcohols (C18:0-C22:0) associated with suberin biosynthesis in Arabidopsis.

The main objectives of the work presented in this thesis were as follows:

a. Identification of FAR enzymes in Arabidopsis that synthesize primary alcohols associated with suberin (Chapter 2).

This objective was approached by studying the spatial and temporal gene expression pattern of the candidate FAR genes using promoter:GUS fusion system under normal and stress conditions. In addition, the single mutant TDNA insertion lines of the candidate genes were analysed for changes in the fatty alcohol composition of root, seed coat, and wound-induced suberin.

b. Study of the substrate specificity of suberin associated FARs in Arabidopsis (Chapters 2 and 4).

This objective was first approached by heterologous expression of candidate FAR genes in yeast and analyzing the lipid composition. The single mutant $\mathrm{T}$ DNA insertion lines of the candidate genes were also analyzed for changes in particular chain length fatty alcohol in the root suberin composition. In addition, the candidate Arabidopsis FAR genes were transiently over- 
expressed in leaves of Nicotiana benthamiana and the lipid composition was analyzed.

c. Study of the effects of reduction of primary fatty alcohol in Arabidopsis plants (Chapter 3).

This objective was approached by generating double and triple mutants of the suberin associated FAR genes and analyzing the suberin composition of the mutant lines in different root fractions. The mutant lines were assessed for alterations in germination rate, root growth and permeability properties.

d. Study of the subcellular localization of the suberin-associated FAR1 enzyme in Arabidopsis. (Chapter 4).

This objective was approached by transiently expressing Arabidopsis FAR1:GFP fusion protein in the leaves of $N$. benthamiana.

e. Identification of a region in the FAR protein domain responsible for the substrate specificity (Chapter 4).

This objective was approached by carrying out domain swaps between the candidate FARs and expressing them heterologously in yeast and analyzing the lipid composition.

f. Study of the regulation of suberin genes under various abiotic stresses (Chapter 5).

This objective was approached by subjecting the Arabidopsis Col-0 seedlings to various abiotic stresses and analyzing the expression levels of various suberin genes, including FARs, using quantitative-RTPCR. 


\section{CHAPTER 2: Three Arabidopsis Fatty Acyl-Coenzyme A Reductases, FAR1, FAR4, and FAR5, Generate Primary Fatty Alcohols Associated with Suberin Deposition.}

Data from this chapter was published in: Domergue, F.*, Vishwanath, S. J.*, Joubès, J., Ono, J., Lee, J., Bourdon, M., Alhattab, R., Lowe, C., Pascal, S., Lessire, R and Rowland, O. (2010). Three Arabidopsis Fatty Acyl-CoA Reductases, FAR1, FAR4, and FAR5, Generate Primary Fatty Alcohols Associated with Suberin Deposition. Plant Physiology, 153: 1539-1554. * Co-first author

\subsection{Introduction}

Suberin is a lipid- and phenolic-based polymer present at the inner face of primary cell walls of various external and internal tissue layers (Franke and Schreiber, 2007). In roots, suberin deposition occurs in the endodermis surrounding the central vasculature, the exodermis near the soil interface, and the peridermis of matured roots and underground storage tubers (Ma and Peterson, 2003). In aerial tissues, suberin deposition occurs in bark tissue, the bundle sheath of $\mathrm{C} 4$ plants, cotton (Gossypium hirsutum) fibers, the chalazae plug of seeds, seed coat integuments, and in response to wounding (Kolattukudy, 2001). Suberin often consists of alternating dark and light bands, termed suberin lamellae, when viewed by transmission electron microscopy. The phenylpropanoid-derived aromatic portion of suberin mostly consists of ferulate, which is a $p$-hydroxycinnamic acid (Bernards et al., 1995). The aliphatic portion of suberin is a polyester largely composed of $\omega$-hydroxy fatty acids, $\alpha, \omega$-dicarboxylic acids, mid-chain oxygenated fatty acids, unsubstituted fatty acids, and primary fatty alcohols (Pollard et al., 2008). The relative abundance of suberin monomer constituents varies considerably between species (Pollard et al., 2008). Another major component of 
the suberin aliphatic domain is glycerol (Moire et al., 1999), which in partial depolymerization experiments is found esterified to $\omega$-hydroxy and $\alpha, \omega-$ dicarboxylic fatty acids (Graca and Pereira, 2000; Graca and Santos, 2006). The overall organization of the interunit linkages, including those involving unsubstituted fatty acids and primary fatty alcohols, remains to be determined.

The composition of suberin indicates that the following enzymatic activities, at least, are required for suberin biosynthesis: $\omega$-carbon oxidation, further oxidation of $\omega$-hydroxy fatty acids to $\alpha, \omega$-dicarboxylic acids, fatty acid elongation of longchain acyl precursors, activation of fatty acids to fatty acyl-CoA thioesters, reduction of acyl chains to primary alcohols, and various acylations, including those involving glycerol, to generate a polyester matrix. The identification of many of the key enzymes has been facilitated by the elucidation of the chemical composition of suberin in the model plant Arabidopsis (Arabidopsis thaliana; Franke et al., 2005) and the isolation of Arabidopsis mutants with altered suberin composition (Pollard et al., 2008). Progress in our understanding of suberin biosynthesis has been recently reviewed (Franke and Schreiber 2007; Pollard et al. 2008; Schreiber 2010; Ranathunge et al., 2011; Beisson et al., 2012; Franke et al., 2012) and discussed in detail in Chapter 1 (General Introduction).

Saturated primary fatty alcohols, typically of chain lengths C18, C20, and C22, are common components of root suberin (Zeier et al., 1999; Franke et al., 2005; Schreiber et al., 2005b; Beisson et al., 2007; Schreiber 2010; Domergue et al., 2010current study). Fatty acyl reductase (FAR) enzymes catalyze the reduction of fatty acyl-CoAs or fatty acyl-ACPs to primary fatty alcohols in a NADPH-dependent 
reaction. This reduction is a two-step process proceeding via an aldehyde intermediate, which can be carried out by a single alcohol forming FAR (Kolattukudy, 1970; Pollard et al., 1979; Vioque and Kolattukudy, 1997). Purification of an alcohol-forming FAR from jojoba (Simmondsia chinensis) embryos facilitated the cloning of the corresponding cDNA, and heterologous expression in bacteria confirmed that reduction of fatty acyl chains to primary alcohols is carried out by a single enzyme in plants (Metz et al., 2000). FAR enzymes related to the jojoba FAR have subsequently been identified and characterized in wheat (Triticum aestivum; Wang et al., 2002), rice (Oryza sativa; Shi et al., 2011), silkmoth (Moto et al., 2003), birds (Hellenbrand et al., 2011), mouse and human (Cheng and Russell, 2004), marine protists and copepod (Teerawanichpan and Qiu, 2010b and 2012), and Arabidopsis (Rowland et al., 2006; Doan et al., 2009; Chen et al., 2011; Doan et al., 2011). Expression of these FARs in various heterologous systems indicated that each has distinct substrate specificity in terms of both acyl chain length and degree of saturation (See General Introduction).

Based on the jojoba FAR sequence, eight genes encoding putative FARs (FAR1-8) (Figure 3.1) have been identified in Arabidopsis. MS2 (FAR2) produces primary alcohols that are incorporated into sporopollenin of the pollen exine layer (Aarts et al., 1997; Dobritsa et al., 2009; Chen et al., 2011), while CER4 (FAR3) generates C24:0-C30:0 primary alcohols present in cuticular wax of aerial tissues (Rowland et al., 2006). The Arabidopsis FAR gene family members have diverse gene expression patterns and the encoded FAR enzymes have diverse substrate specificities (see General Introduction). We demonstrate here that the gene 
expression patterns of FAR1, FAR4, and FAR5 coincide with known sites of suberin deposition and that the encoded enzymes have activities for acyl chain lengths consistent with those of suberin-associated primary alcohols. Furthermore, mutations in each of these genes differentially affected primary alcohol formation in root, seed coat, and wound-induced leaf tissue.

\subsection{Materials and Methods}

\subsubsection{Plant materials and growth conditions}

All Arabidopsis (Arabidopsis thaliana) plants were in the Columbia (Col-0) ecotype background. The following T-DNA insertion lines were obtained from the Salk Institute for Genomic Analysis Laboratory and the Arabidopsis Biological Resource Center (www.arabidopsis.org): SALK_068605 (far1-1), SALK_149469 (far1-2), SALK_000229 (far4-1), SALK_147493 (far4-2), SALK_152963 (far5-1), and SALK_070363 (far5-2). For growing plants, the seeds were surface sterilized by treating with $100 \%$ ethanol for 1 minute, followed by 1 minute incubation in $50 \%$ bleach (containing approximately $6 \%$ sodium hypochlorite and $0.5 \%$ SDS). Seeds were then rinsed several times with sterile distilled water. Sterile seeds were sown on minimal media containing $5 \mathrm{mM} \mathrm{KNO}_{3}, 2.5 \mathrm{mM} \mathrm{KH}_{2} \mathrm{PO}_{4}, 2 \mathrm{mM} \mathrm{MgSO}_{4}, 2 \mathrm{mM}$ $\mathrm{Ca}\left(\mathrm{NO}_{3}\right)_{2}, 50 \mu \mathrm{M} \mathrm{Fe}(\mathrm{EDTA}), 70 \mu \mathrm{M} \mathrm{H} \mathrm{H}_{3} \mathrm{BO}_{3}, 14 \mu \mathrm{M} \mathrm{MnCl}_{2} .4 \mathrm{H}_{2} \mathrm{O}, 0.5 \mu \mathrm{M} \mathrm{CuSO}_{4}, 1 \mu \mathrm{M}$ $\mathrm{ZnSO}_{4} .7 \mathrm{H}_{2} 0,0.2 \mu \mathrm{M} \mathrm{NaMoO}{ }_{4} .2 \mathrm{H}_{2} 0,10 \mu \mathrm{M} \mathrm{NaCl}, 0.01 \mu \mathrm{M} \mathrm{CoCl}_{2} .6 \mathrm{H}_{2} 0$, and $0.7 \%$ agar (Somerville and Ogren, 1982). For experimental analyses, sterilized seeds were sown on MS medium (Sigma) supplemented with $0.7 \%$ agar. The seeds were 
stratified in the dark for 3 to $4 \mathrm{~d}$ at $4^{\circ} \mathrm{C}$ and then transferred to long-day conditions (16-hour-light/8-hour-dark cycle) at $22^{\circ} \mathrm{C}$ or continuous light $\left(90 \mu \mathrm{mol} \mathrm{m}^{-2} \mathrm{~s}^{-1}\right)$ conditions at $20^{\circ} \mathrm{C}$. Seedlings were transferred 10 days to 3 weeks later to a soil vermiculite mixture (Pro-Mix MPV Multipurpose Growing Medium) and further grown at $20^{\circ} \mathrm{C}$ to $22^{\circ} \mathrm{C}$ under long-day conditions in a controlled growth chamber (Conviron model TC26 or Percival chamber model AR66L, Winnipeg, MB).

\subsubsection{Tissue-specific gene expression analysis by quantitative RT-PCR}

RNA was extracted from Arabidopsis tissues using the RNeasy Plant mini kit (Qiagen) according to the manufacturer's instructions, and purified RNA was treated with DNase I. First-strand cDNA was prepared from $1 \mu \mathrm{g}$ of total RNA with SuperScript Reverse Transcriptase II (Invitrogen) and oligo(dT) ${ }_{18}$, according to the manufacturer's instructions. A $0.2-\mathrm{mL}$ aliquot of the total reaction volume $(20 \mu \mathrm{L})$ was used as a template in real-time (quantitative) RT-PCR. Amplification by PCR was performed using the gene-specific primers labeled as FARx-qPCR-x listed in Supplemental Table S5. To ensure that transcript amplification was specific, each set of PCR primers was designed from CATMA sequences (www.catma.org) and specificity was verified by amplification of a single PCR product of the correct size from each set of primers. Quantitative PCR was performed on an iCycler (Bio-Rad) using SYBR Green Master Mix (Bio-Rad) as described previously (Joubés et al., 2008). The relative transcript abundance of $A C T 2, E F-1 a, e I F-4 A-1, U B Q 10$, and $P P 2 A$ in each sample was used to normalize for differences of total RNA amounts Uoubés et al., 2008). 


\subsubsection{Preparation of constructs with promoter-glucuronidase (GUS) fusions}

The expression patterns of FAR1, FAR4, and FAR5 were examined using the $\beta$ glucuronidase (GUS) reporter system. Upstream sequences of approximately $2.5 \mathrm{~kb}$ that included the first three codons of FAR1, FAR4, and FAR5 were amplified by PCR using as templates bacterial artificial chromosomes (BACs) containing the genomic regions of interest. A 2,550-bp fragment of FAR1 upstream sequence was amplified using BAC MIQJ16 as template and primers FAR1-Pro-F and FAR1-Pro-R. A 2,560-bp fragment of FAR4 upstream sequence was amplified using BAC F14L2 as template and primers FAR4-Pro-F and FAR4-Pro-R. A 2,600-bp fragment of FAR5 upstream sequence was amplified using BAC F14L2 as template and primers FAR5-Pro-F and FAR5-Pro-R. The forward (F) and reverse (R) primers contained Sall and BamHI recognition sites, respectively, and the sequences are found in Supplemental Table S5. The amplified products were digested with Sall and BamHI restriction enzymes (Invitrogen) and cloned between the corresponding sites of pBl101 (Clontech) fusing the first few codons from each DNA fragment in frame with the coding region of the GUS gene. These vectors were transformed into DH5 $\alpha$ cells using standard method, and the amplified region was sequenced to confirm that there were no PCRinduced errors. These constructs were then transformed into Agrobacterium tumefaciens (GV3101) cells by electroporation and then introduced into wild-type (Col-0) plants by Agrobacterium-mediated transformation using the floral dip method (Clough and Bent, 1998). Successful transformants were identified by selection on minimal media containing $50 \mu \mathrm{g} / \mathrm{mL}$ kanamycin. The seedlings were 
transplanted to soil and at about 4 weeks of age various aerial tissues were stained to identify lines expressing the reporter gene.

\subsubsection{GUS histochemical assay}

GUS staining solution was composed of $50 \mathrm{mM}$ sodium phosphate, $\mathrm{pH} 7.0,0.5$ $\mathrm{mM}$ potassium ferricyanide, $0.5 \mathrm{mM}$ potassium ferrocyanide, $0.1 \%(\mathrm{v} / \mathrm{v})$ Triton $\mathrm{X}$ -

100 , and $0.5 \mathrm{mg} / \mathrm{mL} \quad 5$-bromo-4-chloro-3-indolyl- $\beta$-D-glucuronide (Gold Biotechnologies, St. Louis MO). Aerial tissues were preincubated in $90 \%$ chilled acetone for $5 \mathrm{~min}$ and then vacuum infiltrated with GUS staining solution. Tissues were incubated in GUS staining solution at $37^{\circ} \mathrm{C}$ for 2 hours (root tissue) or 12 hours (aerial tissues). For whole tissues, samples were cleared in $70 \%(\mathrm{v} / \mathrm{v})$ ethanol following staining and imaged using a stereomicroscope (Zeiss SteREO Discovery V20).

Wounding of transgenic promoter:GUS plants was carried out on stems and leaves of 6-week-old plants grown under long-day conditions (16-hr-light/8-hrdark cycle). Using a needle, injury was made of approximately $1 \mathrm{~cm}$ longitudinally between internodes of the mature stem regions or by puncturing leaves at multiple positions. The plants were returned to the growth cabinet, and the samples were collected after 24 hours and GUS staining was carried out as described above.

\subsubsection{Resin embedding and microtome sectioning}

For sectioning of roots, GUS-stained root tissues were incubated in $25 \mathrm{mM}$ sodium phosphate $(\mathrm{pH} 6.8)$ containing glutaraldehyde $(2 \%)$ at $4^{\circ} \mathrm{C}$ overnight. Following fixation, samples were rinsed for 2 minutes in $50 \%$ ethanol and 
dehydrated in an ethanol series of $50 \%, 70 \%, 95 \%$, and two changes of $100 \%$ ethanol for 30 min each. Dehydrated samples were then infiltrated with $100 \%$ LR White resin (Electron Microscopy Sciences) on a rocker with three changes of $100 \%$ resin, each for an hour. The resin-infiltrated tissues were then mixed with an appropriate quantity of accelerator (supplied with the resin). The mixture was immediately poured into a capsule and allowed to polymerize at room temperature. Ten-micrometer-thick sections were cut on a rotary microtome, mounted under 50\% glycerol, and imaged using a compound microscope (Zeiss Axio Imager M2).

\subsubsection{RNA extraction and cDNA synthesis}

Roots from 15-day-old seedlings and flower and stem tissues were harvested from 6-week-old Arabidopsis thaliana Col-0 plants. Plant tissue was wrapped in aluminum foil and immediately submerged in liquid nitrogen, then stored at $-80^{\circ} \mathrm{C}$. $1.5 \mathrm{ml}$ microcentrifuge tubes and micropestles were pre-chilled in liquid nitrogen and remained chilled throughout the experiment in a rack submerged in liquid nitrogen. The frozen plant tissue was placed in the chilled microcentrifuge tubes and ground briefly using a chilled micropestle. $600 \mu \mathrm{l}$ of TRIzol ${ }^{\circledR}$ reagent (Invitrogen), which is a proprietary mixture of guanidinium thiocyanate (15-40\%) and phenol (30-60\%), was added to the ground-up plant tissue, and the mixture ground to form a fine homogenate. A further addition of $400 \mu \mathrm{l}$ TRIzol reagent was added and briefly ground, and then the homogenized samples were incubated at room temperature for 5 minutes. After the incubation, $200 \mu \mathrm{l}$ of chloroform was added, followed by vigorous shaking for 15 seconds and then incubated at room temperature for 3 minutes. Following the incubation, the samples were centrifuged at $12000 \mathrm{~g}$ for 15 
minutes at $4^{\circ} \mathrm{C}$. The aqueous phase was transferred to a fresh $1.5 \mathrm{ml}$ microcentrifuge tube containing $0.5 \mathrm{ml}$ of isopropyl alcohol, mixed and incubated at room temperature for 10 minutes. After the incubation, the samples were centrifuged at $12000 \mathrm{~g}$ for 10 minutes at $4^{\circ} \mathrm{C}$. The supernatant was poured off leaving the RNA pellet. $1 \mathrm{ml}$ of $75 \%$ ethanol was added to the RNA pellet and mixed by vortexing for 15 seconds. The sample was centrifuged at $7500 \mathrm{~g}$ for 5 minutes at $4^{\circ} \mathrm{C}$, the ethanol was poured off and the RNA pellet was allowed to air dry for 10 minutes at room temperature in the fume hood. The dried RNA was dissolved in $50 \mu$ l of diethylpyrocarbonate (DEPC)-treated water and stored at $-80^{\circ} \mathrm{C}$.

First strand cDNA synthesis was carried out with $1 \mu \mathrm{g}$ of RNA and SuperScript III RT (Invitrogen). The RNA samples were diluted to a concentration of $0.12 \mu \mathrm{g} / \mu \mathrm{l}$ using DEPC-treated water. For each sample, $1 \mu \mathrm{g}(8.5 \mu \mathrm{l})$ of RNA was heated at $70^{\circ} \mathrm{C}$ for 5 minutes and snap chilled on ice. For a final volume of $20 \mu$, the RNA samples $(8.5 \mu l)$ were then added to $11.5 \mu \mathrm{l}$ of the reverse transcriptase reaction mixture $\left(3 \mu \mathrm{M}\right.$ oligo-dT primer, $0.5 \mathrm{mM}$ dNTPs, $5 U$ RNaseOUT ${ }^{\mathrm{m}}$ (Invitrogen), $10 \mathrm{mM}$ DTT, $50 \mathrm{mM}$ Tris- $\mathrm{HCl}(\mathrm{pH} 8.3,75 \mathrm{mM} \mathrm{KCl}, 3 \mathrm{mM} \mathrm{MgCl}$ ) on ice. 50 units of SuperScript III ${ }^{\circledR}$ Reverse Transcriptase (Invitrogen) were added to each reaction and held at $50^{\circ} \mathrm{C}$ for 60 minutes, $72^{\circ} \mathrm{C}$ for 10 minutes, cooled down to $4^{\circ} \mathrm{C}$ and stored at $-20^{\circ} \mathrm{C}$.

\subsubsection{Gene expression analysis by RT-PCR after wounding and salt treatment}

Wounding of stems of 6-week-old wild-type plants (stage 6.3 according to Boyes et al., 2001) was carried out as described above, and tissues were harvested 
after $1,24,48,72$, and 96 hours. As a control, a sample was taken before wounding. For the salt stress study, seedlings were first grown on MS agar plates for 7 days and then transferred to MS agar plates containing 0,50,100, or $200 \mathrm{mM} \mathrm{NaCl}$. Samples were harvested immediately, after $4 \mathrm{~h}$ and after 24 hours.

From tissues harvested in both the wounding and salt stress experiments, RNA was extracted using a phenol-guanidine thiocyanate extraction procedure (Trizol reagent; Invitrogen) as described above. First-strand cDNA was prepared from $1 \mu \mathrm{g}$ of total RNA using SuperScript Reverse Transcriptase III (Invitrogen) and oligo(dT $)_{18}$ as described above. Two microliters of a five-times diluted RT reaction (cDNA) was used as template in a 20- $\mu \mathrm{L}$ PCR using gene-specific primers labeled as $\mathrm{xxxx}-\mathrm{RT}-\mathrm{x}$ in Supplemental Table S5. The gene-specific primers were designed to span introns to differentiate between products amplified from CDNA and PCR products amplified from contaminating genomic DNA. The PCR products were separated on a $1 \%$ agarose gel containing ethidium bromide and visualized using an Alphalmager 2200 (Alpha Innotech, Santa Clara CA). The sizes of the amplified products for FAR1, FAR4, FAR5, RD29A, and GLYCERALDEHYDE-3-PHOSPHATE DEHYDROGENASE C SUBUNIT (GAPC) were 490, 950, 420, 1,000, and $250 \mathrm{bp}$, respectively.

\subsubsection{Characterization of FAR activity in Saccharomyces cerevisiae}

The coding sequences for FAR1, FAR3, FAR4, and FAR5 were amplified from cDNA using primers labeled as FARx-ORF- $\mathrm{x}$ in Supplemental Table S5. The corresponding PCR fragments were cloned into pDONR221 ENTRY vector by the Gateway recombinational cloning technology using the attB $x$ attP (BP) 
recombination sites. Fragments containing open reading frame sequences were subsequently transferred into the pVT102-U-GW Destination vector by LR cloning. To obtain the pVT102-U-GW Destination vector, the Gateway cassette $(1,711 \mathrm{bp}$; Karimi et al., 2002) was amplified by PCR, phosphorylated with T4 polynucleotide kinase, and inserted into the yeast expression vector pVT102-U (Vernet et al., 1987), which had been digested with XhoI and blunt ended with Klenow enzyme. The $S$. cerevisiae strain INVSc1 (Invitrogen) was transformed with yeast expression vectors containing the different open reading frames by a polyethylene glycol/lithium acetate protocol (Ausubel et al., 1995). After selection on minimal medium agar plates without uracil, cells harboring the yeast plasmid were cultivated in liquid minimal medium at $30^{\circ} \mathrm{C}$. Starting from precultures with an $\mathrm{OD}_{600}$ of 0.05 , expression cultures were grown for 5 days at $30^{\circ} \mathrm{C}$ and directly used for fatty acid analysis. Fatty acid methyl esters were obtained by transmethylation at $80^{\circ} \mathrm{C}$ for 1 hour of yeast cell sediments with $0.5 \mathrm{M}$ sulfuric acid in methanol containing $2 \%$ ( $\mathrm{v} / \mathrm{v}$ ) dimethoxypropane and $50 \mu \mathrm{g}$ of heptadecanoic acid (C17:0) as well as $20 \mu \mathrm{g}$ of pentadecanol $(\mathrm{C} 15: 0-\mathrm{OH})$ as internal standards. After cooling, $1 \mathrm{~mL}$ of $\mathrm{NaCl}(2.5 \%$, $\mathrm{w} / \mathrm{v}$ ) was added, and fatty acyl chains were extracted twice in hexane. Extracts were dried under a gentle stream of nitrogen and dissolved into $100 \mu \mathrm{L}$ of $\mathrm{N}, \mathrm{O}-$ bis(trimethylsilyl) trifluoroacetamide:trimethylchlorosilane (BSTFA-TMCS; 99:1), and free hydroxyl groups were derivatized at $80^{\circ} \mathrm{C}$ for 1 hour. Surplus BSTFA-TMCS was evaporated under nitrogen, and samples were dissolved in hexane for analysis using a Hewlett-Packard 5890 Series II gas chromatograph (GC) equipped with an HP-1 column ( $30 \mathrm{~m} \times 0.32 \mathrm{~mm} \times 0.25 \mu \mathrm{m}$ ) and a flame ionization detector. The 
initial temperature of the GC oven was $50^{\circ} \mathrm{C}$ and held for 1 minute, increased at $50^{\circ} \mathrm{C}$ per minute to $200^{\circ} \mathrm{C}$, held for 1 minute at $200^{\circ} \mathrm{C}$, increased again at $10^{\circ} \mathrm{C}$ per minute to $320^{\circ} \mathrm{C}$, and held for 8 minutes at $320^{\circ} \mathrm{C}$. Quantification of fatty acids and fatty alcohols was based on peak areas and the respective internal standard, C17:0 or C15:0-OH. Qualitative analyses were performed using an Agilent 6850 gas chromatograph equipped with an HP-5MS column ( $30 \mathrm{~m} \times 0.25 \mathrm{~mm} \times 0.25 \mu \mathrm{m}$ ) and an Agilent 5975 mass spectrometric detector ( $70 \mathrm{eV}$, mass-to-charge ratio 50-750). The same GC program was used with helium $(1.5 \mathrm{~mL} / \mathrm{min})$ as carrier gas.

\subsubsection{Characterization of T-DNA insertion lines}

T-DNA insertion mutant lines for FAR1, FAR4, and FAR5 were identified from the Sequence-Indexed Library of Insertion Mutations using the Arabidopsis Gene Mapping Tool (http://signal.salk.edu/cgi-bin/tdnaexpress). Homozygous insertion lines were identified from among the segregating plants by PCR screening for both the presence of a T-DNA insertion at the locus and the absence of an intact gene. Genomic DNA was isolated from individual plants and genotyped using genespecific primers labeled as SALK_xxxxxx-TDNA-xx in Supplemental Table S5 and LBb1, which is a T-DNA left border primer. The exact positions of the T-DNA insertions were determined by sequencing the PCR products. For transcript analysis in the mutants, RNA was extracted from root tissues and analyzed by RT-PCR using the same primers and conditions described above for transcript analysis after wounding and salt stress. 


\subsubsection{Analysis of lipid polyester composition (Suberin and Cutin)}

Roots from 6-week-old plants (stage 6.3 according to Boyes et al., 2001) grown on soil were carefully separated from the soil, washed thoroughly in water, and dried on paper towels before delipidation. Roots from mutant plants grown for 5 weeks in tissue culture on vertical plates (MS medium) containing $1.5 \%(\mathrm{w} / \mathrm{v})$ agar were separated from the growing medium and dried on paper towels before delipidation. For seed coat analysis, all the lines were grown together, dry seeds were collected, and about $50 \mathrm{mg}$ of dry seeds was used per replicate. For wounding experiments, four to five leaves from fully developed plants grown on soil for about 10 weeks (stage 8.0 according to Boyes et al., 2001) were injured with a metallic brush, and small holes were produced all along the leaf. Five days later, these leaves, as well as four to five unwounded leaves from the same plant, were harvested and directly used for delipidation.

For delipidation, roots and leaf tissues freshly collected as described above were immersed in hot isopropanol for $10 \mathrm{~min}$ at $80^{\circ} \mathrm{C}$. For seed coat analysis, seeds were ground in cold isopropanol using a mortar and pestle, and then the mixture was transferred to tubes and heated for 30 minutes at $80^{\circ} \mathrm{C}$. After cooling, samples were extensively delipidated by extracting the soluble lipids successively for 24 hours with $\mathrm{CHCl} 3: \mathrm{CH} 3 \mathrm{OH}(2: 1, v / v), \mathrm{CHCl} 3: \mathrm{CH} 30 \mathrm{OH}(1: 1, v / v), \mathrm{CHCl} 3: \mathrm{CH} 30 \mathrm{H}(1: 2$, $v / v$ ), and $\mathrm{CH} 30 \mathrm{H}$, all performed at $4^{\circ} \mathrm{C}$ on a wheel rotating at $33 \mathrm{rpm}$. Each extraction was conducted once for roots and leaves, but twice for seeds. Samples were dried in a fume hood at room temperature for 2 days and then in a desiccator for another 2 days. 
The dried residues were weighed, and 10 to $30 \mathrm{mg}$ of each sample was depolymerized by transmethylation at $85^{\circ} \mathrm{C}$ for 3 hours using $1 \mathrm{M}$ sulfuric acid in methanol spiked with $10 \mu \mathrm{g}$ of heptadecanoic acid (C17:0), $10 \mu \mathrm{g}$ of $\omega$-pentalactone $(\mathrm{C} 15 \omega \mathrm{OH})$, and $10 \mu \mathrm{g}$ of pentadecanol $(\mathrm{C} 15: 0-\mathrm{OH})$ as internal standards. After cooling, $3 \mathrm{~mL}$ of $\mathrm{NaCl}(2.5 \%, \mathrm{w} / \mathrm{v})$ was added, and the released fatty acyl chains were extracted twice in hexane for residual lipids derived from roots and leaves and twice in dichloromethane (DCM) for residual lipids derived from seeds. Extracts were washed once with $3 \mathrm{~mL}$ of saline solution $(200 \mathrm{mM} \mathrm{NaCl}$ and $200 \mathrm{mM}$ Tris, $\mathrm{pH}$ 8.0), dried under a gentle stream of nitrogen, and dissolved in $150 \mu \mathrm{L}$ of BSTFATMCS. Free hydroxyl groups were derivatized at $110^{\circ} \mathrm{C}$ for $30 \mathrm{~min}$, surplus BSTFATMCS was evaporated under nitrogen, and samples were dissolved in hexane for analysis using GC-MS under the same conditions described above for yeast acyl chain analysis except that the first temperature ramp $\left(50^{\circ} \mathrm{C}-150^{\circ} \mathrm{C}\right)$ was done at $25^{\circ} \mathrm{C} /$ minute instead of $50^{\circ} \mathrm{C} /$ minute. Quantification of fatty acids, hydroxyl acids, and fatty alcohols was based on peak areas, which were derived from total ion content, and using the respective internal standards $[\mathrm{C} 17: 0, \omega$-pentalactone, or C15:0-OH).

\subsection{Results}

\subsubsection{FAR1, FAR4, and FAR5 gene expression is associated with suberin} deposition

Arabidopsis contains eight FAR genes that, in agreement with The Arabidopsis Information Resource, we have named FAR1 to FAR8 (Supplemental 
Table S2.1). The tissue-specific gene expression patterns of the FAR genes were determined by quantitative reverse transcription (RT)-PCR using RNA derived from seedlings and young roots from 15-day-old plants grown in tissue culture and using RNA derived from various organs (mature roots, stems, cauline and rosette leaves, flowers, and developing siliques) of 10-week-old plants grown on soil (Figure 2.2). This analysis indicated that FAR3/CER4 is highly expressed in aerial organs of the plant, while FAR2/MS2 expression is restricted to flowers, in agreement with their respective roles in wax biosynthesis (Rowland et al., 2006) and sporopollenin formation (Aarts et al., 1997). The FAR6 gene was found to be mainly expressed in stems (Doan et al., 2011), while the levels of expression for FAR7 and FAR8 were very low under our conditions, suggesting that these latter two genes are expressed at very specific developmental stages. These results are in agreement with DNA microarray data suggesting that FAR6 is expressed in stem epidermis while expression of both FAR7 and FAR8 is restricted to floral organs (Suh et al., 2005; Schmid et al., 2005). FAR1 was widely expressed, most notably in young roots, rosette leaves, and flowers. FAR1 transcripts were also detected in cauline leaves and developing siliques, but not in stems. Finally, FAR4 and FAR5 transcripts were largely restricted to young and mature roots, with slight accumulation detected in seedlings, flowers, and developing siliques.

In order to identify FAR enzymes associated with suberin biosynthesis, we focused our subsequent analyses on the root-expressed FAR1, FAR4, and FAR5 genes. Since FAR3/CER4 was previously shown to be important for the production of C24:0-C30:0 chain length fatty alcohols of cuticular wax (Rowland et al., 2006) 
and its expression in young and mature roots was very low (Figure 2.1), it is unlikely to have a significant role in suberin biosynthesis. The gene expression patterns of FAR1, FAR4, and FAR5 were further investigated by fusing about 2,500 bp of their upstream sequences, relative to the start codon, in frame with the coding region of the GUS reporter gene. The T-DNA plant binary constructs containing the promoter:GUS fusions were used to transform Arabidopsis. Histochemical staining for GUS activity was performed on at least 20 independent $\mathrm{T} 1$ transformants and then repeated in several $\mathrm{T} 2$ or $\mathrm{T} 3$ transgenic lines that showed representative expression patterns. For all three FAR promoters, strong GUS activity was found along the length of primary roots, and cross sections indicated that it was in the endodermal cell layer surrounding the central vasculature (Figure 2.3). Patchy GUS expression was also observed in the peridermal cells of the matured tap roots of FAR1 and FAR4 promoter: GUS transgenic plants, and to a lesser degree with FAR5 promoter: GUS transgenic plants (Figure 2.3). The promoters of FAR1 and FAR4, but not FAR5, drove GUS expression in the micropyle region (hilum) of the seed (Figure 2.3). We also occasionally observed patchy staining in the seed coats of plants expressing the FAR1 and FAR4 promoter:GUS fusions. The root endodermal and peridermal layers, the seed hilum region, which is where sealing of tissue after seed detachment takes place, and the seed coat are sites of suberin deposition. Finally, high levels of GUS activity driven by the FAR1 promoter were also observed in the elongation zone of lateral root tips, cotyledons, the shoot apex, young leaves, petals, filaments of stamens, and the receptacle of siliques (Supplemental Figure S2.2A). FAR4 promoter directed GUS activity in aerial tissues was restricted to filaments of 
stamens and the receptacle of siliques (Supplemental Figure S2.2B), while that driven by the FAR5 promoter was observed only in floral organs of very young unopened buds and the receptacle of siliques (Supplemental Figure S2.2C).

Suberin has also been reported to be produced in response to wounding and abiotic stress (Dean and Kolattukudy, 1976; Kolattukudy, 2001). Six-week-old transgenic plants containing the FAR1, FAR4, or FAR5 promoter:GUS fusion were wounded, and GUS activity was assayed over a 24-hour period. Histochemical staining was evident in wounded, but not unwounded, leaf tissue of transgenic plants containing each of the three promoter:GUS fusions after 24 hours, all along the perimeter of sections that had been punctured (Figure 2.3). The same pattern of staining was also observed when stems of the transgenic plants were wounded (Supplemental Fig. S2.2). GUS activity was not observed immediately after wounding (data not shown), indicating that apparent wound-induced staining was not simply due to increased access of substrate to the reporter enzyme. To further investigate the kinetics of wound-induced gene expression, a detailed time course of wound induction of the endogenous FAR genes was monitored by RT-PCR in wounded stem tissues (Figure 2.4A). At the cycle number used for RT-PCR, the transcripts of all three FARs were not detected in unwounded stems. In contrast, the transcripts of FAR 1 and FAR5 were detectable by RT-PCR at 1 hour after wounding and that of FAR4 at 24 hours after wounding. The transcript levels of all three FAR genes peaked at 48 hours and remained high after 96 hours. We also exposed seedlings to $\mathrm{NaCl}$-induced salt stress $(50,100$, and $200 \mathrm{mM} \mathrm{NaCl})$ and observed the influence on FAR1, FAR4, and FAR5 gene expression by RT-PCR (Figure 2.43B). All 
three genes were expressed at low levels in seedlings grown on plates in tissue culture prior to salt stress, but all showed a rapid induction after 4 hours at all three salt concentrations. Induced expression levels remained high after 24 hours at all three salt concentrations for FAR4 and FAR5, but FAR1 levels remained induced at 24 hours only in the presence of $200 \mathrm{mM} \mathrm{NaCl}$. For comparison, the well characterized RESPONSIVE TO DEHYDRATION29A (RD29A; Yamaguchi-Shinozaki and Shinozaki, 1993) gene was used as a reference for salt stress induction and was strongly up-regulated at $4 \mathrm{~h}$ by all concentrations, but only by $200 \mathrm{mM} \mathrm{NaCl}$ at 24 hours (Figure 2.4B).

\subsubsection{Isolation of single mutants disrupted in the FAR1, FAR4, and FAR5 genes}

We identified two T-DNA insertion lines in each of FAR1, FAR4, and FAR5 to investigate their respective roles in fatty alcohol production associated with suberin deposition. The sites of T-DNA insertion were determined by sequencing, and in some cases, they did not precisely match the sites of insertion reported on the $\mathrm{T}$ DNA Express Arabidopsis Gene Mapping Tool (http://signal.salk.edu/cgibin/tdnaexpress). The far1-1 (SALK_068605) and far1-2 (SALK_149469) alleles have T-DNA inserts in the eighth exon (nucleotide $+2,346$ relative to the start codon) and 5'-untranslated region (nucleotide -94 ), respectively (Figure $2.5 \mathrm{~A}$ ). The transcript levels of FAR1 were nearly absent in both of these lines (Figure 2.5B). The far4-1 (SALK_000229) and far4-2 (SALK_147493) alleles have T-DNA inserts in the third exon (nucleotide +883 ) and 3 '-untranslated region $(87$ bp after the stop codon), respectively (Figure 2.5A). The far4-1 allele was confirmed to be a transcriptional null of FAR4, whereas the far4-2 allele had very slight, if any, effect 
on transcript abundance (Figure 2.5B). The far5-1 (SALK_152963) and far5-2 (SALK_070363) alleles have T-DNA inserts in the second and seventh introns (nucleotides +562 and $+1,966$ ), respectively (Figure $2.5 \mathrm{~A}$ ). The transcript levels of both of these FAR5 alleles were substantially reduced, but nonetheless detectable, indicating that they are likely not null alleles (Figure 2.5B). No obvious developmental phenotypes were visually observed in the homozygous lines of any of these mutant plants when compared with the wild type.

\subsubsection{Alterations of fatty alcohol composition associated with suberin isolated from roots and seed coats of far single mutants}

The pairs of far1, far4, and far5 T-DNA mutant alleles described above were first analyzed for suberin monomer composition in roots of 6-week-old plants grown in soil. Gas chromatography-mass spectrometry (GC-MS) analysis of residual bound lipids after extensive chloroform/methanol extraction of whole roots allowed the identification and quantification of 41 different compounds, of which the major ones are indicated in Figure 2.6A. The major lipid classes usually found in root suberin (i.e. fatty acids, $\alpha, \omega$-dicarboxylic acids, $\omega$-hydroxy acids, and primary fatty alcohols) were detected. 2-hydroxy acids were also detected, but these have been speculated to come from sphingolipids rather than representing suberin monomers (Molina et al., 2006). No significant differences in the total quantity of these lipid classes were observed in the different far mutant lines (Figure 2.6B). However, inspection of the fatty alcohol subclass revealed significant variations among the chain length distributions depending on the mutant (Figure 2.6C; Supplemental 
Table S2.2). The most pronounced phenotype was observed in both far5-1 and far52, where the $\mathrm{C} 18: 0-0 \mathrm{H}$ content was $80 \%$ decreased. This sharp reduction in C18:0$\mathrm{OH}$ was accompanied by a $60 \%$ increase in the amounts of both $\mathrm{C} 20: 0-0 \mathrm{H}$ and $\mathrm{C} 22: 0-0 \mathrm{H}$. In the far1 lines, the amounts of $\mathrm{C} 20: 0-\mathrm{OH}$ and $\mathrm{C} 22: 0-\mathrm{OH}$ were reduced by $20 \%$ and $25 \%$, respectively, while $\mathrm{C} 18: 0-\mathrm{OH}$ content remained about the same. The far4-2 line, which contains a T-DNA insertion in the 3 ' untranslated region and only a slight reduction in FAR4 transcript abundance (Figure 2.5B), had a fatty alcohol composition that was not significantly different from the wild type. In contrast, the far4-1 line, which is a transcriptional null of FAR4, had a $50 \%$ decrease of $\mathrm{C} 20: 0-\mathrm{OH}$. The levels of $\mathrm{C} 18: 0-\mathrm{OH}$ and $\mathrm{C} 22: 0-\mathrm{OH}$ were not significantly altered in far4-1.

There was some variability in the suberin composition data obtained from soil-grown plants. Therefore, we analyzed root suberin composition of wild-type and mutant plants grown on tissue culture medium. Large amounts of roots were easily recovered from plants grown for 5 weeks on vertical plates containing Murashige and Skoog (MS) medium and 1.5\% (w/v) agar. We restricted this analysis to the strongest mutant allele of each FAR: far1-1, far4-1, and far5-1 (Table 2.1; Supplemental Fig. S2.3). As previously observed with soil-grown plants, no significant differences could be detected in the levels of any fatty acids, $\alpha, \omega-$ dicarboxylic acids, or $\omega$-hydroxy acids or in the total suberin load between the three far mutants and the wild type (Table 2.1; Supplemental Fig. S2.3). The absolute amounts of all suberin monomers were generally increased in roots grown in tissue culture in comparison with the amounts measured in roots grown in soil, with the 
exception of 2-hydroxy acids, which were less abundant (Table 2.1; Figure 2.6B). This discrepancy may come from stresses induced by growing the plants in vitro for 5 weeks under continuous light or by soil components that caused artifactual increases in the dry residue weight, thus resulting in reduced total suberin load. Nevertheless, as observed with soil-grown plants, the primary alcohol chain length distributions varied significantly between the different mutants and wild-type plants grown in tissue culture (Table 2.1). Similar to the results obtained from roots grown on soil, the content of $\mathrm{C} 20: 0-\mathrm{OH}$ in far4-1 was reduced by $55 \%$, while C22:0$\mathrm{OH}$ was increased by $36 \%$. In far5-1, there was an $80 \%$ decrease in $\mathrm{C} 18: 0-\mathrm{OH}$ and a $20 \%$ increase of $\mathrm{C} 20: 0-\mathrm{OH}$. Finally, in far1-1, the amount of $\mathrm{C} 22: 0-\mathrm{OH}$ was reduced by $30 \%$, while the other fatty alcohols were not affected. As for soil-grown plants, the total fatty alcohol content was not significantly affected in far1-1 and far4-1 and was only slightly reduced in far5-1 (Table 2.1).

We also analyzed the lipid polyester composition of Arabidopsis wild-type and far mutant seeds (Figure 2.7; Supplemental Table S2.3). Suberin monomers are mostly deposited in the outer integument of the seed coat (Molina et al., 2008). In addition to the components usually found in root suberin, the polyester of seeds contains higher relative amounts of phenolics (mainly trans-ferulic and transsinapic acids), mid-chain hydroxylated fatty acids (10,16-dihydroxy-hexadecanoic and 9,10,18-trihydroxy-octadecenoic acids), diols (1,20-eicosane and 1,22-docosane diols), as well as traces of branched chain compounds (Molina et al., 2006). We did not observe significant variations in the total polyester load of any mutant when compared with the wild type (Supplemental Table S2.3). Similar amounts of the 
different subclasses were found in all four lines, with the exception of primary alcohol and diol levels, which were $42 \%$ and $56 \%$ reduced, respectively, in the far 1 1 line relative to the wild type (Figure 2.7A; Supplemental Table S2.3). Although the total fatty alcohol and diol levels were not affected in far4-1 and far5-1, the amount of $\mathrm{C} 18: 0-0 \mathrm{H}$ was reduced to nearly undetectable levels in far5-1, while the amounts of $\mathrm{C} 20: 0-\mathrm{OH}$ and C20:0-diol were reduced by $68 \%$ and $50 \%$, respectively, in far $4-1$ (Figure 2.7B). In the far1-1 line, the amounts of $\mathrm{C} 22: 0-\mathrm{OH}$ and $\mathrm{C} 22: 0$-diol were reduced by $65 \%$ and $59 \%$, respectively (Figure $2.7 \mathrm{~B}$ ). In addition, the amounts of branched chain $\mathrm{C} 22: 0-\mathrm{OH}$ and $\mathrm{C} 23: 0-\mathrm{OH}$ were significantly reduced in far $1-1$. Since C18:0-OH, C20:0-OH, and C20:0-diol are minor components of the polyester of wildtype seeds compared with $\mathrm{C} 22: 0-\mathrm{OH}$ and $\mathrm{C} 22: 0-\mathrm{diol}$, far1-1 was the only mutant with significantly altered total levels of fatty alcohols and diols (Figure 2.7A). The levels of all other suberin components, including the phenolics, remained about the same between the wild type and the three far mutants.

\subsubsection{Alterations of fatty alcohol composition in leaves of far single mutants after wounding}

Since the transcript levels of FAR1, FAR4, and FAR5 were increased after wounding (Figure 2.4A), we analyzed the levels of primary alcohols in residual bound lipids of leaves before and after wounding. These lipids would collectively represent cutin of the cuticle and possibly suberin deposited in response to wounding. We injured fully developed leaves of 10 -week-old plants with a metallic brush and analyzed 5 days later the content of the residual bound lipids. For each line, unwounded leaves from the same plant were used as the control. This method 
of wounding generated small holes all along the leaf, but in most cases it did not result in dead tissue. After 5 days, total residual bound lipids were slightly increased in wounded leaves of all the lines compared with unwounded leaves, but these differences were not significant according to Student's $t$ test (Supplemental Table S2.4). Some variability came from the method of wounding itself, as it was difficult to precisely control the number of holes per square centimeter of tissue. In addition, our residual bound lipids contained high amounts of palmitic acid $(\mathrm{C} 16: 0)$ and polyunsaturated $\mathrm{C} 18$ fatty acids (C18:2 and C18:3) in comparison with reports in the literature (Bonaventure et al., 2004; Franke et al., 2005), which may have been due to our growing conditions or to incomplete extraction of soluble lipids. Nevertheless, our procedure enabled quantification of the characteristic monomers found in cutin and suberin (residual bound lipids). Wounding treatment did not increase the levels of most major-abundance residual bound lipid components, but the content of some minor-abundance components was significantly increased (Figure 2.8; Supplemental Table S2.4). The C20:0 and C22:0 very-long-chain fatty acids and their corresponding $\omega$-hydroxy fatty acids were significantly induced in wild-type and far mutant lines (Figure 2.8B). Similarly, the amounts of C18:0-C22:0 primary fatty alcohols were clearly induced by the wounding treatment in wild-type plants. In contrast, $\mathrm{C} 18: 0-\mathrm{OH}$ and $\mathrm{C} 22: 0-\mathrm{OH}$ were not increased by wounding in the far5-1 and far1-1 lines, respectively.

Since the mass spectra of the $\mathrm{C} 20: 0$ and C22:0 primary alcohols indicated that other compounds co-eluted with these alcohols, we only considered the major molecular ion of each fatty alcohol molecule for quantification $\left[\mathrm{m}-15, \mathrm{CH}_{3}-\left(\mathrm{CH}_{2}\right)_{\mathrm{n}}-\mathrm{O}\right.$ - 
$\mathrm{Si}\left(\mathrm{CH}_{3}\right)_{2}{ }^{+}$; Supplemental Fig. S2.4], rather than total ion content. With this method, contamination from co-eluting products was eliminated, allowing for more accurate quantification of each fatty alcohol. Using this method of quantification, the total fatty alcohol content was more than doubled by the wounding treatment in the wild type and in each of the far mutants (Figure 2.9A). In the wild type, the content of each chain length of fatty alcohol was more than doubled (Figure 2.9B), with C18:0$\mathrm{OH}$ being the most affected (175\% increase). A similar increase of $\mathrm{C} 18: 0-\mathrm{OH}$ and C22:0-OH was observed in far4-1, but the amount of $\mathrm{C} 20: 0-\mathrm{OH}$ was lower than in the wild type with or without wounding treatment. In far1-1, the amounts of $\mathrm{C} 18: 0-\mathrm{OH}$ and C20:0-OH primary alcohols increased after wounding as in the wild type, but that of $\mathrm{C} 22: 0-\mathrm{OH}$ remained unchanged. The amount of $\mathrm{C} 22: 0-\mathrm{OH}$ in unwounded tissues was lower in far1-1 compared with the wild type. Finally, in far5-1, the C18:0-OH content was not increased by wounding, whereas those of $\mathrm{C} 20: 0-\mathrm{OH}$ and C22:0-OH increased significantly after wounding, each to a much higher level than that of the wild type. Analogous to the decrease of $\mathrm{C} 22: 0-\mathrm{OH}$ and $\mathrm{C} 20: 0-\mathrm{OH}$ in unwounded leaves of far1-1 and far4-1, respectively, the level of $\mathrm{C} 18: 0-0 \mathrm{H}$ in unwounded leaves of far 5-1 was significantly decreased relative to the wild-type.

\subsubsection{Heterologous Expression of FAR1, FAR4, and FAR5 in Yeast}

Since C18:0 to C22:0 primary fatty alcohols are detected in suberin of Arabidopsis roots and seed coats, we examined the activities and substrate specificities of the enzymes encoded by the FAR1, FAR4, and FAR5 genes using Saccharomyces cerevisiae as a heterologous system. The open reading frames were subcloned into the yeast expression vector pVT102U, which places the coding 
sequences under the control of a constitutive promoter, and the resulting constructs were transformed into yeast. The empty vector and pVT102-FAR3 were used as negative and positive controls, respectively. Expression of FAR3/CER4 in yeast results in the production of $\mathrm{C} 24: 0-\mathrm{OH}$ and $\mathrm{C} 26: 0-\mathrm{OH}$, in agreement with Rowland et al. (2006), whereas no fatty alcohols were detected in the empty vector control (Figure 2.10; Table 2.2). Transgenic yeast expressing FAR1, FAR4, or FAR5 produced C18:0 to C22:0 fatty alcohols, indicating that the three proteins are active alcohol-forming fatty acyl-CoA reductases (Figure 2.10; Table 2.2). The major fatty alcohols produced when expressing FAR1 were $\mathrm{C} 18: 0-\mathrm{OH}$ and $\mathrm{C} 22: 0-\mathrm{OH}$, but $\mathrm{C} 16: 0-$

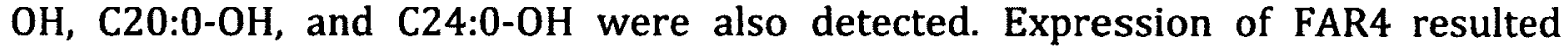
mainly in the production of $\mathrm{C} 20: 0-\mathrm{OH}$ and $\mathrm{C} 18: 0-\mathrm{OH}$, while FAR5 expression led nearly exclusively to the production of $\mathrm{C} 18: 0-0 \mathrm{H}$. The identities of the different fatty alcohols produced were confirmed by GC-MS analyses (Supplemental Fig. S2.4). In no case were unsaturated fatty alcohols detected. Quantification of internal lipids in the yeast strains revealed that expression of FAR5 generated the most fatty alcohol, with $2.14 \mu \mathrm{g}$ per unit optical density (OD; Table 2.2). FAR1 and FAR4 each generated much less, with 0.90 and $0.97 \mu \mathrm{g}$ of fatty alcohol produced per unit OD, respectively, while FAR3 generated $0.60 \mu \mathrm{g}$ of fatty alcohol per unit OD. In contrast, all strains had similar total fatty acid contents (Table 2.2). The high production of $\mathrm{C} 18: 0-\mathrm{OH}$ observed in the case of FAR5 resulted in a lower C18 fatty acid content and a higher C16 fatty acid content. 


\subsection{Discussion}

Suberin is a protective biopolyester that consists of ferulic acid, glycerol, and aliphatic moieties. In this study, we identified three Arabidopsis FAR enzymes, FAR1, FAR4, and FAR5, which are responsible for generating primary fatty alcohols associated with suberin. Evidence for this conclusion is as follows: (1) the gene expression patterns of these three FARs are associated with known sites of suberin deposition; (2) the chain lengths of primary alcohols produced by heterologous expression of these FARs in yeast are consistent with the chain lengths of primary alcohols found in Arabidopsis suberin; and (3) mutants of FAR1, FAR4, and FAR5 are each differentially affected in primary alcohol levels associated with suberin in various tissues.

\subsubsection{FAR1, FAR4, and FAR5 are expressed at sites of suberin deposition.}

Suberin is constitutively deposited in the cell walls of root endodermal cells. The Casparian strip of the root endodermis, which is made up of suberin, lignin, and other primary cell wall components, has a major role in the prevention of apoplastic movement of ions and water from the root cortex to the stele (Enstone et al., 2003). Therefore, we focused our attention on root-expressed FARs to identify those producing fatty alcohols associated with suberin. FAR1, FAR4, and FAR5 were found to be root expressed, consistent with the developmental expression patterns reported using DNA microarrays (Schmid et al., 2005; Supplemental figure S2.1). Expression of the GUS reporter gene under control of the promoters of these rootexpressed FARs in the endodermal cells is similar to that of six other Arabidopsis suberin associated genes: GPAT5, AFST, KCS2/DAISY, KCS20, CYP86A1/HORST, and 
CYP86B1/RALPH (Beisson et al., 2007; Hofer et al., 2008; Compagnon et al., 2009; Lee et al., 2009; Molina et al., 2009). The walls of Arabidopsis root endodermal cells are characterized by suberin lamellae (Franke et al., 2005) and are stained by lipophilic dyes (Franke et al., 2005; Beisson et al., 2007; Compagnon et al., 2009). The promoter activity for all three genes was also observed in the peridermal cells of the matured tap roots. It was recently reported that FATTY ALCOHOL:CAFFEOYLCOA CAFFEOYL TRANSFERASE 1 (FACT1) is responsible for synthesizing alkyl hydroxycinnamates (AHCs) in suberin-associated waxes and that the FACT1 gene is also specifically expressed in endodermal and peridermal root cells (Kosma et al., 2012). In aerial tissues, the receptacle of mature siliques, where abscission of petals takes place, is also stained by these lipophilic dyes and is coincident with KCS2/DAISY and CYP86B1/RALPH gene expression (Compagnon et al., 2009; Franke et al., 2009). Likewise, GUS reporter gene activity driven by all three FAR promoters was observed in silique receptacles. The deposition of suberin in this region is likely important to seal off the area that was formerly attached to the petals. Espelie et al. (1980a) showed that the micropyle-chalazal region of grapefruit (Citrus paradisi) seed is also sealed by suberized cell walls. FAR1 and FAR4 promoter-driven GUS activity was observed in the micropyle region of the Arabidopsis seed coat, similar to that found for GPAT5, KCS2/DAISY, and CYP86B1/RALPH (Beisson et al., 2007; Compagnon et al., 2009; Franke et al., 2009). FAR5 promoter-driven GUS activity was not observed in the micropyle region, suggesting that FAR5 does not generate suberin-associated fatty alcohols at this location. We had difficulties obtaining consistent GUS staining patterns in seed coats, but all three FARs are likely to be 
expressed in the seed coat at some developmental stage, because we observed alterations in fatty alcohol levels in the seed coat lipid polyester in each of the three far mutants, with far1 being the most dramatically affected.

Suberization of cell walls occurs in response to pathogen attack or mechanically induced stress (Dean and Kolattukudy, 1976; Bernards and Lewis, 1998). The polymer likely serves to seal off the tissue to prevent further opportunistic pathogen infections and also to prevent prolonged water or solute leakage. The wound induction kinetics of FAR1, FAR4, and FAR5 were roughly similar, peaking at around 2 days and with induced transcripts remaining high even after 4 days. This gene induction correlated with increased amounts of fatty alcohols in the residual bound lipids of leaves 5 days after wounding. The KCS2/DAISY transcript is similarly up-regulated at 24 hours after wounding (Franke et al., 2009), which likely accounts for the increases in very-long-chain fatty acids at 5 days post wounding. Salt (e.g. $\mathrm{NaCl}$ ) stress leads to increased suberization of endodermal and hypodermal root cell walls in castor bean (Ricinus communis; Schreiber et al., 2005a), and a 2-fold increase in total root suberin occurs in Arabidopsis roots in response to high levels of $\mathrm{NaCl}$ (Franke et al., 2009). Although it was not shown specifically that fatty alcohols are increased by salinity in Arabidopsis roots, this is likely to be the case, since FAR1, FAR4, and FAR5 gene expression levels were increased by exposure to high salt.

The gene expression patterns of FAR4 and FAR5 were largely restricted to sites of suberin deposition, whether constitutive or inducible, and it is thus likely that they serve only to produce fatty alcohols of suberin. FAR1 was found to be more 
widely expressed, such as in flower petals, cotyledons, and young leaves, and may have non-suberin functions. FAR1 is unlikely to have a role in generating fatty alcohols of cuticular wax, because null mutants of far $3 / c e r 4$ are almost completely deficient of all chain-length fatty alcohols normally found in this aerial hydrophobic barrier. The contribution of other FARs to suberin-associated fatty alcohol biosynthesis is likely to be minimal, based on their gene expression patterns, which are not at known sites of suberin deposition. FAR3/CER4, which generates cuticular C24-C30 fatty alcohols in epidermal cells, is expressed at low levels in roots but not specifically in endodermal cells (Rowland et al., 2006). FAR3/CER4 is thus unlikely to significantly influence root suberin composition. Altogether, these data suggest that FAR1, FAR4, and FAR5 represent the three members of the Arabidopsis FAR multigene family that are involved in suberin synthesis.

\subsubsection{Substrate specificities of FAR1, FAR4, and FAR5.}

Fatty alcohols ranging in length from $\mathrm{C} 18: 0-\mathrm{OH}$ to $\mathrm{C} 22: 0-\mathrm{OH}$ have been found in the suberin polyester of Arabidopsis roots and seed coats (Franke et al., 2005; Molina et al., 2006; Beisson et al., 2007; this study). In each of the far mutants in this study, their distribution was differentially affected. These analyses strongly suggest that, in planta, FAR1 is most important for generating C22:0 primary alcohol, FAR4 is most important for generating C20:0 primary alcohol, and FAR5 is most important for generating C18:0 primary alcohol. Expression in yeast confirmed that FAR1, FAR4, and FAR5 produce fatty alcohols ranging from C18:0 to C22:0. These three enzymes are $67 \%$ to $73 \%$ identical and $80 \%$ to $86 \%$ similar at the primary amino acid sequence level (Rowland et al., 2006). In yeast, the apparent substrate 
specificity of FAR5 was particularly stringent for C18 acyl chains, with the other two FARs able to accept a couple of chain lengths. FAR1 mostly generated C22:0 and C18:0 primary alcohols in yeast, while FAR4 mostly generated C20:0 and C18:0 primary alcohols. When FAR1 and FAR3 were expressed in Escherichia coli, both led to the accumulation of $\mathrm{C} 16: 0-0 \mathrm{H}$ and $\mathrm{C} 18: 1-0 \mathrm{H}$ (Doan et al., 2009). This does not fit the plant biochemical phenotype and is in contrast to what was observed here using the yeast system. However, the acyl chains in E. coli are linked to acyl carrier protein, rather than CoA, and this may affect substrate specificity. Expression in yeast, therefore, appears to be a better indicator of in planta substrate specificity than the E. coli system. For example, bacterial expression of FAR1 yielded C16:0-OH and C18:1-OH in similar proportions (Doan et al., 2009), whereas these are not produced when FAR1 is expressed in yeast and instead C18:0-OH and C22:0-OH mainly accumulates (this study). The production of these latter primary alcohols in yeast better reflects the role of FAR1 in producing C22:0 fatty alcohols associated with root, seed coat, and wound-induced suberin. Nevertheless, even in yeast, the available acyl-CoA substrates need to be considered when evaluating FAR substrate specificities. FAR5 preferentially used C18:0-CoA, which is abundant in the acyl-CoA pool of yeast cells (Domergue et al., 2003). In that context, the detection of $\mathrm{C} 18: 0-\mathrm{OH}$ in yeast when expressing FAR1 or FAR4 may be due to the relatively high abundance of $\mathrm{C} 18: 0-\mathrm{CoA}$, since there was no effect on $\mathrm{C} 18: 0-\mathrm{OH}$ in the far1 and far4 mutants. Nevertheless, the fact that no unsaturated fatty alcohols could be detected, despite monounsaturated fatty acids being highly abundant in the yeast acyl-CoA pool (Domergue et al., 2003), strongly suggests that these enzymes only accept 
saturated acyl-CoA chains as substrates. In addition, FAR5 effectively reduced C18:0-CoA while displaying nearly no activity on C16:0-CoA, which is more abundant than C18:0-CoA in the acyl-CoA pool (Domergue et al., 2003). It thus appears that FAR enzymes have strong chain length specificities. Ultimately, measurements of substrate specificity using purified enzymes in vitro will be required for a quantitative assessment of relative substrate specificities.

\subsubsection{Production of primary alcohols associated with suberin is affected in far1, far4, and far5 mutants.}

Individual chain lengths of primary alcohols of root and seed coat suberin were reduced in the far mutants: far1 had a decrease in $\mathrm{C} 22: 0-0 \mathrm{H}$, far 4 had a decrease in $\mathrm{C} 20: 0-\mathrm{OH}$, and far5 had a decrease in $\mathrm{C} 18: 0-\mathrm{OH}$. With the exception of C20:0 and C22:0 diols, which were reduced in the seed coat polyester of far4 and far1, respectively, the other suberin chemical classes were not affected in the different far mutants, indicating that perturbations in primary alcohol accumulation, at least at the levels altered in these mutants, do not affect other constituents. Reciprocally, the suberin mutants gpat5 (Beisson et al., 2007), afst (Molina et al., 2009), and cyp86b1 (Compagnon et al., 2009), have wild-type fatty alcohol levels, despite being significantly affected in at least one other suberin monomer component.

Fatty alcohols have a limited ability to be incorporated into polymers when compared with bifunctional monomers, which may account for the lack of alterations in other polymeric components. Also, a decrease in one chain length of 
fatty alcohol in a far mutant was sometimes accompanied by an increase in another fatty alcohol(s), such that the total fatty alcohol content of the far mutant was similar to the wild-type (e.g. increases of $\mathrm{C} 20: 0-\mathrm{OH}$ and $\mathrm{C} 22: 0-0 \mathrm{H}$ in far5 mutants). Although we observed compensatory effects, we did not detect major increases in the transcript levels of the other two suberin-associated FARs when one was mutated, but there could be changes in protein activities. Alternatively, there may be a certain amount of total fatty alcohols that can be incorporated into or associated with the suberin polymer, such that reduction in one fatty alcohol component allows for the increased accumulation of other chain lengths. That is, more sites of incorporation become available. Suberin associated waxes of roots, which are soluble in organic solvents, contain components that are chemically related to suberin monomers, and in particular alkyl hydroxycinnamate esters, in which the alkyl moieties are C18-C22 saturated primary alcohols (Li et al., 2007b; Molina et al., 2009; Kosma et al., 2012). In a recent study, Kosma et al. (2012) showed that each of the far mutants, far1, far4 and far5, had reduced levels of C22:0, C20:0 and C18:0 alkyl hydroxycinnamates in the suberin associated waxes, respectively.

Wounded plant tissue develops a wound periderm (Yang and Bernards, 2006). We have demonstrated here that wounding increases the levels of some polyester monomer components of Arabidopsis leaves. The components characteristic of cutin were not wound induced, whereas the characteristic very-long-chain components of suberin (i.e. C20:0 and C22:0 chain length fatty acids, $\omega$-fatty acids, and primary alcohols) were increased 5 days after wounding. In most plants, $\alpha, \omega$-dicarboxylic acids are aliphatics unique to suberized tissue and can be used as diagnostic 
markers to differentiate between suberized and cutinized tissues (Matzke and Riederer, 1991). However, Arabidopsis cutin is atypical in that high amounts of $\alpha, \omega$ dicarboxylic acids are present in its cutin (Bonaventure et al., 2004; Franke et al., 2005). Although C18:1 w-hydroxy (co-eluting with C18:0 dicarboxylic acid in our procedure) and $\alpha, \omega$-dicarboxylic acids are major components of suberin, we did not observe any significant increase in these compounds after wounding. This may be due to the fact that in leaf cutin, the metabolism of polyester biosynthesis is different, with C18:2 $\alpha, \omega$-bifunctional components being far more abundant than the corresponding C18:1 molecules. Alternatively, only certain suberin monomers may be increased in wounded leaf tissues of Arabidopsis. As discussed above, the increase in primary alcohols present in the residual lipids of leaves after wounding correlates well with the wound induction of the FAR1, FAR4, and FAR5 genes. Basal levels of C18:0-C22:0 primary alcohols were found in leaves, and these levels were differentially affected in the far mutants, according to the same pattern that was observed in their root suberin compositions. We did not detect transcripts for these FARs in unwounded leaves, but they could be present at low levels. Alternatively, these genes could be active at earlier stages, which was apparent for FAR1, and the fatty alcohols present are due to a small amount of suberin deposited early in plant development or in response to unknown stresses. 


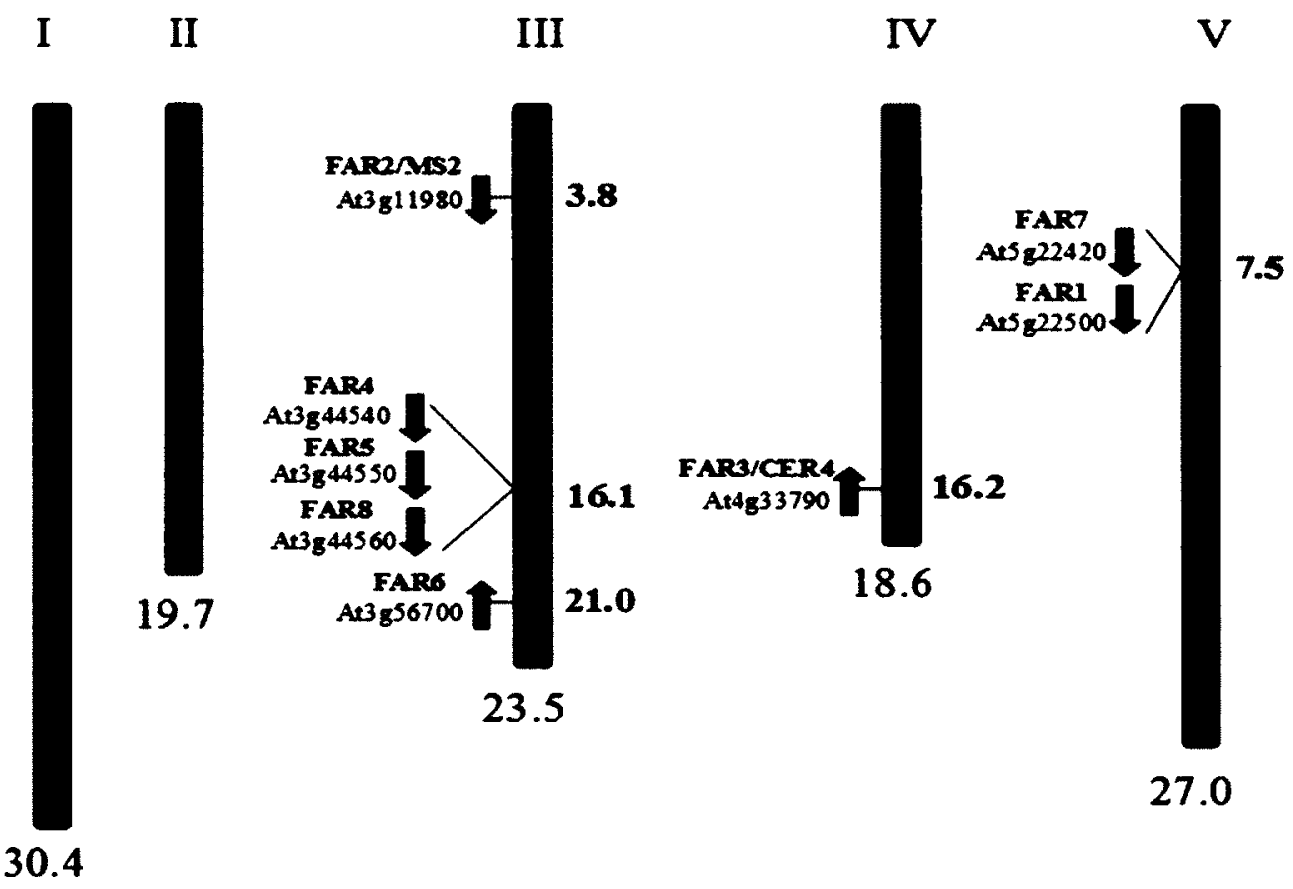

Figure 2.1. The Arabidopsis FAR gene family on chromosomes I-V. The 8 positions and directions of the Arabidopsis FAR genes are shown on chromosomes I, II, III, IV and $\mathrm{V}$. The length of the chromosome is indicated below in mega-basepairs. The black circles represent centromeres. FAR1: AT5G22500, FAR2 (MS2): AT3G11980, FAR3 (CER4): AT4G33790, FAR4: AT3G44540, FAR5: AT3G44550, FAR6: AT3G56700, FAR7: AT5G22420, FAR8: AT3G44560. 


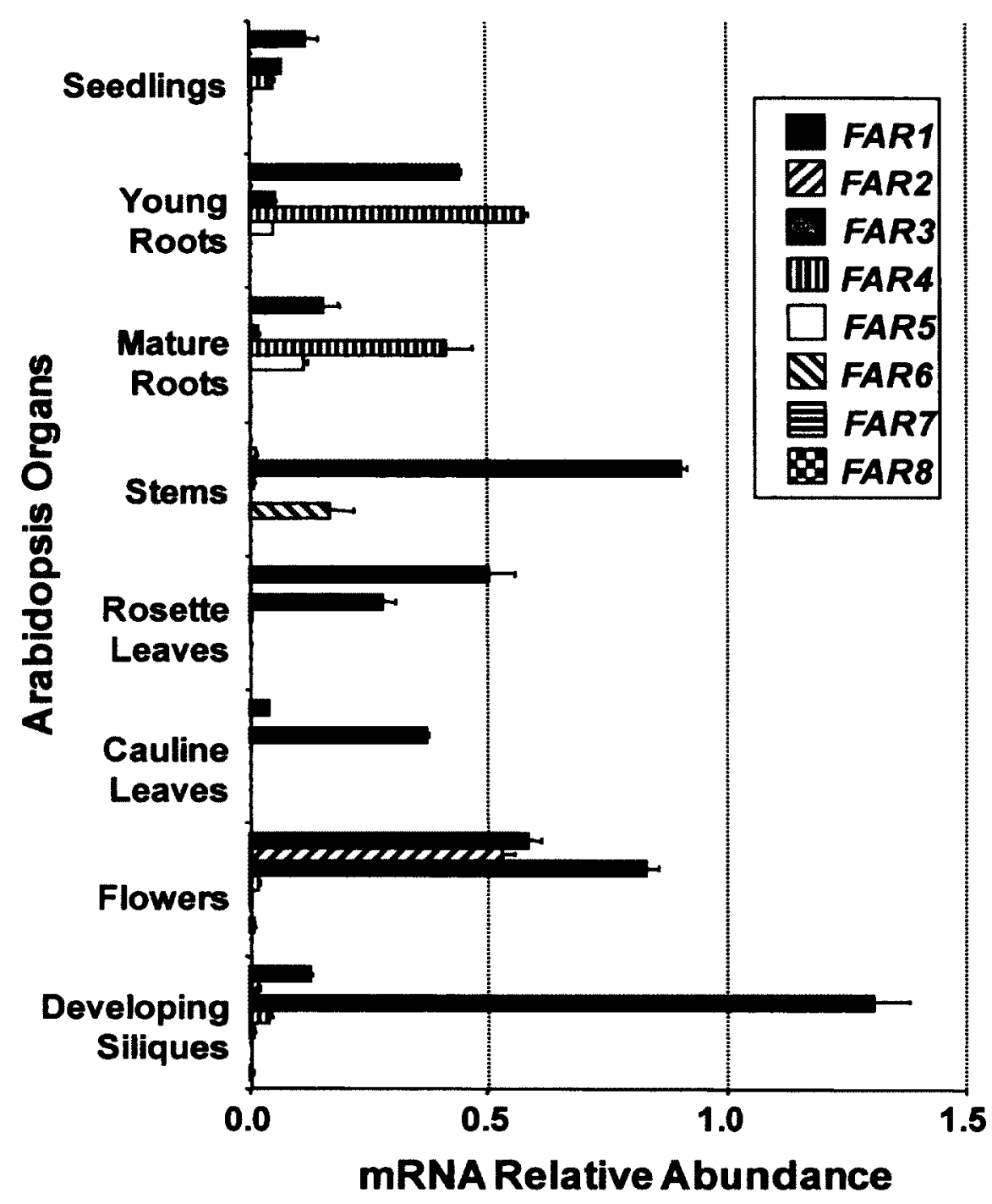

Figure 2.2. Tissue-specific gene expression patterns of Arabidopsis FAR genes. Total RNA was isolated from 15-day-old plants grown in tissue culture (seedlings and young roots), or 10-week-old mature plants grown on soil (mature roots, stems, cauline leaves, rosette leaves, flowers and developing siliques). Gene expression levels were determined by quantitative RT-PCR analysis. Results are presented as relative transcript abundances and normalized through geometric averaging of five constitutively expressed genes ( $A C T 2, E F-1 \alpha, e I F-4 A-1, U B Q 10$ and $P P 2 A$ ). The data represent the means \pm S.D. of three biological replicates. Reprinted with permission of Plant Physiology journal and American Society of Plant Biologists (ASPB). 


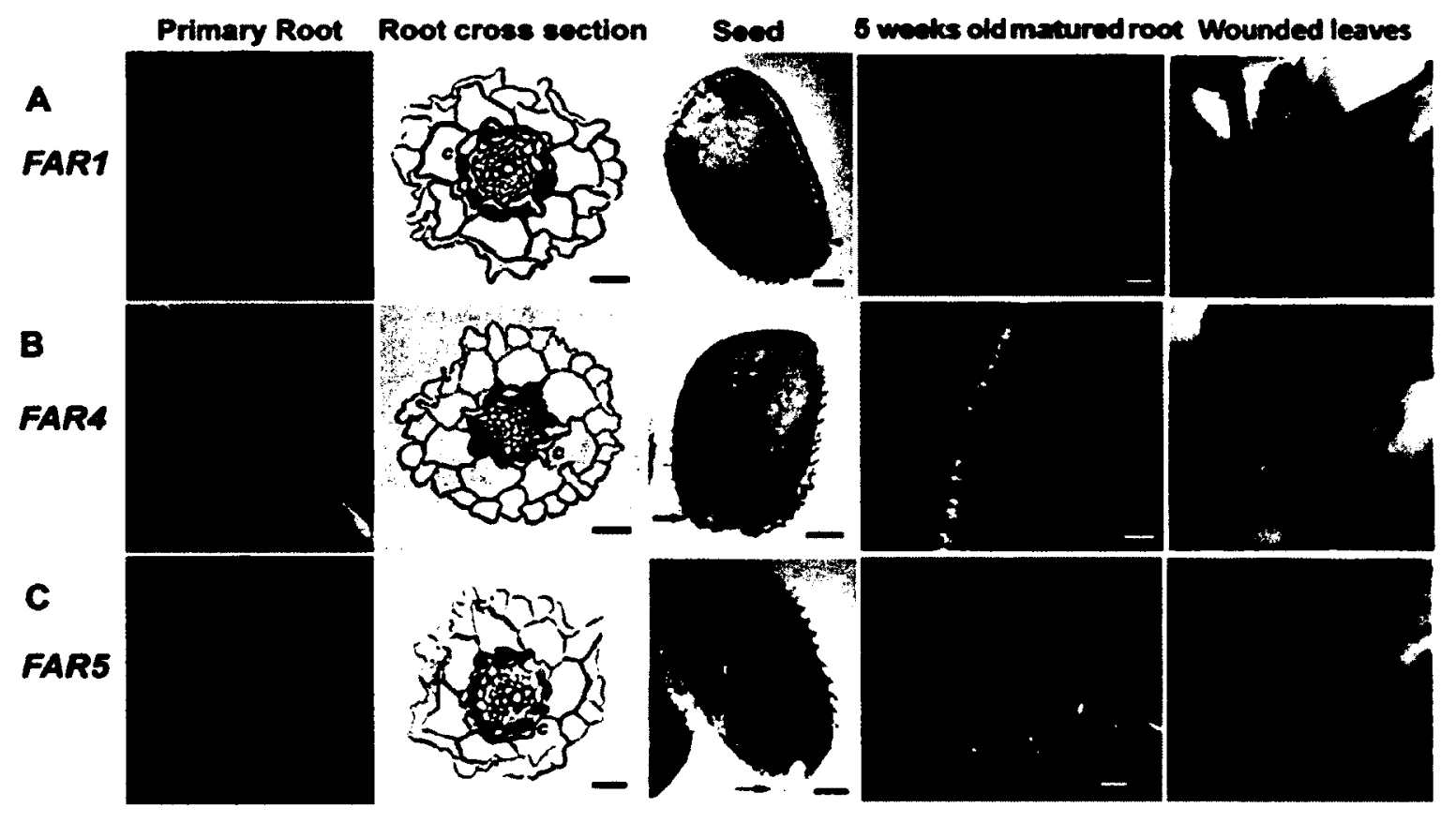

Figure 2.3. Expression patterns of FAR1 (A), FAR4 (B), and FAR5 (C) detected in transgenic promoter:GUS lines. Primary root tissues were collected from 10-day-old seedlings grown in tissue culture. $\mathrm{En}=$ endodermis. $\mathrm{C}=$ cortex. Seeds were collected from recently matured siliques and arrows point to the mycropyle region (hilum). The matured roots were collected from 5 week old plants grown in soil. The leaves of six week-old plants were punctured with a needle and stained for GUS activity 24 hours after wounding. The sizes of the scale bars in the panels for primary root, root cross section, seed; and matured roots and wounded leaves are $100 \mu \mathrm{m}, 25 \mu \mathrm{m}, 10$ $\mu \mathrm{m}$ and $500 \mu \mathrm{m}$, respectively. Reprinted with permission of Plant Physiology journal and American Society of Plant Biologists (ASPB). 


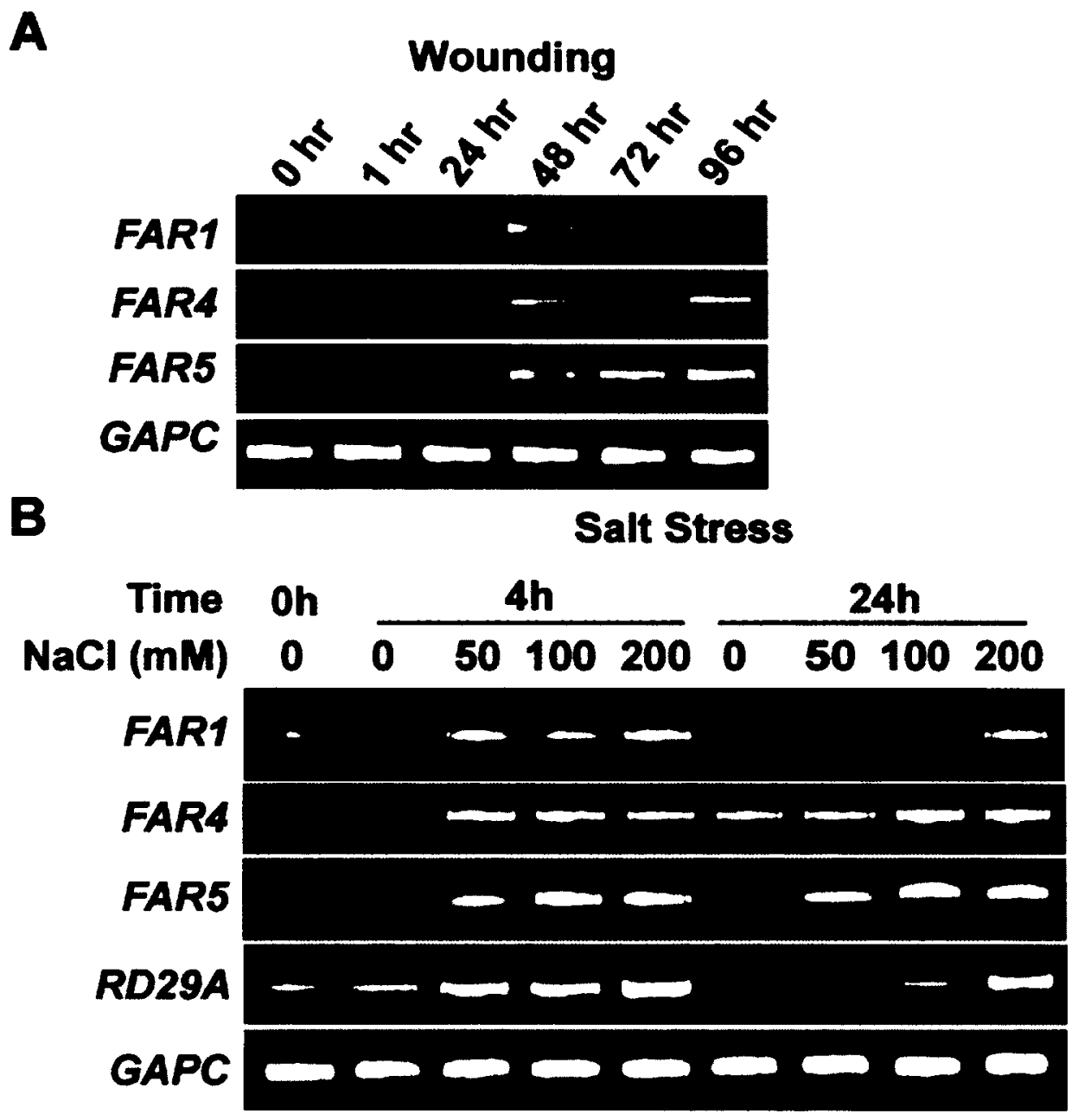

Figure 2.4. Induction of FAR1, FAR4, and FAR5 gene expression by wounding and salt stress. Transcripts were detected by RT-PCR analysis. A, Wounding: Stems of 6week-old plants were slit at multiple positions using a needle and RNA from stems was extracted at the indicated time points. B, Salt stress: 7-day-old seedlings grown on solid MS media were transferred to solid MS media containing different $\mathrm{NaCl}$ concentrations as indicated. RNA samples from whole seedlings were taken at the indicated time points. The Responsive to Dehydration 29A (RD29A) gene was used as a positive control for salt-induced expression. The Glyceraldehyde-3-Phosphate Dehydrogenase $C$ Subunit (GAPC) gene was used as a constitutively expressed control in both (A) and (B). Reprinted with permission of Plant Physiology journal and American Society of Plant Biologists (ASPB). 


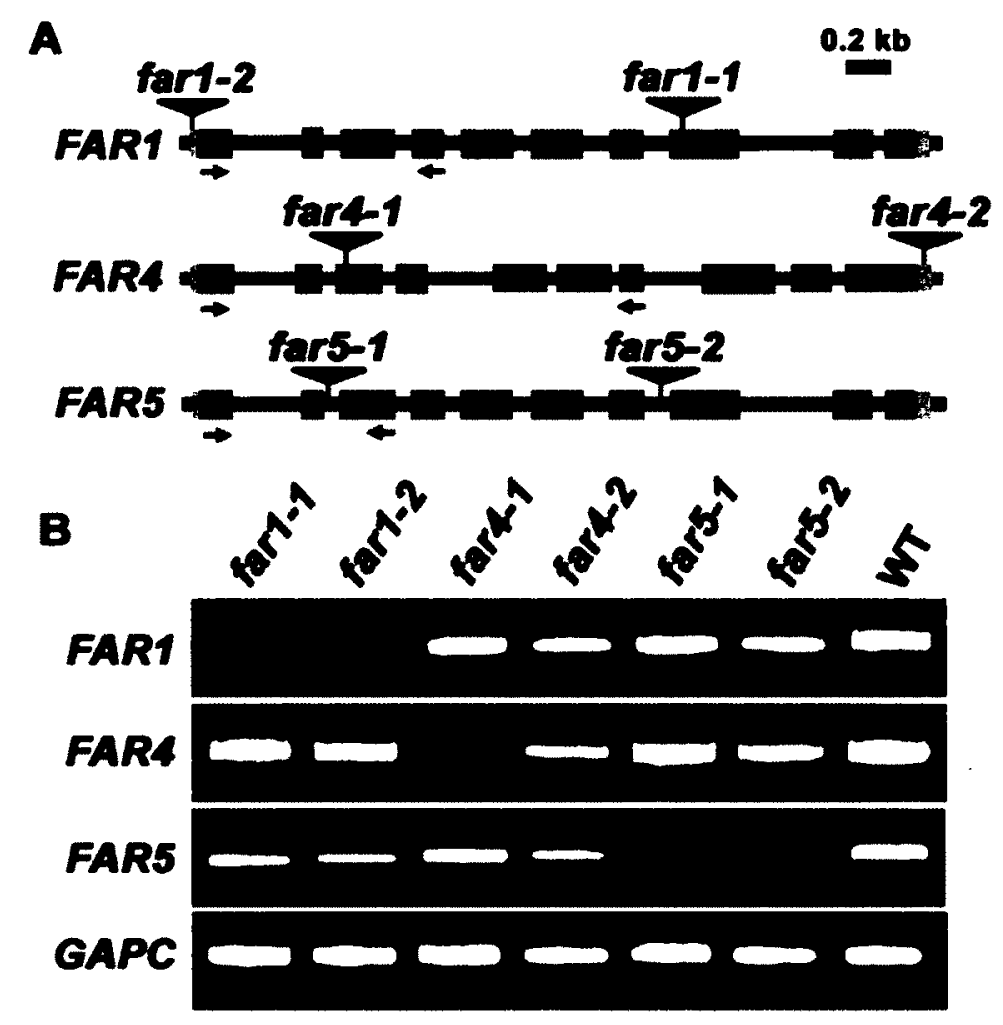

Figure 2.5. T-DNA insertion lines of FAR1, FAR4 and FAR5, and transcript levels in the far mutants. A, Schematics of FAR1, FAR4, and FAR5 gene structures indicating the positions of the T-DNA inserts in each pair of far1, far 4 , and far 5 mutant alleles. Dark grey boxes indicate exons, black lines indicate introns, and light grey boxes indicate $5^{\prime}$ - and 3 '-untranslated regions. The arrows underneath the gene structures are the positions of convergent primers used for RT-PCR to analyze transcript abundance in the homozygous insertion lines. B, RT-PCR analysis of steady-state FAR mRNAs in roots of far mutants compared to wild-type. The GAPC gene was used as a constitutively expressed control. Reprinted with permission of Plant Physiology journal and American Society of Plant Biologists (ASPB). 
Table 2.1. Aliphatic suberin composition in roots of tissue culture-grown wild-type, far1-1, far4-1 and far5-1 plants.

Each value is the mean shown in $\mu \mathrm{g} / \mathrm{mg}$ delipidated dry residue (DR) \pm SD of three to five biological replicates. The amount of each individual chain length of primary alcohol is reported below the total amount of primary alcohols in each genetic background. Reprinted with permission of Plant Physiology journal and American Society of Plant Biologists (ASPB).

\begin{tabular}{|c|c|c|c|c|}
\hline Chemical Class & Col-0 (WT) & far1:1 & far4-1 & far5-1 \\
\hline Fatty Acids & $4.66 \pm 0.71$ & $4.77 \pm 0.58$ & $5.20 \pm 0.12$ & $4.43 \pm 0.15$ \\
\hline$\alpha, \omega$-Dicarboxylic Acids & $4.07 \pm 0.71$ & $3.55 \pm 0.65$ & $3.89 \pm 0.49$ & $3.43 \pm 0.58$ \\
\hline$\omega$-Hydroxy Acids & $6.64 \pm 0.68$ & $6.42 \pm 1.16$ & $6.72 \pm 0.52$ & $6.96 \pm 0.28$ \\
\hline 2-Hydroxy Acids & $0.12 \pm 0.03$ & $0.10 \pm 0.03$ & $0.11 \pm 0.03$ & $0.12 \pm 0.03$ \\
\hline Primary Alcohols & $1.45 \pm 0.11$ & $1.37 \pm 0.24$ & $1.51 \pm 0.22$ & $0.99 \pm 0.19$ \\
\hline $\mathrm{C} 18: 0-\mathrm{OH}$ & $0.68 \pm 0.07$ & $0.75 \pm 0.11$ & $0.75 \pm 0.11$ & $0.15 \pm 0.07$ \\
\hline $\mathrm{C} 20: 0-\mathrm{OH}$ & $0.31 \pm 0.02$ & $0.29 \pm 0.06$ & $0.14 \pm 0.02$ & $0.37 \pm 0.07$ \\
\hline $\mathrm{C} 22: 0-\mathrm{OH}$ & $0.44 \pm 0.04$ & $0.31 \pm 0.08$ & $0.60 \pm 0.09$ & $0.46 \pm 0.07$ \\
\hline $\mathrm{C} 24: 0-\mathrm{OH}$ & $0.02 \pm 0.01$ & $0.02 \pm 0.01$ & $0.03 \pm 0.01$ & $0.02 \pm 0.01$ \\
\hline Total Load & $16.81 \pm 2.12$ & $16.26 \pm 2.27$ & $17.50 \pm 1.38$ & $15.97 \pm 0.90$ \\
\hline
\end{tabular}


Figure 2.6. Aliphatic suberin composition in roots of soil-grown wild-type, far1, far4, and far 5 plants. A, Gas chromatogram of suberin monomers released by depolymerization of solvent-extracted roots of wild-type Arabidopsis plants. Suberin monomers were released by transmethylation and hydroxyl groups silylated before separation by gas chromatography and detection of total ion content with a mass spectrometer. The peaks corresponding to the internal standards (IS) pentadecanol ( $15: 0-\mathrm{OH})$, omega-pentalactone $(\mathrm{C} 15-\omega \mathrm{OH})$ and heptadecanoic acid (C17:0) are indicated, as are each of the major suberin monomers identified by GC-MS. B, Suberin monomer composition, where mean values are shown in $\mu \mathrm{g} / \mathrm{mg}$ delipidated dry residue (DR) $\pm \mathrm{SD}$ of three replicates. Monomers are sorted by compound class along the $\mathrm{x}$-axis. C, Fatty alcohol content of suberin sorted into individual chain lengths, where mean values are shown in $\mu \mathrm{g} / \mathrm{mg}$ delipidated dry residue (DR) of three replicates. Errors bars indicate SD, and significance was assessed by a Student $t$ test $\left(*, \mathrm{P}<0.1 ; * *, \mathrm{P}<0.05 ;{ }^{* * *}, \mathrm{P}<0.01\right)$. Reprinted with permission of Plant Physiology journal and American Society of Plant Biologists (ASPB). (Page 124) 

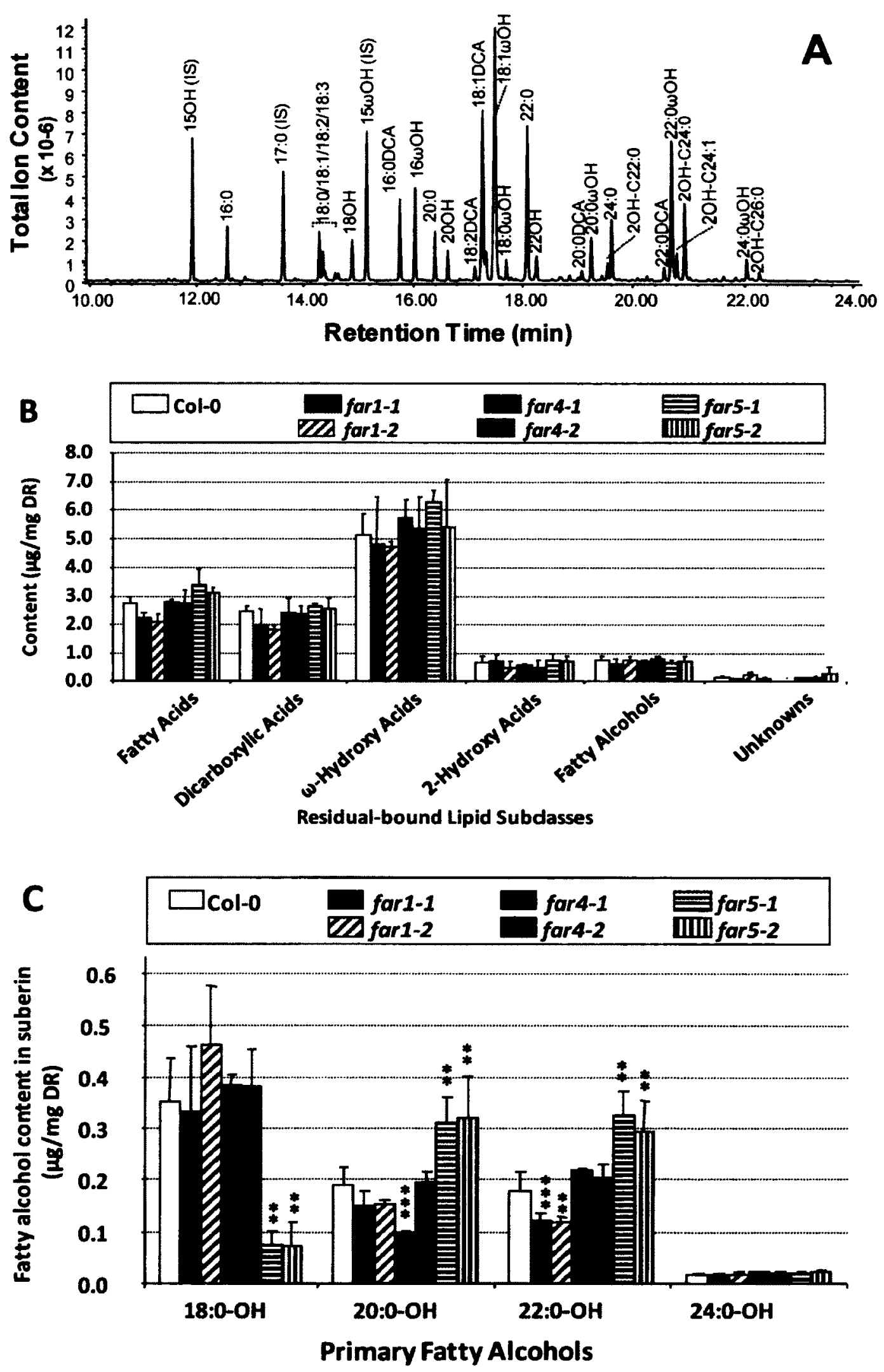


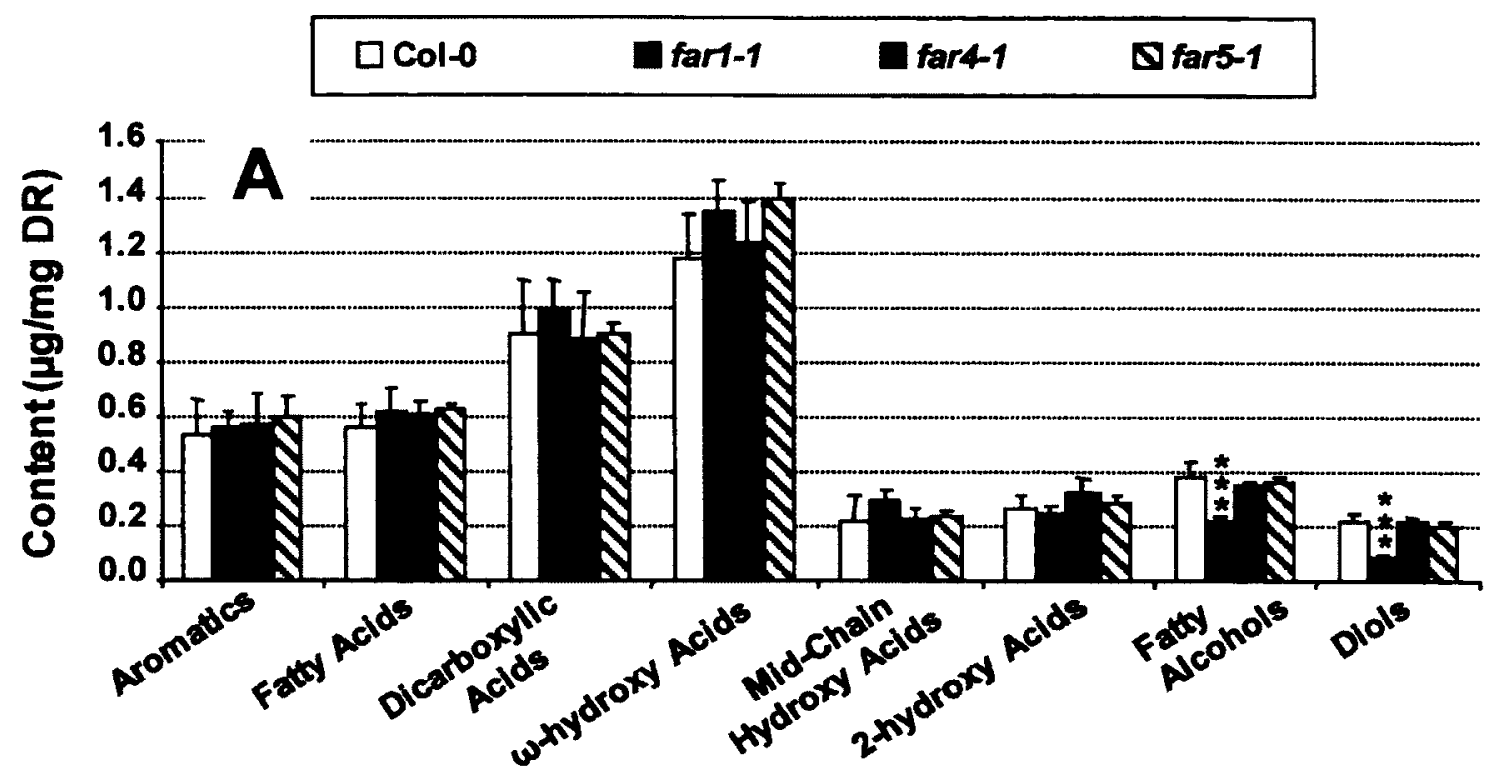

Residual-bound Lipid Subclasses

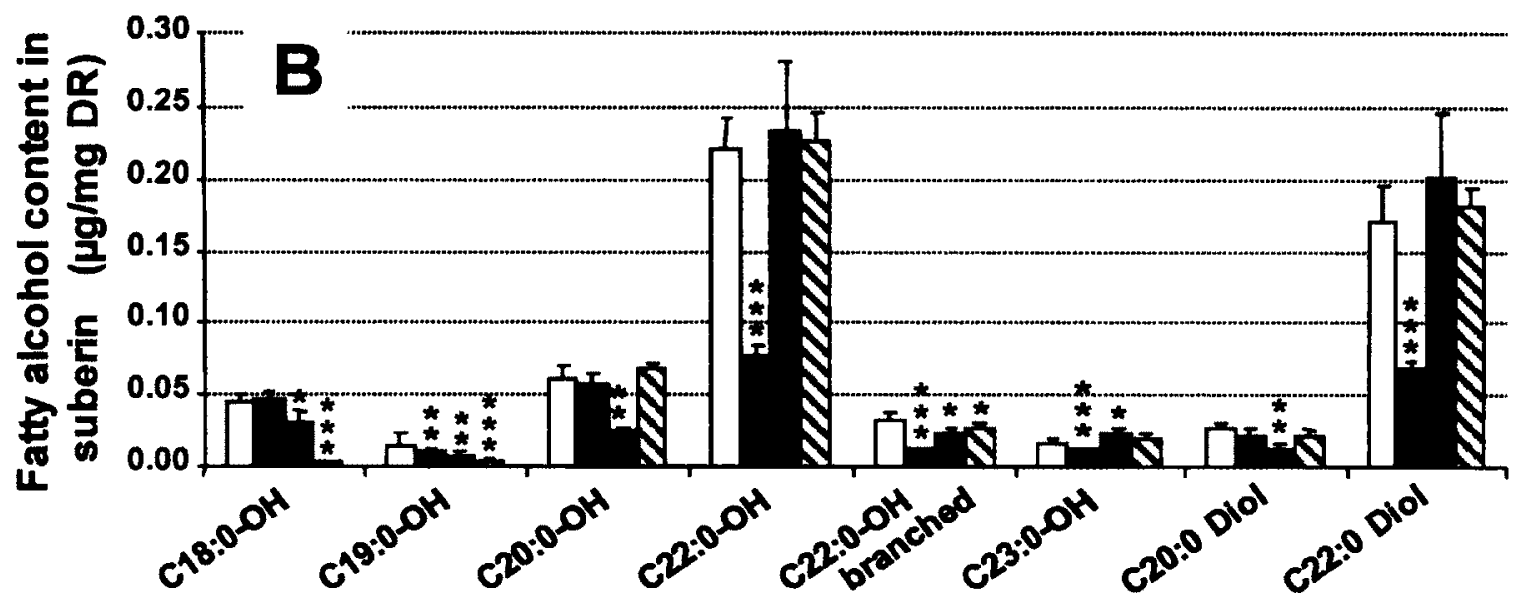

Fatty Alcohols

Figure 2.7. Lipid polyester composition in seeds of wild-type, far1, far4, and far 5 plants. A, Suberin monomer composition, where mean values are shown in $\mu \mathrm{g} / \mathrm{mg}$ delipidated dry residue (DR) \pm SD of five replicates. Monomers are sorted by compound class along the $\mathrm{x}$-axis. $\mathrm{B}$, Chain length distributions of primary fatty alcohols in wild-type, far1-1, far4-1, and far5-1 seed coat suberin. Content of suberin-associated fatty alcohols are sorted into individual chain lengths, where mean values are shown in $\mu \mathrm{g} / \mathrm{mg}$ delipidated dry residue (DR) of five replicates. Errors bars indicate SD, and significance was assessed by a Student $t$ test ${ }^{*}, \mathrm{P}<0.1$; **, $\mathrm{P}<0.05 ;{ }^{* * *}, \mathrm{P}<0.01$ ). Reprinted with permission of Plant Physiology journal and American Society of Plant Biologists (ASPB). 

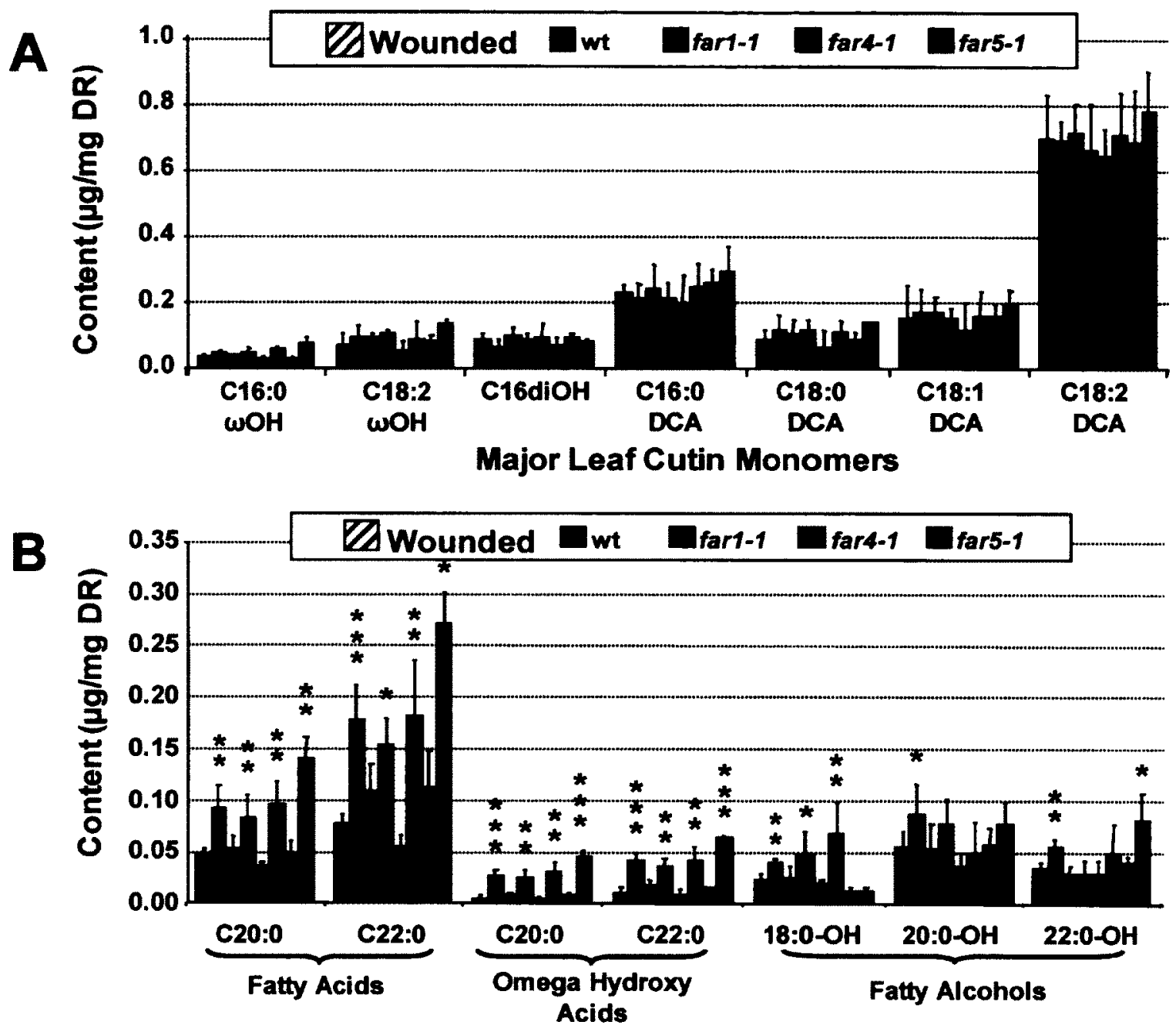

Figure 2.8. Composition of residual bound lipids in unwounded and wounded leaves of wild-type, far1-1, far4-1, and far5-1. A, Amounts of major monomers in residual bound lipids of unwounded and wounded leaves. B, Amounts of fatty acids, very-long-chain omega hydroxy acids, and fatty alcohols present in residual bound lipids in unwounded and wounded leaves. All values are the means shown in $\mu \mathrm{g} / \mathrm{mg}$ delipidated dry weight of residue of three to four replicates. Errors bars indicate SD, and significance was assessed by a Student $t$ test $\left({ }^{*}, \mathrm{P}<0.1 ;{ }^{* *}, \mathrm{P}<0.05 ;{ }^{* * *}, \mathrm{P}<0.01\right)$. Reprinted with permission of Plant Physiology journal and American Society of Plant Biologists (ASPB). 

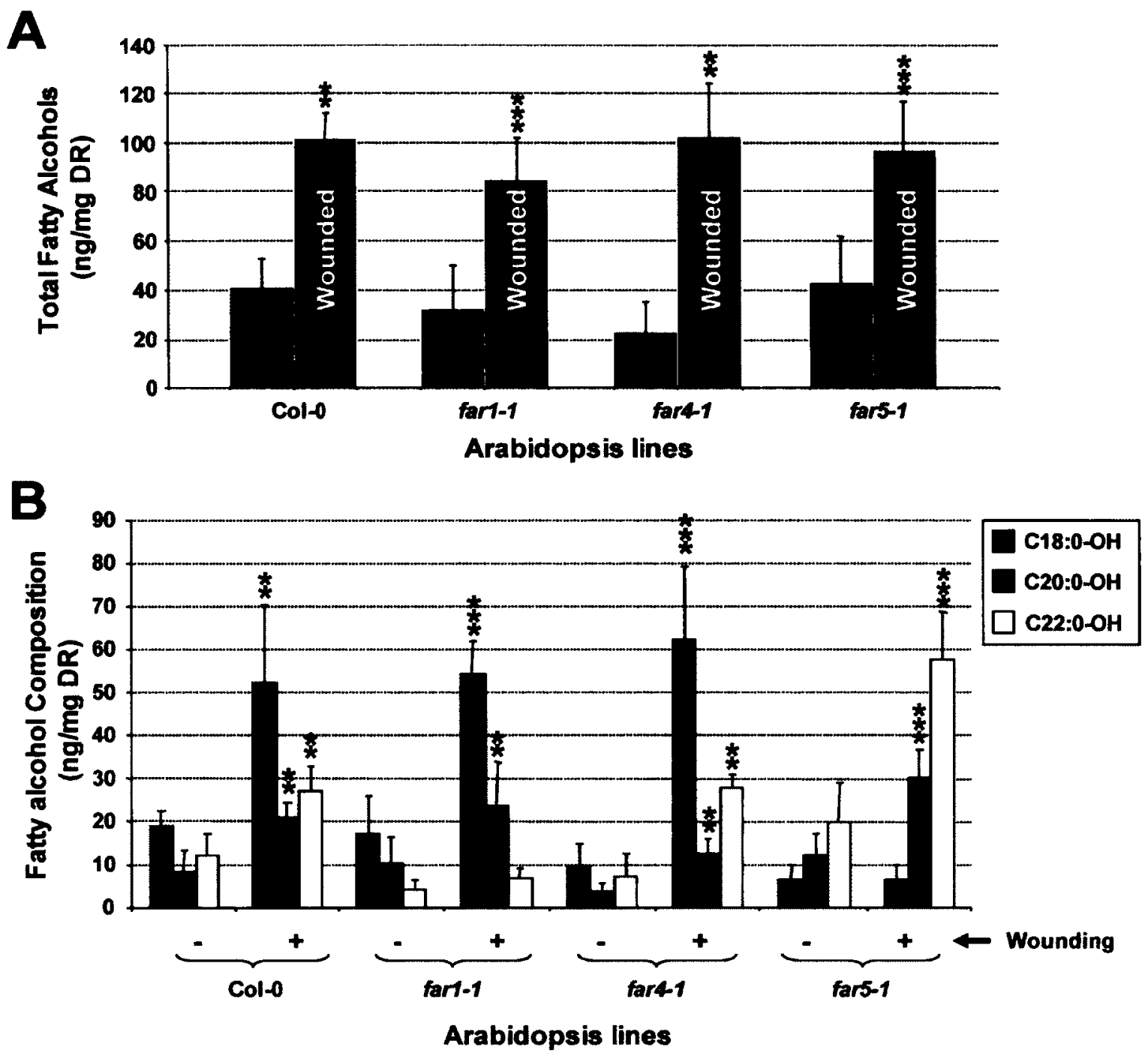

Figure 2.9. Fatty alcohol content of residual bound lipids in unwounded and wounded leaves of wild-type, far1-1, far4-1, and far5-1. A, Total amounts of fatty alcohols in unwounded and wounded leaves. B, Amounts of fatty alcohols sorted by chain length in unwounded and wounded leaves. Quantification of fatty alcohols was based on the major molecular ion of each primary fatty alcohol molecule. All values are the means shown in $\mathrm{ng} / \mathrm{mg}$ delipidated dry weight of residue of four to six replicates. Errors bars indicate $\mathrm{SD}$, and significance was assessed by a Student $t$ test $\left.{ }^{*}, \mathrm{P}<0.1{ }^{* *}, \mathrm{P}<0.05 ;^{* * *}, \mathrm{P}<0.01\right)$. Reprinted with permission of Plant Physiology journal and American Society of Plant Biologists (ASPB). 
Figure 2.10. Fatty acyl-chain profiles of transgenic yeast expressing Arabidopsis FARs. Yeast were transformed with empty-vector control pVT102U (A), or with this vector harbouring FAR3 (B), FAR1 (C), FAR4 (D), or FAR5 (E). Fatty acyl chains were extracted from yeast cells and analyzed by gas chromatography. Major fatty acid peaks are indicated in $(A)$ and retention times of the saturated primary alcohols are indicated by dashed lines with chain lengths indicated above the chromatograms. The peaks corresponding to the internal standards (IS) pentadecanol (C15:0-0TMS) and heptadecanoic acid methyl ester (C17:0) are indicated. Reprinted with permission of Plant Physiology journal and American Society of Plant Biologists (ASPB). (Page 129) 


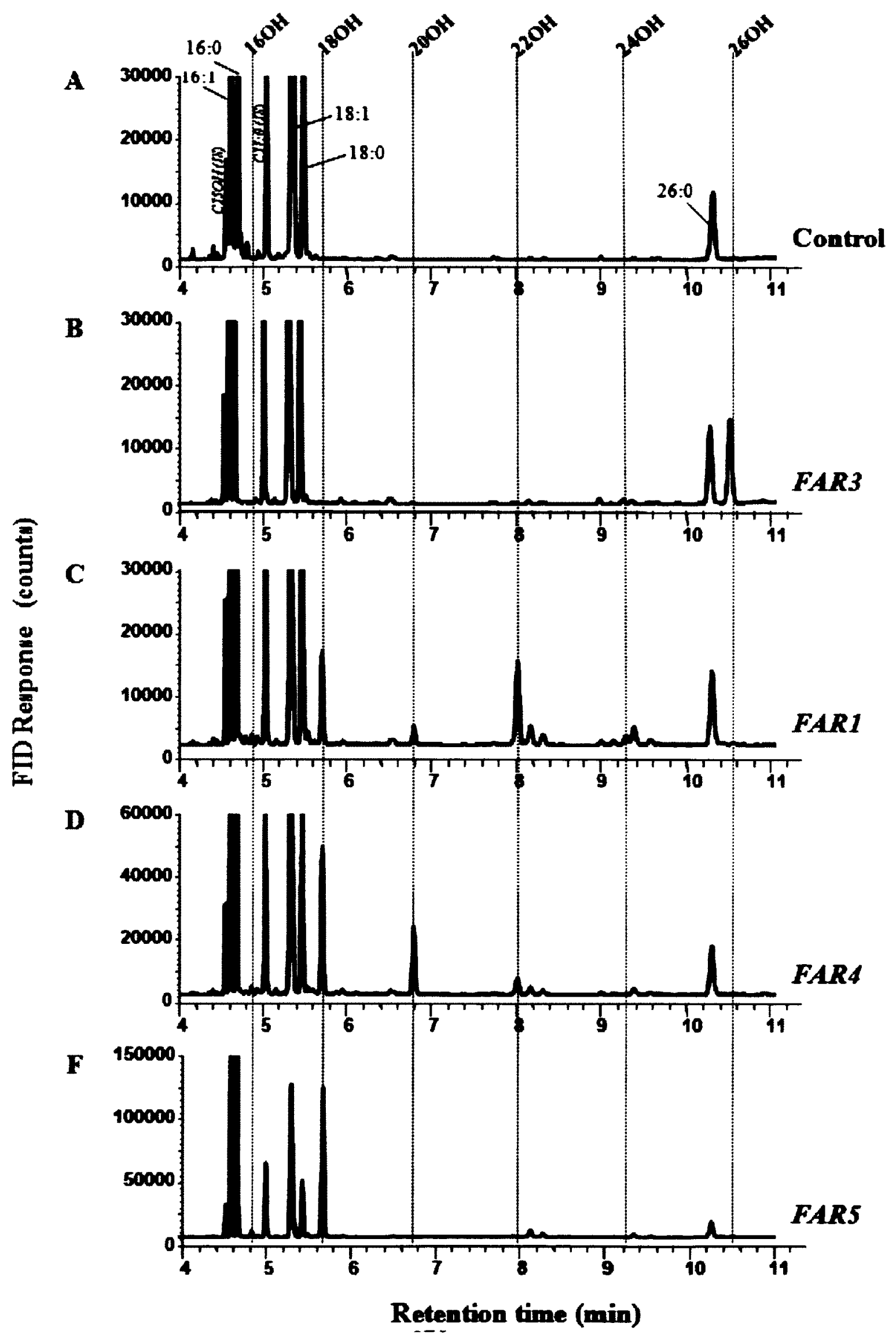


Table 2.2. Internal fatty acyl-chain composition of the different FAR expressing yeast strains.

Fatty acyl-chains from whole cells were prepared, derivatized and analyzed by GC. Data are expressed as $\mu \mathrm{g}$ per unit $\mathrm{OD}_{600}$ and each value is the mean $\pm \mathrm{SD}$ from five biological replicates. ${ }^{1}$ Total fatty acids; ${ }^{2}$ Total fatty alcohols; n.d., not detected. Reprinted with permission of Plant Physiology journal and American Society of Plant Biologists (ASPB).

\begin{tabular}{lrrrrr}
\hline $\begin{array}{c}\text { Fatty Acyl } \\
\text { Chain }\end{array}$ & \multicolumn{1}{c}{$\begin{array}{c}\text { Vector } \\
\text { Control }\end{array}$} & \multicolumn{1}{c}{ FAR1 } & FAR3 & FAR4 & \multicolumn{1}{c}{ FAR5 } \\
\hline Fatty Acids & & & & & \\
$16: 0$ & $1.23 \pm 0.01$ & $1.24 \pm 0.05$ & $1.36 \pm 0.09$ & $1.56 \pm 0.10$ & $1.76 \pm 0.18$ \\
$16: 1$ & $2.85 \pm 0.14$ & $2.53 \pm 0.17$ & $2.90 \pm 0.28$ & $3.28 \pm 0.09$ & $3.34 \pm 0.30$ \\
$18: 0$ & $1.05 \pm 0.15$ & $1.12 \pm 0.07$ & $1.15 \pm 0.12$ & $1.01 \pm 0.07$ & $0.77 \pm 0.06$ \\
$18: 1$ & $4.09 \pm 0.24$ & $3.62 \pm 0.27$ & $3.60 \pm 0.21$ & $3.38 \pm 0.16$ & $2.84 \pm 0.27$ \\
$26: 0$ & $0.18 \pm 0.01$ & $0.18 \pm 0.03$ & $0.29 \pm 0.05$ & $0.15 \pm 0.03$ & $0.11 \pm 0.03$ \\
Total FA 1 & $9.40 \pm 0.81$ & $8.69 \pm 0.77$ & $9.30 \pm 0.97$ & $9.39 \pm 0.84$ & $8.82 \pm 0.99$ \\
Fatty Alcohols & & & & & \\
$16: 0-\mathrm{OH}$ & n.d. & $0.02 \pm 0.00$ & n.d. & $0.01 \pm 0.00$ & $0.06 \pm 0.01$ \\
$18: 0-\mathrm{OH}$ & n.d. & $0.28 \pm 0.03$ & n.d. & $0.52 \pm 0.07$ & $2.07 \pm 0.37$ \\
$20: 0-\mathrm{OH}$ & n.d. & $0.07 \pm 0.01$ & n.d. & $0.32 \pm 0.05$ & $0.01 \pm 0.00$ \\
$22: 0-\mathrm{OH}$ & n.d. & $0.49 \pm 0.10$ & n.d. & $0.11 \pm 0.02$ & n.d. \\
$24: 0-\mathrm{OH}$ & n.d. & $0.04 \pm 0.01$ & $0.02 \pm 0.01$ & n.d. & n.d. \\
26:0-OH & n.d. & n.d. & $0.59 \pm 0.04$ & n.d. & n.d. \\
Total $\mathrm{OH}{ }^{2}$ & n.d. & $0.90 \pm 0.10$ & $0.60 \pm 0.04$ & $0.97 \pm 0.13$ & $2.14 \pm 0.37$ \\
\hline
\end{tabular}




\subsection{Supplemental data}
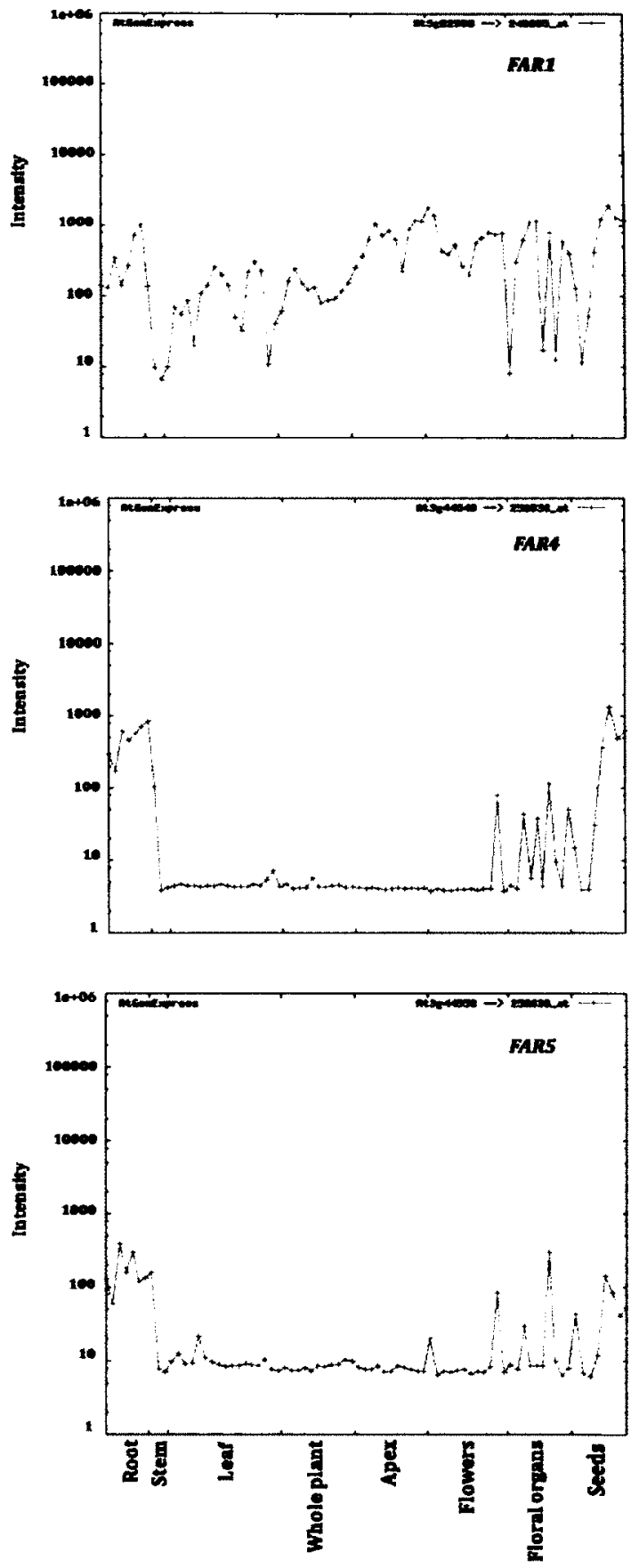

Figure S2.1. The developmental expression pattern of FAR1, FAR4 and FAR5 genes in various plant tissues analysed using DNA microarray (AtGenExpress) data (Schmid et al., 2005). 


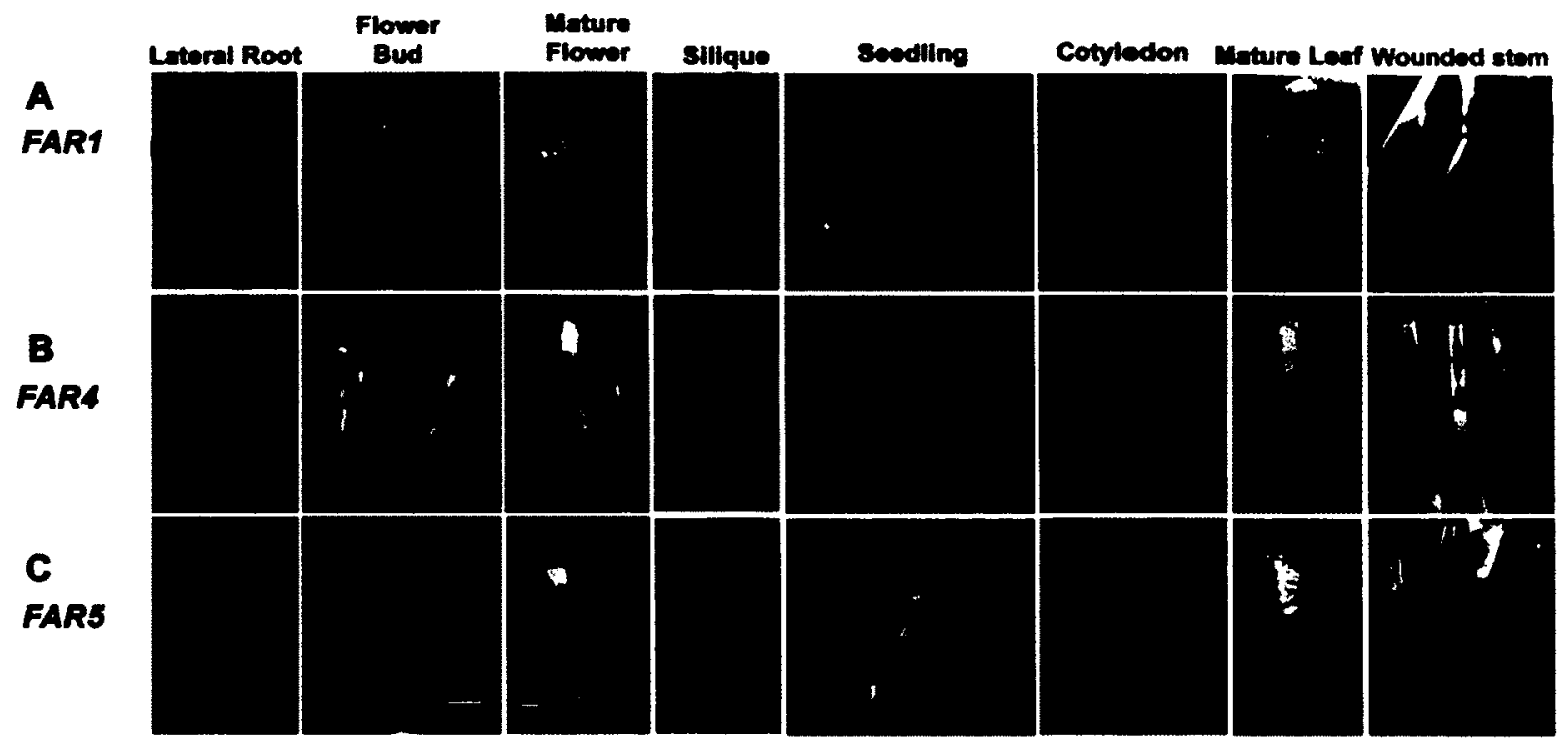

Figure S2.2. Expression patterns of FAR1 (A), FAR4 (B), and FAR5 (C) detected in transgenic promoter:GUS lines. Roots, whole seedlings, and cotyledons were collected from 1-2 week old seedlings grown on vertical plates in tissue culture. Young and mature flowers, siliques, and mature leaves were taken from adult plants grown on soil. The stems of 6-week-old plants were slit with a needle and stained for GUS activity 24 hours after wounding. Reprinted with permission of Plant Physiology journal and American Society of Plant Biologists (ASPB). 


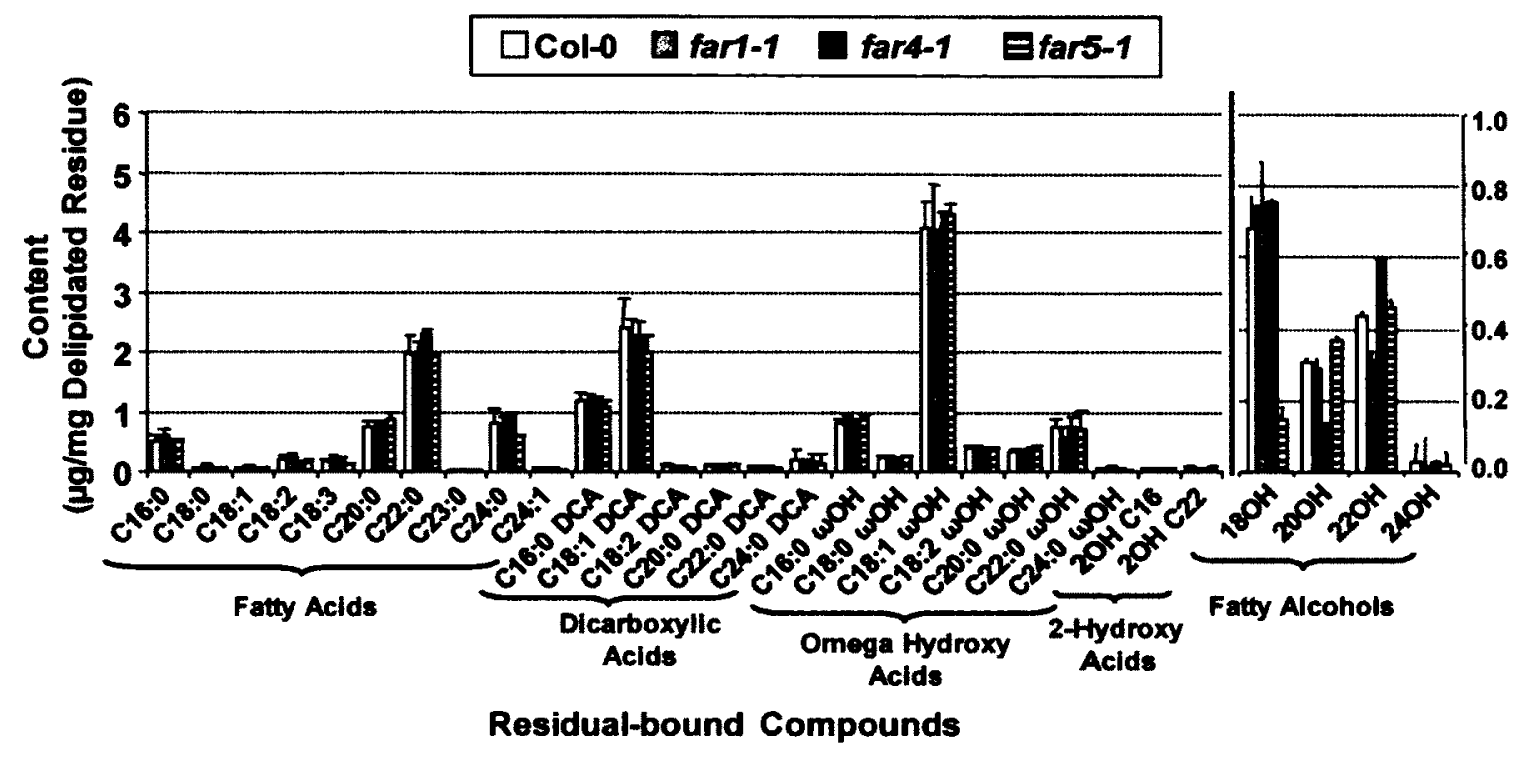

Figure S2.3. Aliphatic suberin composition in roots of tissue culture-grown wildtype, far1-1, far4-1, and far5-1 plants. Suberin monomer composition sorted by chain length and compound class along the x-axis. The amounts of fatty alcohols reported are at a different scale (right side of panel) than the other components (left side of panel). All values are the means shown in $\mu \mathrm{g} / \mathrm{mg}$ delipidated dry residue (DR) of residue \pm SD of three to five replicates. Reprinted with permission of Plant Physiology journal and American Society of Plant Biologists (ASPB). 
Figure S2.4. Identification by gas chromatography-mass spectrometry of primary fatty alcohols produced in transgenic yeast expressing Arabidopsis FAR1. (A) Gas chromatogram of acyl-chains extracted from yeast expressing FAR1. Yeast cells were pelleted by centrifugation and directly transmethylated. Hydroxyl groups were trimethylsilylated (TMS) before separation by gas chromatography and detection of ions with a mass spectrometer. The peaks corresponding to the internal standards (IS) pentadecanol (C15:0-0-TMS) and heptadecanoic acid methyl ester [C17:0) are indicated. Also indicated, the C16:1, C16:0, C18:1, C18:0, and C26:0 fatty acid methyl esters peaks, and the C16:0-0-TMS (B), C18:0-0-TMS (C), C20:0-0-TMS (D), C22:00-TMS (E), and C24:0-0-TMS (F) derivatized primary fatty alcohol peaks. (B-F) Mass spectra of derivatized primary fatty alcohol peaks indicated in panel (A). Reprinted with permission of Plant Physiology journal and American Society of Plant Biologists (ASPB). (Page 135) 


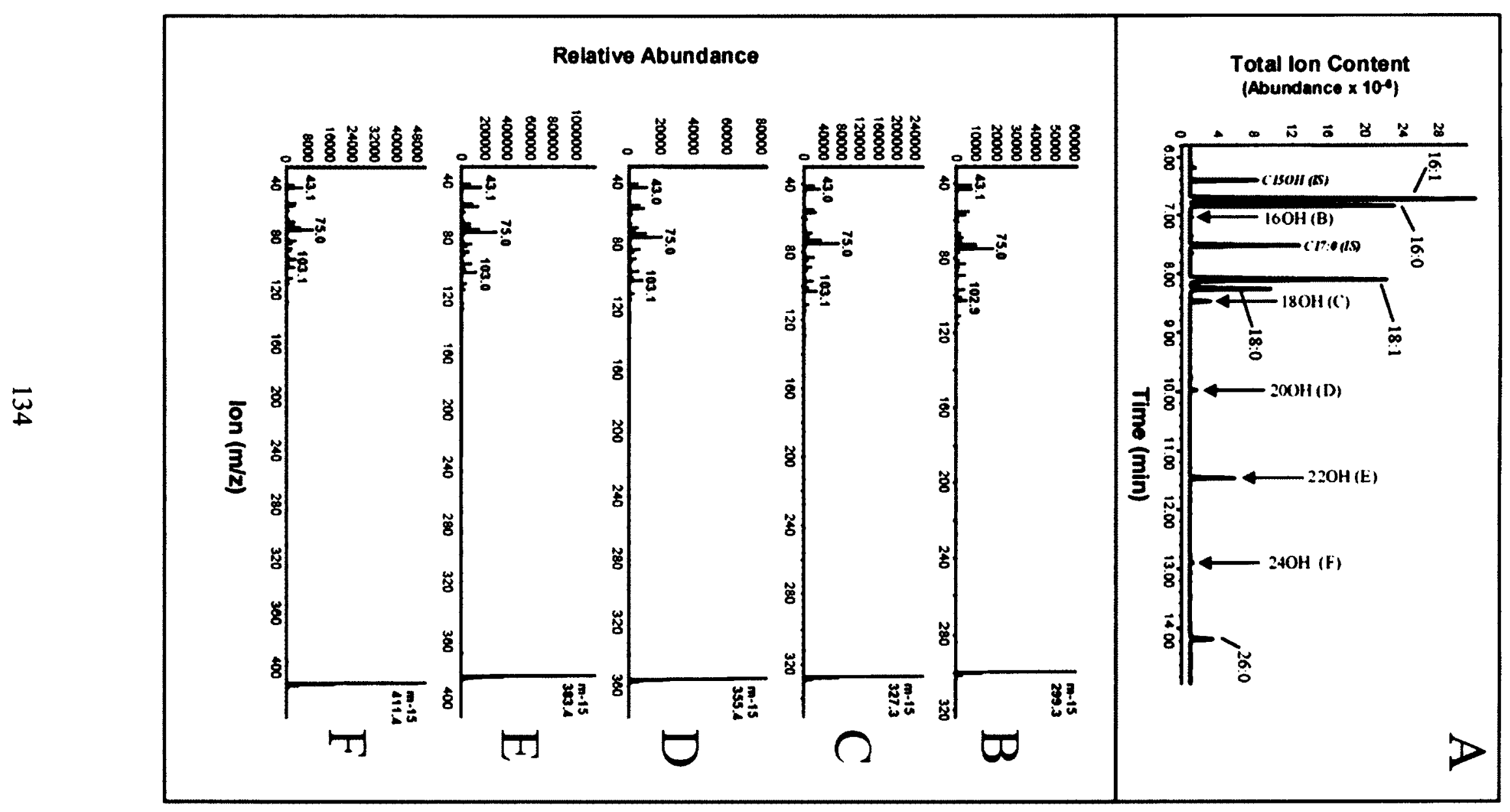


Table S2.1. Fatty Acyl-CoA Reductase (FAR) genes in Arabidopsis thaliana

\begin{tabular}{cccl}
\hline $\begin{array}{c}\text { Gene } \\
\text { Names }\end{array}$ & $\begin{array}{c}\text { Names in } \\
\text { the } \\
\text { literature }\end{array}$ & $\begin{array}{c}\text { Arabidopsis } \\
\text { loci }\end{array}$ & \multicolumn{1}{c}{ References } \\
\hline FAR1 & & At5g22500 & $\begin{array}{l}\text { Doan } \text { et al., 2009 } \\
\text { This study }\end{array}$ \\
FAR2 & MS2 & At3g11980 & $\begin{array}{l}\text { Aarts } \text { et al., 1997 } \\
\text { Doan } \text { et al., 2009 }\end{array}$ \\
& & & $\begin{array}{l}\text { Chen } \text { et al., 2011 } \\
\text { Rowland } \text { et al., 2006 }\end{array}$ \\
FAR3 & CER4 & At4g33790 & Doan et al., 2009 \\
FAR4 & & At3g44540 & This study \\
FAR5 & & At3g44550 & This study \\
FAR6 & & At3g56700 & Doan et al., 2009; \\
& & Doan et al., 2011 \\
FAR7 & & At5g22420 & Doan et al., 2009 \\
FAR8 & & At3g44560 & Doan et al., 2009 \\
\hline
\end{tabular}


Supplemental Table S2.2 Composition of residual bound lipids in whok roots of soil-grown wild-type, farl, far4. and fars plants

Each value is the mean show $\mathrm{n}$ in $\mu \mathrm{g} / \mathrm{mg}$ delipidated dry weight of residue \pm SD of five biological replicates.

\begin{tabular}{|c|c|c|c|c|c|c|c|}
\hline & WT & far $1-1$ & far $1-2$ & fart-1 & far $4-2$ & far $5-1$ & far5-2 \\
\hline \multicolumn{8}{|l|}{ Fatty Acids } \\
\hline Cí6.0 & $0.32 \pm 0.03$ & $0.27 \pm 0.04$ & $0.30 \pm 0.03$ & $0.32 \pm 0.06$ & $0.32 \pm 0.07$ & $0.38 \pm 0.19$ & $0.32 \pm 0.09$ \\
\hline $\mathrm{C} 18: 0$ & $0.05 \pm 0.01$ & $0.04 \pm 0.00$ & $0.06 \pm 0.01$ & $0.08 \pm 0.04$ & $0.05 \pm 0.01$ & $0.10 \pm 0.07$ & $0.07 \pm 0.01$ \\
\hline C18:1 & $0.06 \pm 0.01$ & $0.04 \pm 0.01$ & $0.04 \pm 0.00$ & $0.07 \pm 0.03$ & $0.05+0.02$ & $0.07 \pm 0.02$ & $0.06 \pm 0.02$ \\
\hline $\mathrm{Cl} 18: 2$ & $0.27 \pm 0.06$ & $0.20 \pm 0.06$ & $0.17 \pm 0.08$ & $0.23 \pm 0.05$ & $0.23 \pm 0.09$ & $0.25 \pm 0.11$ & $0.26 \pm 0.12$ \\
\hline $\mathrm{C} 18 \cdot 3$ & $0.17 \pm 0.04$ & $0.14 \pm 0.04$ & $0.13 \pm 0.05$ & $0.22 \pm 0.08$ & $0.17 \pm 0.06$ & $0.21 \pm 0.11$ & $0.21 \pm 0.05$ \\
\hline $\mathrm{C} 20.0$ & $0.39 \pm 0.08$ & $0.36 \pm 0.14$ & $0.38 \pm 0.04$ & $0.49 \pm 0.04$ & $0.47 \pm 0.11$ & $0.59 \pm 0.07$ & $0.49 \pm 0.15$ \\
\hline $\mathrm{C} 22.0$ & $1.08 \pm 0.14$ & $0.87 \pm 0.22$ & $0.77 \pm 0.07$ & $1.02 \pm 0.21$ & $1.07 \pm 0.19$ & $1.28 \pm 0.03$ & $1.19 \pm 0.06$ \\
\hline $\mathrm{C} 23.0$ & $0.04 \pm 0.01$ & $0.02+0.00$ & $0.02 \pm 0.01$ & $0.03 \pm 0.00$ & $0.03 \pm 0.01$ & $0.03 \pm 0.01$ & $0.03 \pm 0.01$ \\
\hline $\mathrm{C} 24: 0$ & $0.30 \pm 0.10$ & $0.21 \neq 0.02$ & $0.17 \pm 0.07$ & $0.21 \pm 0.06$ & $0.26 \pm 0.11$ & $0.27 \pm 0.09$ & $0.35 \pm 0.09$ \\
\hline $\mathrm{C} 24: 1$ & $0.03 \pm 0.00$ & $0.02 \pm 0.00$ & $0.02 \pm 0.01$ & $0.02 \pm 0.00$ & $0.03 \pm 0.00$ & $0.03 \pm 0.01$ & $0.03 \pm 0.00$ \\
\hline C25:0 & $0.02 \pm 0.00$ & $0.01+0.01$ & $0.01 \pm 0.00$ & $0.04 \pm 0.05$ & $0.01 \pm 0.01$ & $0.02+0.01$ & $0.01 \pm 0.00$ \\
\hline C26:0 & $0.02 \pm 0.01$ & $0.03 \pm 0.02$ & $0.02 \pm 0.01$ & $0.03 \pm 0.01$ & $0.02+0.02$ & $0.02 \pm 0.02$ & $0.04 \pm 0.01$ \\
\hline C28:0 & $0.01 \pm 0.00$ & $0.02+0.00$ & $0.01 \pm 0.00$ & $0.01 \pm 0.01$ & $0.01 \pm 0.01$ & $0.00 \pm 0.00$ & $0.01 \pm 0.01$ \\
\hline \multicolumn{8}{|c|}{ Dicarboxylic Acids } \\
\hline C16.0 DCA & $0.65 \pm 0.10$ & $0.52 \pm 0.17$ & $0.55 \pm 0.06$ & $0.66 \pm 0.09$ & $0.65 \pm 0.11$ & $0.72 \pm 0.07$ & $0.65 \pm 0.13$ \\
\hline C18:1 DCA & $1.50 \pm 0.13$ & $1.21 \pm 0.38$ & $1.11 \pm 0.08$ & $1.47 \pm 0.32$ & $1.45 \pm 0.18$ & $1.64 \pm 0.07$ & $1.48 \pm 0.15$ \\
\hline $\mathrm{C} 18.2 \mathrm{DCA}$ & $0.10 \pm 0.01$ & $0.06 \pm 0.01$ & $0.04 \pm 0.02$ & $0.07 \pm 0.03$ & $0.08 \pm 0.03$ & $0.09 \pm 0.03$ & $0.20 \pm 0.21$ \\
\hline C20:0 DCA & $0.10 \pm 0.01$ & $0.08 \pm 0.02$ & $0.07 \pm 0.02$ & $0.10 \pm 0.02$ & $0.09 \pm 0.02$ & $0.12 \pm 0.01$ & $0.09 \pm 0.02$ \\
\hline C22:0 DCA & $0.11 \pm 0.01$ & $0.08 \pm 0.03$ & $0.06 \pm 0.02$ & $0.10 \pm 0.04$ & $0.10 \pm 0.01$ & $0.13 \pm 0.01$ & $0.10 \pm 0.02$ \\
\hline \multicolumn{8}{|c|}{$\omega$-Hydroxy Acids } \\
\hline $\mathrm{Cl6} 0 \omega \mathrm{OOH}$ & $0.61 \pm 0.14$ & $0.57 \pm 0.20$ & $0.64 \pm 0.08$ & $0.71 \pm 0.06$ & $0.66+0.16$ & $0.78 \pm 0.10$ & $0.66 \pm 0.24$ \\
\hline $\mathrm{Cl} 18.0 \omega \mathrm{OH}$ & $0.16 \pm 0.04$ & $0.16 \pm 0.07$ & $0.17 \pm 0.02$ & $0.19 \pm 0.02$ & $0.19 \pm 0.05$ & $0.26 \pm 0.05$ & $0.20 \pm 0.09$ \\
\hline $\mathrm{C} 18: 1 \omega \mathrm{OH}$ & $2.75 \pm 0.46$ & $2.65 \pm 1.07$ & $2.70 \pm 0.20$ & $3.17 \pm 0.40$ & $2.94 \pm 0.71$ & $3.62 \pm 0.41$ & $2.90 \pm 1.01$ \\
\hline $\mathrm{C} 18 \cdot 2 \omega \mathrm{OH}$ & $0.24 \pm 0.04$ & $0.20 \pm 0.07$ & $0.20 \pm 0.01$ & $0.25 \pm 0.02$ & $0.25 \pm 0.03$ & $0.28 \pm 0.03$ & $0.23 \pm 0.05$ \\
\hline $\mathrm{C} 20.0 \omega \mathrm{OH}$ & $0.31 \pm 0.06$ & $0.28 \pm 0.11$ & $0.30 \pm 0.03$ & $0.37 \pm 0.03$ & $0.35 \pm 0.08$ & $0.45 \pm 0.07$ & $0.34 \pm 0.12$ \\
\hline $\mathrm{C} 20: 1 \omega \mathrm{OH}$ & $0.02 \pm 0.00$ & $0.02 \pm 0.01$ & $0.02 \pm 0.00$ & $0.02 \pm 0.01$ & $0.02 \pm 0.00$ & $0.02 \pm 0.00$ & $0.02 \pm 0.00$ \\
\hline $\mathrm{C} 22.0 \omega \mathrm{OH}$ & $0.93 \pm 0.08$ & $0.77 \pm 0.23$ & $0.64 \pm 0.07$ & $0.90 \pm 0.15$ & $0.88 \pm 0.15$ & $1.09 \pm 0.07$ & $0.90 \pm 0.19$ \\
\hline $\mathrm{C} 24.0 \omega \mathrm{OH}$ & $0.09 \pm 0.04$ & $0.08 \pm 0.04$ & $0.04 \pm 0.03$ & $0.06 \pm 0.02$ & $0.06 \pm 0.03$ & $0.07 \pm 0.05$ & $0.11 \pm 0.02$ \\
\hline $\mathrm{C} 26.0 \omega \mathrm{OH}$ & $0.02 \pm 0.01$ & $0.04 \pm 0.05$ & $0.02 \neq 0.01$ & $0.02 \pm 0.01$ & $0.02+0.01$ & $0.02 \pm 0.02$ & $0.03 \pm 0.01$ \\
\hline $\mathrm{C} 28.0 \omega \mathrm{OH}$ & $0.01 \pm 0.01$ & $0.03 \pm 0.03$ & $0.00 \pm 0.01$ & $0.01 \pm 0.01$ & $0.01 \pm 0.01$ & $0.01 \pm 0.01$ & $0.02 \pm 0.01$ \\
\hline \multicolumn{8}{|c|}{ 2-Hydroxy Acids } \\
\hline $\mathrm{Cl} 6: 0-2 \mathrm{OH}$ & $0.03 \pm 0.01$ & $0.03 \pm 0.01$ & $0.02 \pm 0.01$ & $0.03 \pm 0.01$ & $0.02 \pm 0.01$ & $0.03 \pm 0.01$ & $0.03 \pm 0.01$ \\
\hline $\mathrm{C} 22: 0-2 \mathrm{OH}$ & $0.07 \pm 0.03$ & $0.07 \pm 0.03$ & $0.05 \pm 0.03$ & $0.05 \pm 0.01$ & $0.05 \pm 0.03$ & $0.07 \pm 0.03$ & $0.06 \pm 0.04$ \\
\hline $\mathrm{C} 23: 0-2 \mathrm{OH}$ & $0.02 \pm 0.01$ & $0.02 \pm 0.01$ & $0.01 \pm 0.01$ & $0.02 \pm 0.00$ & $0.02 \pm 0.01$ & $0.02 \pm 0.01$ & $0.01 \pm 0.01$ \\
\hline $\mathrm{C} 24: 0-2 \mathrm{OH}$ & $0.30 \pm 0.13$ & $0.32 \pm 0.15$ & $0.19 \pm 0.13$ & $0.21 \pm 0.04$ & $0.22 \pm 0.15$ & $0.28 \pm 0.13$ & $0.27 \pm 0.17$ \\
\hline $\mathrm{C} 24: 1-2 \mathrm{OH}$ & $0.16 \pm 0.03$ & $0.17 \pm 0.00$ & $0.14 \pm 0.04$ & $0.16 \pm 0.01$ & $0.12 \pm 0.03$ & $0.17 \pm 0.04$ & $0.18 \pm 0.01$ \\
\hline $\mathrm{C} 25 \cdot 0-2 \mathrm{OH}$ & $0.03 \pm 0.01$ & $0.02 \pm 0.01$ & $0.01 \pm 0.01$ & $0.02 \pm 0.00$ & $0.02 \pm 0.01$ & $0.02 \pm 0.01$ & $0.02 \pm 0.01$ \\
\hline $\mathrm{C} 25: 1-2 \mathrm{OH}$ & $0.02 \pm 0.01$ & $0.02 \neq 0.00$ & $0.01 \neq 0.01$ & $0.03 \pm 0.01$ & $0.02 \pm 0.00$ & $0.02 \pm 0.00$ & $0.04 \pm 0.04$ \\
\hline $\mathrm{C} 26.0-2 \mathrm{OH}$ & $0.04 \pm 0.02$ & $0.04 \pm 0.02$ & $0.02+0.02$ & $0.03 \pm 0.00$ & $0.03 \pm 0.02$ & $0.03 \pm 0.02$ & $0.04 \pm 0.03$ \\
\hline $\mathrm{C} 26: 1-2 \mathrm{OH}$ & $0.02 \pm 0.00$ & $0.02 \pm 0.01$ & $0.01 \pm 0.01$ & $0.02 \pm 0.00$ & $0.01 \pm 0.01$ & $0.02 \pm 0.01$ & $0.04 \pm 0.04$ \\
\hline \multicolumn{8}{|l|}{ Fatty Aloohols } \\
\hline $\mathrm{Cl} 18.0-\mathrm{OH}$ & $0.35 \pm 0.09$ & $0.32 \pm 0.14$ & $0.46 \pm 0.12$ & $0.38 \pm 0.02$ & $0.38 \pm 0.07$ & $0.07 \pm 0.03$ & $0.07 \pm 0.05$ \\
\hline $\mathrm{C} 20.0-\mathrm{OH}$ & $0.19 \pm 0.03$ & $0.14 \pm 0.04$ & $0.15 \pm 0.01$ & $0.10 \pm 0.01$ & $0.19 \pm 0.02$ & $0.31 \pm 0.05$ & $0.32 \pm 0.08$ \\
\hline $\mathrm{C} 22: 0-\mathrm{OH}$ & $0.18 \pm 0.04$ & $0.12 \pm 0.02$ & $0.12 \pm 0.01$ & $0.22 \pm 0.00$ & $0.20 \pm 0.03$ & $0.33 \pm 0.05$ & $0.29 \pm 0.06$ \\
\hline $\mathrm{C} 24.0 \cdots \mathrm{OH}$ & $0.02 \pm 0.01$ & $0.02 \pm 0.00$ & $0.02 \pm 0.00$ & $0.02 \pm 0.00$ & $0.02 \pm 0.00$ & $0.02 \pm 0.00$ & $0.02 \pm 0.00$ \\
\hline Unknowns & $0.15 \pm 0.06$ & $0.11 \pm 0.04$ & $0.22 \pm 0.16$ & $0.34 \pm 0.34$ & $0.14 \pm 0.03$ & $0.17 \pm 0.06$ & $0.31 \pm 0.27$ \\
\hline Total & $11.91 \pm 1.00$ & $10.41 \pm 2.39$ & $10.1 \pm 0.27$ & $12.91 \pm 0.48$ & $11.91 \pm 1.29$ & $14.11 \pm 0.55$ & $12.73 \pm 1.80$ \\
\hline
\end{tabular}


Supplemen tal Table S2.3. Composition of residual bound lipids in seeds of wild-type, farl -1 , far4-1, and far $5-1$ plants

Each value is the mean shown in $\mu \mathrm{g} / \mathrm{mg}$ delipidated dry weight of residue $\pm \mathrm{SD}$ of three biological replicates.

\begin{tabular}{|c|c|c|c|c|}
\hline & WT & far $1-1$ & far $4-1$ & far5-1 \\
\hline \multicolumn{5}{|l|}{ Aromatics } \\
\hline trans-cinnamic acid & $0.026 \pm 0.013$ & $0.010 \pm 0.003$ & $0.023 \pm 0.006$ & $0.019 \pm 0.004$ \\
\hline cis-ferulic ac id & $0.041 \pm 0.021$ & $0.034 \pm 0.007$ & $0.044 \pm 0.023$ & $0.060 \pm 0.010$ \\
\hline trans-ferulic acid & $0.204 \pm 0.078$ & $0.238 \pm 0.021$ & $0.212 \pm 0.058$ & $0.222 \pm 0.031$ \\
\hline cis-sinapic acid & $0.041 \pm 0.022$ & $0.027 \pm 0.005$ & $0.024 \pm 0.013$ & $0.049 \pm 0.007$ \\
\hline trans-sinapic acid & $0.186 \pm 0.050$ & $0.213 \pm 0.045$ & $0.184 \pm 0.019$ & $0.169 \pm 0.025$ \\
\hline \multicolumn{5}{|l|}{ Fatty Acids } \\
\hline C 16.0 & $0.138 \pm 0.014$ & $0.128 \pm 0.017$ & $0.127 \pm 0.022$ & $0.142 \pm 0.010$ \\
\hline $\mathrm{Cl} 8.0$ & $0.037 \pm 0.005$ & $0.032 \pm 0.005$ & $0.036 \pm 0.008$ & $0.040 \pm 0.002$ \\
\hline $\mathrm{C} 18: 1$ & $0.013 \pm 0.002$ & $0.009 \pm 0.002$ & $0.010 \pm 0.002$ & $0.010 \pm 0.001$ \\
\hline $\mathrm{Cl} 82$ & $0.057 \pm 0.008$ & $0.064 \pm 0.017$ & $0.056 \pm 0.011$ & $0.051 \pm 0.002$ \\
\hline $\mathrm{C} 18: 3$ & $0.047 \pm 0.007$ & $0.048 \pm 0.011$ & $0.042 \pm 0.006$ & $0.050 \pm 0.012$ \\
\hline $\mathrm{C} 20.0$ & $0.032 \pm 0.003$ & $0.028 \pm 0.004$ & $0.026 \pm 0.003$ & $0.028 \pm 0.002$ \\
\hline $\mathrm{C} 20: 1$ & $0.036 \pm 0.015$ & $0.032 \pm 0.009$ & $0.048 \pm 0.025$ & $0.057 \pm 0.006$ \\
\hline C2 1.0 methylated & $0.016 \pm 0.007$ & $0.021 \pm 0.002$ & $0.017 \pm 0.004$ & $0.018 \pm 0.003$ \\
\hline $\mathrm{C} 22.0$ & $0.036 \pm 0.009$ & $0.043 \pm 0.004$ & $0.045 \pm 0.008$ & $0.043 \pm 0.003$ \\
\hline C23.0 & $0.008 \pm 0.002$ & $0.011 \pm 0.003$ & $0.013 \pm 0.004$ & $0.010 \pm 0.001$ \\
\hline $\mathrm{C} 24.0$ & $0.096 \pm 0.018$ & $0.131+0.013$ & $0.123 \pm 0.026$ & $0.125 \pm 0.008$ \\
\hline $\mathrm{C} 26.0$ & $0.031 \pm 0.007$ & $0.030 \pm 0.003$ & $0.034 \pm 0.003$ & $0.030 \pm 0.004$ \\
\hline $\mathrm{C} 26: 1$ & $0.027 \pm 0.010$ & $0.034 \pm 0.002$ & $0.035 \pm 0.009$ & $0.029 \pm 0.003$ \\
\hline \multicolumn{5}{|l|}{ Dicarboxylic Acids } \\
\hline $\mathrm{C} 16.0 \mathrm{DCA}$ & $0.049 \pm 0.011$ & $0.072 \pm 0.008$ & $0.063=0.024$ & $0.070 \pm 0.003$ \\
\hline C1 8:1 DCA & $0.145 \pm 0.024$ & $0.162 \pm 0.012$ & $0.137 \neq 0.035$ & $0.146 \pm 0.009$ \\
\hline C1 8.2 DCA & $0.166 \pm 0.037$ & $0.221 \pm 0.025$ & $0.198=0.035$ & $0.179 \pm 0.004$ \\
\hline $\mathrm{C} 2000 \mathrm{DCA}$ & $0.015 \pm 0.006$ & $0.015 \pm 0.004$ & $0.020=0.008$ & $0.012 \pm 0.001$ \\
\hline $\mathrm{C} 22.0 \mathrm{DCA}$ & $0.111 \pm 0.024$ & $0.115 \pm .013$ & $0.0952 \pm 0.021$ & $0.096 \pm 0.005$ \\
\hline C23.0 DCA & $0.017 \pm 0.008$ & $0.017 \pm 0.004$ & $0.017 \pm 0.004$ & $0.015 \pm 0.001$ \\
\hline $\mathrm{C} 24.0 \mathrm{DCA}$ & $0.347 \pm 0.098$ & $0.383 \pm 0.045$ & $0.358 \pm 0.088$ & $0.392 \pm 0.023$ \\
\hline \multicolumn{5}{|l|}{ w-Hydroxy Acids } \\
\hline $\mathrm{Cl} 6.0 \omega \mathrm{OH}$ & $0.043 \pm 0.007$ & $0.058 \pm 0.006$ & $0.056 \pm 0.009$ & $0.062 \pm 0.004$ \\
\hline $\mathrm{C} 18.0 \omega \mathrm{OH}$ & $0.008 \pm 0.001$ & $0.011 \pm 0.001$ & $0.010 \pm 0.002$ & $0.010 \pm 0.001$ \\
\hline $\mathrm{C} 18: 1 \omega \mathrm{OH}$ & $0.105 \pm 0.011$ & $0.113 \pm 0.011$ & $0.095 \pm 0.018$ & $0.113 \pm 0.006$ \\
\hline $\mathrm{C} 18: 2 \omega \mathrm{OH}$ & $0.062 \pm 0.011$ & $0.066 \pm 0.006$ & $0.056 \pm 0.005$ & $0.052 \pm 0.002$ \\
\hline $\mathrm{C} 20.0 \omega \mathrm{OH}$ & $0.025 \pm 0.002$ & $0.033 \pm 0.006$ & $0.034 \pm 0.006$ & $0.044 \pm 0.004$ \\
\hline C2 $1.0 \omega O H$ methylated & $0.050 \pm 0.008$ & $0.058 \pm 0.005$ & $0.063 \pm 0.013$ & $0.064 \pm 0.002$ \\
\hline $\mathrm{C} 22.0 \omega \mathrm{OH}$ & $0.201 \pm 0.028$ & $0.256 \pm 0.025$ & $0.209 \pm 0.056$ & $0.253 \pm 0.005$ \\
\hline $\mathrm{C} 23.0 \omega \mathrm{OH}$ & $0.017 \pm 0.006$ & $0.019 \pm 0.004$ & $0.023 \pm 0.004$ & $0.024 \pm 0.005$ \\
\hline C23.0 $\omega \mathrm{OH}$ methylated & $0.013 \pm 0.004$ & $0.019 \pm 0.003$ & $0.024 \pm 0.006$ & $0.023 \pm 0.002$ \\
\hline $\mathrm{C} 24.0 \omega \mathrm{OH}$ & $0.644 \pm 0.089$ & $0.713 \pm 0.059$ & $0.640 \pm 0.106$ & $0.755 \pm 0.030$ \\
\hline \multicolumn{5}{|c|}{ Mid-Chain Hydroxy Fatty A cids } \\
\hline $\mathrm{Cl} 6.0 \mathrm{DiOH}$ & $0.023 \pm 0.005$ & $0.035 \pm 0.008$ & $0.026 \pm 0.005$ & $0.037 \pm 0.009$ \\
\hline Cl 8:1 triOH & $0.231 \pm 0.032$ & $0.241 \pm 0.040$ & $0.204 \pm 0.034$ & $0.197 \pm 0.025$ \\
\hline \multicolumn{5}{|l|}{ 2-Hydroxy Acids } \\
\hline $\mathrm{Cl} 6.0-2 \mathrm{OH}$ & $0.018 \pm 0.002$ & $0.022 \pm 0.004$ & $0.028 \pm 0.007$ & $0.023 \pm 0.004$ \\
\hline $\mathrm{C} 20.0-2 \mathrm{OH}$ & $0.008 \pm 0.002$ & $0.011 \pm 0.001$ & $0.011 \pm 0.003$ & $0.010 \pm 0.001$ \\
\hline $\mathrm{C} 22.0-2 \mathrm{OH}$ & $0.034 \pm 0.007$ & $0.022 \pm 0.005$ & $0.033 \pm 0.005$ & $0.028 \pm 0.005$ \\
\hline $\mathrm{C} 23.0-2 \mathrm{OH}$ & $0.016 \pm 0.005$ & $0.016 \pm 0.001$ & $0.017 \pm 0.002$ & $0.016 \pm 0.002$ \\
\hline $\mathrm{C} 24.0-2 \mathrm{OH}$ & $0.093 \pm 0.015$ & $0.087 \pm 0.006$ & $0.104 \pm 0.014$ & $0.103 \pm 0.011$ \\
\hline
\end{tabular}




\begin{tabular}{|c|c|c|c|c|}
\hline \multicolumn{5}{|c|}{$\begin{array}{l}\text { Supplem en tal Table } \$ 2.3 \text { continued. Composition of residual bound lipids in seeds of wild-type, } \\
\text { far } 1-1, \text { far4-1, and far } 5-1 \text { plants }\end{array}$} \\
\hline \multicolumn{5}{|c|}{$\begin{array}{l}\text { Each value is the mean shown in } \mu \mathrm{g} / \mathrm{mg} \text { delipidated dry weight of residue } \pm \mathrm{SD} \text { of three } \\
\text { biological replicates. }\end{array}$} \\
\hline & WT & farl-1 & far $4-1$ & far $5-1$ \\
\hline $\mathrm{C} 25: 1-2 \mathrm{OH}$ & $0.014 \pm 0.003$ & $0.008 \pm 0.002$ & $0.022 \pm 0.008$ & $0.013 \pm 0.002$ \\
\hline $\mathrm{C} 25: 0-2 \mathrm{OH}$ & $0.01 \pm 0.03$ & $0.010 \pm 0.001$ & $0.018 \pm 0.003$ & $0.014 \pm 0.002$ \\
\hline $\mathrm{C} 26.0-2 \mathrm{OH}$ & $0.034 \pm 0.011$ & $0.039 \pm 0.011$ & $0.056+0.009$ & $0.050 \pm 0.005$ \\
\hline $\mathrm{C} 26: 1-2 \mathrm{OH}$ & $0.025 \pm 0.005$ & $0.027 \pm 0.009$ & $0.040 \pm 0.006$ & $0.031 \pm 0.006$ \\
\hline \multicolumn{5}{|l|}{ Fatty Alcohols } \\
\hline $\mathrm{Cl} 8.0-\mathrm{OH}$ & $0.045 \pm 0.006$ & $0.047 \pm 0.006$ & $0.032 \pm 0.007$ & $0.004 \pm 0.001$ \\
\hline $\mathrm{Cl} 9.0-\mathrm{OH}$ & $0.016 \pm 0.008$ & $0.011 \pm 0.003$ & $0.008 \pm 0.002$ & $0.004 \pm 0.002$ \\
\hline $\mathrm{C} 20: 0-\mathrm{OH}$ & $0.061 \pm 0.010$ & $0.058 \pm 0.007$ & $0.025 \pm 0.003$ & $0.068 \pm 0.003$ \\
\hline C21:0-OH methylated & $0.033 \pm 0.004$ & $0.012 \pm 0.001$ & $0.024 \pm 0.004$ & $0.028 \pm 0.003$ \\
\hline $\mathrm{C} 22: 0-\mathrm{OH}$ & $0.219 \pm 0.022$ & $0.077 \pm 0.008$ & $0.232 \pm 0.049$ & $0.227 \pm 0.018$ \\
\hline $\mathrm{C} 23.0-\mathrm{OH}$ & $0.018 \pm 0.003$ & $0.013 \pm 0.001$ & $0.023 \pm 0.005$ & $0.021 \pm 0.002$ \\
\hline $\mathrm{C} 20.0 \mathrm{diOL}$ & $0.026 \pm 0.005$ & $0.022 \pm 0.007$ & $0.013 \pm 0.005$ & $0.021 \pm 0.005$ \\
\hline $\mathrm{C} 22.0 \mathrm{diOL}$ & $0.169 \pm 0.026$ & $0.070 \pm 0.004$ & $0.201 \pm 0.044$ & $0.180 \pm 0.014$ \\
\hline Unknowns & $0.208 \pm 0.022$ & $0.271 \pm 0.010$ & $0.266 \pm 0.027$ & $0.230 \pm 0.024$ \\
\hline Total & $4.46 \pm 0.37$ & $4.56 \pm 0.41$ & $4.70 \pm 0.25$ & $4.68 \pm 0.19$ \\
\hline
\end{tabular}




\begin{tabular}{|c|c|c|c|c|c|c|c|c|}
\hline \multicolumn{9}{|c|}{$\begin{array}{l}\text { Supplemental Table S2.4. Composition of nesidual bound lipi } \\
\text { Each value is the mean shown in } \mu g \mathrm{mg} \text { delipidated dry weig } \\
(-) \text { denotes unwounded leaves, }(+) \text { denotes wounded leaves }\end{array}$} \\
\hline & WT (-) & WT $(+)$ & farl-l(-) & farl-l $1+)$ & fart-l (-) & fart-l $(+)$ & fars-l(-) & far $5-1(+)$ \\
\hline \multicolumn{9}{|l|}{ Fatty Acids } \\
\hline $\begin{array}{l}\text { C16:0 } \\
\text { C18:0 }\end{array}$ & $\begin{array}{l}0.843 \pm 0.114 \\
0.100 \pm 0.043\end{array}$ & $\begin{array}{l}1.100 \pm 0.034 \\
0.120 \pm 0.014\end{array}$ & $\begin{array}{l}0.900 \pm 0.190 \\
0.107 \pm 0.041\end{array}$ & $\begin{array}{l}1.248 \pm 0.179 \\
0.136 \pm 0.087\end{array}$ & $\begin{array}{l}0.701 \pm 0.128 \\
0.061 \pm 0.014\end{array}$ & $\begin{array}{l}0.858 \pm 0.210 \\
0.086 \pm 0.025\end{array}$ & $\begin{array}{l}0.847 \pm 0.089 \\
0.091 \pm 0.017\end{array}$ & $\begin{array}{l}0.968 \pm 0.149 \\
0.126 \pm 0.009\end{array}$ \\
\hline $\begin{array}{l}\mathrm{C} 18: 1 \\
\mathrm{C} 18: 2\end{array}$ & $\begin{array}{l}0.043 \pm 0.022 \\
0.176 \pm 0.112\end{array}$ & $\begin{array}{l}0.027 \pm 0.010 \\
0.181 \pm 0.043\end{array}$ & $\begin{array}{l}0.049 \pm 0.023 \\
0.139 \pm 0.046\end{array}$ & $\begin{array}{l}0.032 \pm 0.024 \\
0.212 \pm 0.087\end{array}$ & $\begin{array}{l}0.031 \pm 0.022 \\
0.128 \pm 0.018\end{array}$ & $\begin{array}{l}0.030 \pm 0.024 \\
0.173 \pm 0.083\end{array}$ & $\begin{array}{l}0.028 \pm 0.006 \\
0.117 \pm 0.048\end{array}$ & $\begin{array}{l}0.039 \pm 0.009 \\
0.175 \pm 0.010\end{array}$ \\
\hline C18:3 & $0.301 \pm 0.193$ & $0.281 \pm 0.099$ & $0.283 \pm 0.089$ & $0.349 \pm 0.091$ & $0.225 \pm 0.105$ & $0.243 \pm 0.031$ & $0.231 \pm 0.092$ & $0.303 \pm 0.014$ \\
\hline $\mathrm{C} 20: 0$ & $0.048 \pm 0.005$ & $0.092 \pm 0.022$ & $0.053 \pm 0.012$ & $0.084 \pm 0.021$ & $0.035 \pm 0.005$ & $0.096 \pm 0.023$ & $0.050=0.010$ & $0.140 \pm 0.021$ \\
\hline $\begin{array}{l}\mathrm{C} 22: 0 \\
\mathrm{C} 23: 0\end{array}$ & $\begin{array}{l}0.078 \pm 0.009 \\
0.030 \pm 0.025\end{array}$ & $\begin{array}{l}0.178 \pm 0.034 \\
0.032 \pm 0.015\end{array}$ & $\begin{array}{l}0.109 \pm 0.027 \\
0.030 \pm 0.006\end{array}$ & $\begin{array}{l}0.153 \pm 0.026 \\
0.027 \pm 0.009\end{array}$ & $\begin{array}{l}0.055 \pm 0.011 \\
0.025 \pm 0.011\end{array}$ & $\begin{array}{l}0.182 \pm 0.054 \\
0.026 \pm 0.012\end{array}$ & $\begin{array}{l}0.112 \pm 0.036 \\
0.036 \pm 0.005\end{array}$ & $\begin{array}{l}0.271 \pm 0.030 \\
0.031 \pm 0.005\end{array}$ \\
\hline $\begin{array}{l}\mathrm{C} 24: 0 \\
\mathrm{C} 24: 1\end{array}$ & $\begin{array}{l}0.156 \pm 0.050 \\
0.029 \pm 0.012\end{array}$ & $\begin{array}{l}0.185 \pm 0.026 \\
0.028 \pm 0.011\end{array}$ & $\begin{array}{l}0.251 \pm 0.096 \\
0.038 \pm 0.015\end{array}$ & $\begin{array}{l}0.191 \pm 0.029 \\
0.042 \pm 0.008\end{array}$ & $\begin{array}{l}0.167 \pm 0.052 \\
0.019 \pm 0.006\end{array}$ & $\begin{array}{l}0.181 \pm 0.049 \\
0.023 \pm 0.009\end{array}$ & $\begin{array}{l}0.238 \pm 0.087 \\
0.040 \pm 0.010\end{array}$ & $\begin{array}{l}0.223 \pm 0.059 \\
0.026 \pm 0.010\end{array}$ \\
\hline C25:0 & $0.030 \pm 0.007$ & $0.037 \pm 0.008$ & $0.047 \pm 0.008$ & $0.036 \pm 0.005$ & $0.030 \pm 0.013$ & $0.036 \pm 0.014$ & $0.035 \pm 0.002$ & $0.034 \pm 0.009$ \\
\hline $\mathrm{C} 26: 0$ & $0.046 \pm 0.010$ & $0.050 \pm 0.010$ & $0.089 \pm 0.038$ & $0.065 \pm 0.027$ & $0.046 \pm 0.017$ & $0.053 \pm 0.007$ & $0.081 \pm 0.040$ & $0.065 \pm 0.022$ \\
\hline $\mathrm{C} 28: 0$ & $0.020 \pm 0.005$ & $0.017 \pm 0.004$ & $0.033 \pm 0.018$ & $0.022 \pm 0.010$ & $0.013 \pm 0.004$ & $0.014 \pm 0.003$ & $0.025 \pm 0.012$ & $0.022 \pm 0.014$ \\
\hline \multicolumn{9}{|c|}{ Dicarboxylic Acids } \\
\hline $\begin{array}{l}\mathrm{C} 16: 0 \mathrm{DCA} \\
\mathrm{C} 18: 0 \mathrm{DCA}\end{array}$ & $\begin{array}{l}0.226 \pm 0.025 \\
0.086 \pm 0.031\end{array}$ & $\begin{array}{l}0.211 \pm 0.045 \\
0.111 \pm 0.049\end{array}$ & $\begin{array}{l}0.241 \neq 0.075 \\
0.102+0.047\end{array}$ & $\begin{array}{l}0.213 \pm 0.048 \\
0.114 \pm 0.033\end{array}$ & $\begin{array}{l}0.194 \pm 0.089 \\
0.068 \pm 0.044\end{array}$ & $\begin{array}{l}0.246 \pm 0.072 \\
0.110 \pm 0.035\end{array}$ & $\begin{array}{l}0.261=0.038 \\
0.088=0.022\end{array}$ & $\begin{array}{l}0.295 \pm 0.075 \\
0.139 \pm 0.003\end{array}$ \\
\hline C18:1 DCA & $0.150 \pm 0.103$ & $0.168 \pm 0.074$ & $0.167 \pm 0.051$ & $0.153 \pm 0.031$ & $0.118 \pm 0.082$ & $0.156 \pm 0.077$ & $0.158 \pm 0.034$ & $0.190 \pm 0.048$ \\
\hline C18:2 DCA & $0.696 \pm 0.137$ & $0.690 \pm 0.062$ & $0.715 \pm 0.092$ & $0.665 \pm 0.141$ & $0.647 \pm 0.082$ & $0.710 \pm 0.129$ & $0.686=0.162$ & $0.784 \pm 0.118$ \\
\hline \multicolumn{9}{|c|}{ (1)-Hydro xy Acids } \\
\hline $16: 0 \omega \mathrm{OH}$ & $0.031 \pm 0.011$ & $0.044 \pm 0.006$ & $0.037 \pm 0.004$ & $0.048 \pm 0.014$ & $0.030 \pm 0.006$ & $0.054 \pm 0.009$ & $0.029 \pm 0.002$ & $0.074 \pm 0.018$ \\
\hline $18: 2 \omega \mathrm{OH}$ & $0.071 \pm 0.034$ & $0.097 \pm 0.032$ & $0.096 \pm 0.011$ & $0.105 \pm 0.009$ & $0.051 \pm 0.029$ & $0.088 \pm 0.052$ & $0.080 \pm 0.018$ & $0.133 \pm 0.015$ \\
\hline $20: 0 \omega \mathrm{OH}$ & $0.004 \pm 0.005$ & $0.026 \pm 0.006$ & $0.008+0.003$ & $0.024 \pm 0.008$ & $0.005 \pm 0.002$ & $0.031 \pm 0.010$ & $0.008 \pm 0.002$ & $45 \pm 0.007$ \\
\hline $\begin{array}{l}22: 0 \mathrm{wOH} \\
16: 0 \mathrm{diOH}\end{array}$ & $\begin{array}{l}0.010 \pm 0.006 \\
0.087 \pm 0.016\end{array}$ & $\begin{array}{l}0.041 \pm 0.009 \\
0.069 \pm 0.017\end{array}$ & $\begin{array}{l}0.017 \pm 0.006 \\
0.100 \pm 0.023\end{array}$ & $\begin{array}{l}0.036 \pm 0.008 \\
0.088 \pm 0.020\end{array}$ & $\begin{array}{l}0.009 \pm 0.005 \\
0.095 \pm 0.039\end{array}$ & $\begin{array}{l}0.041 \pm 0.014 \\
0.071 \pm 0.025\end{array}$ & $\begin{array}{l}0.015 \pm 0.001 \\
0.097 \pm 0.007\end{array}$ & $\begin{array}{l}0.064 \pm 0.002 \\
0.079 \pm 0.008\end{array}$ \\
\hline \multicolumn{9}{|c|}{ 2-Hydroxy Acids } \\
\hline $\mathrm{C} 16: 0-2 \mathrm{OH}$ & $0.059 \pm 0.033$ & $0.062 \pm 0.019$ & $0.051 \pm 0.011$ & 0.050 & .004 & 002 & \pm 0.014 & .010 \\
\hline $\mathrm{C} 20: 0-2 \mathrm{OH}$ & $0.035 \pm 0.026$ & $0.038 \pm 0.018$ & 0.0 & 0.019 & 13 & 05 & 13 & 014 \\
\hline $\mathrm{C} 22: 0-2 \mathrm{OH}$ & $0.125+0.047$ & $0.131+0.021$ & $0.145 \pm 0.074$ & $0.104 \pm 0.022$ & $0.080+0.022$ & $0.093 \pm 0.009$ & $0.111 \pm 0.042$ & $0.094 \pm 0.053$ \\
\hline $\mathrm{C} 23: 0-2 \mathrm{OH}$ & $0.033 \pm 0.019$ & $0.034 \pm 0.013$ & $0.034 \pm 0.004$ & $0.027 \pm 0.003$ & $0.020 \pm 0.005$ & $0.022 \pm 0.007$ & $0.023 \pm 0.006$ & $0.021 \pm 0.009$ \\
\hline $\begin{array}{l}\mathrm{C} 23: 1-2 \mathrm{OH} \\
\mathrm{C} 24: 0-2 \mathrm{OH}\end{array}$ & $\begin{array}{l}0.029 \pm 0.003 \\
0.467 \pm 0.087\end{array}$ & $\begin{array}{l}0.028 \pm 0.007 \\
0.483 \pm 0.113\end{array}$ & $\begin{array}{l}0.026 \pm 0.006 \\
0.480 \pm 0.148\end{array}$ & $\begin{array}{l}0.026 \pm 0.014 \\
0.446 \pm 0.068\end{array}$ & $\begin{array}{l}0.017 \pm 0.008 \\
0.398 \pm 0.123\end{array}$ & $\begin{array}{l}0.017 \pm 0.006 \\
0.389 \pm 0.027\end{array}$ & $\begin{array}{l}0.026 \pm 0.010 \\
0.444 \pm 0.141\end{array}$ & $\begin{array}{l}0.024 \pm 0.027 \\
0.411 \pm 0.182\end{array}$ \\
\hline $\mathrm{C} 24: 1-2 \mathrm{OH}$ & $0.160 \pm 0.068$ & $0.144 \pm 0.023$ & $0.212 \pm 0.100$ & $0.195 \pm 0.037$ & $0.171 \pm 0.073$ & $0.153 \pm 0.044$ & $0.212 \pm 0.055$ & $0.146 \pm 0.063$ \\
\hline $\mathrm{C} 25: 0.2 \mathrm{OH}$ & $0.039 \pm 0.026$ & $0.043 \pm 0.020$ & $0.043 \pm 0.008$ & $0.040 \pm 0.006$ & & & $0.030 \pm 0.006$ & $0.032+0.014$ \\
\hline C25:1-2OH & $0.025 \pm 0.014$ & $0.025 \pm 0.003$ & $0.022 \pm 0.005$ & $0.027 \pm 0.013$ & $0.017 \pm 0.015$ & $0.014 \pm 0.014$ & $0.020 \pm 0.003$ & $0.014 \pm 0.024$ \\
\hline $\mathrm{C} 26: 0-2 \mathrm{OH}$ & $0.108 \pm 0.069$ & $0.112 \pm 0.037$ & $0.150 \pm 0.047$ & $0.115 \pm 0.037$ & $0.085 \pm 0.034$ & $0.084 \pm 0.010$ & $0.105 \pm 0.045$ & $0.103 \pm 0.055$ \\
\hline $\mathrm{C} 26: 1-2 \mathrm{OH}$ & $0.024 \pm 0.010$ & $0.021 \pm 0.006$ & $0.027 \pm 0.010$ & $0.023 \pm 0.002$ & $0.019=0.018$ & $0.019 \pm 0.010$ & $0.024 \pm 0.003$ & $0.019 \pm 0.015$ \\
\hline \multicolumn{9}{|l|}{ Fatty Alcohols } \\
\hline $18: 0-\mathrm{OH}$ & $0.024 \pm 0.006$ & $0.041+0.003$ & $0.025+0.012$ & $0.048 \pm 0.022$ & $0.020 \pm 0.003$ & $0.069 \pm 0.029$ & $0.013 \pm 0.004$ & $0.013+0.004$ \\
\hline $20: 0-\mathrm{OH}$ & $0.054 \pm 0.015$ & $0.087 \pm 0.029$ & $0.054 \pm 0.024$ & $0.076 \pm 0.025$ & $0.036 \pm 0.013$ & $0.050 \pm 0.030$ & $0.056 \pm 0.017$ & $0.078 \pm 0.020$ \\
\hline $22: 0-\mathrm{OH}$ & $0.034 \pm 0.007$ & $0.055 \pm 0.007$ & $0.029 \pm 0.007$ & $0.028 \pm 0.013$ & $0.028 \pm 0.015$ & $0.050 \pm 0.028$ & $0.041 \pm 0.006$ & $0.082 \pm 0.026$ \\
\hline Unknowns & $0.182 \pm 0.050$ & $0.172+0.034$ & $0.258 \pm 0.077$ & $0.235 \pm 0.058$ & $0.166 \pm 0.074$ & $0.185 \pm 0.159$ & $0.188 \pm 0.010$ & $0.274 \pm 0.025$ \\
\hline Total & $4.88=1.66$ & $5.28 \pm 0.96$ & $5.34 \pm 1.01$ & $5.58 \pm 1.02$ & $4.02 \pm 1.35$ & $4.78 \pm 1.62$ & $4.80 \pm 0.87$ & $5.49 \pm 0.25$ \\
\hline
\end{tabular}


Table S2.5. Sequences of DNA primers used for PCR

\begin{tabular}{|c|c|}
\hline FAR1-qPCR-F & ACAGCTCATTCGGGAGACAC \\
\hline FAR1-qPCR-R & GTAACGAGCCGTGAAATCGT \\
\hline FAR2-qPCR-F & TGGATTTGATGTTGGAAGCA \\
\hline FAR2-qPCR-R & GGGATCGATTCTGTTTGGTC \\
\hline FAR3-qPCR-F & ACCGTGGACCAACAAAGAAG \\
\hline FAR3-qPCR-R & GCAATCAAGTAGCGTATGGTCA \\
\hline FAR4-qPCR-F & TGCGGTTGAAAAGAAAGGAG \\
\hline FAR4-qPCR-R & CGGGAATATGAATGGTCGTC \\
\hline FAR5-qPCR-F & GAGTTGGTGATGAGATTGGTAGAG \\
\hline FAR5-qPCR-R & CTTCTTAAGCACGTGTGTGACG \\
\hline FAR6-qPCR-F & TGTGGTGTCCCAGAGTTCAA \\
\hline FAR6-qPCR-R & TCCAATGGAAAGTCACACAGA \\
\hline FAR7-qPCR-F & CAGTTTACCATGTCGGCTCA \\
\hline FAR7-qPCR-R & CCATTACGTCCGACAAGAGG \\
\hline FAR8-qPCR-F & GCCAAGCAGAAAGAAGAGGA \\
\hline FAR8-qPCR-R & AGTGATGAGGCCAGGAATGT \\
\hline \multicolumn{2}{|c|}{ W. } \\
\hline FAR1-Pro-F & AGAGGTCGACGAAGCATCATTGGAGATGGTC \\
\hline FAR1-Pro-R & AGAGGATCCTTGAACACAATTGGATTCCATTG \\
\hline FAR4-Pro-F & AGAGTCGACCCTTCTTGAAGGTCAAGCCT \\
\hline FAR4-Pro-R & AGAGGATCCCTGAATGCAATTGGAGTCCAT \\
\hline FAR5-Pro-F & AGAGTCGACTGAAGTTGATCAGGTTGACAAT \\
\hline FAR5-Pro-R & AGAGCATCCTTCAACACAATTGAGTTCCATTG \\
\hline \multicolumn{2}{|c|}{ H. } \\
\hline FAR1-RT-F & CTACGTAGTGAGGTTATGGAGATCG \\
\hline FAR1-RT-R & CGCTTATATACGCGGTTGAGACATG \\
\hline FAR4-RT-F & CTTGGAATGTCAAGGGCAAAGCTTC \\
\hline FAR4-RT-R & TGTCCACAGGTATAAGATCAAGGAC \\
\hline FAR5-RT-F & CGCACAGAGGTATTTGAGAAAGAAC \\
\hline
\end{tabular}




\begin{tabular}{|c|c|}
\hline FAR5-RT-R & CAAGCCCAACATCATATCTTTCGTC \\
\hline GAPC-RT-F & TCAGACTCGAGAAAGCTGCTAC \\
\hline GAPC-RT-R & GATCAAGTCGACCACACGG \\
\hline RD29A-RT-F & GGAAGAGTCGGCTGTTTCAG \\
\hline RD29A-RT-R & GTGCTCTGTTTTGGCTCCTC \\
\hline FAR1-ORF-F & $\begin{array}{l}\text { AAAAAGCAGGCTACATAATGGAATCCAATTGTGTTCAATTTCTCG } \\
\text { G }\end{array}$ \\
\hline FAR1-ORF-R & $\begin{array}{l}\text { AGAAAGCTGGGTATTATTGTTTAAGCACATGGGTGATGAGGCCAG } \\
\text { GAATGTGGG }\end{array}$ \\
\hline FAR3-ORF-F & AAAAAGCAGGCTACATAATGTCGACAGAAATGGAGGTCG \\
\hline FAR3-0RF-R & AGAAAGCTGGGTACTCGAGTTAGAAGACATACTTAAGCAGCCC \\
\hline FAR4-ORF-F & AAAAAGCAGGCTACATAATGGACTCCAATTGCATTCAGTTCCTCC \\
\hline FAR4-ORF-R & AGAAAGCTGGGTATTATTTTTTGAGTACATAGGTGATGAGGCCGG \\
\hline FAR5-ORF-F & $\begin{array}{l}\text { AAAAAGCAGGCTACATAATGGAACTCAATTGTGTTCAATTTCTTC } \\
\text { GAAACAAGACG }\end{array}$ \\
\hline FAR5-ORF-R & AGAAAGCTGGGCTATCACTTCTTAAGCACGTGTGTGACGAGTCC \\
\hline SALK_068605-TDNA-LP & 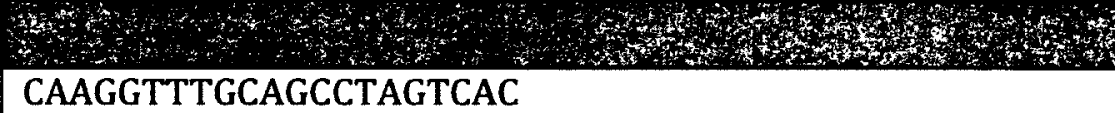 \\
\hline SALK_068605-TDNA-RP & TGTTACAACACTGTTGCGGTG \\
\hline SALK_149469-TDNA-LP & TGTTGCAATAAATGAAATGAACAG \\
\hline SALK_149469-TDNA-RP & TACCTTGCACGACTATGTCCC \\
\hline SALK_000229-TDNA-LP & TGTATTCATCAAACCAATTGATCC \\
\hline SALK_000229-TDNA-RP & TTGCGATGGTGAACTACTTCC \\
\hline SALK_147493-TDNA-LP & GTTGACCCGCTTCTTCTTCTC \\
\hline SALK_147493-TDNA-RP & AAACCAAATGTTGAAAGAGAAAAC \\
\hline SALK_152963-TDNA-LP & TTCTTGCAACGTCCTTAGCTG \\
\hline SALK_152963-TDNA-RP & AAAGGTGGTATATAAAATTTCTTGTAGC \\
\hline SALK_070363-TDNA-LP & AAAGAACTCGGAATGGAAAGG \\
\hline SALK_070363-TDNA-RP & GGGCAAATTAGCTTAAGTACGC \\
\hline LBb1 & GCGTGGACCGCTTGCTGCAAC \\
\hline
\end{tabular}




\section{CHAPTER 3: Biosynthesis and functions of free and combined fatty alcohols associated with suberin}

\subsection{Introduction}

Suberin is an important extracellular lipid polyester barrier that plays a critical role in survival of plants by protecting them against various biotic and abiotic stresses. Suberin is deposited between at the inner face of primary cell walls next to the plasma membrane (Kolattukudy, 1980; Franke and Schreiber, 2007). Suberin is typically found as lamellae, alternating dark and light bands, in root endodermis, mature exodermis and peridermis of secondary growth roots, and in the peridermis of underground storage tubers (Nawrath, 2002). Suberin is also found in seed coats (Espelie et al., 1980a; Molina et al., 2006, 2008), and deposited in response to wounding stress by injured plant cells (Kolattukudy, 2001a; Chapter 2 of this thesis). It acts as a hydrophobic barrier to control the movement of water, gases and solutes, and also contributes to the strength of the cell wall (Ranathunge et al., 2011). Suberin is a polymer consisting of aliphatic and phenolic suberin monomers as well as glycerol (Bernards, 2002). The predominant aliphatic components are $\omega$-hydroxyacids, $\alpha, \omega$-dicarboxylic acids, fatty acids, and primary fatty alcohols with chain lengths ranging from $\mathrm{C} 16$ to $\mathrm{C} 28$. The predominant aromatic components are $p$-hydroxycinnamic acids, especially ferulic acid (Bernards et al., 1995; Pollard et al., 2008; Ranathunge et al., 2011).

The suberin polymer is insoluble in organic solvents without depolymerization, but it has been found in association with solvent-extractable waxes. Waxes are complex mixtures of lipids extractable by brief immersion of 
tissues in chloroform. In potato periderm, suberin-associated waxes consist mostly of linear very-long-chain aliphatics up to C32 (Schreiber et al., 2005). Arabidopsis root waxes, also proposed to be associated with the suberin polymer, consist of fatty acids (C16-C22), alkanes (C29:0 and C31:0) and their mid-chain oxidized (keto or hydroxy) derivatives, monoacylglycerols (C20 and C22 acyl chains), fatty alcohols (C18:0-C22:0), and alkyl hydroxycinnamates (C18:0-C22:0 fatty alcohols linked with hydroxycinnamates, mainly coumarate and caffeate) (Li et al., 2007; Kosma et al., 2012). The alkyl hydroxycinnamates are the major components of Arabidopsis root wax, present primarily in 6-7-week old mature taproots with a fully developed periderm (Kosma et al., 2012). It has been suggested that the light bands in the suberin lamellar structure represent a wax layer (Soliday et al., 1979), but how the suberin and wax components are organized in the lamellated suberin secondary cell wall is a matter of debate (Graça and Santos, 2007). Aliphatic suberin and suberinassociated waxes are considered the major contributors to the diffusion resistance of the suberized cell walls to radial water and solute transport (Soliday et al., 1979; Espelie et al., 1980b; Zimmermann et al., 2000; Hose et al., 2001; Schreiber et al., 2005; Ranathunge and Schreiber, 2011).

The relative abundance of aliphatic suberin monomer composition varies considerably between plant species and developmental stages (Kolattukudy and Dean 1974; Thomas et al., 2007; Pollard et al., 2008; Krishnamurthy et al., 2009). The composition of suberin in different tissues from a variety of plant species has been well documented (Holloway 1983; Matzke and Riederer 1991; Zeier and Schreiber 1998, 1999; Zeier et al., 1999; Graca and Santos, 2007). Fatty alcohols 
(alkan-1-ols and $\alpha, \omega$-diols) constitute about 1-8 $\%$ of the total suberin monomer mass recovered after transesterification (Pollard et al., 2008). Studies on suberin of the wound-induced periderm in $S$. tuberosum tuber as well as the exodermis of onion and Iris germanica revealed the presence of insoluble (polymerized) and soluble (unpolymerized) aliphatic monomers (Yang and Bernards, 2006; Meyer et al., 2011a; 2011b).

In the previous chapter, we reported that Arabidopsis FAR1, FAR4, and FAR5 are responsible for synthesis of C18:0-C22:0 primary fatty alcohols found in the suberin polymer. It was subsequently shown using far single mutants that FAR5, FAR4, and FAR1 generate the C18:0, C20:0, and C22:0 fatty alcohol components, respectively, that make up alkyl hydroxycinnamates in root waxes (Kosma et al., 2012). The far single mutant lines, reduced in one particular chain length of primary alcohol, did not show a significant reduction in the total fatty alcohol load of the root suberin polyester and the mutant plants had no obvious developmental or physiological defects (Chapter 2 of this thesis). In these far single mutants, the total amounts of free and combined fatty alcohols are not drastically reduced, which may account for the lack of obvious physiological defects.

In this chapter, we report the distribution of primary fatty alcohols and the other suberin monomer classes in insoluble (suberin polymer) and soluble (nonpolymerized) fractions from the mature roots of Arabidopsis thaliana. We report generation of far double and far triple mutants and their characterization with respect to the composition of suberin polymer, soluble fraction, and suberinassociated root waxes. We also report that a significant reduction in total fatty 
alcohols load in seed coat suberin leads to an increased permeability of seed coat and also an increased sensitivity to abscisic acid (ABA).

\subsection{Materials and Methods}

\subsubsection{Plant materials and growth conditions}

Arabidopsis thaliana plants were in the Columbia (Col-0) ecotype background. The following T-DNA insertion lines were obtained from the Arabidopsis Biological Resource Center (www.arabidopsis.org): SALK_149469 (far1-2), SALK_000229 (far4-1) and SALK_152963 (far5-1) (lines described in Chapter 2). The seeds were surface sterilized by treating with $100 \%$ ethanol for 1 minute; followed by 1 minute incubation in $50 \%$ bleach (contained approximately $6 \%$ sodium hypochlorite and $0.5 \%$ SDS). Seeds were mixed well by tapping the microcentrifuge tubes and after discarding the $50 \%$ bleach solution; the seeds were rinsed several times with sterile distilled water. For experimental analyses, surface sterilized seeds were sown on Murashige and Skoog (MS) medium (Murashige and Skoog, 1962) (4.5g/L MS salts + $0.5 \mathrm{~g} / \mathrm{L}$ MES (2-[N-morpholino]ethanesulfonic acid), Sigma) with the pH adjusted to 5.7 and supplemented with $0.7 \%$ agar. Plates with the seeds were sealed with micropore tape and seeds were stratified in the dark for 3 to 4 days at $4^{\circ} \mathrm{C}$ and then transferred to long-day conditions (16-h-light/8-h-dark cycle) at $22^{\circ} \mathrm{C}$ or continuous light $\left(90 \mu \mathrm{mol} \mathrm{m}^{-2} \mathrm{~s}^{-1}\right)$ conditions at $20^{\circ} \mathrm{C}$. Approximately 2 weeks later, seedlings were transferred to a soil-vermiculite mixture (Pro-Mix MPV Multipurpose Growing Medium), fertilized with $1 \mathrm{~g} / \mathrm{L}$ 20-20- 
20 fertilizer (Plant-Prod, Brampton, ON), and further grown at $20^{\circ} \mathrm{C}$ to $22^{\circ} \mathrm{C}$ and $60 \%$ relative humidity, under long-day conditions in a controlled growth chamber (Conviron model TC26 or Percival chamber model AR66L).

\subsubsection{Generation of far 1 far 4 and far1 far 5 double mutants}

In the Arabidopsis genome, FAR4 and FAR5 are on chromosome 3 while FAR1 is on chromosome 5 . The far 1-2 far4-1 and far1-2 far5-1 double mutants were made using conventional genetic crossing. Homozygous insertion lines for both the genes were identified among the segregating plants in the F2 generation by PCR screening for the presence of a T-DNA insertion at both loci. Genotyping PCR was carried out using genomic DNA isolated from the leaves of segregating plants, as described in Chapter 2, Section 2.3.9.

\subsubsection{Generation of artificial microRNA (amiRNA) lines.}

Artificial microRNA silencing (Schwab et al., 2006; Ossowski et al., 2008) was used to generate far 4 far 5 double and far 1 far 4 far 5 triple mutant lines. For the artificial microRNAs, the key 21 nucleotides of the naturally occurring Arabidopsis miR319a were replaced with sequences specific for FAR4 or FAR5. Two amiRNAs each for FAR4 and FAR5 that hybridized to different parts of the respective transcripts were designed using WMD2-Web MicroRNA Designer (Schwab et al., 2010; http://wmd3.weigelworld.org/cgi-bin/webapp.cgi). The amiRNAs for FAR4 and FAR5 were selected based on established rules for miRNA targeting, which includes: no mismatches between the amiRNA and target in the seed region of the amiRNA (between positions 2 and 12), one or two mismatches at the amiRNA 3'-end 
(positions 18-21), nucleotide ' $\mathrm{A}$ ' at the cleavage site (position +10 ), and an absolute hybridization energy between -35 and $38 \mathrm{kcal} / \mathrm{mole}$. The WMD2 oligo tool provided the following amiRNA specific primers: FAR4-amiX-I, FAR4-amiX-II, FAR4-amiX-III, and FAR4-amiX-IV for FAR4-amiRNAX, and FAR5-amiX-I, FAR5-amiX-II, FAR5-amiXIII, and FAR5-amiX-IV for FAR-amiRNAX (X represents amiRNA sequence 1 or 2 selected for each gene) (for primer sequences, see Appendix Table 2). In addition to these amiRNA specific primers, the template plasmid specific primers, pRS300-A and pRS300-B, were also obtained. The plasmid pRS300 (obtained from www.weigelworld.org) contains the endogenous Arabidopsis miR319a microRNA precursor in a pBluescript SK backbone, and this was used as template to amplify three separate fragments of the amiRNA: the $5^{\prime}$ arm fragment, the central loop fragment, and the 3' arm fragment, of sizes $272 \mathrm{bp}, 171 \mathrm{bp}$ and $298 \mathrm{bp}$, respectively (Figure 3.1a). The primer combinations used for amplifying each fragment is outlined in Table 3.1 .

PCRs a, b, and c (Figure 3.1b, Table 3.1) were carried out using iProof High Fidelity Polymerase (BioRad) at an annealing temperature of $52^{\circ} \mathrm{C}$ and extension at $72^{\circ} \mathrm{C}$ for 40 seconds, and for 40 cycles. Each of these three initial PCR products was gel-purified from a $1 \%$ agarose gel using the Promega Wizard® SV Gel and PCR Clean Up system according to the manufacturer's instructions. The purified amiRNA fragments were subsequently fused in an overlap PCR reaction (Figure 3.1b, Table 3.1, Reaction d) using the plasmid specific primers, pRS300-A and pRS300-B, to generate the final full length amiRNA precursor fragment. The PCR reaction was carried out using iProof High Fidelity Polymerase (BioRad) at an annealing 
temperature of $55^{\circ} \mathrm{C}$, extension at $72^{\circ} \mathrm{C}$ for 90 seconds, and for 40 cycles. The final amiRNA precursor PCR products for FAR4 and FAR5 were run on a $1 \%$ agarose gel at 85 volts and fragments excised from the gel and purified as described before using the Promega Wizard@ SV Gel and PCR Clean Up system.

The purified 701bp full length precursor amiRNAs for FAR4 and FAR5 and the pBluescript II SK+ plasmid were digested with restriction enzymes EcoRI and $X b a \mathrm{l}$ in a $37^{\circ} \mathrm{C}$ water bath for 2 hours, and gel purified using Promega Wizard® SV Gel and PCR Clean Up system. The purified digested products were ligated together between the corresponding sites in the pBluescript vector using a 3:1 insert-vector ratio to generate pBluescript-FAR4_amiRNA $(1$ and 2$)$ and pBluescriptFAR5_amiRNA (1 and 2). The ligation reactions were carried out at $16^{\circ} \mathrm{C}$ using $\mathrm{T} 4$ DNA ligase (Invitrogen) for 4 hours. The ligation mixtures were transformed into $E$. coli DH5 $\alpha$ ultracompetent cells. Transformed cells were screened on LB+Ampicilin $(100 \mu \mathrm{g} / \mathrm{ml})$ plates and the clones containing FAR4/5_amiRNA inserts were selected by colony PCR using universal M13 forward and reverse primers and also confirmed by diagnostic restriction test digestions. The inserts were sequenced to make sure there were no PCR-induced mutations.

\section{Construction of plant binary vectors pBAR1:35S:FAR(4/5)-amiRNA:}

Construction of the plant binary vectors pBAR1:35S:FAR(4/5)-amiRNA were done by step wise insertion of individual FAR(4/5)-amiRNA fragments first into the pBAR1 binary vector, followed by insertion of a $35 \mathrm{~S}$ promoter into each plasmid. The FAR4/5-amiRNA fragments were excised out of pBluescript:FAR(4/5)-amiRNA plasmids using $E c o R I$ and $X b a \mathrm{I}$ restriction enzymes. The digested products were gel 
purified using the Promega Wizard® SV Gel and PCR Clean Up system, followed by ligation into the corresponding sites of pBAR1 to create the constructs pBAR:FAR4amiRNA (1 and 2) and pBAR:FAR5-amiRNA (1 and 2). The ligations were then transformed into $E$. coli DH5 $\alpha$ ultracompetent cells. Positive clones were selected by colony PCR using the primers amiFAR (4 or 5) forward-III and NOS terminator reverse (Supplementary Table S3.4).

The cauliflower mosaic virus (CaMV) 35S promoter was amplified using iProof High Fidelity polymerase from the pCAMBIA-1301 vector (Hajdukiewicz et al., 1994) with the pCambia35S-Prom-F and pCambia35S-Prom-R primers (See Supplementary Table-S3.4] creating a PCR product with HindIII at the upstream end and EcoRI at the downstream end of the $35 \mathrm{~S}$ promoter. The gel purified $35 \mathrm{~S}$ promoter insert and the pBAR:FAR(4/5)-amiRNA plasmids were digested with HindIII and EcoRI restriction enzymes. These were then ligated and transformed into ultracompetent $E$. coli cells. The screening was carried out on LB+kanamycin $(50 \mu \mathrm{g} / \mathrm{ml})$ plates and the positive clones were selected based on colony PCR followed by diagnostic test digests.

\section{Generation of amiRNA transgenic plants:}

Following verification of the DNA sequence, the vectors were transformed into Agrobacterium tumefaciens GV3101:pMP90, a strain based on C58 and pTiC58 genotypes (Koncz and Schell, 1986), by electroporation using a MicroPulser Electroporator (BioRad, Mississauga ON). Wild-type Arabidopsis plants were transformed using the $A$. tumefaciens strain by the floral dip method (Clough and Bent, 1998; Zhang et al., 2006). Successful Arabidopsis transformants containing the 
T-DNA harbouring 35S-FAR(4/5)-amiRNA were selected on minimal media containing $10 \mu \mathrm{g} / \mathrm{ml}$ 'BASTA' (DL-Phosphinothrycin, Duchefa Biochemie/Gold biotechnology, USA). Alternatively, the T1 seedlings were sown on soil in a flat and screened by spraying BASTA herbicide (Farnam Companies, Inc, Phoenix AZ) on seedlings 4,7 , and 10 days after sowing. The surviving positive transformants were transplanted to single pots and were assessed for silencing of FAR4 or FAR5 transcripts by RT-PCR as described below.

\subsubsection{RNA extraction and cDNA synthesis}

RNA extraction was carried out using the RNAqueous ${ }^{\circledR}$ Kit (Applied Biosystems-Ambion AM1912). Roots ( $50-60 \mathrm{mg}$ ) from 15-day-old seedlings grown vertically on MS medium (Sigma) were collected and ground to a fine powder in liquid nitrogen using a mortar and pestle. The ground tissues were transferred to sterile microcentrifuge tubes using a pre-chilled metal spatula. $700 \mu \mathrm{l}$ of lysis/binding solution (supplied in the kit) was added, and then mixed rapidly. To this homogenised lysate, an equal volume of $64 \%$ ethanol $(700 \mu \mathrm{l})$ was added and mixed gently by inverting the tube several times. This lysate/ethanol mixture was then drawn through a filter cartridge (supplied in the kit) by centrifuging at 12,000 $\mathrm{x} \mathrm{g}$ for 1 minute. The flow-through was discarded and the filter cartridge was washed twice with $700 \mu \mathrm{l}$ of wash solution \#1 and then with $500 \mu \mathrm{l}$ of wash solution $\# 2 / 3$ (supplied in the kit) by centrifuging at $12,000 \mathrm{x}$ for 30 seconds. The filter cartridge was placed in a fresh collection tube and $50 \mu \mathrm{l}$ of pre-heated elution solution was added to the centre of the cartridge filter using a pipette. The eluate was collected by centrifuging at $12,000 \mathrm{x}$ g for 30 seconds at room temperature. The 
collected eluate was subjected to DNase treatment using the TURBO DNA-free ${ }^{\mathrm{TM}}$ kit (Applied Biosystems-Ambion AM1907). To the eluate containing total RNA, 0.1 volumes 10X TURBO DNase buffer and $1 \mu \mathrm{l}$ TURBO DNase was added, mixed gently and incubated at $37^{\circ} \mathrm{C}$ for $20-30$ minutes. A 0.1 volume of DNase inactivation reagent (supplied in kit) was added, mixed well, and incubated at room temperature for 5 minutes. The tube was centrifuged at $10,000 \times \mathrm{g}$ for 2 minutes and the supernatant (RNA) was transferred to a fresh sterile tube. RNA yield and quality was assessed using a NanoDrop spectrophotometer (NanoDrop Technologies, Inc., USA) and also by running an aliquot on a $1 \%$ agarose gel containing $37 \%$ formaldehyde/MOPS buffer.

First strand cDNA synthesis

For each sample, $1 \mu \mathrm{g}(8.5 \mu)$ of total RNA, diluted using diethyl pyrocarbonate (DEPC)-treated water, was heated at $70^{\circ} \mathrm{C}$ for 5 minutes and snap chilled on ice. For a final volume of $20 \mu l$, the RNA samples $(8.5 \mu l)$ were then added to $11.5 \mu \mathrm{l}$ of the reverse transcriptase reaction mixture on ice to give final concentrations of $2 \mu \mathrm{M}$ oligo-dT primer, $0.5 \mathrm{mM}$ dNTPs, 5U RNaseOUT ${ }^{\mathrm{TM}}$ (Invitrogen), $10 \mathrm{mM}$ DTT, $50 \mathrm{mM}$ Tris- $\mathrm{HCl}(\mathrm{pH}$ 8.3), $75 \mathrm{mM} \mathrm{KCl}$, and $3 \mathrm{mM} \mathrm{MgCl} 2$. Fifty units of SuperScript ${ }^{\circledR}$ III Reverse Transcriptase (Invitrogen) were added to each reaction and held at $50^{\circ} \mathrm{C}$ for 60 minutes, $72^{\circ} \mathrm{C}$ for 10 minutes, cooled down to $4^{\circ} \mathrm{C}$ and stored at $-20^{\circ} \mathrm{C}$.

\subsubsection{Transcript analysis by quantitative RT-PCR.}

Quantitative PCR was performed on the StepOnePlus ${ }^{\circledR}$ real time PCR system (Applied Biosystem) using Power SYBR Green qPCR mastermix (Applied 
Biosystems). The cDNA from the reverse transcriptase reaction was diluted ten times with nuclease free water. Each qPCR reaction contained: $5 \mu \mathrm{l}$ of $5 \mathrm{X}$ Power SYBR mix, $2 \mu \mathrm{l}$ of primer mix ( $5 \mu \mathrm{M}$ each of forward and reverse primer), $2 \mu$ of the diluted cDNA and $1 \mu \mathrm{l}$ of sterile water (for primer sequences, see Supplementary Table- S3.4). Transcripts were quantified as relative abundance using the comparative Ct method [delta-delta Ct: $2^{-\Delta \Delta \mathrm{Ct}}$ (Pfaffl, 2001; 2006)]. The geometric mean of the relative transcript abundance of the endogenous controls ACTIN2 (At1g49240), eIF4a1 (At3g13920), and GAPC (At3G04120) in each sample was used to normalize for differences of total RNA amounts between the samples. Two biological replications were carried out for each sample, with each reaction having three technical replicates.

\subsubsection{Lipid analysis}

\subsubsection{Root delipidation}

Roots were collected from 4-week-old plants grown under long-day conditions (16-h-light/8-h-dark cycle) at $22^{\circ} \mathrm{C}$ and on plates containing MS medium (4.5g/L MS salts, $0.5 \mathrm{~g} / \mathrm{L}$ MES, pH set to $5.7,7.5 \%$ Agar). Each mutant line was analysed in three replicates, with each replicate representing a pool of approximately 20 plants. Roots were dried on blotting paper (kimwipes) before the delipidation process. Freshly collected roots were then immersed in $4 \mathrm{ml}$ hot isopropanol and incubated for 30 minutes at $85^{\circ} \mathrm{C}$ in an oven incubator. After cooling, samples were extensively delipidated by extracting the soluble lipids successively for 24 hours each with $4 \mathrm{ml}$ chloroform:methanol $(2: 1, \mathrm{v} / \mathrm{v}), 4 \mathrm{ml}$ 
chloroform:methanol $(1: 1, \mathrm{v} / \mathrm{v}), 4 \mathrm{ml}$ chloroform:methanol $(1: 2, \mathrm{v} / \mathrm{v})$, and $4 \mathrm{ml}$ $100 \%$ methanol, all performed at room temperature on a tube rotator with the wheel rotating at $60 \mathrm{rpm}$. All the organic solvents used were spectro grade from Caledon, USA. Samples were dried in a fume hood at room temperature for 2-3 days and then in a desiccator for another 2 days before using for suberin analysis.

For the soluble fractions of the samples, each delipidation step (isopropanol and the chloroform:methanol fractions) was collected in the same $9 \mathrm{ml}$ glass tube by evaporating the newly collected solvent under a gentle stream of nitrogen gas each day. The resulting pooled fraction containing all soluble lipids was stored at $4^{\circ} \mathrm{C}$ until further analysis as described below.

\subsubsection{Root suberin polyester and soluble fraction analysis}

Acidic transmethylation with methanolic sulphuric acid

The solvent extracted dried residues of roots were weighed and 10 to $30 \mathrm{mg}$

of each sample was depolymerized by transmethylation at $85^{\circ} \mathrm{C}$ for $3 \mathrm{~h}$ using $2 \mathrm{ml}$ of $5 \%$ sulfuric acid in methanol. The soluble fractions were processed in the same way. $10 \mu \mathrm{g}$ each of heptadecanoic acid (C17:0), pentadecanol (C15:0-OH), C15hydroxypentadecanoic acid ( $\omega-\mathrm{OH}-\mathrm{C} 15: 0)$ and 2-hydroxy tetradecanoic acid (2OHC14:0) were added as internal standards (all internal standards were from SigmaAldrich, USA). After cooling, $2 \mathrm{ml}$ of $\mathrm{NaCl}(2.5 \%, \mathrm{w} / \mathrm{v})$ and $2.5 \mathrm{ml}$ of dichloromethane (DCM) was added and mixed vigorously for 5 minutes followed by centrifugation at $800 \mathrm{x} \mathrm{g}$ for 5 minutes at room temperature to allow phase separation. The lower DCM phase was transferred to a fresh glass tube. An additional $2.5 \mathrm{ml}$ of DCM was added to the original mixture for a second extraction. The pooled DCM extracts were 
then washed with $2 \mathrm{~mL}$ of Tris- $\mathrm{NaCl}$ solution $(100 \mathrm{mM}$ Tris base $\mathrm{pH} 8.0$ in $0.09 \%$ $\mathrm{NaCl}$ ) and the lower DCM phase was collected in a fresh GC tube, avoiding any aqueous phase. The lower organic phase was evaporated under a gentle stream of nitrogen, and taken forward for derivatization.

Preparation of trimethylsilyl derivatives

The free hydroxyl groups were trimethylsilylated (TMS) by incubating the transmethylated samples with $100 \mu \mathrm{L}$ of $99 \%$ BSTFA ( $N, O$-bis(trimethylsilyl)trifluoroacetamide) $+1 \%$ TMS (Supelco/Sigma-Aldrich, USA), at $110^{\circ} \mathrm{C}$ for $15 \mathrm{~min}$. After cooling, the solvent was evaporated under nitrogen and the product was dissolved in $100 \mu \mathrm{L}$ heptane:toluene $(1: 1 \mathrm{v} / \mathrm{v})$ for GC-FID and GC-MS analysis under the conditions described below in Section 3.3.6.5.

\subsubsection{Root waxes analysis}

For root wax extractions, seven-week-old Arabidopsis plants were grown in soil-less potting medium [mixture of Promix PGX soil-less medium (Premier Horticulture), vermiculite, and perlite $(1: 1: 1, v / v)]$ in a growth chamber at $21^{\circ} \mathrm{C}$ to $22^{\circ} \mathrm{C}, 40 \%$ to $60 \%$ humidity, a $16 / 8$-hour light/dark cycle, and a fluorescent light intensity of 80 to $100 \mathrm{mmol} \mathrm{m}^{-2} \mathrm{~s}^{-1}$. Each replicate consisted of roots from 12-20 plants. Arabidopsis root waxes were extracted by rapid dipping of roots in chloroform for 1.5 minutes to maximize alkyl hydroxycinnamate extraction. After extraction, internal standards were added to chloroform extracts, which were pentadecanoic acid (C15:0), tricosan-1-ol (C23:0-OH), octacosane (C28:0), tridecyl (C13:0)-ferulate and heptadecyl (C17:0)-coumarate, for quantification of the respective chemical compounds. The chloroform extracts were filtered through 
glass wool and then evaporated under a gentle stream of nitrogen gas to dryness. The dried extracts were then derivatized (silylated) with $100 \mu \mathrm{L}$, each, of pyridine and N,O-bis(trimethylsilyl)trifluoroacetamide (BSTFA) (Supelco/Sigma-Aldrich, USA) at $110^{\circ} \mathrm{C}$ for $10 \mathrm{~min}$. Derivatized samples were evaporated to dryness (without heating) and dissolved in heptane:toluene $(1: 1, v / v)$ for analysis by gas chromatography (GC-FID/GC-MS).

\subsubsection{Seed coat suberin analysis}

For seed coat delipidation, 50-60 mg of dry seeds were ground in isopropanol using a mortar and pestle, and then the mixture was transferred to glass GC tubes and heated for $30 \mathrm{~min}$ at $85^{\circ} \mathrm{C}$ in an oven incubator. After cooling, samples were extensively delipidated by extracting the soluble lipids successively for $24 \mathrm{~h}$ with chloroform:methanol $(2: 1, \mathrm{v} / \mathrm{v})$, chloroform:methanol $(1: 2, \mathrm{v} / \mathrm{v})$, and $100 \%$ methanol, all performed at room temperature on a tube rotator (Boekel Scientific) with a wheel rotating at $60 \mathrm{rpm}$. This was followed by a series of washes with water (1 hour), $2 \mathrm{M} \mathrm{NaCl}(1$ hour), water ( 1 hour), and $100 \%$ methanol (1 hour). The delipidation was continued with chloroform:methanol $(1: 2, v / v)$, chloroform:methanol $(2: 1, v / v)$, and $100 \%$ methanol for 24 hours each. Samples were dried in a fume hood at room temperature for 2-3 days and then in a desiccator for another 2 days before using for suberin analysis. Seed coat suberin analysis was carried out using acid catalyzed transmethylation and derivatization as described above in section 3.3.6.2. 


\subsubsection{Gas chromatography}

The methyl ester/TMS derivatives from the suberin and soluble fractions were quantified with a Varian-3900 Gas Chromatograph equipped with a flame ionization detector (GC-FID) and a HP-5MS capillary column (30m x $0.25 \mathrm{~mm}$ inner diameter, $0.25 \mathrm{~mm}$ film thickness; Agilent). A $1 \mu \mathrm{l}$ aliquot of the sample dissolved in heptane:toluene (1:1) was injected in splitless mode. The temperature of the injector was held at $250^{\circ} \mathrm{C}$ and the column oven temperature was held at $50^{\circ} \mathrm{C}$ for 1 minute and then increased from $50^{\circ} \mathrm{C}$ to $200^{\circ} \mathrm{C}$ at a rate of $25^{\circ} \mathrm{C}$ per minute, followed by a 1 minute hold, and then it was ramped up again at the rate of $10^{\circ} \mathrm{C}$ per minute to a final temperature of $320^{\circ} \mathrm{C}$, which was held for 8 minutes. The total run time was 28 minutes. The flame ionization detector (FID) was held at $350^{\circ} \mathrm{C}$ and high purity helium was used as the carrier gas at a flow rate of $1.5 \mathrm{ml}$ per min. Quantification of monomers was based on peak areas in the GC-FID chromatograms, identified using their retention time, and using the peak area of the respective internal standards $(\mathrm{C} 17: 0,2 \mathrm{OH}-\mathrm{C} 14: 0$, or $\mathrm{C} 15: 0-\mathrm{OH})$. The identity of peaks was verified by GC-MS at the Laboratoire de Biogenese Membranaire, UMR 5200 CNRS, University of Bordeaux.

Root waxes were analyzed on an Agilent 6850 gas chromatograph equipped with an Agilent 5975 mass spectrometer at Michigan State University. Splitless injection was used with a 30-m HP5-MS column. Temperature settings were as follows: inlet $350^{\circ} \mathrm{C}$, detector $320^{\circ} \mathrm{C}$, oven temperature program was set to $130^{\circ} \mathrm{C}$ for $2 \mathrm{~min}$ and increased to $325^{\circ} \mathrm{C}$ at a rate of $5^{\circ} \mathrm{C}$ per minute. The helium flow rate was set at $1.5 \mathrm{~mL}$ per minute. 


\subsubsection{Phenotypic analyses}

\subsubsection{Seed coat permeability test}

For seed coat permeability tests, tetrazolium red assays were used (Debeaujon et al., 2000; Beisson et al., 2007). Dried Arabidopsis seeds were incubated in the dark in an aqueous solution of $1 \%(\mathrm{w} / \mathrm{v})$ tetrazolium red $(2,3,5-$ triphenyltetrazolium chloride; Sigma Chemicals No.T-8877) at $30^{\circ} \mathrm{C}$ for 4 to $48 \mathrm{~h}$. The seeds were observed for change in colour and imaged using a stereo microscope (Model-Discovery.V20, Carl Zeiss, USA). The quantitative formazan assay was carried out as described by Molina et al., (2008). If the tetrazolium salts penetrates into the seed, they are reduced by the embryo to a red precipitate called formazans. Seed samples (50 $\pm 1 \mathrm{mg}$ each) of Arabidopsis wild-type and mutants were incubated in $500 \mu \mathrm{l} 1 \%$ aqueous solution of 2,3,5-triphenyltetrazolium chloride (TTC) at $30^{\circ} \mathrm{C}$ for $4 \mathrm{hr}, 24 \mathrm{hr}, 48 \mathrm{hr}, 72 \mathrm{hr}$ and $120 \mathrm{hr}$ in darkness. After incubation, the samples were washed twice with water, resuspended in $1 \mathrm{ml} 95 \%$ ethanol and finely ground with mortar and pestle to extract formazans. The final volume was adjusted to $2 \mathrm{ml}$ with $95 \%$ ethanol, immediately centrifuged for $3 \mathrm{~min}$ at $15,000 \mathrm{xg}$ and the supernatant was recovered. This procedure was performed quickly to avoid reaction of TTC with the embryo cells after seed disruption. Formazan concentration was determined by measuring the absorbance at $485 \mathrm{~nm}$ (Candler $e t$ al., 1997). Each sample was assayed in triplicates. 


\subsubsection{Germination percentage assay}

Germination rates of the Arabidopsis wild-type and far mutant seeds were observed on MS medium (4.3g/L MS salts, $0.5 \mathrm{~g} / \mathrm{L} \mathrm{MES,} \mathrm{pH} \mathrm{set} \mathrm{to} 5.7,7.5 \%$ Agar; Sigma) supplemented with (+)-abscisic acid (ABA) (Phyto Technology laboratories, Shawnee, Mission, KS-662802) at concentrations of $0 \mu \mathrm{M}, 0.5 \mu \mathrm{M}, 1 \mu \mathrm{M}$ and $10 \mu \mathrm{M}$. Wild-type and far mutant seeds were harvested immediately after maturity from plants of the same age and grown under the same conditions. Mature seeds were sown in triplicate on petri plates (100 seeds per plate) and were transferred to a controlled growth chamber (see Plant Materials and Growth Conditions above) after cold treatment in the dark for 3 days at $4^{\circ} \mathrm{C}$. Germination was scored at 4 days after transfer to the growth chamber. Seeds that showed penetration of the radicle through the seed coat were counted as germinated seeds. The test was done with two separate batches of seeds harvested from plants grown at different times. In addition, the germination rate of Arabidopsis wild-type and far mutant seeds was assessed on MS medium (Sigma) supplemented with $\mathrm{NaCl}$ at concentrations of 0 $\mathrm{mM}, 50 \mathrm{mM}, 100 \mathrm{mM}$ and $200 \mathrm{mM}$, using the same conditions as described above.

\subsubsection{Primary root growth assay}

For measurement of root growth under salt stress, 4-day-old seedlings grown on MS medium (Sigma) were transferred to MS plates containing $0 \mathrm{mM}$ and $150 \mathrm{mM} \mathrm{NaCl}$, and were grown in an upright orientated position. The root length was measured every alternate day for the next 14 days. 


\subsection{Results}

\subsubsection{Distribution of lipids and phenolics in suberin polymer and soluble lipid fractions of Arabidopsis roots}

Lipid-phenolic extracts from roots can be divided into three categories. First, root waxes present in mature taproots, which are extracted by rapid dipping of roots in chloroform solvent (Li et al., 2007; Kosma et al., 2012). Second, the soluble fraction that is obtained by exhaustive solvent extraction of roots, which contains non-polymerized lipids including those making all the cellular membranes, precursor aliphatics potentially targeted for polymerization into the suberin polyester, and unpolymerized phenolics. Third, the residual-bound (insoluble) fraction that remains after exhaustive solvent extraction, which contains the monomers that are part of the suberin polymer.

We first analyzed the distribution of lipids between the soluble and residualbound fraction of 4-week-old wild-type plants grown in tissue culture, focussing on lipids and phenolics that are associated with the suberin polymer (Figure 3.2A). The suberin polyester was depolymerized using acid-catalyzed transmethylation, and the soluble fraction was processed in the same way. Amongst the various lipid constituents, $>90 \%$ of $\alpha, \omega$-dicarboxylic acids and $\omega$-hydroxy fatty acids were found to be associated with the suberin polymer, while the majority $(>90 \%)$ of the fatty acids and phenolics were found in the soluble fraction. The sterols were exclusively found in the soluble fraction. Out of the total, nearly $55 \%$ of very-long-chain (>C18) fatty acids and $36 \%$ of the 2-hydroxy fatty acids were found in the suberin polymer. However, the 2-hydroxy fatty acids that are detected in the suberin polymer using 
acid-catalyzed depolymerisation are likely derived from highly insoluble sphingolipids rather than representing suberin monomers (Molina et al., 2006). Fatty alcohols were found partitioned between the polymer and soluble fractions, with $23 \%$ found in the polymer and $77 \%$ found in the soluble fraction (Figure $3.2 \mathrm{~A}$ and B). Amongst the fatty alcohol chain lengths, a higher percentage of $\mathrm{C} 18: 0-\mathrm{OH}$ $(\sim 36 \%)$ was found to be associated with polymer than the longer chain lengths C20:0-OH ( $\sim 29 \%$ bound) and C22:0-OH ( $\sim 16 \%$ bound) (Table 3.2$)$.

\subsubsection{Isolation of double and triple mutants disrupted in the FAR1, FAR4, and FAR5 genes}

We next wanted to analyze the compositions of the soluble and residual bound (polymeric) fractions in mutants with major deficiencies of fatty alcohols and this required the generation of double and triple far mutants. The FAR4 and FAR5 genes are located adjacent to one another on chromosome 3, while FAR1 is located on chromosome 5 (Chapter 2, Figure 2.1). The far1 far4 and far1 far5 double mutants were generated by conventional genetic crossing. Since FAR4 and FAR5 are positioned in tandem, it was not feasible to identify a double mutant of these genes by crossing the single mutants as the size of the population required to identify a recombination event precisely in between the two adjacent genes would be massive. Therefore, artificial microRNA (amiRNA)-mediated gene silencing technique (Schwab et al., 2006; Ossowski et al., 2008) was used to silence these genes. MicroRNAs (miRNAs) are small (typically 21-25 nucleotides in length), naturally occurring, single-stranded noncoding RNAs involved in gene regulation by translational repression and/or mRNA cleavage (Schwab et al., 2006). Artificial 
miRNAs are much more transcript specific compared to short interfering RNAs (siRNA) because they do not trigger transitive silencing. In transitive silencing, siRNAs not only trigger cleavage and destruction of target gene, but also serve as primers for RNA-dependent RNA polymerases that produce secondary siRNAs, which in turn can affect other genes not targeted by the original siRNAs (Schwab et al., 2006).

The Web MicroRNA Designer Platform (wmd2.weigelworld.org) was used to design amiRNAs specific for FAR5. The designed amiRNA sense and antisense strands were complementary to the $3^{\prime}$ ends of the FAR5 transcripts. For example, based on the alignment, the annealing of the FAR5 amiRNA sequence- 1 would cause cleavage of the FAR5 transcript at position +1110 , producing a non-functional truncated mRNA (Figure 3.3A). Gene silencing mediated by amiRNAs is known to occur in a quantitative fashion (Ossoswski et al, 2008), where use of stronger promoters is required for effective gene silencing. The amiRNAs for each gene was thus expressed under the strong, constitutive $35 \mathrm{~S}$ promoter. Constructs with FAR5amiRNA were introduced into wild-type, far4, and far1 far4 Arabidopsis plants. A similar strategy was used to silence the FAR4 gene and constructs with FAR4amiRNA was introduced into wild-type, far 5 and far1 far5. This silencing approach allowed for the generation of double far 4 far 5 and triple far 1 far 4 far 5 plants.

The effects of plant miRNAs are evident at the transcript level (Ossoswski $e t$ al, 2008). None of the FAR4-amiRNA lines showed silencing of the FAR4 gene by RTPCR, while FAR5-amiRNA lines exhibited silencing although with significant variability between individual plants in the $\mathrm{T} 2$ generation analysis (data not 
shown). Further screening and selection of silenced lines was carried out with the FAR5-amiRNA sequence-1 carrying lines. Two homozygous lines each of the Col-0, far4 and far1 far 4 transgenic plants expressing FAR5-amiRNA sequence-1 were selected by identifying pure-breeding lines for the linked herbicide resistance marker ( $B A R$ gene). The transcriptional down-regulation of FAR5 gene was confirmed in the 35S:FAR5amiRNA plants by RT-PCR using RNA derived from young roots of 15-days-old T4 seedlings grown in tissue culture (Figure 3.3C).

A promising far5ami single mutant line (denoted far $5^{*}$; it is marked by an asterisk to distinguish amiRNA-silenced FAR5 lines from far5 T-DNA insertion lines), a far 4 far5* double mutant line, and two independent far 1 far 4 far $5^{*}$ triple mutant lines, along with the other far single and far double mutants were selected and taken forward for further quantitative transcript analysis. Quantitative RT-PCR carried out on total RNA $(0.1 \mu \mathrm{g})$ isolated from the roots of 15 -day-old T5 seedlings revealed a significant reduction ( $>90 \%$ ) of FAR1, FAR4 and FAR5 transcripts in far12, far4-1 and far5-1 T-DNA lines as expected. Further, there was near absence of FAR1 transcript in far 1 far 4 and far 1 far 5 double mutant lines; near absence of FAR4 transcripts in far 1 far 4 and far 4 far $5^{*}$ double mutant lines; and near absence of FAR5 transcripts in far 1 far 5 double mutant lines. FAR5 transcript levels in most of the FAR5-amiRNA lines were found to be reduced greater than $70 \%$, although none of them were reduced to the levels observed with far5-1 (Figure 3.4). The triple far 1 far 4 far5*ami lines showed a $>95 \%$ reduction in FAR1 and FAR4 levels, with the FAR5 transcript levels reduced by $70-80 \%$. The FAR5-amiRNA mediated silencing is highly specific to FAR5 as the transcript levels of FAR1 and FAR4 remained 
unaffected in wild-type plants expressing FAR5-amiRNA. The well silenced far5*ami single line, the well silenced far 4 far $5^{*}$ double mutant line, and two independent well-silenced far 1 far 4 far $5 *$ triple mutant lines, along with the other far single and far double mutants were taken forward for further lipid analysis.

\subsubsection{Alterations of fatty alcohol composition associated with suberin polyester and soluble fraction of roots of far single, double, and triple mutants}

We then analyzed for alterations in root suberin polymer and root soluble lipid compositions in the far single mutants (far1, far4, far5 and far5*), far double mutants (far 1 far 4 , far 1 far 5 and far 4 far $\left.5^{*}\right)$, and far triple mutants (far 1 far 4 far $5^{*}$ lines 1 and 2). There were no significant differences in the quantities of most monomer classes in the suberin polymer in the different far mutant lines in comparison to wild-type, with the exception of fatty alcohols (Figure 3.5). The far single mutants showed $\sim 30 \%$ reduction, the far double mutants showed $\sim 50 \%$ reduction, while the far triple mutants showed $\sim 65-70 \%$ reduction in the total fatty alcohol content (Figure 3.6A). There were also significant variations in the fatty alcohol chain length distributions depending on the mutant (Figure 3.6B; Supplemental Table S3.1). In Col-0 plants, there are nearly equal amounts of C18:0, C20:0 and C22:0 fatty alcohols in root suberin. Among the far single mutants, far1 showed a significant $42 \%$ reduction in $\mathrm{C} 22: 0-0 \mathrm{H}$, far 4 showed a significant $77 \%$ reduction in $\mathrm{C} 20: 0-\mathrm{OH}$, while the levels of the other two chain length fatty alcohols were not significantly altered in far 1 and far 4 single mutant lines. The far 5 single mutant showed a significant $97 \%$ reduction of $\mathrm{C} 18: 0-\mathrm{OH}$, accompanied by a 
significant increase in $\mathrm{C} 20: 0-\mathrm{OH}$. These results are consistent with our previous findings that FAR1, FAR4, and FAR5 are primarily involved in the production of $\mathrm{C} 22: 0-\mathrm{OH}, \mathrm{C} 20: 0-\mathrm{OH}$, and $\mathrm{C} 18: 0-\mathrm{OH}$ chain lengths, respectively. However, in addition, these data emphasize that there is partial overlap of FAR1 and FAR4 in producing $\mathrm{C} 20: 0-\mathrm{OH}$ and $\mathrm{C} 22: 0-\mathrm{OH}$, but a near absence of overlap with FAR5 such that nearly all the C18:0-OH found in the root suberin polymer is synthesized by FAR5. The $f a r 5^{*}$ amiRNA single mutant line showed about a $71 \%$ reduction in C18:0-0H, while the levels of other two chain length alcohols (C20:0-OH and C22:0OH) remained unaffected confirming the high sequence specific silencing of FAR5 by the FAR5-amiRNA and that the artificial microRNA silencing is not causing a null phenotype. This is in agreement with the transcriptomic data (Figure 3.4). The double mutant far1 far 4 showed $78 \%$ reduction in $\mathrm{C} 20: 0-\mathrm{OH}$ and $80.5 \%$ reduction in $\mathrm{C} 22: 0-\mathrm{OH}$ content compared to the wild-type control. The double mutant far1 far5 showed $97 \%$ reduction in $\mathrm{C} 18: 0-\mathrm{OH}$ content, but had only a $46 \%$ reduction in the $\mathrm{C} 22: 0-\mathrm{OH}$ content (Figure 3.6B). It is likely that FAR4 in the far1 far5 double mutant is contributing towards the production of $\mathrm{C} 22: 0-\mathrm{OH}$ fatty alcohols. The far 4 far5* line showed $91 \%$ and $72 \%$ reduction in $\mathrm{C} 18: 0-\mathrm{OH}$ and $\mathrm{C} 22: 0-\mathrm{OH}$ levels, respectively. The triple mutant far 1 far 4 far $5^{*}$ line-1 showed around a 52\% reduction in $\mathrm{C} 18: 0-\mathrm{OH}$, while the levels of $\mathrm{C} 20: 0-\mathrm{OH}$ and $\mathrm{C} 22: 0-\mathrm{OH}$ was reduced by $80 \%$ and $91 \%$, respectively. Similar reduction levels were observed in the second

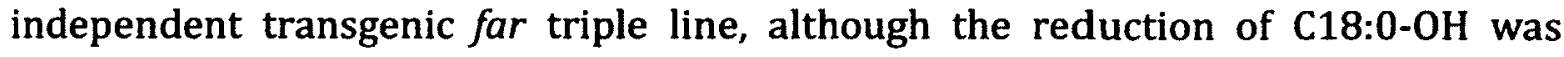
around $46 \%$, indicating variation in the degree of silencing of FAR5 between individual lines. It should be noted that the silencing effect of FAR5-amiRNA was less 
strong in the far triple $(<50 \%)$ and $f a r 5^{*}$ single mutant lines than in the far 4 far $5^{*}$ double mutant line (>90\%), but additional screening of lines did not result in lines that were better silenced. Altogether, the far triple mutant lines had reductions in all three chain lengths of primary fatty alcohols $(\mathrm{C} 18: 0-\mathrm{OH}, \mathrm{C} 20: 0-\mathrm{OH}$, and $\mathrm{C} 22: 0-\mathrm{OH})$, which thereby resulted in a significant reduction in the total fatty alcohol load (Figure 3.6A).

As reported above, the soluble fraction from the roots of wild-type plants has a higher amount of fatty alcohols compared to the suberin polymer fraction. There was about three times more $\mathrm{C} 18: 0-\mathrm{OH}(0.59 \mu \mathrm{g} / \mathrm{mg}$ DR), five times more $\mathrm{C} 20: 0-\mathrm{OH}$ $(0.73 \mu \mathrm{g} / \mathrm{mg} \mathrm{DR})$ and six times more $\mathrm{C} 22: 0-0 \mathrm{H}(1.02 \mu \mathrm{g} / \mathrm{mg} \mathrm{DR})$ in the soluble fraction compared to the suberin polymer (Figure 3.7 ). There was a $56 \%$ reduction in $\mathrm{C} 22: 0-\mathrm{OH}, 65 \%$ reduction in $\mathrm{C} 20: 0-\mathrm{OH}$ and $93 \%$ reduction in $\mathrm{C} 18: 0-\mathrm{OH}$ in far1, far 4 and far 5 single mutants, respectively. The far $5^{*}$ line showed a $55 \%$ reduction in C18:0-0H. Among the far double mutants: far 1 far 4 showed a reduction of $76.7 \%$ and $86.3 \%$ in $\mathrm{C} 20: 0-\mathrm{OH}$ and $\mathrm{C} 22: 0-\mathrm{OH}$, respectively; far 1 far 5 showed a reduction of $91.5 \%$ and $60.3 \%$ in $\mathrm{C} 18: 0-\mathrm{OH}$ and $\mathrm{C} 22: 0-\mathrm{OH}$, respectively; and far4 far5* showed a reduction of $88.6 \%$ and $61.6 \%$ in $\mathrm{C} 18: 0-\mathrm{OH}$ and $\mathrm{C} 20: 0-\mathrm{OH}$ respectively. The far triple mutant far 1 far 4 far $5^{*}$ line- 1 showed reductions in the levels of all three chain length fatty alcohols, with C18:0-0H reduced by $70 \%, \mathrm{C} 20: 0-0 \mathrm{H}$ reduced by $90 \%$ and $\mathrm{C} 22: 0-\mathrm{OH}$ reduced by $90.2 \%$. Similar levels of reduction were observed for the three chain lengths of fatty alcohols in the second independent transgenic line of far triple mutant: $\mathrm{C} 18: 0-\mathrm{OH}$ was reduced by $65 \%, \mathrm{C} 20: 0-\mathrm{OH}$ was reduced by $85 \%$, and C22:0-OH was reduced by $90 \%$. The alteration in the fatty alcohol level in the far 
triple mutants was more prominent in the soluble fraction compared to the suberin polymer fraction.

The total fatty alcohol loads, which comprise both the suberin polymer and soluble fractions for wild-type and far mutants, are presented in Table 3.3. In Col-0 roots, $\mathrm{C} 22: 0-\mathrm{OH}$ is the most abundant chain length fatty alcohol and constitutes about $42 \%$ of the total fatty alcohol load. The shorter chain length fatty alcohols, $\mathrm{C} 18: 0-\mathrm{OH}$ and $\mathrm{C} 22: 0-\mathrm{OH}$, constitute about $27 \%$ and $31 \%$ of the total fatty alcohol load, respectively (Table 3.3). Quantification of individual chain length fatty alcohols in wild-type roots and their partitioning between soluble and polymer fraction showed that about $24 \%$ of the total $\mathrm{C} 18: 0-\mathrm{OH}, 17.4 \%$ of the total $\mathrm{C} 20: 0-\mathrm{OH}$ and $14.6 \%$ of the total $\mathrm{C} 22: 0-\mathrm{OH}$ is bound to the suberin polymer, while the rest is found in the soluble fraction. In contrast, a higher percentage of all three chain length fatty alcohols were bound the far 1 far 4 far $5 *$ triple mutant. In this triple mutant, $32 \%$ of $\mathrm{C} 18: 0-\mathrm{OH}, 28.7 \%$ of $\mathrm{C} 20: 0-\mathrm{OH}$ and $18.5 \%$ of $\mathrm{C} 22: 0-\mathrm{OH}$ were found to be part of the suberin polymer (Table 3.4 ).

\subsubsection{Alterations of fatty alcohol composition and alkyl hydroxycinnamates in root waxes of far double and far triple mutants}

Suberin-associated root waxes are isolated by rapid immersion of Arabidopsis roots in organic solvents (e.g. chloroform). Arabidopsis root waxes are rich in saturated C18:0-C22:0 alkyl esters of $p$-hydroxycinnamic acids ( $p$-coumaric, ferulic and caffeic acids), collectively called alkyl hydroxycinnamates (AHCs). The other important components of root waxes are sterols, primary alcohols, free fatty acids, and monoacylglycerols (Li et al., 2007; Kosma et al., 2012). Kosma et al., 
(2012) reported that FAR1, FAR4 and FAR5 are responsible for production of C22:0, C20:0 and C18:0 alcohols, respectively, that make up the AHCs of Arabidopsis. A study of spatial gene expression patterns using promoter:GUS transgenic plants revealed that these FAR genes are expressed in the peridermal cells of the old matured Arabidopsis tap roots (Chapter 2, Section 2.4.1), where the suberinassociated root waxes are thought to be concentrated. We further investigated the effects on root waxes components in six-week-old far double and far triple mutants grown in a soil-less potting medium. The root waxes in wild type (Col-0) Arabidopsis plants were largely composed of alkyl hydroxycinnamates (78.5\%), of which alkyl caffeates were the most abundant class (49.7\%), followed by alkyl coumarates $(24.1 \%)$ and alkyl ferulates (4.7\%) (Figure 3.8). The total root wax load was significantly reduced $(48-78 \%)$ in the far double and triple mutant lines compared to wild-type (Figure 3.8A). No significant differences were observed in the quantities of free fatty acids, sterols and monoacylglycerols (Supplemental Table 3.2). However, it was found that primary fatty alcohols and AHC levels were drastically affected. The primary fatty alcohols in the root waxes showed significant reduction in a chain length specific manner as with the root suberin and soluble fractions. The far1 far 4 double mutants showed a significant reduction in $\mathrm{C20:0- \textrm {OH }}$ and $\mathrm{C} 22: 0-\mathrm{OH}$; far1 far5 had a reduction in $\mathrm{C} 18: 0-\mathrm{OH}$ and $\mathrm{C} 22: 0-\mathrm{OH}$; and far4 far5* showed reduced levels of $\mathrm{C} 18: 0-\mathrm{OH}$ and $\mathrm{C} 20: 0-0 \mathrm{H}$. The triple mutant far1 far4 far ${ }^{*}$ had a $45 \%$ reduction in $\mathrm{C} 18: 0-0 \mathrm{H}$ and more than $90 \%$ reduction in both $\mathrm{C} 20: 0$ and C22:0 alcohols (Figure 3.8B). A similar reduction pattern was observed in all three subsets of AHCs (alkyl coumarates, alkyl ferulates, and alkyl caffeates) (Figure 3.8B). 
The far1 far4 double mutant had major reductions in C20:0 (85\%) alkyl coumarates and lacked C22:0 alkyl coumarates. The far1 far4 double mutant lacked alkyl ferulates and alkyl caffeates of $\mathrm{C} 20: 0$ and $\mathrm{C} 22: 0$ chain lengths. The far1 far5 double mutant lacked C18 alkyl hydroxycinnamates and had reduced levels of both C20:0 and C22:0 AHCs. The far 4 far $5^{*}$ double mutant line showed a complete lack of C18:0 AHCs, along with a lack of C20:0 alkyl coumarates and alkyl ferulates and reduced levels of C20:0 alkyl caffeates. The far 1 far 4 far $5^{*}$ triple mutant showed a dramatic reduction in all three C18:0-C22:0 AHCs. There were no alkyl ferulates and alkyl caffeates detected in the far triple mutant line. Along with alkyl coumarates, the far triple mutants line showed a $66 \%$ and $95 \%$ reduction in the $\mathrm{C} 18$ and $\mathrm{C} 20$ chain length coumarates, respectively, while it lacked C22 chain length coumarates (Figure 3.8B). These results confirm that FAR1, FAR4 and FAR5 are involved in the production of fatty alcohols necessary for alkyl hydroxycinnamate synthesis, meaning that the C18:0-C22:0 fatty alcohols are shared between the suberin and root wax biosynthetic pathways.

\subsubsection{Alterations of fatty alcohol composition associated with seed coat suberin of far single, double, and triple mutants}

Suberin is present in the Arabidopsis seed coat (Molina et al., 2008). The predominant monomers found in the seed coat polyester include fatty alcohols, ferulates, C18:1 and C18:2 $\omega$-hydroxy fatty acids, C22 and C24 $\omega$-hydroxy fatty acids and saturated C18-C24 $\alpha, \omega$-dioic acids monomers (Molina et al., 2008). The FAR genes under study have been observed to express in the Arabidopsis seed coat and the single mutants affect fatty alcohols in a chain-length specific manner (Chapter 
2). We further analyzed the composition of seed coat suberin polyester in the double and triple far mutants in comparison to wild-type and the single mutant lines (Figure 3.9, Supplemental Table 3.3). There were no significant differences observed in the amounts of most suberin monomer subclasses (fatty acids, $\alpha, \omega$-dicarboxylic acids, and $\omega$-hydroxy acids) in the seeds of any of the far mutants compared to wildtype (Figure 3.9A, Supplemental Table 3.3). In the wild-type seed coat, C22:0-OH and C22:0-diol are the major components amongst the fatty alcohols, being present at levels about twice that of $\mathrm{C} 18: 0-\mathrm{OH}, \mathrm{C} 20: 0-\mathrm{OH}$ and C20:0-diols (Figure 3.9B). A similar chain length-specific reduction pattern of fatty alcohols was observed in seed coat suberin as that observed with root suberin in the various far mutants. The far1 mutant had $64 \%$ and $62.2 \%$ reductions in $\mathrm{C} 22: 0-\mathrm{OH}$ and C22:0-diol, respectively, and the far 4 mutant had $84.6 \%$ and $41.4 \%$ reductions in $\mathrm{C} 20: 0-\mathrm{OH}$ and C20:0-diol, respectively. The far5 T-DNA insertion line had a 95\% reduction in C18:0-0H, while the far $5^{*}$ artificial microRNA line had about a $60 \%$ reduction in C18:0-OH (Figure 3.9B). Among the far double mutants, far1 far4 had a reduction of both $\mathrm{C} 20: 0-\mathrm{OH}$ and $\mathrm{C} 22: 0-\mathrm{OH}$ by $80 \%$ and $92.8 \%$, respectively, accompanied by reductions in C20:0-diol and C22:0-diol by $46.6 \%$ and $62.4 \%$, respectively. The far 1 far5 was affected in C18:0-OH (reduced by $87.8 \%$ ) and C22:0-0H (reduced by $64 \%$ ) and showed $68 \%$ reduction in C22:0-diol levels. The far 4 far5* line showed about a $90.6 \%$ reduction in $\mathrm{C} 18: 0-0 \mathrm{H}$ and $78 \%$ reduction in $\mathrm{C} 20: 0-\mathrm{OH}$ along with $\mathrm{C} 20: 0$-diol reduced by $50 \%$. The triple mutant far 1 far 4 far $5^{*}$ (only line 1 analyzed) was the most affected and showed a significant reduction in all three chain length fatty alcohols by $57 \%, 88.3 \%$, and $92.3 \%$ for $\mathrm{C} 18: 0-\mathrm{OH}, \mathrm{C} 20: 0-\mathrm{OH}$, and $\mathrm{C} 22: 0-\mathrm{OH}$, 
respectively. In addition, the amounts of C20:0-diol and C22:0-diol was reduced by $46 \%$ and $69 \%$, respectively (Figure $3.9 \mathrm{~B}$ ). Overall, the far double and triple mutant lines had significantly altered total levels of fatty alcohols and diols, with the far1 far 4 far $5^{*}$ triple mutant (line 1) having the most drastic reduction in the total fatty alcohol load (75\% reduction compared to wild-type) (Supplemental Table 3.3).

\subsubsection{Alteration in seed coat permeability and germination percentage in far double and far triple mutants}

The isolated single, double and triple mutants for Arabidopsis FAR1, FAR4, and FAR5, had no obvious developmental phenotype under non-stressed conditions. The far single, double and triple mutants were studied for possible effects of reduced suberin related fatty alcohols on germination ability, seedling establishment, and root growth rate under salt stress conditions $(0 \mathrm{mM}$ vs. $100 \mathrm{mM}$ vs. $150 \mathrm{mM} \mathrm{NaCl}$ salt), but no significant differences were observed (Supplementary Figure S3.1A,B). The suberin polyester is also an important barrier in seed coats (Beisson et al., 2008). Since the seed coat suberin polyester of some of the far mutants were significantly affected in both fatty alcohols and diols, which together represent about $17 \%$ of the total suberin monomer load, we tested the effect of altered polyester monomer composition on the permeability of seed coat. The kinetics of tetrazolium salt uptake by mature seeds of wild-type and the far double and far triple mutants was studied using the triphenyl tetrazolium chloride reduction method and spectrophotometric determination of absorbance of formazans extracts at $485 \mathrm{~nm}$ as described by Molina et al. (2008). Tetrazolium red is a cationic dye that is widely used in seed viability testing (Moore, 1962; 
McDonald, 1975). Tetrazolium salts are amphipathic cations, which, after penetrating the dead cells of the seed coat, are reduced to a red-coloured insoluble precipitate made up of formazans by active dehydrogenases (NADH-dependant reductases) in the embryo of seeds (Berridge et al., 1996). The gpat5-1 mutant line was reported to have increased seed coat permeability (Beisson et al., 2005) and this mutant was used as a positive control. Seeds of far double and triple mutants showed a significant increase in accumulation of formazans after 24 hours incubation compared to wild-type seeds (Figure 3.10A). Triple mutant lines were more affected in seed coat permeability compared to the double mutants. After 120 hours of incubation, a 10-fold increase in formazans was observed in gpat5-1 seeds, a 3-fold increase in the far double mutant seeds, a 6-fold increase in far1 far4 far5* line-1 seeds, and a 4 -fold increase in far 1 far 4 far $5^{*}$ line- 2 seeds compared to wildtype seeds. In addition, seed samples incubated in $1 \%$ tetrazolium red at $30^{\circ} \mathrm{C}$ for duration of $\mathbf{4 8}$ hours were observed and imaged with a stereomicroscope. The wildtype seeds showed no perceptible red staining, while all the gpat5-1 seeds exhibited an intense red staining. The far single mutants did not show any significantly altered seed coat permeability compared to the control Col-WT. Most of the seeds in far double mutant lines also did not show much penetration of tetrazolium salts at this time point, whereas the far triple mutants showed red staining of seeds to various degrees. The far 1 far 4 far $5^{*}$ line-1 was the most affected where more than $90 \%$ of the seeds showed a change in colour (Figure 3.10B).

Since seed coat permeability was affected, we examined the effects of the hormone abscisic acid (ABA) on germination percentage of these far singles, far 
double and far triple mutant seeds. ABA has been reported to inhibit Arabidopsis seed germination by restricting the availability of energy and metabolites. (Garciarrubio et al., 1997). Matured seeds of wild-type and far mutants placed in MS medium lacking $\mathrm{ABA}$ or supplemented with $\mathrm{ABA}$ at concentrations of $0.5 \mu \mathrm{M}, 1.0 \mu \mathrm{M}$ and $10 \mu \mathrm{M}$, and then scored for germination percentage after four days (Figure 3.11). In the absence of $A B A$, all the lines showed $100 \%$ germination except the far1 and $\operatorname{far}^{*}$ single mutant line which showed a germination rate of $96 \%$ and $98 \%$, respectively. A significant reduction of $60 \%$ was observed in the germination rate of far double mutants at $0.5 \mu \mathrm{M} \mathrm{ABA}$, while the far triple mutant was further affected at only $15 \%$ germination in $0.5 \mu \mathrm{M}$ ABA compared to the wild-type seeds. At $1.0 \mu \mathrm{M}$ ABA concentration, wild-type seeds showed about $50 \%$ germination rate, while far single mutants and far double mutants showed about $35 \%$ and $20 \%$ of germination rate, respectively. The far 1 far 4 far $5 *$ triple mutant line and the gpat5-1 line showed a severe reduction of $>95 \%$ in germination rate at $1.0 \mu \mathrm{M} \mathrm{ABA}$. Further, at a concentration of $10 \mu \mathrm{M} \mathrm{ABA}$, none of the far mutant seeds showed any germination, while wild-type seeds showed about $6 \%$ germination. 


\subsection{Discussion}

Suberin is an insoluble apoplastic biopolymer deposited during secondary cell wall differentiation in specialized internal cell layers (e.g. root endodermis and bundle sheath of monocots) and external cell layers (e.g. periderm of roots and stems that have undergone secondary growth), as well as in response to environmental cues (e.g. wounding, salt stress) (Ranathunge et al., 2011). Suberin prevents the uncontrolled flow of water, gases and ions, and provides resistance to pathogens (Enstone et al., 2003, Franke and Schreiber, 2007; Franke et al., 2012). The suberin polymer is mainly composed of $\omega$-hydroxyacids and $\alpha, \omega$-dicarboxylic acids with lower amounts of fatty acids and primary alcohols; typically ranging in carbon chain length from $\mathrm{C} 16-\mathrm{C} 24$, although there is variability between species and between tissues (Pollard et al., 2008). Glycerol is also part of the aliphatic suberin polyester (Graca and Santos, 2007; Beisson et al., 2012). The phenolic portion of the suberin polyester is rich in $p$-hydroxycinnamic acid derivatives, primarily ferulic acid (Bernards, 2001). In the current work, we analyzed the composition of lipids in three root lipid fractions. Two fractions were from 4-week-old wild type (Col-0) Arabidopsis plants grown in tissue culture: (1) the residual-bound (insoluble) fraction, which includes all the monomers released from the suberin polymer following depolymerisation, and (2) the soluble-lipid fraction obtained by exhaustive solvent extraction of roots, which includes all the monomers that were not cross-linked (unpolymerised) to either aliphatic or phenolic components of the suberin polymer, but also various lipids not associated with suberin. A third fraction was root waxes, rich in alkyl hydroxycinnamates, obtained by dipping of 6-week-old 
soil-grown Arabidopsis roots in chloroform for 1 minute. These root waxes are concentrated in the top 4-6 $\mathrm{cms}$ of the tap root, but are absent in roots from seedlings grown for 4 weeks in vitro (Kosma et al., 2012).

We observed a distribution of suberin-type monomers between the suberin polymer and the soluble fraction in Arabidopsis roots, which is consistent with reports described in other plants such as potato and Iris germanica (Yang and Bernards, 2006; Meyer et al., 2011a and 2011b). A large proportion (>90\%) of the $\omega$-hydroxy fatty acids and $\alpha, \omega$-dicarboxylic acids, which are typically the two most abundant suberin monomer classes, were found in the residual-bound insoluble fraction with only a small amount in the soluble fraction. Dean and Kolattukudy (1977) reported an abundance of $\omega$-hydroxy fatty acids and $\alpha, \omega$-dioic acids in the insoluble fraction of wound healing Solanum tuberosum tuber periderm, while these were detected in trace amounts in the soluble fraction. Yang and Bernards (2006) quantified the insoluble and soluble aliphatic monomers in the wound-induced periderm of $S$. tuberosum tubers as they developed over seven days and found that the insoluble fraction mostly consisted of $\omega$-hydroxy fatty acids, $\alpha, \omega$-dioic acids, and fatty acids, while the soluble fraction contained only trace amounts of $\omega$-hydroxy fatty acids and $\alpha, \omega$-dioic acids, but greater amounts of very-long-chain fatty acids $(>\mathrm{C} 20)$. Primary fatty alcohols ranging in chain length from C16 to C28 were reported in both soluble and insoluble fractions, with C26:0 and C28:0 primary alcohols being the abundant ones (Yang and Bernards, 2006). The polymerized monomers were reported to increase with aging of the wound-induced periderm, while the total amount of soluble aliphatic compounds remained relatively constant 
throughout the time course period. Meyer et al. (2011a) reported quantification of soluble and insoluble aliphatic monomers in a study of maturing exodermis of Iris germanica roots under different growth conditions. The soluble fraction was reported to consist mostly of fatty acids, alkanes, fatty alcohols, and ferulic acid, while the insoluble fraction (suberin polymer) was mostly fatty acids, $\alpha, \omega$-dioic acids, $\omega-\mathrm{OH}$ fatty acids, and ferulic acid. Primary fatty alcohols with a chain length distribution of $12: 0,14: 0,16: 0$ and $18: 0$ constituted about $1.3-3.4 \%$ of the total soluble aliphatics (Meyer et al., 2011a). In a similar study on onion root exodermis, Meyer et al. (2011b) reported absence of C18:1 $\alpha, \omega$-dioic acids and C18:1 $\omega-\mathrm{OH}$ fatty acids in the soluble fraction of Allium cepa roots. The increase in $\omega$-hydroxy fatty acids along the root zones measured in corn and Arabidopsis was found to correlate with substantial decrease in fatty acids (Zeier et al., 1999; Hofer et al., 2008). Due to the two opposite functional groups, $\omega$-hydroxy acids are chemically ideally suited to form a polyester and such related compositional changes indicates that $\omega$-hydroxylation is an important step in suberization of cell walls (Hofer et al., 2008). The results indicate that most of the C18:1 fatty acids are oxidized to $\omega$ hydroxy fatty acids and $\alpha, \omega$-dicarboxylic acids, and are rapidly incorporated into the developing suberin polyester (Meyer et al., 2011a). Fatty alcohols (C14:0-C28:0) were reported to account for $7-12 \%$ of the soluble fraction, while in the suberin fraction, only C22:0 fatty alcohol monomer was detected, which made up just $3 \%$ of the suberin fraction across all exodermal maturation zones (Meyer et al., 2011b). It is not known how fatty alcohols are incorporated into the suberin polymer. A large fraction of monomers with single functional group should preclude an extended or 
cross-linked aliphatic polymer, since monofunctional aliphatics terminate the ester polymer chain and this may account for their relatively low abundance in the polymer itself.

Fatty alcohols detected in the soluble fraction might be precursors for the suberin polymer, which remained because all the potential incorporation sites in the developing suberin polymer were filled. However, considering the relatively large amounts of total fatty alcohol in the soluble fractions, it is more likely that the free primary alcohols have additional roles to play in plants. It is currently not clear if the fatty alcohols in the soluble fraction are non-covalently associated with the suberin polymer or even if they are extracellular. Soliday et al. (1979) proposed that waxes associated with the suberin polymer, rather than the polymerized lipids, constitute the major diffusion barrier formed during wound healing in potato tuber. It was found that the treatment of wounded potato tuber tissue with trichloroacetate, an inhibitor of fatty-acid chain elongation, severely inhibited accumulation of hydrocarbons and fatty alcohols associated with the suberized layer, but had very little effect on the deposition of the major aliphatic components of the suberin polymer. This preferential inhibition of wax synthesis disrupted the formation of lamellar structure of suberin resulting in severe loss in the diffusion resistance of the tissue to water vapours (Soliday et al. 1979). This indicates that very-long chain aliphatics, possibly including very-long-chain fatty alcohols, found in suberin-associated waxes play an important role in rendering diffusion resistance and barrier formation. Free long-chain fatty alcohols have been found to have 
antibacterial action (Masao et al., 1987), and thus fatty alcohols may have an important role in biotic interactions rendering pathogen resistance.

As reported in Chapter 2, the Arabidopsis far single mutants showed reductions in primary alcohols in accordance with the individual chain length specificity of each FAR enzyme, such that far1 was reduced in $\mathrm{C} 22: 0-\mathrm{OH}$, far4 was reduced in $\mathrm{C} 20: 0-0 \mathrm{H}$, and far 5 was reduced in $\mathrm{C} 18: 0-\mathrm{OH}$ in the root and seed coat suberin. Among the far double mutants, far1 far4 had significant reductions in $\mathrm{C} 20: 0-\mathrm{OH}$ and $\mathrm{C} 22: 0-\mathrm{OH}$, far1 far5 had significant reductions in $\mathrm{C} 18: 0-\mathrm{OH}$ and $\mathrm{C} 22: 0-\mathrm{OH}$, and far4 far $5^{*}$ had significant reductions in $\mathrm{C} 18: 0-\mathrm{OH}$ and $\mathrm{C} 20: 0-\mathrm{OH}$ in the root and seed coat suberin. The fatty alcohol composition of root suberin showed greater reduction of $\mathrm{C} 22: 0-\mathrm{OH}$ in far1 far4 when compared to far1 and far1 far5 mutant lines. This indicates overlapping specificities of FAR1 and FAR4. On the other hand, the far1 far 5 and far 5 , far 4 far $5^{*}$ lines showed $>90 \%$ reduction in the C18:0-OH levels. This implies that FAR5 is highly specific to C18:0-CoA, and that FAR1 and FAR4 do not significantly contribute to $\mathrm{C} 18: 0-\mathrm{OH}$ production in planta. The far triple mutant far 1 far 4 far5* showed a significant reduction in all three chain length fatty alcohols and had $\sim 70 \%$ reduction in the total fatty alcohol load in root suberin compared to wild-type plants. Although fatty alcohol compositions were systematically affected in the triple far mutant, the other main lipid polyester monomers (unsubstituted fatty acids, $\omega$-hydroxyacids, and $\alpha, \omega$-dicarboxylic acids) were not significantly altered even in the far triple mutants, indicating that fatty alcohols do not influence the incorporation of other suberin monomer classes. 
Alkyl hydroxycinnamates are present in the suberized periderm of both aboveground (bark) and underground (tuber and taproot) plant organs (Schreiber et al., 2005; Freire et al., 2007, Kosma et al., 2012). However, their exact localization, both in terms of extra- versus intracellular deposition, and lateral heterogeneity, is not clear. Kosma et al. (2012) compared the spatial distribution of waxes along the root axis of Arabidopsis and reported a nearly 5-fold enrichment of root wax aliphatics from the taproot (primary root; $3-5 \mathrm{~cm}$ below the rosette) compared with younger roots, including a nearly 9-fold enrichment in alkyl hydroxycinnamates. Kosma et al. (2012) reported chain length specific reductions in alkyl hydroxycinnamates of root waxes in far 1 , far4, and far5 mutants, whereas far5 mutant lacked all C18 alkyl hydroxycinnamates, far4 mutant had reduced levels of C20 alkyl hydroxycinnamates, and far1 mutant had reduced levels of C22 alkyl hydroxycinnamates. We report here that the primary alcohols and alkyl hydroxycinnamates in root waxes are drastically decreased in the far double and far triple mutant lines compared to the wild-type. The reductions in free and combined fatty alcohols were accompanied with a significant increase in the amount of free fatty acids in the far triple mutant lines possibly due to accumulation of fatty acids as a result of decreased fatty acyl reductase activity. The alkyl hydroxycinnamates in the root waxes showed reductions in a chain length specific manner as observed earlier in the root suberin polymer and soluble fractions of far mutants. The presence of C22 alkyl hydroxycinnamates in far1 far 5 double mutant and their complete absence in the far1 far4 double mutant confirms partial complementation of FAR1 by FAR4 and vice versa. The complete absence of C18 alkyl 
hydroxycinnamates in far 1 far5 and far4 far $5^{*}$ double mutants indicates no complementation of FAR5 by either FAR1 or FAR4 and suggests that all C18:0 fatty alcohol is synthesized by FAR5. It is possible that these waxes play a role in augmenting the barrier properties of suberin, but other functions are more likely. Hydroxycinnamic acids have been reported to show considerable antimicrobial activity. Caffeic acid has been found to inhibit Clostridium botulinum (Bowles and Miller, 1994) and Ganoderma Boninense (Chong et al., 2009), and ferulic and pcoumaric acids are known to be active against Saccharomyces cerevisiae (Baranowski et al., 1980). Baranowski and Nagel (1983) found that the alkyl hydroxycinnamates; caffeate esters, methyl $p$-coumarate, and methyl ferulate possessed antibacterial activity toward Pseudomonas fluorescens, a gram negative bacterium. Long-chain fatty alcohols have also been reported to have antibacterial action (Masao et al., 1987). 1-Dodecanol and 1-tridecanol were reported to have the highest antibacterial activity among the various long-chain fatty alcohols tested against Staphylococcus aureus (Toqashi et al., 2007). Accumulation of alkyl ferulates in the wax fraction of wound periderm suggests their role in pathogen interaction (Bernard and Lewis, 1992; Nicholson and Hammerschmidt, 1992; Schreiber et al., 2005b). Thus, free fatty alcohols and fatty alcohols combined with hydroxycinnamates possibly have a role in protecting plant roots from soil-borne plant pathogens. Further studies on far double and far triple mutants with respect to pathogen susceptibility will provide a better understanding of their potential roles in resisting pathogenic microbes. 
In Arabidopsis, the seed coat constitutes about $20 \%$ of mature dry seed weight (Li et al., 2006) and is associated with complex physiological processes such as seed dormancy and germination (Haughn and Chaudhury, 2005). Suberization is present over the entire seed coat of matured seeds (Molina et al., 2008). The inner seed coat polyester has been reported to be rich in cutin-type monomers, while typical suberin monomers are abundant in the outer integument (oi1 layer) of the seed coat in Arabidopsis and Brassica napus (Espelie et al., 1979; Molina et al., 2008). In Arabidopsis seeds, the major C16 and C18 monomers of suberin are $\omega$ hydroxy fatty acids and $\alpha, \omega$-dicarboxylic acids, while the major C20-C26 monomers were C22:0-OH, C22:0-diol, 22-hydroxydocosanoic acid, 24-hydroxytetracosanoic acid and tetracosane-1,24-dioic acids (Molina et al., 2006). C22:0-OH and C22:0-diol together constituted about $60 \%$ of the total fatty alcohol load in the seed suberin polyester. The analysis of seed coat suberin polyester in far single, far double and far triple mutants showed reductions of fatty alcohols in a chain length specific manner similar to that of root suberin. Among the double mutants, far1 far 4 and far 1 far 5 were the most affected, while the triple mutant had the highest $(\sim 80 \%)$ reduction in total fatty alcohol load. The tetrazolium salt (formazans) assays showed increased seed coat permeability in far double and far triple mutants compared to wild-type seeds. The far single mutants however, did not show any effect on seed coat permeability when tested for tetrazolium salt penetration into the seeds (data not shown) possibly because reduction in individual chain length fatty alcohol would have a little effect on the permeability properties. The far triple mutant was the most severely affected in seed coat permeability as there was about a $\sim 75 \%$ 
reduction in the fatty alcohol load in far triple mutant compared to far double mutants with $40-60 \%$ reduction and far 1 single mutant with $\sim 30 \%$ reduction in the total fatty alcohol load, indicating that permeance is proportional to the reduction in fatty alcohol load. The altered diffusion resistance of the suberin polyester may be more evident in seeds than in roots because in the root suberin polymer primary alcohols only constitute $\sim 5 \%$ of the total suberin monomer load, whereas in the seed coat suberin polyester primary alcohol, including diols, represent $\sim 17 \%$ of the total suberin monomer load. In most plant extracellular lipid barriers, waxes play a critical role as a barrier for water passage (Molina et al., 2008). In seed coats, however, waxes are minor components (Beisson et al., 2007; Shao et al., 2007). Additionally, it was found using the tetrazolium dye test that permeability is almost entirely dependent on lipid polyester composition and not dependant on waxes (comparing waxed vs. dewaxed seed) (Molina et al., 2008). Furthermore, Arabidopsis mutants $a p-2-1, a p 2-7, a t t 1, f a t B$ and gpat5, which have altered seed polyester monomers composition (Debeaujon et al., 2000; Western et al., 2001; Molina et al., 2008; Beisson et al., 2007), showed increased permeability in dye penetration assays, indicating that lipid polyesters deposited in the seed coat play important roles in controlling permeability to tetrazolium dyes (Beisson et al., 2007). It has been shown that seed coat polyesters may also influence pathogen resistance in soybean and Arabidopsis (Shao et al., 2007; Beisson et al., 2007).

Abscisic acid (ABA) is generally known for its inhibitory action on physiological and biological functions. However, Cottle and Kolattukudy (1982) reported induction of suberization in cell walls of potato by $A B A$ treatment in potato 
medium tissue culture and found several fold increase in polymeric aliphatic and aromatic components along with hydrocarbons and fatty alcohols in the suberinassociated waxes in potato. It was also indicated that ABA induces certain key enzymes involved in suberization. However, a mechanism of action of ABA induced suberization at the molecular level is not well understood. Several studies on potato tubers have suggested that the abscisic acid (ABA) stress signal response may be involved in regulation of wound healing (Soliday et al., 1979; Lulai et al., 2008). In a recent study, an increased amount of $A B A$ was detected in the stem scar tissue of tomato fruits during wound healing and that the suberization is positively correlated with $\mathrm{ABA}$ content (Leide et al., 2012). This clearly indicates the in vivo involvement of $\mathrm{ABA}$ in the suberization-based wound healing process. $\mathrm{ABA}$ is an endogenous inhibitor of precocious germination during seed formation. It has been suggested previously that $A B A$ interferes with seed germination by changing the water status of the seed so that water uptake is inhibited similar to that of an osmotic stress (Schopfer et al., 1979). Garciarrubio et al. (1997) reported that ABA affects the germination of mature seeds and the growth of nascent seedlings of Arabidopsis thaliana by preventing the degradation of the seed storage proteins and restricting the availability of energy and metabolites. In our study, the germination rate of far mutant seeds decreased with increasing concentration of $\mathrm{ABA}$ in the media compared to wild-type seeds. It is possible that such a phenotype is evident due to increased exposure of the far mutant seed embryo to the ABA imbibed as a result of altered seed coat permeability. The triple mutant seeds were the most severely affected and were comparable to the effect with gpat5 seeds. These results 
indicate that fatty alcohols play a major role in developing diffusion resistance in seed coat suberin polyester.

In the far single mutants the total fatty alcohol levels in root aliphatic suberin are not much lower than wild-type, which may explain the absence of any obvious developmental or physiological phenotypes in these mutants. Further reduction of fatty alcohol loads in double and triple mutants did not obviously affect root growth even under salt stress and osmotic stress conditions (data not shown). The fatty alcohols in root suberin could not be completely eliminated due to the partial silencing of the FAR5 gene. There is also a possibility that complete reduction of fatty alcohols is lethal. On the other hand, an accumulation of fatty alcohols inside the cell also might be toxic to the cell, particularly in the absence of an efficient excretion mechanism. Such toxicity could also be the reason why we could not generate overexpression lines any of the FAR genes. Nonetheless, the double and triple far mutant lines reported will provide a means to further investigate for physiological defects using sensitive tests to measure water potential and ion mobility within roots. Pathogen susceptibility tests may also provide a better understanding of the biological role of fatty alcohols in plants. 


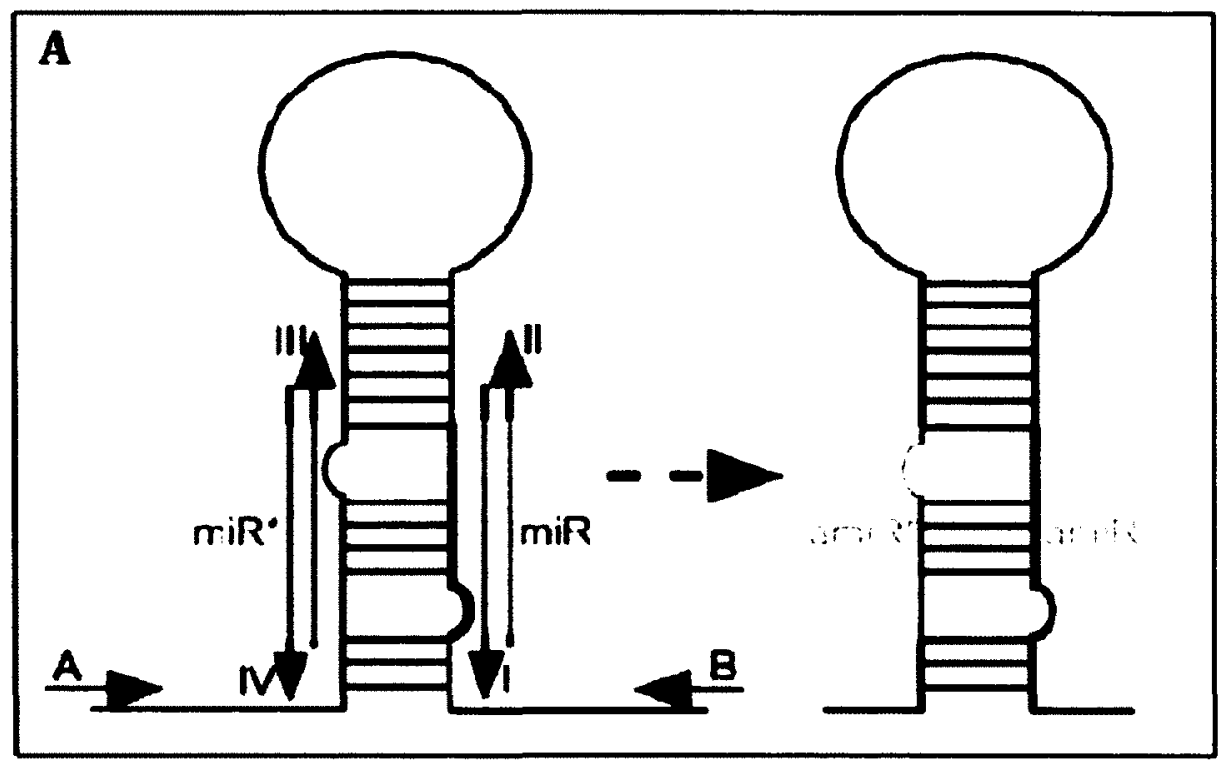

B.

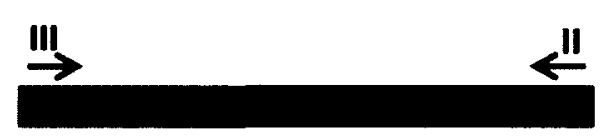

(b) Loop fragment

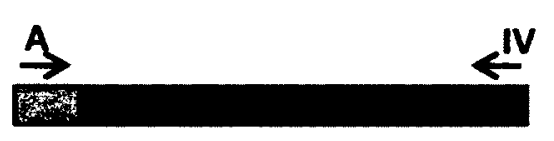

(a) ami*RNA fragment

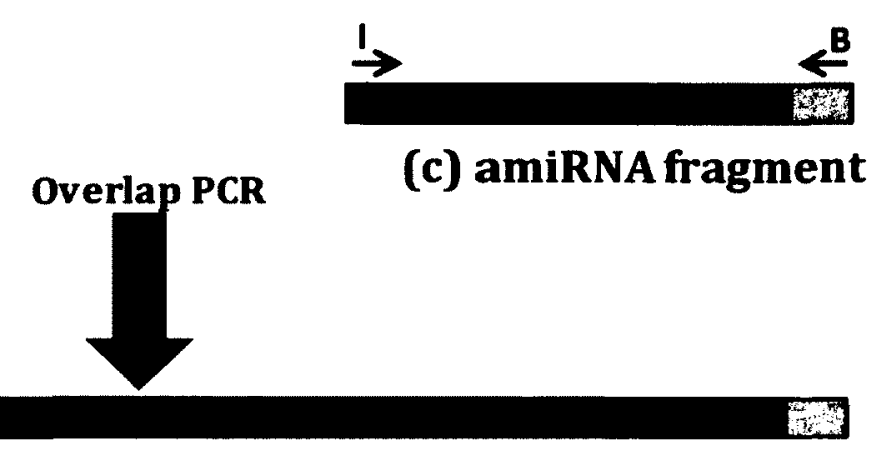

(d) ami*RNA:loop:amiRNA

Figure 3.1. Schematic showing PCR amplification of artificial miRNA precursors. (A) Amplification of the amiRNA fragments from the template plasmid pRS300 containing endogenous Arabidopsis MIR319a precursor. Diagram is not to scale. (B) Overlapping PCR strategy to generate the full length ami*RNA-loop-amiRNA fragment. A first round amplifies fragments (a) to (c), which are listed in the Table 3.1 below. These are subsequently fused in an overlap PCR (d). Gene specific oligonucleotides were: I: amiRNA forward; II: amiRNA reverse; III: amiRNA* forward; IV: amiRNA* reverse. Oligonucleotides A and B are based on the template plasmid sequence and are located outside of the multiple cloning site of pRS300 to generate the full artificial miRNA precursor DNA sequence. Modified from Ossowski et al., (2008). Image reproduced with permission from (C) The Plant Journal, 2008. 
Table 3.1. The oligonucleotide primer combination used for amplification of each amiRNA fragment, the template used, and their expected PCR product sizes to form the full-length amiRNA sequences (adapted from Ossowski et al., 2005). This strategy applies to the synthesis of both FAR4_amiRNA-Seq1 and FAR4_amiRNASeq2, as well as FAR5_amiRNA-Seq1 and FAR5_amiRNA-Seq2 (see Appendix Table 2 for primer sequences).

\begin{tabular}{|l|c|c|c|c|}
\hline \multicolumn{1}{|c|}{ Reaction } & $\begin{array}{c}\text { Forward } \\
\text { primer }\end{array}$ & $\begin{array}{c}\text { Reverse } \\
\text { primer }\end{array}$ & Template & $\begin{array}{c}\text { Size of PCR } \\
\text { product (bp) }\end{array}$ \\
\hline (a)5'arm- ami*RNA antisense & A & IV & pRS300 & 272 \\
\hline (b) Central loop & III & II & pRS300 & 171 \\
\hline (c) 3' arm-amiRNA sense & I & B & pRS300 & 298 \\
\hline (d) Loop structured amiRNA & A & B & a + b +c & 701 \\
\hline
\end{tabular}



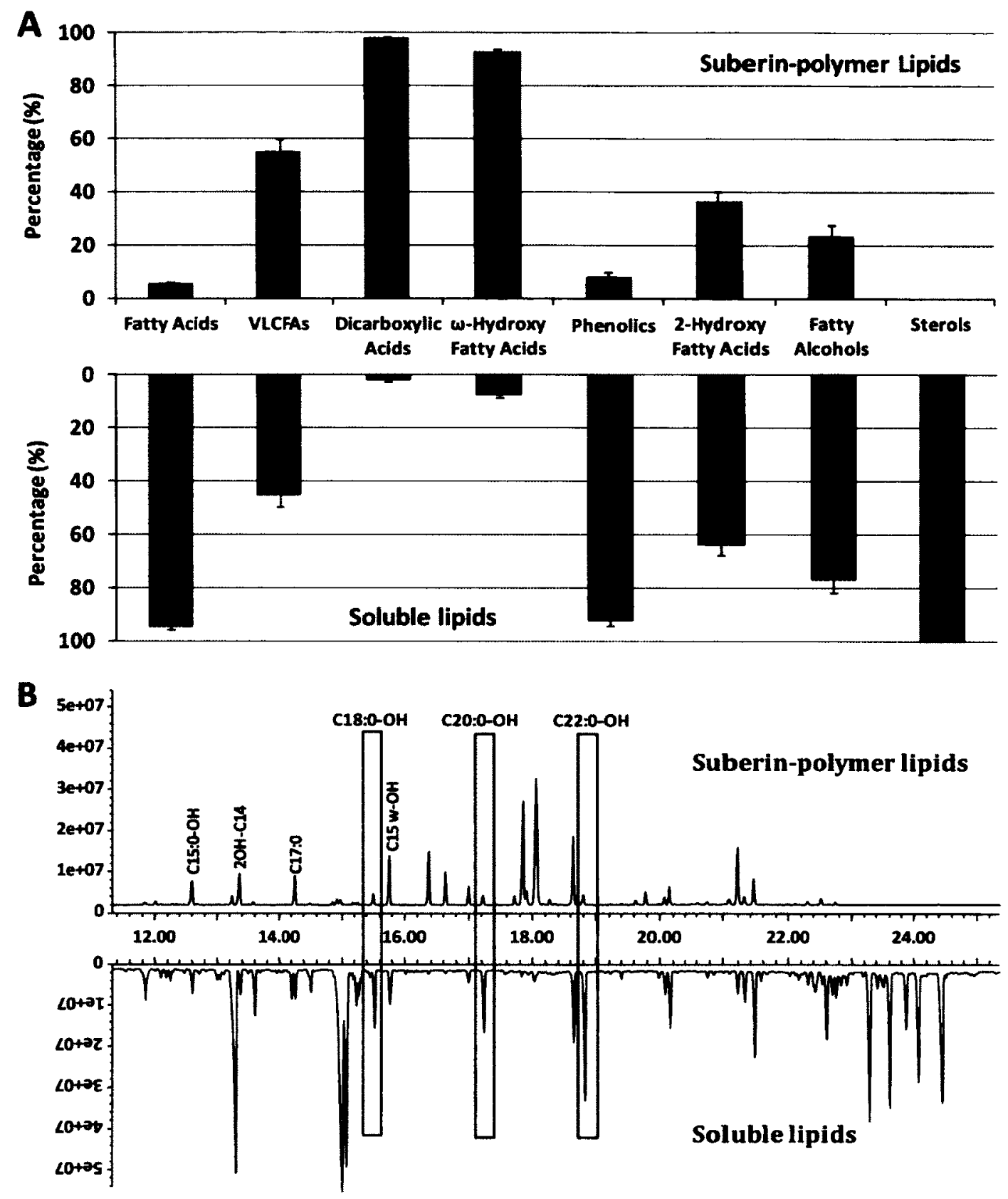

Figure 3.2. (A) Distribution of aliphatics (fatty acids and derivatives), phenolics and sterols in suberin polymer and soluble fraction of roots from 4-week-old wild-type plants, shown as percentage (\%) of total lipid content. Fatty acids $=\mathrm{C} 16 / \mathrm{C} 18$; VLCFAs $=$ very-long-chain fatty acids ( $>\mathrm{C} 18)$. Each value is the mean of three to four biological replicates. (B) GC chromatogram with peak relevant to fatty alcohol identified (in blocks) shown in both suberin polymer and soluble lipids fractions of roots of wild-type plants. 
Table 3.2. Fatty alcohol composition in insoluble suberin polymer fraction versus soluble fraction of roots of 4-week-old tissue culture-grown wild-type plants. Each value is the mean shown in $\mu \mathrm{g} / \mathrm{mg}$ dry residue $\pm S D$ of three to four biological replicates.

\begin{tabular}{|l|l|ll|}
\hline Fatty alcohol & \multicolumn{2}{|c|}{ Polymer fraction } & \multicolumn{2}{|c|}{ Soluble fraction } \\
\hline $\mathrm{C} 18: \mathrm{O}-\mathrm{OH}$ & $0.30 \pm 0.04$ & $0.53 \pm 0.13$ \\
\hline $\mathrm{C} 20: \mathrm{O}-\mathrm{OH}$ & $0.33 \pm 0.06$ & $0.82 \pm 0.10$ \\
\hline $\mathrm{C} 22: \mathrm{O}-\mathrm{OH}$ & $0.40 \pm 0.12$ & $2.03 \pm 0.12$ \\
\hline Total & $1.03 \pm 0.22$ & $3.38 \pm 0.35$ \\
\hline
\end{tabular}




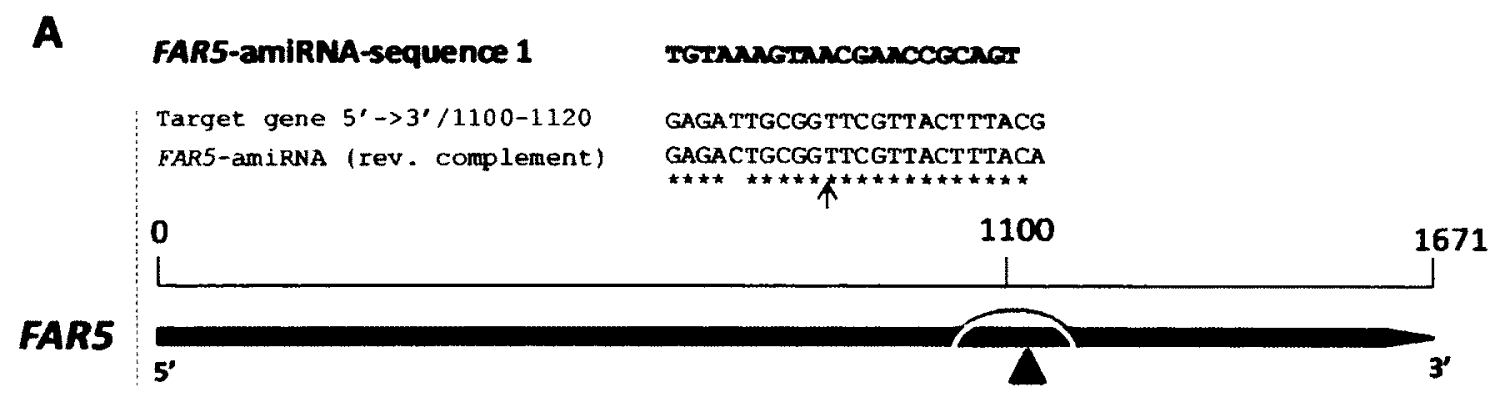

B
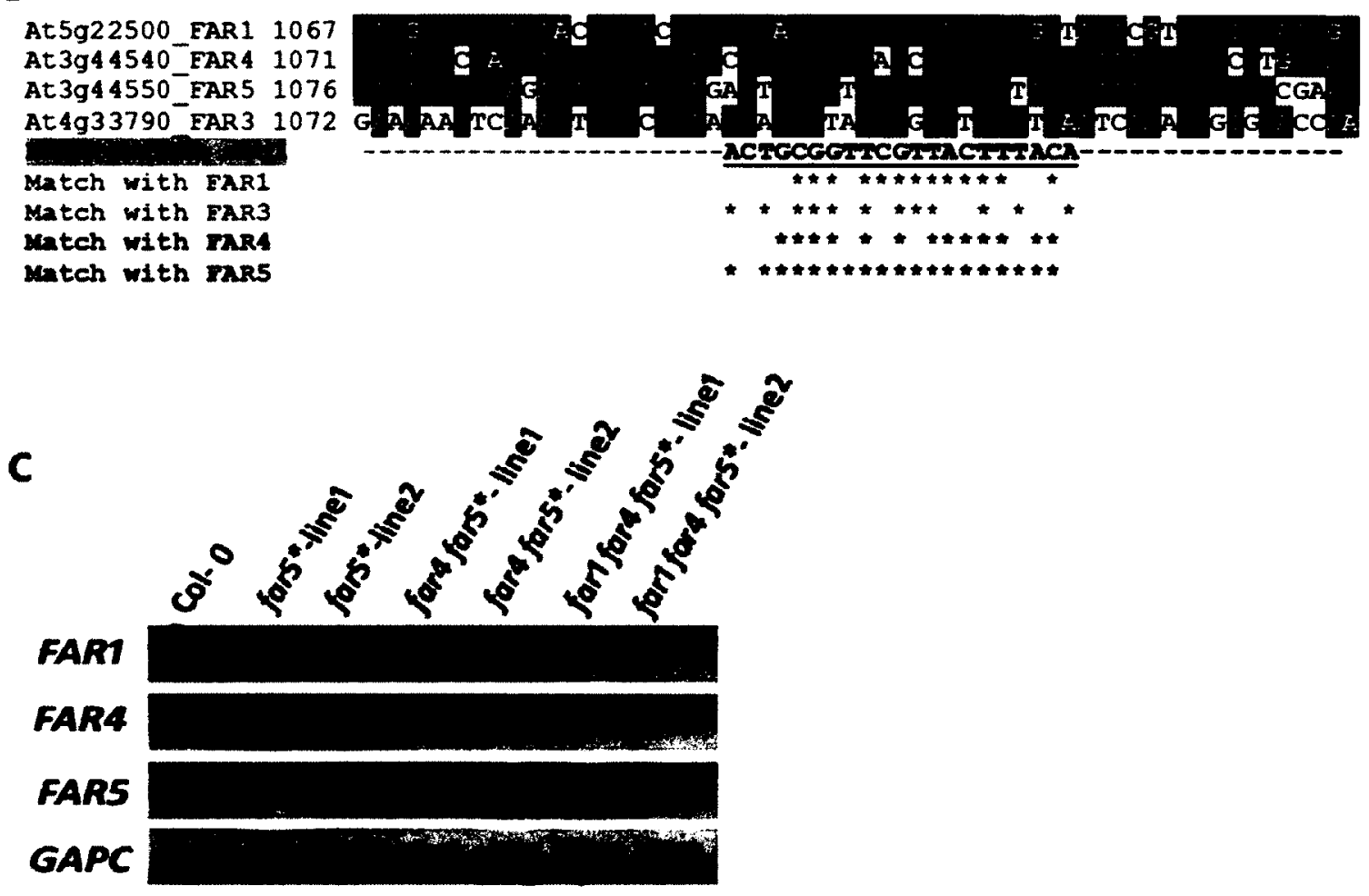

Figure 3.3. (A) Schematic showing the FAR5-amiRNA sequence-1 and the cleavage site on FAR5 cDNA sequence (red arrow). (B) The FAR5-amiRNA-sequence1 (reverse compliment) aligned with the coding sequence of root-expressing FARs: FAR1, FAR3, FAR4, and FAR5 gene. Ideal amiRNA sequences have no mismatches in positions 2 and 12, and only have 1 or 2 mismatches in positions $18-21$ (Ossowski et al., 2008). Cross-hybridization of FAR5-amiRNA-sequence1 with FAR1, FAR3 and FAR4 is unlikely because of several mismatches in the 5'-end of the amiRNA and the coding sequence. (C) Semi-quantitative RT-PCR transcript analysis of two independent transgenic lines of far 5 single, far 4 far 5 double, and far 1 far 4 far 5 triple mutants with FAR5-amiRNA-1 (*denotes the FAR5-amiRNA lines). Total RNA was isolated from roots of 15-days-old T4 seedlings grown in tissue culture. GAPC was used as a constitutively expressed loading control in panel $\mathrm{C}$. 


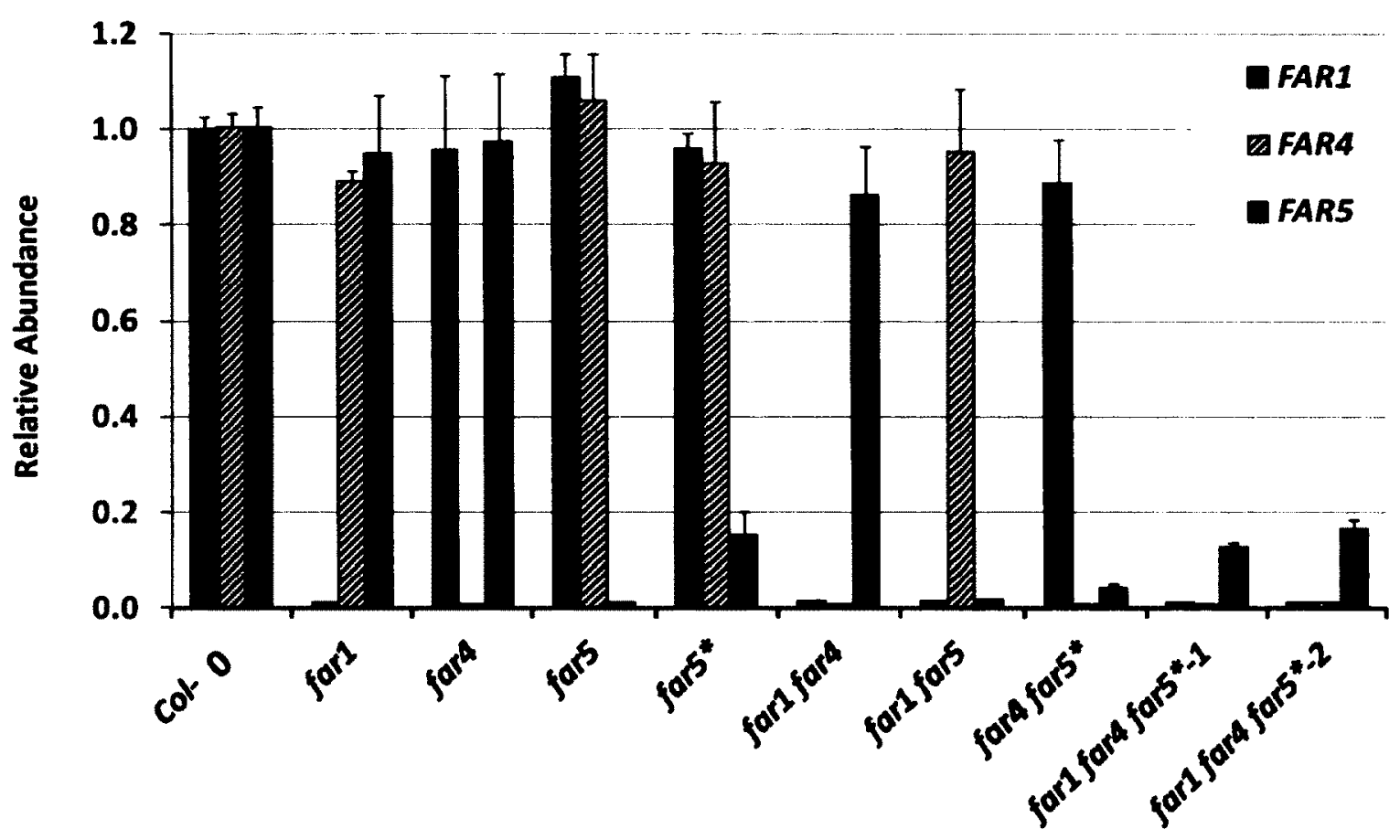

Figure 3.4. Transcript levels of FAR genes in wild type, far1, far4, far5, and far double, and far triple mutant lines determined by quantitative RT-PCR (*denotes FAR5 artificial-micro-RNA lines; 1 and 2 denotes two independent transgenic far triple mutant lines). Total RNA $(0.1 \mu \mathrm{g})$ isolated from the roots of 2-week-old T5 seedlings grown on MS agar plates was used for RT-PCR. Results are presented as relative transcript abundances to Col-0 and normalized through geometric averaging of three constitutively expressed genes (ACT2, GAPC and eIF-4A-1). The data represent means of two biological replicates, each with three technical replicates. 


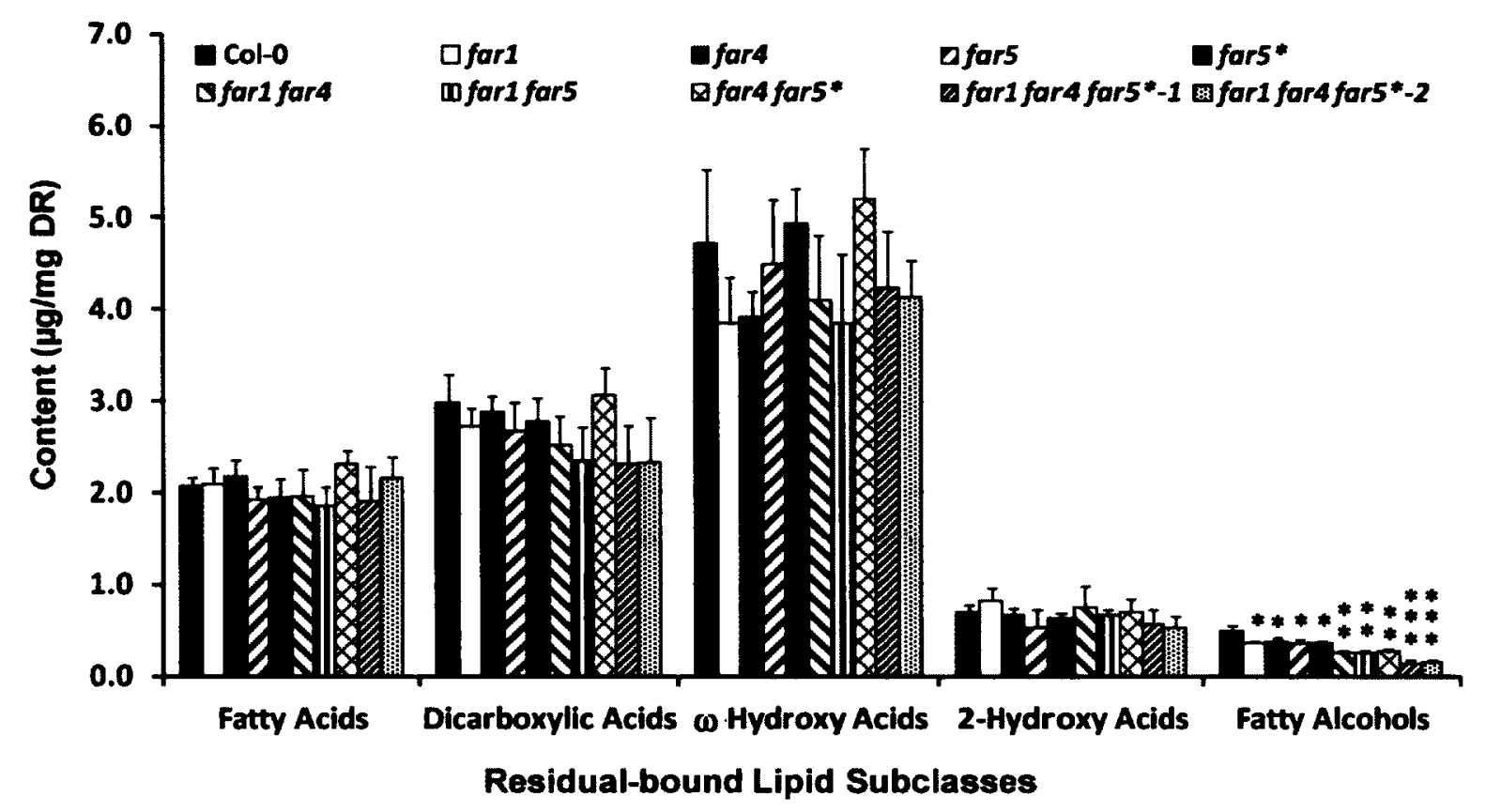

Figure 3.5. Aliphatic suberin residual-bound monomer composition in roots of wild-type, far1, far4, far5, far5* and far double, and far triple mutant plants (* denotes the lines with FAR5-amiRNA; 1 and 2 denotes two independent transgenic far triple mutant lines) where mean values are shown in $\mu \mathrm{g} / \mathrm{mg}$ delipidated dry residue (DR) \pm SD of three replicates. Suberin monomers were released by depolymerization of solvent-extracted roots of wild-type Arabidopsis and far mutant plants. The monomers were transmethylated and hydroxyl groups silylated before separation by GC and detection by flame ionization detector (FID). The pentadecanol (C15:0-OH), C15-hydroxypentadecanoic acid ( $\omega-\mathrm{OH}-\mathrm{C} 15: 0)$, and heptadecanoic acid (C17:0) were used as internal standards (IS) for quantification of various suberin monomers. Lipid classes of monomers are sorted by compound class along the $\mathrm{x}$-axis (different chain lengths added together). Errors bars indicate $\mathrm{SD}$, and significance was assessed by a Student $t$ test $\left({ }^{*}, \mathrm{P}<0.05 ;{ }^{* *}, \mathrm{P}<0.01\right.$; ${ }^{* * *}$, $\mathrm{P}<0.001$ ). 

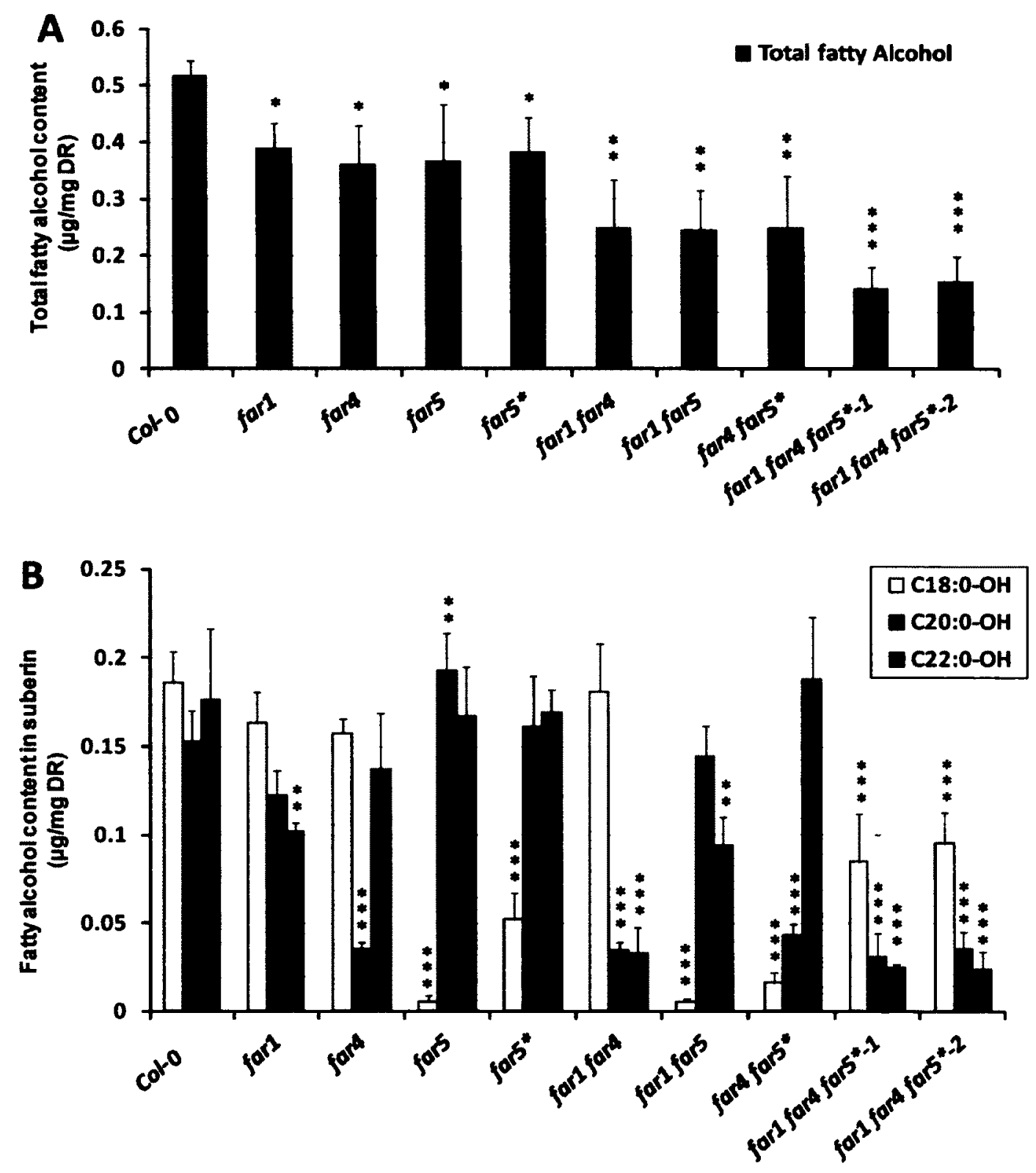

Figure 3.6. (A) Total fatty alcohol content of suberin in roots of wild-type, far1, far4, far 5 , far $5^{*}$ as well as far double and far triple mutant plants ( ${ }^{*}$ denotes the lines with FAR5-amiRNA; 1 and 2 denotes two independent transgenic far triple mutant lines). (B) Fatty alcohols sorted into individual chain lengths, where mean values are shown in $\mu \mathrm{g} / \mathrm{mg}$ delipidated dry residue (DR) of three replicates. Fatty alcohols were released by acid-catalysed transmethylation and hydroxyl groups' silylated before separation by GC and detection by flame ionization detector (FID). The pentadecanol (C15:0-OH) was used as the internal standard (IS) for quantification of fatty alcohols. Errors bars indicate SD, and significance was assessed by a Student $t$ test $\left({ }^{*}, \mathrm{P}<0.05,{ }^{* *}, \mathrm{P}<0.01 ;{ }^{* * *}, \mathrm{P}<0.001\right)$. 


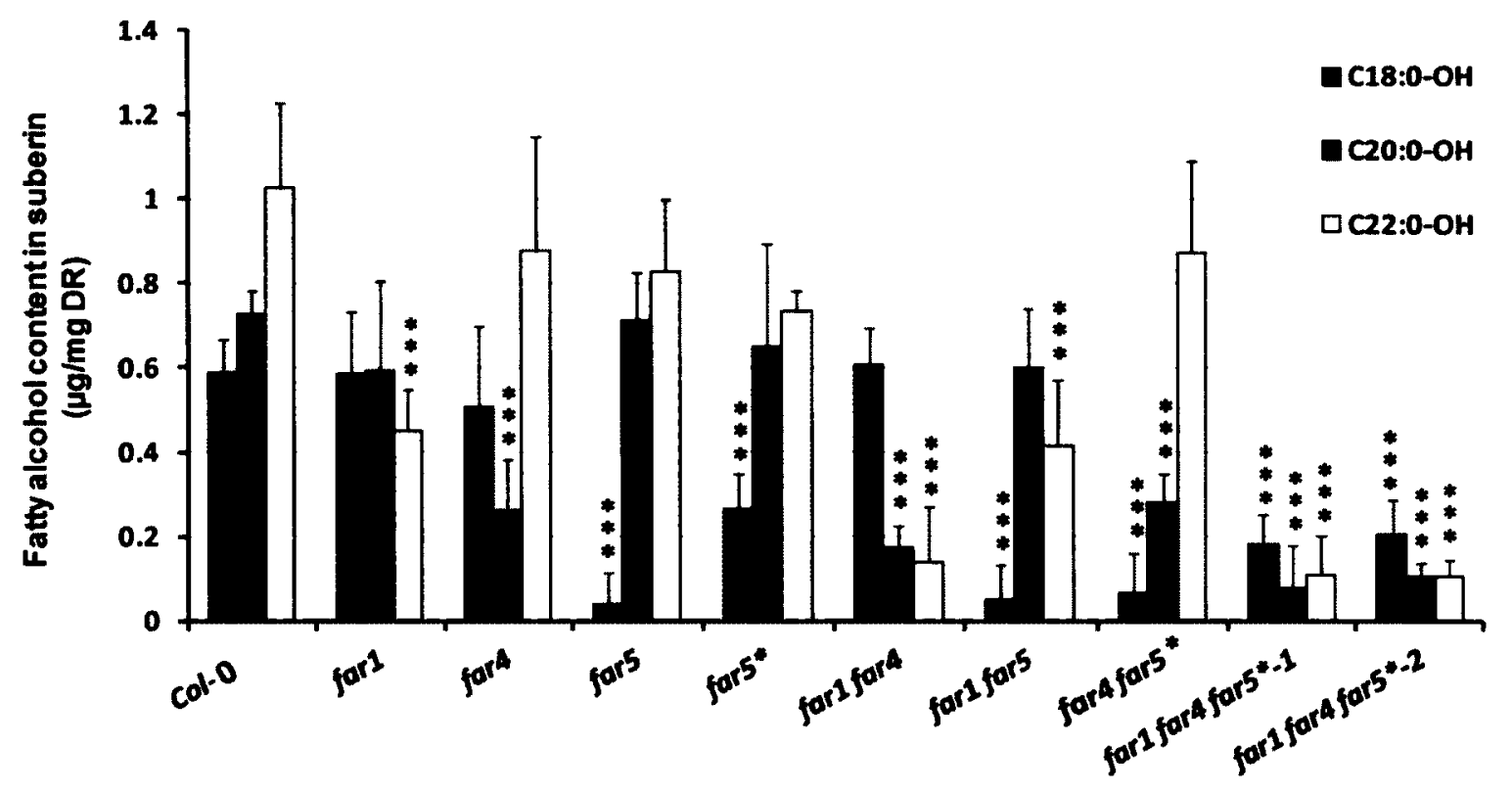

Figure 3.7. Fatty alcohol content of suberin in root delipidation (soluble) fraction of wild-type, far 1, far4, far5, far5* as well as far double and far triple mutant plants ( ${ }^{*}$ denotes the lines with FAR5-amiRNA; 1 and 2 denotes two independent transgenic far triple mutant lines). Fatty alcohols were released by acid-catalysed transmethylation and hydroxyl groups' silylated before separation by GC and detection by flame ionization detector (FID). The pentadecanol (C15:0-OH) was used as the internal standard (IS) for quantification of fatty alcohols. Fatty alcohols are sorted into individual chain lengths, where mean values are shown in $\mu \mathrm{g} / \mathrm{mg}$ delipidated dry residue (DR) of three replicates. Errors bars indicate SD, and significance was assessed by a Student $t$ test $\left(* *, \mathrm{P}<0.05,{ }^{* * *}, \mathrm{P}<0.01\right)$. 
Table 3.3. Total primary fatty alcohol loads (suberin polymer bound and delipidated soluble fraction combined) and total quantity of particular chain length fatty alcohol in the roots of 4-weeks-old tissue culture-grown wild-type plants. Each value is the mean shown in $\mu \mathrm{g} / \mathrm{mg}$ dry residue $\pm \mathrm{SD}$ of three biological replicates. ${ }^{*}$ indicates the lines with FAR5-amiRNA; 1 and 2 are two independent transgenic FAR5-amiRNA far triple mutant lines).

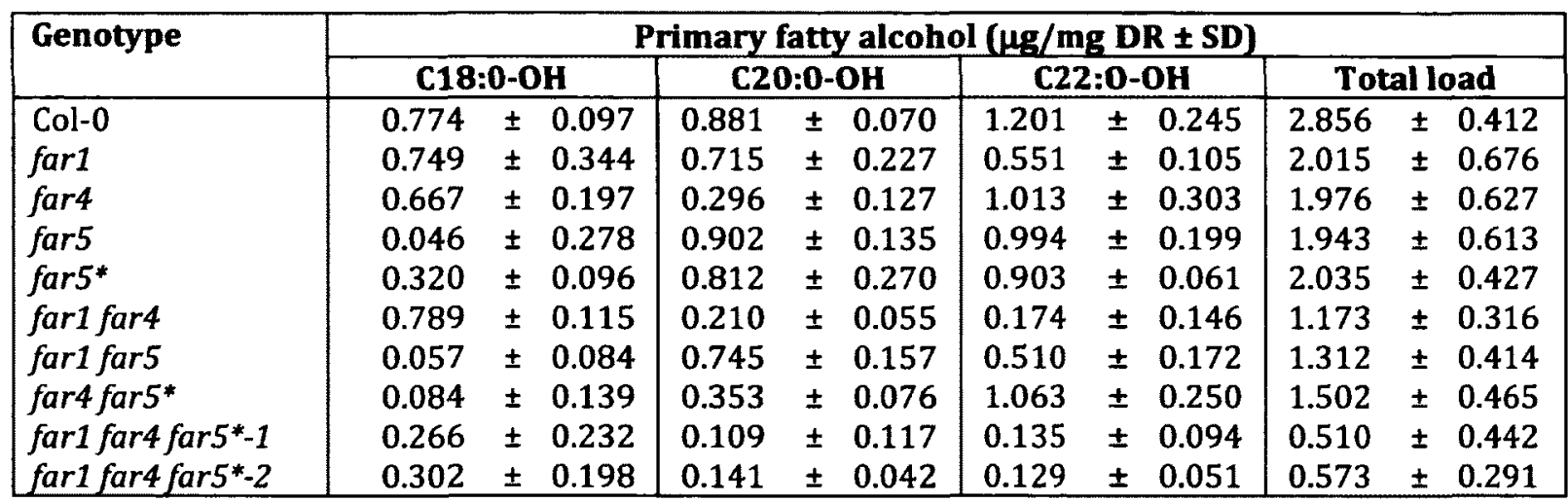

Table 3.4. Percent of fatty alcohol bound to suberin polymer in the roots of 4-weekold, tissue culture grown wild-type and far mutant plants. (*indicates the lines with FAR5-amiRNA; 1 and 2 are two independent transgenic FAR5-amiRNA far triple mutant lines).

\begin{tabular}{|l|c|c|c|}
\hline \multirow{2}{*}{ Genotype } & \multicolumn{3}{|c|}{ Primary fatty alcohol (\% Bound) } \\
\cline { 2 - 4 } & C18:0-0H & C20:0-OH & C22:0-0H \\
\hline Col-0 & 24.03 & 17.38 & 14.67 \\
far1 & 21.77 & 17.13 & 18.54 \\
far4 & 23.62 & 11.98 & 13.56 \\
far5 & 12.03 & 21.39 & 16.82 \\
far5 & 16.37 & 19.90 & 18.73 \\
far1 far4 & 22.95 & 16.64 & 18.98 \\
far1 far5 & 9.95 & 19.39 & 18.51 \\
far4 far5* & 19.78 & 12.21 & 17.71 \\
far1 far4 far5*-1 & 31.99 & 28.70 & 18.48 \\
far1 far4 far5 $5^{*}-2$ & 31.60 & 25.20 & 18.60 \\
\hline
\end{tabular}



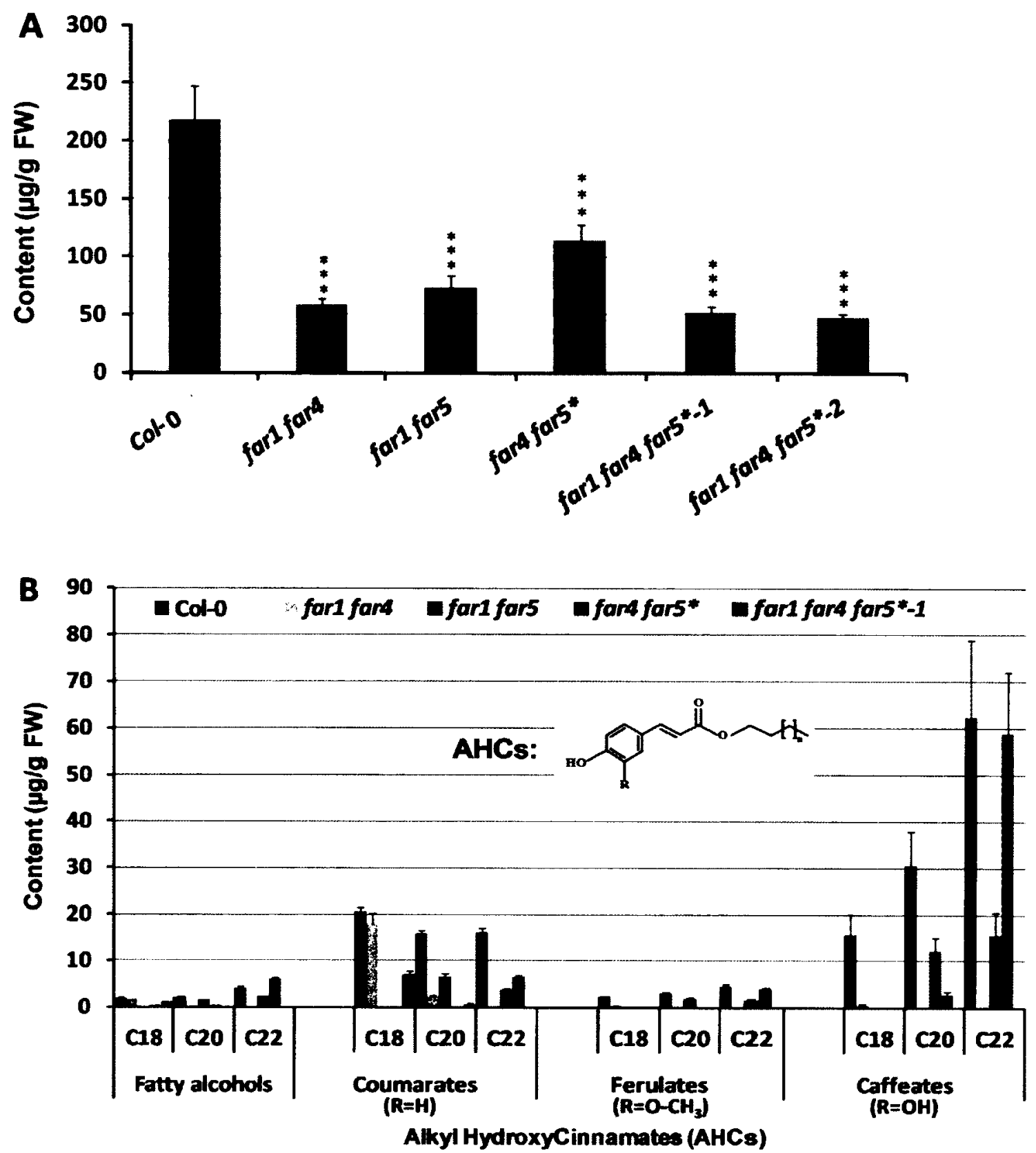

Figure 3.8. Levels of total amounts of waxes (A), and primary fatty alcohols and alkyl hydroxycinnamates (AHCs) (B) in root waxes of wild-type, far double, and far triple mutant plants (* denotes FAR5 artificial-micro-RNA lines). AHCs are sorted into individual chain lengths, where mean values are shown in $\mu \mathrm{g} / \mathrm{g}$ fresh weight (FW) of four replicates. Errors bars indicate SD. 

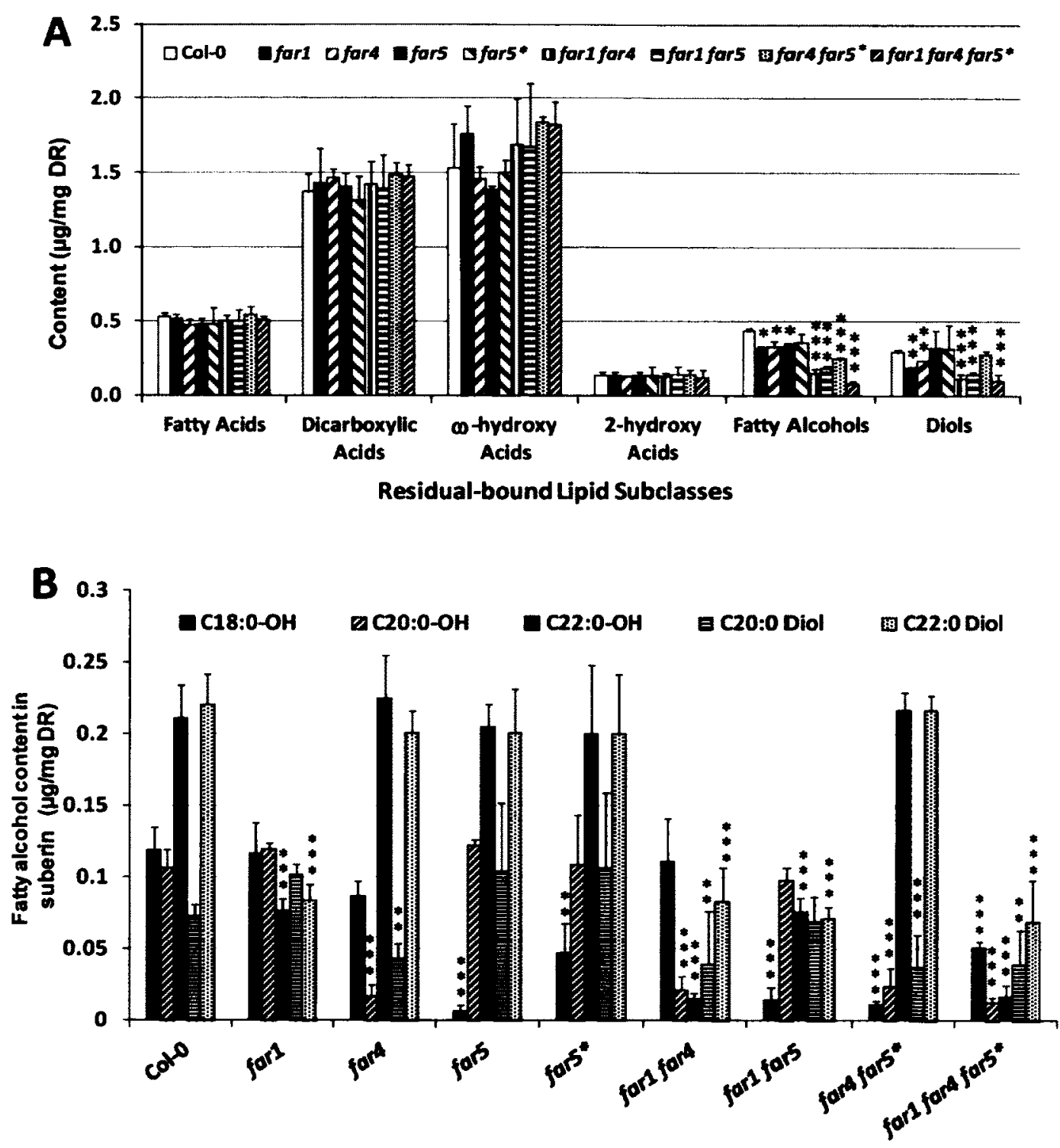

Figure 3.9. (A) Suberin polyester composition in seed coat of wild-type, far1, far4, far5, as well as far double and far triple mutant plants (*denotes the lines with FAR5-amiRNA). Suberin monomers were released by depolymerization of solvent-extracted seeds of wild-type Arabidopsis and far mutant plants. Monomers were released by acid-catalysed transmethylation and hydroxyl groups silylated before separation by GC and detection by flame ionization detector (FID). Lipid classes of monomers are sorted by compound class along the $x$-axis. The pentadecanol (C15:0-OH), C15-hydroxypentadecanoic acid ( $\omega-\mathrm{OH}-\mathrm{C} 15: 0)$, and heptadecanoic acid (C17:0) were used as the internal standards (IS) for quantification of various suberin monomers. (B) Amounts of seed coat suberin-associated fatty alcohols, sorted into individual chain lengths in Col-0 and far mutants. The pentadecanol $(\mathrm{C} 15: 0-0 \mathrm{H})$ was used as internal standards (IS) for quantification of fatty alcohols. The values in $\mu \mathrm{g} / \mathrm{mg}$ delipidated dry residue (DR) are means of three replicates \pm SD. Significance was assessed by a Student $t$ test $\left({ }^{*}, P<0.05\right.$; **, $\left.\mathrm{P}<0.01 ;{ }^{* * *}, \mathrm{P}<0.001\right)$. 


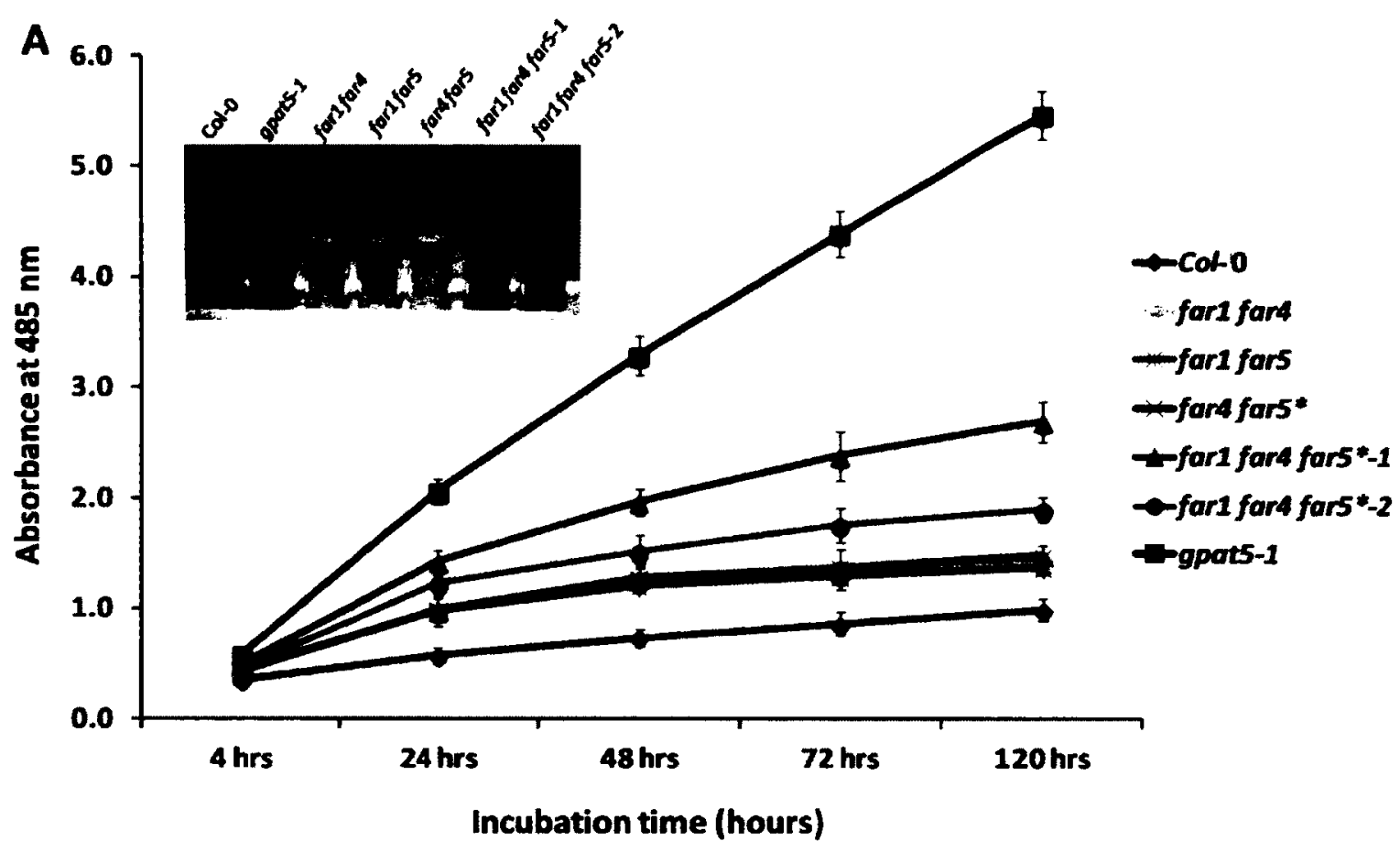

B

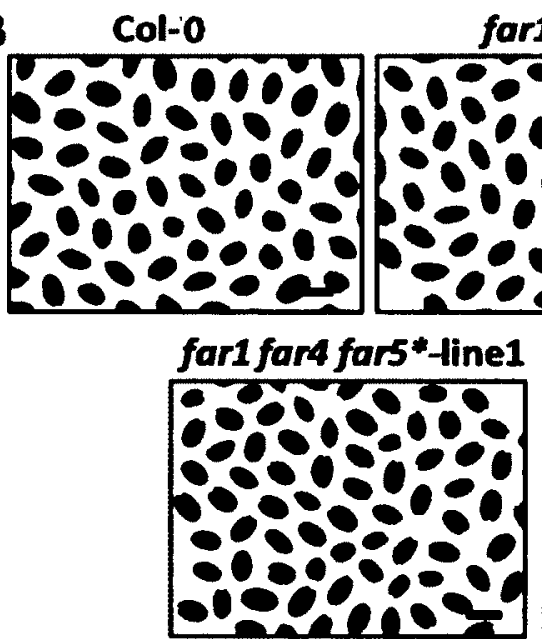

far1 far4 far1 far5 far4far5*

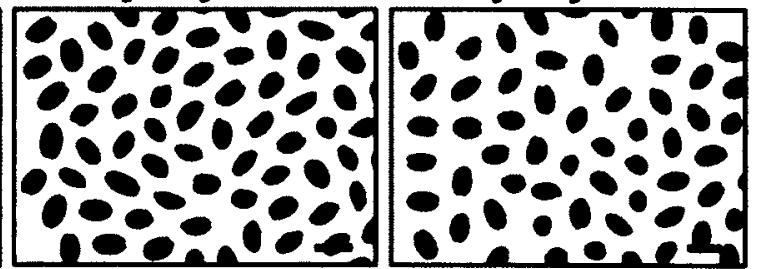

gpat5
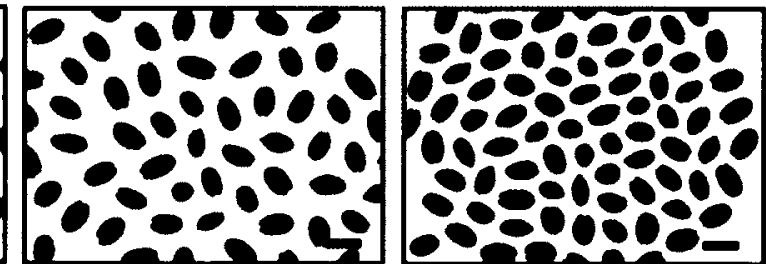

Figure 3.10. Tetrazolium penetration assays. (A) Time course of formazans produced by reduction of tetrazolium salts by the embryos of Arabidopsis wild-type (WT) and far double and triple mutants. The higher amount of formazan accumulation indicates increased seed coat permeability (inset shows the extracted formazans at $72 \mathrm{hr}$ time point). The data is mean value of three replicates shown as measure of absorbance at $485 \mathrm{~nm}$. Errors bars indicate SD (*denotes FAR5 artificialmicro-RNA lines; 1 and 2 denotes two independent far triple mutant lines). (B) Staining pattern in seeds of Col-0 and far mutants incubated in 1\% tetrazolium red at $30^{\circ} \mathrm{C}$ and imaged at the end of $48 \mathrm{hrs}$ (scale bar $=500 \mu \mathrm{m}$ ). 


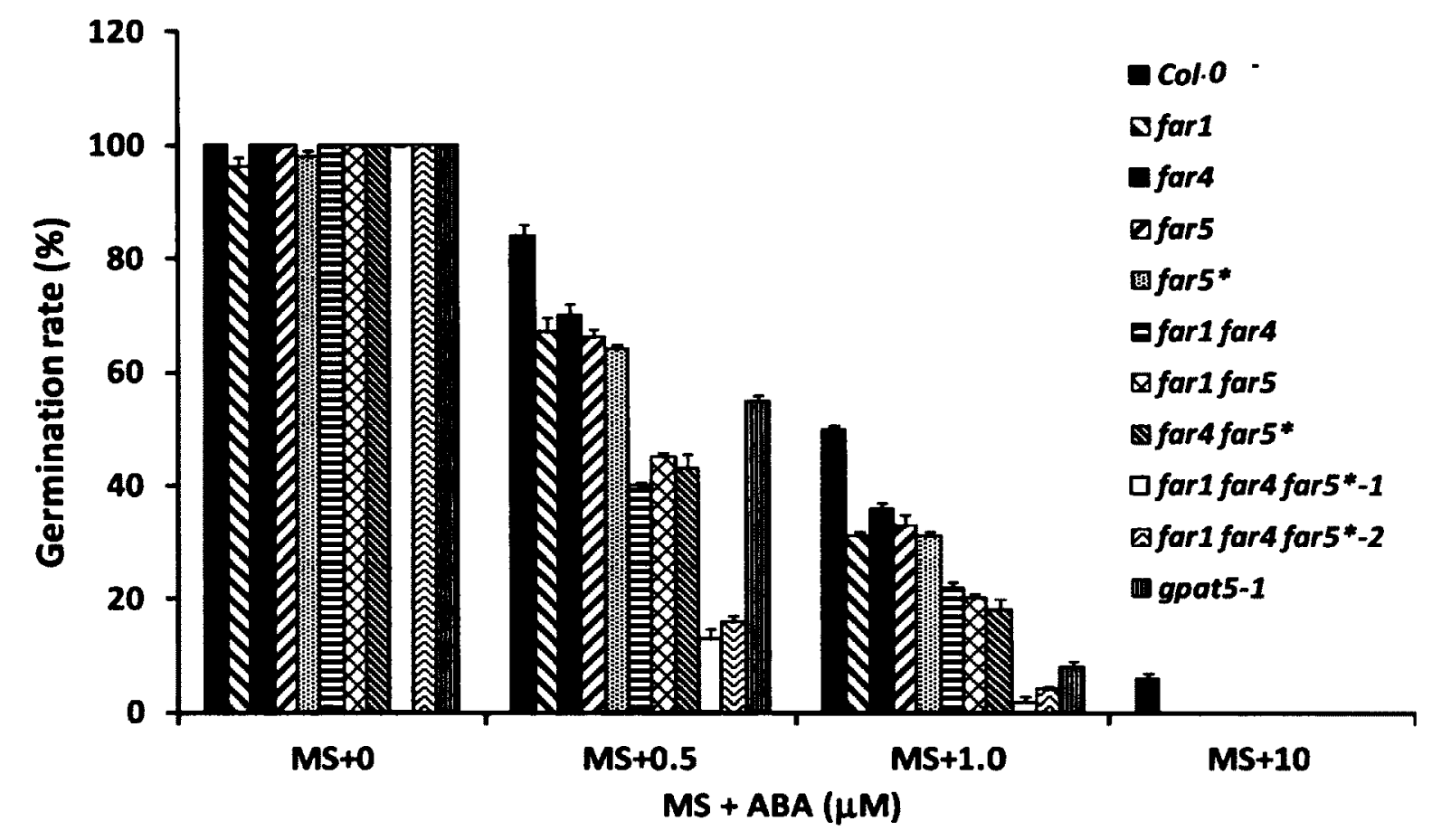

Figure 3.11. Seed germination rate of wild type, far 1 , far4, far 5 , and far double, and far triple mutants subjected to varying abscisic acid (ABA) concentration. The seed germination percentage was recorded 4 days after placing seeds on MS plates supplemented with $\mathrm{ABA}$ at concentrations of $0.5 \mu \mathrm{M}, 1.0 \mu \mathrm{M}$ and $10 \mu \mathrm{M}$. Values are the means of total 6 replications from two independent experiments, each with three replicates of 100 seeds per plate and carried out using different seed batches. The data is shown in percentage of total seeds sown. Errors bars indicate SD. ( ${ }^{*}$ denotes FAR5-amiRNA lines). 


\subsection{Supplemental data}

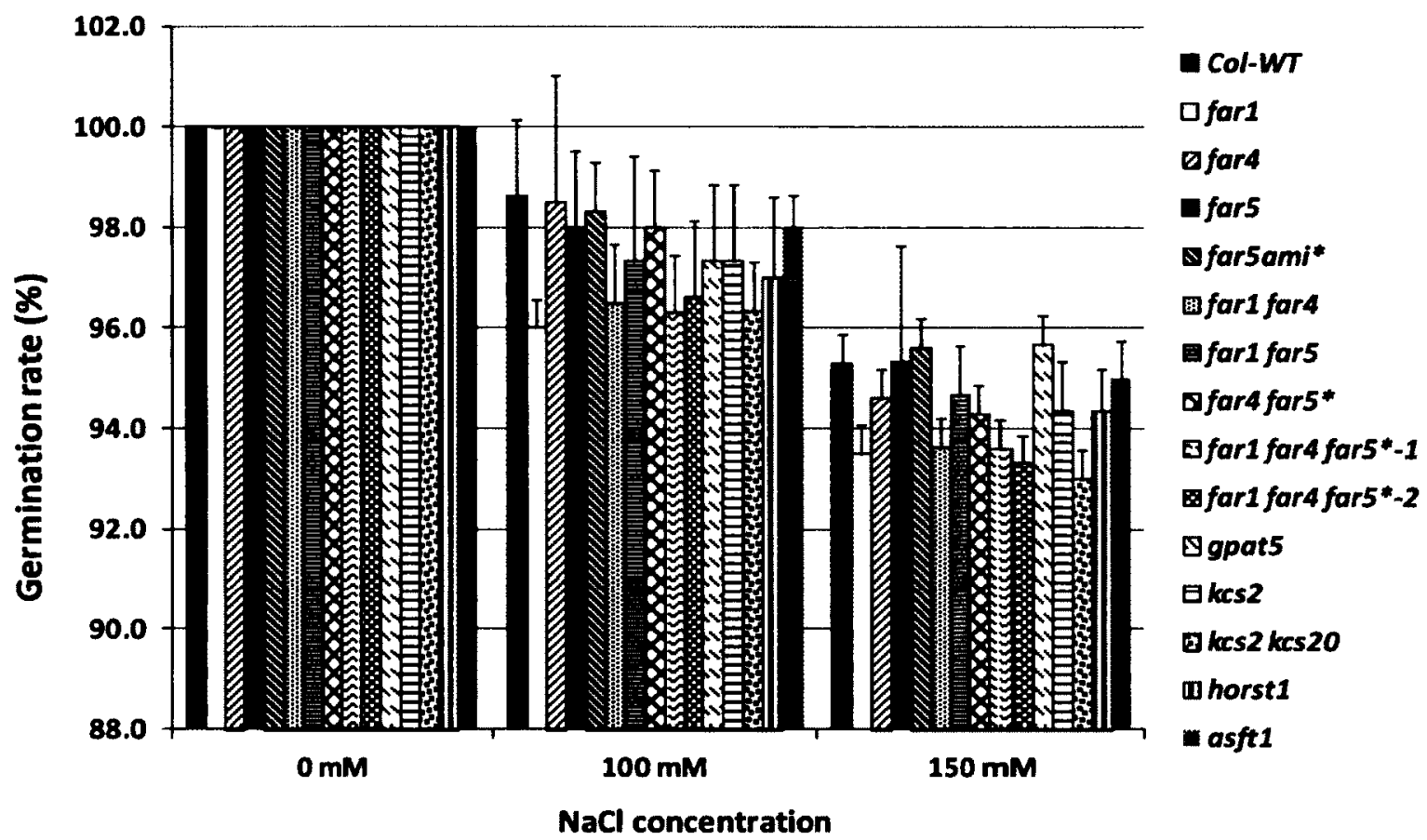

Figure S3.1. Seed germination rate of wild type, far1, far4, far5, far double, and far triple mutants, and other suberin mutants subjected to varying salt $(\mathrm{NaCl})$ concentration. The seed germination percentage was recorded 4 days after placing seeds on MS plates supplemented with $\mathrm{NaCl}$ at concentrations of $0 \mathrm{mM}, 100 \mathrm{mM}$ and $150 \mathrm{mM}$. Values are the means of total 6 replications from two independent experiments, each with three replicates of 100 seeds per plate and carried out using different seed batches. The data is shown in percentage of total seeds sown. Errors bars indicate SD. (*denotes FAR5-amiRNA lines). 

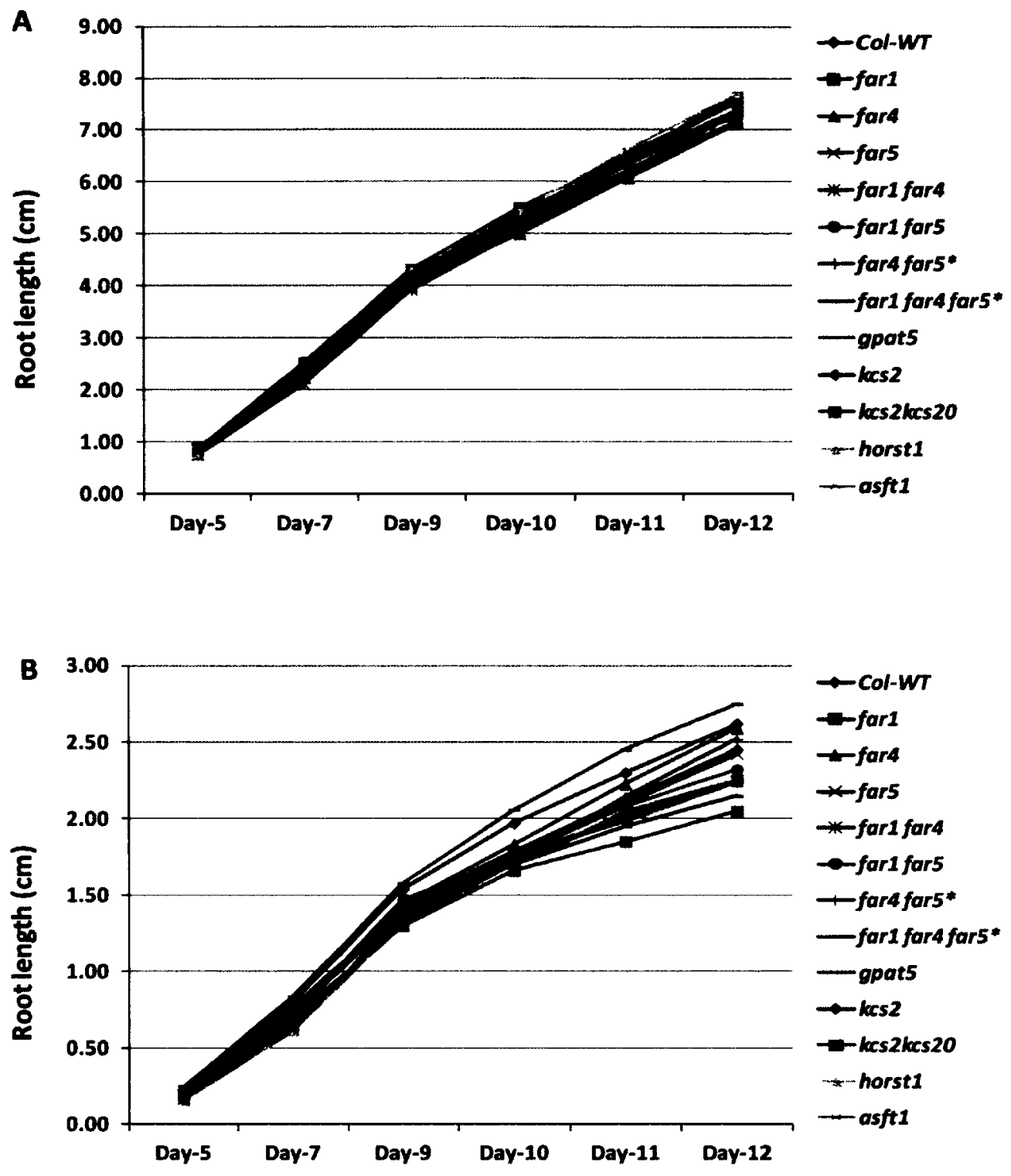

Figure S3.2. Primary root growth of wild type, far1, far4, far5, far double, and far triple mutants, and other suberin mutants subjected to salt $(\mathrm{NaCl})$ stress. The root length was recorded every alternate day after placing seeds on MS plates supplemented with $\mathrm{NaCl}$ at concentrations of $0 \mathrm{mM}(\mathrm{A})$ and $100 \mathrm{mM}(\mathrm{B})$. Values are the means of total 10 seedlings from three replications. The data is shown in centimeter of root length. 
Table S3.1: Composition of residual bound lipids in roots of wild-type, far1, far4, far5 and far double and far triple mutant plants. Each value is the mean shown in $\mu \mathrm{g} / \mathrm{mg}$ delipidated dry weight of residue $\pm \mathrm{SD}$ of three biological replicates.

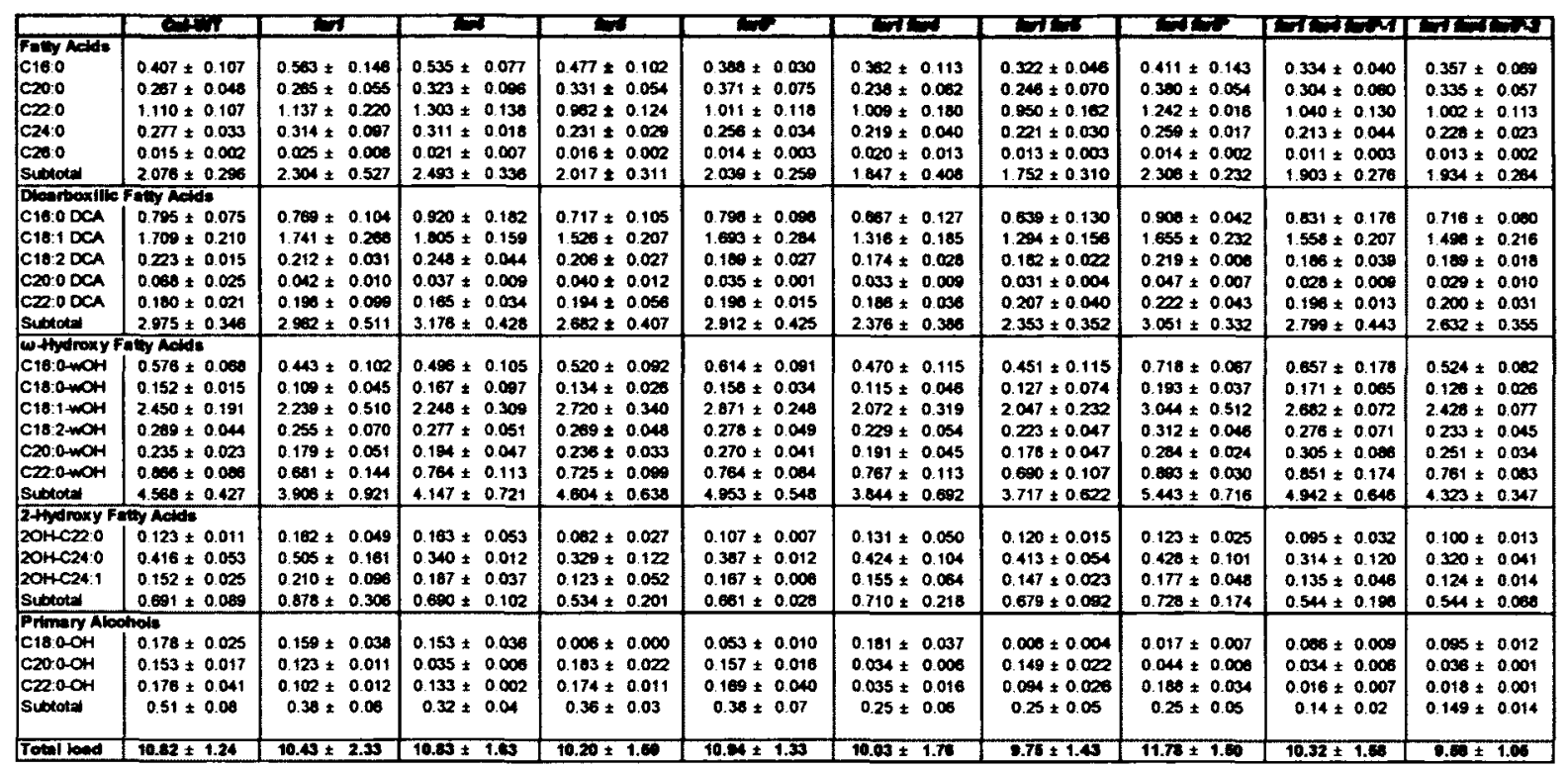


Table-S3.2: Composition of suberin-associated waxes in roots of wild-type, and far double and far triple mutant plants. Each value is the mean shown in $\mu \mathrm{g} / \mathrm{mg}$ delipidated fresh weight of residue $\pm S E$ of three biological replicates.

\begin{tabular}{|c|c|c|c|c|c|c|}
\hline & Oort & $\operatorname{sing}$ & 16 & $\ln \sin ^{\circ}$ & 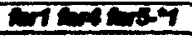 & nombers \\
\hline Alkan-1-ols & $\begin{array}{l}1.959 \pm 0.277 \\
2.187 \pm 0.287 \\
4.240 \pm 0.420 \\
\end{array}$ & $\begin{array}{l}1.573 \pm 0.082 \\
0.277 \pm 0.027 \\
0.119 \pm 0.013 \\
\end{array}$ & $\begin{array}{l}0.000 \pm 0.000 \\
1.598 \pm 0.166 \\
2.355 \pm 0.099 \\
\end{array}$ & $\begin{array}{l}0.352 \pm 0.166 \\
0.482 \pm 0.047 \\
6.052 \pm 0.253 \\
\end{array}$ & $\begin{array}{l}1.105 \pm 0.078 \\
0.252 \pm 0.031 \\
0.150 \pm 0.030 \\
\end{array}$ & $\begin{array}{l}0.904 \pm 0.043 \\
0.180 \pm 0.018 \\
0.158 \pm 0.040\end{array}$ \\
\hline $\begin{array}{l}C 16 \\
C 18 \\
C 20 \\
C 22\end{array}$ & $\begin{array}{l}4.220 \pm 0.366 \\
1.276 \pm 0.589 \\
0.155 \pm 0.125 \\
0.569 \pm 0.336 \\
\end{array}$ & $\begin{array}{l}5.374 \pm 0.688 \\
1.020 \pm 0.275 \\
0.081 \pm 0.064 \\
1.116 \pm 0.377 \\
\end{array}$ & $\begin{array}{l}4.506 \pm 1.040 \\
1.810 \pm 1.178 \\
0.016 \pm 0.006 \\
0.463 \pm 0.083\end{array}$ & $\begin{array}{l}5.338 \pm 0.661 \\
2.189 \pm 0.957 \\
0.448 \pm 0.135 \\
1.190 \pm 0.348 \\
\end{array}$ & $\begin{array}{l}5.771 \pm 0.783 \\
2.679 \pm 1.204 \\
0.425 \pm 0.234 \\
4.028 \pm 1.539\end{array}$ & $\begin{array}{l}5.654 \pm 0.618 \\
2.788 \pm 1.028 \\
0.396 \pm 0.139 \\
4.565 \pm 0.873\end{array}$ \\
\hline $\begin{array}{l}\text { C28:1 } \\
\text { C29:1 }\end{array}$ & $\begin{array}{r}4.642 \pm 0.475 \\
16.058 \pm 2.212 \\
\end{array}$ & $\begin{array}{r}3.629 \pm 0.685 \\
14.840 \pm 1.353 \\
\end{array}$ & $\begin{array}{r}3.869 \pm 0.159 \\
11.729 \pm 0.845 \\
\end{array}$ & $\begin{array}{r}4.768 \pm 0.098 \\
14.723 \pm 1.530 \\
\end{array}$ & $\begin{array}{r}4.054 \pm 0.250 \\
14.327 \pm 1.127 \\
\end{array}$ & $\begin{array}{r}3.786 \pm 0.347 \\
13.182 \pm 1.715 \\
\end{array}$ \\
\hline \begin{tabular}{rr|} 
MACs & in2-c22 \\
m1 $(3)-C 22$ \\
\end{tabular} & $\begin{array}{l}3.444 \pm 0.507 \\
8.111 \pm 3.853 \\
\end{array}$ & $\begin{array}{l}4.413 \pm 0.383 \\
4.400 \pm 0.443 \\
\end{array}$ & $\begin{array}{l}2.904 \pm 0.345 \\
2.438 \pm 0.146 \\
\end{array}$ & $\begin{array}{l}3.253 \pm 0.208 \\
2.819 \pm 0.231 \\
\end{array}$ & $\begin{array}{l}4.828 \pm 0.570 \\
4.849 \pm 0.178 \\
\end{array}$ & $\begin{array}{l}4.189 \pm 0.278 \\
4.183 \pm 0.259 \\
\end{array}$ \\
\hline Coumarates & $\begin{array}{l}20.560 \pm 0.997 \\
15.851 \pm 0.740 \\
16.174 \pm 0.859 \\
\end{array}$ & $\begin{array}{r}18.121 \pm 2.103 \\
2.460 \pm 0.274 \\
0.000 \pm 0.000 \\
\end{array}$ & $\begin{array}{l}0.000 \pm 0.000 \\
6.593 \pm 0.602 \\
3.862 \pm 0.191 \\
\end{array}$ & $\begin{array}{l}0.000 \pm 0.000 \\
0.000 \pm 0.000 \\
6.605 \pm 0.299 \\
\end{array}$ & $\begin{array}{l}8.680 \pm 0.424 \\
0.906 \pm 0.130 \\
0.000 \pm 0.000 \\
\end{array}$ & $\begin{array}{l}6.963 \pm 0.807 \\
0.811 \pm 0.125 \\
0.000 \pm 0.000 \\
\end{array}$ \\
\hline $\begin{array}{l}C 18 \\
C 20 \\
c 22 \\
\end{array}$ & $\begin{array}{l}2.435 \pm 0.077 \\
3.173 \pm 0.159 \\
4.722 \pm 0.485 \\
\end{array}$ & $\begin{array}{l}0.439 \pm 0.057 \\
0.000 \pm 0.000 \\
0.000 \pm 0.000 \\
\end{array}$ & $\begin{array}{l}0.000 \pm 0.000 \\
1.972 \pm 0.220 \\
1.633 \pm 0.252 \\
\end{array}$ & $\begin{array}{l}0.000 \pm 0.000 \\
0.000 \pm 0.000 \\
4.125 \pm 0.201 \\
\end{array}$ & $\begin{array}{l}0.000 \pm 0.000 \\
0.000 \pm 0.000 \\
0.000 \pm 0.000 \\
\end{array}$ & $\begin{array}{l}0.000 \pm 0.000 \\
0.000 \pm 0.000 \\
0.000 \pm 0.000 \\
\end{array}$ \\
\hline $\begin{array}{l}C 18 \\
C 20 \\
C 22\end{array}$ & $\begin{array}{l}15.672 \pm 4.268 \\
30.475 \pm 7.315 \\
62.300 \pm 16.772 \\
\end{array}$ & $\begin{array}{l}0.663 \pm 0.240 \\
0.000 \pm 0.000 \\
0.000 \pm 0.000 \\
\end{array}$ & $\begin{array}{r}0.000 \pm 0.000 \\
12.225 \pm 2.997 \\
15.546 \pm 5.072 \\
\end{array}$ & $\begin{array}{c}0.000 \pm 0.000 \\
2.928 \pm 0.657 \\
58.996 \pm 13.072\end{array}$ & $\begin{array}{l}0.298 \pm 0.145 \\
0.000 \pm 0.000 \\
0.000 \pm 0.000 \\
\end{array}$ & $\begin{array}{l}0.173 \pm 0.038 \\
0.000 \pm 0.000 \\
0.000 \pm 0.000 \\
\end{array}$ \\
\hline Total & $218.224 \pm 29.185$ & $58.525 \pm 4.641$ & $73.519 \pm 10.099$ & $114.268 \neq 13.484$ & $52.351 \pm 5.422$ & $47.933 \pm 3.257$ \\
\hline
\end{tabular}


Table-S3.3: Composition of residual bound lipids in seed coat of wild-type, far1, far4, far 5 and far double and far triple mutant plants. Each value is the mean shown in $\mu \mathrm{g} / \mathrm{mg}$ delipidated dry weight of residue \pm SD of three biological replicates.

\begin{tabular}{|c|c|c|c|c|c|c|c|c|c|}
\hline & EFT & 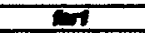 & $=$ & 2 & 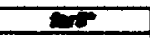 & 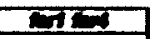 & 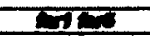 & 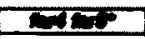 & $n=I-\mu$ \\
\hline Fatty Ack & $020 \pm$ & $68 \pm 0.016$ & $153 t$ & $144 \div 0.037$ & $154+0.007$ & $171 \pm 0.031$ & $01+009$ & & \\
\hline C20:0 & $0.05 \pm 0.00$ & $049 \pm 0.012$ & $54 \pm 0.003$ & $050 \pm 0.002$ & $.054 \pm 0.020$ & $0.047 \pm 0.000$ & $0.043 \pm 0.005$ & $0.047 \pm 0.002$ & $\begin{array}{l}0.173 \pm 0.006 \\
0.045 \pm 0.001\end{array}$ \\
\hline C22:0 & $0.11 \div 0.01$ & $105 \pm 0.016$ & $.096 \pm 0.007$ & $.092 \pm 0.002$ & $088 \pm 0.036$ & $.080 \pm 0.028$ & $0.091 \neq 0.011$ & $119 \neq 0.003$ & $0.102 \pm 0.013$ \\
\hline C24:0 & $0.14 \pm 0.01$ & $0.152 \neq 0.018$ & $0.143 \pm 0.025$ & $0.164 \pm 0.016$ & $129 \pm 0.030$ & $146 \pm 0.027$ & $0.126 \pm 0.010$ & $141 \pm 0.010$ & $0.142 \pm 0.013$ \\
\hline 2060 & $0.04 \pm 0.00$ & $0.040 \pm 0.010$ & $0.026 \pm 0.009$ & $0.029 \pm 0.008$ & $0.055 \pm 0.033$ & $0.059 \pm 0.019$ & $0.037 \pm 0.006$ & $.047 \pm 0.011$ & $0.047 \pm 0.016$ \\
\hline blotal & $0.53 \pm 0.06$ & $51 \pm 0.07$ & $47 \pm 0.0$ & \pm 0.06 & $48 \pm 0.13$ & $50 \pm 0.10$ & $0 \pm 0.08$ & $54+003$ & $51 \pm 0.05$ \\
\hline \multicolumn{2}{|c|}{ Dicarboxilic Faty Aclds } & & & & & & & & \\
\hline $16: 0 \mathrm{~L}$ & $0.135 \pm 0.011$ & $2 \pm 0.027$ & .004 & 0.013 & $5 \pm 0.031$ & $3 \pm 0.009$ & $135 \pm 0.024$ & $155 \pm 0.011$ & $67 \pm 0.026$ \\
\hline 5 & $2 \pm 0.064$ & $12 \pm 0.080$ & $6 \pm 0.021$ & $6 \pm 0.034$ & $58 \pm 0.055$ & $34 \pm 0.047$ & 0.256 & $292 \pm 0.010$ & $0.285 \pm 0.016$ \\
\hline A & $0.810 \neq 0.029$ & $0.794 \pm 0.092$ & $0.877 \pm 0.047$ & $31 \pm 0.055$ & $0.733 \pm 0.072$ & $0.754 \pm 0.065$ & $0.832 \pm 0.121$ & $839 \pm 0.063$ & $0.835 \pm 0.027$ \\
\hline A & $0.027 \pm 0.004$ & $.020 \pm 0.006$ & $0.022 \pm 0.001$ & $0 \pm 0.004$ & $0.022 \pm 0.004$ & $0.020 \pm 0.002$ & $.024 \pm 0.004$ & $.033 \pm 0.001$ & $0.021 \pm 0.010$ \\
\hline & $0.144 \pm 0.017$ & $168 \pm 0.029$ & $0.173 \pm 0.006$ & $5 \pm 0.028$ & $0.131 \pm 0.058$ & $0.187 \pm 0.022$ & $146 \pm 0.024$ & $164 \pm 0.012$ & $0.160 \pm 0.011$ \\
\hline Sublotal & $1.368 \pm 0.126$ & $25 \pm 0.246$ & $.462 \pm 0.0$ & $9 \neq 0.135$ & $09 \pm 0.220$ & $418 \pm 0.165$ & $393 \pm 0.228$ & $483 \pm 0.098$ & $.468 \neq 0.090$ \\
\hline \multicolumn{2}{|c|}{ wthriroxy Fatiy Acida } & & & & & & & & \\
\hline cis:o & $7 \pm 0.013$ & $3 \pm 0.028$ & $.151 \pm 0.008$ & $7 \pm 0.014$ & $88 \pm 0.035$ & $13 \pm 0.028$ & $0.183 \pm 0.029$ & $.168 \pm 0.006$ & $74 \pm 0.022$ \\
\hline$H$ & $0.296 \pm 0.030$ & $0.288 \pm 0.021$ & $274 \pm 0.021$ & $1 \pm 0.001$ & $268 \pm 0.022$ & $28 \pm 0.057$ & $0.241 \pm 0.072$ & $286 \pm 0.003$ & $299 \pm 0.057$ \\
\hline H & $0.294 \pm 0.059$ & $0.350 \pm 0.080$ & $0.203 \pm 0.007$ & $7 \pm 0.008$ & $4 \pm 0.019$ & $3 \pm 0.061$ & $0.333 \pm 0.076$ & $364 \pm 0.009$ & $0.350 \pm 0.029$ \\
\hline H & $0.389 \pm 0.200$ & $0.492 \pm 0.081$ & $0.380 \pm 0.017$ & $6 \pm 0.022$ & $0 \pm 0.006$ & $0.384 \pm 0.139$ & $455 \pm 0.175$ & $562 \pm 0.021$ & $0.522 \pm 0.032$ \\
\hline D- & $0.059 \pm 0.007$ & $0.065 \pm 0.002$ & $064 \pm 0.003$ & $6 \pm 0.007$ & $067 \pm 0.005$ & $12 \pm 0.005$ & 011 & 003 & $74 \pm 0.013$ \\
\hline OH & $0.346 \pm 0.027$ & $0.378 \pm 0.023$ & $0.387 \pm 0.028$ & $0 \pm 0.012$ & $373 \pm 0.024$ & $0.379 \pm 0.026$ & $0.407 \pm 0.069$ & $368 \pm 0.005$ & $.404 \pm 0.029$ \\
\hline Subtota & $1.531 \pm 0.335$ & $1.756 \pm 0.235$ & $1.459 \pm 0.003$ & $7 \pm$ & $99 \pm 0.111$ & $1.684 \pm 0.316$ & $1.673 \pm 0.432$ & $.838 \pm 0.047$ & $1.823 \pm 0.182$ \\
\hline \multicolumn{2}{|c|}{ 2+tydroxy Fathy Aclds } & & & & & & & & \\
\hline & $0.026 \pm$ & 0 & 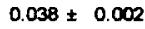 & $0+2-2$ & 0.025 & 07 & 111 & 114 & 0 \\
\hline & $3 \pm 0.0$ & $0.098 \pm 0.0$ & $090 \pm$ & \pm & \pm & 24 & 049 & 20 & \pm 0.028 \\
\hline Sublota: & $0.139 \pm$ & $38 \pm 0.025$ & $128 \pm 0.005$ & $32 \pm$ & $34 \pm 0.067$ & $125 \pm$ & $141 \pm 0.060$ & $142 \pm 0.034$ & $122 \pm 0.055$ \\
\hline \multicolumn{2}{|c|}{ Primany Ncohols } & & & & & & & & \\
\hline C18:0-OH & $0.119 \pm 0.016$ & $16 \pm 0.022$ & $0.087 \pm 0.010$ & $06 \pm 0.002$ & $0.047 \pm 0.021$ & $0.111 \pm 0.030$ & 0.009 & .003 & $0.051 \pm 0.005$ \\
\hline :O-OH & $0.106 \neq 0.013$ & $0.119 \pm 0.003$ & $0.016 \neq 0.001$ & $21 \pm 0.005$ & $0.109 \pm 0.035$ & $0.021 \pm 0.010$ & $0.098 \pm$ & 0.0 & $0.012 \neq 0.001$ \\
\hline 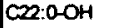 & $0.211 \pm 0.023$ & $0.077 \pm 0.008$ & $0.225 \pm 0.030$ & $0.205 \pm 0.0$ & $0.200 \pm 0.048$ & $0.015 \pm$ & 0.076 & 03 & $0.016 \neq 0.009$ \\
\hline diOL & $0.072 \pm 0.0$ & $0.101 \pm 0$. & $0.042 \pm 0.0$ & $3 \pm 0.0$ & $0.106 \pm 0.053$ & $9 \pm 0.038$ & 018 & 024 & $0.039 \pm 0.024$ \\
\hline $\mathrm{C22} \mathrm{diOL}$ & $0.220 \pm 0.022$ & $0.083 \pm 0.002$ & $0.200 \neq 0.016$ & $0.201 \pm 0.031$ & $0.199 \pm 0.072$ & $0.083 \pm 0.024$ & $0.071 \pm 0.009$ & $0.216 \pm 0.010$ & $0.088 * 0.030$ \\
\hline Subtotal & $0.73 \pm 0.0$ & 0. & $0.57 \pm 0.0$ & $0.64 \pm 0.1$ & $6 \pm 0.23$ & $0.27 \pm 0.10$ & $0.33 \pm$ & .05 & $0.19 \pm 0.07$ \\
\hline ond & $4.30+0.62$ & $4.33+0.62$ & $4.38 \pm 0.28$ & $4.34 \pm 0.40$ & $4.38 \pm 0.75$ & $4.30 \pm$ & $4.23 \pm$ & 0.01110 .20 & $.11 \pm 0.4$ \\
\hline
\end{tabular}


Supplementary Table-S3.4: Sequences of the final amiRNAs targeting FAR4 and FAR5 genes as well as the corresponding oligonucleotides primers used to generate these amiRNAs from the pRS300 plasmid template that contains the Arabidopsis miR319a backbone.

\begin{tabular}{|c|c|c|}
\hline FAR4 & $\begin{array}{l}\text { Sequence-1: } \\
\text { TCTATAATTACCAAGAAGCGT }\end{array}$ & $\begin{array}{l}\text { I) gaTCTATAATTACCAAGAAGCGTtctctctttgtattcc } \\
\text { II) gaACGCTTCTTGGTAATTATAGAtcaaagagaatcaatga } \\
\text { III) gaACACTTCTTGGTATTTATAGTtcacaggtcgtgatatg } \\
\text { IV) gaACTATAAATACCAAGAAGTGTtctacatatatattcct }\end{array}$ \\
\hline FAR4 & $\begin{array}{l}\text { Sequence-2: } \\
\text { TATCAACGGATATATCACGGG }\end{array}$ & $\begin{array}{l}\text { I) gaTATCAACGGATATATCACGGGtctctcttttgtattcc } \\
\text { II) gaCCCGTGATATATCCGTTGATAtcaaagagaatcaatga } \\
\text { III) gaCCAGTGATATATCGGTTGATTtcacaggtcgtgatatg } \\
\text { IV) gaAATCAACCGATATATCACTGGtctacatatatattcct }\end{array}$ \\
\hline FAR5 & $\begin{array}{l}\text { Sequence-1: } \\
\text { TGTAAAGTAACGAACCGCAGT }\end{array}$ & $\begin{array}{l}\text { I) gaTGTAAAGTAACGAACCGCAGTtctctcttttgtattcc } \\
\text { II) gaACTGCGGTTCGTTACTTTACAtcaaagagaatcaatga } \\
\text { III) gaACCGCGGTTCGTTTCTTTACTtcacaggtcgtgatatg } \\
\text { IV) gaAGTAAAGAAACGAACCGCGGTtctacatatatattcct }\end{array}$ \\
\hline FAR5 & $\begin{array}{l}\text { Sequence-2: } \\
\text { TCATATGCAATAATCACGCCG }\end{array}$ & $\begin{array}{l}\text { I) gaTCATATGCAATAATCACGCCGtctctcttttgtattcc } \\
\text { II) gaCGGCGTGATTATTGCATATGAtcaaagagaatcaatga } \\
\text { III) gaCGACGTGATTATTCCATATGTtcacaggtcgtgatatg } \\
\text { IV) gaACATATGGAATAATCACGTCGtctacatatatattcct }\end{array}$ \\
\hline \multicolumn{2}{|c|}{ pRS300 specific oligo primers } & $\begin{array}{l}\text { A) CTG CAAGGCGATTAAGTTGGGTAAC } \\
\text { B) GCG GATAACAATTTCACACAGGAAACAG }\end{array}$ \\
\hline NOS t & inator reverse & CCGGCAACAGGATTCAATCTTAAG \\
\hline
\end{tabular}




\section{CHAPTER 4: Substrate Specificities and Subcellular Localisation Studies of Arabidopsis Fatty Acyl Reductases.}

\subsection{Introduction}

Fatty acyl reductase (EC:1.2.1.n2) enzymes catalyze the reduction of fatty acyl-CoAs or fatty acyl-ACPs to fatty alcohols with or without the release of a fatty aldehyde intermediate (Rowland and Domergue, 2012). In plants, FARs exhibit the single enzyme two-step reaction and are dependent on $\mathrm{NAD}(\mathrm{P}) \mathrm{H}$ for activity (Kolattukudy, 1970; Pollard et al., 1979; Rowland et al., 2006; Doan et al., 2011). Plant FARs are about 500 amino acids in length, excluding a potential plastid targeting sequence. The plant FARs all share a NAD(P)H-binding Rossmann fold domain (Rao and Rossmann, 1973) (Figure 1.10 of Chapter 1). The Rossmann fold is found in many redox enzymes and dehydrogenases (Arthur, 1995). The Rossmann fold binds a mononucleotide and is comprised of a $\beta \alpha \beta \alpha \beta$ motif (Arthur, 1995). The dinucleotide-binding (i.e. nicotinamide adenine dinucleotide or NAD) Rossmann fold structure consists of two such mononucleotide binding motif repeats and form a parallel six-stranded $\beta$-sheet flanked by two pairs of alpha helices in the topological order beta-alpha-beta-alpha-beta (Arthur, 1995). The Rossmann fold proteins typically exhibit a consensus motif of GXXGXX(G/A), in which the first two glycines participate in $\mathrm{NAD}(\mathrm{P}) \mathrm{H}$-binding, and the third glycine or an alanine facilitates close packing of a helix to a beta-strand (Rao and Rossmann, 1973). Typically, Rossmann fold-containing proteins contain a second domain that is responsible for specifically binding a substrate and catalyzing a particular enzymatic reaction (Marchler-Bauer et al., 2011). Along these lines, plant FARs contain a fatty 
acyl-CoA reductase or 'FAR_C' domain at their C-terminus. The FAR_C domain is also annotated as "Male Sterility 2" or "Sterile" domain because it was first reported in the MALE STERILITY2 (MS2/FAR2) predicted protein from Arabidopsis thaliana (Aarts et al., 1997). This domain is unique to the FAR subfamily of reductases (Aarts et al., 1997; Rowland et al., 2006; Doan et al., 2009). The function of this domain is unknown and no structural information on the FAR_C domain has been reported.

FARs have distinct substrate specificities with regard to acyl chain length and degree of saturation (see Table 1.3 of Chapter 1 ). The relatively well characterized Arabidopsis FAR protein family have chain length specificities that range from $\mathrm{C} 16$ to C30. Arabidopsis FAR2/MS2 and FAR6 encode plastidial isoforms and use C16:0 fatty acyl-ACP as substrate (Doan et al., 2011; Chen et al., 2012). The rest of the FARs in Arabidopsis are thought to be microsomal enzymes acting on acyl-CoA substrates (Rowland and Domergue, 2012). FAR3/CER4 is specific for C24:0-C30:0 acyl-CoAs and was found to be in the endoplasmic reticulum when expressed in yeast although in planta subcellular localization was not reported (Rowland et al., 2006). FAR1, FAR4 and FAR5, when heterologously expressed in transgenic yeast, produce saturated fatty alcohols ranging from C18:0 to C22:0, where FAR1 mostly produces $\mathrm{C} 22: 0-\mathrm{OH}$ and C18:0-OH, FAR4 mostly produces $\mathrm{C} 20: 0-\mathrm{OH}$ and $\mathrm{C} 18: 0-\mathrm{OH}$, and FAR5 produced almost exclusively C18:0-OH (Chapter 2 of this thesis). This indicates that FAR1, FAR4, and FAR5 have distinct but overlapping chain length specificities.

Little is known about the specific amino acids that are important for activity or those that confer distinct substrate specificity to a particular FAR enzyme. 
Altering substrate specificity is one of the most frequently required property changes in protein engineering of enzymes. Most of the enzymes in nature are highly specific to a particular substrate. Enzyme engineering to switch the specificity towards novel substrates, expanding substrate specificity, and narrowing substrate specificity are often industrially important modifications. Primary fatty alcohols and wax esters (fatty alcohols combined with fatty acids) are of high industrial value (Rowland and Domergue, 2012). Knowledge of the amino acids important for determining activity and/or substrate specificity of plant FARs could thus provide information useful for engineering novel FARs with high activities and/or desired substrate specificities. These engineered FARs could be expressed in transgenic oilseed crops or microbes for production of fatty alcohols of desired chain lengths.

FAR1, FAR4, and FAR5 share $68-74 \%$ identity at the amino acid level with FAR1 and FAR4 having the highest identity among the three (74\%) (Figure 4.1; Table 4.1). This high degree of sequence similarity is advantageous in determining domains or even individual amino acids important for substrate specificity using, for example, a domain swapping approach. In this chapter, I examine the substrate specificity determinants of Arabidopsis FAR1, FAR4 and FAR5 using yeast as a heterologous system and provide information on the amino acid regions responsible for substrate specificity. The substrate specificities were also examined in planta using a leaf transient assay system. In addition, the endoplasmic reticulum localization of FAR1 fused to green fluorescent protein was demonstrated by transient expression in Nicotiana benthamiana leaves. 


\subsection{Materials and Methods}

\subsubsection{Cloning of FAR1, FAR4, FAR5 and FAR chimeric genes into yeast expression vector}

The full length FAR1, FAR4 and FAR5 open reading frames were amplified using flower cDNA as a template. The FAR wild-type sequences as well as domain swaps were amplified by PCR using iProof ${ }^{\mathrm{T}}$ High-Fidelity DNA polymerase (BioRad). Each PCR contained 1X High-Fidelity iProof Buffer (containing $1.5 \mathrm{mM} \mathrm{MgCl}_{2}$ ), $0.2 \mathrm{mM}$ dNTPs, $1-5 \mathrm{ng}$ DNA template, $0.5 \mu \mathrm{M}$ of each Primer (Forward and Reverse), and 0.5 Units of iProof DNA Polymerase. PCR cycling conditions included an initial denaturation step of $98^{\circ} \mathrm{C}$ for 30 seconds, followed by 30 cycles of $98^{\circ} \mathrm{C}$ for 15 seconds (denaturation), $57^{\circ} \mathrm{C}$ for 30 seconds (annealing), and $72^{\circ} \mathrm{C}$ for 1 minute (extension), with a final single step of $72^{\circ} \mathrm{C}$ for 5 minutes to ensure fully elongated products. DNA products were amplified using the primers: FAR1_BamHI_Forward and FAR1_XhoI_Reverse, FAR4_BamHI_Forward and FAR4_Xhol_Reverse, FAR5_BamHI_Forward and FAR5_XhoI_Reverse. See supplementary table for primer sequences. FAR chimeras (domain swaps) were generated by swapping the 5 ' end of the open reading frame ( $\mathrm{N}$-terminal end of the protein corresponding to the Rossmann fold domain) and the $3^{\prime}$ end of the open reading frame (C-terminal end of the protein corresponding to the FAR_C domain) between the FAR1, FAR4 and FAR5

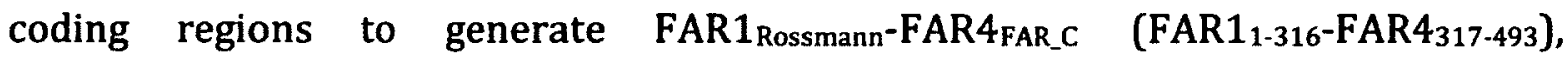

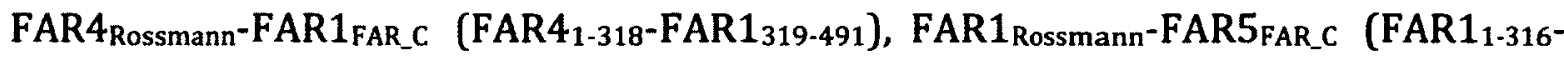
FAR5 317-496), FAR5 Rossmann $_{\text {-FAR1 }}$ FAR_C $_{\text {(FAR5 1-319-FAR1 }}$ 320-491), FAR4 $_{\text {Rossmann-FAR5 }}$ FAR_C

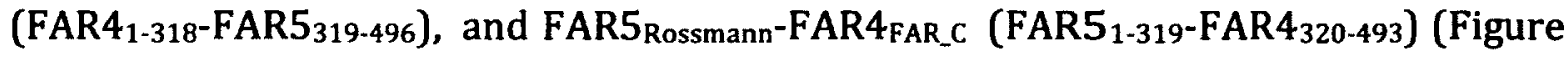


4.2a and b). The FAR domain swaps were made by overlapping extension PCR, consisting of two separate PCR reactions as described by Heckman and Pease (2007). Four additional chimeras, FAR4 $25 \%$-FAR1 $1_{75 \%}$ (FAR4 $_{1-123}$-FAR1 $1_{124-492}$ ), FAR4 $4_{50 \%-}$

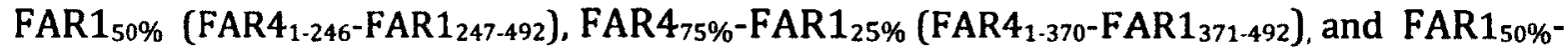
FAR4 ${ }_{50 \%}$ (FAR1 1-245-FAR $1_{246-492}$ ) (Figure $4.2 \mathrm{~b}$ ), were prepared using overlap PCR and cloned into pDONR221 entry vector by Dr. Frédéric Domergue (Centre National de la Recherché Scientifique, Laboratoire de Biogenèse Membranaire, Université Victor Ségalen Bordeaux, Bordeaux, France). The FAR4 $25 \%$-FAR1 $1_{55}$, FAR4 ${ }_{50 \%}$-FAR1 $1_{50 \%}$, and FAR4 ${ }_{75} \%$-FAR $1_{25 \%}$ fragments were amplified from the vector templates using FAR4_BamHI_Forward and FAR1_Xhol_Reverse primers. The FAR1 $50 \%$-FAR4 $4_{50 \%}$ fragment was amplified from the vector template using FAR1_BamHI_Forward and FAR4_XhoI_Reverse primers. Two PCR programs were designed depending on the size of the amplicon. For shorter $(<1000 \mathrm{bp})$ fragments, the cycling conditions, denoted iProof PCR 1 Program, were: initial denaturation step at $98^{\circ} \mathrm{C}$ for 30 seconds, 30 cycles at $98^{\circ} \mathrm{C}$ for 15 seconds, $57^{\circ} \mathrm{C}$ for 20 seconds, and $72^{\circ} \mathrm{C}$ for 30 seconds, with a final single step of $72^{\circ} \mathrm{C}$ for 5 minutes. For longer $(>1000 \mathrm{bp})$ fragments, the cycling conditions, denoted iProof PCR 2 Program, were: initial denaturation step at $98^{\circ} \mathrm{C}$ for 30 seconds, 30 cycles at $98^{\circ} \mathrm{C}$ for 15 seconds, $57^{\circ} \mathrm{C}$ for 30 seconds, and $72^{\circ} \mathrm{C}$ for 1 minute, with a final single step of $72^{\circ} \mathrm{C}$ for 5 minutes.

A modified version of yeast expression vector pYES2 (Invitrogen) that contains histidine (x6) and T7 epitope tags for protein purification and detection, respectively, was used to express proteins of interest in Saccharomyces cerevisiae. It also contains a URA3 gene for selection in yeast, a $2 \mu \mathrm{m}$ origin of replication for high- 
copy plasmid maintenance, and the GAL1 promoter for high level inducible protein expression. The wild-type and chimeric FAR1, FAR4 and FAR5 open reading frames were cloned between the BamHI and XhoI sites of the modified version of pYES2 (pYES2-His6x/T7) vector. All restriction digests were for approximately 2 hours at $37^{\circ} \mathrm{C}$ using 10 units of enzyme per $\mu \mathrm{g}$ of DNA. The digested DNA was then isolated by the "Freeze and Squeeze" method. The digested insert DNA molecules were ligated into digested pYES2-His6x/T7 in a reaction containing $1 \mathrm{x}$ ligase buffer (50mM Tris- $\mathrm{HCl}, 10 \mathrm{mM} \mathrm{MgCl}, 1 \mathrm{mM} \mathrm{ATP,} 10 \mathrm{mM}$ DTT pH 7.5), $\sim 50 \mathrm{ng}$ of vector, 235ng of insert and 1 Unit of T4 DNA Ligase) for 1 hour at room temperature. Ligations were transformed into ultra-competent DH5 $\alpha$ Escherichia coli cells using standard procedures. The positive colonies were selected on LB plates supplemented with $100 \mu \mathrm{g} / \mathrm{ml}$ of ampicillin and confirmed by colony PCR using T7_Forward primer and CYC_Terminator_Reverse primer (See supplementary table 4.4 for primer sequences). Plasmids were isolated from E. coli cells using the standard mini-prep plasmid isolation procedure. A diagnostic digest was performed on the potentially correct clones using the restriction enzymes used to insert the gene of interest into the vector, as well as unique restriction sites present within the gene. For sequencing, the inserts were amplified by PCR in a $50 \mu \mathrm{l}$ reaction: $10 \mathrm{mM}$ $\mathrm{KCl}, 10 \mathrm{mM}\left(\mathrm{NH}_{4}\right)_{2} \mathrm{SO}_{4}, 20 \mathrm{mM}$ Tris- $\mathrm{HCl}$ pH $8.3,0.1 \%$ Triton $\mathrm{X}-100,2 \mathrm{mM} \mathrm{MgCl}$, 0.25mM dNTPs, $0.25 \mathrm{mM}$ T7_Forward Primer, $0.25 \mathrm{mM}$ CYC_Terminator_Reverse Primer, $0.25 \mu \mathrm{l} \mathrm{Taq}$ Polymerase and 10ng of template. Sequencing was performed at Eurofins MWG-Operon (Hunstville, Alabama) using T7 and CYC primers, as well as gene specific primers. 


\subsubsection{Yeast transformation}

The yeast expression constructs were transformed into yeast using the lithium acetate (LiAc)/single strand (SS) carrier DNA/polyethylene glycol (PEG) method (Gietz and Woods, 2002). $5 \mathrm{ml}$ of YPAD media (1\% yeast extract, 2\% peptone, $2 \% \mathrm{D}$-glucose, $0.01 \%$ adenine hemisulphate, $1.5 \%$ agar) was inoculated with the yeast strain W303-1A (MATa his341 leu2 trp1-289 ura3-52) and incubated at $30^{\circ} \mathrm{C}$ and $250 \mathrm{rpm}$ for 16 hours. The yeast cells were harvested the following day by centrifugation at $4000 \mathrm{rpm}$ for 5 minutes at room temperature. The pellet was resuspended in $240 \mu \mathrm{l}$ of sterile $50 \%(\mathrm{w} / \mathrm{v})$ PEG 3500 , and then transferred to a sterile $1.5 \mathrm{ml}$ microcentrifuge tube. $36 \mu \mathrm{l}$ sterile $1 \mathrm{M}$ LiAc, $50 \mu \mathrm{l}$ boiled SS carrier DNA $(2.0 \mathrm{mg} / \mathrm{ml}), 5 \mu \mathrm{l}$ plasmid DNA $(100 \mathrm{ng}-1 \mu \mathrm{g})$, and $29 \mu \mathrm{l}$ of sterile $\mathrm{H}_{2} \mathrm{O}$ were then added to the yeast suspension. The mixture was mixed by flicking until resuspended, and then incubated in a $42^{\circ} \mathrm{C}$ water bath for 1 hour while being mixed by flicking periodically during the incubation period to prevent separation of the constituents. After the incubation period, the tubes were centrifuged at $21100 \mathrm{~g}$ for 30 seconds at room temperature, resuspended in $200 \mu$ l of sterile water, and $100 \mu l$ was plated onto SD Ura plates $(0.674 \%$ yeast nitrogen base, $2 \%$ D-glucose, $0.01 \%$ adenine, $0.002 \%$ histidine, $0.002 \%$ tryptophan, $0.01 \%$ leucine, $2 \%$ agar) to select for transformants. The plates were incubated at $30^{\circ} \mathrm{C}$ for 3 days.

\subsubsection{Induction of FAR expression in yeast and lipid extraction}

Individual yeast transformants containing FAR1, FAR4 or FAR5 yeast expression constructs (including domain swap chimeras) were selected on SD Ura- 
plates and then individual colonies inoculated in triplicate cultures in $3 \mathrm{ml} \mathrm{SD} \mathrm{Ura-}$ liquid media $(0.674 \%$ yeast nitrogen base, $2 \%$ D-glucose, $0.01 \%$ adenine, $0.002 \%$ histidine, $0.002 \%$ tryptophan, $0.01 \%$ leucine). The cultures were grown for 24 hours at $30^{\circ} \mathrm{C}$ and $250 \mathrm{rpm}$. The $\mathrm{OD}_{600}$ was measured for each yeast culture and the amount of culture required to obtain an $\mathrm{OD}_{600}$ of 0.4 in $3 \mathrm{ml}$ of SG Ura- media ( $0.674 \%$ yeast nitrogen base, $2 \%$ galactose, $0.01 \%$ adenine, $0.002 \%$ histidine, $0.002 \%$ tryptophan, $0.01 \%$ leucine) was centrifuged at $2880 \mathrm{~g}$ (4000 rpm) for 10 minutes at room temperature. The supernatants were discarded and the pellets were washed with $5 \mathrm{ml}$ of distilled water, vortexed, and then centrifuged again at $2880 \mathrm{~g}$ for 10 minutes at room temperature. The supernatants were discarded and the pellets were resuspended in $3 \mathrm{ml}$ of SG Ura- media and induced for 2 days at $30^{\circ} \mathrm{C}$ and $250 \mathrm{rpm}$ for protein expression.

For lipid extraction, the $\mathrm{OD}_{600}$ was measured for each galactose-induced yeast culture grown and $2 \mathrm{ml}$ of each yeast culture was transferred to a glass $(13 \mathrm{~mm}$ $\mathrm{x} 100 \mathrm{~mm}$ ) GC tube and centrifuged at $4500 \mathrm{~g}$ for 10 minutes. The supernatant was poured into a fresh glass tube. The supernatant was extracted once with $2 \mathrm{ml}$ of 2:1 chloroform/methanol $(\mathrm{v} / \mathrm{v})$ containing $10 \mu \mathrm{g}$ of 1-pentadecanol $(\mathrm{C} 15: 0-0 \mathrm{H})$ as an internal standard, vortexed, and centrifuged at $4500 \mathrm{~g}$ for 5 minutes. The lower organic phase was transferred to a new GC tube. The supernatant was extracted once more with $2 \mathrm{ml}$ of $2: 1$ chloroform/methanol $(\mathrm{v} / \mathrm{v})$ and once with $2 \mathrm{ml}$ of chloroform as described above. All of the organic phases were combined, washed with $5 \mathrm{ml}$ of $0.9 \% \mathrm{NaCl}(\mathrm{w} / \mathrm{v})$, and evaporated to dryness under a gentle stream of nitrogen gas at $37^{\circ} \mathrm{C}$ for $15-20$ minutes. The supernatant extractions and the yeast 
pellets from the centrifugation step were resuspended in $3 \mathrm{ml}$ of $1 \mathrm{M}$ methanolic- $\mathrm{HCl}$ and $3 \mathrm{ml}$ of $1 \mathrm{M}$ methanolic- $\mathrm{HCl}$ containing $10 \mu \mathrm{g}$ 1-pentadecanol (C15:0-OH), respectively, by vortexing and incubated at $80^{\circ} \mathrm{C}$ for 90 minutes. $1 \mathrm{ml}$ of $0.9 \% \mathrm{NaCl}$ was added to the samples after the pellet and supernatant samples cooled to room temperature and then the lipids were extracted with $500 \mu$ l of hexane. The top organic phase was transferred to a new glass tube. The liquid-liquid extraction was repeated with an additional $500 \mu$ l of hexane and the top organic phase was transferred to the first extraction. The samples were evaporated to dryness under nitrogen gas for $15-20$ minutes at $37^{\circ} \mathrm{C}$. The lipids were derivatized with $50 \mu \mathrm{l}$ of $\mathrm{N}, \mathrm{O}$-bis (trimethylsilyl) trifluoroacetamide : trimethylchlorosilane (BSTFA+TMS, 99:1) at $80^{\circ} \mathrm{C}$ for 90 minutes.

\subsubsection{Gas chromatography analysis (Yeast samples)}

The samples derivatized in BSTFA were directly analyzed by gas chromatography on a Varian GC-450 Gas Chromatograph equipped with a flame ionization detector (GC-FID) and fitted with a HP-1MS capillary column (30m x 0.25 $\mathrm{mm}$ inner diameter, $0.25 \mu \mathrm{m}$ film thickness; Varian, Santa Clara, CA). A $1 \mu \mathrm{L}$ aliquot of the sample was injected in split mode (10:1 split ratio). The temperature of the injector was held at $250^{\circ} \mathrm{C}$ and the column oven temperature was held initially at $150^{\circ} \mathrm{C}$ for 5 minute and then increased to $300^{\circ} \mathrm{C}$ at a rate of $10^{\circ} \mathrm{C}$ per minute, which was held for 8 minutes. The total run time was of 28 minutes. High purity helium was used as the carrier gas at a flow rate of $2 \mathrm{ml}$ per min. The flame ionization 
detector (FID) was held at $350^{\circ} \mathrm{C}$. Quantification of fatty alcohols was determined by comparing peak areas to that of 1-pentadecanol $(\mathrm{C} 15: 0-\mathrm{OH})$ internal standard.

\subsubsection{SDS-PAGE and western blot analysis}

Yeast cultures were handled and FAR protein expression induced with galactose as described above. The protein extraction from the yeast pellet was carried as described by Kushnirov (2000). After 48 hours of incubation, the $\mathrm{OD}_{600}$ of each culture was measured and the yeast was harvested to obtain a pellet with 1.5 $\mathrm{OD}_{600}$ units of cells by centrifuging at $2880 \mathrm{~g}$ at room temperature for 5 minutes. The supernatant was discarded, the pellet was resuspended in $100 \mu$ l of water, and transferred to a $1.5 \mathrm{ml}$ microcentrifuge tube. $100 \mu \mathrm{l}$ of $0.2 \mathrm{M} \mathrm{NaOH}$ was then added to each sample and incubated for 5 minutes at room temperature. After incubation, the samples were centrifuged at $21100 \mathrm{~g}$ for 5 minutes, and the pellet was resuspended in $50 \mu$ l SDS samples buffer $(62.5 \mathrm{mM} \mathrm{NaPO} 4 \mathrm{pH} 7.0 ; 10 \%$ glycerol; $2 \%$ SDS; $5 \% 2-$ mercaptoethanol; spatula tip of bromophenol blue). The samples were then boiled for 3 minutes and centrifuged again for 5 minutes at $14800 \mathrm{rpm}$. These samples were used for sodium dodecyl sulphate-polyacrylamide gel electrophoresis (SDSPAGE) and Western blot analysis. The samples were run on SDS-PAGE gels in duplicates with a $12 \%$ resolving gel $(12 \%$ acrylamide:bis-acrylamide $37.5: 1$, $0.375 \mathrm{M}$ Tris- $\mathrm{HCl} \mathrm{pH} 8.8,0.1 \%$ SDS, $0.05 \%$ APS, $0.005 \%$ TEMED) and $4 \%$ stacking gel (4\% acrylamide:bis-acrylamide $37.5: 1,0.1 \mathrm{M}$ Tris- $\mathrm{HCl} \mathrm{pH} 6.8,0.1 \%$ SDS, $0.05 \%$ APS, $0.005 \%$ TEMED). $10 \mu \mathrm{l}$ of each sample was run on the gel for 30 minutes at $50 \mathrm{~V}$ and 1.5 hours at $100 \mathrm{~V}$ in $1 \mathrm{X}$ running buffer $(25 \mathrm{mM}$ Tris, $186 \mathrm{mM}$ glycine, $0.1 \%$ SDS, 
pH8.3). One of the gels was stained with Coomassie blue stain $(0.25 \%$ Coomassie Brilliant Blue R-250, 10\% glacial acetic acid, 50\% methanol) for 1 hour while shaking on a nutator, then de-stained overnight in de-staining solution $25 \%$ methanol, 7\% glacial acetic acid). The SDS-PAGE gel was then imaged with an Alpha Imager2200 (Alpha Innotech). The other gel was transferred to $0.45 \mu \mathrm{m}$ nitrocellulose membrane in chilled transfer buffer $(25 \mathrm{mM}$ Tris Base, $192 \mathrm{mM}$ Glycine, $20 \%$ methanol) for 1 hour at $350 \mathrm{~mA}$ at $4^{\circ} \mathrm{C}$. The nitrocellulose membrane was then blocked overnight in blocking solution (5\% fat free skim milk, TBS-T pH $7.6\left(25 \mathrm{mM}\right.$ Tris Base, $137 \mathrm{mM} \mathrm{NaCl}, 0.1 \%$ Tween-20)) at $4^{\circ} \mathrm{C}$. The following day the membrane was rinsed twice with TBS-T solution and incubated with a 1:50 000 dilution of T7-Tag( ${ }^{\circledR}$ Monoclonal Mouse Antibody (Novagen) in $25 \mathrm{ml}$ blocking solution for 1 hour with continuous swirling on a platform shaker. The membrane was washed with TBS-T for $1 \times 15$ minutes and then $4 \times 5$ minutes while shaking, replacing with fresh TBS-T each time. The membrane was then incubated with 1:50 000 dilution of horseradish peroxidase (HRP)-conjugated Anti-Mouse Secondary antibody (Novagen) in $25 \mathrm{ml}$ blocking solution for 1 hour while shaking. The membrane was then washed again with TBST for $1 \mathrm{x} 15$ minutes and then $4 \mathrm{x}$ 5 minutes while shaking. The membrane was then covered with $200 \mu \mathrm{l}$ of $1: 1$ Lumigen TMA-6 Solution A (solution containing Tris Buffer in $3.2 \% \mathrm{v} / \mathrm{v}$ ethanol) and Lumigen TMA-6 Solution B (proprietary substrate in Tris Buffer), which are part of the Amersham ${ }^{\text {TM }}$ ECL $^{\text {TM }}$ Advance Western Blotting Detection Kit (GE Healthcare) for 5 minutes in dark. Immunodetection was performed on a FluorChem Q Mulitimage III imaging system (Alpha Innotech) using the Chemi-Super Protocol 
$($ Excitation $=$ none, Emission $=$ Chemi, Speed $/$ Resolution $=$ Normal $/$ Ultra $)$ and the auto-exposure time.

\subsubsection{Preparation of constructs for FAR over-expression}

To ectopically overexpress FAR1, FAR4 or FAR5, the cDNA sequences were cloned into a vector downstream of the cauliflower mosaic virus $35 \mathrm{~S}$ promoter. The FAR1, FAR4 and FAR5 cDNAs were excised from pYES2:FAR1, pYES2:FAR4 and pYES2:FAR5 plasmids, respectively, using EcoRI and XbaI restriction enzymes. The recipient pHANNIBAL vector (Wesley et al., 2001) containing the $35 \mathrm{~S}$ promoter was digested with the same restriction enzymes. The digested pHANNIBAL::35S vector fragment and digested FAR1, FAR4 and FAR5 cDNA fragments were gel purified by the following phenol extraction ('Freeze and Squeeze method') procedure. Following electrophoresis, a UV box was used to image the DNA fragments and the large fragment associated with the pHANNIBAL::35S vector and the smaller FAR cDNA fragment was excised with a razor. The gel fragment was crushed with a syringe into a microcentrifuge tube and TE saturated phenol (Bioshop) added in a 1 $\mu \mathrm{l} / \mathrm{mg}$ of gel ratio. The mixture was vortexed for 1 minute before being frozen in liquid nitrogen. The aqueous layer was separated by centrifugation at $13000 \mathrm{rpm}$ for 15 minutes and the top layer transferred to a fresh microcentrifuge tube. Further purification of the DNA was carried out by the addition of an equal volume of 25:24:1 (v/v) phenol:chloroform:isoamyl alcohol (Bioshop). After 1 minute of mixing, the sample was centrifuged at $13000 \mathrm{rpm}$ for 10 minutes. The aqueous layer was transferred to a fresh vial and the DNA precipitated with 3M sodium acetate ( $\mathrm{pH} \mathrm{5.3)} \mathrm{and} 2 \mathrm{x}$ volume of absolute ethanol. The mixture was centrifuged at 
$13000 \mathrm{rpm}$ for 15 minutes at $4^{\circ} \mathrm{C}$. The DNA pellet was further washed with cold $70 \%$ ethanol, air dried, and resuspended in $50 \mu \mathrm{l}$ of nanopure water. The FAR1, FAR4 and FAR5 coding sequences were ligated into pHANNIBAL::35S using T4 DNA ligase to generate pHANNIBAL::35S-FARx $(x=1,4$ or 5$)$. The vectors were transformed into chemically competent $E$. coli DH5 $\alpha$ cells. Five $\mu$ l of each ligation was incubated with $50 \mu \mathrm{l}$ of thawed competent cells on ice for 30 minutes and heat shocked for 30 seconds at $42^{\circ} \mathrm{C}$ followed by a 2 minute incubation on ice. The cells were incubated at $37^{\circ} \mathrm{C}$ in $1 \mathrm{ml}$ of LB media for 1 hour and plated on $\mathrm{LB}$ media with $1.5 \%$ agar containing $100 \mu \mathrm{g} / \mathrm{ml}$ ampicillin. E. coli colonies containing the plasmid with an insert were identified through colony PCR. Plasmids were then purified from $5 \mathrm{ml}$ of overnight culture using a standard miniprep procedure. The 35S:FARx:OCST ( $x=1,4$ or 5 ; OCST= octopine synthase gene terminator) fragments were excised from pHANNIBAL::35S-FARx vector using NotI restriction enzyme and gel purified using the procedure described above. These fragments were subsequently ligated into the NotI restriction site of pART27 binary vector (Gleave et al., 1992). The vector pART27 was digested with NotI and dephosphorylated before gel purification. The fragment 35S:FARx:OCST was ligated into pART27 vector using T4 DNA ligase and transformed into chemically competent $E$. coli DH5 $\alpha$ cells as described above. The cells were selected on LB plates with $50 \mu \mathrm{g} / \mathrm{ml}$ spectinomycin and the colonies containing the plasmid with an insert were identified through colony PCR (see Appendix for primers). The pART27:35S:FARx:OCST vector plasmids were isolated from $5 \mathrm{ml}$ of overnight culture using a standard miniprep procedure and were transformed into GV3101 
Agrobacterium tumefaciens cells by electro-transformation using a MicroPulser Electroporator (BioRad, Mississauga ON). The Agrobacterium was used to introduce the T-DNA regions of the binary plasmids into wild-type Arabidopsis plants by the floral dip method (Zhang et al., 2006) and transiently into Nicotiana benthamiana leaves as described below.

\subsubsection{Plant material and growth conditions}

Nicotiana benthamiana seeds were obtained from Dr. Owen Rowland (Carleton University, Ottawa, Canada). All seeds were surface sterilized in a microcentrifuge tube using $100 \%$ ethanol, mixed by inversion 5 times, followed by incubation in $50 \%$ bleach $/ 0.5 \%$ SDS solution, mixed by inversion 5 times, rinsed with sterile deionized water 3 times with mixing by inversion 10 times, and then suspended in sterile $0.1 \%$ agarose. The seeds were then plated on AT-agar ( $2 \mathrm{mM}$ $\mathrm{MgSO}_{4}, 2.5 \mathrm{mM} \mathrm{KH}_{2} \mathrm{PO}_{4}, 50 \mu \mathrm{M} \mathrm{Fe}(\mathrm{EDTA}), 2 \mathrm{mM} \mathrm{Ca}\left(\mathrm{NO}_{3}\right)_{2}, 5 \mathrm{mM} \mathrm{KNO}_{3}$, micronutrients $10 \mu \mathrm{M} \mathrm{H}_{3} \mathrm{BO}_{3}, 14 \mu \mathrm{M} \mathrm{MnCl}_{2}, 0.5 \mu \mathrm{MuSO}_{4}, 1 \mu \mathrm{M} \mathrm{ZnSO}{ }_{4}, 0.2 \mu \mathrm{M} \mathrm{NaMoO}{ }_{4}, 10 \mu \mathrm{M} \mathrm{NaCl}$, and $10 \mathrm{nM} \mathrm{CoCl}$, and solidified with $0.7 \%(\mathrm{w} / \mathrm{v})$ agar in sterile petri plates). The seeds were stratified on AT-agar for 3 days at $4^{\circ} \mathrm{C}$ and in the dark. Plates were sealed with micropore tape and placed in a growth chamber (Conviron, model TC 26) at $22^{\circ} \mathrm{C}$ under continuous light $\left(90 \mu \mathrm{mol} \mathrm{m}^{-2} \mathrm{~s}^{-1}\right)$ conditions for $7-10$ days (until the first true leaves appeared). Seedlings were transplanted to soil (Pro-Mix MPV Multipurpose Growing Medium) containing 20-20-20 fertilizer, covered with cellophane for $\mathbf{5}$ days, and grown in a growth chamber (Percival, model AR66L) at $22^{\circ} \mathrm{C}$ and $80 \%$ relative humidity under long day conditions. 


\subsubsection{Transient expression of Arabidopsis FAR1, FAR4 and FAR5 in Nicotiana}

\section{benthamiana leaves}

For transient over-expression of FAR1, FAR4 and FAR5, the Agrobacterium cells carrying pART27:35S:FARx ( $x=1,4$ or 5$)$ were co-infiltrated with Agrobacterium cells carrying 35S:p19 into the leaves of 4 to 6 week old $N$. benthamiana plants according to the procedure of Sparkes et al. (2006). The p19 protein of tomato bushy stunt virus (TBSV) prevents the onset of posttranscriptional gene silencing (PTGS) in the infiltrated tissues and allows for high levels of transient protein expression (Voinnet et al., 2003). The Agrobacterium tumefaciens cells carrying pART27 binary vector harboring either FAR1, FAR4 or FAR5 as well as the 35S:p19 and pVKH18:35S:GFPC (Dean et al., 2007) constructs were grown overnight at $28^{\circ} \mathrm{C}, 240 \mathrm{rpm}$ agitation in $5 \mathrm{ml} \mathrm{LB}$ medium supplemented with various antibiotics. The Agrobacteria carrying pART27:35S:FAR binary vector were grown in LB medium with $50 \mu \mathrm{g} / \mathrm{ml}$ rifampicin, $50 \mu \mathrm{g} / \mathrm{ml}$ gentamycin and 50 $\mu \mathrm{g} / \mathrm{ml}$ spectinomycin. For the culture of Agrobacteria harbouring pVKH:35S:GFPC, $50 \mu \mathrm{g} / \mathrm{ml}$ of kanamycin was used instead of spectinomycin. For the culture of Agrobacteria harbouring 35S:p19, $50 \mu \mathrm{g} / \mathrm{ml}$ of kanamycin, $50 \mu \mathrm{g} / \mathrm{ml}$ rifampicin and $50 \mu \mathrm{g} / \mathrm{ml}$ tetracycline was used. When the OD reached 0.5 to 1 , acetosyringone was added to a final concentration of $100 \mu \mathrm{M}$ to induce the vir operon of $A$. tumefaciens. Bacteria were grown for an additional 3 hours and harvested by centrifugation at $3000 \mathrm{~g}$ for $10 \mathrm{~min}$ at room temperature and washed twice with $2 \mathrm{ml}$ of infiltration

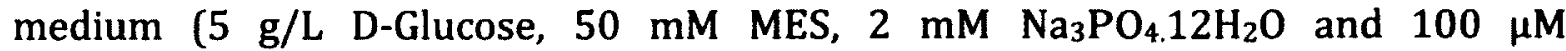
acetosyringone). Diluted cultures carrying a 1:1 mix of pART27:35S:FARx and 
35S:p19 at a final $\mathrm{OD}_{600}$ of 0.1 each were prepared in fresh infiltration medium and used for injection. Agrobacterium harbouring only p19 and GFP constructs was used as the negative control. The large third or fourth leaf down from the apical meristem was infiltrated with Agrobacteria (one construct/leaf) from the underside using a 1 $\mathrm{ml}$ syringe without a needle as described by Sparkes et al. (2006). The plants were placed back in the growth chamber after infiltration. After 4 to 5 days, the infiltrated leaves were examined for GFP expression. The infiltrated leaves were excised; fresh weight measured, and then used for surface wax, cutin and total lipid measurements.

\subsubsection{Cutin analysis of Nicotiana benthamiana leaves}

About three mature rosette leaves of $200 \mathrm{mg}$ total fresh weight were harvested into chloroform rinsed glass vials for delipidation. The vials were filled with hot isopropanol and incubated at $80^{\circ} \mathrm{C}$ for 1 hour. Once cooled, the isopropanol was replaced with a $2: 1(\mathrm{v} / \mathrm{v})$ solution of chloroform:methanol for 24 hours. The samples were then sequentially incubated in $1: 1(\mathrm{v} / \mathrm{v})$ chloroform:methanol, 1:2 (v/v) chloroform:methanol, and $100 \%$ methanol for 24 hours each with changes twice a day. Samples were dried in the fumehood for 2 days and in a dessicator for further 2 days. The dried tissues were weighed and depolymerized by incubation in $1 \mathrm{M}$ sulfuric acid in methanol for 3 hours at $85^{\circ} \mathrm{C}$. The samples were spiked with 10 $\mu \mathrm{g}$ heptadecanoic acid $(\mathrm{C} 17: 0)$ and $10 \mu \mathrm{g}$ pentadecanol $(\mathrm{C} 15: 0-\mathrm{OH})$ as internal standards. Once cooled, $3 \mathrm{~mL}$ of $2.5 \% \mathrm{NaCl}(\mathrm{w} / \mathrm{v})$ was added to each sample and the released fatty acyl chains were extracted twice in hexane. The hexane fraction was 
washed with $3 \mathrm{~mL}$ of saline solution (200 mM NaCl and $200 \mathrm{mM}$ Tris, pH8.0) and then transferred to a fresh vial and dried under a gentle stream of nitrogen gas. Samples were derivatized in $100 \mu \mathrm{L}$ of BSTFA+TMCS at $110^{\circ} \mathrm{C}$ for 30 minutes. The BSTFA+TMCS was evaporated under nitrogen and then the sample was dissolved in heptane:toluene (1:1) for gas chromatography-flame ionization detection (GC-FID) (method described in Chapter 2). Quantification of fatty acids, hydroxyl acids, and fatty alcohols was based on peak areas, which were derived from using the respective internal standards.

\subsubsection{Surface wax analysis of Nicotiana benthamiana leaves}

Surface wax composition of $N$. benthamiana leaves was analyzed by gas chromatography and using a flame ionization detector (GC-FID). Infiltrated leaf tissue of approximately $1 \mathrm{~cm}^{2}$ area was harvested and immersed for 30 seconds in 8 $\mathrm{ml}$ of spectro grade chloroform (Caledon) containing 1 $\mu \mathrm{g}$ 1-pentadecanol (C15:0$\mathrm{OH})$ and $1 \mu \mathrm{g}$ tetracosane (C24:0 alkane) (Sigma-Aldrich) as internal standards. The chloroform was evaporated to dryness at $37^{\circ} \mathrm{C}$ under a gentle stream of nitrogen gas. The samples were derivatized BSTFA+TMCS (99:1) (Sigma Aldrich), and derivatized at $80^{\circ} \mathrm{C}$ for 90 minutes. A $1 \mu \mathrm{L}$ aliquot of the sample was then analyzed by gas chromatography as described in section 4.3.14. Quantification of fatty acids, fatty alcohols and alkanes was based on peak areas, which were derived from using the respective internal standards. 


\subsubsection{Total internal lipid analysis of Nicotiana benthamiana leaves}

Total lipid extraction from $N$. benthamiana leaves was carried out using a modified Bligh and Dyer method (Bligh and Dyer, 1959). $200 \mathrm{mg}$ of the harvested leaves were homogenized in liquid nitrogen and transferred into a GC tube. The ground leaf material was mixed with $3.75 \mathrm{ml}$ of methanol:chloroform $(2: 1 \mathrm{v} / \mathrm{v})$ and $1 \mathrm{ml}$ of $1 \mathrm{mM}$ EDTA in $0.15 \mathrm{M}$ acetic acid by vortexing. To this mixture, $1.25 \mathrm{ml} \mathrm{CHCl}_{3}$ and $1.25 \mathrm{ml} 0.88 \%(\mathrm{w} / \mathrm{v}) \mathrm{KCl}$ was added and vortexed again. This was centrifuged at $3000 \mathrm{rpm}$ for 5 minutes and the lower chloroform phase was transferred to a fresh GC tube. The collected $\mathrm{CHCl}_{3}$ phase was dried under a stream of $\mathrm{N}_{2}$. The extracted lipids were transmethylated using $3 \mathrm{ml}$ of $1 \mathrm{M}$ methanolic- $\mathrm{HCl}$ containing $10 \mu \mathrm{g} 1$ pentadecanol $(\mathrm{C} 15: 0-0 \mathrm{H})$ and $10 \mu \mathrm{g}$ heptadecanoic acid $(\mathrm{C} 17: 0)$ as internal standards. Derivatization using BSTFA was carried out as described in Section 4.3.6 above, before analysis by gas chromatography-flame ionization detection. Quantification of fatty acids and fatty alcohols was based on peak areas, which were derived from using the respective internal standards.

\subsubsection{Gas chromatography analysis (plant samples)}

\subsubsection{Surface wax sample analysis}

The samples were analyzed by gas chromatography on a Varian-GC-450 gas chromatograph equipped with a flame ionization detector (GC-FID) and fitted with a HP-1MS capillary column $(30 \mathrm{~m} \times 0.25 \mathrm{~mm}$ inner diameter, $0.25 \mu \mathrm{m}$ film thickness; Varian, Santa Clara, CA). One $\mu$ l of sample was injected in splitless mode and helium 
carrier gas was set to a constant flow rate of $2 \mathrm{ml}$ per minute. The injector temperature was held at $250^{\circ} \mathrm{C}$ and the oven temperature was initially held at $150^{\circ} \mathrm{C}$ for 10 minutes and then ramped to $300^{\circ} \mathrm{C}$ at a rate of $4^{\circ} \mathrm{C}$ per minute, and held for 10 minutes. The total run time was 57.5 minutes. The FID was held at $325^{\circ} \mathrm{C}$. Peaks were quantified on the basis of their FID ion current.

\subsubsection{Total internal lipids and cutin sample analysis}

The samples were analyzed by gas chromatography on a Varian 3900 GC-FID fitted with a HP-5MS capillary column $(30 \mathrm{~m} \times 0.25 \mathrm{~mm}$ inner diameter, $0.25 \mu \mathrm{m}$ film thickness; Agilent). One $\mu$ of sample was injected in splitless mode and helium carrier gas was set to a constant flow rate of $2 \mathrm{ml}$ per minute. The temperature of the injector was held at $250^{\circ} \mathrm{C}$ and the column oven temperature was held initially at $150^{\circ} \mathrm{C}$ for 5 minute and then increased to $300^{\circ} \mathrm{C}$ at a rate of $10^{\circ} \mathrm{C}$ per minute, which was held for 8 minutes. The total run time was 28 minutes. The FID was set at $325^{\circ} \mathrm{C}$. Peaks were quantified on the basis of their FID ion current.

\subsubsection{Preparation of AtFAR1-GFP fusion expression construct}

Complementary DNA (cDNA) was made using root RNA extracted from Arabidopsis thaliana (Col-0) plants. The RNA samples were diluted to a concentration of $0.12 \mu \mathrm{g} / \mu \mathrm{l}$ using DEPC-treated water. For each sample, $1 \mu \mathrm{g}$ ( $8.5 \mu \mathrm{l}$ ) of RNA was heated at $70^{\circ} \mathrm{C}$ for 5 minutes and snap chilled on ice. For a final volume of $20 \mu \mathrm{l}$, the RNA samples $(8.5 \mu \mathrm{l})$ were then added to $11.5 \mu \mathrm{l}$ of the reverse transcriptase reaction mixture $(3 \mu \mathrm{M}$ oligo-dT primer, $0.5 \mathrm{mM}$ dNTPs, $5 \mathrm{U}$ 
RNaseOUT $^{\text {TM }}$ (Invitrogen), 10mM DTT, 50mM Tris- $\mathrm{HCl}$ (pH 8.3, 75mM KCl, 3mM $\mathrm{MgCl}_{2}$ ) on ice. 50 units of SuperScript III ${ }^{\circledR}$ Reverse Transcriptase (Invitrogen) were added to each reaction and held at $50^{\circ} \mathrm{C}$ for 60 minutes, $72^{\circ} \mathrm{C}$ for 10 minutes, cooled down to $4^{\circ} \mathrm{C}$, and stored at $-20^{\circ} \mathrm{C}$. FAR1 was amplified from Arabidopsis root cDNA using primers FAR1-GW_Forward and FAR1-GW_Reverse (see supplementary table for primer sequence) and verified by DNA sequencing. The corresponding PCR fragment (Full length FAR1 cDNA-STOP GW) was cloned into pDONR ${ }^{\mathrm{TM}} 221$ ENTRY vector by GATEWAY ${ }^{\circledR}$ recombination cloning technology using the attB $\mathrm{x}$ attP (BP) recombination sites resulting in pDONR221:FAR1-stop (FAR1 lacks stop codon) vector. Fragments were then transferred into the pK7YWG2 DESTINATION vector (Karimi et al., 2002) by LR cloning, resulting in a 3'-transcriptional fusion between the cDNA and the GFP gene. The resultant construct produced 35S promoter driven, FAR1-GFP fusion protein. Constructs were transferred into the Agrobacterium tumefaciens strain GV3101.

\subsubsection{Transient expression of AtFAR1-GFP in $N$. benthamiana leaves and analysis by confocal microscopy}

Leaves of three-week-old $N$. benthamiana plants were infiltrated with Agrobacterium carrying pK7YWG2:AtFAR1-GFP vector as described in Section 4.3.8. The infiltration was carried out in the absence of p19 to avoid overaccumulation of fluorescent protein in the cells. HDEL-GFP and Rubisco-GFP were used as ER and plastid positive markers, respectively (Napier et al., 1992; Gomord and Faye, 1996). 35S-GFP and empty vector were used as controls. Three days post-infiltration, a thin 
strip of $N$. benthamiana leaves injected with Agrobacterium cell suspension containing pK7YWG2:AtFAR1-GFP vector was cut and mounted on a glass slide with fluoromount G (Electron Microscopy Sciences) and imaged using a confocal microscope (Zeiss LSM510 Meta Confocal). Excitation was with an Argon/2 laser at a wavelength of $488 \mathrm{~nm}$. Emission was between $505-565 \mathrm{~nm}$ for imaging of GFP and $650 \mathrm{~nm}$ for imaging of chlorophyll autofluorescence.

\subsection{Results}

\subsubsection{Heterologous expression of Arabidopsis FAR1, FAR4, and FAR5 and FAR1/4/5 domain swap chimeras in yeast}

Plant FARs contain two distinct domains: a Rossmann fold NAD(P)H-binding domain and a FAR_C domain (Figure 1.12) according to the protein structure prediction software Conserved Domains Database (CDD) (Marchler-Bauer et al., 2010). We used a domain swapping approach to determine regions important for the substrate specificities of FAR1, FAR4 and FAR5. There is a relatively high amino acid sequence identity between FAR1 and FAR4 (74\%), FAR1 and FAR5 (68\%) and FAR4 and FAR5 (69\%) (Table 4.1), which facilitated this approach. Six of the FAR chimeras were generated by swapping the Rossmann fold NAD(P)H-binding domain and the FAR_C domain between FAR1, FAR4 and FAR5 to give FAR1 Rossmann- $_{\text {- }}$

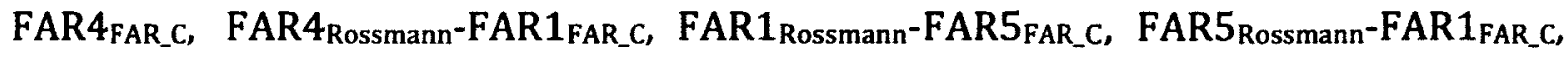
FAR4 $4_{\text {Rossmann-FAR5 }}$ FAR_c, and FAR5 $_{\text {Rossmann }}$-FAR4 FAR_c $_{-}$(see Methods for exact amino acid positions of junctions). Another set of four FAR chimeras were generated 
between FAR1 and FAR4 only, containing fusions at junctions located $25 \%, 50 \%$, and $75 \%$ along the polypeptides to give FAR4 ${ }_{25 \%}$-FAR1 $75 \%$, FAR4 ${ }_{50 \%}$-FAR1 $1_{50 \%}$, FAR475\%-FAR1 $25 \%$, and FAR1 ${ }_{50 \%}$-FAR450\% (see Methods for exact amino acid positions of junctions). The genes were placed under the control of the GAL1 promoter for high-level, rapid induction in yeast. The proteins were expressed with an $\mathrm{N}$-terminal T7 epitope tag for detection by Western blotting.

Western blot analysis indicated that wild-type FAR1, FAR4, and FAR5, and the FAR chimeric proteins accumulated to varying levels in the yeast cells, with no cross-reacting protein in the empty vector control (Figure 4.3). FAR1, FAR4 and FAR5 accumulated to relatively high levels in yeast and migrated at positions of their predicted molecular weights of $55.5 \mathrm{kDa}, 56.2 \mathrm{kDa}$ and $56.4 \mathrm{kDa}$, respectively, assuming no post-translational modifications. The FAR chimera proteins were also all in the 56-58 KDa molecular weight range. FAR1 $1_{\text {Rossmann }}$-FAR4 $4_{\text {FAR_c, }}$ FAR1 Rossmann $^{-}$ FAR5 FAR_c, $_{-}$and FAR4 $4_{\text {Rossmann }}$-FAR5 FAR_c $_{-}$chimeras accumulated to very low levels relative to the other FAR proteins, which corresponded with little accumulation of fatty alcohol in these yeast strains (see below). FAR5 Rossmann $_{\text {-FAR4 }}$ FAR_c and FAR450\%FAR1 $1_{50 \%}$ chimeras accumulated to levels intermediate that of the three low level FARs and the other high-level accumulating FARs, which included the wild-types,

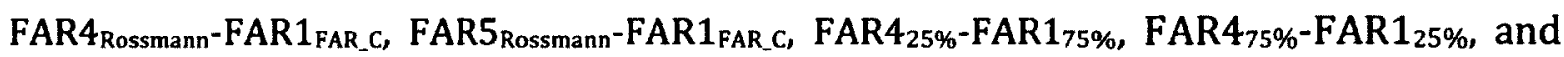
FAR1 ${ }_{50 \%}-$ FAR4 $_{50} \%$.

The lipids from the yeast cells expressing the wild-type and chimeric FARs were extracted and analyzed by gas chromatography, focussing on fatty alcohol production (Figure 4.4). The fatty alcohols were produced using the endogenous 
acyl-CoAs pools in S. cerevisiae as substrates (i.e. no feeding of fatty acids). Yeast normally do not accumulate any fatty alcohols. FAR1 mainly produced $\mathrm{C} 22: 0-\mathrm{OH}$ $\left(0.45 \mu \mathrm{g} / \mathrm{OD}_{600}\right.$ unit), and to a lesser extent $\mathrm{C} 18: 0-\mathrm{OH}, \mathrm{C} 20: 0-\mathrm{OH}$ and $\mathrm{C} 24: 0-\mathrm{OH}$. FAR4 mainly produced $\mathrm{C} 20: 0-\mathrm{OH}\left(0.39 \mu \mathrm{g} / \mathrm{OD}_{600}\right.$ unit $)$ and to a lesser extent $\mathrm{C} 18: 0-$ $\mathrm{OH}$ and $\mathrm{C} 22: 0-\mathrm{OH}$. FAR5 mainly produced $\mathrm{C} 18: 0-\mathrm{OH}\left(\sim 3.2 \mu \mathrm{g} / \mathrm{OD}_{600}\right.$ unit $)$ and to a lesser extent $\mathrm{C} 16: 0-\mathrm{OH}$ and $\mathrm{C} 20: 0-\mathrm{OH}$ (Figure 4.5). These results are in agreement with our previous results reported in Chapter 2, although the chain-length specificity appeared stricter here possibly due to the lesser time of FAR expression prior to harvesting ( 2 days of growth and genes were under inducible GAL1 promoter, rather than 5 days of growth and genes under strong constitutive promoter as used in Chapter 2 experiments). Among the FAR chimeras, yeast

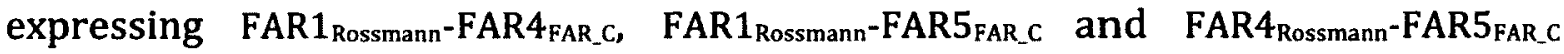
showed very low accumulation of any fatty alcohols likely due to their very low protein accumulation as measured by Western blot analysis (Figure 4.3). The

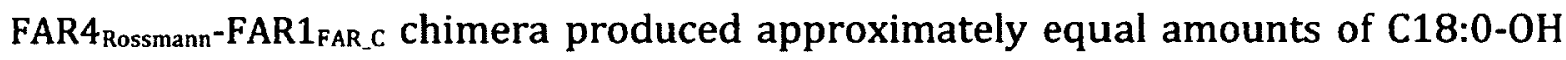
$\left(0.16 \mu \mathrm{g} / \mathrm{OD}_{600}\right.$ unit) and $\mathrm{C} 22: 0-\mathrm{OH}\left(0.12 \mu \mathrm{g} / \mathrm{OD}_{600}\right.$ unit), followed by $\mathrm{C} 20: 0-\mathrm{OH}$ $\left(0.05 \mu \mathrm{g} / \mathrm{OD}_{600}\right.$ unit). This chimera produced $88.3 \%$ lower $\mathrm{C} 20: 0-0 \mathrm{H}$ compared to native FAR4 and $74.4 \%$ lower C22:0-OH compared to native FAR1, but it produced $36 \%$ and $73.3 \%$ higher amounts of $\mathrm{C} 18: 0-\mathrm{OH}$ when compared to native FAR4 and FAR1 respectively. The FAR5 Rossmann $_{\text {-FAR1 }}$ FAR_C $_{\text {C }}$ chimera showed high specificity to C18:0 acyl chain. The fatty alcohol profile was similar to native FAR5 in producing mostly C18:0-OH followed by $\mathrm{C} 16: 0-\mathrm{OH}$ and $\mathrm{C} 20: 0-\mathrm{OH}$, although the total $\mathrm{C} 18: 0-\mathrm{OH}$ produced was lower by $87.5 \%$ compared to native FAR5. When compared to native 
FAR1, a very small amount of $\mathrm{C} 22: 0-\mathrm{OH}\left(0.01 \mu \mathrm{g} / \mathrm{OD}_{600}\right.$ unit $)$ was detected in yeast

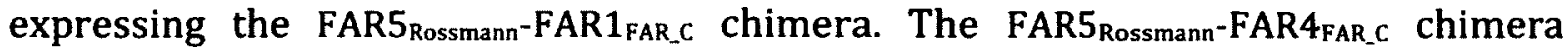
produced similar amounts of $\mathrm{C} 18: 0-\mathrm{OH}$ and $\mathrm{C} 20: 0-\mathrm{OH}$ in contrast to the quite strict specificities of native FAR4 or FAR5. However, FAR5 Rossmann $_{\text {-FAR4 }} 4_{\text {FAR_c }}$ produced $88 \%$ lower $\mathrm{C} 18: 0-\mathrm{OH}$ and $42.9 \%$ lower $\mathrm{C} 20: 0-\mathrm{OH}$ when compared to native FAR5 and FAR4, respectively. The FAR4 $_{25 \%}$-FAR $1_{75 \%}$ and FAR4 ${ }_{50 \%}$-FAR1 $1_{50 \%}$ chimeras mostly produced $\mathrm{C} 22: 0-\mathrm{OH}$, with approximately equally low amounts of $\mathrm{C} 18: 0-\mathrm{OH}$ and C20:0-OH. In FAR4 $4_{50 \%}-\mathrm{FAR} 1_{50 \%}$ chimera expressing yeast, when compared to native FAR4, the C20:0-OH produced was lower by $\sim 87.8 \%$, while $\mathrm{C} 22: 0-0 \mathrm{H}$ was reduced by $\sim 43.5 \%$ when compared to native FAR1. FAR4 $4_{75 \%}$-FAR $1_{25 \%}$ did not result in significant accumulation of any chain length of fatty alcohol, although the $\mathrm{C} 18: 0-\mathrm{OH}$, $\mathrm{C} 20: 0-\mathrm{OH}$ and $\mathrm{C} 22: 0-\mathrm{OH}$ fatty alcohols it did generate were in similar amounts. The FAR1 $1_{50 \%}$-FAR $4_{50 \%}$ chimera showed high specificity to C20:0 acyl chain, although it produced $42.5 \%$ lower amounts of $\mathrm{C} 20: 0-0 \mathrm{H}$ relative to the native FAR4. The C22:0$\mathrm{OH}$ produced was comparable to the amount produced by native FAR4, although compared to native FAR1, it produced $81 \%$ lower $\mathrm{C} 22: 0-0 \mathrm{H}$.

\subsubsection{Transient overexpression of Arabidopsis FAR1, FAR4 and FAR5 in Nicotiana benthamiana leaves}

We wanted to investigate further the in planta specificities of FAR1, FAR4, and FAR5 by examining transgenic Arabidopsis lines overexpressing these proteins, as well as use these lines to test for any phenotypic changes. Transgenic Arabidopsis lines containing T-DNAs with FAR1, FAR4 and FAR5 open reading frames under the 
control of $35 \mathrm{~S}$ promoter were generated. However, none of the lines had a major increase in transcript levels, nor showed any phenotype when compared to wildtype control. As an alternative approach, we examined the effects of overexpressing FAR1, FAR4, and FAR5 using Agrobacterium-mediated transient assays in leaves of Nicotiana benthamiana. Leaves were co-infiltrated with Agrobacteria containing the same 35S:FAR constructs described above and Agrobacteria containing a binary construct with $\mathrm{p} 19$ also under control of the $35 \mathrm{~S}$ promoter. $\mathrm{p} 19$ is a viral protein that suppresses gene silencing (Voinnet et al., 2003) and will thus increase and prolong the expression of transgenes in plant host cells. GFP was used as an expression marker to monitor the transient expression levels. The leaf samples were collected 4-5 days post infiltration and analyzed for alterations in total lipids, surface waxes, and leaf lipid polyester (cutin).

Analysis of total internal lipids from infiltrated $N$. benthamiana leaves showed that primary alcohols were produced in leaves infiltrated with FAR1, FAR4 and FAR5 genes, in contrast to the insignificant alcohol production detected with infiltrations involving negative control (empty vector) (Figure 4.6, Table S4.2). Leaves expressing FAR1 showed highest accumulation of total primary alcohols $(9.5$ fold increase), followed by FAR4 (5.6 fold increase), and FAR5 (4.3 fold increase). The transient over-expression of FAR1 predominately generated $\mathrm{C22:0-0H,} \mathrm{which}$ was $78 \%$ of the total primary alcohol load in transformed leaves. The transient overexpression of FAR4 predominately generated C20:0-0H, which was $66 \%$ of the total primary alcohol load in transformed leaves. The transient over-expression of FAR5 predominately generated $\mathrm{C} 18: 0-\mathrm{OH}$, which was $64.5 \%$ of the total primary alcohol 
load in transformed leaves. In addition, a significant decrease in C16:0 fatty acid was observed in the internal lipids of leaves expressing FAR1, FAR4 or FAR5, while a significant decrease in C18:0 fatty acid was additionally seen in leaves expressing FAR5.

Similar results were observed in the analysis of chloroform-extracted surface wax composition of infiltrated $N$. benthamiana leaves. The leaves infiltrated with empty vector showed only minor quantities of fatty alcohol in the surface wax, with C22:0-OH being the predominant one and the total fatty alcohol load was 0.016 $\mu \mathrm{g} / \mathrm{cm}^{2}$. In comparison to the negative control (empty vector), leaves transiently overexpressing FAR1 showed highest accumulation of primary alcohols (15.5 fold increase), followed by FAR4 ( 4 fold increase), and FAR5 (1.8 fold increase) (Figure 4.7, Table-S4.3). The transient over-expression of FAR1 showed a significant increase in accumulation of $\mathrm{C} 22: 0-\mathrm{OH}(96.4 \%)$, which constituted $\sim 92.8 \%$ of the total primary alcohol load in cuticular waxes of transformed leaves. The transient overexpression of FAR4 showed a significant increase in accumulation of C20:0-OH (93\%), which constituted $\sim 71.7 \%$ of the total primary alcohol load in cuticular waxes of transformed leaves. The transient over-expression of FAR5 showed a significant increase in $\mathrm{C} 18: 0-\mathrm{OH}(90 \%)$, which constituted $\sim 64.1 \%$ of the total primary alcohol load in cuticular waxes of transformed leaves. In addition, a significant decrease in C16:0 and C18:0 fatty acids were observed in surface lipids of $N$. benthamiana leaves expressing FAR1, FAR4 or FAR5. However, the levels of alkanes were unaffected. 
We also analyzed the composition of leaf cutin polymer in $N$. benthamiana leaves transiently expressing FAR1, FAR4 or FAR5 genes. Surprisingly, no significant alterations were observed in the leaf cutin composition of leaves expressing any of the Arabidopsis FARs compared to leaves infiltrated with empty vector (Figure 4.8).

\subsubsection{Subcellular localisation of Arabidopsis FAR1}

Arabidopsis FAR1, FAR4, and FAR5 have been proposed to be located at the endoplasmic reticulum (Rowland and Domergue, 2012), but no experimental evidence yet exists. To determine the subcellular localisation of FAR1, a transient expression assay was carried out in Nicotiana benthamiana leaves and the subcellular location of FAR1 fused at its C-terminus with green fluorescent protein (GFP) was examined by laser scanning confocal microscopy. The FAR1-GFP transcript was driven by the cauliflower mosaic virus (CaMV) $35 \mathrm{~S}$ promoter. The vector control expresses free GFP, which localizes to the cytoplasm and nucleus (no ER localization). A GFP fusion containing an $\mathrm{N}$-terminal secretory signal and a Cterminal HDEL endoplasmic reticulum (ER) retention signal (GFP-HDEL) was used as an ER positive marker. HDEL (His-Asp-Glu-Leu) is a C-terminal tetrapeptide found in yeast and plants, which retains proteins that are part of the secretory pathway in the endoplasmic reticulum (Napier et al., 1992). The FAR1-GFP fusion clearly showed fluorescence associated with the reticulations of cortical ER, similar to that of fluorescence observed with GFP-HDEL (Figure 4.9). 


\subsection{Discussion}

\subsubsection{Substrate specificities of plant FARs are governed by both the Rossmann fold domain and the FAR_C domain}

We previously studied the substrate specificities of Arabidopsis FAR1, FAR4 and FAR5 by heterologously expressing them in yeast as well as by analysing the suberin compositions of T-DNA mutant lines of these genes (Chapters 2 and 3 of this thesis). The results indicated that FAR1 is highly specific for C22:0-CoA, FAR4 is highly specific for C20:0-CoA, and FAR5 is highly specific for C18:0-CoA. Here, we attempted to gain insights into the protein regions responsible for governing the substrate specificities of FAR1, FAR4 and FAR5 by a domain-swapping approach, wherein the Rossmann fold and FAR_C domains were swapped between FAR1 and FAR4, FAR1 and FAR5, and FAR4 and FAR5. With the exception of FAR1 ${ }_{\text {Rossmann- }}$ FAR4 $_{\text {FAR }_{-} \text {, }}$ FAR1 $1_{\text {Rossmann }}$-FAR5 FAR_C $_{-}$FAR4 Rossmann $_{\text {-FAR5 }}$ FAR_c, $_{\text {, }}$ which were unstable in yeast for reasons that are unclear, the chimeric proteins were found to be variably active. The FAR5 Rossmann-FAR1 $_{\text {FAR_c }}$ chimera displayed FAR5-like specificity for C18:0-CoA, although with 8-fold less fatty alcohol produced in yeast than native FAR5 indicating a reduction in enzyme specific activity. This would suggest a major role for the Rossmann fold domain in determining substrate specificity. However, the FAR5 $_{\text {Rossmann-FAR4 }}$ FAR_C $_{-}$chimera showed specificity towards both C18:0 and C20:0 acyl chain substrates, indicating that the FAR_C domain contributes to substrate specificity. This chimera produced much less C18:0-OH than native FAR5 (8-fold lower), while the $\mathrm{C} 20: 0-\mathrm{OH}$ levels were similar to that produced by native FAR4. 
Similarly, the FAR4 $4_{\text {Rossmann }}$ FAR1 $1_{\text {FAR }}$ chimera produced 7-fold less C20:0-OH than native FAR4, while C22:0-OH levels remained almost the same as that produced by native FAR1. These results indicate that FARs with broader specificities are possible, but that they may be compromised in their activities for one or more of the chain lengths. This may explain in part why three FARs are involved in the production of C18:0, C20:0 and C22:0 suberin-associated fatty alcohols, rather than a single FAR with broad specificity, as then production of the three chain lengths are maximized.

Among the other set of chimeras involving quarterly divisions of FAR4 and FAR1, FAR4 ${ }_{25 \%}$-FAR1 $75 \%$, and FAR4 ${ }_{50 \%}$-FAR1 $1_{50 \%}$ produced similar fatty alcohol profiles in yeast as that of native FAR1 with high specificities towards C22:0-CoA. The FAR4 ${ }_{75 \%}$-FAR $1_{25 \%}$ chimera resulted in a 10-fold decrease in C22:0-OH levels without any increase in other chain length fatty alcohols. On the other hand, the FAR1 $1_{50 \%-}$ FAR4 ${ }_{50 \%}$ chimera showed a specificity profile similar in yeast to that of the native FAR4 enzyme, although with slightly reduced C20:0-0H production. Taken together, these results indicate that the differential specificities of FAR4 and FAR1 for C20:0 and C20:0 acyl chains, respectively, is largely determined by the region between amino acids 235 and 385 spanning part of the N-terminal Rossmann fold domain and the FAR_C-terminal domain. This region is moderately less conserved between plants FARs relative to the other regions of the proteins (Figure 4.1). It is likely, however, that amino acids outside of this central region can influence chain length specificity as interactions between amino acid residues in the folded up protein likely dictates the precise ability to bind a particular acyl-CoA chain. Site directed substitutions focusing on the 235 to 385 region of FARs can be used to further 
identify individual amino acid residues that have key roles in governing the chainlength specificity.

However, it is difficult to rationalize these results in detail without the aid of three-dimensional protein structure information. An X-ray crystal or nuclear magnetic resonance (NMR) structure of a FAR protein has not yet been reported. From the utilitarian point of view, the tertiary structure is very important for the understanding of molecular mechanisms. The highest sequence identity for a solved protein structure to model FAR protein structure is only $26 \%$. In addition, this structure of a non-homologous protein only includes the Rossmann fold domain and lacks the FAR_C domain determined here to be important for chain-length substrate specificity. Determining the structure of a FAR should be a future priority in FAR enzyme characterization. This would be most feasible with a soluble, plastid FAR, such as FAR2/MS2 or FAR6 from Arabidopsis. Other membrane-associated FARs (e.g. FAR1, FAR4, and FAR5) may be modelled from the soluble FAR threedimensional structure. Additionally, comparative structure-function analysis or directed evolution of these enzymes would provide further insights into the amino acids responsible for conferring substrate specificity (from inspection of active sequences). This structural information would allow for the engineering FAR enzymes with desired activities and specificities for the production of primary fatty alcohols of commercial value. 


\subsubsection{FAR1, FAR4 and FAR5 specificities in planta and influence of acyl-CoA pool on fatty alcohol production}

Substrate specificity studies carried out in a heterologous system (e.g. yeast) and using endogenous acyl pools of substrate do not always match the in planta specificities as the availabilities and relative amounts of substrates present in the expression system can significantly influence the products produced. Although $S$. cerevisiae is quite a reliable heterologous system to gain insights into the substrate specificities of plants FARs especially compared to E. coli (Doan et al., 2009; Rowland and Domergue, 2012), the yeast endogenous subcellular pool of available acyl chains still governs to a certain extent the chemical composition of primary alcohols produced. In contrast, heterologous expression in Nicotiana benthamiana leaves may provide more accurate information on the substrate specificities as the composition of the acyl pools between $N$. benthamiana and Arabidopsis tissues is likely to be more similar. We therefore carried out transient overexpression of FAR1, FAR4 and FAR5 in $N$. benthamiana leaves to further study their substrate specificities. In agreement with our previous results, the overexpression of FAR1, FAR4 and FAR5 resulted in significant accumulation of predominantly C22:0-OH, C20:0-OH and C18:0-0H, respectively, in both total lipids and cuticular waxes of $N$. benthamiana leaves. This confirms that each of these FARs has fairly high specificity to one particular chain length. Nonetheless, FAR1 also slightly increased the levels of $\mathrm{C} 20: 0-\mathrm{OH}$ and $\mathrm{C} 24: 0-\mathrm{OH}$ in leaves; FAR4 slightly increased the levels of $\mathrm{C} 18: 0-\mathrm{OH}$ and C22:0-OH, and FAR5 slightly increased the levels of C16:0-0H and C20:0-OH compared to control, indicating that the specificities are not entirely strict. FAR5 
when expressed in yeast resulted in production of nearly exclusively C18:0-0H (>90\%) with minor quantities of $\mathrm{C} 16: 0-\mathrm{OH}$ and $\mathrm{C} 20: 0-\mathrm{OH}$. However, in the total lipids of $N$. benthamiana leaves expressing FAR5, C18:0-OH only constituted $64.5 \%$ of total alcohol load, with $\mathrm{C} 16: 0-\mathrm{OH}$ and $\mathrm{C} 20: 0-\mathrm{OH}$ constituting the rest. This indicates that each FAR has overlapping specificity in planta and can produce more than one particular chain length of fatty alcohol in plants. This further supports the notion of compensatory effects of one FAR in the absence of another to maintain the total primary fatty alcohol load and composition in the suberin polymer (Chapter 2).

When expressed in yeast, FAR5 appeared to have the highest activity since it produced six times more product, i.e. C18:0-OH $(\sim 3 \mu \mathrm{g} / \mathrm{OD}$ unit) compared to FAR1 and FAR4 producing $\mathrm{C} 22: 0-\mathrm{OH}$ and $\mathrm{C} 20: 0-\mathrm{OH}$, respectively (each $\sim 0.5 \mu \mathrm{g} / \mathrm{OD}$ unit). However, analysis of total lipids and cuticular waxes of $N$. benthamiana leaves overexpressing the FARs indicated that FAR1 had the highest activity compared to FAR4 and FAR5. The expression of FAR1 resulted in 2-6 times more product (C22:0$\mathrm{OH})$ compared to FAR4 $(\mathrm{C} 20: 0-\mathrm{OH})$ and FAR5 $(\mathrm{C} 18: 0-\mathrm{OH})$. This is likely due to differences in the endogenous acyl pools of the expression systems. The acyl pool in yeast cells is abundant in C16:0-CoA and C18:0-CoA, while $N$. benthamiana leaves has relatively higher amounts of longer chain lengths $(>\mathrm{C} 18: 0-\mathrm{CoA})$ in the acyl pool (Doan et al., 2011). Thus, the apparent activity is determined in part by the abundance of relevant acyl-CoA substrates in the expression system. Direct biochemical and kinetic analysis using in vitro assays with purified FAR enzyme must be carried out for accurate quantification of enzyme activity and substrate specificity. However, these in vitro assays have only recently been developed for the 
soluble plastid FARs (Chen et al., 2011; Doan et al., 2011) and in vitro enzymatic activities of the insoluble, microsomal membrane bound FARs (e.g. FAR1, FAR4, and FAR5) have not yet been reported.

We expected that the fatty alcohols produced by the overexpressed FARs in $N$. benthamiana leaves would be found in the lipid polyester (cutin) as these chain lengths are found in cutin of wild-type Arabidopsis, albeit at low levels (Chapter 2). We also tested for their accumulation in cuticular waxes, although fatty alcohols of surface waxes are usually longer (C24:0 or longer) (Rowland et al., 2006). Although there was significant accumulation of fatty alcohols in total lipids, overexpression of FAR1, FAR4 or FAR5 resulted in no change in the cutin polyester composition of $N$. benthamiana leaves. There may be no enzyme activity to facilitate incorporation of fatty alcohols into the cutin polymer in $N$. benthamiana leaves and/or the sites of incorporation are outcompeted by other monomers such as omega-hydroxy fatty acids. However, there were fatty alcohols at the cuticle in the wax fraction. The surface wax composition of $N$. benthamiana leaves overexpressing FAR1 showed 6 times higher accumulation of $\mathrm{C} 22: 0-0 \mathrm{H}$, compared to the levels of $\mathrm{C} 20: 0-\mathrm{OH}$ and C18:0-OH observed in leaves over-expressing FAR4 or FAR5, respectively. Analysis of primary alcohols in cuticular waxes from Arabidopsis stems and leaves has shown that they are all saturated with 20 carbons or longer (C22:0-C30:0), and alcohols with 26 carbons are the most frequent species (Rowland et al., 2006; Lai et al., 2007). The higher amount of $\mathrm{C} 22: 0-\mathrm{OH}$ in cuticular wax indicates that only verylong chain fatty alcohols $(>\mathrm{C} 22)$ are secreted efficiently and transported to the 
surface for deposition into the waxes, while shorter chain fatty alcohols (C18:0C20:0-OH) are perhaps not efficiently secreted.

We attempted to stably overexpress FAR1, FAR4 and FAR5 in Arabidopsis. However, we were unable to identify transgenic lines exhibiting significant overexpression. It is possible that accumulation of fatty alcohols in the absence of an efficient secretion system could be toxic and detrimental for cell viability. Therefore, there would be selection against the overexpressing lines. An alternative approach would be to generate inducible (e.g. dexamethasone) overexpression Arabidopsis lines.

\subsubsection{Arabidopsis FAR1 is localised to the Endoplasmic Reticulum (ER)}

Arabidopsis FAR2/MS2 and FAR6 as well as rice DPW have been localized to plastids using FAR-GFP fusions (Chen et al., 2011; Shi et al., 2011; Doan et al., 2012). However, there had been no definitive in planta subcellular localization reports for the non-plastid FARs. Arabidopsis FAR1, FAR3/CER4, FAR4, FAR5, and FAR8 were predicted to be ER-localized and to contain 2-3 transmembrane domains (Rowland and Domergue, 2012). The jojoba embryo FAR enzyme was found to be associated with microsomal membrane fractions highly enriched in ER membranes, although there was the possibility of plasma or other membrane contamination (Metz et al., 2000). A FAR3/CER4-GFP fusion was found to be in the endoplasmic reticulum when expressed in yeast (Rowland et al., 2006), but attempts to express FAR3/CER4-GFP fusions in plants were unsuccessful (Owen Rowland, personal communication). We found here that FAR1-GFP is localized to the ER when transiently expressed in $N$. benthamiana leaves, and it is likely that FAR4 and FAR5 
also reside in the ER due to their high degree of sequence relatedness with FAR1. FAR4 and FAR1 use very-long chain fatty acyl-CoA substrates (C20:0- and C22:0CoA, respectively), which are produced by the ER-localized fatty acid elongase. A close subcellular proximity of FARs to the site of synthesis of their cognate substrates would allow for efficient channelling. In addition, other suberin biosynthetic enzymes have been found to reside in the ER. CYP86A1/HORST and CYP86B1/RALPH, which are responsible for generation of long-chain and very-long chain $\omega$-hydroxyacids and $\alpha, \omega$-dicarboxylic acids, respectively, have also been localized to the ER (Hofer et al., 2008; Campagnon et al., 2009). GPAT5 is predicted to be ER membrane localized (Beisson et al., 2005), and ER localization has been experimentally demonstrated for the highly related GPAT1 and GPAT8 enzymes that are involved in cutin formation (Zheng et al., 2003; Gidda et al., 2009). Predictions based on the ARAMEMNON database (Schwacke et al., 2003) localize DAISY/KCS2 to endomembranes. Overall, these results strongly indicate that most, or perhaps all, of the suberin monomer biosynthetic steps occur in the endoplasmic reticulum.

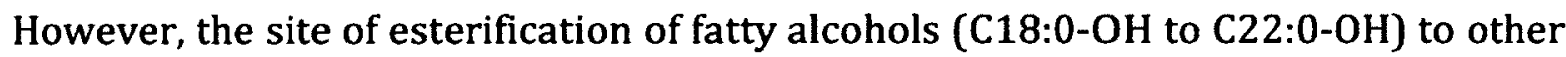
suberin monomers or hydroxycinnamic acids of the phenylpropanoid pathway (generating AHCs) remains to be identified. The site of suberin polymerization is likely to be extracellular. An extracellular cutin polymerase, which is part of the GDSL-motif lipase/hydrolase family, has recently been reported in tomato (Yeats et al., 2012). By analogy, a related extracellular GDSL lipase may be involved in suberin polymerization. 
Table 4.1: Amino Acid Sequence Identity between the Arabidopsis FAR family and Jojoba FAR

\begin{tabular}{|c|c|c|c|c|c|c|c|c|c|}
\hline $\begin{array}{c}\text { \% } \\
\text { Identity }\end{array}$ & FAR1 & FAR2 & FAR3 & FAR4 & FAR5 & FAR6 & FAR7 & FAR8 & $\begin{array}{c}\text { Jojoba } \\
\text { FAR }\end{array}$ \\
\hline FAR1 & - & 39 & 51 & 74 & 68 & 34 & 61 & 64 & 51 \\
\hline FAR2 & 39 & - & 39 & 38 & 39 & 41 & 38 & 38 & 39 \\
\hline FAR3 & 51 & 39 & - & 50 & 50 & 33 & 47 & 49 & 54 \\
\hline FAR4 & 74 & 38 & 50 & - & 69 & 35 & 61 & 65 & 51 \\
\hline FAR5 & 68 & 39 & 50 & 69 & - & 35 & 62 & 85 & 51 \\
\hline FAR6 & 34 & 41 & 33 & 35 & 35 & - & 32 & 35 & 32 \\
\hline FAR7 & $\mathbf{6 1}$ & $\mathbf{3 8}$ & 47 & 61 & 62 & 32 & - & 60 & 47 \\
\hline FAR8 & 64 & 38 & 49 & $\mathbf{6 5}$ & $\mathbf{8 5}$ & 35 & 60 & - & 49 \\
\hline $\begin{array}{c}\text { Jojoba } \\
\text { FAR }\end{array}$ & 51 & 39 & 54 & 51 & 51 & 32 & 47 & 49 & - \\
\hline
\end{tabular}

* Based on the full length CDSs from the TAIR website for FAR1 (At5g22500), FAR2 (At3g11980), FAR3 (At4g33790), FAR4 (At3g44540), FAR5 (At3g44550), FAR6 (At3g56700), FAR7 (At5g22420), FAR8 (At3g44560) and the protein sequence for the Jojoba FAR (AAD38039) obtained from NCBI (Carnegie Institution of Washington Department of Plant Biology, 2008; U.S. National Library of Medicine. (2009); European Bioinformatics Institute, 2010). 
Arabidopsis_FAR1
Arabidopsis_FAR4
Arabidopsis_EAR5

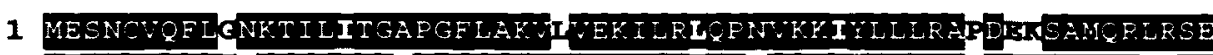

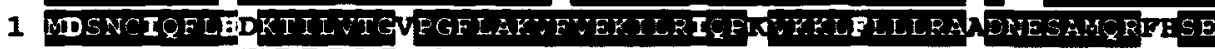

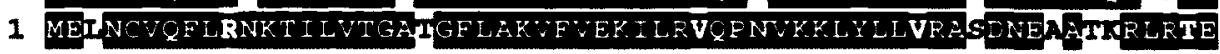

Arabidopsis_FAR1 Arabidopsis_FAR4

ArabidopBiB_FAR5
61 WMEIDLFKVRNNLGEDNLNALEREK IVEVPGD I I INLGLYDTDLIQR MASEE I I I I INI

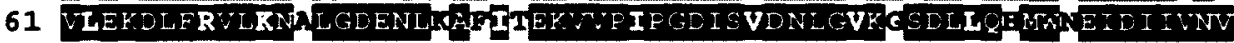

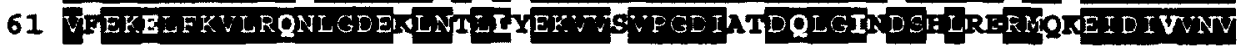

Arabidopa1B_FARI 121 AATTNFDERYDIGLGINTFGALNULNEAKKCVYGQLLLHVSTAYISGERPGLLIEKPFKM Arabidopais_FAR4 121 AATTNFDERYDVGLSVNTFGPLNVLNFARCVYGQLLLHVSTATVRGEKSGLEEEKIFHM Arabidopgis_FAR5 121 AATNNFDERYDVGLGINTFGALNVLNARKCVYVELLLHVSTAYVCGEKPGLIPEKPFIM

Arab1dopaiB_FAR1 181 GETLSGD-REDDINIEHDLMKCKLKELQE--CSDEEISQTMKDFGMARAKLHGWPNTYVF Arabidopa1B_FAR4 181 GETLNGA-RALVIETEVELMKQKLKELQAQNCSEEE I SQSMKDLGMSRAKI_HGWPNTYVF Arabidopa18_FAR5 181 EEIRWEWGLQLDINLERELMKQRLKELNEODCSEEDITLSMKELGMERAKLHGWPNTYVF

Arabidops18_FAR1 238 TKAMGEMLYGKYRENLPLVIIRPTMITSTIAEPFPGWIEGLRTLDSVIVAYGKGRLKCFL Arabidops18_FAR4 240 TKSMGEMLLGYRENLFIVIIRPTMITSTFISEFFPGWIEGLRTIDSVIVAYGKGRLKCFL Arabidops1a_FAR5 241 TKSMGEMLLGKWKENLPLVI IRPTMI TSTLSEPFPGWIEGLRTVDSVIIAYGKGVLKCFL Arab1dopa18_EAR1 298 ADSNSVFDEIPADMVVNAMVAAATAFSGDTGIQAIYHVGSSCKNPVTFGQLHDFTARYFA Arab1dopsis_FAR4 300 ADPNSVIDLI PVDMUANAMVTAAAIHAGKIGSOVYHVGSSCKNPITFEQI HDIAASYFT Arab1dopsis_FAR5 301 VDVNSVCDMI PVDMVANAMITAAANHAGGSGVEVYHVGS SEONPVTFGEI HEI AVRYFT

Arabidops 18_FAR1 358 KRPLICRNGSPIIVVKGTILSTMAQFSLYMTLRYKLPLQILRLINIVYPWSEGDNYSDLS

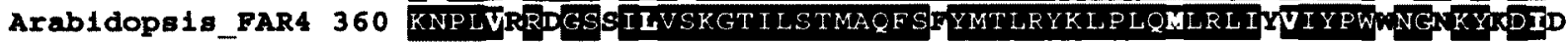
Arab1dopsis_FAR5 361 KNPLRSRNGSI TIVSKVRFIPTMALFSLYMTLRYK LP LQHLK LVD I I Y PWRNGDKYGDKN

Arabidopsis_FAR1 418 RKIRLAMRLVELYQPYLLFKGIFDDLNTERLRMKRKE--NIKEIDGSFEF DPKS IDWDYY Arab1dopsis_FAR4 420 RKIRLAMRLVDLYRPYVLFKGIFDDINTEKLRLKRKE--TWKEWYGLFEFDPKSIDWEDY

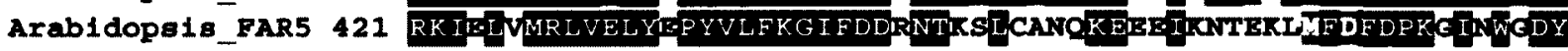

Arabldope1s_FAR1 476 ITRIHIPGLITHVLKR

Arabidope is FAR4 478 TTIFIPGLITYVLKK

Arabldopsis_FAR5 481 ITNIHFGGLVTHVLKK

Figure 4.1. Alignment of Arabidopsis FAR1, FAR4 and FAR5 protein sequences. Identical residues $=$ black, physicochemical similar residues $=$ grey. The alignment was constructed using ClustalW2 and BOXSHADE 3.21Server. Full length CDSs were obtained from the TAIR website for FAR1 (At5g22500), FAR4 (At3g44540), FAR5 (At3g44550). The residues denoted with stars correspond to the predicted active site motif. 


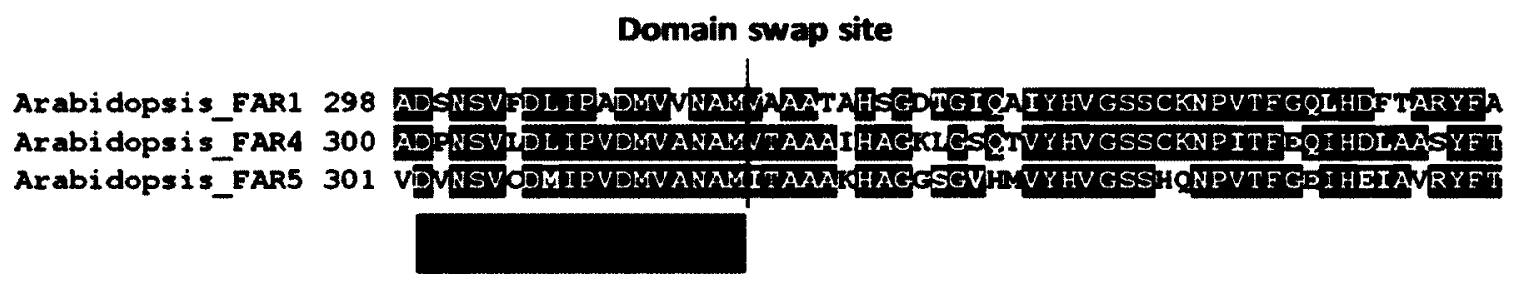

Figure 4.2a. Domain swap site at the junction of the Rossmann fold domain and the FAR_C domain (annotated FAR_C domain starts at 389, 391 and 392 a.a. residue for FAR1, FAR4, and FAR5 repectively] is shown in the alignment of FAR1, FAR4 and FAR5 protein sequences. 


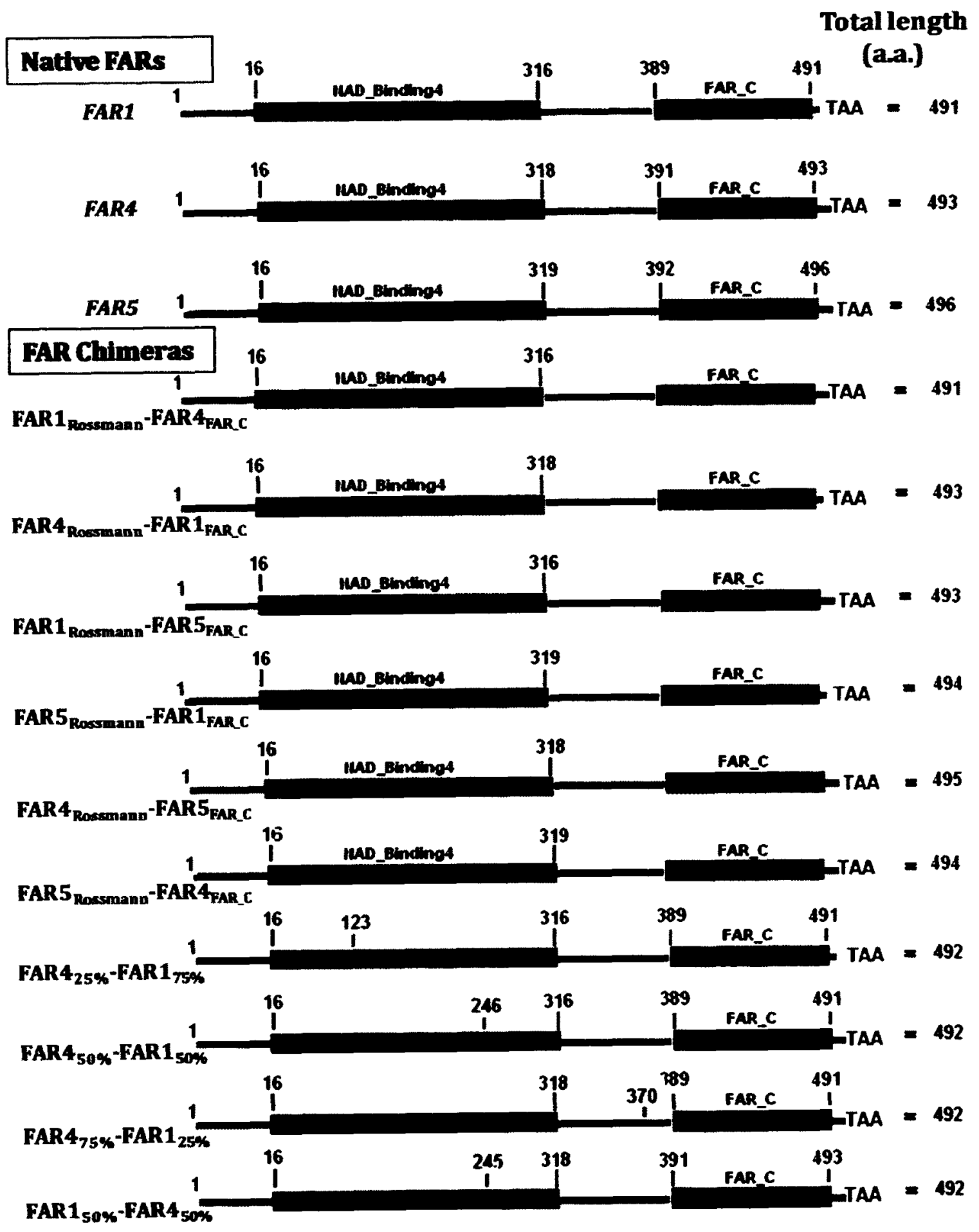

Figure 4.2b. Schematic showing domains of native FAR1, FAR4 and FAR5 protein and the resultant FAR chimeras after domain swaps. 


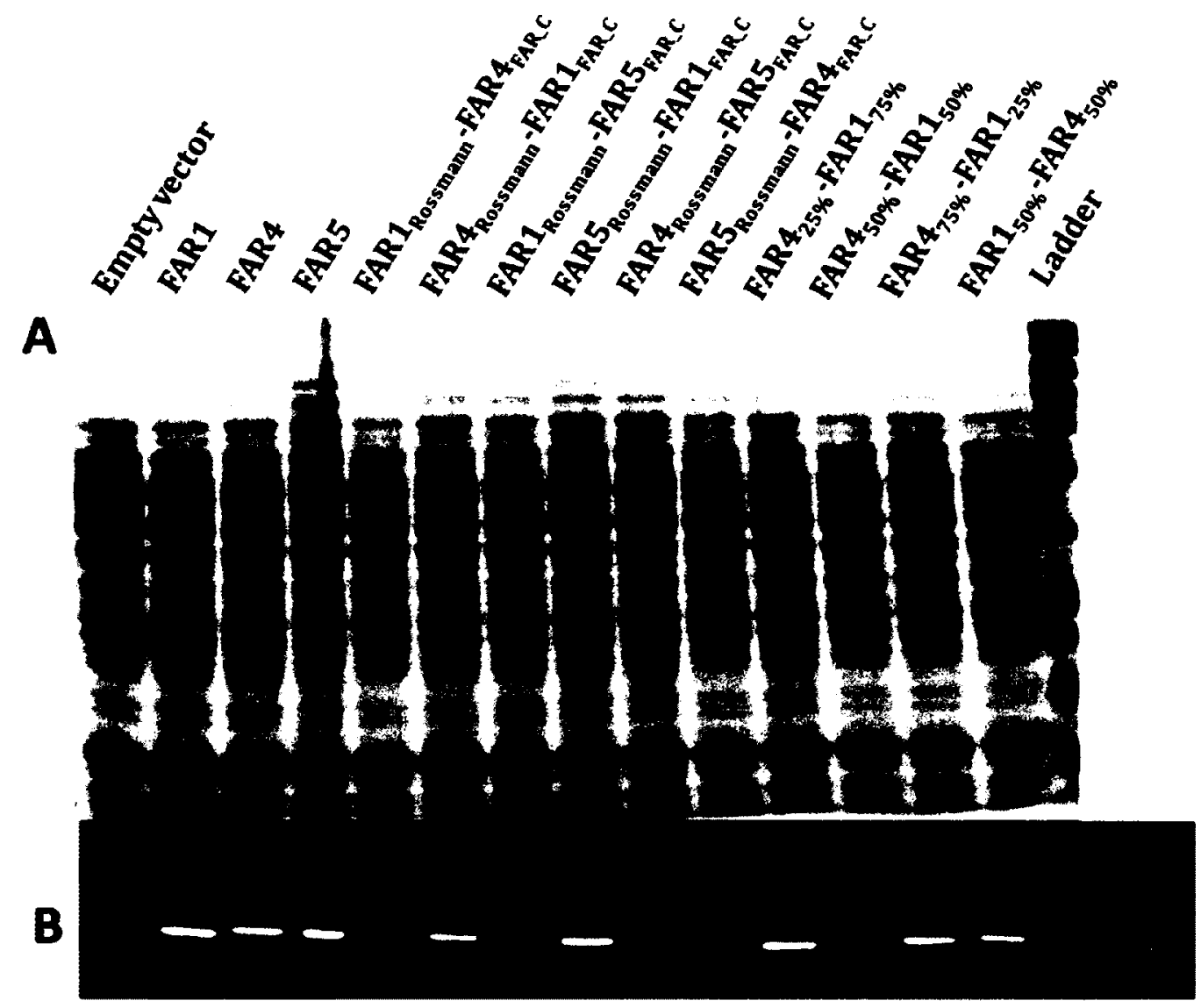

Figure 4.3. Expression of T7-epitope tagged Arabidopsis FAR1, FAR4, FAR5 and FAR chimeras in yeast. (A) SDS-PAGE gel stained with Coomassie Brilliant Blue. (B) Western blot analysis of proteins detected with T7 Tag monoclonal mouse primary antibody. The FAR wild-types and chimeras were in the expected 56-58 KDa molecular weight range. 


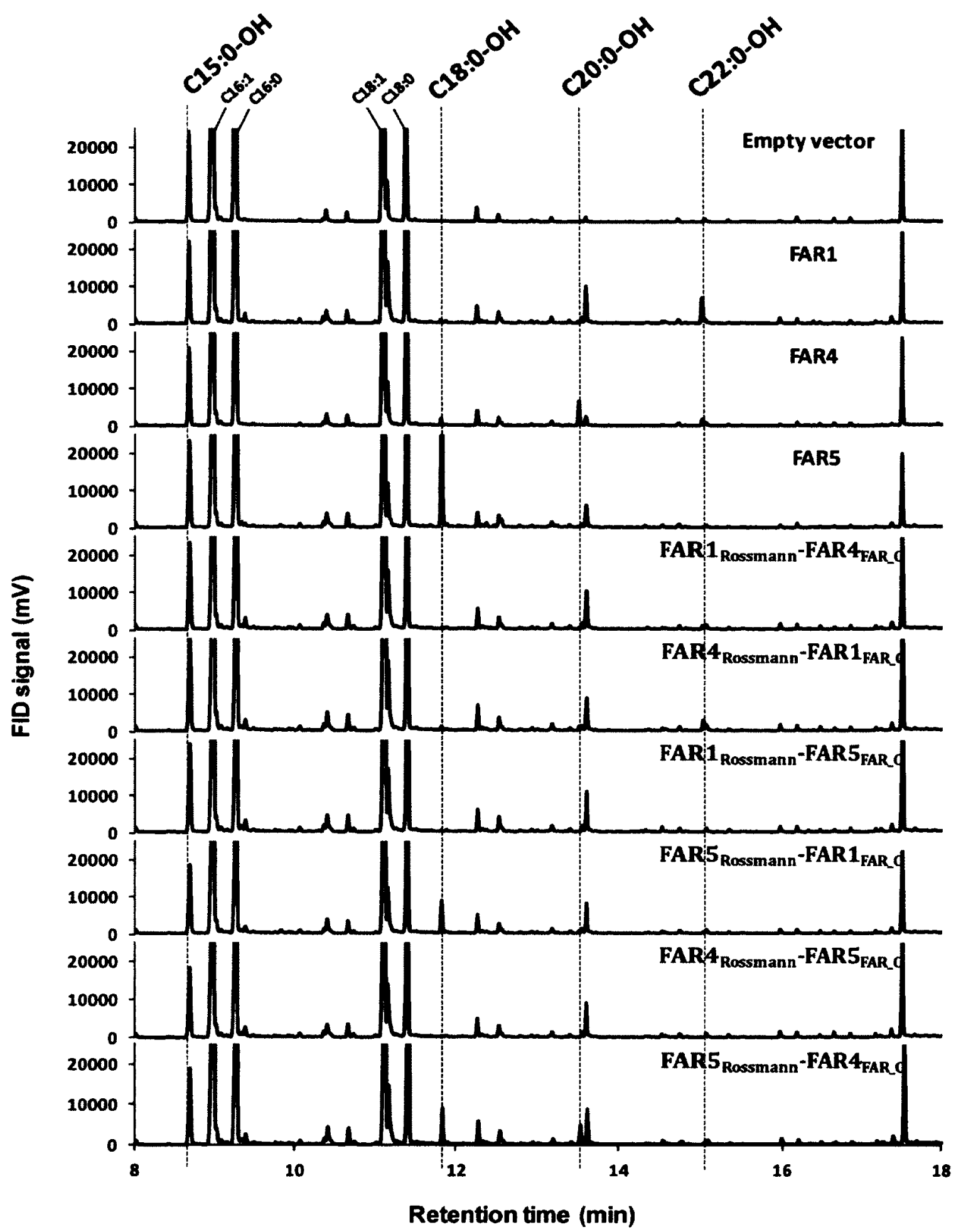




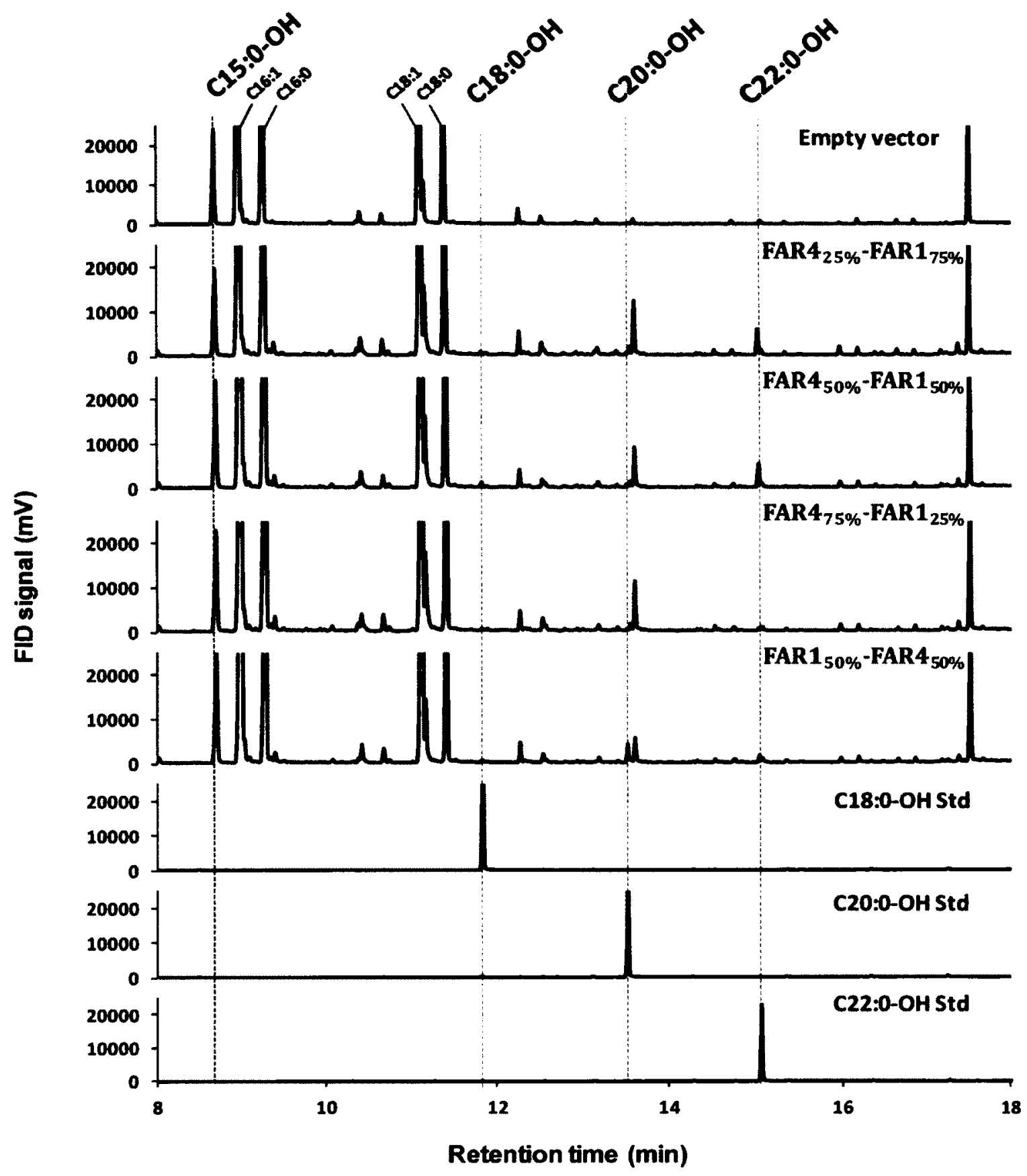

Figure 4.4. GC-FID chromatograms (zoomed in) of lipids extracted from transgenic yeast expressing tagged Arabidopsis FAR1, FAR4, FAR5, and FAR chimeras. Lipids were extracted from the yeast cell pellets and analyzed by gas chromatography. The primary alcohol peaks of interest are indicated by the dashed lines and denoted above the chromatograms. The peak indicated by $\mathrm{C} 15: 0-\mathrm{OH}$ is the internal standard (IS) (1-pentadecanol). FID = Flame Ionization Detection. 


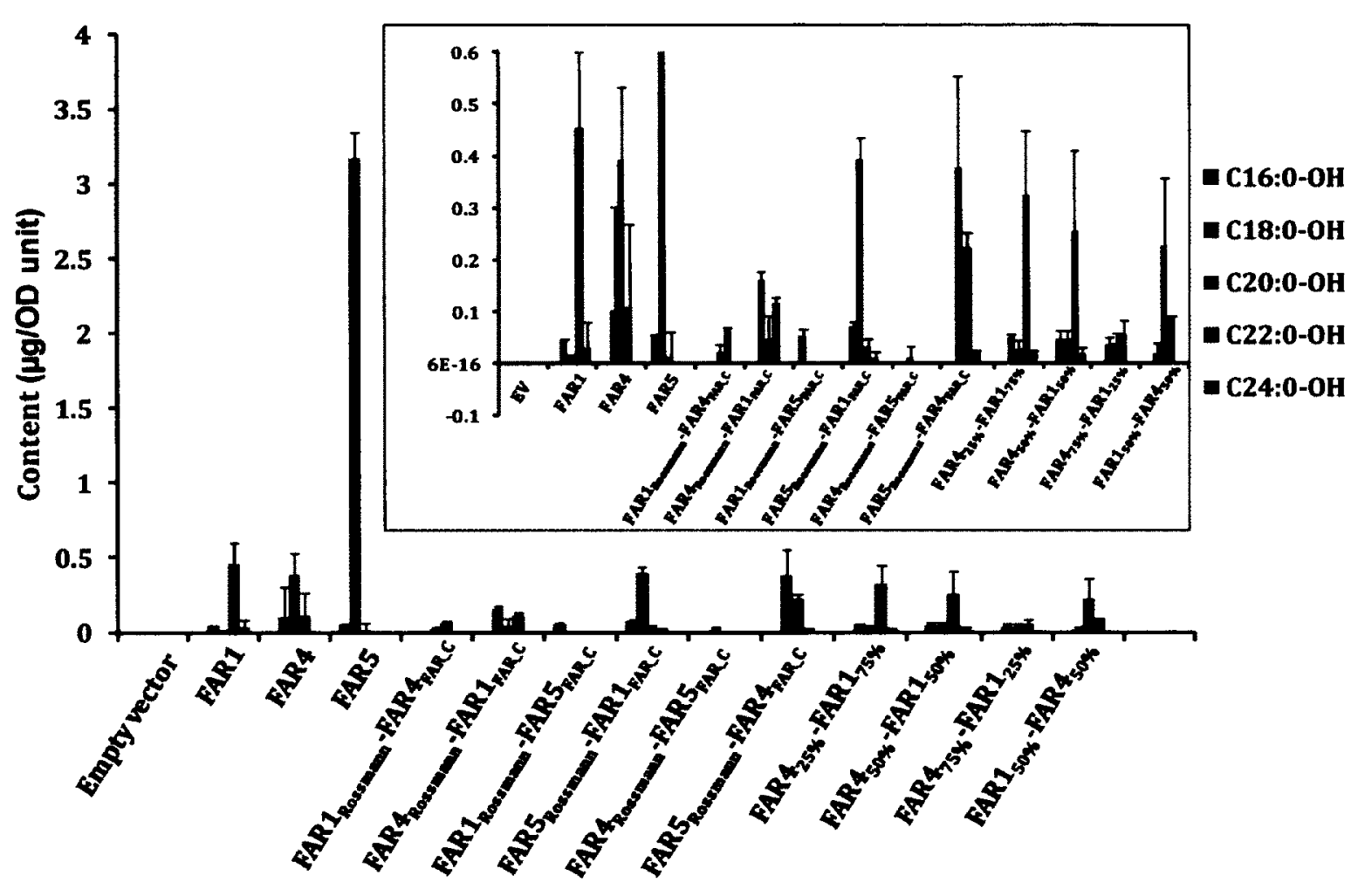

Figure 4.5. Internal fatty alcohol composition of yeast cells expressing Arabidopsis FAR1, FAR4, FAR5 and FAR chimeras measured by gas chromatography (GC-FID). The inset shows the fatty alcohol levels at a magnified scale. The pentadecanol (C15:0-OH) was used as an internal standard for quantification. The data represent means of 3 replicates where values are expressed in $\mu \mathrm{g} / \mathrm{OD}_{600}$ Unit (error bars are \pm standard deviation). EV: Empty vector. 


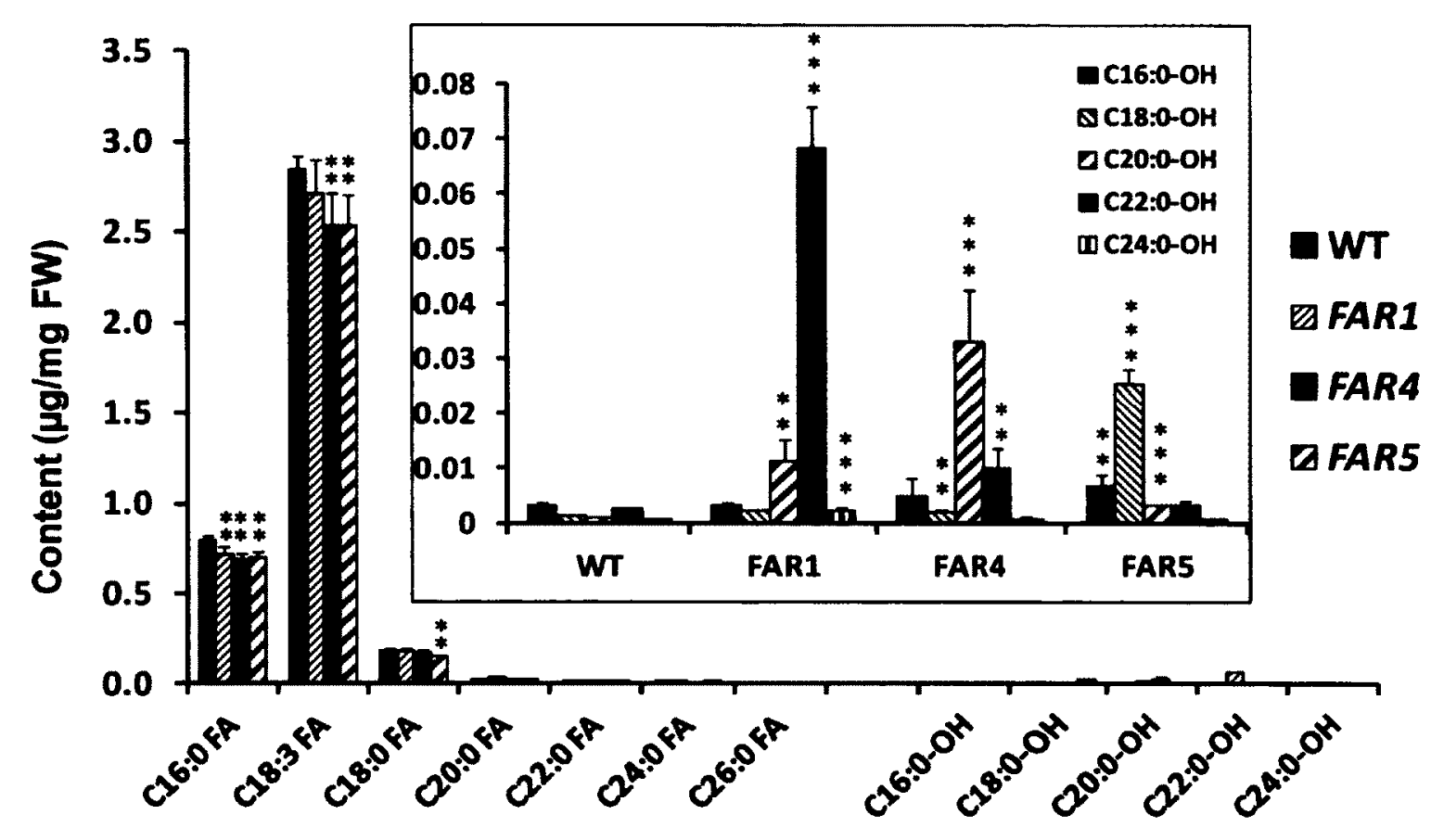

Figure 4.6. Composition of total internal lipids in Nicotiana benthamiana leaves overexpressing FAR1, FAR4 and FAR5. The total lipids are sorted into individual chain lengths, where mean values of three replicates are shown in $\mu \mathrm{g} / \mathrm{mg}$ fresh weight (FW). The inset shows the alterations in fatty alcohol composition sorted into chain length in WT and each of FAR1, FAR4 and FAR5 over-expressing leaf samples. Total lipids were extracted from leaves using a methanol:chloroform extraction procedure (modified method of Bligh and Dyer, 1959). The monomers were transmethylated and hydroxyl groups silylated before separation by gas chromatography and detection by flame ionisation detector (FID). The pentadecanol (C15:0-OH) and heptadecanoic acid (C17:0) were used as the internal standards (IS) for quantification of fatty alcohols and fatty acids, respectively. Errors bars indicate $S D$, and significance was assessed by a Student $t$ test $\left({ }^{* *}, \mathrm{P}<0.05 ;{ }^{* * *}, \mathrm{P}<0.01\right)$. 


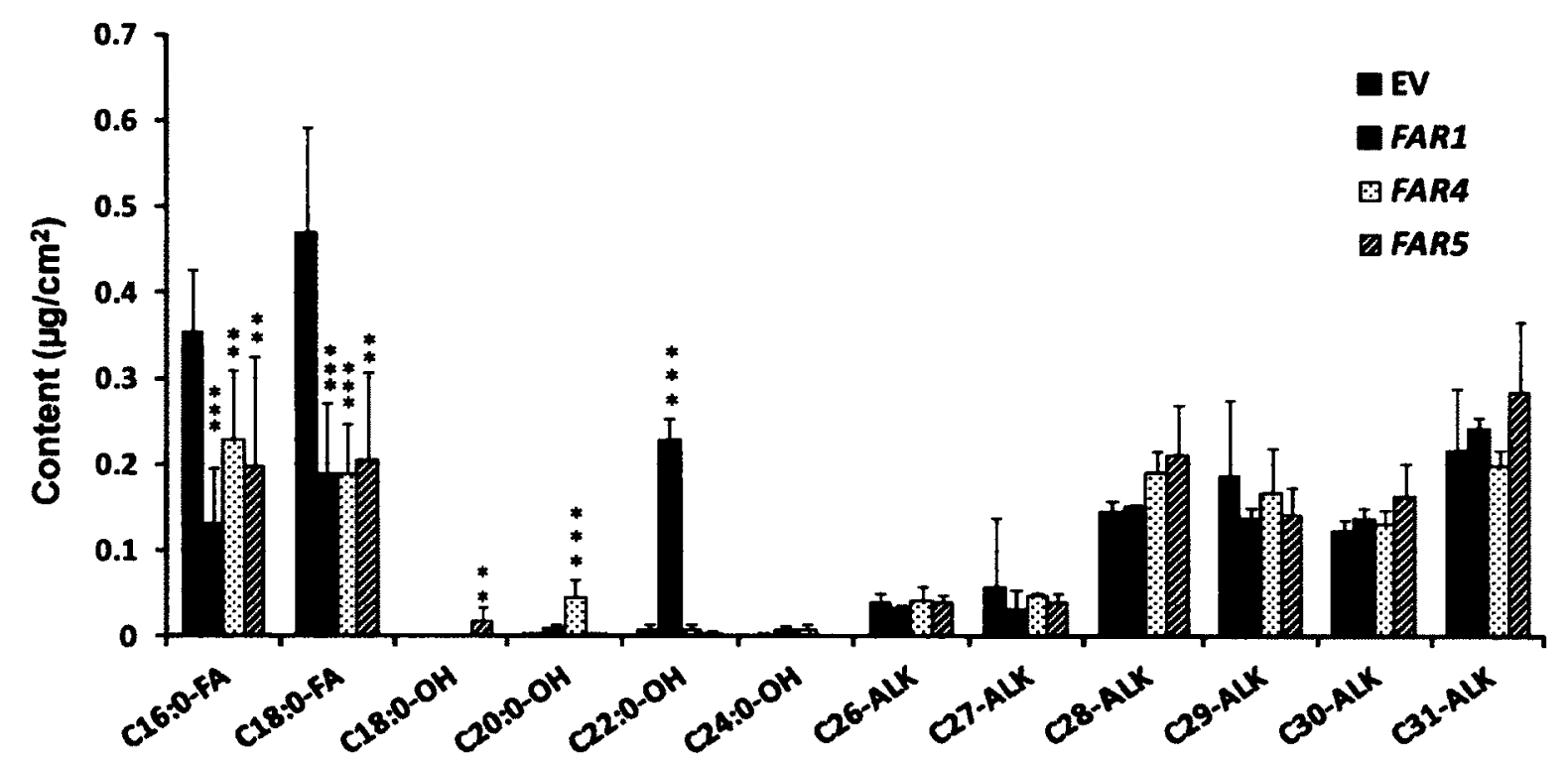

Figure 4.7. Composition of surface waxes of Nicotiana benthamiana leaves transiently overexpressing Arabidopsis FAR1, FAR4 and FAR5. The waxes are sorted into individual chain lengths (FA=fatty acid, ALK=alkane), where mean values of three replicates are shown in $\mu \mathrm{g} / \mathrm{cm}^{2}$. The lipids were extracted in chloroform and the levels of cuticular waxes were quantified by GC-FID using pentadecanol ( $\mathrm{C} 15: 0-\mathrm{OH})$ and tetracosane (C24:0-ALK) as internal standards. Errors bars indicate $\mathrm{SD}$, and significance was assessed by a Student $t$ test $\left({ }^{* *}, \mathrm{P}<0.05\right.$; ${ }^{* * *}$, $\mathrm{P}<0.01)$. 


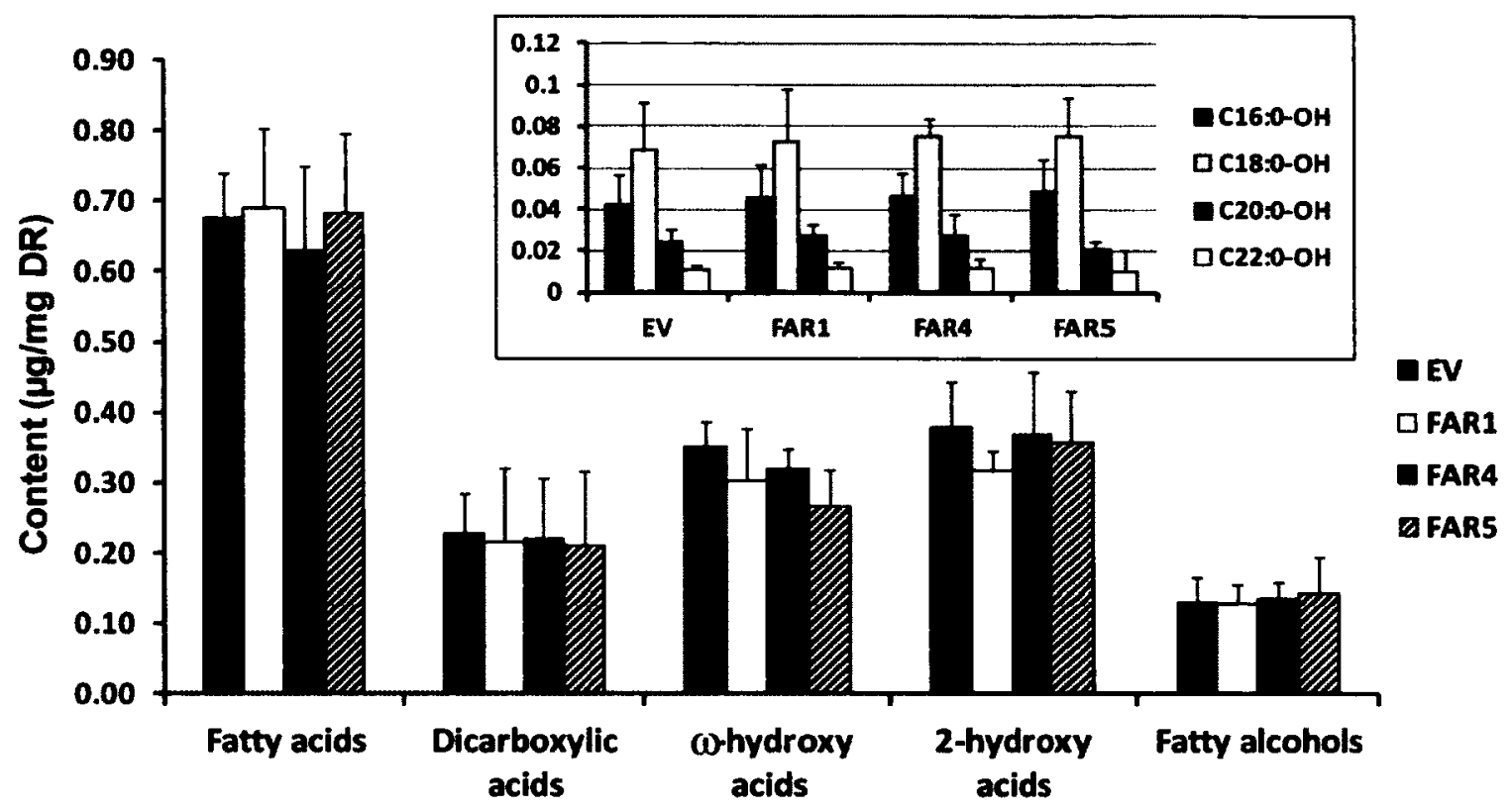

Figure 4.8. Composition of cutin polyester of Nicotiana benthamiana leaves transiently overexpressing Arabidopsis FAR1, FAR4 and FAR5, where mean values of three replicates are shown in $\mu \mathrm{g} / \mathrm{mg}$ dry residue (DR). Cutin monomers were released by depolymerization of solvent-extracted leaf tissue. The monomers were transmethylated and hydroxyl groups silylated before separation by GC and detection by flame ionisation detector (FID). Pentadecanol (C15:0-OH), C15hydroxypentadecanoic acid ( $\omega-0 \mathrm{HC} 15: 0)$, and heptadecanoic acid (C17:0) were used as the internal standards (IS) for quantification of various cutin monomers. Lipid classes of monomers are sorted by compound class along the $\mathbf{x}$-axis (different chain lengths added together). The inset shows fatty alcohol composition sorted into chain length in WT (empty vector) and each of FAR1, FAR4 and FAR5 overexpressing leaf samples. Errors bars indicate SD, and significance was assessed by a Student $t$ test $\left(* *, \mathrm{P}<0.05 ;{ }^{* * *}, \mathrm{P}<0.01\right)$. 

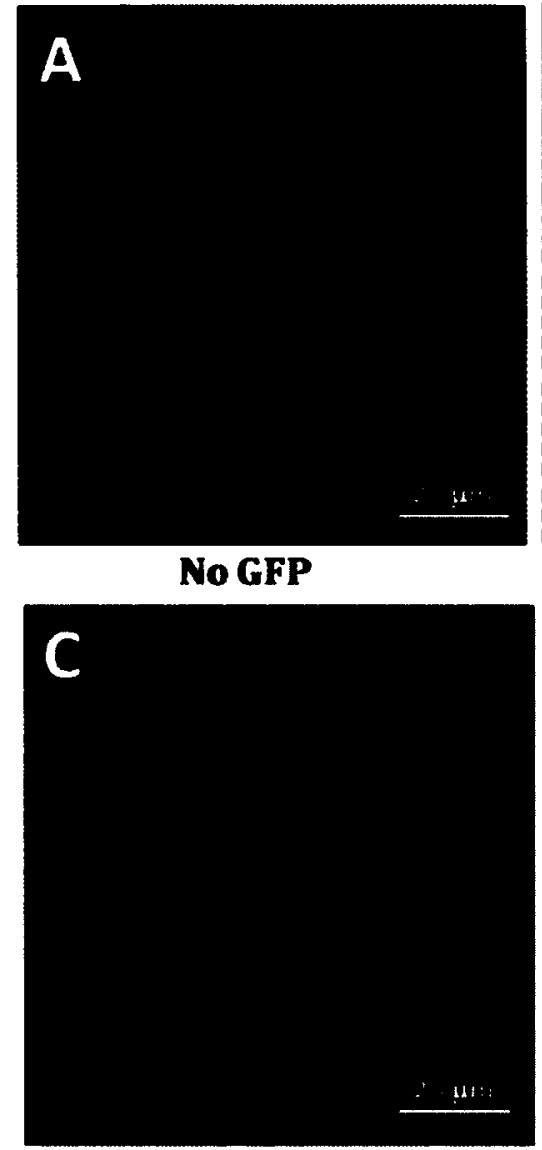

35S:GFP-HDEL

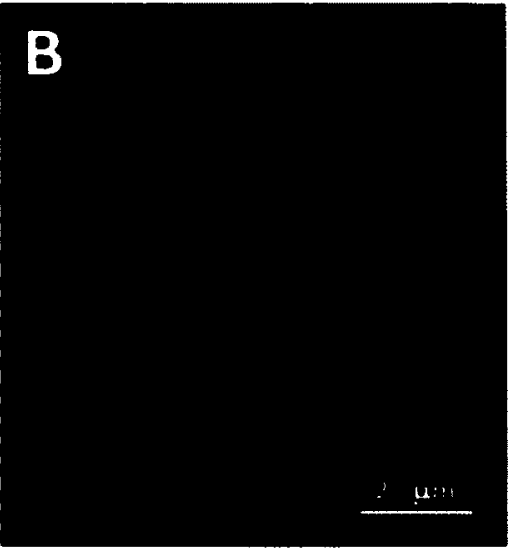

35S:GFP

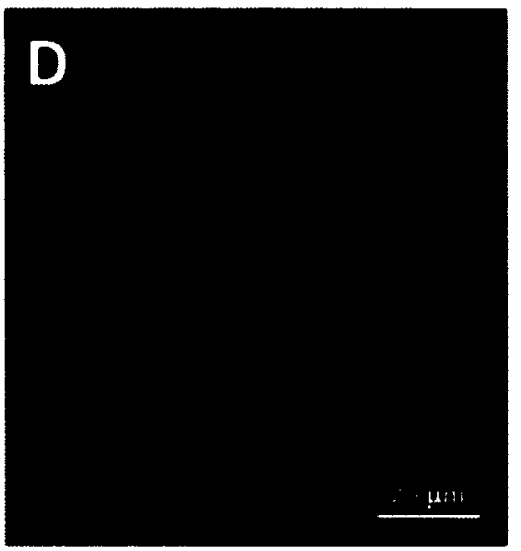

35S:FAR1-GFP

Figure 4.9. Localisation of FAR1-GFP fusion protein to the endoplasmic reticulum (ER) in Nicotiana benthamiana leaves. Free GFP localizes to the cytoplasm and nucleus. GFP containing an $\mathrm{N}$-terminal secretory signal and a C-terminal HDEL tetrapeptide ER retention signal was used as a positive marker. Fluorescence was imaged by laser scanning confocal microscopy. 


\section{Supplementary Tables:}

Table S4.1: Composition of fatty alcohols in transgenic yeast cells expressing Arabidopsis FAR1, FAR4 and FAR5. Each value is the mean shown in $\mu \mathrm{g} / \mathrm{OD}_{600} \pm \mathrm{SD}$ of three biological replicates.

\begin{tabular}{|c|c|c|c|c|c|c|}
\hline & areach & anoci & Co:pord & enon & Cinou & 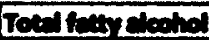 \\
\hline EV & $0 \pm 0$ & $0 \pm 0$ & $0 \pm 0$ & $0 \pm 0$ & $0 \pm 0$ & $0 \pm 0$ \\
\hline FAR1 & $0 \pm 0$ & $0.043 \pm 0.004$ & $0.016 \pm 0.002$ & $0.454 \pm 0.145$ & $0.031 \pm 0.051$ & $0.545 \pm 0.202$ \\
\hline FARA & $0 \pm 0$ & $0.103 \pm 0.200$ & $0.394 \pm 0.139$ & $0.109 \pm 0.159$ & $0 \pm 0$ & $0.605 \pm 0.498$ \\
\hline FARS & $0.051 \pm 0.005$ & $3.171 \pm 0.171$ & $0.015 \pm 0.045$ & $0 \pm 0$ & $0 \pm 0$ & $3.238 \pm 0.222$ \\
\hline FARI & $0 \pm 0$ & $0 \pm 0$ & $0.024 \pm 0.012$ & $0.063 \pm 0.006$ & $0 \pm 0$ & $0.086 \pm 0.019$ \\
\hline 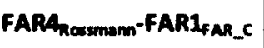 & $0 \pm 0$ & $0.161 \pm 0.016$ & $0.046 \pm 0.046$ & $0.116 \pm 0.012$ & $0 \pm 0$ & $0.323 \pm 0.074$ \\
\hline$F A R 1_{\text {Rosememn-FAR5 }}-F M_{-} C$ & $0.053 \pm 0.013$ & $0 \pm 0$ & $0 \pm 0$ & $0 \pm 0$ & $0 \pm 0$ & $0.053 \pm 0.013$ \\
\hline 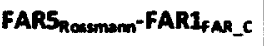 & $0.072 \pm 0.007$ & $0.395 \pm 0.039$ & $0.033 \pm 0.013$ & $0.011 \pm 0.011$ & $0 \pm 0$ & $0.511 \pm 0.071$ \\
\hline FAR4 & $0 \pm 0$ & $0.013 \pm 0.021$ & $0 \pm 0$ & $0 \pm 0$ & $0 \pm 0$ & $0.013 \pm 0.021$ \\
\hline 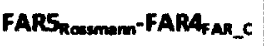 & $0 \pm 0$ & $0.377 \pm 0.177$ & $0.225 \pm 0.025$ & $0.023 \pm 0.003$ & $0 \pm 0$ & $0.626 \pm 0.206$ \\
\hline${\text { FARA } 25 \times-F A R 1_{75 \%}}$ & $0 \pm 0$ & $0.051 \pm 0.005$ & $0.028 \pm 0.018$ & $0.325 \pm 0.125$ & $0.022 \pm 0.002$ & $0.425 \pm 0.149$ \\
\hline FARA $_{500 x}-F A R 1_{50 \%}$ & $0 \pm 0$ & $0.048 \pm 0.018$ & $0.048 \pm 0.018$ & $0.255 \pm 0.155$ & $0.020 \pm 0.011$ & $0.370 \pm 0.201$ \\
\hline FAR45X-FAR1 25X & $0 \pm 0$ & $0.035 \pm 0.015$ & $0.038 \pm 0.018$ & $0.057 \pm 0.027$ & $0 \pm 0$ & $0.131 \pm 0.061$ \\
\hline FAR1 $_{\text {sox }}$-FAR4 & $0 \pm 0$ & $0.019 \pm 0.019$ & $0.228 \pm 0.128$ & $0.086 \pm 0.006$ & $0 \pm 0$ & $0.334 \pm 0.154$ \\
\hline
\end{tabular}


Table S4.2: Composition of internal lipids in $N$. benthamiana leaves expressing Arabidopsis FAR1, FAR4 and FAR5. Each value is the mean shown in $\mu \mathrm{g} / \mathrm{mg}$ fresh weight \pm SD of three biological replicates.

\begin{tabular}{|c|c|c|c|c|}
\hline Aver: & Emptr vector (WT) & OX-ALFAR1 & Ox-AY7AR4 & Ox-AuFAis \\
\hline \multicolumn{5}{|l|}{ Fatty acids } \\
\hline C16:0FA & $0.800 \pm 0.018$ & $0.711 \pm 0.048$ & $0.689 \pm 0.035$ & $0.696 \pm 0.042$ \\
\hline C18:3 FA & $2.851 \pm 0.065$ & $2.708 \pm 0.188$ & $2.540 \pm 0.171$ & $2.531 \pm 0.175$ \\
\hline C18:0 FA & $0.185 \pm 0.015$ & $0.181 \pm 0.018$ & $0.175 \pm 0.012$ & $0.154 \pm 0.006$ \\
\hline C20:0 FA & $0.028 \pm 0.002$ & $0.033 \pm 0.003$ & $0.027 \pm 0.001$ & $0.025 \pm 0.002$ \\
\hline C22:0 FA & $0.017 \pm 0.001$ & $0.018 \pm 0.002$ & $0.015 \pm 0.002$ & $0.017 \pm 0.003$ \\
\hline C24:0 FA & $0.014 \pm 0.000$ & $0.011 \pm 0.000$ & $0.011 \pm 0.000$ & $0.012 \pm 0.001$ \\
\hline C26:0 FA & $0.007 \pm 0.001$ & $0.007 \pm 0.000$ & $0.006 \pm 0.001$ & $0.010 \pm 0.005$ \\
\hline Subtotal & $3.902 \pm 0.103$ & $3.669 \pm 0.260$ & $3.464 \pm 0.223$ & $3.445 \pm 0.233$ \\
\hline \multicolumn{5}{|l|}{ Fatty alcohols } \\
\hline C16:0-OH & $0.003 \pm 0.001$ & $0.003 \pm 0.000$ & $0.005 \pm 0.003$ & $0.007 \pm 0.002$ \\
\hline C18:0-OH & $0.001 \pm 0.000$ & $0.002 \pm 0.000$ & $0.002 \pm 0.000$ & $0.025 \pm 0.003$ \\
\hline $\mathrm{C20:0} \mathrm{OH}$ & $0.001 \pm 0.000$ & $0.011 \pm 0.004$ & $0.033 \pm 0.009$ & $0.003 \pm 0.000$ \\
\hline C22:O-OH & $0.003 \pm 0.000$ & $0.068 \pm 0.008$ & $0.010 \pm 0.004$ & $0.003 \pm 0.001$ \\
\hline $\mathrm{C} 24: 0-\mathrm{OH}$ & $0.001 \pm 0.000$ & $0.002 \pm 0.000$ & $0.001 \pm 0.000$ & $0.001 \pm 0.000$ \\
\hline Subtotal & $0.009 \pm 0.001$ & $0.088 \pm 0.012$ & $0.051 \pm 0.017$ & $0.039 \pm 0.006$ \\
\hline
\end{tabular}


Table S4.3: Composition of surface wax in $N$. benthamiana leaves expressing Arabidopsis FAR1, FAR4 and FAR5. Each value is the mean shown in $\mu \mathrm{g} / \mathrm{mg}$ fresh weight \pm SD of three biological replicates.

\begin{tabular}{|c|c|c|c|c|}
\hline Avera? & Empty vector (WM) & Ox-Atrapd & Ox-AtFARA & Ox-Atrans \\
\hline \multicolumn{5}{|l|}{ Fatty acids } \\
\hline C16:0 FA & $0.354 \pm 0.074$ & $0.131 \pm 0.064$ & $0.230 \pm 0.080$ & $0.197 \pm 0.129$ \\
\hline C18:0 FA & $0.470 \pm 0.122$ & $0.189 \pm 0.083$ & $0.190 \pm 0.058$ & $0.205 \pm 0.103$ \\
\hline Subtotal & $0.824 \pm 0.196$ & $0.321 \pm 0.147$ & $0.419 \pm 0.138$ & $0.401 \pm 0.232$ \\
\hline \multicolumn{5}{|l|}{ Fatty alcohols } \\
\hline C18:0-OH & $0.002 \pm 0.001$ & $0.001 \pm 0.001$ & $0.002 \pm 0.001$ & $0.019 \pm 0.015$ \\
\hline $\mathrm{C} 20: 0-\mathrm{OH}$ & $0.003 \pm 0.001$ & $0.009 \pm 0.006$ & $0.047 \pm 0.020$ & $0.004 \pm 0.002$ \\
\hline $\mathrm{C} 22: 0-\mathrm{OH}$ & $0.008 \pm 0.007$ & $0.228 \pm 0.025$ & $0.009 \pm 0.006$ & $0.005 \pm 0.002$ \\
\hline C24:0-OH & $0.003 \pm 0.002$ & $0.007 \pm 0.006$ & $0.008 \pm 0.006$ & $0.002 \pm 0.001$ \\
\hline Subtotal & $0.016 \pm 0.011$ & $0.246 \pm 0.039$ & $0.065 \pm 0.034$ & $0.029 \pm 0.020$ \\
\hline \multicolumn{5}{|l|}{ Alkanes } \\
\hline C27-ALK & $0.040 \pm 0.011$ & $0.033 \pm 0.004$ & $0.043 \pm 0.015$ & $0.041 \pm 0.009$ \\
\hline C26-ALK & $0.057 \pm 0.081$ & $0.031 \pm 0.024$ & $0.048 \pm 0.002$ & $0.041 \pm 0.010$ \\
\hline C29-ALK & $0.145 \pm 0.013$ & $0.151 \pm 0.003$ & $0.192 \pm 0.023$ & $0.212 \pm 0.056$ \\
\hline C28-ALK & $0.187 \pm 0.088$ & $0.138 \pm 0.013$ & $0.168 \pm 0.051$ & $0.141 \pm 0.032$ \\
\hline C30-ALK & $0.124 \pm 0.012$ & $0.137 \pm 0.013$ & $0.132 \pm 0.016$ & $0.163 \pm 0.039$ \\
\hline C31-ALK & $0.218 \pm 0.072$ & $0.243 \pm 0.014$ & $0.199 \pm 0.019$ & $0.285 \pm 0.082$ \\
\hline Subtotal & $0.771 \pm 0.276$ & $0.732 \pm 0.069$ & $0.782 \pm 0.126$ & $0.884 \pm 0.229$ \\
\hline
\end{tabular}


Table S4.4: List of primers used for generation of FAR chimera constructs.

\begin{tabular}{|c|c|}
\hline Primer Name & $\begin{array}{c}\text { Primer sequence } \\
\left(5^{\circ} \rightarrow 3^{\prime}\right)\end{array}$ \\
\hline FAR1_BamHI_Forward & GAGGGATCCATGGAATCCAATTGTGTTCAAT \\
\hline FAR1_Xhol_Reverse & GCGCTCGAGTTATTGTTTAAGCACATGGGTGA \\
\hline FAR4_BamHI Forward & GAGGGATCCATGGACTCCAATTGCATTCAG \\
\hline FAR4_Xhol Reverse & GCGCTCGAGTTATTTTTTGAGTACATAGGTGAT \\
\hline FAR5_BamHI_Forward & GAGGGATCCATGGAACTCAATTGTGTTCAAT \\
\hline FAR5_Xholl_Reverse & GCGCTCGAGTCACTTCTTAAGCACGTGTG \\
\hline FAR1-FAR4-mid_F1 & GTAGTAAATGCGATGGTCACGGCTGCGGCAATACA \\
\hline FAR1-FAR4-mid_R1 & TGCCGCAGCCGTGACCATCGCATTTACTACCATGT \\
\hline FAR4-FAR1-mid_F1 & GTCGCAAACGCAATGGTTGCAGCCGCGACAGCTCA \\
\hline FAR4-FAR1-mid_R1 & TGTCGCGGCTGCAACCATTGCGTTTGCGACCATGT \\
\hline FAR1-FAR5-mid_F1 & GTAGTAAATGCGATGATCACGGCTGCAGCCAAACA \\
\hline FAR1-FAR5-mid_R1 & GGCTGCAGCCGTGATCATCGCATTTACTACCATGT \\
\hline FAR5-FAR1-mid_F1 & GTGGCAAACGCAATGGTTGCAGCCGCGACAGCTCA \\
\hline FAR5-FAR1-mid_R1 & TGTCGCGGCTGCAACCATTGCGTTTGCCACCATAT \\
\hline FAR4-FAR5-mid_F1 & GTCGCAAACGCAATGATCACGGCTGCAGCCAAACA \\
\hline FAR4-FAR5-mid_R1 & GGCTGCAGCCGTGATCATTGCGTTTGCGACCATGT \\
\hline FAR5-FAR4-mid_F1 & GTGGCAAACGCAATGGTCACGGCTGCGGCAATACA \\
\hline FAR5-FAR4-mid_R1 & TGCCGCAGCCGTGACCATTGCGTTTGCCACCATAT \\
\hline
\end{tabular}




\begin{tabular}{|l|l|}
\hline pHANNIBAL-35S-F & CCCACTATCCTTCGCAAGACCCTTC \\
\hline pHANNIBAL-Intron-R & GCTAGTATATCATCTTACATGTTCGATC \\
\hline pHANNIBAL-Intron-F & GGATTGATTACAGTTGGGAAATTGGG \\
\hline pHANNIBAL-OCST-R & CGGTAAGGATCTGAGCTACACATGC \\
\hline FAR1-GW-F & GGAGATAGAACCATGGAATCCAATTGTGTTCAATTTCTCGG \\
\hline FAR1-GW-R & AGAAAGCTGGGTATTGTTTAAGCACATGGGTGATGAGGCC \\
\hline GAPC_RT_Forward & TCAGACTCGAGAAAGCTGCTAC \\
\hline GAPC_RT_Reverse & GATCAAGTCGACCACACGG \\
\hline T7_Forward & TAATACGACTCACTATAGGG \\
\hline CYC_Terminator_Reverse & ACCTAGACTTCAGGTTGTCT \\
\hline
\end{tabular}




\section{CHAPTER 5: Transcriptional regulation of Arabidopsis FAR1, FAR4, and FAR5 and other suberin biosynthetic genes under abiotic stress conditions.}

\subsection{Introduction}

Plants can experience many different kinds of abiotic stresses, including high salt concentrations, extremes of temperature (low or high), and water shortage (drought). Plants exhibit adaptive changes in both physiological and biochemical processes as well as anatomical changes in response to abiotic stresses (Patakas, 2012). Regulation of biochemical and physiological reactions is generally achieved at the level of gene expression (up- and down-regulation of transcripts), protein stability, and post-translational modifications of proteins in a tissue-specific manner.

Suberization is a cell wall modification that contributes to the adaptation towards unfavorable environmental conditions. Suberin is a protective plant biopolymer that is usually deposited wherever the plant needs a persistent barrier (Franke et al., 2009, 2012) (Also see General Introduction, Chapter 1). Suberin modifies the chemical and physical properties of the plant cell wall (Franke et al., 2009). It functions to prevent water and nutrient loss, gas and solute transport, acts as thermal insulation, as well as a barrier against pathogenic attack, and to seal wounded tissues (Bernards, 2002; Franke et al., 2009; Graca and Pereira, 2000, Ranathunge et al., 2011). The formation of suberin lamellae as a barrier to water movement in radial and transverse walls of the endodermis and exodermis is often associated with the responses to stress such as water deficit and aging of the plant 
(Patakas, 2012). Several studies have shown an important role of suberin in controlling water and solute losses from the roots under abiotic stresses (Krishnamurthy et al., 2009, 2011; Song et al., 2011; Ranathunge and Schreiber, 2011).

Suberin polymer is composed of aliphatic and aromatic components (See General Introduction, Chapter 1). The specialized barrier properties of suberin are the result of its chemical composition and its spatial organization in different plant tissues. For example, the aliphatics in suberin and its associated waxes are hydrophobic in nature, and are located between the primary cell wall and plasma membrane. This imparts a defined hydrophobic characteristic to the walls and establishes it as a structure that restricts water and solute flow through the transcellular pathway (Soliday et al., 1979; Vogt et al., 1983; Zimmermann et al., 2000; Schreiber et al., 2005b). Conversely, the phenolic compounds that are embedded in the cell wall, makes this structure restrictive to apoplastic microbial penetration (Kolattukudy 1980; Lulai and Corsini, 1998).

The developmental and stress induced deposition of suberin has been associated with specifically localized gene expression patterns, mostly from studies in Arabidopsis. In studies using promoter-reporter gene fusions, several Arabidopsis genes involved in suberin production, such as GPAT5, CYP86A1/HORST, CYP86B1/RALPH, ASFT/AtHHT, KCS2/DAISY, KCS20, FAR1, FAR4, and FAR5, were found to co-localise in the root endodermis, a known site of suberin deposition (Beisson et al., 2005; Hofer et al., 2008; Compagnon et al., 2009; Franke et al., 2009; Molina et al., 2009; Lee et al., 2009; Chapter 2 of this thesis). GPAT5, ASFT, FAR1, 
FAR4, and FAR5 are expressed in the root periderm (Beisson et al., 2005; Molina et al., 2009; Chapter 2). ASFT was shown to be expressed in the outer seed integument (Molina et al., 2009), and GPAT5, KCS2/DAISY, FAR1 and FAR4 were shown to be preferentially expressed in the seed hilum region (Beisson et al., 2005; Franke et al., 2009; Chapter 2). In studies using semi-quantitative RT-PCR, several of these genes, FAR1, FAR4, FAR5, DAISY/KCS2 and KCS20, were also found to be induced by wounding, osmotic stress, and salt stress (Lee et al., 2009; Chapter 2). Suberin deposition is thus spatially restricted, varies temporally, and is inducible by environmental stimuli. However, the gene regulatory networks and underlying molecular mechanisms governing suberin biosynthesis under stress conditions have been largely unexplored.

We carried out a study of the regulation of FAR1, FAR4, FAR5 and five other major suberin biosynthetic genes viz., GPAT5, CYP86A1/HORST, CYP86B1/RALPH, $K C S 2 / D A I S Y$ and $A S F T 1$, by quantifying their transcript levels under the influence of abscisic acid and abiotic stresses that are known to induce suberization. The study provides information into the regulation of suberin genes at the transcriptional level in response to environmental cues. 


\subsection{Materials and Methods}

\subsubsection{Plant material}

Arabidopsis thaliana plants were in the Columbia (Col-0) ecotype background. The seeds were surface sterilized as described before in Chapter 2. For experimental analyses, sterilized seeds were sown on Murashige and Skoog (MS) medium (4.5g/L MS salts $+0.5 \mathrm{~g} / \mathrm{L}$ MES, Sigma) (Murashige and Skoog, 1962) with $\mathrm{pH}$ set to 5.7 , supplemented with $0.7 \%$ agar and poured in petri-plates. The plates were sealed with micropore tape and seeds were stratified in the dark for 3 to 4 days at $4^{\circ} \mathrm{C}$ and then transferred to a growth chamber with long-day conditions (16h-light/8-h-dark cycle) at a light intensity of $90 \mu \mathrm{mol}$ photons $\mathrm{m}^{-2} \mathrm{~s}^{-1}$ and $22^{\circ} \mathrm{C}$ temperature. The seedlings were grown vertically for 2 weeks. Fifteen-day-old seedlings were subjected to various abiotic stresses for 24 hours as described below.

\subsubsection{Abiotic stress treatments}

The stress treatments were all initiated 4 hours after dark/light transition to exclude differential circadian effects, which could superimpose stress-induced gene expression. For stress treatments, the plates with seedlings were transferred from the growth chamber to the laboratory where all treatments were performed and then returned to the growth chamber and incubated for 24 hours before harvesting. For harvesting, the seedlings were removed from the surface of the media in the plate and the roots were cut off. From one plate, root material of $\sim$ ten plants was harvested and pooled to minimize physiological differences between single plants. 
The entire harvest procedure was completed in less than $5 \mathrm{~min}$ and the root samples were immediately ground in liquid nitrogen for RNA extraction. The total RNA for control samples were isolated from non-stressed plants, which were handled in exactly the same way but were not exposed to stress conditions. ABA, cold, osmotic, salt, and wounding stress applications were each performed as described below.

\subsubsection{ABA (abscisic acid) stress}

15-day-old seedlings grown vertically were subjected to ABA stress by pouring $10 \mathrm{ml}$ of $10 \mu \mathrm{M} \mathrm{ABA}$ solution into the petri plates, thereby submerging the roots growing on the surface of the media. The plates were incubated for 24 hours in a horizontal position.

\subsubsection{Cold stress}

15-day-old seedlings grown vertically were subjected to cold stress $\left(4^{\circ} \mathrm{C}\right)$ by placing the petri plates with the seedlings on ice in a tray for rapid cooling, kept in the growth chamber under long-day conditions. The plates were incubated for 24 hours in a horizontal position.

\subsubsection{Osmotic stress}

15-day-old seedlings grown vertically were subjected to osmotic stress by pouring $10 \mathrm{ml}$ of $150 \mathrm{mM}$ Mannitol solution into the petri plates, thereby submerging the roots growing on the surface of the media. The plates were incubated for 24 hours in a horizontal position. 


\subsubsection{Salt stress}

15-day-old seedlings grown vertically were subjected to salt stress by pouring $10 \mathrm{ml}$ of $200 \mathrm{mM} \mathrm{NaCl}$ solution into the petri plates, thereby submerging the roots growing on the surface of the media. The plates were incubated for 24 hours in a horizontal position.

\subsubsection{Wound stress}

Wounding was carried out on stems of 6-week-old plants grown under longday conditions (16-hr-light/8-hr-dark cycle). Using a needle, injury was made of approximately $1 \mathrm{~cm}$ by slitting longitudinally between internodes of the mature stem regions. The plants were returned to the growth chamber under long-day conditions and the samples were collected after 24 hours. As a control, a sample was taken prior to wounding.

\subsubsection{RNA extraction}

RNA extraction was carried out using RNAqueous ${ }^{\circledR}$ Kit (Applied Biosystems). Roots ( $\sim 50-60 \mathrm{mg}$ ) from seedlings or stem tissues (wounding stress) were collected and ground up using a pestle and liquid nitrogen. The ground tissue was transferred to a sterile microcentrifuge tube using a pre-chilled metal spatula and $700 \mu \mathrm{l}$ of Lysis/Binding solution (supplied in the kit) was added, and then mixed rapidly. To this homogenised lysate, an equal volume of $64 \%$ ethanol $(700 \mu \mathrm{l})$ was added and mixed gently by inverting the tube several times. This lysate/ethanol mixture was then drawn through a filter cartridge (supplied in the kit) by centrifuging at RCF 
$12,000 \mathrm{x}$ for 1 minute. The flow through was discarded and the filter cartridge was washed twice with $700 \mu \mathrm{l}$ of Wash solution \#1 and then with $500 \mu \mathrm{l}$ of Wash solution \#2/3 (supplied in the kit), by centrifuging at RCF $12,000 \mathrm{xg}$ for 30 seconds. The filter cartridge was placed in a fresh collection tube and $50 \mu l$ of pre-heated elution solution was added to the centre of the cartridge filter using a pipette. The eluate was collected by centrifuging at RCF $12,000 \mathrm{x}$ g for 30 seconds at room temperature.

Further, the collected eluate was subjected to DNase treatment using TURBO DNA-free ${ }^{\mathrm{TM}}$ kit (Applied Biosystems-Ambion AM1907). To the eluate containing total-RNA, 0.1 volumes 10X TURBO DNase buffer $(\sim 5 \mu \mathrm{l})$ and $1 \mu \mathrm{l}$ TURBO DNase was added, mixed gently, and incubated at $37^{\circ} \mathrm{C}$ for $20-30$ minutes. Later, 0.1 volume resuspended DNase inactivation reagent $(\sim 5 \mu \mathrm{l})$ was added, mixed well, and incubated at room temperature for 5 minutes. The tube was centrifuged at $10,000 \mathrm{~g}$ for 2 minutes and the supernatant (RNA) was transferred to a fresh sterile tube. RNA yield and purity was assessed using a NanoDrop spectrophotometer (NanoDrop Technologies, Inc., USA) and RNA integrity was checked by running an aliquot on a $1 \%$ agarose (in formaldehyde (37\%)/MOPS buffer) gel.

\subsubsection{First strand cDNA synthesis}

For each sample, $1 \mu \mathrm{g}(8.5 \mu \mathrm{l})$ of total RNA, diluted using DEPC-treated water, was heated at $70^{\circ} \mathrm{C}$ for 5 minutes and snap chilled on ice. For a final volume of $20 \mu \mathrm{l}$, the total RNA samples $(8.5 \mu \mathrm{l})$ were added to $11.5 \mu \mathrm{l}$ of the reverse transcriptase reaction mixture containing $2 \mu \mathrm{M}$ oligo-dT primer, $0.5 \mathrm{mM}$ dNTPs, $5 \mathrm{U}$ RNaseOUT ${ }^{\mathrm{TM}}$ 
(Invitrogen), $10 \mathrm{mM}$ DTT, $50 \mathrm{mM}$ Tris- $\mathrm{HCl}$ (pH 8.3), $75 \mathrm{mM} \mathrm{KCl}, 3 \mathrm{mM} \mathrm{MgCl} 2$, and 50 units of SuperScript ${ }^{\circledR}$ III Reverse Transcriptase (Invitrogen) on ice. The reaction was held at $50^{\circ} \mathrm{C}$ for 60 minutes, $72^{\circ} \mathrm{C}$ for 10 minutes, cooled down to $4^{\circ} \mathrm{C}$ and stored at $20^{\circ} \mathrm{C}$.

\subsubsection{Quantitative PCR}

Quantitative PCR was performed on the StepOnePlus ${ }^{\circledR}$ real time PCR system (Applied Biosystem) using Power SYBR ${ }^{\circledR}$ Green qPCR mastermix (Applied Biosystems). The cDNA samples were diluted ten times with nuclease free water. Each qPCR reaction contained $5 \mu$ of $5 X$ Power SYBR mix, $2 \mu$ of primer mix ( $5 \mu \mathrm{M}$ of each forward and reverse primer), $2 \mu \mathrm{l}$ of the diluted cDNA and $1 \mu \mathrm{l}$ of sterile water, in a total $10 \mu l$ reaction. The $\mathrm{qPCR}$ amplification was performed with gene specific primers (for primer sequences - see Supplementary Table S5.1). PCR efficiency ranged from $90 \%$ to $105 \%$. The amplicon size ranged from $110-124 \mathrm{bp}$. The thermal profile consisted of 1 cycle at $95^{\circ} \mathrm{C}$ for 10 minutes followed by 40 cycles at $95^{\circ} \mathrm{C}$ for 15 seconds and $60^{\circ} \mathrm{C}$ for 30 seconds. For each run, data acquisition was done at the end of each cycle using StepOne software version 2.1 (Applied Biosystems). Melt curves were obtained with 1 cycle at $95^{\circ} \mathrm{C}$ for 15 seconds and $60^{\circ} \mathrm{C}$ for 1 minute to confirm the specificity of the primers.

Transcripts were quantified as relative abundance using a comparative $\mathrm{Ct}$ method [delta-delta Ct: $2^{-\Delta \Delta \mathrm{Ct}}$ (Pfaffl, 2001; 2006)]. Housekeeping genes, ACTIN2 (ACT2) and EUKARYOTIC TRANSLATION INITIATION FACTOR 4A ISOFORM 1 (elF4A1), were used as endogenous genes. The geometric mean of the relative transcript abundance of the two endogenous controls was used to normalize for 
differences of total RNA amounts between the samples according to the method described by Vandesompele et al. (2002). The abiotic stress-responsive gene RD29A (Yamaguchi-Shinozaki and Shinozaki, 1993; Msanne et al., 2011) was used as a positive general-stress induced control for the experiment. Two biological replications were carried out for each sample, with each reaction having three technical replicates.

\subsubsection{Accession numbers}

Sequence data of the genes studied in this chapter can be found in the Arabidopsis Genome Initiative (TAIR) or GenBank/EMBL databases under the following accession numbers: GPAT5, At3g11430; CYP86A1, At5g58860; CYP86B1, At5g23190; KCS2/DAISY, At1g04220; AFST, At5g41040; FAR1, At5g22500; FAR4, At3g44540; FAR5, At3g44550; GAPC, At3G04120; ACT2, At1g49240; eIF4A1, At3g13920; and RD29A, At5g52310.

\subsection{Results}

Several Arabidopsis suberin-involved genes have been shown to be induced in response to various abiotic stresses, but in separate studies and under various growth conditions. To directly compare the regulation of expression of suberin genes under a set of defined abiotic stress conditions, we quantified transcript levels of eight known suberin genes, FAR1, FAR4, FAR5, GPAT5, CYP86A1/HORST, CYP86B1/RALPH, KCS2/DAISY and ASFT1, in 15-day-old seedlings subjected to ABA, cold, osmotic, and salt stresses as well as 6-week-old plants subjected to wounding 
of stems. RD29A has been shown to be highly induced under all these abiotic stresses (Yamaguchi-Shinozaki and Shinozaki, 1993; Msanne et al., 2011) and so $R D 29 A$ induction was used as a positive control for treatment efficiency. Results discussed below are from a single biological replication. A second biological replication showed a similar trend in transcript fold change (data not shown).

Treatment with $10 \mu \mathrm{M}$ ABA resulted in a 24-fold increase of $R D 29 A$ transcript level compared with mock-treated plants. Under ABA stress, all eight suberin genes studied were found to be induced at various levels, with $K C S 2$ having the highest induction (32-fold increase), followed by GPAT5 (19-fold increase). FAR1, FAR4 and FAR5 showed 12-fold, 7-fold and 5-fold increase, respectively, compared with the mock-treated plants (Figure 5.1; Supplementary Table S5.2). CYP86A1 was found to be the least induced and showed only a 2.7 -fold increase in transcript level.

Cold treatment $\left(4^{\circ} \mathrm{C}\right)$ for 24 hours led to a 20 -fold increase of $R D 29 A$ transcript abundance compared with the mock-treated plants. Among the suberin genes, GPAT5 showed the highest induction (4.3-fold increase), followed by FAR5 (3.9-fold increase), KCS2 (3.9-fold increase), and ASFT1 (3.5-fold increase) compared with the mock-treated plants (Figure 5.2; Supplementary table S5.2). However, cold treatment resulted in decreased expression of the FAR1 (0.4-fold), FAR4 (0.9-fold), and CYP86A1 (0.8-fold), genes, indicating that transcription of these genes were not significantly affected by cold stress.

Osmotic stress induced by treatment with $150 \mathrm{mM}$ mannitol also resulted in an increase of transcript levels of all eight suberin genes studied; however, 
compared to other abiotic stresses, induction due to mannitol stress was relatively low. After 24 hours of treatment with mannitol, $R D 29 A$ showed a 4 -fold increase in transcript level. Among the suberin genes, KCS2 showed the highest induction (3.3fold increase), followed by FAR5 (2.7-fold increase), and FAR1 (2.3-fold increase) compared with mock-treated plants (Figure 5.3; Supplementary Table S5.2). Transcripts level of GPAT5 and FAR4 showed a 2.2-fold increase. CYP86A1 was not significantly induced by mannitol stress.

Treatment of the seedlings with $200 \mathrm{mM} \mathrm{NaCl}$ for 24 hours resulted in an increase of $R D 29 A$ transcript levels by 33 -fold as compared with the untreated plants. The expression of GPAT5, FAR1 and ASFT1 transcripts increased 8.3, 4.9 and 4.8-fold, respectively, in response to salt stress (Figure 5.4; Supplementary table S5.2). Among the fatty acyl reductase genes, FAR1 was the most induced (4.9-fold), followed by FAR5 (3.9-fold), and then FAR4 (3.1-fold). Both, CYP86A1 and CYP86B1 however, showed no change in transcript levels under salt stress.

We also analyzed the modulation of suberin gene expression under wounding stress in stem tissue of 6-week-old plants. Wounding treatment led to a 24-fold increase of ASFT1 transcript abundance, higher than the increase of RD29A transcript level (12-fold increase) compared with the non-wounded plants (Figure 5.5; Supplementary Table S5.2). Among fatty acyl reductase genes, FAR1 was the most induced (9.1-fold increase), followed by FAR5 (6.9-fold increase), and then FAR4 (5-fold increase). Other suberin genes also showed a considerably higher (1217 fold) accumulation of transcripts in response to wounding stress. 


\subsection{Discussion}

Plants respond to stresses as individual cells and synergistically as a whole organism. The extracellular stress signal is often first perceived at the membrane level by membrane receptors (e.g. receptor-like kinases and ion channels), which then activate large and complex signaling pathways intracellularly including the generation of secondary signal molecules such as $\mathrm{Ca}^{2+}$, inositol phosphates (InsP), reactive oxygen species (ROS), and ABA (Tuteja et al., 2007). The stress signal can then induce multiple stress responsive genes, the products of which ultimately lead to plant adaptation to stress tolerance directly or indirectly. ABA is a phytohormone that regulates plant water balance critical for plant growth and development (Tuteja et al., 2007). $\mathrm{ABA}$ is also considered a plant stress hormone since various abiotic stresses induce ABA synthesis, which in turn plays an important role in integrating various stress signals and controlling downstream stress responses (Swamy and Smith, 1999). Plant stress responses can be broadly divided into ABA-dependent and ABA-independent processes (Tuteja et al., 2007).

Suberin deposition has been observed in response to many biotic and abiotic stresses (Schreiber et al., 2005a; Yang and Bernards, 2006; Pinto et al., 2009; Ranathunge et al., 2011). Suberin amounts in castor bean (Ricinus communis) roots were reported to be significantly increased (2-fold) in the endodermal cell wall in the presence of $\mathrm{NaCl}$, likely to reinforce the apoplastic transport barrier in roots to minimize $\mathrm{NaCl}$ uptake (Schreiber et al., 2005a). In addition to $\mathrm{NaCl}$, other environmental stress factors such as polyethylene glycol-induced osmotic stress and heavy metal stress have been shown to result in 1.5-3-fold increases in total suberin 
in corn roots, without any effect on suberin composition (Schreiber et al., 1999; Zeier, 1998). In response to pathogen- or mechanically-induced injuries, many plants undergo suberization as part of an active sealing of plant tissues (Bernards and Lewis, 1998; Kolattukudy, 2001a). Several studies on potato tubers have suggested that the abscisic acid ( $A B A)$ stress signal response may be involved in the regulation of wound healing (Soliday et al., 1978; Lulai et al., 2008). Wound-induced suberization of tomato fruit surfaces has been described by Dean and Kolattukudy (1976). Agrobacterium-induced Arabidopsis tumors were found to accumulate high amounts of $A B A$ and form a suberized periderm-like protective layer that protects the tumors against desiccation (Efetova et al., 2007). It was reported that treatment of 2-week-old Arabidopsis seedlings with exogenous ABA $(10 \mu \mathrm{M})$ increased the root suberin content by $1.5-2$-fold in comparison to water-treated control seedlings (Efetova et al., 2007). In a study using potato tuber tissue culture treated with 10 $\mu \mathrm{M}$ ABA for 2 days, a 3-fold increase in the aliphatic components and a 4-fold increase in the aromatic components of suberin was reported (Cottle and Kollatukudy, 1982). In the same study, a 9-fold increase in fatty alcohol levels associated with waxes of potato periderm suberin was reported. There was a relatively small increase in the activity of $\omega$-hydroxy-fatty acid dehydrogenase in the tissue cultures treated with ABA (Cottle and Kollatukudy, 1982). It is notable that the levels of Arabidopsis cutin monomers are largely unaffected by ABA treatments (Kosma et al., 2009). 
It has been demonstrated that several suberin biosynthetic genes are upregulated during stress-regulated suberization (Lee et al., 2009; Chapter 2), and we investigated this further by focusing on eight Arabidopsis suberin biosynthetic genes including FAR1, FAR4, and FAR5. We compared their stress responsive gene expression patterns to the well characterized $R D 29 A$ gene, which is induced by various abiotic stresses (Msanne et al., 2011). The RD29A promoter contains several dehydration-responsive element (DREs) and one abscisic-acid-responsive element (ABRE) motif (Zhang et al. 2004). In our study, RD29A showed a significantly higher induction in all the abiotic stress treatments confirming the treatment efficiency. $R D 29 A$ showed a much higher induction in response to salt and cold stress treatment, compared to the suberin genes.

Among the fatty acyl reductase genes, FAR1, FAR4 and FAR5 were found to be induced under the abiotic stress treatments with the exception of cold treatment. Under cold treatment, FAR1 and FAR4 were not significantly affected, while FAR5 showed about 4-fold induction. It is difficult to speculate why this would be, but it is possible that the fatty alcohol chain-length distribution in suberin is important for its specific functions. Having three FARs each mostly specific for a particular chainlength allows for such control of the chain distribution in suberin. FAR1 was the most induced among the three by ABA, salt stress, and wound stress. Up-regulation of the FAR1, FAR4 and FAR5 transcripts was previously observed in response to wounding, resulting in about a doubling of the total fatty alcohols level in the leaf lipid polyester (Chapter 2). FAR1 is responsible for the synthesis of mainly C22:0- 
$\mathrm{OH}$ and its higher induction might suggest that fatty alcohols of longer chain length are preferentially synthesized for stress related suberization.

Glycerol is one of the major constituents of suberin and has been indicated to have a structural role, linking aliphatics to the aromatic components allowing the formation of three-dimensional cross-linked network (Moire et al., 1999; Graça and Pereira, 2000a, 2000b; Graça and Santos, 2006). Moire et al. (1999) reported accumulation of glycerol in suberin of wound-healing potato tuber at the same rate as the $\alpha, \omega$-dicarboxylic fatty acids. GPAT5 encodes a glycerol-3-phosphate acyltransferase and catalyzes the transfer of acyl groups from acyl-CoAs to glycerol3-phosphate (Beisson et al., 2007). GPAT5 is a key suberin biosynthetic enzyme involved in the formation of very-long-chain (C22 - C24) oxygenated suberin monomers in Arabidopsis roots and seed coat (Beisson et al., 2007). GPAT5 was shown to work in synergy with fatty acid $\omega$-hydroxylases (CYP86A1 or CYP86B1) for suberin synthesis (Beisson et al., 2007). These oxidized fatty acid-containing acylglycerols are suggested to be substrates for the polymerization reactions (Beisson et al., 2007; Yeats et al., 2012). It is thus evident that active suberization would involve increased GPAT5 activity. In our study, GPAT5 was found to be induced in all the stress conditions.

One major reaction in early suberin biosynthesis is fatty acid elongation to give $\mathrm{C} 20$ and C22 very-long-chains. Very-long-chains monomers are less abundant in suberin than C16 and C18 long-chain monomers, but nonetheless they are present at high amounts, especially compared to cutin that nearly completely lacks very-long-chain monomers. KCS2/DAISY encodes a $\beta$-ketoacyl-CoA synthases (KCS) 
of the fatty acid elongase (FAE). The KCS catalyzes the initial step in FAE synthesis converting fatty acyl-CoA to fatty $\beta$-ketoacyl-CoA. Arabidopsis KCS2/DAISY is involved in elongation of C20 and C20 precursors to very-long chain (C22 and C24) fatty acids, which are characteristic of Arabidopsis root suberin (Franke et al., 2009). KCS2/DAISY is partially redundant with KCS20 (Lee et al., 2009). Franke et al. (2009) reported induction of $K C S 2 / D A I S Y$ transcript after 4 hours by $50 \mathrm{mM} \mathrm{NaCl}$ treatment and this induction increased with increasing concentrations of $\mathrm{NaCl}$ treatment $(100 \mathrm{mM}$ and $200 \mathrm{mM})$. This concentration-dependent induction was also observed after a $24-\mathrm{h}$ treatment, except for the $200 \mathrm{mM} \mathrm{NaCl}$ application, in which KCS2/DAISY expression was reduced to control levels. In a similar study, Lee et al. (2009) reported induction of $K C S 2 / D A I S Y$ by approximately 3-, 4-, 30- and 65 -fold following application of $\mathrm{NaCl}$, mannitol, $\mathrm{ABA}$ or drought stress, respectively, for 6 hours. KCS2/DAISY is also induced by $10-12$-fold upon inoculation by avirulent bacterial strains Xcc147 in leaves of Arabidopsis (Raeffele et al., 2008). In our study, KCS2/DAISY was found to be highly induced in response to ABA, mannitol and wounding treatment at 24 hours, consistent with these earlier studies.

CYP86A1/HORST and CYP86B1/RALPH are NADPH-dependent cytochrome P450 monooxygenases that catalyze the hydroxylation of fatty acids at the $\omega$ position. CYP86A1/HORST is involved in the synthesis of long-chain C16 and C18 $\omega-$ hydroxy fatty acids in root suberin (Hofer et al., 2008), while CYP86B1/RALPH is a very long chain fatty acid hydroxylase involved in the synthesis of C22- and C24 $\omega$ hydroxy fatty acids in root and seed coat suberin (Compagnon et al., 2009; Molina et al., 2009). CYP86A1 was determined to be especially important in the early 
deposition of suberin in the primary endodermis (Hofer et al., 2008). Duan and Schuler (2005) reported a transient 3-fold up-regulation of CYP86A1 in seedlings after 3 hours of $A B A$ treatment, and a 1.9-fold up-regulation of CYP86A1 in response to cold treatment but only at a later 27 hour time-point. Inspection of the CYP86A1 promoter revealed the presence of $A B A$ response element (ABRE)-like elements (dehydration/low temperature) and AtMYC2 elements (drought/ABA), consistent with its induction in response to ABA, mannitol (drought), and wounding (Duan and Schuler, 2005). In our study, cold treatment caused down-regulation of CYP86A1 expression at 24 hours, which is inconsistent with the findings of Duan and Schuler (2005). However, the experimental conditions and timing were not identical between the two studies, and the 2005 study also did not use quantitative real-time PCR. CYP86B1 showed a similar overall pattern of induction as CYP86A1, with the exception for cold treatment, where CYP86B1 showed about 2-fold induction. CYP86B1 had highest induction (16-fold) in response to wounding stress. In general, CYP86B1 was more induced compared to CYP86A1 in all abiotic stresses. It is possible that very-long-chain $\omega$-hydroxy acids and dicarboxylic acids are preferentially deposited over long-chains under abiotic stress conditions, but measurements of suberin composition under these stress conditions has not yet been performed.

Arabidopsis ASFT (aliphatic suberin feruloyl transferase) is part of the BAHD family of acyltransferases, catalyzing the transfer of ferulate from feruloyl-CoA to $\omega$ hydroxy fatty acids and fatty alcohols (Molina et al., 2009). It thus links the phenolic portion of suberin (i.e. ferulate) with the aliphatic portion of suberin. In our study, 
ASFT1 was found to be induced in response to all abiotic stresses, showing the highest induction (24-fold) in response to wounding stress. It was also the most induced under wounding stress at the 24 hour time point, compared to other suberin genes studied. In a time course study of wound healing potato (Solanum tuberosum) tuber periderm, Bernards and Lewis (1992) reported that alkyl ferulate esters begin to accumulate 3-5 days after wounding. In a study by Lulai and Corsini (1998), it was shown that potato tubers developing resistance to bacterial (Erwinia spp.) and fungal (Fusarium spp.) infection, was related to the differential deposition of the two major suberin components (phenolics and aliphatics). The study revealed that aromatic suberin deposition begins before aliphatic suberin deposition. Total resistance to infection by $E$. carotovora occurred after completion of phenolic deposition on the outer tangential wall of the first layer of cells (2-3 days), while total resistance to fungal infection was attained after completion of aliphatic deposition within the first layer of suberizing cells (5-7 days), indicating a distinct control of the aromatic and aliphatic branches of the suberin biosynthetic machinery (Lulai and Corsini, 1998). It is currently unknown whether Arabidopsis ASFT1 and the aliphatic suberin biosynthetic genes are controlled by common transcription factor(s), but it has been reported that AFST1 is expressed earlier in root development than the aliphatic suberin biosynthetic genes using promoter:GUS transgenic lines (Naseer et al., 2012). A more detailed time course study would be required to determine whether ASFT1 is expressed earlier than the other genes under abiotic stresses. 
At the 24 hour time point, treatment with exogenous ABA showed much higher induction of all suberin genes compared to other abiotic stress we studied. It is possible that $\mathrm{ABA}$ being a stress signal is perceived quickly by the seedlings to activate downstream stress-responsive genes within $24 \mathrm{hrs}$. Other abiotic stress would take longer to induce the ABA signaling cascade, resulting in lower induction of stress-responsive (suberin) genes at the 24 hour time-point. The relatively low induction of suberin biosynthesis genes under cold stress may be a result of a much slower response of the plants to cold stress than the other stresses. It is possible that at the $24 \mathrm{hr}$ time point, most of the suberin genes are still in the process of induction. A time course of suberin gene expression over a broader range will give a better understanding of their regulation in response to abiotic stresses.

The transcription factors involved in controlling suberin biosynthesis are currently unknown. Members of MYB, WRKY, and NAC transcription factor families have been postulated to be involved because they are preferentially expressed in tissues undergoing suberisation (Kilian et al., 2007) and are the most frequent regulatory proteins that are co-expressed with suberin-biosynthetic genes (Obayashi et al., 2009; Ranathunge et al., 2011). These types of transcription factors are also upregulated in suberising phellem tissue of cork oak (Soler et al., 2007). MYBs are involved in the biosynthesis of phenylpropanoids (Zhou et al., 2009) as well as fatty acid elongation (Raffaele et al., 2008). A whole-genome DNA microarray analysis of a MYB30 overexpressing line revealed 14 putative transcriptional targets encoding putative lipid metabolic enzymes, with $\mathrm{KCS} 2$ being one among them (Raffaele et al., 2008). In another study, MYB96, which is a part of an ABA signaling 
cascade in the drought resistance response (Seo et al., 2009), was found to modulate cuticular wax biosynthesis in response to drought by regulating cuticular wax biosynthetic genes (Seo et al., 2011), but no association with suberin was reported.

It was observed that Arabidopsis suberin biosynthetic genes were generally, but not always, induced in response to abiotic stress. There were differential expression patterns of the suberin genes, depending on the particular stress. The functional significance of these alterations awaits further analysis. From these observations, we conclude that transcriptional control plays a major role in the regulation of suberin biosynthesis during abiotic stresses. More detailed transcriptome profiling, perhaps using DNA microarrays or next generation sequencing, may provide important insights into the dynamics of the transcriptional changes that accompany abiotic stress treatments and a detailed time course study will help in understanding transcriptional regulation of suberin genes during abiotic stresses. Identification of the transcription factors involved in regulating suberin biosynthesis genes will be an important future direction and would provide a means to control suberin deposition perhaps for the development of more stress resistant crops. 


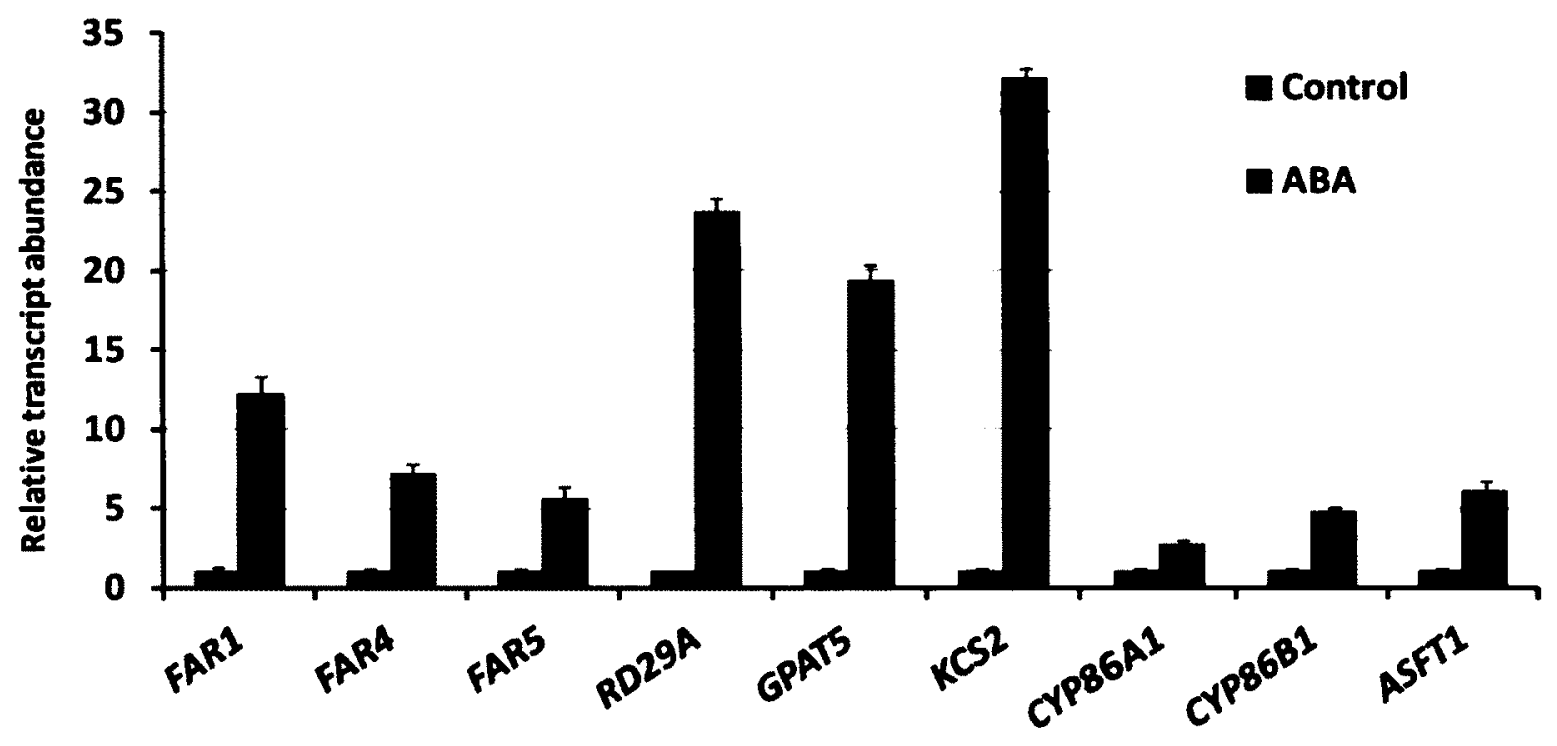

Figure 5.1. Modulation of expression (fold change) of FAR1, FAR4, and FAR5 and five other suberin genes in the Arabidopsis root tissue subjected to ABA stress $(10 \mu \mathrm{M})$ for 24 hours. The transcript levels were determined by quantitative PCR analysis. Abiotic-stress responsive gene $R D 29 A$ was used as a positive control for treatment efficiency. The results are presented as relative transcript abundances and normalized through geometric averaging of two constitutively expressed genes (ACT2 and eIF4A-1). The data represent the means \pm S.D. of three technical replicates. 


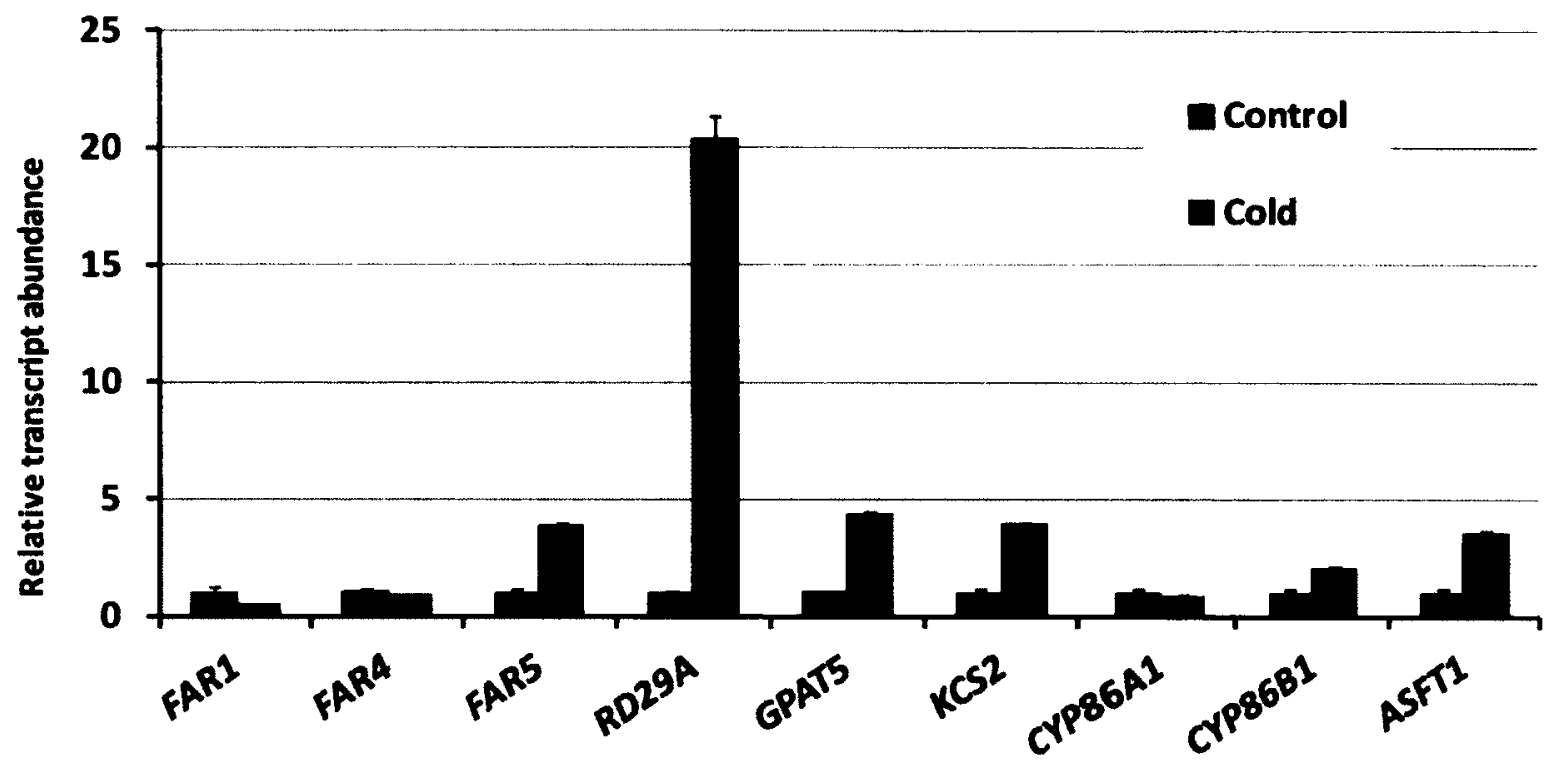

Figure 5.2. Modulation of expression (fold change) of FAR1, FAR4, and FAR5 and five other suberin genes in the Arabidopsis root tissue subjected to cold stress $\left(4^{\circ} \mathrm{C}\right.$ ) for 24 hours. The transcript levels were determined by quantitative PCR analysis. Abiotic-stress responsive gene $R D 29 A$ was used as a positive control for treatment efficiency. The results are presented as relative transcript abundances and normalized through geometric averaging of two constitutively expressed genes (ACT2 and eIF4A-1). The data represent the means \pm S.D. of three technical replicates. 


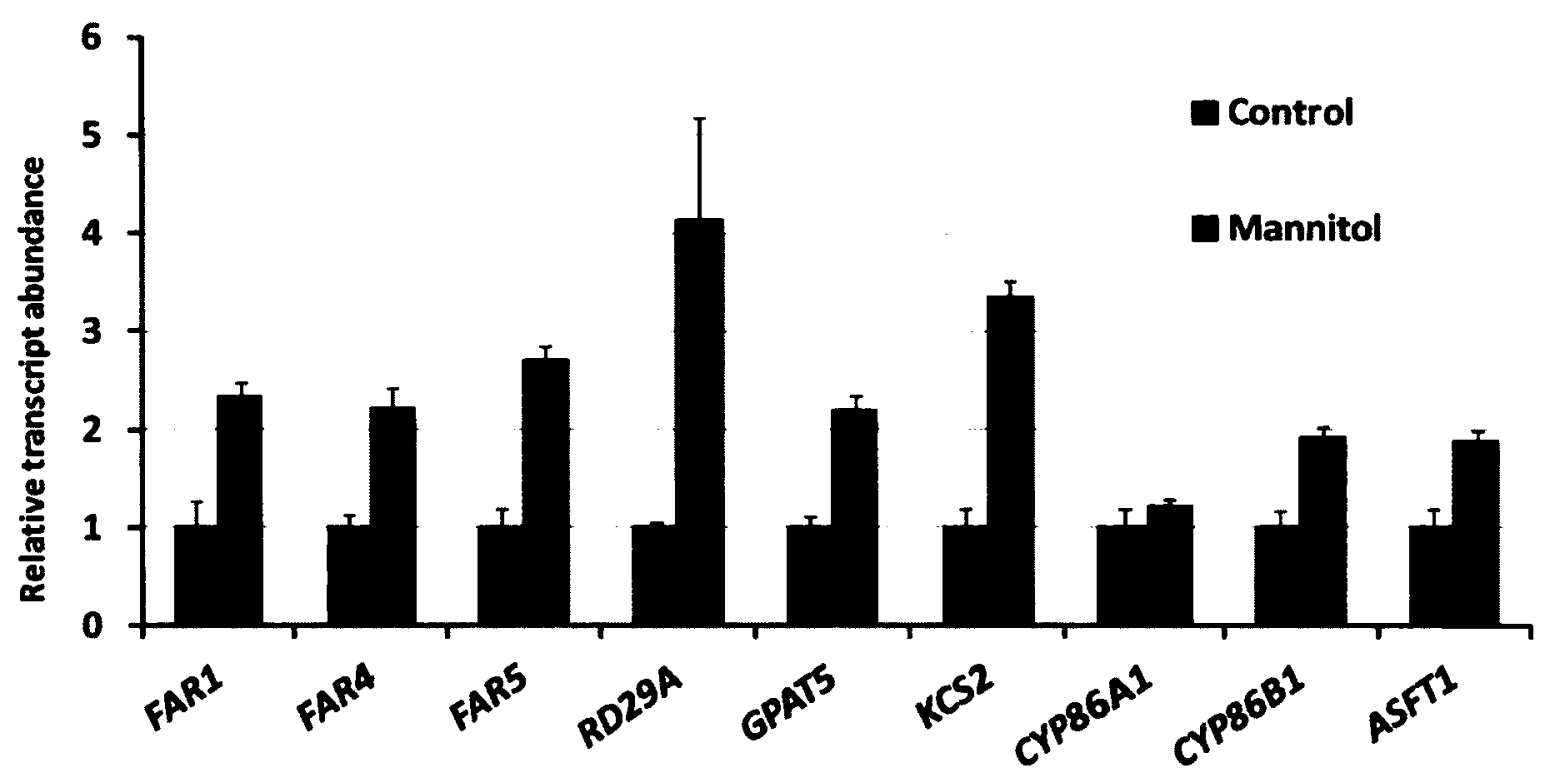

Figure 5.3. Modulation of expression (fold change) of FAR1, FAR4, and FAR5 and five other suberin genes in the Arabidopsis root tissue subjected to osmotic stress (150mM Mannitol) for 24 hours. The transcript levels were determined by quantitative PCR analysis. Abiotic-stress responsive gene $R D 29 A$ was used as a positive control for treatment efficiency. The results are presented as relative transcript abundances and normalized through geometric averaging of two constitutively expressed genes ( $A C T 2$ and $e I F 4 A-1)$. The data represent the means \pm S.D. of three technical replicates. 


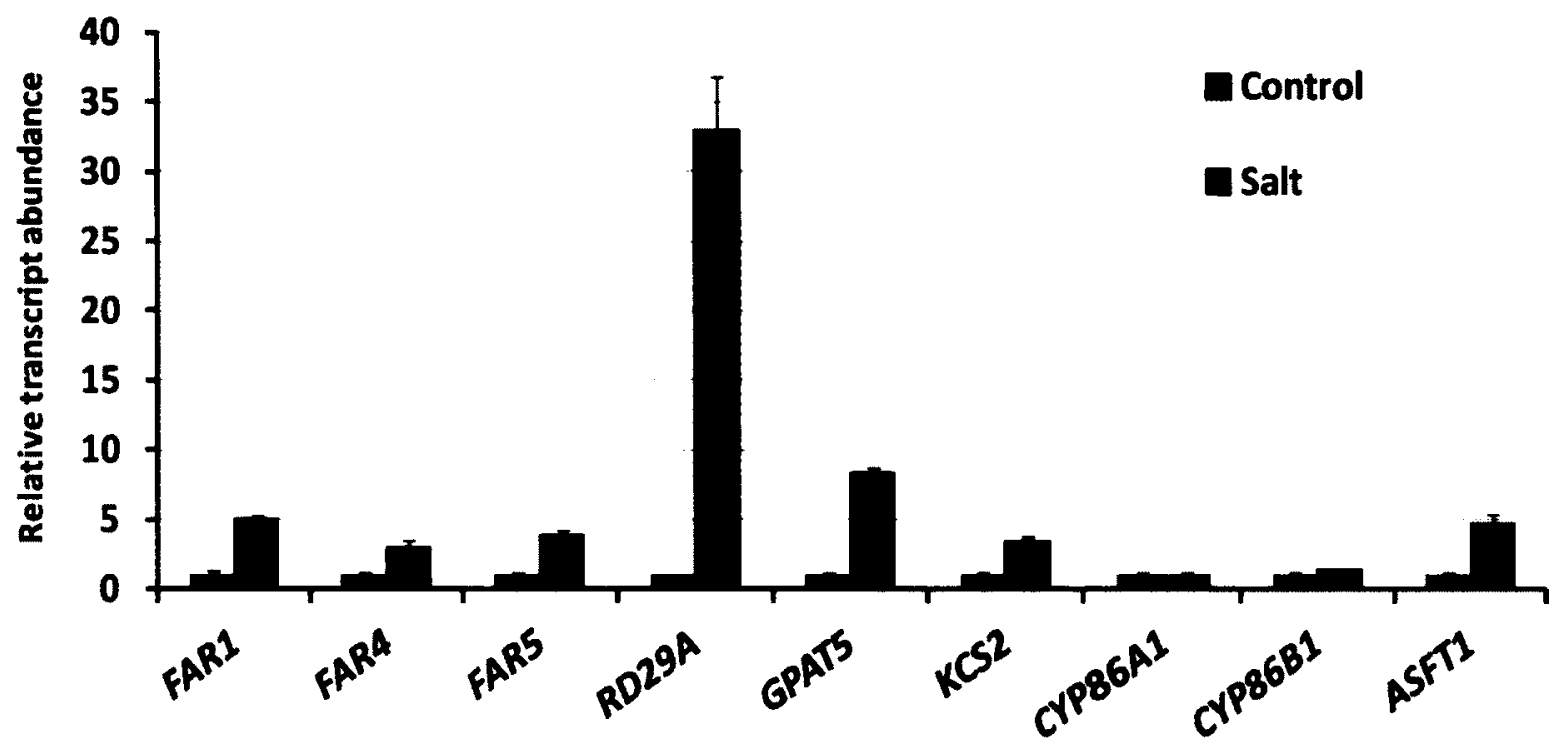

Figure 5.4. Modulation of expression (fold change) of FAR1, FAR4, and FAR5 and five other suberin genes in the Arabidopsis root tissue subjected to salt stress (200 $\mathrm{mM} \mathrm{NaCl}$ ) for 24 hours. The transcript levels were determined by quantitative PCR analysis. Abiotic-stress responsive gene RD29A was used as a positive control for treatment efficiency. The results are presented as relative transcript abundances and normalized through geometric averaging of two constitutively expressed genes (ACT2 and elF4A-1). The data represent the means \pm S.D. of three technical replicates. 


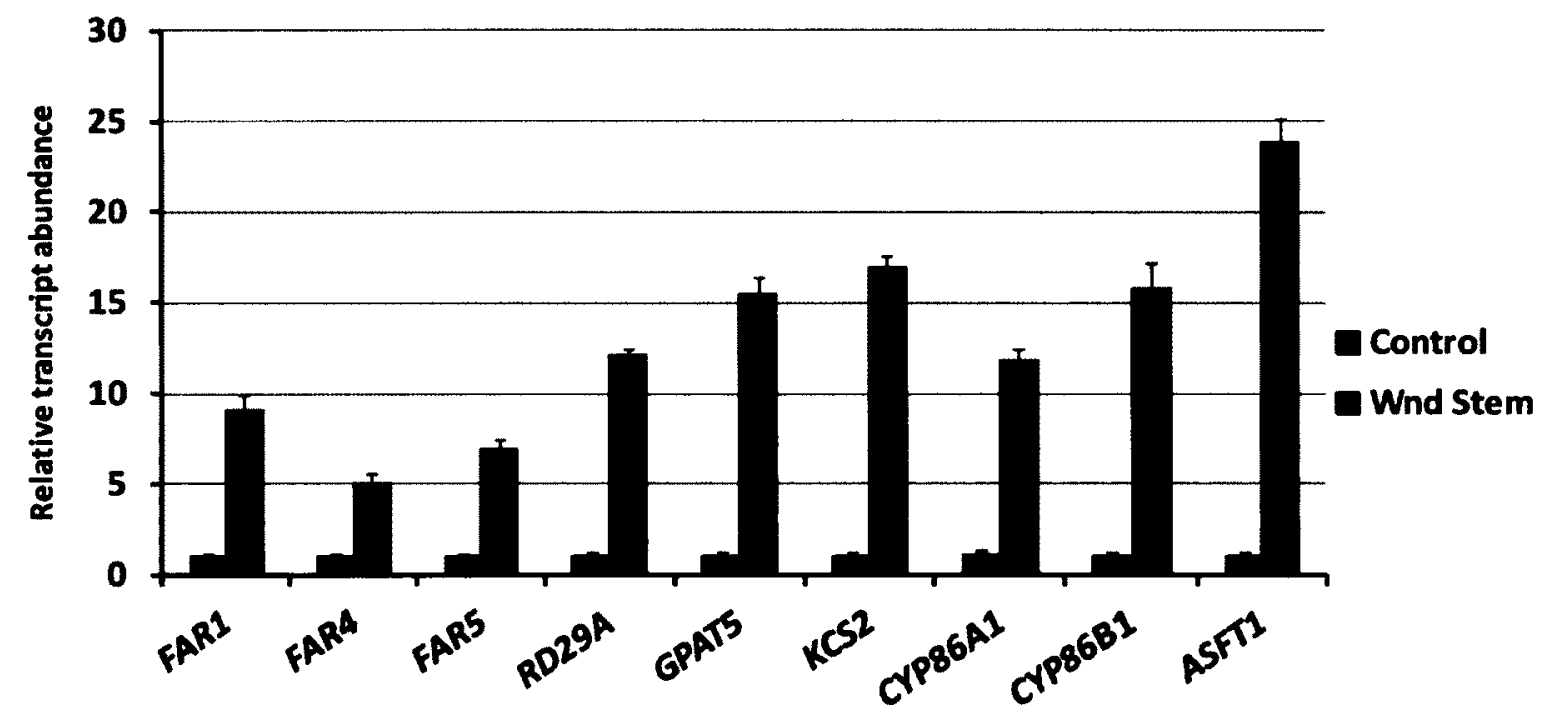

Figure 5.5. Modulation of expression (fold change) of FAR1, FAR4, and FAR5 and five other suberin genes in the Arabidopsis stem tissue subjected to wounding stress for 24 hours. The transcript levels were determined by quantitative PCR analysis. Abiotic-stress responsive gene $R D 29 A$ was used as a positive control for treatment efficiency. The results are presented as relative transcript abundances and normalized through geometric averaging of two constitutively expressed genes (ACT2 and eIF4A-1). The data represent the means \pm S.D. of three technical replicates. 


\subsection{Supplemental tables}

Table S5.1. Modulation of expression of FAR1, FAR4, and FAR5 and five other suberin genes in 15-day-old Arabidopsis seedlings subjected to different stress conditions. The gene expression levels were determined by quantitative-PCR analysis. Results are presented as relative transcript abundance, and the relative transcript abundance of the gene of interest in the control sample was arbitrarily defined as 1 . The general abiotic stress-inducible $R D 29 A$ gene was used as a control for treatment efficiency. The data represent means \pm SD of three replicates.

\begin{tabular}{|c|c|c|c|c|c|c|c|}
\hline & $\begin{array}{c}\text { Control } \\
\text { (roots) }\end{array}$ & $\begin{array}{c}\text { ABA } \\
(10 \mu \mathrm{M})\end{array}$ & $\begin{array}{c}\text { Cold } \\
(4 \circ \mathrm{C})\end{array}$ & $\begin{array}{c}\text { Mannitol } \\
(150 \mathrm{~mm})\end{array}$ & $\begin{array}{c}\text { NaCl } \\
(200 \mathrm{~mm})\end{array}$ & $\begin{array}{c}\text { Control } \\
\text { (stem) }\end{array}$ & $\begin{array}{c}\text { Wound } \\
\text { (stem) }\end{array}$ \\
\hline FARI & $1.0 \pm 0.3$ & $12.2 \pm 1.1$ & $0.4 \pm 0.1$ & $2.3 \pm 0.1$ & $4.9 \pm 0.3$ & $1.0 \pm 0.2$ & $9.1 \pm 0.8$ \\
\hline FAR4 & $1.0 \pm 0.1$ & $7.1 \pm 0.7$ & $0.9 \pm 0.1$ & $2.2 \pm 0.2$ & $3.1 \pm 0.4$ & $1.0 \pm 0.1$ & $5.0 \pm 0.6$ \\
\hline FAR5 & $1.0 \pm 0.2$ & $5.6 \pm 0.7$ & $3.9 \pm 0.1$ & $2.7 \pm 0.1$ & $3.9 \pm 0.4$ & $1.0 \pm 0.1$ & $6.9 \pm 0.5$ \\
\hline RD29A & $1.0 \pm 0.1$ & $23.6 \pm 0.9$ & $20.3 \pm 1.0$ & $4.1 \pm 1.0$ & $32.9 \pm 3.9$ & $1.0 \pm 0.2$ & $12.1 \pm 0.3$ \\
\hline GPAT5 & $1.0 \pm 0.1$ & $19.3 \pm 1.0$ & $4.3 \pm 0.1$ & $2.2 \pm 0.1$ & $8.3 \pm 0.3$ & $1.0 \pm 0.2$ & $15.5 \pm 1.0$ \\
\hline KCS2 & $1.0 \pm 0.2$ & $32.1 \pm 0.6$ & $3.9 \pm 0.1$ & $3.3 \pm 0.2$ & $3.4 \pm 0.5$ & $1.0 \pm 0.2$ & $17.0 \pm 0.6$ \\
\hline CYP86AI & $1.0 \pm 0.2$ & $2.7 \pm 0.3$ & $0.8 \pm 0.1$ & $1.2 \pm 0.1$ & $0.9 \pm 0.2$ & $1.0 \pm 0.3$ & $11.9 \pm 0.6$ \\
\hline CYP86B1 & $1.0 \pm 0.2$ & $4.7 \pm 0.3$ & $2.0 \pm 0.1$ & $1.9 \pm 0.1$ & $1.3 \pm 0.1$ & $1.0 \pm 0.2$ & $15.7 \pm 1.5$ \\
\hline ASFT1 & $1.0 \pm 0.2$ & $6.0 \pm 0.7$ & $3.5 \pm 0.1$ & $1.9 \pm 0.1$ & $4.8 \pm 0.5$ & $1.0 \pm 0.2$ & $23.8 \pm 1.4$ \\
\hline
\end{tabular}


Table S5.2. List of primers used for the study.

\begin{tabular}{|l|l|}
\hline \multicolumn{1}{|c|}{ Primer Name } & \multicolumn{1}{c|}{$\begin{array}{c}\text { Primer sequence } \\
(5 \rightarrow 3)\end{array}$} \\
\hline FAR1_AtRT3-Fwd & CCTGGTTTTCTTGCCAAGGTTTTG \\
\hline FAR1_AtRT3-Rev & CGTTGCATGGCTGATTTTTCGTC \\
\hline FAR4_AtRT2-Fwd & CCCGGTTGGATCGAAGGGTTAAGA \\
\hline FAR4_qRT_Rev & GATGAACCATCGCGTCTAACA \\
\hline FAR5_AtRT3-Fwd & GCATAAAGAAAATCTCCCCTCGTC \\
\hline FAR5_AtRT3-Rev & TCCATATGCAATAATCACGCTGTCG \\
\hline GPAT5_qRT-Fwd & ACCGTGTCGCTAATTGTTTGTTGG \\
\hline GPAT5_qRT-Rev & TCTCCGGGATTGGTGCATGAATATG \\
\hline KCS2_qRT-Fwd & CTCAGCGTGTCGGTATCTTCACAGA \\
\hline KCS2_qRT-Rev & GATTAGGCGGGACACGTAAGAGAGC \\
\hline CYP86A1_qRT-Fwd & CGCTGCGTTTATACCCTTCTGTGC \\
\hline CYP86A1_qRT-Rev & TCTTCGCCCCAAATTGTTTTCATAC \\
\hline CYP86B1_qRT-Fwd & CTTCAGTTCCGTGGATCACAAAG \\
\hline CYP86B1_qRT-Rev & GACCCATTGCGTAAATCGCGTA \\
\hline ASFT1_qRT-Fwd & GTTCGATGGAATTGGAGCTATGGAG \\
\hline ASFT1_qRT-Rev & CTTTGGAGGGTTTCGAGCATTGAG \\
\hline ACT2_qRT_Rev & CATACTCTGCCTTAGAGATCCACA \\
\hline
\end{tabular}




\begin{tabular}{|l|l|}
\hline eIF4A1_qRT_Fwd & CTGATTTTGACCCGTCGTCT \\
\hline elF4A1_qRT_Rev & AAGACAAACAACAAAGCCGAAT \\
\hline & \\
\hline RD29A_qPCR_Fwd & TGTCGACGAGAAGCAAAGAA \\
\hline RD29A_qPCR_Rev & CGGGAAAACAACTCCTGATT \\
\hline
\end{tabular}




\section{CHAPTER 6: Conclusions and Future directions}

Suberin is a protective hydrophobic barrier produced by various internal and external cell layers of plants, such as the endodermis of roots and the mature peridermis of roots and shoots (Kolattukudy, 2001a; Bernards, 2002). It plays important roles at the plant-environment interface by preventing the loss of water and solutes to the environment as well as helping plants cope with various abiotic stresses and pathogens (Franke and Schreiber, 2007; Schreiber, 2010; Ranathunge et al., 2011) (See General Introduction, Chapter 1). The complexities of suberin synthesis and its regulated deposition must be understood before the suberization of plant tissues can be manipulated to achieve optimal barrier properties in crop plants. The characterization of suberin mutants and suberin biosynthetic enzymes offers the opportunity to develop more comprehensive structural models that can be used to further rationally alter suberin for applications. Many key suberin biosynthetic genes have been identified in the recent past (Chapter 1 ), including in this thesis. However, many aspects of suberin biosynthesis, regulation and transport are still not well understood.

FARs are responsible for an $\mathrm{NAD}(\mathrm{P}) \mathrm{H}$-dependent reaction that reduces fatty acyl-CoAs or fatty acyl-ACPs to primary fatty alcohols via an unreleased fatty aldehyde intermediate. Evolutionarily related FARs have been cloned and characterized from many species of plants, protists, and animals (Chapter 1). There is much variation in the substrate specificities of these FARs according to acyl chain length and/or degree of saturation. The free fatty alcohols may then be combined 
with other molecules to give, for example, wax esters, alkyl hydroxycinnamates, or ether lipids. Free and combined fatty alcohols are utilized in a variety of biological roles such as energy storage, pheromones, neuronal membrane lipids, and protective surface lipids (Chapter 1). Saturated primary fatty alcohols of chain lengths C18, C20 and C22 are common components of suberin (Franke et al., 2005; Rowland and Domergue, 2012; This Study) and account for about 6-7\% of the aliphatic suberin polymer in Arabidopsis root and seed coat (Pollard et al., 2008) and up to $15 \%$ in potato tuber periderm (Schreiber et al., 2005b). However, the structural incorporation and biological roles of these fatty alcohols in suberin are currently not well understood.

\subsection{Major findings of the thesis work}

One of the main objectives of this thesis work was to identify and characterize the fatty acyl reductase(s) in Arabidopsis thaliana that synthesize C18:0-C22:0 primary fatty alcohols associated with suberin. Another key objective was to understand the biological roles of suberin-associated primary alcohols (free or combined) in roots and seed coats, particularly in relation to the barrier properties of suberin. The study involved a multidisciplinary approach combining molecular genetics, analytical chemistry, cell biology, and physiological studies to address these objectives. The major findings of this work are summarized below.

In Chapter 2, it was demonstrated that Arabidopsis FAR1, FAR4, and FAR5 are specifically expressed in root endodermal cells and the hilum region of the seeds. Also, these three genes are transcriptionally induced by wounding and salt stress. 
These patterns of gene expression coincide with known sites of suberin deposition (Kolattukudy, 2001b; Ranathunge et al., 2012). A set of mutants with T-DNA insertions in FAR1, FAR4, or FAR5 were characterized and it was found that the suberin compositions of roots and seed coats were modified in each far mutant. Specifically, C18:0-OH was reduced in far5-1, C20:0-OH was reduced in far $4-1$, and C22:0-0H was reduced in far1-1. The composition of polymer-bound lipids of leaves before and after wounding was analyzed and it was found that the basal levels of C18:0-C22:0 primary alcohols in wild-type leaves were increased by wounding. In contrast, $\mathrm{C} 18: 0-\mathrm{OH}$ and $\mathrm{C} 22: 0-\mathrm{OH}$ were not increased by wounding in far5-1 and far1-1 mutants, respectively. Therefore, it was discovered that FAR1, FAR4, and FAR5 generate the fatty alcohols found in root, seed coat, and wound-induced leaf tissue in a substrate-specific manner. Heterologous expression of FAR1, FAR4, and FAR5 in yeast resulted in the preferential production of $\mathrm{C} 22: 0-\mathrm{OH}, \mathrm{C20:0-0H}$, and C18:0-0H, respectively. These data indicate that FAR1, FAR4, and FAR5 are active alcohol-forming FARs with distinct, but overlapping, chain length specificities. In addition, transient over-expression of these three FARs in $N$. benthamiana leaves confirmed that these FARs have almost strict specificity in planta to one chain length, rather than broad chain-length specificities (Chapter 4).

In order to study the effect of lack of suberin-associated C18:0-C22:0 primary alcohols in plants, I generated far 1 far 4 and far1 far 5 double mutants as well as far 4 far5* double and far1 far 4 far5* triple mutant lines using an artificial-microRNA gene silencing technique (Chapter 3). We carried out suberin analysis of different root fractions viz. soluble fraction (unpolymerised) and insoluble fraction 
(polymerized; suberin) to study the distribution of suberin monomers. Saturated C18:0-C22:0 fatty alcohols were found in both these root fractions, with the majority of the fatty alcohols in the soluble fraction. Analysis of suberin in roots and seed coats of far double and triple mutants showed chain length-specific reductions in fatty alcohols and total fatty alcohol levels were reduced. Primary alcohols are also found in a combined state in root waxes, specifically as C18:0-C22:0 alkyl hydroxycinnamates (AHCs) (Li et al., 2007). The far double and triple mutants showed chain-length specific reduction in AHCs in suberin-associated root waxes, with far 1 far 4 and far 1 far 4 far $5^{*}$ each nearly devoid of AHCs.

The primary role of suberized cells appears to be that of controlling water and solute transport (Bernards, 2002), but it has also been postulated that suberized cells act as preformed, as well as wound-induced, antimicrobial barriers (Kolattukudy, 2001). An Arabidopsis mutant called enhanced suberin1 (esb1) is characterized by increased root suberin, and this was correlated with reduced daytime transpiration rates and increased water use efficiency (Baxter et al., 2009). The Arabidopsis gpat5-1 mutant has an approximately 50\% decrease in aliphatic suberin in roots and seed coats, and this is correlated with increases in both root and seed coat permeabilities (Beisson et al., 2007). We carefully monitored for developmental abnormalities in the different far mutants, especially in the development of their roots, but we did not detect any obvious developmental differences in the single far mutants when compared with wild-type. We also did not detect major reductions in germination rates or seed coat permeability, even with far1-1, which had the largest reduction in fatty alcohol levels associated with seed 
coat suberin. Since primary fatty alcohols constitute less than $9 \%$ of the total aliphatic content in Arabidopsis and as each far single mutant was affected in only one primary alcohol chain length with the overall amount of fatty alcohol remaining insignificantly changed (Chapter 2), it was not unexpected that far single mutants did not show a dramatic effect on permeability. Surprisingly, the far triple mutants with $\sim 74 \%$ reduction in total fatty alcohol load in root and seed coat suberin, also did not show any alterations in germination percentage or root growth under salt stress conditions (Chapter 3). However, far triple mutant seeds showed increased seed coat permeability and were affected in germination when subjected to abscisic acid, indicating that primary alcohols play an important role in the barrier properties of seed coat suberin. We were not able to generate a far triple mutant line that had a complete absence of C18:0-C22:0 primary alcohols due to partial silencing of the FAR5 gene, which is a limitation of the current study. It is likely that a complete elimination of fatty alcohol content from root suberin is required to see a major effect on the barrier properties. It is possible, however, that the absence of primary alcohols may be detrimental for the survival of the plant and that isolating such a mutant line is not possible.

There is currently little information regarding what amino acid residues determine the substrate specificities of FAR enzymes, both with regard to chain length and degree of acyl-chain saturation. Primary amino acid sequence alignments of the Arabidopsis FAR family revealed that there is a high degree of amino acid identity between FAR1 and FAR4 (74\%), FAR4 and FAR5 (69\%), and FAR1 and FAR5 (68\%) (Chapter 2). The high amino acid similarity between these FARs and 
the finding that they have distinct specificities means that the three FARs are an excellent platform for identifying protein domains and individual amino acids that are important for catalytic activity as well as substrate specificity. In Chapter 4, using a domain-swapping approach, I successfully narrowed down an important 150 amino acid region between residues 235 and 385 that significantly influences chain-length substrate specificity in FAR1, FAR4 and FAR5 enzymes. The FAR5FAR4 chimera showed the possibility of engineering FARs with broad specificity, although there may be a compromise in enzymatic turnover. This limitation may be overcome with additional protein engineering. These results represent a first step towards the generation of engineered FARs with altered specificities.

In Chapter 4, by transiently expressing the FAR1-GFP fusion protein in $N$. benthamiana leaves and using confocal laser scanning microscopy, I provided evidence for the localization of FAR1 to the endoplasmic reticulum (ER). Other suberin biosynthetic enzymes, such as CYP86A1 and CYP86B1, have also been localized to the ER (Hofer et al., 2008; Campagnon et al., 2009). Based on gene ontology, GPAT5 and KCS2/DAISY have been predicted to be ER localized. Together, this suggests that the major biosynthetic steps in suberin biosynthesis, including acyl reduction, occur in the endoplasmic reticulum. However, stable Arabidopsis transgenic lines expressing a FAR1:GFP/YFP fusion protein under the control of its respective native promoter and complementing the respective far1 mutant will provide better evidence of specific ER localization. 
In Chapter 5, using quantitative RT-PCR, I studied the expression of Arabidopsis FAR1, FAR4, and FAR5 and other suberin biosynthetic genes in response to $\mathrm{ABA}$ and various abiotic stresses. Such a comparative study has not been reported in the literature before. I showed that there is a rapid and mostly coordinated response of suberin genes to these environmental stressors.

\section{Future directions}

\section{What are the physiological roles of primary alcohols associated with root suberin?}

Overall, this thesis work resulted in the identification of three FAR enzymes that are involved in synthesis of primary alcohols associated with suberin. A major outstanding question that still needs to be addressed regards the specific in planta roles of fatty alcohols and alkyl hydroxycinnamates associated with suberin in plant physiology and plant-environment interactions.

Although the far triple mutant seeds showed increased permeability to tetrazolium salts and $A B A$, there was no dramatic effect on the normal growth and development of far triple mutant seedlings under normal or salt stress conditions. This absence of a developmental and physiological phenotype raises the question of the importance of fatty alcohols in root suberin. However, if the role of fatty alcohols in suberin is limited, why then are fatty alcohols consistently found to be associated with suberin? Also, why does the plant require three complementary proteins when just a single FAR with broader substrate specificity would be sufficient? The presence of three genes having similar spatiotemporal gene expression patterns, 
shared transcriptional regulation, and coding for enzymes with complementary catalytic activities strongly suggests that fatty alcohols have fundamental functions in suberin polyester structure and/or function. Further studies involving physiological experiments with double or triple mutants (far1, far4, and/or far5) may unravel important functions for primary alcohols in suberin assembly and suberin permeability characteristics (this was initiated in Chapter 3 of this thesis). The far 4 far $5^{*}$ amiRNA line showed a better silencing compared to the far 1 far 4 far5*amiRNA triple mutant line (Chapter 3 ). I have further made crosses between far1 far 4 and far 4 far 5 *amiRNA lines to generate a better silenced far triple mutant line, which is crucial for carrying out further phenotypic experiments. A series of physiological experiments such as measuring transpiration rates, wilting resistance under drought conditions, water use efficiency, and resistance to salt stress conditions can be performed to analyze the effect of reduced fatty alcohol level in far double and triple mutant seedlings. The effect on ion uptake by roots and translocation of solute through the endodermis and peridermis to shoots in far double and triple mutants can be studied by carrying out elemental analysis, particularly total $\mathrm{Ca}^{2+}, \mathrm{Mn}^{2+}, \mathrm{Zn}^{2+}, \mathrm{K}^{+}$and $\mathrm{Na}^{+}$, in the whole rosettes of seedlings grown in hydroponic medium containing these elements, using inductively coupled plasma-atomic emission spectroscopy (ICP/AES) (Baxter et al., 2009; Compagnon et al., 2009). Further, a detailed correlative study of hydraulic conductivity and solute permeability in far double and triple mutant plants using root pressure probes may reveal a better understanding of role of primary alcohols in the barrier properties of suberin in roots. Recently, Ranathunge and Schreiber (2011) used such an approach 
to study the correlation between root permeabilities with changes in aliphatic suberin amounts and composition in Arabidopsis roots, using two of the suberin mutants, horst1 (Hofer et al., 2008) and esb1 (Baxter et al., 2009). It was reported that the amount of suberin may not always correlate with permeability, and the microstructure and monomer arrangement in the suberin biopolymer might also play an important role in controlling the permeability (Ranathunge and Schreiber, 2011). Further, transmission electron microscopy (TEM) of root endodermal cells should be carried out in far triple mutants to observe for any disruption of suberin lamellar structure. The far triple mutant seeds were affected in germination percentage under the influence of $\mathrm{ABA}$, likely due to increase permeability of the seeds. It will be useful in studying the effect of seed coat polyester composition and suberin load on germination and dormancy under salinity and osmotic stress conditions.

Long-chain fatty alcohols have been found to have antibacterial action (Masao et al., 1987) and hydroxycinnamic acid derivatives have also been reported to show considerable antimicrobial activity (Baranowski and Nagel, 1983; Bowles and Miller, 1994; Chong et al., 2009). Thus, free fatty alcohols and fatty alcohols combined with hydroxycinnamates may have an important role in protecting plant roots from soil-borne pathogens. Further studies on far double and triple mutants with respect to pathogen susceptibility will provide a better understanding of roles of fatty alcohols in resisting pathogenic microbes. The mutants can be tested for altered susceptibility to pathogens by subjecting them to inoculums of root 
pathogens, such as Erwinia carotovora (bacteria), Pythium irregulare (oomycete), Ralstonia solanacerarum (bacteria) and Fusarium oxysporium (fungus).

\section{How are primary alcohols incorporated into the suberin polymer?}

The mechanism of fatty alcohol incorporation into the suberin polymer is still not clear. Primary alcohols, being mono-functional, are dead-end products for polymerization (Rowland and Domergue, 2012). In partial depolymerization studies, primary alcohols have been reported to be found as alkyl feruloyl (phenolic) esters (Moire et al., 1999; Graca et al., 2000). Detailed profiling of cross-linked phenolic components may provide clues to the linkages or identification of anchor molecules of primary alcohols. Further, quantification of alkyl phenolic esters (i.e. ferulates) in the suberin polymer fraction of far mutants needs to be carried out to assess the effects of reduced primary alcohol load on suberin-linked phenolics. These experiments should be done using partial depolymerization conditions to detect linkages and assessed by GC-MS and/or NMR spectroscopy.

In the classic two-domain suberin model (after Bernards, 2002; reviewed in Chapter 1; Graca, 2007), it is held that ferulic acid covalently links the suberin aliphatic polyester to the neighboring polyaromatics, as it may form carboxyl-ester bonds with aliphatic monomers and non-ester radical coupled bonds with other phenolics including those of lignin (Graca and Santos, 2007; Serra et al., 2010a). Recent characterization of loss-of-function mutants in ASFT/HHT (Arabidopsis) and FHT (potato) with major reductions in ester-linked ferulic acid in the suberin polyester showed no disruption of the lamellar ultrastructure observed by TEM 
(Molina et al., 2009; Serra et al., 2010b). These findings challenge the current suberin model and alternatives roles for ferulate in suberin structure and its properties need to be considered. Generating a quadruple mutant of far 1 far 4 far $5^{*}$ with asft1, and then assessing suberin composition, ultrastructure by TEM, and permeability will help in understanding the roles of primary alcohols and alkyl ferulates. Alternatively, transient co-overexpression of FAR1/4/5 and ASFT1 in Nicotiana benthamiana and/or Arabidopsis leaves will help to understand the effects of increased accumulation of alkyl ferulates in the suberin polyester. For such experiments, production of anti-FAR and anti-ASFT antibodies will be useful to confirm the expression of transgenic FAR and AFST proteins.

\section{How are suberin biosynthetic genes, including FAR1, FAR5, and FAR5,} transcriptionally regulated?

Suberin biosynthesis and deposition is strictly regulated at the developmental level (Ranathunge et al., 2012). Suberin genes (KCS2/DAISY, KCS20, FAR1, FAR4, FAR4) have also been found to be induced under various abiotic stresses (Chapter 5). A more detailed time-course gene expression study of FARs and other suberin genes should be carried out to understand the dynamics of regulation of suberin biosynthesis and deposition. This may reveal which genes are tightly co-regulated and which may be under the control of other transcriptional regulators. Identification and characterization of transcription factors involved in suberin biosynthesis regulation should be a future priority. 
What structural features and specific amino acids dictate the substrate specificities of FARs?

Knowledge of the three-dimensional structure of an enzyme is very important to understand its structure/function properties and catalytic mechanisms. An X-ray crystal or NMR structure of a FAR protein has not yet been reported. Such information will help immensely in engineering of FAR enzymes for industrial uses through enhancement of catalytic activity, modification of substrate specificity, and redirection of subcellular location. Determining the structure of a FAR should be a future priority in FAR enzyme characterization. This would be most feasible with a soluble, plastid FAR, such as FAR2 or FAR6 from Arabidopsis. Other membrane-associated FARs (e.g. FAR5) may be modeled from the soluble FAR three-dimensional structure. However, if they are integral membrane protein, then it may be difficult to model outside of catalytic region.

A detailed in vitro characterization of the substrate specificities and enzymatic activities of purified Arabidopsis FAR enzymes will help in better understanding the relationship of enzyme specificities in governing the primary alcohol composition in specific plant tissues. FAR in vitro assays using radio-labelled acyl-CoA or acyl-ACP substrates have been previously reported (Wang and Kolattukudy, 1995; Metz, et al., 2000; Teerawanichpan et al., 2010). Since most FARs are not active in soluble form, microsome preparations from yeast heterologously expressing these enzymes can be used for in vitro assays. An in vitro FAR assay should be developed for a detailed kinetic analysis of these FARs and the promising FAR chimeras using yeast microsome preparation. FAR1, FAR4 and FAR5 are 
predicted to be membrane bound enzymes, and thus isolation of pure enzymes from transgenic $E$. coli would be difficult. This may be solved by fusing them to $E$. coli maltose binding protein (MBP), which should enhance their solubility while hopefully maintaining their biologically active conformations (Kapust and Waugh, 1999). Recently, Doan et al. (2011) reported in vitro characterization of Arabidopsis FAR6 enzyme fused to MBP and expressed in E. coli cells. Commercial gateway vectors pDESTHis 6 MBP (Nallamsetty et al., 2005) can be used for heterologous FAR protein expression and affinity tag purification.

Using a domain swapping approach, I identified a region between residues 235 and 385 that influences chain-length substrate specificity in FAR1, FAR4 and FAR5 enzymes (Chapter 4). Further internal domain swaps between this region of the proteins, as well as detailed site-directed mutagenesis should be carried out to identify specific amino acids that are important for catalytic activity and substrate specificity. For example, amino acid exchanges between FAR5 and FAR1 will reveal a.a.'s important for chain length specificity (C18:0 vs. C22:0 specificity). Substrate specificity studies in the current work was carried out analyzing the fatty alcohols generated by heterologous expression of FAR1, FAR4, FAR5, and FAR chimeras utilizing endogenous yeast acyl-CoA pools. Feeding experiments can be carried out to gain further insights into the specificity distributions using saturated and monosaturated fatty acids from $\mathrm{C} 12$ to $\mathrm{C} 24$. Similar studies can be carried out on other FAR combinations. For example, Arabidopsis FAR3/CER4 (Rowland et al., 2006) and the Jojoba embryo FAR (Metz et al., 2000) share 54\% amino acid sequence identity and have overlapping substrate specificity with regards to chain 
length but the preferred substrates differ with regards to degree of saturation. FAR3/CER4 preferentially reduces C24:0-C30:0 acyl-CoAs, while jojoba FAR converts C22:1 and C24:1 acyl-CoAs to primary alcohols (Rowland et al., 2006; Metz et al., 2000). Amino acid exchanges between FAR3/CER4 and jojoba FAR will reveal a.a.'s important for degree of saturation specificity (i.e. C24:0 vs. C24:1). Yeast would need to be fed C24:1 fatty acid as they do not naturally accumulate this acid, while they do make small amounts of C24:0 that can be converted to C24:0 fatty alcohol by FAR3/CER4 (Rowland et al., 2006). Wax esters with short chain length unsaturated fatty alcohols have low melting point properties, which is desirable for some of the industrial applications. Thus, engineering of highly active FAR enzymes with medium chain-length specificity and activity for monounsaturated substrates (e.g. producing C12:1, C14:1, C16:1, or C18:1 fatty alcohols) is an important biotechnological goal. Furthermore, engineering of a FAR enzymes showing high activity towards more than one chain length may be useful for some applications. In the current study, the FAR4-FAR5 chimera showed specificity to both C18:0 and C20:0 chain lengths.

Directed evolution is another approach that can be used to modify FAR enzymes for altered catalytic efficiencies, thermal stability, substrate specificities, and/or to create a novel function (Tao and Cornish, 2002). For example, a library of mutated Arabidopsis FAR5 coding sequences (the native FAR5 protein has C18:0 acyl-CoA substrate specificity) can be created and further screened for activity in converting C18:1 (monounsaturated) fatty acyl-CoA to C18:1 fatty alcohol by a yeast heterologous system. A quadruple knockout yeast strain (H1246) that is devoid of 
neutral lipid (e.g. triacylglycerol) biosynthesis could be used for selection purposes (Sandager et al., 2002). It has been found that this yeast strain has a greatly reduced growth rate in medium supplemented with fatty acids, which can be rescued by heterologous expression of triacylglycerol synthesizing enzymes (Siloto et al., 2009). This system can be used for screening active FARs by introducing them into $H 1246$ yeast expressing a wax synthase. Only those variants of FARs that are capable of converting the exogenous fatty acid (e.g. C18:1 fatty acid) to a fatty alcohol followed by incorporation into wax esters by the action of the wax synthase will rescue the yeast cells. Directed evolution of these enzymes, would provide further insights into FAR enzymology and protein domains responsible for conferring substrate saturation specificity.

\subsection{Industrial application of suberin and FARs}

There are many current and potential industrial applications of suberin. Suberin is the main component of cork, which is obtained from the bark of the cork tree (Quercus suber) (Silva et al., 2005). The impermeability, buoyancy, and elasticity of cork make it an effective bottle stopper, which finds most use in the wine industry. Cork is also an essential component of shuttlecocks and baseballs, and can be used to make bulletin boards, fishing rod handles, and thermal insulation in house materials. The cork industry is generally regarded as sustainable and environmentally friendly. The chemical composition of suberin is complex and this provides many other applications that could be commercially exploited (Kolattukudy, 2001d; Gandini et al., 2006). Suberin monomers can be utilized in 
various high-value industrial applications such as in inks, paints, coatings, drying oils, plasticizers, wetting agents, viscosity modifiers, substitutes to fats in oils, as well as precursors to produce polyurethanes, polyesters and polyamides (Gandini et al., 2006). Suberin is composed of a wide variety of oxygenated fatty acids (e.g. saturated $\omega$-hydroxy fatty acids and $\alpha, \omega$-diacids) that can also be used to synthesize speciality chemicals such as cosmetics and aromas ( $\mathrm{Li}$ and Beisson, 2009). Attempts to develop greener synthetic pathways for polymers by polymerization of suberin monomer mixtures have been made (Sousa et al., 2008). Sousa et al. (2011) reported the successful in vitro synthesis and characterization of two types of biobased polymers prepared by the polycondensation or polytransesterification of aliphatic fragments of suberin from cork and birch.

Free fatty alcohols have many industrial applications, being used for example as additives in cleaning detergents, cosmetics, pharmaceutical formulations, food products (typically acting as thickening agents), textiles, and coatings (Houston, 1984; Rowland and Domergue, 2012). Currently, fatty alcohols are mostly synthesized from petrochemical sources, but this is limited by high production costs and is environmentally unfriendly. Fatty alcohols esterified with a fatty acid, i.e. wax esters, are also valuable, as they have a variety of commercial applications such as high-performance industrial lubricants and automobile transmission fluids (Carlsson, 2006). Wax esters were extracted in the past from the spermaceti organ in the heads of sperm whales, but this led to the near extinction of sperm whales and hunting is now banned. Other natural plant sources of wax esters are jojoba seed oil and carnauba wax (Rowland and Domergue, 2012). An alternative method 
for production of commercially valuable fatty alcohols and wax esters is metabolic engineering of high-yielding industrial oilseed crops (e.g. Crambe abyssinica or Camelina sativa). This can be accomplished by expressing FARs and wax synthases of desired specificities in seeds of target oilseed crops. High levels of wax ester accumulation (up to $70 \%$ of oil weight in seeds) in transgenic Arabidopsis seeds was observed on co-expressing jojoba embryo FAR and a jojoba wax synthase along with a fatty acid elongase (KCS) from Lunaria annua (Lassner et al., 1999; Lardizabal et al., 2000). Such work is currently being pursued by an international group of plant lipid scientists (http://icon.slu.se/ICON/), and as part of this group we provided the Arabidopsis FAR cDNA sequences (e.g. FAR1, FAR4, and FAR5) and associated knowledge of the encoded protein activities. Transgenic approaches may also be feasible with an engineered microbe, such as $E$. coli, cyanobacteria, microalgae, or an oil-accumulating yeast (e.g. Yarrowia lipolytica). Many FAR enzymes from various plant and animal species with different substrate specificities according to both acyl chain length and degree of saturation have now been identified (See Chapter 1, Section 1.3). The chain length and degree of saturation of the primary alcohols significantly influence the physical properties of the wax esters. For example, shorter chain and unsaturated wax esters have considerably more industrial applications because they have a very low melting point (Rowland and Domergue, 2012). The engineering of FAR enzymes, as described above, will help in generating a diverse array of novel, high-value fatty alcohol and wax ester products. The work carried out in this thesis represents a step forward in this area of biotechnology, but 
further exploration of FAR enzyme engineering will be important to generate the enzymes of desired activities.

\subsection{Concluding remarks}

In conclusion, three fatty acyl reductases that synthesize C18:0-C22:0 primary alcohols associated with the suberin polyester have been identified. The substrate specificity of each of the FAR enzymes was elucidated by heterologous expression in yeast and by analysis of suberin in far single, double, and triple mutant plant lines. The information from this thesis work has advanced our understanding of the suberin biosynthetic pathway and provided insights into the importance of fatty alcohols in suberin structure and function. This knowledge of suberin biosynthesis and regulation will enable biotechnologists to alter suberin composition and/or load to improve tolerance of crops to abiotic and biotic stresses. Further, this study provides important information about the amino acid regions imparting FAR substrate specificities. This information can be useful in future protein engineering of FAR enzymes with desired substrate specificities, which could enable the production of tailor-made, commercially valuable fatty alcohols and wax esters in an industrial oilseed crop or transgenic microbe. 


\section{References}

Aarts, M.G., Hodge, R., Kalantidis, K., Florack, D., Wilson, Z.A., Mulligan, B.J., Stiekema, W.J., Scott, R., and Pereira, A. (1997). The Arabidopsis MALE STERILITY 2 protein shares similarity with reductases in elongation/condensation complexes. Plant Journal 12: 615-623.

Ausubel, F. M., Brent, R., Kingston, R. E., Moore, D. D., Seidman, J. G., Smith, J. A., Struhl, K., Albright, L. M., Coen, D. M. and Varki, A. (1995). Current Protocols in Molecular Biology. John Wiley \& Sons, New York.

Agrawal, V. and Kolattukudy, P. (1978a). Purification and characterization of a wound-induced $\omega$-hydroxyfatty acid:NADP oxidoreductase from potato tuber disks (Solanum tuberosum L.). Archives of Biochemistry and Biophysics 191: 452-465.

Arabidopsis Genome Initiative. (2000). Analysis of the genome sequence of the flowering plant Arabidopsis thaliana. Nature 408: 796-815.

Ariizumi, T., Hatakeyama, K., Hinata, K., Sato, S., Kato, T., et al. (2003). A novel malesterile mutant of Arabidopsis thaliana, faceless pollen-1, produces pollen with a smooth surface and an acetolysis-sensitive exine. Plant Mol. Biol. 53: 107-16.

Ariizumi, T. and Toriyama, K. (2011). Genetic Regulation of Sporopollenin Synthesis and Pollen Exine Development. Annu. Rev. Plant Biol. 62: 437-460.

Arthur, M. L. (1995). NAD-binding domains of dehydrogenases. Current Opinion in Structural Biology 5: 775-783.

Bargel, H., Koch, K., Cerman, Z. and Neinhuis, C. (2006) Structure-function relationships of the plant cuticle and cuticular waxes - a smart material? Funct Plant Biol. 33: 893-910.

Baranowski, J. D and Nagel, C. W. (1983). Properties of Alkyl Hydroxycinnamates and Effects on Pseudomonas fluorescenst. Applied and Environmental Microbiology 45: 218-222.

Baranowski, J. D., P. M. Davidson, C. W. Nagel, and Brane, A. L. (1980). Inhibition of Saccharomyces cerevisiae by naturally occurring hydroxycinnamates. J. Food Sci. 45: 592-594.

Barrena, V. G. and Wilson, Z. A. (2006). Altered tapetal PCD and pollen wall development in the Arabidopsis ms1 mutant. Journal of Experimental Botany 57: 2709-2721. 
Baxter, I, Hosmani, P. S, Rus, A., Lahner, B., Borevitz, J. O., Muthukumar, B., Mickelbart, M. V., Schreiber, L., Franke, R. B and Salt, D. E. (2009). Root suberin forms an extracellular barrier that affects water relations and mineral nutrition in Arabidopsis. PLoS Genet. 5: 1000492

Beisson, F., Li, Y., Bonaventure, G., Pollard, M., and Ohlrogg, J.B. (2007). The acyltransferase GPAT5 is required for the synthesis of suberin in the seed coat and root of Arabidopsis. Plant Cell 19: 351-368.

Beisson, F., Li-Beisson, Y. and Pollard, M. (2012). Solving the puzzles of cutin and suberin polymer biosynthesis. Current Opinion in Plant Biology 15: 329-337

Bernard, A., Domergue, F., Pascal, S., Jetter, R., Renne, C., Faure, J. D., Haslam, R. P., Napier, J. A., Lessire, R. and Joubes, J. (2012). Reconstitution of plant alkane biosynthesis in yeast demonstrates that Arabidopsis ECERIFERUM1 and ECERIFERUM3 are core components of a very-long-chain alkane synthesis complex. Plant Cell 24: 3106-3118.

Bernard, A. and Joubès, J. (2013). Arabidopsis cuticular waxes: Advances in synthesis, export and regulation. Progress in Lipid Research 52: 110-129.

Bernards, M. A. (2002). Demistifying Suberin. Can. J. Bot. 80: 227-240.

Bernards, M. A., Fleming, W. D., Llewellyn, D. B., Priefer, R., Yang, X., Sabatino, A. and Plourde, G. L. (1999). Biochemical characterization of the suberizationassociated anionic peroxidase of potato. Plant Physiology 121: 135-145.

Bernards, M. A. and Lewis, N. G. (1992). Alkyl ferulates in wound-healing potatotubers. Phytochemistry 31: 3409-3412.

Bernards, M. A. and Lewis, N. G. (1998). The macromolecular aromatic domain in suberized tissue: a changing paradigm. Phytochemistry 47: 915-933.

Bernards, M. A., Lopez, M. L., Zajeck, J. and Lewis, N. G. (1995). Hydroxycinnamic acid-derived polymers constitute the polyaromatic domain of suberin. Journal of Biological Chemistry 270: 7382-7386.

Berridge, M.V., Tan, A.S., McCoy, K.D. and Wang, R. (1996). The biochemical and cellular basis of cell proliferation assays that use tetrazolium salts. Biochemica, 4: $15-20$.

Bianchi, G., Avato, P. and Salamini, F. (1984). Surface waxes from grain, leaves and husks of maize (Zea Mays L.). Cereal Chemistry 61: 45-47.

Bligh, E. G. and Dyer, W. J. (1959). A rapid method for total lipid extraction and purification. Can. J. Biochem. Physiol. 37: 911-917. 
Bonaventure, G., Salas, J. J., Pollard, M. R., and Ohlrogge, J. B. (2003). Disruption of the $F A T B$ gene in Arabidopsis demonstrates an essential role of saturated fatty acids in plant growth. Plant Cell 15: 1020-1033.

Bonaventure, G., Beisson, F., Ohlrogge, J. and Pollard, M. (2004). Analysis of the aliphatic monomer composition of polyesters associated with Arabidopsis epidermis: occurrence of octadeca-cis-6, cis-9-diene-1,18-dioate as the major component. Plant J. 40: 920-930.

Borg-Olivier , O, and Monties, B. (1993). Lignin, suberin, phenolic acids and tyramine in the suberized, wound-induced potato periderm. Phytochemistry 32: 601606.

Bowles, B.L. and Miller, A.J. (1994). Caffeic acid activity against Clostridium botulinum spores. Journal of food science. 59: 6039-6043.

Boyes, D. C., Zayed, A. M., Ascenzi, R., McCaskill, A. J., Hoffman, N. E., Davis, K. R., Gorlach, J. (2001). Growth stage-based phenotypic analysis of Arabidopsis: a model for high throughput functional genomics in plants. Plant Cell 13: 14991510.

Brooks. J. and Shaw, B. (1978). Sporopollenin: a review of its chemistry, palaeochemistry and geochemistry. Grana 17: 91-97.

Browse, J., McCourt, P. J. and Somerville, C. R. (1986). Fatty acid composition of leaf lipids determined after combined digestion and fatty acid methyl ester formation from fresh tissue. Analytical Biochemistry 152: 141-145.

Cameron, J. N. and Carlile, M. J. (1978). Fatty acids, aldehydes and alcohols as attractants for zoospores of Phytophthora palmivora. Nature 271: 448-449.

Candler, J.W., Abrams, S.R. and Bartels, D. (1997). The effect of ABA analogs on callus viability and gene expression in Craterostigma plantagineum. Physiol. Plant. 99, 465-469.

Carlsson, A. (2006). Production of Wax Esters in Crambe, CLC Press, Newbury, UK.

Cassier, P. and Lensky, Y. (1995). Ultrastructure of the wax gland complex and secretion of beeswax in the worker honey bee Apis mellifera L. Apidologie 26: $17-26$.

Cheesborough, T. M. and Kolattukudy, P. E. (1984). Alkane biosynthesis by decarbonylation of aldehydes catalyzed by a particulate preparation from Pisum sativum. Proceedings of the National Academy of Sciences USA 81: 66136617. 
Chen, W., Yu, X. H., Zhang, K. J., Shi, S., De Oliveira, Schreiber, L., Shanklin, J., and Zhang, D. (2011). Male Sterile2 encodes a plastid-localized fatty acyl carrier protein reductase required for pollen exine development in Arabidopsis. Plant Physiol. 157: 842-853.

Cheng, J. B. and Russell, D. W. (2004a). Mammalian wax biosynthesis: I. Identification of two fatty acyl-Coenzyme A reductases with different substrate specificities and tissue distributions. The Journal of Biological Chemistry, 279: 37789-37797.

Cheng, J. B. and Russell, D. W. (2004b). Mammalian wax biosynthesis: II. Expression cloning of wax synthase cDNAs encoding a member of the acyltransferase enzyme family. The Journal of Biological. Chemistry, 279: 37798-37807.

Chong, K. P., Rossall, S. and Atong, M. (2009). In Vitro Antimicrobial Activity and Fungitoxicity of Syringic Acid, Caffeic Acid and 4-hydroxybenzoic Acid against Ganoderma Boninense. Journal of Agricultural Science 1: 15-20.

Clarke, M. R. (1978). Physical Properties of Spermaceti Oil in the Sperm Whale. Journal of the Marine Biological Association of the United Kingdom, 58: 19-26.

Clough, S. J. and Bent, A. F. (1998) Floral dip: a simplified method for Agrobacterium-mediated transformation of Arabidopsis thaliana. Plant J. 16: 735-743.

Compagnon, V., Diehl, P., Benveniste, I., Meyer, D., Schaller, H., Schreiber, L., Franke, R. and Pinot, F. (2009). CYP86B1 is required for Very Long Chain $\omega$ hydroxyacid and $\alpha, \omega$-dicarboxylic acid synthesis in root and seed suberin polyester. Plant Physiol. 150: 1831-1843.

Costaglioli, P., Joubes, J., Garcia, C., Stef, M., Arveiler, B., Lessire, R. and Garbay, B. (2005). Profiling candidate genes involved in wax biosynthesis in Arabidopsis thaliana by microarray analysis. Biochimica et Biophysica Acta (BBA) Molecular and Cell Biology of Lipids 1734: 247-258.

Cottle, W and Kolattukudy, P. E. (1982). Abscisic Acid Stimulation of Suberization. Plant Physiol. 70: 775-780.

Dean, B. B. and Kolattukudy, P. E. (1976). Synthesis of Suberin during Woundhealing in Jade Leaves, Tomato Fruit, and Bean Pods. Plant Physiol. 58, 411416.

Dean, B. B. and Kolattukudy, P. E. (1977) Biochemistry of Suberization Incorporation of [1-C-14]Oleic Acid and [1-C-14]Acetate into Aliphatic Components of Suberin in Potato-Tuber Disks (Solanum tuberosum). Plant Physiology. 59: 48-54. 
Dean, H. G., Zheng, H., Tewari J., Huang, J., Young, D. S., Hwang, Y. T., Western, T. L., Carpita, N. C., McCann, M. C., Mansfield, S. D. and Haughn, G. W. (2007). The Arabidopsis MUM2 Gene Encodes a $\beta$-Galactosidase required for the Production of Seed Coat Mucilage with Correct Hydration Properties. Plant Cell 19: 4007-4021.

Debeaujon, I., Leon-Kloosterziel, K.M., and Koornneef, M. (2000). Influence of the testa on seed dormancy, germination, and longevity in Arabidopsis. Plant Physiol. 122: 403-414.

DeBono, A., Yeats, T. H., Rose, J. K. C., Bird, D., Jetter, R., Kunst, L., and Samuels, L. (2009). Arabidopsis LTPG Is a glycosylphosphatidylinositol-anchored lipid transfer protein required for export of lipids to the plant surface. Plant Cell 21: 1230-1238.

Dixon, R. A., Chen, F., Guo, D. and Parvathi, K. (2001). The biosynthesis of monolignols: a "metabolic grid", or independent pathways to guaiacyl and syringyl units? Phytochemistry 57: 1069-1084.

Doan, T. T., Carlsson, A. S., Hamberg, M., Bulow, L., Stymne, S., and Olsson, P. (2009). Functional expression of five Arabidopsis fatty acyl-CoA reductase genes in Escherichia coli. J. Plant Physiol. 166: 787-796.

Doan, T. T., Domergue, F., Fournier, A. E., Vishwanath, S. J., Rowland, O., Moreau, P., Wood, C. C., Carlsson, A. S., Hamberg, M., and Hofvander, P. (2011). Biochemical characterization of a chloroplast localized fatty acid reductase from Arabidopsis thaliana, Biochimica et Biophysica Acta-Molecular and Cell Biology of Lipids 1821: 1244-1255.

Dobritsa, A. A., Shrestha, J., Morant, M., Pinot, F., Matsuno, M., Swanson, R., Møller, B. L., and Preuss, D. (2009). CYP704B1 Is a Long-Chain Fatty Acid v-Hydroxylase Essential for Sporopollenin Synthesis in Pollen of Arabidopsis. Plant Physiology. 151: 574-589.

Domergue, F., Abbadi, A., Ott, C., Zank, T. K., Zahringer, U., and Heinz, E. (2003). Acyl carriers used as substrates by the desaturases and elongases involved in very long-chain polyunsaturated fatty acids biosynthesis reconstituted in yeast. J. Biol. Chem. 278: 35115-35126.

Domergue, F., Vishwanath, S.J., Joubès, J., Ono, J., Lee, J., Bourdon, M., Alhattab, R., Lowe, C., Pascal, S., Lessire, R., and Rowland, 0. (2010). Three Arabidopsis Fatty Acyl-CoA Reductases, FAR1, FAR4, and FAR5, Generate Primary Fatty Alcohols Associated with Suberin Deposition. Plant Physiology 153: 1539-1554. 
Domínguez. E., Mercado, J. A., Quesada, M. A. and Heredia, A. (1999). Pollen sporopollenin: degradation and structural elucidation. Sex Plant Reproduction, 12: 171-178.

Domínguez, E., Cuartero, J. \& Heredia, A. (2011). An overview on plant cuticle biomechanics. Plant Science 181: 77-84.

Duan, H. and Schuler, M. A. (2005). Differential expression and evolution of the Arabidopsis CYP86A subfamily. Plant Physiology 137: 1067-1081.

Efetova, M., Zeier, J., Riederer, M., Lee, C., Stingl, N., Mueller, M., Hartung, W., Hedrich, R. and Deeken, R. (2007). A Central Role of Abscisic Acid in Drought Stress Protection of Agrobacterium-Induced Tumors on Arabidopsis. Plant Physiology 145: 853-862.

Eggink, G., Engel, H., Vriend, G., Terpstra, P., and Witholt, B. (1990). Rubredoxin Reductase of Pseudomonas oleovorans tructural Relationship to Other Flavoprotein Oxidoreductases Based on One NAD and Two FAD Fingerprints. Journal of Molecular Biology. 212: 135-142.

Enstone, D.E., Peterson, C.A., and Ma, F. (2003). Root endodermis and exodermis: Structure, function, and responses to the environment. J. Plant Growth Regul. 21: 335-351.

Espelie, K. E., Davis, R. W. and Kolattukudy, P. E. (1980a). Composition, ultrastructure and function of the cutin- and suberin-containing layers in the leaf, fruit peel, juice-sac and inner seed coat of grapefruit (Citrus paradisi Macfed.). Planta 149: 498-511.

Espelie, K. E., Dean, B. D. and Kolattukudy, P. E. (1979). Composition of lipid-derived polymers from different anatomical regions of several plant species. Plant Physiol. 64: 1089-1093.

Espelie, K.E., and Kolattukudy, P.E. (1979a). Composition of the aliphatic components of suberin of the endodermal fraction from the first internode of etiolated Sorghum seedlings. Plant Physiol. 63: 433-435.

Espelie, K.E., and Kolattukudy, P.E. (1979b). Composition of the aliphatic components of suberin from the bundle sheaths of Zea mays leaves. Plant Sci. Lett. 15: 225-230.

Espelie, K. E. and Kolattukudy, P. E. (1985). Purification and characterization of an abscisic acid-inducible anionic peroxidase associated with suberization in potato (Solanum tuberosum). Arch. Biochem. Biophys. 240: 539-545. 
Espelie, K.E., Sadek, N.Z., and Kolattukudy, P.E. (1980b). Composition of suberinassociated waxes from the subterranean storage organs of seven plants, parsnip, carrot, rutabaga, turnip, red beet, sweet potato and potato. Planta 148: 468-476.

Fixter, L. M., Nagi, M. N., McCormack, J. G. and Fewson, C. A. (1986). Structure, distribution and function of wax esters in Acinetobacter calcoaceticus. J. Gen. Microbiol. 132: 3147-3157.

Franke, R., Briesen, I., Wojciechowski, T., Faust, A., Yephremov, A., Nawrath, C., and Schreiber, L. (2005). Apoplastic polyesters in Arabidopsis surface tissues-A typical suberin and a particular cutin. Phytochemistry 66: 2643-2658.

Franke, R., Dombrink, I. and Schreiber, L. (2012). Suberin goes genomics: use of a short living plant to investigate a long lasting polymer. Frontiers in Plant Science 3: 1-8.

Franke, R., Hofer, R., Briesen, I., Emsermann, M., Efremova, N., Yephremov, A and Schreiber, L. (2009). The DAISY gene from Arabidopsis encodes a fatty acid elongase condensing enzyme involved in the biosynthesis of aliphatic suberin in roots and the chalaza-micropyle region of seeds. The Plant Journal 57: 8095.

Franke, R. and Schreiber, L. (2007). Suberin - a biopolyester forming apoplastic plant interfaces. Current Opinion in Plant Biology, 10: 252-259.

Freire, C. S. R., Silvestre, A. J. D. and Neto, C. P. (2007). Demonstration of long-chain $n$ alkyl caffeates and delta7-steryl glucosides in the bark of Acacia species by gas chromatography-mass spectrometry. Phytochem Anal. 18: 151-156.

Gandini, A., Neto, C.P. and Silvestre, A.J.D. (2006). Suberin: A promising renewable resource for novel macromolecular materials. Prog. Polym. Sci. 31: 878-892.

Garciarrubio, A., Legaria, J. P. and Covarrubias, A. A. (1997). Abscisic acid inhibits germination of mature Arabidopsis seeds by limiting the availability of energy and nutrients. Planta 203: 182-187.

Gavel, Y. and Von Heijne, G. (1990). A conserved cleavage-site motif in chloroplast transit peptides. FEBS Letters 261: 455-458.

Gau, J-Y, Yu, X-H and Liu, C-J (2009). A hydroxycinnamoyltransferase responsible for synthesizing suberin aromatics in Arabidopsis. Proc. Natl. Acad. Sci. USA 106: 18855-18860.

Gidda, S. K., Shockey, J. M., Rothstein, S. J., Dyer, J. M. and Mullen, R. T. (2009). Arabidopsis thaliana GPAT8 and GPAT9 are localized to the ER and possess distinct ER retrieval signals: functional divergence of the dilysine ER retrieval motif in plant cells. Plant Physiol. Biochem. 47: 867-879. 
Gietz, R.D. and Woods, R.A. (2002). Yeast Transformation by the LiAc/SS Carrier DNA/PEG Method. In: Yeast Protocol. 2nd ed. Totowa, NJ: Humana Press Inc. p. 107-120.

Gleave, A.P. (1992). A versatile binary vector system with a T-DNA organisational structure conducive to efficient integration of cloned DNA into the plant genome. Plant Mol. Biol. 20:1203-1207.

Gomord, V. and Faye, L. (1996) Signals and mechanisms involved in intracellular transport of secreted proteins in plants. Plant Physiol. Biochem. 34: 165-181.

Graça, J. (2010). Hydroxycinnamates in suberin formation. Phytochem. Rev. 9: 85-91.

Graça, J. and Lamosa, P. (2010). Linear and branched poly( $\omega$-hydroxyacid) esters in plant cutins. Journal of Agricultural and Food Chemistry. 58: 9666-9674.

Graça, J. and Pereira, H. (2000a). Suberin structure in potato periderm: glycerol, long-chain monomers, and glyceryl and feruroyl dimers. Journal of Agricultural and Food Chemistry 48: 5476-5483.

Graça, J. and Pereira, H. (2000b). Methanolysis of bark suberins: Analysis of glycerol and acid monomers. Phytochem. Anal. 11: 45-51.

Graça, J., Schreiber, L., Rodrigues, J. and Pereira, H. (2002). Glycerol and glyceryl esters of omega-hydroxyacids in cutins. Phytochemistry 61: 205-215.

Graça, J. and Santos, S. (2006). Glycerol-derived ester oligomers from cork suberin. Chem. Phys. Lipids 144: 96-107.

Graça, J. and Santos, S. (2007). Suberin: a biopolyester of plants' skin. Macromolecular Biosciences 7: 128-135.

Greer, S., Wen, M., Bird, D., Wu, X., Samuels, L., Kunst, L. and Jetter, R. (2007). The cytochrome P450 enzyme CYP96A15 is the midchain alkane hydroxylase responsible for formation of secondary alcohols and ketones in stem cuticular wax of Arabidopsis. Plant Physiol. 145: 653-667.

Gupta, N. C., Jain, K. P., Bhat, S. R. and R. Srinivasan. (2012). Upstream sequence of fatty acyl-CoA reductase (FAR6) of Arabidopsis thaliana drives woundinducible and stem-specific expression. Plant Cell Rep. 31: 839-850.

Hajdukiewicz, P., Svab, Z. and Maliga, P. (1994). The small, versatile pPZP family of Agrobacterium binary vectors for plant transformation. Plant Mol. Biol. 25: 989-994. 
Hajra, A. K. (1983). in Ether Lipids: Biochemical and Biomedical Aspects, eds. Mangold, H. K. and Paltauf, F. NY: Academic Press p. 85-106.

Hargrove J, Phillip G and Diane KH. (2004). Nutritional Significance and Metabolism of Very Long Chain Fatty Alcohols and Acids from Dietary Waxes. Experimental Biology and Medicine 229: 215-226.

Haughn, G. and Chaudhury, A. (2005). Genetic analysis of seed coat development in Arabidopsis. Trends Plant Sci. 10, 472-477.

Heckman, K. L and L. R. Pease. (2007). Gene splicing and mutagenesis by PCR-driven overlap Extension. Nature Protocols 2: 924-934.

Held, B., Wang, H., Wurtele, E. and Colbert, J. (1993). An mRNA putatively coding for an $O$-methyltransferase accumulates preferentially in maize roots and is located predominantly in the region of the endodermis. Plant Physiology 102: 1001-1008.

Hellenbrand, J., Biester, E. M., Gruber, J., Hamberg, M., and Frentzen, M. (2011). Fatty acyl-CoA reductases of birds. BMC Biochemistry 12: 64 .

Hofer, R., Briesen, I., Beck, M., Pinot, F., Schreiber, L. and Franke, R. (2008). The Arabidopsis cytochrome P450 CYP86A1 encodes a fatty acid omegahydroxylase involved in suberin monomer biosynthesis. J. Expt. Bot. 59: 23472360.

Hofvander, P., Doan, T. T. and Hamberg, M. (2011). A prokaryotic acyl-CoA reductase performing reduction of fatty acyl-CoA to fatty alcohol. FEBS Letters 585: 3538-3543.

Holloway, P. (1983). Some variations in the composition of suberin from the cork layers of higher plants. Phytochemistry 22: 495-502.

Honsho, M., Asaoku, S. and Fujiki, Y. (2010). Posttranslational regulation of fatty Acyl-CoA reductase 1, Far1, controls ether glycerophospholipid synthesis. J. Biol. Chem. 285: 8537-8542.

Hooker, T. S., Millar, A. A. and Kunst, L. (2002). Significance of the expression of the CER6 condensing enzyme for cuticular wax production in Arabidopsis. Plant Physiol. 129: 1568-80.

Hose, E., Clarkson, D. T., Steudle, E., Schreiber, L. and Hartung, W. (2001). The exodermis - a variable apoplastic barrier. Journal of Experimental Botany 52: 2245-2264. 
Houston, C. A. (1984). Marketing and economics of fatty alcohols. Journal of the American Oil Chemists' Society 61: 179-184.

Isaacson, T., Kosma, D.K., Matas, A.J., Buda, G.J., He, Y., Yu, B., Pravitasari, A., Batteas, J.D., Stark, R. E., Jenks, M. A. and Rose, J. K. (2009). Cutin deficiency in the tomato fruit cuticle consistently affects resistance to microbial infection and biomechanical properties, but not transpirational water loss. Plant J. 60: 363377.

Jenks, M. A., Joly, R. J., Peters, P. J., Rich, P. J., Axtell, J. D., and Ashworth, E. A. (1994). Chemically induced cuticle mutation affecting epidermal conductance to water vapor and disease susceptibility in Sorghum bicolor (L.) Moench. Plant Physiology 105: 1239-1245.

Jenks, M. A., Tuttle, H. A., Eigenbrode, S. D., and Feldmann, K. A. (1995). Leaf epicuticular waxes of the Eceriferum mutants in Arabidopsis. Plant Physiology 108: 369-377.

Jetter, R. and Kunst, L. (2008). Harnessing plant biomass for biofuels and biomaterials: Plant surface lipid biosynthetic pathways and their utility for metabolic engineering of waxes and hydrocarbon biofuels. The Plant Journal 54: 670-683.

Jetter, R., Kunst, L. and Samuels, A. L. (2006). Composition of plant cuticular waxes. Biology of the Plant Cuticle. Annual Review of Plant Biology 23: 145-175.

Kapust, R.B. and Waugh, D.S. (1999). Escherichia coli maltose-binding protein is uncommonly effective at promoting the solubility of polypeptides to which it is fused. Protein Sci. 8: 1668-1674.

Karimi, M., Inze, D. and Depicker, A. (2002). Gateway vectors for Agrobacterium mediated plant transformation. Trends Plant Sci. 7: 193-195.

Kavanagh, K.L., Jörnvall, H., Persson, B. and U. Oppermann. (2008). Medium- and short-chain dehydrogenase/reductase gene and protein families: the SDR superfamily: functional and structural diversity within a family of metabolic and regulatory enzymes. Cellular and Molecular Life Sciences 65: 3895-3906.

Kilian, J., Whitehead, D., Horak, J., Wanke, D., S. Weinl, O. Batistic, C. Angelo, E. Bornberg-Bauer, J. Kudla, and K. Harter. (2007). The AtGenExpress global stress expression data set: protocols, evaluation and model data analysis of UV-B light, drought and cold stress responses. Plant J. 50: 347-363.

Kolattukudy, P.E. (1970). Reduction of Fatty Acids to Alcohols by Cell-Free Preparations of Euglena gracilis. Biochemistry 9: 1095-1102. 
Kolattukudy, P. E. (1971). Enzymatic Synthesis of Fatty Alcohols in Brassica oleracea. Archives of Biochemistry and Biophysics 142: 701-709.

Kolattukudy, P.E. (1980). Biopolyester Membranes of Plants: Cutin and Suberin. Science 20: 990-1000.

Kolattukudy, P. E. (1981). Structure, biosynthesis and biodegradation of cutin and suberin. Ann. Rev. Plant Physiology 81: 539-546.

Kolattukudy, P. E. (1987). Lipid-derived defensive polymers and waxes and their role in plant-microbe interaction. In The Biochemistry of Plants, ed. PK Stumpf, EE Conn, 9: 291-314. New York: Academic.

Kolattukudy, P. E. (2001a). Plant Cuticle and Suberin. Encyclopedia of Life Sciences (c) 2001, John Wiley \& Sons, Ltd.

Kolattukudy, P. E. (2001b). Polyesters in Higher plants. Advances in Biochemical Engineering/Biotechnology, Vol. 71:1-49.

Kolattukudy, P.E. (2001c). Cutin from plants, In Biopolymers, Steinbüchel, A. and Doi, Y., Eds.; Weinhem Wiley-VCH; Vol.3a pp. 1-40.

Kolattukudy, P.E. (2001d). Suberin from plants, In Biopolymers, Steinbüchel, A. and Doi, Y., Eds.; Weinhem Wiley-VCH: Vol.3a pp. 41-73.

Kolattukudy, P. E. and Dean, B. (1974). Structure, gas chromatographic measurement, and function of suberin synthesized by potato tuber tissue slices. Plant Physiology 54: 116-121.

Kolattukudy, P. E and Espelie, K. E. (1989). Chemistry, biochemistry and function of suberin and associated waxes. In: Rowe J (ed) Natural products of woody plants, chemicals extraneous to the lignocellulosic cell wall. Springer, Berlin Heidelberg New York, p 304.

Koncz, C. and Schell, J. (1986). The promoter of the T-DNA gene 5 controls the tissue-specific expression of chimaeric gene carried by a novel type of Agrobacterium binary vector. Mol. Gen. Genet. 204: 383-396.

Kosma, D. K., Molina, l., Ohlrogge, J. B. and Pollard, M. (2012). Identification of an Arabidopsis Fatty Alcohol:Caffeoyl-Coenzyme A Acyltransferase Required for the Synthesis of Alkyl Hydroxycinnamates in Root Waxes. Plant Physiology 160: 237-248.

Krishnamurthy, P., Ranathunge, K., Franke, R., Prakash, H. S., Schreiber, L. and Mathew, M. K. (2009). The role of root apoplastic transport barriers in salt tolerance of rice (Oryza sativa L.). Planta 230: 119-134. 
Krishnamurthy, P., Ranathunge, K., Nayak, S., Schreiber, L. and M. K. Mathew. (2011). Root apoplastic barriers block $\mathrm{Na}+$ transport to shoots in rice (Oryza sativa L.). Journal of Experimental Botany 62: 4215-4228.

Kunst, L. and Samuels, A. L. (2003). Biosynthesis and secretion of plant cuticular wax. Prog. Lipid Res. 42: 51-80.

Kunst, L., Samuels, A. L. and Jetter, R. (2004). The plant cuticle: formation and structure of epidermal surfaces. In Plant lipids - Biology, utilisation and manipulation. Murphy, D. ed. Oxford, UK: Blackwell, pp. 270-302.

Kunst, L. and Samuels, L. (2009). Plant cuticles shine: advances in wax biosynthesis and export. Curr. Opin. Plant Biol. 12: 721-727.

Kurdyukov, S., Faust, A., Nawrath, C., Bar, S., Voisin, D., Efremova, N., Franke, R., Schreiber, L., Saedler, H., Metraux, J.P. and Yephremov, A. (2006a). The epidermis-specific extracellular BODYGUARD controls cuticle development and morphogenesis in Arabidopsis. Plant Cell 18: 321-339.

Kurdyukov, S., Faust, A., Trenkamp, S., Bar, S., Franke, R., Efremova, N., Tietjen, K., Schreiber, L., Saedler, H. and Yephremov, A. (2006b). Genetic and biochemical evidence for involvement of HOTHEAD in the biosynthesis of long-chain alphaomega-dicarboxylic fatty acids and formation of extracellular matrix. Planta 15: 1-15.

Kushnirov, V. V. (2000). Rapid and reliable protein extraction from yeast. Yeast 16: 857-860.

Lai, C., Kunst, L. and Jetter, R. (2007). Composition of alkyl esters in the cuticular wax on inflorescence stems of Arabidopsis thaliana cer mutants. The Plant Journal 50: 189-96.

Lardizabal, K. D., Metz, J. G., Sakamoto, T., Hutton, W.C., Pollard, M. R and Lassner, M. W. (2000). Purification of a jojoba embryo wax synthase, cloning of its cDNA, and production of high levels of wax in seeds of transgenic Arabidopsis. Plant Physiol. 122: 645-56.

Larkin, M. A., Blackshields, G., Brown, N. P., Chenna, R., McGettigan, P. A., McWilliams, H., Valentin, F., Wallace, I. M., Wilm, A., Lopez, R., Thompson, J. D., Gibson, T. J., and Higgins, D. G. (2007). ClustalW and ClustalX version 2. Bioinformatics 23: 2947-2948.

Larsson, K. E., Kjellberg, J. M., Tjellström, H. and Sandelius, A. S. (2007). LysoPC acyltransferase/PC transacylase activities in plant plasma membrane and plasma membrane-associated endoplasmic reticulum. BMC Plant Biology 7: 64 doi:10.1186/1471-2229-7-64. 
Lassance, J.M., Groot, A.T., Liénard, M.A., Antony, B., Borgwardt, C., Andersson, F., Hedenström, E., Heckel, D.G. and Löfstedt, C. (2010). Allelic variation in a fattyacyl reductase gene causes divergence in moth sex pheromones. Nature 466: 486-489.

Lassner, M.W., K. Lardizabal, and J.G. Metz. 1999. Producing wax esters in transgenic plants by expression of genes derived from jojoba. In: J. Janick (ed.), Perspectives on new crops and new uses. ASHS Press, Alexandria, pp. 220-224.

Lee, S.B., Jung, S.J., Go, Y.S., Kim, H.U., Kim, J.K., Cho, H.J., Park, O.K. and Suh, M.C (2009) Two Arabidopsis 3-ketoacyl CoA synthase genes, KCS20 and KCS2/DAISY, are functionally redundant in cuticular wax and root suberin biosynthesis, but differentially controlled by osmotic stress. Plant J. 60: 462475.

Leide, J., Hildebrandt, U., Hartung, W., Riederer, M and Vogg, G. (2012). Abscisic acid mediates the formation of a suberized stem scar tissue in tomato fruits. New Phytologist 194: 402-415.

Li, Y. and F. Beisson. (2009). The biosynthesis of cutin and suberin as an alternative source of enzymes for the production of bio-based chemicals and materials Biochimie 91: 685-691.

Li, Y., Beisson, F., Koo, A.J.K., Molina, I., Pollard, M. and Ohlrogge, J. (2007a) Identification of acyltransferases required for cutin synthesis and production of cutin with suberin-like monomers. Proc. Natl. Acad. Sci. U. S. A. 104: 1833918344.

Li, Y., Beisson, F., Ohlrogge, J. and Pollard, M. (2007b) Monoacylglycerols are components of root waxes and can be produced in the aerial cuticle by ectopic expression of a suberin associated acyltransferase. Plant Physiol. 144: 12671277.

Li, Y., Beisson, F., Pollard, M. and Ohlrogge, J. (2006). Oil content of Arabidopsis seeds: the influence of seed anatomy, light and plant-to-plant variation. Phytochemistry 67: 904-915.

Li, F., Wu, X., Lam, P., Bird, D., Zheng, H., Samuels, L., Jetter, R and Kunst, L. (2008). Identification of the Wax Ester Synthase/Acyl-Coenzyme A:Diacylglycerol Acyltransferase WSD1 Required for Stem Wax Ester Biosynthesis in Arabidopsis. Plant Physiology 148: 97-107.

Lü, S., Song, T., Kosma, D. K., Parsons, E. P., Rowland, O., and Jenks, M. A. (2009). Arabidopsis CER8 encodes LONG-CHAIN ACYL-COA SYNTHETASE 1 (LACS1) that has overlapping functions with LACS2 in plant wax and cutin synthesis. Plant Journal. 59: 553-564. 
Lulai, E. and Corsini, D. (1998). Differential deposition of suberin phenolic and aliphatic domains and their roles in resistance to infection during potato tuber (Solanum tuberosum L.) wound healing. Physiological and Molecular Plant Pathology 53: 209-222.

Lulai, E.C. and Freeman, T.P. (2001) The importance of phellogen cells and their structural characteristics in susceptibility and resistance to excoriation in immature and mature potato tuber (Solanum tuberosum L.) periderm. Ann. Bot. 88: 555-561.

Lulai, E. C., Suttle, J. C. and Pederson, S. M. (2008). Regulatory involvement of abscisic acid in potato tuber wound-healing. Journal of Experimental Botany 59: $1175-1186$.

Ma, H. (2005). Molecular genetic analyses of microsporogenesis and microgametogenesis in flowering plants. Annual Review of Plant Biology 56: 393-434.

Ma, F. S and Peterson, C. A (2003). Current Insights into the development, structure, and chemistry of the endodermis and exodermis of roots. Can. J. Bot. 81: 405421.

Marchler-Bauer, A., S. Lu, J.B. Anderson, F. Chitsaz, M.K. Derbyshire, C. DeweeseScott, J.H. Fong, L.Y. Geer, R.C. Geer, N.R. Gonzales, M. Gwadz, D.I. Hurwitz, J.D. Jackson, Z. Ke, C.J. Lanczycki, F. Lu, G.H. Marchler, M. Mullokandov, M.V. Omelchenko, C.L. Robertson, J.S. Song, N. Thanki, R.A. Yamashita, D. Zhang, N. Zhang, C. Zheng, S.H. Bryant. (2010). CDD: a Conserved Domain Database for the functional annotation of proteins. Nucleic Acids Research 39: 225-229.

Masao H, Kumi M and Sumitra H. (1987). Effect of long chain fatty acids and fatty alcohols on streptococcus mutans. Chem. Pharma. Bull. 35: 3507-3510.

Matern, U. and Kneusel, R. E. (1988). Phenolic Compounds in Plant Disease Resistance. Phytoparasitica 16: 153-170.

Matzke, K. and Reiderer, R. (1991). A comparative study into the chemical constitution of cutins and suberins from Picea abies (L.) Karst., Quercus robur L., and Fagus sylvatica L.. Planta 185: 233-245.

McDonald, M. B. (1975). A review and evaluation of seed vigor tests. Proc. Assoc. Off. Seed Anal. 65: 109-139.

Meiering, A. G., Paroschy, J. H., Peterson, R. L., Hostetter, G. and Neff, A. (1980). Mechanical freezing injury in grapevine trunks. Am. J. Enol. Vitic. 31: 81-89. 
Menezes, J.C.J.M.D.S., Kamat, S. P., Jose, A. S. C., Gaspar, A., Garrido, J. and Borges, F. (2011). Synthesis and antioxidant activity of long chain alkyl Hydroxycinnamates. European Journal of Medicinal Chemistry 46: 773-777.

Metz, J. G., Pollard, M. R, Anderson, L., Hayes, T. R. and Lassner, M. W. (2000). Purification of a jojoba embryo fatty acyl-CoenzymeA reductase and expression of its cDNA in high erucic acid rapeseed. Plant Physiol. 122: 635644.

Meyer, C. J., Peterson, C. A. and M. A. Bernards. (2011a). A comparison of suberin monomers from the multiseriate exodermis of Iris germanica during maturation under differing growth conditions. Planta 233: 773-786.

Meyer, C. J., Peterson, C. A. and M. A. Bernards. (2011b). Spatial and temporal deposition of suberin during maturation of the onion root exodermis. Botany 89: 119-131.

Meyer, C. J., Seago, J. L. and Peterson, C. A. (2009). Environmental effects on the maturation of the endodermis and multiseriate exodermis of Iris germanica roots. Ann. Bot. 103: 687-702.

Millar, A. A., Clemens, S., Zachgo, S., Giblin, E. M., Taylor, D. C. and Kunst. L. (1999). CUT1, an Arabidopsis gene required for cuticular wax biosynthesis and pollen fertility, encodes a very-long-chain fatty acid condensing enzyme. Plant Cell 11: 825-838.

Miwa, T. K. (1971). Jojoba oil wax esters and derived fatty acids and alcohols: gas chromatographic analyses. Journal of the American Oil Chemists Society, 48: 259-264.

Moire, L., Schmutz, A., Buchala, A., Yan, B., Stark, R. E. and Ryser, U. (1999). Glycerol is a suberin monomer: New experimental evidence for an old hypothesis. Plant Physiol. 119: 1137-1146.

Molina, I., Bonaventure, G., Ohlrogge, J. and Pollard, M. (2006). The lipid polyester composition of Arabidopsis thaliana and Brassica napus seeds. Phytochemistry 67: $2597-2610$.

Molina, I., Ohlrogge, J. B. and Pollard, M. (2008). Deposition and localization of lipid polyester in developing seeds of Brassica napus and Arabidopsis thaliana. The Plant Journal 53: 437-449.

Molina, I., Beisson-Li, Y., Beisson, F., Ohlrogge, J. B. and Pollard, M. (2009). Identification of an Arabidopsis feruloyl-CoA transferase required for suberin synthesis. Plant Physiol. 151: 1317-1328. 
Moore, R. P. (1962). Tetrazolium as a universally acceptable quality test of viable seed. Proc. Int. Seed Test. Assoc. 27: 795--805.

Moto, K., Yoshiga, T., Yamamoto, M., Takahashi, S., Okano, K., Ando T, Nakata, T. and Matsumoto, S. (2003). Pheromone gland-specific fatty-acyl reductase of the silkmoth, Bombyx mori. Proc. Natl. Acad. Sci. USA 100: 9156-9161.

Msanne, J., Lin, J., Stone, J. M. and T. Awada. (2011). Characterization of abiotic stress-responsive Arabidopsis thaliana RD29A and RD29B genes and evaluation of transgenes. Planta 234: 97-107.

Murashige, T. and Skoog, F. (1962). A revised medium for rapid growth and bioassays with tobacco tissue cultures. Plant Physiol. 15: 473-497.

Nagan, N. and Zoeller, R. A. (2001). Plasmalogens: biosynthesis and functions. Progress in Lipid Research. 40: 199-229.

Nallamsetty, S., Austin, B. P., Penrose, K. J. and Waugh, D. S. (2005). Gateway vectors for the production of combinatorially-tagged His6-MBP fusion proteins in the cytoplasm and periplasm of Escherichia coli, 14, Cold Spring Harbor Laboratory Press, 2005, pp. 2964-2971.

Napier, R. M., Fowke, L. C., Hawes, C., Lewis, M. and Pelham, H. R. (1992). Immunological evidence that plants use both HDEL and KDEL for targeting proteins to the endoplasmic reticulum. J. Cell Sci. 102: 261-271.

Naseer, S., Lee, Y., Lapierre, C., Franke, R., Nawrath, C. and N. Geldner. (2012). Casparian strip diffusion barrier in Arabidopsis is made of a lignin polymer without suberin. Proceedings of the National Academy of Sciences USA, 109: 10101-10106.

Nawrath, C. (2002). The biopolymers cutin and suberin. In The Arabidopsis Book (Somerville, C.R. and Meyerowitz, E.M., eds). Rockville, MD: American Society of Plant Biologists, doi: 10.1199/ tab.0021, pp. 1-14.

Nawrath, C. (2006). Unraveling the complex network of Cuticular structure and function. Curr. Opin. Plant Biol. 9: 281-287.

Negrel J, Pollet, B. and Lapierre, C. (1996). Ether-linked ferulic acid amides in natural and wound periderms of potato tuber. Phytochemistry 43: 1195-1199.

Nicholson, R. L. and Hammerschmidt, R. (1992). Phenolic compounds and their role in disease resistance. Annu. Rev. Phytopathol. 30: 369-389. 
Obayashi, T., Hayashi, S., Saeki, M., Ohta, H. and Kinoshita K. (2009). ATTED-II provides coexpressed gene networks for Arabidopsis. Nucleic Acids Res. 37: 987-991.

Ohlrogge, J. and J. Browse. (1995). Lipid Biosynthesis. Plant Cell 7: 957-970.

Ohlrogge, J.B. and Jaworski, J. G. (1997). Regulation of fatty acid synthesis. Annual Review of Plant Physiology and Plant Molecular Biology 48: 109-136.

Ohlrogge, J. B., Pollard, M. R. and Stumpf, P. K. (1978). Studies on Biosythesis of Waxes by Developing Jojoba Seed Tissue. Lipids 13: 203-210.

Ossowski, S., Fitz, J., Schwab, R., Riester, M., and Weigel, D. (2005). WMD2-Web MicroRNA Designer. http://wmd2.weigelworld.org.

Ossowski, S., Schwab, R. and Weigel, D. (2008). Gene silencing in plants using artificial microRNAs and other small RNAs. The Plant Journal 53: 674-690.

Panikashvili, D., Savaldi-Goldstein, S., Mandel, T., Yifhar, T., Franke, R. B., Hofer, R., Schreiber, L., Chory, J. and A. Aharoni. (2007). The Arabidopsis DESPERADO/AtWBC11 transporter is required for cutin and wax secretion, Plant Physiol. 145: 1345-1360.

Panikashvili, D., Shi, J. X., Bocobza, S., Franke, R. B., Schreiber, L. and Aharoni, A. (2010). The Arabidopsis DSO/ABCG11 transporter affects cutin metabolism in reproductive organs and suberin in roots. Mol. Plant 3: 563-575.

Patakas, A. (2012). Abiotic stress-induced morphological and anatomical changes in plants. P. Ahmad and M.N.V. Prasad (eds.), Abiotic Stress Responses in Plants: Metabolism, Productivity and Sustainability, DOI 10.1007/978-1-4614-0634.

Pfaffl, M. W. (2001). A new mathematical model for relative quantification in realtime RT-PCR. Nucleic Acids Research 29: 2002-2007.

Pfaffl, M. W. (2006). Relative quantification. in: Real-time PCR. Published by International University Line (Editor: T. Dorak), pg. 63-82.

Piffanelli, P., Ross, J. H. E., and Murphy, D. J. (1998). Biogenesis and function of the lipidic structures of pollen grains. Sexual Plant Reproduction 11: 65-80

Pollard, M., McKeon, T., Gupta, L. M. and Stumpf, P. K. (1979). Studies on biosynthesis of waxes by developing jojoba seed. II. The demonstration of wax biosynthesis by cell-free homogenates. Lipids 14: 651-662.

Pollard, M., Beisson, F., Li, Y and Ohlrogge, J. B. (2008). Building lipid barriers: biosynthesis of cutin and suberin. Trends in Plant Science 13: 236-246. 
Post-Beittenmiller, D. (1996). Biochemistry and molecular biology of wax production in plants. Annual Review of Plant Physiology and Plant Molecular Biology 47: 405-430.

Priestley, J. H. and E. E. North. (1922). Physiological studies in plant Anatomy. The New Phytologist 21: 8-138.

Raffaele, S., Vailleau, F., Leger, A., Joubes, J., Miersch, O., Huard, C., Elisabeth B., Mongrand, S., Domergue, F., and Roby, D. (2008). A MYB transcription factor regulates very-long-chain fatty acid biosynthesis for activation of the hypersensitive cell death response in Arabidopsis. The Plant Cell 20: 752-767.

Ranathunge, K. and Schreiber, L. (2011). Water and solute permeabilities of Arabidopsis roots in relation to the amount and composition of aliphatic suberin. Journal of Experimental Botany 62: 1961-1974.

Ranathunge, K., Schreiber, L. and Franke, R. (2011). Suberin research in the genomics era-New interest for an old polymer. Plant Science 180: 399-413.

Ranathunge, K., Thomas, R.H., Fang, X., Peterson, C.A., Gijzen, M., and Bernards, M.A. (2008). Soybean root suberin and partial resistance to root rot caused by Phytophthora sojae. Phytopathology 98: 1179-1189.

Rao, S. and Rossmann, M. (1973). Comparison of super-secondary structures in proteins. J. Mol. Biol. 76: 241-56.

Rautengarten, C., Ebert, B., Ouellet, M., Nafisi, M., Baidoo, E.E., Benke, P., Stranne, M., Mukhopadhyay, A., Keasling, J.D., Sakuragi, Y., et al. (2012). Arabidopsis deficient in cutin ferulate encodes a transferase required for feruloylation of $\omega$ hydroxy fatty acids in cutin polyester. Plant Physiol. 158: 654-665.

Reiderer, M and Muller, C. (2006). Biology of the plant cuticle. Annual Plant Reviews. Vol. 23: pp1-6.

Richardson, J. S. (1981) The anatomy and taxonomy of protein structure. Advances in Protein Chemistry 34: 167-339.

Rossmann, M. G., Moras, D. and Olsen, K. W. (1974), Chemical and biological evolution of a nucleotide-binding protein. Nature 250: 194-199.

Rowland, 0. and Domergue, F.. (2012) Plant fatty acyl reductases: Enzymes generating fatty alcohols for protective layers with potential for industrial applications. Plant Science. 193-194: 28-38. 
Rowland, 0., Lee, R., Franke, R., Schreiber, L., and Kunst, L. (2007). The CER3 wax biosynthetic gene from Arabidopsis thaliana is allelic to WAX2/YRE/FLP1. FEBS Letters 581: 3538-3544.

Rowland, O., Zheng, H., Hepworth, S.R., Lam., P., Jetter, R., and Kunst, L. (2006). CER4 encodes an alcohol-forming fatty acyl-coenzyme $A$ reductase involved in cuticular wax production in Arabidopsis. Plant Physiol. 142: 866-877.

Sabba, R.P. and Lulai, E.C. (2002) Histological analysis of the maturation of native and wound periderm in potato (Solanum tuberosum L.) tuber. Ann. Bot. 90: 110.

Saitou N. and Nei M. (1987). The neighbor-joining method: A new method for reconstructing phylogenetic trees. Molecular Biology and Evolution 4: 406-425.

Sakuradani, E., Zhao, L., Haslam, T. M. and Kunst, L. (2013). The CER22 gene required for the synthesis of cuticular wax alkanes in Arabidopsis thaliana is allelic to CER1. Planta 237: 731-738.

Samuels, L., Kunst, L., and Jetter R. (2008) Sealing plant surfaces: Cuticular wax formation by epidermal cells. Annu. Rev. Plant Biol., 59: 683-707.

Sandager, L., Gustavsson, M. H., Stahl, U., Dahlqvist, A., Wiberg, E., Banas, A., Lenman, M., Ronne, H. and Stymne, S. (2002). Storage lipid synthesis is nonessential in yeast. J. Biol. Chem. 277: 6478-6482.

Sanders, P. M., Bui, A. Q., Weterings, K., McIntire, K. N., Hsu, Y. C., Lee, P. Y., Truong, M. T., Beals, T. P., and Goldberg, R. B. (1999). Anther developmental defects in Arabidopsis thaliana male-sterile mutants. Sexual Plant Reproduction 11: 297322.

Schirmer, A., Rude, M. A., Li, X., Popova, E. and del Cardayre S. B. (2010). Microbial biosynthesis of alkanes. Science 329: 559-562.

Schmid, M., Davison, T. S., Henz, S. R., Pape, U. J., Demar, M., Vingron, M., Schölkopf, B., Weigel, D. and Lohmann, J. U. (2005). A gene expression map of Arabidopsis thaliana development. Nat. Genet. 37: 501-506.

Schmutz, A., Jenny, T. and Ryser, U. (1994). A caffeoyl-fatty acid glycerol ester from wax associated with green cotton fiber suberin. Phytochemistry 36: 13431346.

Schnurr, J., Shockey, J. M., de Boer, G.-J., and Browse, J. (2002). Fatty acid export from the chloroplast. Molecular characterization of a major plastidial acylcoenzyme A synthetase from Arabidopsis. Plant Physiology 129: 1700-1709. 
Schnurr, J., Shockey, J. and Browse, J. (2004). The acyl-CoA synthetase encoded by LACS2 is essential for normal cuticle development in Arabidopsis. Plant Cell 16: 629-642.

Schopfer, P., Bajracharya, D and C. Plachy. (1979). Control of seed germination by abscisic acid. Plant Physiol. 64: 822-827

Schreiber, L. (2010) Transport barriers made of cutin, suberin and associated waxes. Trends in Plant Science 15: 546-553.

Schreiber, L., Franke, R. and Hartmann, K. (2005a). Effects of NO3 deficiency and $\mathrm{NaCl}$ stress on suberin deposition in rhizo and hypodermal (RHCW) and endodermal cell walls (ECW) of castor bean (Ricinus communis L.) roots. Plant and Soil 269: 333-339.

Schreiber, L., Franke, R., Hartmann, K. (2005b). Wax and suberin development of native and wound periderm of potato (Solanum tuberosum L.) and its relation to peridermal transpiration. Planta 220: 520-530.

Schreiber, L., Franke, R., Hartmann, K. D., Ranathunge, K. and Steudle, E. (2005c). The chemical composition of suberin in apoplastic barriers affects radial hydraulic conductivity differently in roots of rice (Oryza sativa L. cv. IR64) and corn (Zea mays L. cv. Helix). J. Exp. Bot. 56: 1427-1436.

Schreiber, L., Franke, R. and Lessire R (2005d). Biochemical characterization of elongase activity in corn (Zea mays L.) roots. Phytochemistry 66:131-138.

Schwab, R., Ossowski, S., Riester, M., Warthmann, N., and Weigel, D. (2006). Highly specific gene silencing by artificial microRNAs in Arabidopsis. Plant Cell 18: 1121-1133.

Schwacke, R., Schneider, A., van der Graaff, E., Fischer, K., Catoni, E., Desimone, M., Frommer, W.B., Flugge, U.I. and R. Kunze, (2003). ARAMEMNON, a novel database for Arabidopsis integral membrane proteins. Plant Physiol. 131: 1626.

Scott, R. J., Spielman, M. and Dickinson, H. G. (2004). Stamen structure and function. The Plant Cell 16: 46-60.

Seo, P. J., Xiang, F., Qiao, M., Park, J. Y., Lee, Y. N., Kim, S. G., Lee, Y. H., Park, W. J. And Park, C. M. (2009). The MYB96 Transcription Factor Mediates Abscisic Acid Signaling during Drought Stress Response in Arabidopsis. Plant Physiology 151: 275-289. 
Seo, P. J. Lee, S. B., Suh, M. C., Park, M., Go, Y. S. and C. Park. (2011). The MYB96 transcription factor regulates cuticular wax biosynthesis under drought conditions in arabidopsis. The Plant Cell 23: 1138-1152.

Serra, 0., Figueras, M., Franke, R., Prat, S. and M. Molinas. (2010a). Unraveling ferulate role in suberin and periderm biology by reverse genetics. Plant Signaling \& Behavior 5: 953-958.

Serra, 0, Hohn, C, Franke, R, Prat, S, Molinas, M, Figueras, M (2010b). A feruloyl transferase involved in the biosynthesis of suberin and suberin-associated wax is required for maturation and sealing properties of potato periderm. Plant J. 62: 277-290.

Serra, 0., Soler, M., Hohn, C., Franke, R., Schreiber, L., Prat, S., Molinas, M and Figueras, M. (2009b). Silencing of StKCS6 in potato periderm leads to reduced chain lengths of suberin and wax compounds and increased peridermal transpiration. J. Expt Botany. 60: 697-707.

Serra, O., Soler, M., Hohn, C., Sauveplane, V., Pinot, F., Franke, R., Schreiber, L., Prat, S., Molinas, M. and Figueras, M (2009a). CYP86A33-Targeted Gene Silencing in Potato Tuber Alters Suberin Composition, Distorts Suberin Lamellae, and Impairs the Periderm's Water Barrier Function. Plant Physiol. 149: 1050-1060.

Shao, S., Meyer, C. J., Ma, F., Peterson, C. A. and Bernards, M. A. (2007). The outermost cuticle of soybean seeds: chemical composition and function during imbibition. Journal of Experimental Botany 58, 1071-1082.

Shi, J., H. Tan, X. H. Yu, Y. Liu, W. Liang, K. Ranathunge, R. B. Franke, L. Schreiber, Y. Wang, G. Kai, J. Shanklin, H. Ma, and Zhang, D.. (2011). Defective pollen wall is required for anther and microspore development in rice and encodes a fatty acyl carrier protein Reductase. Plant Cell 23: 2225-2246.

Sieber, P., Schorderet, M., Ryser, U., Buchala, A., Kolattukudy, P., Metraux, J.-P., and Nawrath, C. (2000). Transgenic Arabidopsis plants expressing a fungal cutinase show alterations in the structure and properties of the cuticle and post genital organ fusions. Plant Cell 12: 721-738.

Siloto, R.M.P., Truksa, M., Brownfield, D., Good, A.G., and Weselake, R.J. (2009). Directed evolution of acyl-CoA:diacylglycerol acyltransferase: Development and characterization of Brassica napus DGAT1 mutagenized libraries. Plant Physiology and Biochemistry 47: 456-461.

Silva, S., Sabino, M., Fernandes, E., Correlo, V., Boesel, L., Reis, R. (2005). Cork: properties, capabilities and applications. Int. Matr. Rev. 50: 345-365. 
Soler, M., Serra, O., Molinas, M., Huguet, G., Fluch, S. and Figueras, M. (2007). A genomic approach to suberin biosynthesis and cork differentiation. Plant Physiology 144: 419-431.

Soliday, C. L., Kolattukudy, P. E. and Davis, R. W. (1979). Chemical and ultrastructural evidence that waxes associated with the suberin polymer constitute the major diffusion barrier to water vapor in potato tuber (Solanum tuberosum L.). Planta 146: 607-614.

Soll, J. and Tien, R. (1998). Protein translocation into and across the chloroplastic envelope membranes. Plant Molecular Biology 38: 191-207

Song, Y., Ye, L. and N. Nii. (2011). Effects of soil water availability on development of suberin lamellae in the endodermis and exodermis and on cortical cell wall thickening in red bayberry (Myrica rubra Sieb. et Zucc.) tree roots. Scientia Horticulturae 129: 554-560.

Sousa, A. F., Gandini, A., Silvestre, A. J. D. and Neto, C. P. (2008). Synthesis and characterization of novel biopolyesters from suberin and model comonomers. Chem. Sus. Chem. 1: 1020-1025.

Sousa, A. F., Gandini, A., Silvestre, A. J. D., Neto, C. P., Pinto, J. J. C .C., Eckerman, C. and B. Holmbom. (2011). Novel suberin-based biopolyesters: from synthesis to properties. Journal of Polymer Science Part A: Polymer Chemistry 49: 22812291.

Souza C. de Azevedo, Kim, S. S., Koch, S., Kienow, L, Schneider K, et al. (2009). A novel fatty Acyl-CoA synthetase is required for pollen development and sporopollenin biosynthesis in Arabidopsis. Plant Cell 21: 507-525.

Sparkes, I. A., Runions, J., Kearns, A. and C. Hawes. (2006). Rapid, transient expression of fluorescent fusion proteins in tobacco plants and generation of stably transformed plants. Nature Protocols 1: 2019-2025.

Staehelin, L. A. (1997). The plant ER: a dynamic organelle composed of a large number of discrete functional domains. Plant J. 11: 1151-1165.

Stark, R. E., Sohn, W., Pacchiano, R. A., Albashir, M. and J. R. Garbow. (1994). Following suberization in potato wound periderm by histochemical and solidstate C-13 nuclear-magnetic-resonance methods, Plant Physiol. 104: 527-533.

Steudle, E. and Peterson, C. A. (1998). How does water get through roots? Journal of Experimental Botany 49: 775-788. 
Suh, M. C., Samuels, A. L., Jetter, R., Kunst, L., Pollard, M., Ohlrogge, J. and Beisson, F. (2005). Cuticular lipid composition, surface structure, and gene expression in Arabidopsis stem epidermis. Plant Physiol. 139: 1649-1665.

Swamy, P. M. and Smith, B. (1999). Role of abscisic acid in plant stress tolerance. Current Science 76: 1220-1227.

Tao, H. and Cornish, V. W. (2002). Milestones in directed enzyme evolution. Curr. Opin. Chem. Biol. 6: 858-864.

Tamura K., Peterson D., Peterson N., Stecher G., Nei M., and Kumar S. (2011). MEGA5: Molecular Evolutionary Genetics Analysis using Maximum Likelihood, Evolutionary Distance, and Maximum Parsimony Methods. Molecular Biology and Evolution 28: 2731-2739.

Teerawanichpan, P., Robertson, A. J. and Qiu, X. (2010a). A fatty acyl-CoA reductase highly expressed in the head of honey bee (Apis mellifera) involves biosynthesis of a wide range of aliphatic fatty alcohols. Insect Biochemistry and Molecular Biology. 40: 641-649.

Teerawanichpan, P. and Qiu, X. (2010b). Fatty Acyl-CoA Reductase and Wax Synthase from Euglena gracilis in the Biosynthesis of Medium-Chain Wax Esters. Lipids 45: 263-273.

Teerawanichpan, P. and Qiu, X. (2012). Molecular and functional analysis of three fatty acyl-CoA reductases with distinct substrate specificities in copepod Calanus finmarchicus, Marine Biotechnology 14: 227-236.

Thomas, R., Fang, X., Ranathunge, K., Anderson, T. R., Peterson, C. A. and Bernards, M. A. (2007). Soybean root suberin: anatomical distribution, chemical composition, and relationship to partial resistance to Phytophthora sojae. Plant Physiol. 144: 299-311.

Tillman, J. A., Seybold, S. J., Jurenka, R. A. and Blomquist, G. J. (1999). Insect pheromones-an overview of biosynthesis and endocrine regulation. Insect Biochem. Mol. Biol. 29: 481-514.

Tuteja, N. (2007). Abscisic Acid and Abiotic Stress Signaling. Plant Signaling \& Behavior 2: 135-138.

U.S. National Library of Medicine. (2009). National Center for Biotechnology Information.Available:http://www.ncbi.nlm.nih.gov/protein/AAD38039? $\log \$$ =ACCN. Last accessed: October 3, 2012. 
Vandenburg, L. E. and E. A. Wilder. (1970). The structural constituents of Carnauba wax. Journal of the American Oil Chemists Society 47: 514-518.

Vandesompele, J. Preter, k. D., Pattyn, F., Poppe B., Roy, N. V., Paepe, A. D. and F. Speleman. (2002). Accurate normalization of real-time quantitative RT-PCR data by geometric averaging of multiple internal control genes. Genome Biology 3: research 0034.1-0034.11.

Vernet, T., Dignard, D. and Thomas, D. Y. (1987). A family of yeast expression vectors containing the phage $\mathrm{f} 1$ intergenic region. Gene 52: 225-233.

Vioque, J and Kolattukudy, P. E. (1997). Resolution and purification of an aldehydegenerating and an alcohol-generating fatty acyl-CoA reductase from pea leaves (Pisum sativum L.). Archives of Biochemistry and Biophysics 340: 64-72.

Vogt, E., Schonherr, J. and Schmidt, H. (1983). Water permeability of periderm membranes isolated enzymatically from potato tubers (Solanum tuberosum L.). Planta 158: 294-301.

Voinnet, O., Rivas, S., Mestre, P and D. Baulcombe. (2003). An enhanced transient expression system in plants based on suppression of gene silencing by the p19 protein of tomato bushy stunt virus. The Plant Journal 33: 949-956.

Von Wettstein-Knowles, P. (1974). Gene mutation in barley inhibiting the production and use of $\mathrm{C} 26$ chains in epicuticular wax formation, FEBS Letters, 42: 187-191.

Von Wettstein-Knowles, P. (2012). Plant Waxes. In: eLS. John Wiley \& Sons, Ltd: Chichester. DOI: 10.1002/9780470015902.a0001919.pub2

Waeltermann, M., Stoeveken, T. and Steinbuechel, A. (2007). Key enzymes for biosynthesis of neutral lipid storage compounds in prokaryotes: Properties, function and occurrence of wax ester synthases/acyl-CoA : diacylglycerol acyltransferases. Biochimie 89: 230-242.

Wang, $X$ and Kolattukudy, P.E. (1995a). Solubilization and purification of aldehydegenerating fatty acyl-CoA reductase from green alga Botryococcus braunii. FEBS Letters. 370: 15-18.

Wang, X. and Kolattukudy, P. E. (1995b). Solubilization, purification and characterization of fatty acyl-CoA reductase from duck uropygial gland. Biochem. Biophys. Res. Commun. 208: 210-215. 
Wang, A., Xia, Q., Xie, W., Dumonceaux, T., Zou, J., Datla, R. and Selvaraj, G. (2002). Male gametophyte development in bread wheat (Triticum aestivum L.): molecular, cellular, and biochemical analyses of a sporophytic contribution to pollen wall ontogeny. The Plant Journal 30: 613-623.

Wellesen, K., Durst, F., Pinot, F., Benveniste, I., Nettesheim, K., Wisman, E., SteinerLange, S., Saedler, H., and Yephremov, A. (2001). Functional analysis of the LACERATA gene of Arabidopsis provides evidence for different roles of fatty acid omega-hydroxylation in development. Proceedings of the National Academy of Sciences of the United States of America. 98: 9694-9699.

Wesley, S. V., Helliwell, C. A., Smith, N. A., Wang, M. B., Rouse, D. T., Liu, Q., Gooding, P. S., Singh, S. P., Abbott, D., Stoutjesdijk, P. A., Robinson, S. P., Gleave, A. P., Green, A. G. and Waterhouse, P. M. (2001). Construct design for efficient, effective and high-throughput gene silencing in plants. Plant J. 27:581-590.

Western, T.L., Burn, J., Tan, W.L., Skinner, D.J., Martin-McCaffrey, L., Moffat, B.A. and Haughn, G.W. (2001). Isolation and characterization of mutants defective in seed coat mucilage secretory cell development in Arabidopsis. Plant Physiol. 127: 998-1011.

Wierenga, R. K., Terpstra, P., and Hol, W. G. J. (1986). Prediction of the occurrence of the ADP-binding $\beta \alpha \beta$-fold in proteins, using an amino acid sequence fingerprint. Journal of Molecular Biology 187: 101-107.

Xiao, F., Goodwin, S. M., Xiao, Y., Sun, Z., Baker, D., Tang, X., Jenks, M. A., and Zhou, JM. (2004). Arabidopsis CYP86A2 represses Pseudomonas syringae type III genes and is required for cuticle development. The EMBO Journal 23: 29032913.

Yamaguchi-Shinozaki, K. and Shinozaki, K. (1993). Arabidopsis DNA encoding two desiccation-responsive rd29 genes. Plant Physiol. 101: 1119-1120.

Yang, W. L. and Bernards, M. A. (2006). Wound-induced metabolism in potato (Solanum tuberosum) tubers. Plant Signaling and Behavior 1: 59-66.

Yang, W. L. and Bernards, M. A. (2007). Metabolite profiling of potato (Solanum tuberosum L.) tubers during wound-induced suberization. Metabolomics 3: 147-159.

Yeats, T. H., Laetitia, B. B., Martin, Viart, H. M. F., Isaacson, T., He, Y., Zhao, L., Matas, A. J., Buda, G. J., Domozych, S. D., Clausen, M. H. and Rose, J. K. C. (2012). The identification of cutin synthase: formation of the plant polyester cutin. Nature Chemical Biology 8: 609-611. 
Yunoki, K., R. Musa, M. Kinoshita, H. Tazaki, Y. Oda, M. Ohnishi. (2004). Presence of higher alcohols as ferulates in potato pulp and its radical-scavenging activity, Bioscience, Biotechnology, and Biochemistry 68: 2619-2622.

Zeier, J., Goll, A., Yokoyama, M., Karahara, I. and Schreiber, L. (1999a). Structure and chemical composition of endodermal and rhizodermal/hypodermal walls of several species. Plant Cell Environ. 22: 271-279.

Zeier, J., Ruel, K., Ryser, U. and Schreiber, L. (1999b). Chemical analysis and immunolocalisation of lignin and suberin in endodermal and hypodermal/rhizodermal cell walls of developing maize (Zea mays L.) primary roots. Planta 209: 1-12.

Zeier, J. and Schreiber, L. (1997). Chemical composition of hypodermal and endodermal cell walls and xylem vessels isolated from Clivia miniata: identification of the biopolymers lignin and suberin. Plant Physiol. 113: 12231231.

Zeier, J. and Schreiber, L. (1998). Comparative investigation of primary and tertiary endodermal cell walls isolated from the roots of five monocotyledoneous species: chemical composition in relation to fine structure. Planta 206: 349361.

Zhang, J. Z., Creelman, R. A. and Zhu, J. K. (2004). From laboratory to field. Using information from Arabidopsis to engineer salt, cold, and drought tolerance in crops. Plant Physiol. 135: 615-621.

Zhang, X., Henriques, R., Lin, S., Niu, Q., and Chua, N. (2006). Arabidopsis-mediated transformation of Arabidopsis thaliana using the floral dip method. Nature Protocols 1: 641-646.

Zheng H., Rowland, 0., and Kunst, L. (2005). Disruptions of the Arabidopsis enoylCoA reductase gene reveal an essential role for very-long-chain fatty acid synthesis in cell expansion during plant morphogenesis. The Plant Cell 17: 1467-1481.

Zheng, Z. F., Xia, Q., Dauk, M., Shen, W. Y., Selvaraj, G. and Zou, J. T. (2003). Arabidopsis AtGPAT1, a member of the membrane-bound glycerol-3phosphate acyltransferase gene family, is essential for tapetum differentiation and male fertility. The Plant Cell 15: 1872-1887.

Zhou, J., Lee, C., Zhong R. and Ye, Z. H. (2009). MYB58 and MYB63 are transcriptional activators of the lignin biosynthetic pathway during secondary cell wall formation in Arabidopsis. The Plant Cell 21: 248-266. 
Zimmermann, H. M., Hartmann, K., Schreiber, L. and Steudle, E. (2000). Chemical composition of apoplastic transport barriers in relation to radial hydraulic conductivity of corn roots (Zea mays L.). Planta 210: 302-311. 